\title{
TEXT-BOOK OF PHYSICS
}

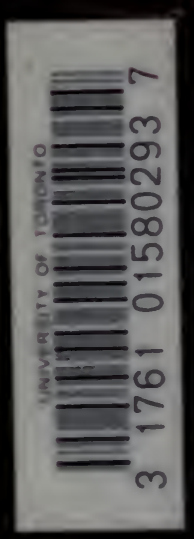

BLECTRIGITY AND MAGNETISW 


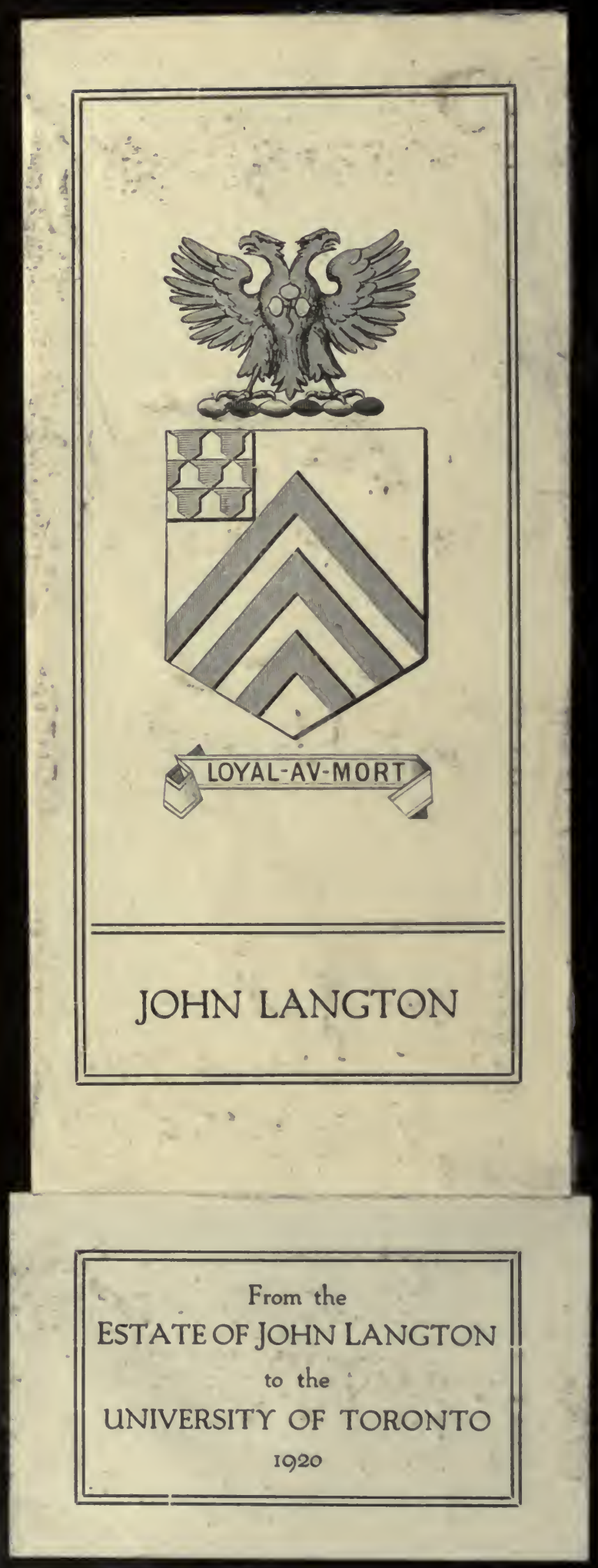


- 

Digitized by the Internet Archive in 2007 with funding from Microsoft Corporation 
A

TEXT-B00K OF PHYSICS

\section{ELECTRICITY AND MAGNETISM}




\title{
A TEXT $=$ BOOK OF PHYSICS
}

By J. H. POYNTING, Sc.D., F.R.S.

Professor of Physics, Birmingham University

And Sir J. J. THOMSON, M.A., F.R.S. Professor of Experimental Physics in the University of Cambridge.

In Five Volumes. Large 8vo. Sold Separately.

Volume I. Sixth Edition, Revised, Fully Illustrated.

\section{PROPERTIES OF MATTER}

ConTents. - Gravitation. - The Acceleration of Gravity. - Elasticity. - Stresses and Strains. -Torsion.-Bending of Kods.-Spiral Springs. -Collision.-Compressibility of Liquids.-Pressures and Volumes of Gases.-Thermal Effects Accompanying Strain.-Capillarity.-Surface Tension.-Laplace's Theory of Capillarity.-Diffusion of Liquids.-Diffusion of Gasses.Yiscosity of Liquids. I I NDX.

"We regard this book as quite indispensable not merely to teachers but to physicists of every grade above the lowest."-University Correspondent.

Volume II. Fifth Edition, Revised, Fully Illustrated.

\section{S O U N D}

Contents. - The Nature of Sound and its chief Characteristics. - The Velocity of Sound in Air and other Media,-Reflection and Refraction of Sound.-Frequency and Pitch of Notes.Resonance and Forced Oscillations.-Analysis of Vibrations. - The Transverse Vibrations of Stretched Strings or Wires.-Pipes and other Air Cavities.-Rods.-Plates.-Membranes.Vibrations maintained by Heat.-Sensitive Flames and Jets.-Musical Sand.-The Superposition of Waves. - Index.

"The work . . may be recommended to anyone desirous of possessing an EASY LP.TODate StANDARD TrEatise on Acoustics."-Literature.

Volume III. Fourth Edition, Revised, Fully Illustrated.

\section{E A T}

ContEnts. - Temperature. - Expansion of Solids. - Liquids.-Gases, - Circulation and Con. vection.-Quantity of Heat; Specific Heat.-Conductivity.-Forms of Energy; Conservation ; Mechanical Equivalent of Heat.-The Kinetic Theory.-Change of State ; Liquid, Vapour.Critical Points.-Solids and Liquids. -Atmospheric Conditions.-Radiation.-Theory of Ex. changes. - Radiation and Temperature. - Thermodynamics.-Isothermal and Adiabatic Changes. -Thermody namics of Changes of State, and Solutions. -Thermodynamics of Radiation.-Index.

"Well up-to-date, and extremely clear and exact throughout. . . . As clear as it would be possible to make such a text-book."-Nature.

Volume IV. In Three Parts. Parts I \& II, pp. i-xiv + 246. Part III at press.

\section{ELECTRICITY \& MAGNETISM}

\author{
In Preparation. Volume V \\ LI G I T
}

LONDON: CHARLES GRIFFIN \& CO. Ltd., ExeTER Street, Strand. Philadelphia : J. B. LIPPINCOTt COMPANY 


\title{
TEXT-BOOK OF PHYSICS
}

BY

J. H. POYNTING, Sc.D., F.R.S.

HON. SC.D. VICTORIA UNIVERSITY

LATE PELLOW OP TRINITY COLLEGE, CAMBRIDGE; MASON PROFESSOR

OF PHYSICS IN THE USIVERSITY OF BIRMINGIIAM

AND

SrR J. J. THOMSON, O.M., M.A., F.R.S. FELLOW OP TRINITY COLLEGE, CAMBRIDGE; CAVENDISII PROFESSOR OY EXPERIMENTAL PHYSICS IN THE UNIVERSITY OF CAMBRIDGE; PROFESSOR OF NATURAL PHILOSOPHY AT THE ROYAL INSTITUTION

\section{ELECTRICITY AND MAGNETISM}

\author{
PARTS I AND II
}

\section{STATIC ELECTRICITY AND MAGNETISM}

\author{
WITH ILLUSTRATIONS
}

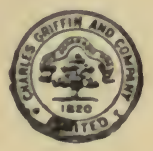

LONDON

CHARLES GRIFFIN AND COMPANY LIMITED PHILADELPHIA : J. B. LIPPINCOTT COMPANY 
I BRAR

NOV 11967 


\section{PREFA CE}

Ir was intended that the subject of Magnetism and Electricity should be a volume complete in itself, of the Series forming a 'TextBook on Physics, but it has been found necessary to divide it into two. 'The present volume contains an account of the chief phenomena of electric and magnetic systems when they are respectively charged and magnetised. 'The effects of changes in the systems are only considered statically, after the changes a:e effected, and the systems have become steady again. 'The phenomena accompanying the progress of change belong to electric current or electromagnetism and will be treated in another volume.

'The view of electric action taken by Faraday and largely developed in mathematical form by Maxwell still holds good. Faraday showed that we must regard the material medium between electrified bodies as in an altered condition. In this volume the quantity which is taken as measuring the alteration is termed "electric strain." The term is adopted in preference to "electric displacement" or "electric polarisation," in that it does not suggest or imply any special hypothesis. When the term is first found useful, it is not necessary to form a hypothesis as to the nature of the alteration, though the somewhat vague representation of it as the beginning of chemical separation, the drawing apart of two equally and oppositely charged portions of atoms or molecules, which Faraday appears to adopt, is helpful. 'The electron theory is, substantially, a much more highly developed and dynamical form of Faraday's theory. But the electron theory was suggested by the phenomena of electric discharge, and is so largely a current theory that it is more appropriately considered in the companion volume. In this volume it is only introduced occasionally in magnetism. 
The experiments are selected chiefly for their value in establishing the fundamental principles, and no attempt is made to give a complete account of experimental methods. For fuller details the reader is referred to Wiedemann's Die Lehre zon der Elektricität, Graetz's Handbuch der Elcktricität und des Magnetismus, Winkelmann's Handbuch der Physik, or the Encyclopectia Britannicu, 11th edition.

The mathematical development is only carried so far as is needed for the account of the experiments described. 'The aim is to build firmly the foundation on which the mathematical theory may be raised.

The companion volume is now at press and will be issued shortly.

J. H. P. 


\section{CON'TEN'TS}

\section{PAR'T I: S'IA'TIC ELEC'TRICITY}

\section{CHAP'IER I \\ GENERAL ACCOUNT OF COMMON PHENOMENA}

Electrification by friction-Actions between bodies both electrified by frictionTwo kinds of electrification-Law of electric mechanical action-Electric actions not magnetic-Conduction and insulation-Gold-leaf electroscopeElectrification by induction-Detection of kind of charge by the electroscope-Nature of induced electrification-Only the outer surface of a conductor is charged-The two electrifications always occur together, inducing each other-Discharge of electrification-Frictional machines-Ease of discharge from points-Lightning conductors-Electrophorus-Bennett's doubler - Belli's machine-Holiz's machine-Wimslıurst's machine-The Leyden jar -Franklin's jar-liesidual charge

Pp. 1-23

\section{CIIAP'TER II}

\section{QUANTITY OF ELECTRIFICATION}

Use of the electroscope to indicate equality of charge-The two electrifications always appear or disappear in equal amounts whether the electrification is by friction, by conduction, by induction, or by supply from currentElectrification resides only on the outside surface of a conductor unless it contains insulated charged bodies-An insulated charged conductor inside a hollow conductor induces an equal and opposite charge on the inside and an equal like charge on the outside-Imagined construction of multiples and submultiples of an arbitrary unit of charge-Imagined method of measuring any cliarge in terms of this unit

Pp. 24-30

\section{CHAP'IER III \\ PROPOSITIONS APPLYING TO "INVERSE SQUARE" SYSTEMS}

The inverse square law-The field-Unit quantity-Intensity-Force between quantities $m_{1}$ and $m_{2}$-Lines of force and tubes of force-Gauss's theoremIf a tube of force starts from a given cliarge it cither continues indefinitely 
or if it ends it ends on an equal and opposite charge-The product intensity $x$ cross-section is constant along a tube of force which contains no chargeIf a tube of force passes through a charge and $q$ is the charge within the tube, the product intensity $\times$ cross-section changes by $4 \pi q$-The intensity outside a conductor is $4 \pi \sigma$-Representation of intensity by the number of lines of force through unit area perpendicular to the lines-Number of lines starting from unit quantity-The normal component of the intensity at any surface is equal to the number of lines of force passing through unit area of the surface-Fluid displacement tubes used to prove the properties of tubes of force-Spherical shell uniformly charged-Intensity outside the shellIntensity inside the hell-Intensity at any point in the axis of a uniformly charged circular disc-Intensity due to a very long uniformly charged cylinder near the midale-Potential-The resolute of the intensity in any direction in terms of potential variation-Equipotential surfaces-The energy of a system in terms of the charges and potentials-The potential due to a uniformly charged sphere at points without and within its surface

Pp. 31-46

\section{CHAPTER IV}

\section{THE FIELD CONSIDERED WITH REGARD TO THE INDUCTION OR ELECTRIC STRAIN PRODUCED IN IT}

The two kinds of charge always present and facing cach other-Electric action in the medium-Electric strain-No strain in conductors when the charges are at rest-Direction of electric strain-The electric strain just outside a conductor is normal to the surface of the conductor-Magnitude of electric strain-Variation of electric strain with distance from the charged bodies : The inverse square law-Lines and tubes of strain-Unit tube-Results deduced from the inverse square law-The transference of tubes of strain from one charge to another when the charged bodies move-Molecular hypothesis of electric strain

I'p. 47-60

\section{CHAP'IER V}

\section{THE FORCE ON A SMALL CHARGED BODY IN THE FIELD, AND THE PULL OUT- WARDS PER UNIT AREA ON A CHARGED CONDUCTING SUREACE}

The inverse square law for the force deduced from that for electric strainCoulumb's direct measurements-General statement of the inverse square law -Electric intensity - Unit quantity-Relation between electric intensity and 
electric strain-Intensity just outside the surface of a conductor-Lines and tubes of force-Outward pull per unit area on a charged conducting surface - Note on the method of investigating the field in chapters III-V

Pp. 61-71

\section{CHAP'IER VI}

\section{ELECTRICAL LEVEL OR POTENTIAL. THE ENERGY IN ELECTRIFIED SYSTEMS}

Electrical level-Putential at a point-Equipotential surfaces-Intensity expressed in terms of rate of change of potential-General nature of level surfaces-Energy of an electrified system in terms of charges and polentials -Unit cells-Number of unit cells in a system double the number of units of energs-Distribution of energy in a system

Pp. $72-79$

\section{CHAP'TER VII}

\section{POTENTIAL AND CAPACITY IN CERTAIN ELECTRIFIED SYSTEMS. SOME METHODS OF MEASURING POTENTIAL AND CAPACITY}

Definition of capacity-Splere in the middle of large room-Two concentric splieres-Two parallel plane conducting plates-Two long co-axial cylinders - Two long thin equal and parallel cylinders - Long thin cylinder parallel to a conducting plane-Instruments to measure potential difference-Quadrant electrometer-Attracted disc electrometer-Practical methods of measuring potential-Reduction of the potential of a conductor to that of a given point in the air-Simple methods of measuring capacity-Capacity of a Leyden jar-Capacity of a gold-leaf electroscope

Pp. 80-98

\section{CHAP'TER VIII}

\section{THE DIELECTRIC. SPECIFIC INDUCTIVE CAPACITY. RESIDUAL EFFECTS}

Specific inductive capacity- Faraday's experiments - Effect of specific inductive capacity on the relations between electric quantities-Conditions to be satisfied where tubes of strain pass from one dielectric to another-Law of refraction-Capacity of a condenser with a plate of dielectric insertedThe effect of placing a dielectric sphere in a previously uniform fieldlosidual charge and discharge-Mechanical model 


\section{CHAP'TER IX}

\section{RELATION OF SPECIFIC INDUCTIVE CAPACITY TO REFRACTIVE INDEX. THE MEASURE- IMENT OF SPECIFIC INDUCTIVE CAPACITY}

The relation between specific inductive capacity and refractive index in the electro-magnetic theory of light-Determinations of specific inductive capacity-Bo'tzmann's condenser method for solids-His experiments witl crystalline sulphur-Hopkinson's experiments-Boltzman's's experiments on gases-Specific inductive capacity of water, alcohol, and other electrolstes -Experiments of Cohn and Arons, Rosa, Heerwagen, and Nernst-Experiments of Dewar and Fleming at low temperatures-Drude's experiments with electric waves

Pp. 120-133

\section{CHAPIER X}

\section{STRESSES IN THE DIELECTRIC}

Tension along the lines of strain-Pressure transverse to the lines of strain-. Value of the pressure in a simple case-This value will maintain equilibrium in any case-There may be other solutions of the problem-These stresses will not produce equilibrium if $K$ is not uniform-Quincke's experimentsGeneral expression for the force on a surface due to the electric tension and pressure-The electric stress system is not an elastic stress system and is not accompanied by ordinary elastic strains

Pp. 134-141

\section{CHAP'TER XI}

\section{ALTERATIONS OBSERVED IN THE DIELEC- TRIC WHEN IT IS SUBJECTED TO ELECTRIC STRAIN}

Electric expansion in glass-Maxwell's electric stresses do not explain the effect -Electric expansion of liquids-Electric double refraction: The Kerr effect

Pp. 142-147

\section{CHAPTER XII}

\section{PYROELECTRICITY AND PIEZOELECTRICITY}

Pyroelectricity-Historical notes-Analogous and antilogous poles-Some methods of investigating pyroelectricity-Gaugain's researches-Lord Kelvin's theory of pyroelectricity-Voigt's experiment on a broken crystal-Piezoelectricity -The discovery by the brothers Curie-The piezoelectric electrometer- 
Voigt's theory connecting pyroelectricity and piezoelectricity-Electric deformation of crystals-Lippmann's theory-Verification by the CuriesDielectric subjected to uniform pressure-Change of temperature of a pyro. electric crystal on changing the potential

Pp. 148-163

\section{PAR'T II : MAGNE'TISM}

\section{CHAPTER XIII \\ GENERAL ACCOUNT OF MAGNETIC ACTIONS}

Natural and artificial magnets-Poles-Mechanical action of poles on each other - The two poles have opposite mechanical actions-In a well-magnetised bar the poles are near the ends-The two poles alwass accompany each other in a magnet-The two poles are equal in strength-The magnetic action takes place equally well through most media-Induced magnetisation-Magnetisation can take place without contact-Retentivity and permanent magnetism -The earth is a great magnet-Magnetisation induced by the earthMethods of magnetisation-Single touch-Divided touch-Double touch -The electro-magnetic method-Electro-magnets-Ball-ended magnetsHorseshoe magnets-Compound magnets-Distribution of magnetisationConsequent poles-Magnetisation chiefly near the surface-Saturation and supersaturation-Preservation of magnetisation-Armatures-Portative force -Nickel and cobalt magnets-The magnetic field-Lines of force mapped by iron filings

Pp. 165-180

\section{CHAP'TER XIV \\ GENERAL ACCOUNT OF MAGNETIC ACTIONS-contimued}

Relation between magnetisation and the magnetising force producing it-The hysteresis loop--Susceptibility and permeability-Magnetisation and temperature-Permeability and temperature-Change of length on magnetisation -Magnetisation and strain

Pp. 181-191

\section{CHAP'TER XV}

\section{MOLECULAR HYPOTHESIS OF THE CONSTITUTION OF MAGNETS}

Molecular hypothesis-Ewing's theory and model-Dissipation of energy in a hysteresis cycle-Temperature and magnetisation-Attempts to explain the constitution of molecular magnets

Pp. 192-202 


\section{CHAPIER XVI}

\section{GENERAL ACCOUNT OF MAGNETIC QUALITIES OF SUBSTANCES OTHER THAN IRON AND STEEL}

Faraday's classification of all bodies as either paramagnetics or diamagneticsGeneral law-Explanation of the action of the medium by an extension of Archimedes' principle-Ferromagnetics, paramagnetics, and diamagnetics

Pp. 203-207

\section{CHAPTER XVII}

\section{THE INVERSE SQUARE LAW}

Variation of the force due to a pole as the distance from the pole is varied-The inverse square law-Magnetic measurements founded on the law-Coulomb's experiments-Unit magnetic pole-Strength of pole $m$-Magnetic intensity - Geometrical construction for the direction of the intensity in the field of a bar magnet-Gauss's proof of the inverse square law-Deflection method of verification-Moment of a magnet-Comparison of moments-Some conse. quences of the inverse square law-Application of Gauss's theorem-Flux of force-Unit tubes- $\Lambda$ pole $m$ sends out $4 \pi m$ unit tubes-Potential

Pp. 208-218

\section{CHAP'TER XVIII}

\section{SOME MAGNETIC FIELDS}

Small magnets-Magnetic shells-Uniform sphere

Pp. 219-228

\section{CHAP'TER XIX}

\section{INDUCED MAGNETISATION}

Magnetic induction-Induction and intensity in air-Lines and tubes of induction in air-Induction within a magnetised body-Hydraulic illustration-Equations expressing continuity of potential and continuity of induction tubesRepresentation of induced magnetisation by an imaginary distribution of magnetic poles acting according to the inverse square law-Magnetic susceptibility-Imagined investigation of $\mathrm{B}$ and $\mathrm{H}$ within iron-Permeability and the molecular theory-Induction in a sphere of uniform permeability $\mu$ placed in a field in air uniform before its introduction-Long straight wire placed in a field in air uniform before its introduction-Circular iron wire in a circular field-Energs per cubic centimetre in a magnetised body with constant permeability - The energy supplied during a cycle when the permeability 
varies-Calculation of induced magnetisation is only practicable when the permeability is constant-Diamagnetic bodies-Magnetic induction in a body when the surrounding medium has permeability differing from unity

Pp. 229-252

\section{CHAPTER XX}

\section{FORCES ON MAGNETISED BODIES. STRESSES IN THE MEDIUM}

The forces on a body of constant permeability $\mu$ placed in a magnetic field in air - Illustrations-The forces on a body of permeability $\mu_{2}$ placed in a field in a medium of permeability $\mu_{1}$-Forces on an elongated bar in a uniform field in air when its permeability differs very slightly from 1-The magnetic moment of a small paramagnetic or diamagnetic body placed in a magnetic field-Time of vibration of a small needle with $\mu-1$ very small suspended in the centre of the field between the poles of a magnet-Stresses in the medium which will account for the forces on magnetic bodies-The stresses on an element of surface separating media of permeabilities $\mu_{1}$ and $\mu_{2}$

Pp. 2:3-265

\section{CHAP'TER XXI}

\section{THE MEASUREMENT OF PERMEABILITY AND THE ALLIED QUALITIES IN FERRO- MAGNETIC BODIES}

Magnetometer method-Ballistic method-Magnetisation in very weak fieldsMagnetisation in very strong fields-Ewing's isthmus method-Nickel and cobalt-The hysteresis loop and the energy dissipated in a cycle-Mechanical model to illustrate hysteresis

Pp. 266-281

\section{CHAPTER XXII}

\section{MEASUREMENTS OF SUSCEPTIBILITY AND PERMEABILITY OF PARAMAGNETIC AND DIAMAGNETIC SUBSTANCES}

Faraday's experiments-Rowland's experiment-Experiments of v. Ettingshausen -Curie's experiments-Curie's law - Wills's experiment-Townsend's experiment-Pascal's experiments-The electron theory-The magneton 


\section{CHAPTER XXIII}

\section{TERRESTRIAL MAGNETISM}

T'he direction of the earth's lines of force at a given place-To find the declination -To find the dip or inclination-The earth inductor-The intensity of the field-To determine the horizontal intensity $\mathrm{H}$-The vibration experimentThe deflection experiment-Recording instruments-Results-Sketch of Gauss's theory of terrestrial magnetism Pp. 301-319

\section{CHAP'TER XXIV MAGNETISM AND LIGHT}

The Faraday rotation-Faraday's successors-Verdet's constant and its variation with wave lingth-Effect of rise of temperatnre-Absolute values of Verdet's constant-Rotation by gases-Rotation by films of iron, nickel, and cobaltRepresentation of the rotation by two equal circularly polarised rars with opposite rotations travelling with different velocities-An electron theory of the rutation-Confirmations of the theors-Magnetic double refraction when the light ray is perpendicular to the lines of force-Voigt's theory-Magnetic double refraction in colloids - Magnetic double refractiou in pure liquidsThe Kerr magnetic effect-I'he Zeeman effect-Lorentz's theory-Zeewan's verification 


\title{
PART I : S'TA'TIC ELEC'TRICITY
}

\author{
CHAPTER I \\ GENERAL ACCOUNT OF COMMON \\ PHENOMENA
}

\begin{abstract}
Electrification by friction-Actions between bodies both electrified by friction-Two kinds of electrification-Law of electric mechanical action -Electric actions not magnetic-Conduction and insulation-Gold-leaf electroscope-Electrification by induction-Detection of kind of charge by the electroscope-Nature of induced electrification-Only the outer surface of a conductor is charged-The two electrifications al ways occur together, inducing each other-Discharge of electrification-Frictional machines-Ease of discharge from points-Lightning conductorsElectrophorus-Bennett's doubler-Belli's machine-Holtz's machineWimshurst's machine-The Leyden jar-Franklin's jar-Residual charge.
\end{abstract}

Electrification by friction. When a stick of sealing-wax has been rubbed with any dry woollen material it is found to attract dust, small pieces of paper, bran, or other very light particles. A dry glass rod after being rubbed with silk acts in a similar way, and the property is shared by many other substances.

When showing this peculiar action the surface of the rubbed rod is said to be electrified, to have undergone electrification, or to possess electricity, this term being derived from the Greek $\eta_{\lambda} \epsilon \kappa \tau \rho \circ \nu$ -amber, a substance which was known to the ancients to possess these electrical properties.

Many very simple and interesting experiments may be made on electric actions. We may, for instance, attract egg-shells, paper rings, wood laths suspended by silk threads, \&c., by electrified rods of sealing-wax, resin, ebonite, and glass. Perhaps the most striking experiment is the electrification of dry paper. If several sheets of paper are thoroughly dried before the fire or by ironing with a hot iron, and are, while still quite hot, laid in a pile and stroked sharply with the finger-nails, they adhere very closely together. If separated, they for a short time show electrification to a very high degree, and attract small pieces of paper with great violence, adhere to the wall if brought near to it, and so on.

In making these elementary expcriments, we find that for different substances certain "rubbers" are the most eflicient. 
'Thus, resinous materials, such as sealing-wax or vulcanite, should be rubbed with wool or fur, and glass with silk.

Actions between bodies both electrified by friction. We may study electric actions between bodies in more detail by means of some such apparatus as the following, which we may term a disc electroscope. 'Two discs, $a, b$ (Fig. 1), are attached to the ends of a vulcanite or varnished glass rod, pivoted and free to turn on an upright support; $a$ is glass and $b$ is vulcanite; $c, d$ are two other discs, of glass and vulcanite respectively, provided with vulcanite handles. On rubbing $a$ and $c$ with silk, and $b$ and $d$ with wool or fur, all the surfaces are electrified. On presenting the glass disc $c$ to the glass disc $a$, we find that it repels it. We find also that the vulcanite disc $d$ repels the disc $b$, or that the similar bodies repel

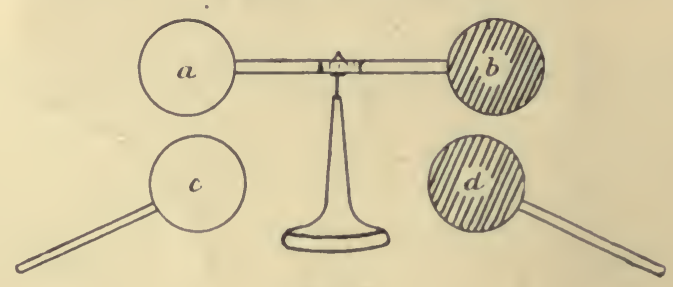

FiG. 1.

each other when electrified. On the other hand, $c$ attracts $b$, and $d$ attracts $a$.

Two kinds of electrification: vitreous or positive, resinous or negative. We have, then, two distinct kinds of electrification-that of the rubbed glass and that of the rubbed vulcanite. Further experiments show that there are only these two kinds, and they are often termed, from their simplest modes of production, vitreous and resinous respectively. But it is to be observed that when acting on a given electritied body they exert opposite forces, which tend to neutralise each other. Thus the detached discs $c$ and $d$ may be so electrified that if held close together in front of $a$ or of $b$ they will produce no motion, their two actions being equal and opposite.

Hence, in regard to their mechanical actions, we may apply to the two electrifications the terms positive and negative. Vitreous or glass electrification is always taken as positive, and therefore resinous electrification is negative.

We may now make the following statement:

Law of electric mechanical action. Bodies if similarly electrified repel each other, and if oppositely electrified attract each other.

Two bodies rubbed together are oppositely electrified. 'This may easily be proved by means of the suspended glass and rulcanite discs (Fig. 1), electrifying these by 
their appropriate rubbers. Each rubber, if very dry and held at the end of a vulcanite handle, will be found to repel the disc rubbed by the other, while it attracts its own disc. Or if two other discs which electrify each other be rubbed together and presented to the glass disc, one attracts and the other repels it.

It must not be supposed that a given substance is always electrified in the same way. The nature of its electrification depends on its physical condition and upon the rubber used. It is found that substances can be arranged approximately in order, thus

Cat's fur,
Glass,
Wool,
Feathers,
Wood,
Paper,
Ebonite,
Silk,
- Shellac.

So that if any one of these substances is rubbed by one higher in the list it is negatively electrified, while if rubbed by one lower in the list it is positively electrified. 'The order in such a list must not be taken as quite fixed, since change in physical condition may alter the position of a substance in the list. A polished surface, for instance, appears to increase the tendency to positive electrification, so that while polished glass is near the head of the list, roughened glass may be low down.

Electric actions not magnetic. We may at once distinguish electric from magnetic action by the fact that iron, nickel, and cobalt are, with all the other metals, absent from the above list.

Conduction and insulation. At one time it was supposed that the metals and other substances not showing electric attraction after friction were non-electric, differing fundamentally from substances such as shellac and glass, which were alone regarded as being capable of electrification. But afterwards it was shown that the real distinction is in the degree to which a substance will allow electrification to spread over or through it. Shellac and dry glass keep the electrification for a longer or shorter time on the surface where it is first developed, and are therefore termed insulators. Metals allow it to spread over their whole surfaces or to be communicated to other bodies which they touch, and are therefore termed conductors. If a metal rod is held by an insulating handle it may be electrified easily by friction with fur, but if it is touched by the hand, a conductor, it instantly loses its electrification.

'Ihere is no doubt a fundamental difference between conductors and perfect insulators. But we term many bodies insulators which are really very slow conductors, and we may have every intervening 
stage between moderate insulation, such as that of wool or that of baked wood, and good conduction, such as that of the metals. Sometimes a body may be in itself an insulator, but may have a tendency to condense water from the air on its surface, and the film of water will form a conductor. 'This explains the difficulty experienced in keeping electrification on glass or the still greater difficulty in keeping it on paper. Sometimes the surface of the insulator decomposes. 'This often happens with vulcanite and glass, and the products of decomposition form a conducting layer. The insulation of glass may be improved by immersion for a short time in boiling water. Dust may also prevent insulation by forming a continuous coating of conducting matter on the surface of a body.

The hygroseopic tendency of glass greatly impairs its efficiency as an insulator except in a very dry atmosphere. It is, therefore, necessary in electrical experiments with glass either to replace the

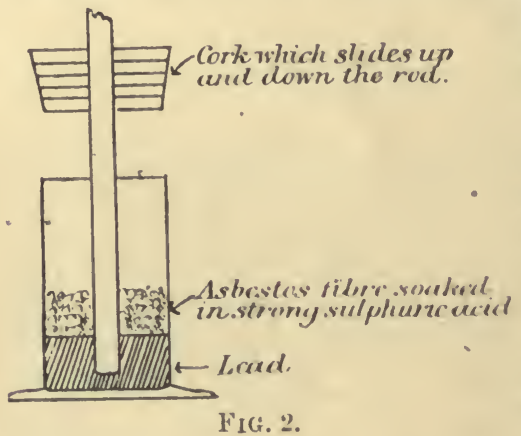
glass surface by another less hygroscopic or to take special precautions to keep the surface dry. Often the glass is covered with shellac varnish or with sulphur. A very simple plan to preserve the insulating property of a vertical glass rod is to allow it to end in a lead foot fitting tightly at the bottom of a narrow glass jar (Fig. 2). Above the lead is placed some asbestos fibre moistened with strong sulphuric acid. Sliding on the rod is a cork which may be brought down to close the jar when not in use. 'The sulphuric acid dries the air of the jar thoroughly, and the glass is therefore also dry. When in use the cork is slightly raised so that there is no connection between the upper and possibly damp part of the rod and the jar except through the rod itself, which is insulating.

Gold-leaf electroscope. 'The two electrified discs $a, b$ (Fig. 1) will serve as indicators of charge on another body if that charge is considerable. For a charged body will repel one or other of the discs. But a much more delicate test of the existence of electrification is given by the grold-leaf electroscope, which in its original form consists essentially of two strips of gold leaf hanging from the lower end of an insulated metal rod in a glass case, the upper end of the rod projecting above the case. Fig. 3 is a common type of instrument. $g, g$ are two long, narrow strips of gold leaf hanging from the end of the brass rod $r$, which is fixed in an insulating bar of vulcanite $r$ stretching across the case. 'The rod passes freely through a large hole in the top of the case, 
which can be closed by the cork $c$ when the instrument is not in use, in order to prevent the entrance of dust. $\quad p, p$ are two brass side plates which can be arranged at any distance on each side of the gold leaves, the sensitiveness of the instrument being greater the nearer the plates are to the leares. If the wood of the case is non-conducting the side plates should be connected by wire to earth. The reason for this will be seen when we have considered the phenomena of induction. 'The top of the rod may conveniently

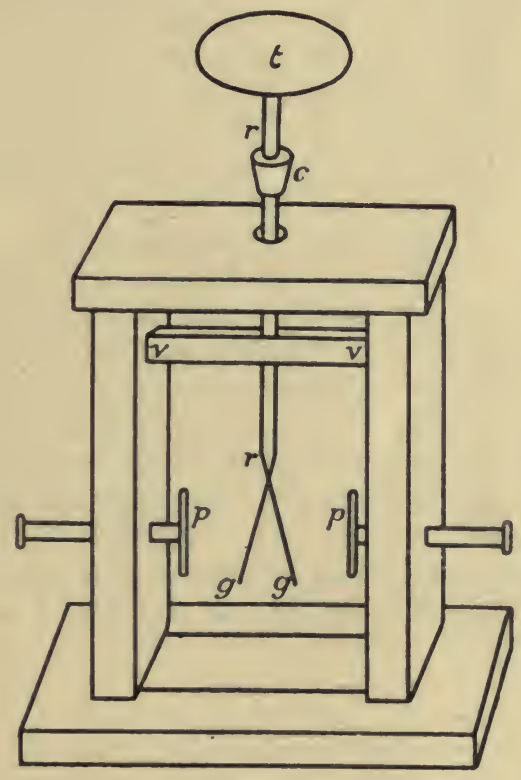

FIG. 3.

end in a metal table $t$. If this is touched by an electrified borly some of the electrification is communicated through it to the rod and leaves, and the leaves, being similarly electrified, repel each other and stand out as in the figure.

An important improvement in the construction of the gold-leaf electroscope which is now usual consists in prolonging the central rod by a thin metal strip, and having only one gold leaf which is attached to the strip near its upper end. The fixed vertical strip then takes the place of the second gold leaf. On electrification the gold leaf stands out from the strip. Fig. 4 represents a form of the instrument suitable for lantern projection. It is convenient to have a glass scale engraved on one of the glass sides.

Electrification by induction. We have described above the charging of the electroscope by contact with an electrified body. But contact is not necessary. On bringing an electrificd 
body near the electroscope the leaves diverge, or the single leaf stands out from the strip, showing that they have undergone electrification. The electrification thus produced at a distance without contact is said to be induced.

Detection of kind of charge by the gold-leaf electroscope. If the electroscope be slightly charged, say by rubbing the table with flannel or fur, it will indicate the

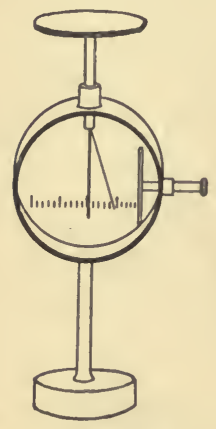

FIf. 4. kind of charge on any body brought near to the table. If, for example, an excited ebonite rod held over the table makes the leaf or leaves stand out further, an excited glass rod makes them fall nearer together, and vice versa. We shall see later how these actions may be explained. Here we take them as the results of direct experiment and shall use them to test the nature of induced electrification.

Nature of induced electrification. It may be shown easily that whenever an electrified body is brought near an insulated conductor it induces electrification of the kind opposite to its own in the nearer part, and of the kind similar to its own in the further part of the insulated body, as in Fig. 5, in which A is a body on an insulating support, and is electrified, say, positively, while B is a conductor standing on an insulating support. We may verify this by means of a proof plane (Fig. 6), which is merely a small metal dise at the end of an insulating rod.
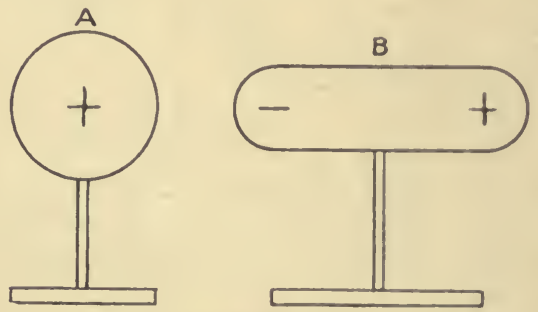

FIG. 5.

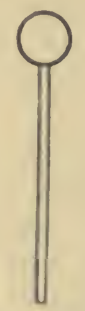

FIG. 6.

When the metal disc is laid on an electrified surface it virtually forms, if small enough, part of the surface, and is electrified in the same way as the surface round it. When lifted from the surface it preserves its electrification, and we may directly test the kind by means of the gold-leaf electroscope.

Testing the electrification at each end of B (Fig. 5), we find that on the end nearer $A$ to be negative, and that on the end farther from it to be positive. If we now withdraw A, B returns to its ordinary state, the ends showing no electrification. 'Ihus positive and negative have come together and have neutralised each other. 
'This gives us an additional reason for ascribing the algebraic signs + and - to the two kinds of electrification. Not only do they show opposite mechanical actions, but when they come together in proper proportion the resultant effect is non-electrification.

We may describe induction, perhaps, more easily by materialising our conception of electrification, regarding it as something which we call electricity, in addition to and possessed by the matter, rather than of as a condition of the matter. A neutral body we may regard as possessing amounts of the two electrifications practically unlimited and so mixed together as to neutralise each other. Each electricity must be endowed with an action of repulsion on its own kind and of attraction on the opposite kind. 'This, we must note, is not a repetition of the statement on p. 2 of the mechanical action between electrified bodies. One gives the nature of the force between portions of electrified matter tending to move matter' the other gives the actions between electrifications tending to move electricity even though the matter is kept at rest.

Using this new conception, we may say that the + electricity on A (Fig. 5) decomposes the neutral mixture on $\mathbf{B}$, drawing the opposite - nearer to itself and repelling the like + , which we may term complementary, away to the other end.

This mode of description is merely provisional. We shall see later that we must suppose the medium round the electrified bodies to take part in the phenomena, and when we try to assign to the medium its share in the action the above conception of electrification ceases to be adequate. But if we use it, not as a hypothesis but rather as an illustration to aid us for a time in arranging the ascertained facts of electric induction, it will be at least quite harmless.

If we touch $\mathrm{B}$ with the finger or with any other conductor, so breaking down its insulation, $B$ becomes part of the general conducting system, consisting of the table, floor, walls, and so on, and then either the positive electricity can get further away from A and so leaves B, or A draws up towards itself more negative from the surrounding conductors on to B. Whichever process takes place the effect is the same, viz. B loses all its positive charge and is only negatively electrified. If now we break the communication with the earth, $B$ retains this electricity even if $A$ is removed; on the removal of $\mathbf{A}$ the electricity on $\mathbf{B}$ is redistributed, and negative will be found on either end of it.

We may now explain the action of an electrified body in the fundamental experiment of the attraction of light bodies, such as bits of paper or bran. The electrified body acts by induction on these light bndies, electrifying their nearer surfaces with the kind opposite to its own, the complementary charge of the same kind going either to the further side or passing away to the table or whatever the particles rest upon. The bodies thus oppositely electrified attract each other and the particles fly to the electrified 
surface. Frequently they only adhere for a moment and are then repelled. This is especially noticeable with pith balls suspended by insulating silk threads. They rush to the electrified surface and then rush away, standing out as in Fig. 7. 'The explanation of this repulsion is that on touching the electrified surface,

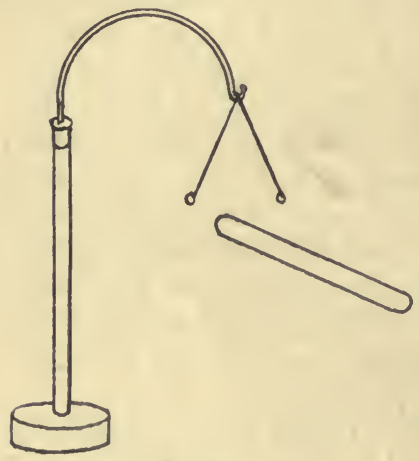

FIG. 7. they yield up their opposite electrification, being slight conductors, and gather from the surface some of its own electrification; being now electrified in the same sense as the surface, they are repelled from it.

The gold-leaf electroscope may be used to illustrate some of the phenomena of induction. Let us use the form with two leaves. If a negatively electrified rod, say of vulcanite, be held above the table of the electroscope as in Fig. 8, the table is electrified positively and the leaves negatively. The negative in the leaves induces positive in the side plates, their negative going off through the conducting communication to the table or floor, or, let us say generally, to the earth symbolised by the plate E. To be sure that we get rid of this negative charge it is advisable to connect the side plates to the gas- or water-pipes by wires.

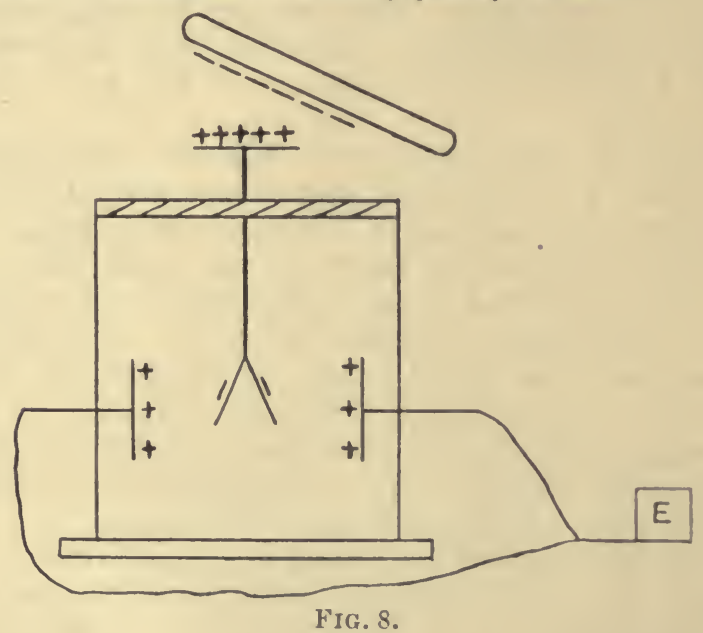

The gold leaves stand out from each other. We described this before as due to repulsion of the like electrifications ; but we may now replace this provisional description by saying that each leaf is pulled by the plate near it which is oppositely e'ectrified, and there is still an action of this kind, even if, as in 
many electroscopes, there are no special side plates. In that case the surface of the glass may be dusty or damp and so become oppositely electrified. If the glass is perfectly insulating the opposite electrification is induced in the nearest conducting surfaces and the leaves are still pulled out by the attractions of those surfaces rather than pushed apart by mutual repulsion. But the nearer the oppositely electrified surfaces the stronger its outward pull. Hence the advantage of having adjustable side plates, which may be brought very near the leaves when the charge is small. If while the electrified rod is over the table of the electroscope we either touch the table or allow the leaves to diverge so far that they touch the side plates, the negative from the leaves either passes away to "the earth," i.e. to the surrounding conductors, or is neutralised by a further supply of positive brought up from the earth, and, ceasing to be electrified, the leaves fall together. On breaking the earth communication the positive on the table above remains, and on removing the vulcanite this positive spreads over the rod and leaves, and induces negative in the side plates. 'These again attract the leaves, which are once more drawn apart and remain apart as long as the insulation is preserved.

We can now see how an electroscope, left charged with positive in this way, may be used as a detector of the kind of charge possessed by another body. If a positively electrified body is brought near its table, then the table is negatively electrified by induction and positive is sent down into the already positively electrified leaves, and they are forced still further apart. If a negatively electrified body is brought near the table, it is positively electrified and either this positive is drawn up to it from the leaves or negative is sent down to the leaves, and in either case they are less strongly positively electrified and fall together somewhat. If the body is strongly charged with negative and is brought nearer, a point may be reached when the positive in the leaves is just neutralised and they come together. If the body approaches still nearer than this point, then there is a balance of negative in the leaves over and above that neutralising the positive previously there, and the leaves diverge once more. It will easily be seen how the indications are modified if the initial charge of the electroscope is negative.

Only the outer surface of a conductor is electrified unless there are insulated charged bodies within it. 'This may be proved as follows: A narrow, deep, tin can, is placed on a block of paraffin to serve as insulator and is charged with electricity either by contact or induction. If we place a proof plane in contact with the inside of the can and then remove the plane to an electroscope no charge is detected, however strongly the outside may be charged. In fact, the can acts as a screen protecting the inside space from outside electrification, and the more nearly it is closed the more complete is the protection. 'The tendency of electrification to the 
outside of a conductor may be shown in another way. Placing the can on the paraffin, uncharged, we may introduce within it a charged conductor, such as a proof plane or a metal ball with an insulating handle. On lowering the charged body well within the can and allowing it to touch the inside of the can it becomes part of the inside surface. Its electrification at once leaves it, and on taking it out of the can it is found to be entirely discharged. By aid of the proof plane we can show that there is no electrification on the inside of the can. But before contact the inside of the can was electrified by induction, the state of affairs being represented by Fig. 9 ; the + on the insulated body within the can induces - on the inner surface and the complementary + is drawn to the outside. But we may still say that the can acts as a complete screen between the inside and the outside. For the + charge on the outside may be

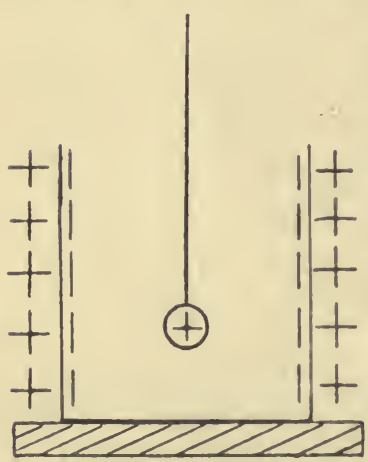

FjG. 9. shown to remain in the same position wherever the + body is placed within the conductor so long as it is sone distance below the top.

We may, for example, conduct the experiment with the can on the table of a gold-leaf electroscope so that the charge on the outside extends to the leares. 'Their divergence remains the same, however the body within the can may be moved about. When it is well below the top, the + on the outside is really related now to a - charge which it induces on the surface of the nearest conductors, the table, wall, \&c.

The two electrifications always occur together, inducing each other; or electrification is always inductive. The analysis of these simple cases of induction prepares us for the general statement that all electrification is accompanied by induction, i.e. that whenever we have one kind of electrification we have somewhere, facing it, the other kind, and the bodies whose surfaces are subjected to these related charges are being pulled towards each other. In the next chapter we shall describe experiments which verify this, and shall show also that the two related electrifications are always equal in quantity, so that if any conducting connection be made between the oppositely electrificd surfaces the electrifications coming together exactly neutralise each other. 'Thus in the experiment represented in Fig. 9, the + on the inside body is equal to the - on the inside of the can. 'The + on the outside of the can induces equal - on the inside of the walls of the room. If the body touches the can the first + and - come together and only the outside + is left inducing the - on the walls. Now, connecting the walls to the can by a wire or by the body, 
these two charges come together and also neutralise each other and no electrification of either kind remains.

When electrification is produced by friction the two electrifications are at first one on the rubber and the other on the rubbed bodies. When the two bodies are widely separated they may no longer act only on each other, but on the walls. Thus a charged ebonite rod which after being rubbed with wool is held up in the middle of a room has its opposite + on the arm of the holder, on the table, or on the walls. If two rods both negatively electrified are held up near each other they appear to repel each other, but our further study will show that we ought rather to regard them as drawn apart by the opposite walls, which are positively electrified by induction.

Discharge of electrification. When an insulated electrified body is touched by the finger or by a conductor connected with the ground the electrification passes away and the body is said to be discharged. We have described this discharge as if the charge merely went to the earth. We can now see more of the true nature of the process. 'The electrification of the body, when insulated, is accompanied by opposite electrification of the walls, floor, and surrouncling conductors, and we may regard the two charges as tending to come together, but unable to pass through the separating insulating medium. When a conducting bridge is made the two charges spread along or through it and unite to neutralise each other. Frequently they are able to break down the insulation of the medium if the electrification is considerable, and a spark occurs. In all cases of electric discharge the energy of the electrified system is dissipated in the discharge, either as heat in the conductors, or as light, heat, and sound, and perhaps chemical energy in the spark.

Methods of producing electrification in large quantity. Frictional machines. Many different forms of machine have been devised for producing electrification continuously by friction, and for a long time they were in common use. As they are now superseded for most purposes by induction machines, it will suffice if we describe one form-Winter's plate machine. A full account of the mode of constructing and using this and other frictional machines will be found in Harris's Frictional Electricity.

In Fig. $10 p$ is a circular glass plate mounted on a horizontal axis, which can be turned by the handle $h$ in the direction of the arrow-head. 'The plate passes at the lowest point betwcen two pads of silk or leather, stuffed with wool, and these serve as rubbers. 'Their power of exciting electrification is greatly increased if an amalgam, usually of mercury, zinc, and tin, is mixed with lard and smeared over the surfaces. The pads are backed with wond and connected to earth, either through the woodwork of the machine or, if this is not sufficient, directly by a wire to the 
gas or water pipes. At the level of the horizontal radius the plate passes between two metal combs $c$ - the collectors. These combs have sharp teeth pointing to the plate and separated from it by a small interval to allow of free running. 'Two silk flaps $s$ attached by their lower left-hand edges to the rubbers extend nearly to the combs and are held so as loosely to cover the section of the plate between rubber and collector. 'The combs are comnected to

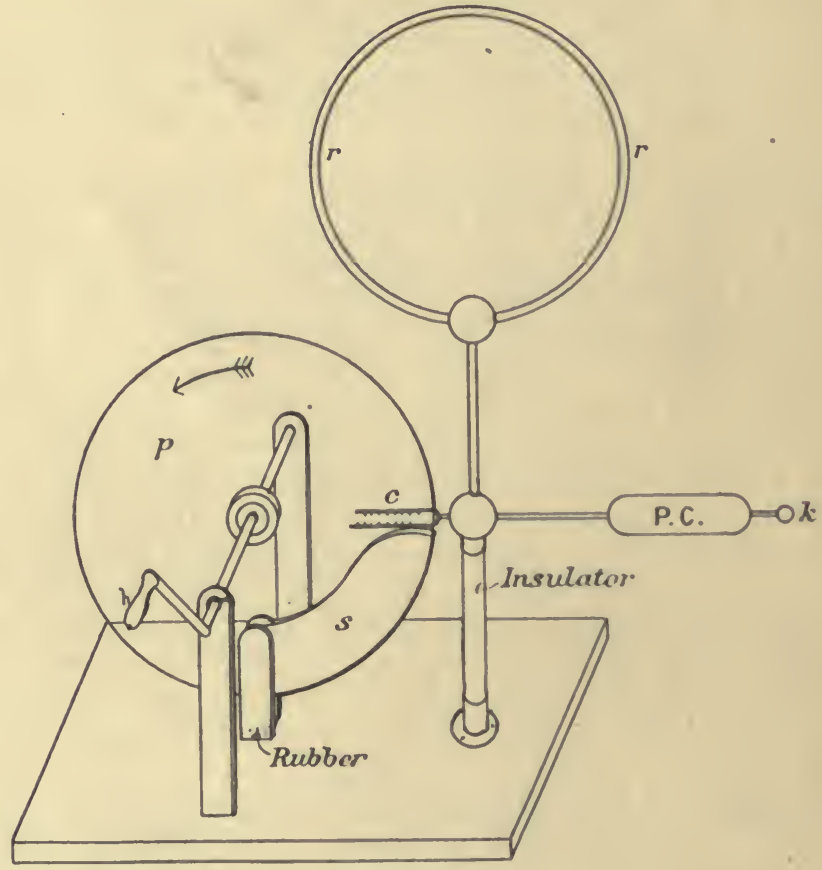

Fig. 10.

a metal body PC, called the prime conductor, which is supported by an insulating pillar and is terminated by a small knob $k$. $r r$ represents a wooden ring with a metal wire as core. 'This ring was introduced by Winter and is named after him Winter's ring.

If the plate is turned the glass is electrified positively and the rubbers negatively. The + on the glass surface is carried round, the silk flaps appearing to protect it in some way till it comes under the combs. There it acts inductively, and draws - into the points while the complementary + goes into the rest of the prime conductor. Now electrification is discharged through the air from points with great ease, so that the - passes from the points on to the glass plate, which is thus neutralised, leaving the prime conductor charged with + . Sparks may then be drawn from the knob $k$, their length and brilliancy increasing rapidly with the size 
of the plate, the excellence of its surface and of the surface of the rubbers. 'The advantage of the particular structure chosen for the ring is not very clear. It certainly makes the sparks brighter, and this is probably explained by the fact that it increases the capacity of the prime conductor so that more electricity is stored in it before a discharge occurs, and more, therefore, passes in the discharge.

The ease of discharge from points. If a very sharp metal point is placed on the prime conductor it is found that discharge takes place from it continuously, the air or clust in the air acting to convey the charge to the surrounding and oppositely charged conductors. We need not here discuss the mode of discharge. It is sufficient at this stage to say that the electrification on any conductor tends to accumulate at any projecting point on the conductor and-to a greater amount per unit area the sharper the point. It appears then that even with comparatively weak charges the crowding of the electrification on to a point may be so great that the air is unable to insulate, and discharge occurs through it. 'The point appear's in the dark to glow. If the hand be held over the point the impact of the electrified air streaning from it is distinctly felt. A sort of Barker's mill may easily be made to work by means of this point discharge. A light " whirl "represented in ligg. 11, consisting of a number of spokes with ends pointed and bent tangentially, is piroted on a vertical metal support on the prime conductor of a machine. When the machine is turned the whirl rotates with the points directed backwards. We may explain the motion thus: Were the points covered with some efficient insulator the whirl would be in equilibrium, the pull on the strongly electrified points being neutralised by the pulls over the more weakly electrified remainder of the surface. But is the points are not insulated the charge is constantly streaming off them and so the pull on then is diminished. 'The pull

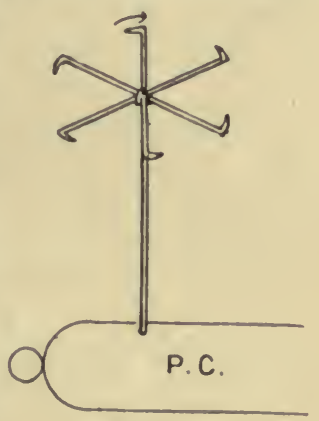

FIG. 11. on the rest of the surface is therefore not fully compensated and the whirl rotates. Or, without considering the arrangement of forces, it is enough to say that forward momentum is generated in the air streaming from the point, and that the equal backward momentum is manifested in the retrcat of the point.

Lightning conductors. 'The efficiency of lightning conductors depends on the case of discharge from points. A flash of lightning is merely a spark discharge on a grand scale between oppositely electrified clouds or between a cloud and the earth. suppose that a cloud is highly charged. 'The under' surface induces opposite elestrification on the ground underneath it, and if the 
mutually inducing charges rise above a certain degree a flash or flashes may ensue between the clouds and conducting projections from the earth, such as buildings, trees, or conducting rocks. But if there be a lightning conductor with a sharp point directed upwards and well connected below with the earth, the earth charge may pass off continuously by the point towards the cloud and may neutralise the charge there, or at any rate prevent it from rising to sparking intensity. We may illustrate this action of a lightning conductor by placing a sharply pointed earth-connected metal rod near the prime conductor of a machine. If the machine is now turned the discharge from the point to the prime conductor prevents the gathering of a charge in the latter, and this may be shown by the fact that a gold-leaf electroscope placed near the prime conductor is nearly unaffected. Here the prime conductor may be taken to represent a cloud and the sharp point a lightning conductor. If the point is now covered by a round metal knob the continuous discharge from it ceases and the charge on the prime conductor gathers till there is a spark from it to the knob. 'The electroscope indicates the gathering of the charge and its dispersal in the spark.*

Induction machines. The electrophorus. The clectrophorus is exceedingly useful for the production of a series of small charges. It consists of a flat cake (RR, Fig. 12) of some resinous material, say of vulcanite, resting on a metal plate SS, called the

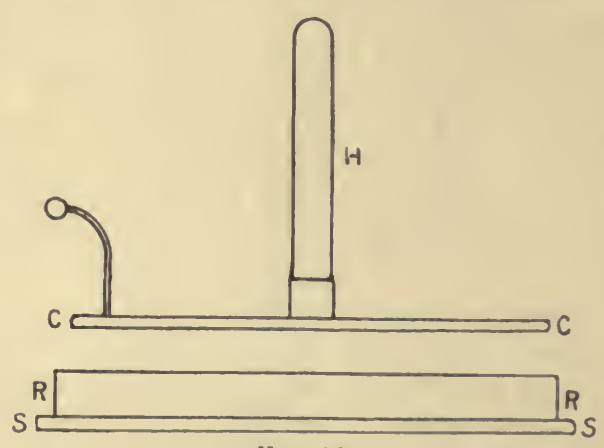

Fig. 12.

sole plate, and a movable metal cover $\mathrm{CC}$, furnished with an insulating handle $\mathrm{H}$. 'The surface of the ebonite is first electrified by friction, say by beating with cat-skin. The negative charge thus developed induces a positive charge on the upper surface of the sole, which, lying on the table, is earth-connected. 'The cover is now placed on the ebonite. Since the two surfaces are not perfect planes they are not absolutely in contact except at

* For an account of the phenomena of lightning discharges and the use of lightning conductors, see Sir Oliver Lorlge's Lightning C'onductor's and Lightning
ciucurds. 
a few points. 'The charged ebonite therefore acts inductively across the intervening layer of air on the cover, and some positive is induced on its under surface and the complementary negative on its upper surface. 'The cover is now touched with the finger and so earth-connected. 'The positive charge on the sole can now get very much nearer to the upper surface of the ebonite and we may regard it as passing round through the earth and the body of the operator to the cover, which receives nearly all the charge previously in the sole. 'The negative charge in the cover we may regard either as neutralised by the addition of positive or as passing away through the operator. Now removing the finger, the cover is insulated, and on lifting it by the insulating handle it contains a positive charge which is quite large enough to be useful for many purposes. The charge on the ebonite still remains practically undiminished and at once induces another charge on the sole. By discharging the cover and replacing it on the ebonite the process may be repeated, the original charge of the ebonite sufficing to give a great number of charges to the cover. Though the original charge is thus the exciter of all the succeeding charges, it must not be supposed that it is also the source of their energy. 'Ihe cover' when placed on the ebonite and charged oppositely is attracted by it, and in lifting the cover up again more work must be done than that required merely to raise its weight, and it is this excess of work which gives to the charge on the cover the energy it possesses. 'The operator therefore supplies the energy of each charge in the act of raising the cover.

As illustrating the fact that the charge on the ebonite acts inductively through a thin layer of air on the ordinary cover, it is interesting to note that if a layer of mercury be poured on to the cake and touched to earth the cake is discharged. Evidently the mercury fits so closely on to the electrified surface that the two electrifications are able to unite.

It is important to notice that the charge taken away on raising the cover is nearly all derived from the sole. When the cover is first placed on the cake a small electrification of the cover, + below, -above, no doubt occurs. But the greater part of the charge on the cake is still occupied in inducing the charge on the sole. When the cover is touched the sole charge rushes up into the cover, and really forms the charge carried away when the cover is raised. 'This may easily be proved by placing the electrophorus when excited on an insulating pillar. If the cover is put on the cake and touched, the sole charge cannot now pass into the cover and a very small result is obtained.

The electrophorus is a convenient instrument only when a small quantity of electricity is required. For though any number of nearly equal charges can be obtained on the cover in succession from the same electrification of the resinous cake, and may be communicated to a suitably arranged conductor, it would be both 
troublesome and slow to gather together any large quantity in this way.

Bennett's Doubler.-Very soon after the invention of the electrophorus means were devised for increasing the output, the earliest being Bennett's Doubler, invented in 1786 for the purpose of increasing very weak charges. It consisted of three metal plates A B C, Fig. 13, varnished as indicated by the black lines, $A$ on the upper side, $B$ on both sides, and $C$ on the under side, the varnish acting as an insulator. $\mathbf{B}$ and $\mathbf{C}$ were provided with insulating handles. Let us suppose the following operation to be

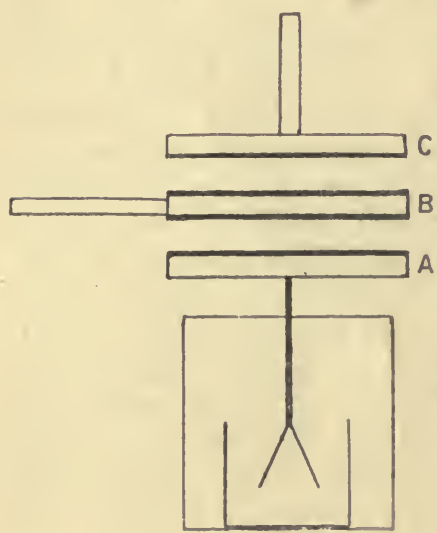

FIG. 13. gone through: A is placed on an insulator, which we may suppose to be an electroscope as that will serve as an indicator of increasing charge ; $\mathbf{B}$ is placed on $\mathrm{A}$. If a conducting body charged, say, positively touches $A$, while $\mathbf{B}$ is touched to carth, almost all the charge will gather on $A$, for there it can be quite close to the opposite negative charge induced on the under side of $\mathbf{B}$. 'This negative charge is nearly equal to that on $A$. Now insulate $B$ and remove the body; let 13 be raised and brought against $\mathrm{C}$; touch $\mathrm{C}$ to earth. A positive charge nearly equal to the negative on $\mathrm{B}$ or the positive on A gathers on $\mathrm{C}$. Insulate C and replace $B$ on $A$; touch $B$ to earth; touch $C$ to $A$, when nearly the whole of its charge will pass to $A$, being added to and thus almost doubling the original charge. Repeating the process after each series of operations, the charge on $\mathrm{A}$ is nearly doubled and may soon be made a very large multiple of its original value, as the electroscope will show. The disadvantage of this form of apparatus is that it requires considerable mamipulation, and it was soon followed by instruments in which the process was carried out mechanically by the turning of a handle. Probably they were not very efficient, for they seem to have been considered merely as curiosities. One machine, invented by Belli in 1531, should have secured more notice. It. appears, however, to have been neglected, and it was not until the independent invention of machines by Varley, 'Thomson, 'I'oepler, and Holtz, about the year's 1860-65, that general attention was directed to the subject. Since that time various forms of machines, all dependent on induction or " influence "and not on friction, have been devised. These are termed Influence Machines, and have now practically displaced the old frictional machines as sources of electrification. As, perhaps, the simplest in principle we shall first describe Belli's machine. 
Belli's machine.* In the form here described there are two metal plates, the field plates A B, bent, as in Fig. 14, like the covers of a book and fixed on insulating pillars. The movable part consists of two circular" metal plates, the "carriers" C D, at the extremities of a glass arm. By turning a handle these can be revolved on a horizontal axis so that they pass through the spaces partially enclosed by the field plates. Projecting from the inside of each field plate is a spring $s$, which each carrier tonches as soon as it is completely within the field plates. After contacts with the springs

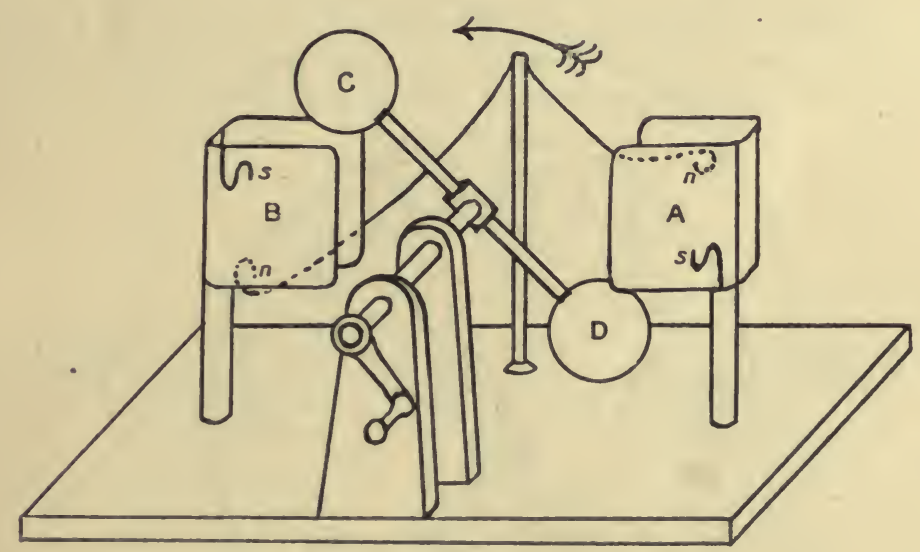

FIG. 14.

are broken the carriers come in contact with the two ends of a wire $n n$, the "neutralising rod" of later machines, which puts them in electric communication while still within the field plates. To understand the action of the machine let us suppose that to begin with there is a positive charge on $\mathbf{A}$ and that the carrier plates start from the position of contact with the wire $n n, \mathrm{C}$ being within $\mathrm{A}$ and $\mathrm{D}$ within $\mathrm{B}$. Then negative electricity is induced on $\mathrm{C}$ and positive is sent to $\mathrm{D}$. These charges are carried round until $\mathrm{C}$ touches the spring $s$ within $\mathrm{B}$, and $\mathrm{D}$ touches that within $\mathrm{A}$. Since the carriers are almost enclosed by the field plates and are in contact with them, their charges, tending to get to the outside, pass almost entirely to the field plates, increasing the + charge on A and giving a - charge to $\mathbf{B}$. The carriers then move on to contact with the ends of the wire $n n$, when $\mathrm{D}$ will be negatively and $\mathrm{C}$ positively electrified by induction. Further rotation brings $\mathrm{C}$ again within $\mathrm{A}$ and $\mathrm{D}$ within $\mathrm{B}$, and on contact with $s s$ the charges go to increase those on the field plates. Thus the charges on the field plates rapidly increase.

* Di una nuora meniera di macchina elletrica, Ann. Sci. Lomb. Veneto, I. (1831), p. 111. 
It is not always necessary to electrify a field plate to set the machine in action. 'There is frequently some charge to begin with, perhaps due to friction of a carrier against a spring, and if the two plates are not electrified equally the machine will increase the larger charge and soon make the smaller charge of the opposite sign to it, if it was not so initially.

In 1860 C. F. Varley* invented a machine similar in principle to that of Belli, and later Lord Kelvint devised a modification, the "replenisher" used to charge the jar in his Quadrant Electrometer.

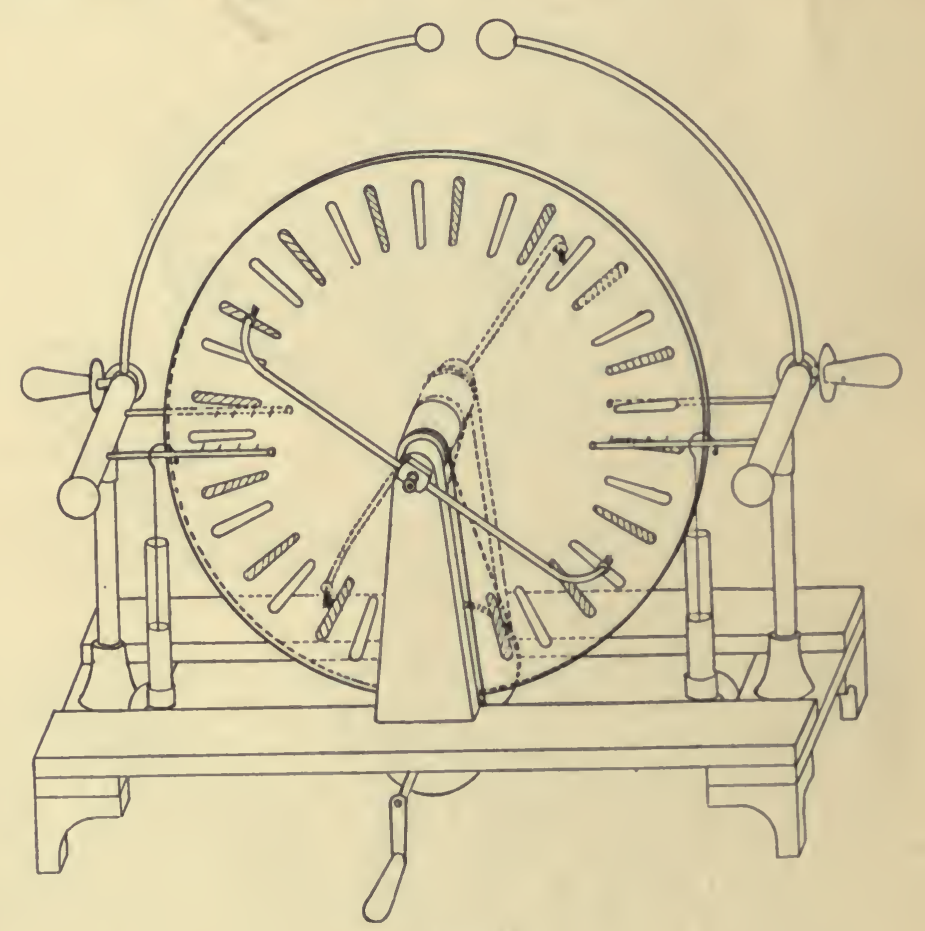

FIG. 15.

Other important developments were made in 'Toepler's machine and Holtz's machines.t We shall only describe the machine usually employed in this country and introduced by Wimshurst in 1883, a modification of the previous machines somewhat more certain and convenient in its working.

The Wimshurst machine. The common form of this machine is represented in Fig. 15. It consists of two exactly similar glass plates which are rotated close to each other with equal

* Specification of Patent, Jan. 27, 1860, No. 206.

+ Papers on Electrostatics and Magnetism, p. 270.

I A full account of Toepler's and Holtz's machines is given in Gray's Electrical Influence Machine'. 
GENERAL ACCOUN' OF COMMON PHENOMENA '1 9

relocities in opposite directions on the same axes. On the outer faces of the plates are equal numbers of thin brass sectors arranged radially at equal intervals. On the horizontal diameter are the collecting combs connected with the discharging knobs which are placed above the plates. Two small Leyden jars have their inside coatings connected one to each of the collecting combs, while their outside coatings are connected with each other. At $45^{\circ}$ or thereabouts to the horizontal, the best position being found by trial, are two neutralising rods, one opposite each face, and nearly at right angles to each other. These terminate in wire brushes which touch the sectors as they revolve. 'To start the machine most easily, the discharging knobs are placed nearly in contact and the handle is turned. After a few revolutions of the plates, if the

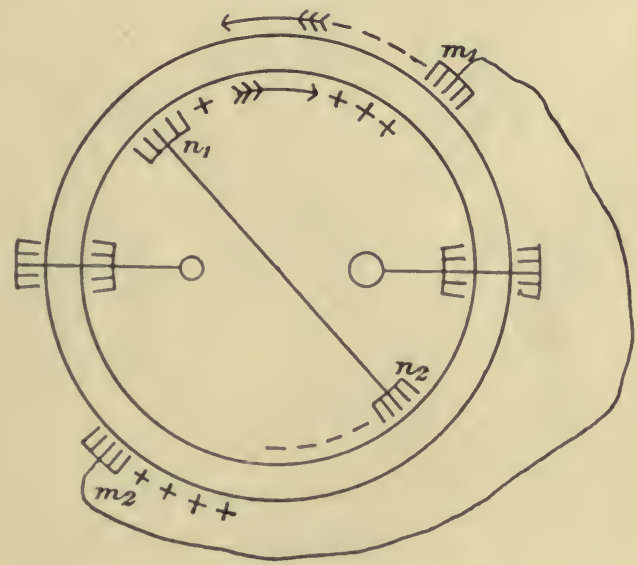

FIG. 16.

machine is fairly dry and dust-free, sparks will pass. 'The knobs may now be separated gradually, and the sparks will continue to pass till the limiting length is passed. Even when the machine will not excite itself it may readily be set in action by holding an electrified body near one of the brushes on the far side of the plates from it, and then rotating the plates. The Wimshurst is one of the most easily worked and most regular of the influence machines. 'The explanation of its working can be given most readily by aid of a diagram in which, as represented in Fig. 16, the plates are replaced by co-axial cylinders. We may then distinguish them as inner and outer. Let us first leave out of account the discharging circuit and suppose only that we have the two neutralising rods $m_{1} m_{2}, n_{1} n_{2}$. I.et the plates revolve as in the figure, and let us suppose that in some way, say by friction as it passes the brush $n_{1}$, a sector at the highest part of the inner plate is slightly positively electrified. As this comes opposite $m_{1}$ it induces - on the sectors of the outer plate under $m_{1}$, and sends + on to the 
sectors of the outer plate under $m_{2}$. The smaller the sectors and the more numerous they are, the greater will be the quantity of - induced through $m_{1}$ by the + on the inner plate, and it may be much more in total amount than the original + if a sufficient number of sectors pass $m_{1}$ while the inducing charge is near that brush. Now the - passing round on the outer plate arrives opposite $n_{1}$ when the + on the inner arrives at $n_{2}$, and, meanwhile, the + given to the outer plate through $\mathrm{m}_{2}$ arrives opposite $n_{2}$, and under these charges not only is the $t$ on the inner plate under $n_{2}$ neutralised, but a - charge is induced on it there while $a+$ charge is given to the sectors under $n_{1}$. These are carried on, and when under $m_{1} m_{2}$ they induce - and + on the outer plate, while when under $n_{1} n_{2}$ they are reversed.

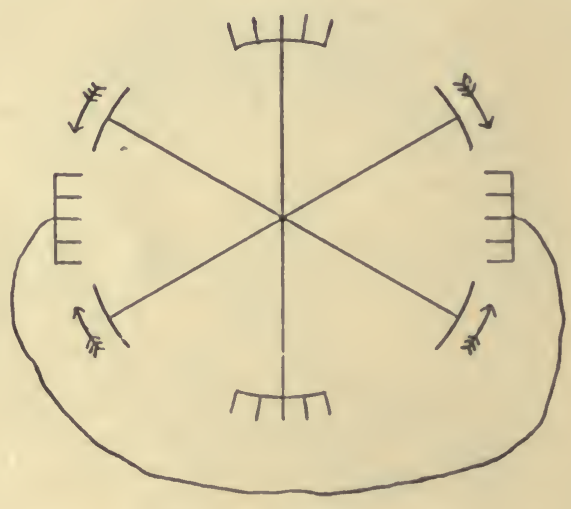

FIG. 17.

The charges on the different parts of the plates are therefore as in the figure, and if the sectors are sufficiently numerous the charges will mount up until the increase is balanced by leakage. To understand the necessity of a number of sectors it is sufficient to consider the case represented diagrammatically in Fig. 17, where there are on each plate only two sectors at the opposite ends of a diameter and so arranged that they meet under the brushes. Then if one inner sector receives a charge $+Q$ the most it can do is to induce $-\mathbf{Q}$ on the outer sector when the latter is under a brush, the opposite sector being charged with $+Q$. These + and charges cannot do more than induce $-\mathbf{Q}$ and $+\mathbf{Q}$ when in turn the inner plate sectors are under the brushes, and so on, so that the charges do not increase however long the rotation is continued.

The addition of the discharging circuit does not alter the nature of the action; it only leads to an earlier reversal of the charges on the sectors. Thus the + on the inner plate and the + on the outer plate running in opposite directions past the right-hand 
GENERAL ACCOUNT OF COMMON PHENOMENA 21

combs will clearly tend to draw out - and leave + on the righthand discharging rod. On the left-hand rod, - will be left, and if the knobs are not far apart the charges may soon be sufficient to spark across. But if the knobs are so distant that no sparks pass, the charges on them may soon reach a steady state, and no more electrification being taken from the combs, the machine works just as if the discharging circuit were removed, and increase is balanced by leakage.

Storage of electrification. The Leyden jar. The Leyden jar is a device for collecting and storing large quantities of electrification. It usually consists of a glass jar, as shown in Fig. 18 , lined inside and out with tinfoil to perhaps three-quarters of its height; the neck of the jar is closed by a cork, or preferably

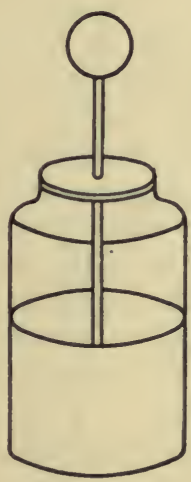

FIG. 18.

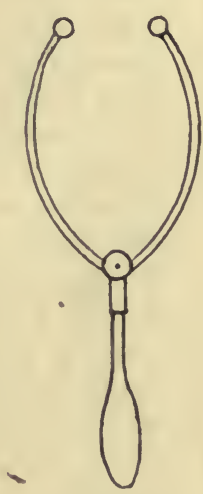

FIG. 19.

some insulating material, through which passes a metal rod terminated above in a knob and below in a chain, which puts it in communication with the inner coating of tinfoil. If the jar is held in the hand, the outer coat is comnected to earth, and if the knob is applied to the prime conductor of a machine, the positive charge from the prime conductor passes into the jar and collects on the outside of the inner coating. 'There it induces a negative charge on the inside of the outcr coating. If the knob is held at some distance from the prime conductor the jar is charged by a series of sparks, and the successive rushes of negative electricity to the outer coating through the arm may frequently be felt. If the source gives large charges in each spark, the rushes through the arm would be painful, and the arm must be replaced by an earth-comnected wire.

'To discharge the jar it is only necessary to connect the two coatings, or to connect the inside to earth. As it is disagreeable to the operator to form part of the comnecting circuit it is usual to employ a discharger, consisting of metal tongs provided 
with an insulating handle (Fig. 19). 'The knobs being separated a proper distance, one is first brought against the outer coating, and the other is then brought near the knob of the jar; the discharge takes place by means of a spark more or less brilliant, according to the charge in the jar. If the tongs are brought first against the knob, discharge sometimes occurs through the operator if there is faulty insulation in the handle. It is therefore advisable to observe the order above described. With a given machine as source of electrification the maximum charge collecting in a jar, which we may take as indicating its electrical capacity, is proportional, as we shall prove later, to the area of the coating, and inversely as the thickness. It depends also on the nature of the insulating material of which the jar is made. It would, for example, be greater for an ebonite jar than for a paraffin jar of the

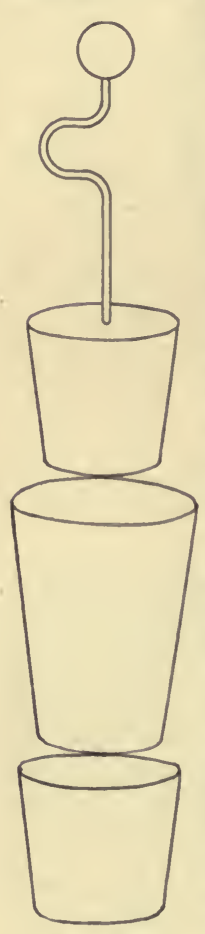

FIG. 20. same dimensions. Sometimes, instead of a jar, a flat pane of glass is used, coated on its two sides. We may evidently regard this as a jar opened out.

Franklin's jar. If the coating is not absolutely in contact with the glass the charge resides on the surface of the glass, passing, no doubt, through the intervening thin layer of air. This was proved by Franklin by means of a jar with movable coatings. Fig. 20 represents the separated parts of such a jar. 'The lowest is a tin cup to form the outer coating. 'I'he middle is a glass beaker which fits into the cup, and the upper is a tin cylinder to form the immer coating when dropped into the beaker. After charging the jar we may remove first the outer coating, and then the immer by an insulating hook so as to prevent earth comnection before the coating is remored from the jar. 'The two coatings will now be found free from charge. After building up the jar again, however, a discharge may be obtained on connecting the coatings, showing that when the coatings were remored the two surfaces of the glass still retained the charges. 'This may seem at first sight inconsistent with the behaviour of the electrophorus, in which the charge on the cover does not pass across the intervening air to the surface of the ebonite. But it must be remembered that in the jar the charge is in general far greater than in the electrophorus, and its tendency to get orer the intervening air space is therefore greater.

Residual charge. If a Leyden jar is charged, left for a short time, and then discharged, it appears to be entirely free from electrification. But if left again for a short time another charge of the same kind as the previous one is found to have 
GENERAL ACCOUN'T OF COMMON PHENOMENA 23

ppeared, but much less in quantity, and another spark may be obtained. Leaving it again for a time after discharge, another but still smaller charge gathers and a third spark may be obtained, and so on. These successive charges are termed residual charges, and the successive sparks residual discharges. We shall give the probable explanation of the phenomenon later. 


\title{
CHAPTER II
}

\section{QUANTITY OF ELECTRIFICATION}

\begin{abstract}
Use of the electroscope to indicate equ uality of charge-The two electrifications ailways appear or disappear in equal amounts whether the electritication is by friction, by conduction, by induction, or by supply from current -Electrification resides only on the outside surface of a conductor unless it contains insulated charged bodies-An insulated charged conductor inside a hollow conductor induces an equal and opposite charge on the inside and an equal like charge on the outside-linagined construction of inultiples and submultiples of an arbitrary unit of charge-Imagined method of measuring any charge in terms of this unit.
\end{abstract}

WE shall now describe a series of experiments, some illustrating the general laws of the production and distribution of electrification, and others showing that we may make definite and consistent measurements of quantity of electrification.

Use of the electroscope to indicate equality of charge. If a deep and narrow metal vessel, such as an ordinary round tin canister, is placed on the upper table of a gold-leaf electro. scope, it may be used to detect a very slight charge of electrification on a body, by the introduction of the body within the can. It is not necessary to communicate any of the charge to the can, for it will act inductively, calling up an opposite charge on the inside of the can and sending a like charge to the gold leaves.*

As the body is being lowered within the can the divergence of the leaves rapidly increases at first, but soon it approaches a limit, which it does not exced, and the leaves remain practically fixed however the body be moved about within the can, so long as it does not come near the open top.

If the can be closed by a lid with only a small hole for the insulating holder of the body to pass through, the body may be brought quite close to the top, without change in the divergence of the leaves. 'Thus a change in the position of a charged body within a closed can does not affect the indications of an electroscope connected with the outside of the can. 'This result, which is

* In the experiments described in this chapter the gold-leaf electroscope may be replaced by the Quadrant Electrometer described on $\mu .87$, the can being insulated and connected with one pair of quadrants. Much greater sensitiveness is thus obtained, but the gold-leaf electroscope uay be made sufticiently sensitive for purposes of demonstration by bringing the side plates nearer the leaves, and it is much more easily used than the Quadrant Elcctrometer. 
of great importance, may be verified with great exactness by connecting the can to earth until the charged body is inserted. The induced like electrification on the outside of the can is discharged and the leaves remain together. 'They are then in the most sensitive position, and the sensitiveness may be still further increased by approach of the side plates. If there were any change in the effect on the leaves due to change of position of the inside body it would now be noted most easily.

Here we have a definite effect due to a charged body, which may be easily observed, and one which is consistent. For on successive withdrawals and insertions the same divergence of the leaves is obtained, and the exact position of the charge within the can is unimportant. We may, therefore, use this effect to compare two different electrifications as to quantity. If two charged bodies inserted successively, produce the same divergence, they are to be regarded as having equal charges. If the divergence is not the same the greater divergence corresponds to the greater charge. Again, if two oppositely electrified bodies are inserted in the can at the same time, they tend to send opposite electrifications into the leaves and so to neutralise each the effect of the other. If the leares do not diverge at all, the charges are to be regarded as equal in amount though opposite in kind. We have thus defined what we mean by equal charges on different bodies, whether like or opposite in kind, and we can now state the fundamental law of the production and disappearance of electricity, viz. :

'T'he two electrifications always exist in equal amounts. If any amount of one appears or disappears, an equal amount of the other appears or discippears at the same time.

'The truth of this law may be tested by the following simple experiments with the gold-leaf electroscope and can.

Electrification by friction. If a rod of sealing-wax and a rod of glass are rubbed together within the can the leaves do not diverge. 'They are both electrified, however, for on withdrawing one of them the effect of the other is at once evident. But since, before the withdrawal of one, the leaves did not diverge, it follows that the two kinds of electrification are produced in equal amounts, one on the sealing-wax and the other on the glass. We may use other pairs of substances, and in every case we shall find that the two bodies rubbed together are electrified oppositely with equal amounts.

Electrification by conduction. If we first lower an insulated charged conductor into the space inside the can, touching the can to earth meanwhile so that the leaves remain together and in the position most sensitive to variation of charge, and if we then lower a second insulated uncharged conductor into the can till it touches the first, we know that conduction takes place from the first to the second. But the electroscope gives no indication; then the total amount of electrification remains the same, though 
now it is on two bodies. Again, charging the second body oppositely to the first before inserting it into the can, and keeping the can untouched to earth until both conductors are within it but not in contact, if we now bring them together the smaller charge will be entirely neutralised by a part of the larger and will disappear, while the rest of the larger cliarge will spread over both bodies. But the electroscope remains unaffected by this process, or the algebraic sum is still the same. 'T'he two kinds have, therefore, disappeared in equal amounts.

Electrification by induction. Returning to the first of the two preceding experiments, while the second uncharged body was being lowered into the can it became electrified by induction on approaching the charged body. 'This we know from our gencral experiments on induction, described in Chapter I. Since the electroscope gave no indication of change of amount, it is evident that equal and opposite amounts must have been induced on the body. The unlike was near the first body, the like remote from it. On contact the unlike was neutralised by some of the charge on the first body. So that it is again evident that the two kinds disappeared in equal amounts.

Electric current. We shall see hereafter that the electric current as produced by voltaic cells or dynamos may be regarded as a conduction of electrification along the wire in which it occurs. The quantities conducted even in a short time are usually enormously great compared with the quantities with which we deal in experiments with the gold-leaf electroscope on charges produced by friction or induction. Making a small voltaic cell, and comecting the plates together by a wire so that the current flows, let us insert it within the electroscope can. No vestige of charge is shown. Hence the two kinds are being produced and are disappearing in equal quantities.

The distribution of electrification on conductors. Electrification resides only on the outside surface of conductors, unless, being holloz", they contain insulated charged bodies.

If the deep can of the previous experiments, or preferably a hollow insulated metal globe with a small opening to the inside. is charged in any way, a proof plane inserted inside through the opening by an insulating holder and brought into contact with the inside shows no trace of electrification on withdrawal. Or, if a charged conductor is brought within the hollow conductor and touched to it, it is entirely discharged. On contact it forms part of the inside of the hollow conductor and can no longer keep its electrification.

Faraday (Exp. Res. vol. i. $\$ \$ 1173-4)$ made experiments on a large scale to show that the charge of a conductor resides on the outside. A cube with each edge $12 \mathrm{ft}$. loner, consisting of a light wooden framework corered in with paper, was made thoroughly conducting by copper wire and bands of tinfoil. It was insulated 
and charged by a powerful machine till " large sparks and brushes were darting off from every part of its outer surface." Faraday "went into the cube and lived in it, and using lighted candles, electrometers, and all other tests of electrical states," he was unable to detect any charge within the cube.

Faraday devised a very simple and interesting method of showing that charge resides only on the outer surface of a conductor by an experiment known as the "butterfly-net experiment." A conical muslin bag (Fig. 20A) which may be 10 or 12 inches long is fitted on to a ring about 4 inches in diameter, placed on the top of an insulating pillar with a base fixed to the table. 'The muslin is preferably slightly starched. After it is starched two

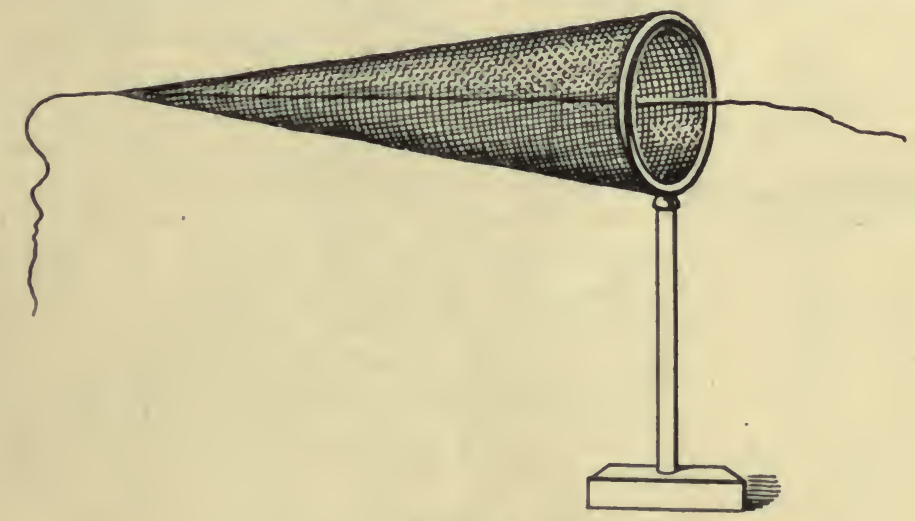

Fig. $20 \mathrm{~A}$.

insulating silk threads are attached to the apex, one inside, one out, so that the bag may be easily turned inside out merely by pulling one of the threads. A charge is then communicated to the bag, and it can be detected on a proof plane which has been applied to the outer surface while no charge is thus detected on the imner surface. 'The bag is then pulled inside out by one of the silk threads, and the outside, which was previously the inside, is found to possess the whole charge.

When an insulated charged conductor is brought within a hollow conductor it induces an equal and opposite charge on the inside of the hollow conductor, while an equal like charge goes to the outside.

Let Fig. 21 represent a deep can on the table of an electroscope and let a positively charged insulated conductor be inserted within the can. Outside the can and in the leaves there is a positive charge, as may be shown by the collapse of the gold leaves on bringing near the can a negatively electrified body, say an ebonite rod. Inside the can there is a negative charge, for if we touch the can to earth to discharge the outside positive electrification and then withdraw the charged hody from within, the 
outside of the can and the gold leaves become negalively electrified. We may at once prove this by again bringing up the ebonite rod, when the leaves will diverge still further. Now, discharging the electroscope and again inserting in the can

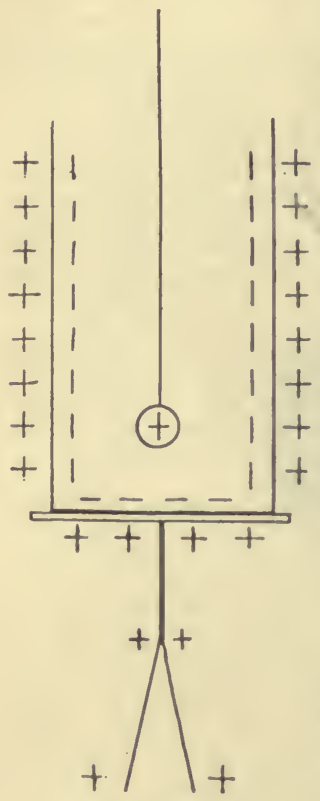

Fig. 21. the positively charged body, we know from our experiments on induction that the quantities on the inside and the outside surfaces of the can are equal, though opposite. 'Touching the charged body to the inside of the can, it is entirely discharged, and at the same time the inside charge of the can disappears, while the outside charge is unaffected. Hence the inside charge must have been exactly equal in amount, though opposite in kind, to the charge on the inserted body. Faraday used the cube already described to show that a charge within a conductor induces an equal and opposite charge on the inner surface of the conductor. For this purpose he passed a long glass tube through the wall of the cube so far that it reached well within it. A wire from a large electric machine passed through the tube, and by discharge from the end of the wire the air within the cube could be highly electrified. If while the machine was being worked the cube remained insulated, the outside became strongly charged. If it was put to earth this charge was conducted away. If at the moment that the machine was stopped the cube was insulated again, no charge gathered on the outside, showing that the electrification of the air within was exactly neutralised by the charge which it induced on the inner surface of the cube.

Imagined construction of multiples and submultiples of an arbitrary unit of charge. Before proceeding further with the account of electric induction it may assist us if we consider a method of making quantitative measurements of charge which depends upon the foregoing experiments. 'The method is impracticable owing to the impossibility of perfect insulation, but, as a conception, it is a legitinate deduction from the principles to which we have been led, and it serves to show that we can attach a definite meaning to the term "quantity of electricity."

Let us suppose that perfect insulation is possible and that we have a conductor on a perfectly insulating stand. Let us impart to A, Fig. 22, some arbitrary positive charge. With a perfect insulation this charge will remain on $\Lambda$ for erer, and so being definite and consistent we may choose it as the unit of charge. 
Let a small can $B$ have a lid furnished with an insulating handle $h$ and let the under side of the lid have some arrangement by which the base of $\mathrm{A}$ can be attached when $\mathrm{A}$ is inverted. Let $\mathbf{H}$ be an insulating handle by which the can may be lifted up. Let an insulating base plate be fixed to the can. Let $\mathrm{C}$ and $\mathrm{D}$ be two other cans similar in general plan to B, but large enough to contain it. Let a metal piece $S$ be fastened to the lid of each of the larger cans so as just to touch the smaller can when inside.

We shall now show that we may give to $\mathrm{C}$ a charge equal to

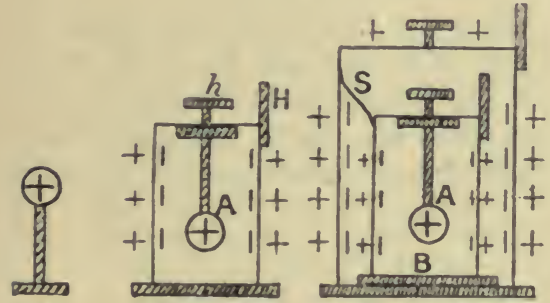

B

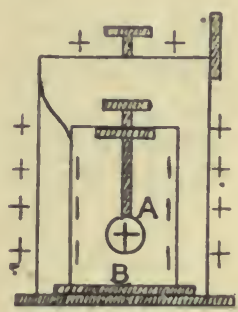

C

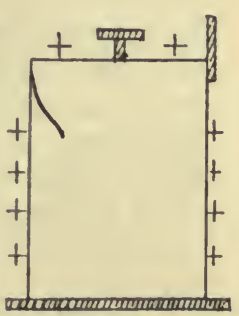

C

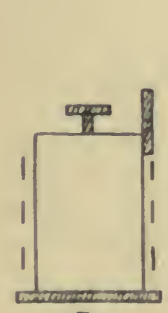

B

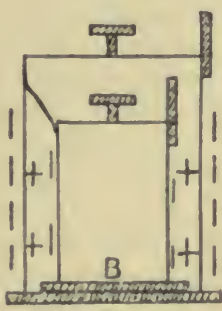

D

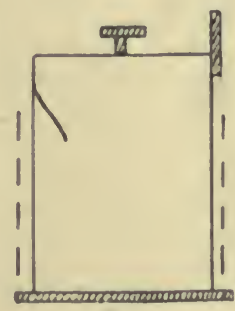

D

Fig. 22.

any positive multiple of the unit, and to $\mathrm{D}$ a charge equal to any negative multiple. To charge $\mathrm{C}$ with a positive multiple, place the unit within $\mathrm{B}$, place $\mathrm{B}$ within $\mathrm{C}$, and put the lid on $\mathrm{C}$, taking care not to touch the conductors in any case, but moving them always by their insulating handles. 'Then following out the process by the aid of the upper row of diagrams in Fig. 22, it is easy to see that when $\mathrm{B}$ is taken out of $\mathrm{C}, \mathrm{C}$ will have a positive unit on the outside. On taking $\mathrm{A}$ out of $\mathrm{B}, \mathrm{B}$ may be inserted in $\mathrm{D}$, as represented in the lower row, and its negative charge is imparted to $D$. On taking $B$ out of $D, B$ is free from charge. As A has its original charge we may repeat the process any number of times, each repetition adding a positive unit to $\mathrm{C}$ and a negative unit to D. We may imagine the preparation in this way of a series of multiples of the unit, both positive and negative, by having a number of cans like $\mathrm{C}$ and $\mathrm{D}$. By making a number of exactly similar cans, charging one with a unit and sharing this unit 
with another, then sharing one of the half units with another, and so on, we may suppose sub-multiples prepared on the scale $\frac{1}{2}, \frac{1}{4}, \frac{1}{8}$, \&r.

Imagined method of measuring any charge in terms of the unit. We may now use these quantities somewhat as we use the weights of a balance. Suppose that we wish to measure the quantity of electricity on a given body, let us place it insulated within an insulated can connected to an electroscope. Then let us place within the can and also insulated from it quantities of the opposite kind of electricity until the leaves of the electroscope fall together. 'Then the two kinds are equal in amount. It is evident that we may keep the can to earth while we are inserting the charged body and the opposite quantities and then test their equality by lifting them all out at the same time. If the opposite charges are unequal the electroscope will at once show a charge. 


\title{
CHAP'TER III
}

\section{PROPOSITIONS APPLYING TO "INVERSE SQUARE" SYSTEMS}

\begin{abstract}
The inverse square law-The field-Unit quantity-Intensity-Force between quantities $m_{1}$ and $m_{2}$-Lines of force and tubes of force-Gauss's theorein-If a tube of force starts from a given charge it either continues indefinitely, or if it ends it ends on an equal and opposite charge-The product intensity $\times$ cross-section is constant along a tube of force which contains no charge-If a tube of force passes through a charge and $q$ is the charge within the tube, the product intensity $\times$ cross-section changes by $4 \pi q$-The intensity outside a conductor is $4 \pi \sigma$-Representation of intensity by the number of lines of force through unit area perpendicular to the lines-Number of lines starting from unit quantity-The normal component of the intensity at any surface is equal to the number of lines of force passing through unit area of the surface-Fluid displacement tubes used to prove the properties of tubes of force-Spherical shell uniformly charged - Intensity outside the shell-Intensity inside the shell-Intensity at any point in the axis of a uniformly chargel circular disc-Intensity due to a very long uniformly charged cylinder near the iniddle-Potential-The resolute of the intensity in any direction in terms of potential variation-Equipotential surfaces-The energy of a system in terms of the churges and potentials-The potential due to a uniformly charged sphere at points without and within its surface.
\end{abstract}

The inverse square law. In the chapters following this we shall show that certain actions at any point in a space containing electric charges may be calculated on the supposition that each element of charge exerts a direct action at the point proportional to the element, and inversely proportional to the square of the distance of the point from it. 'The same method of calculation holds for magnetic and for gravitative systems. It is to be noted that the supposition of direct action according to the inverse square law is adopted merely for the purposes of calculation. Experiments show that it gives correct results, but, as we shall see, it does not give us any insight into the real physical actions occurring in the system.

'There are certain propositions which are mathematical consequences of the inverse square law, and it will be convenient to prove these before we discuss the experimental verifications. 'These propositions hold good alike for electric, magnetic, and gravitative systems.

We shall prove the propositions on the assumption that we are 
dealing with an electric system in which the charges are separated by air. Some of the propositions will, however, apply at once to magnetic and gravitative systems if we use corresponding units of measurement.

The field. 'The space in which the action of a system is manifested is termed its field.

Force between quantities $m_{1}$ and $m_{2}$. Experiments which are described in Chapter $V$ show that a small body charged with $m_{1}$ acts upon a small body charged with $m_{2}$ and distant $d$ from it with a force proportional to $m_{1} m_{2} / d^{2}$, whatever be the unit in terms of which we measure the charges.

Unit quantity. 'The inverse square law enables us to fix on the following convenient unit: If two small bodies charged with equal quantities would act on each other with a force of 1 dyne if placed $1 \mathrm{~cm}$. apart in air, each body has the unit charge on it.

This unit is termed the Electrostatic or E.S. unit. If two bodies have charges $m_{1}$ and $m_{2}$ in E.S. units and are $d \mathrm{~cm}$. apart, the inverse square law shows that the force between them is $m_{1} m_{2} / d^{2}$ degrees.

Intensity of field. The intensity at a point is the force which would act on a small body placed at the point if the body carried a mit charge.

'The intensity due to a charge $m$ at a distance $d$ is $m / d^{2}$.

Lines of force and tubes of force. If a line is drawn in the field so that the tangent to it at any point is in the direction of the intensity at that point, the line is termed a line of force. A bundle of lines of force is termed a tube of force. Or we may think of a tube of force as enclosed by the surface obtrined by drawing the lines of force through every point of a small closed curve.

We shall now prove a theorem due to Gauss which enables us to obtain the intensities in certain cases very simply, and which also shows us that lines and tubes of force indicate for us the magnitude

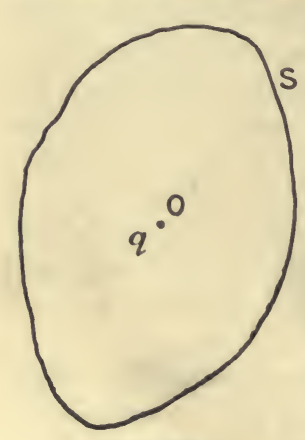

FIG. 2:. as well as the direction of the intensity at every point in their length.

Gauss's theorem. If we take any closed surface $S$, and if $N$ is the resolved part of the intensity normal to the surface at the element $d S$, positive when outwards, negative when inwards, then $\int \mathrm{N} d \mathbf{S}=4 \pi \mathrm{Q}$, where $\mathrm{Q}$ is the quantity of charge within the surface $S$. Any charge without the surface makes on the whole no contribution to $\int \mathrm{N} d \mathbf{S}$.

'To prove this let us consider an element of charge $q$, situated at a point $\mathrm{O}$ within a closed surface, such as is indicated by $\mathrm{S}$ in Fig. 23. Let an elementary cone of solid angle dw be drawn from $O$, intercepting an element of area $d \mathbf{S}$ of the surface $\mathrm{S}$. Let this cone 
be represented on a larger scale in lig. 24, in which AHBK represents $d \mathbf{S}$. The intensity at $d \mathbf{S}$ may be taken as $q / \mathrm{OA}^{2}$ outwards along the axis of the cone, and if $\theta$ is the angle which the axis makes with the normal to AHBK, then $\mathrm{N}=\frac{q \cos \theta}{\mathrm{OA}^{2}}$, and $\mathbf{N} d \mathbf{S}=\frac{q d \mathbf{S} \cos \theta}{\mathrm{OA}^{2}}$.

But if we draw ALCM, a section of the cone $d \omega$, through $\mathbf{A}$

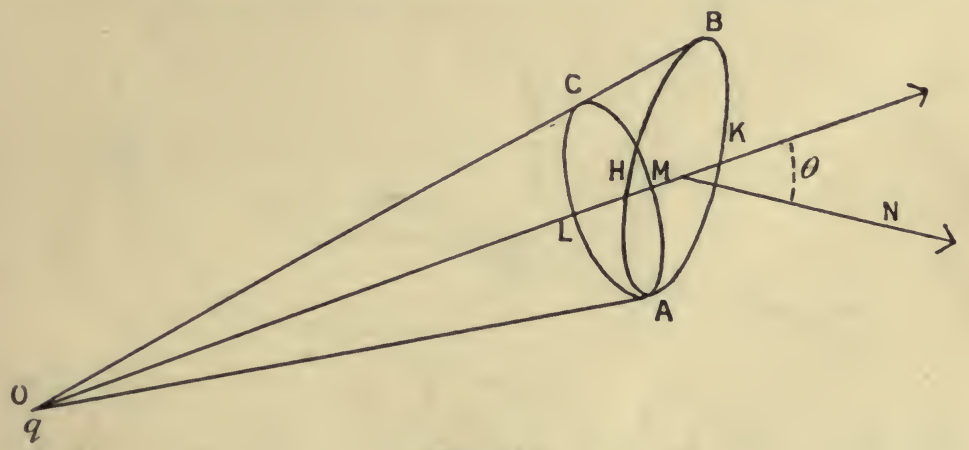

Fig. 21.

and perpendicular to the axis, the angle between AHBK and ALCM is $\theta$, and since div is so small that $\mathrm{BC}$ is practically parallel to $\mathrm{AO}, \mathrm{ALC} \mathrm{M}=d \mathrm{~S} \cos \theta$.

Hence

$$
\begin{aligned}
\mathbf{N} d \mathbf{S} & =\frac{q \mathrm{ALCM}}{\mathrm{OA}^{2}} \\
& =q d \omega .
\end{aligned}
$$

If we now sum up for every element of the surface, the contribution of $q$ is

$$
\int \mathbf{N} d \mathbf{S}=\int q d \omega=q \int d \omega=4 \pi q
$$

since the total solid angle round $q$ is $4 \pi$.

The normal intensity at any point of $S$ due to any number of elements of charge is equal to the sum of the normal intensities due to the separate elements. Hence for the whole charge $\mathbf{Q}$ within $\mathbf{S}$ we shall have to add up all the quartities such as $4 \pi q$, and so

$$
\int \mathrm{N} d \mathrm{~S}=4 \pi \mathrm{Q} \text {. }
$$

Now let $q$ be outside the surface at $\mathrm{O}$, Fig. 25, and let an elementary cone $d_{\boldsymbol{u}^{\prime}}$ cut $\mathrm{S}$ in $d \mathrm{~S}_{1}$ at $\mathrm{A}$, and $d \mathrm{~S}_{2}$ at $\mathbf{B}$. Let the intensities at $\mathrm{A}$ and $\mathbf{B}$ normal to the surface be $\mathbf{N}_{1} \mathbf{N}_{2}$ respectively. Then, as we have just proved, $\mathbf{N}_{\mathbf{1}} l \mathrm{~S}_{1}=q d \omega$, while at $\mathbf{B}, \mathbf{N}$ being in wards and negative, $\mathrm{N}_{2} d \mathrm{~S}_{2}=-q d \omega$. 'The two, therefore, neutralise each other. 'I'his 
will hold good for every elementary cone drawn from $O$ to cut $S$, and therefore an element of charge $q$ without $\mathrm{S}$ on the whole makes no contribution to $\int \mathrm{N} d \mathrm{~S}$, and only the charge within S counts.

We have taken the simple case in which each cone drawn from a point inside cuts the surface once, and each cone from the out-

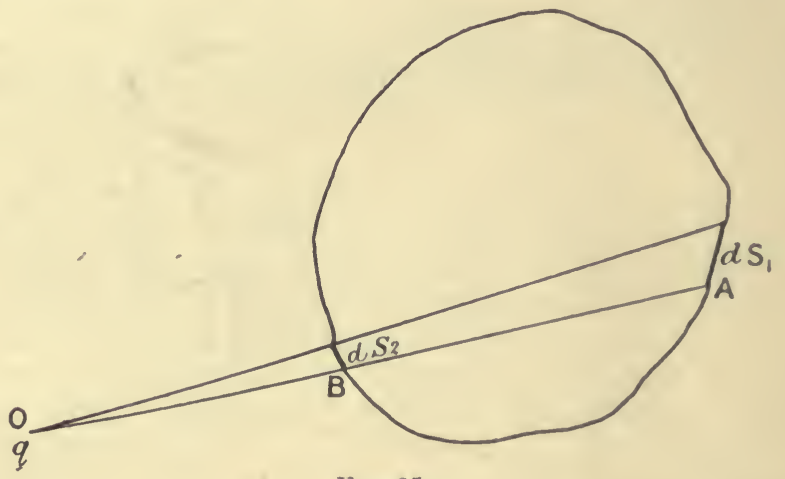

FIG. 25.

side cuts it twice, but it is easy to see that the results are true if the surface is re-entrant, so that it is cut any odd number of times in the first case and any even number of times in the second case.

We shall now apply Gauss's theorem to tubes of force.

If a tube of force starts from a given charge it either continues indefinitely, or if it ends it does so on an equal and opposite charge. Let a tube start from a quantity $q$ on an element of surface $\mathrm{S}_{1}$, Fig. 26. In describing the tube as starting from $q$ we imply that within $\mathrm{S}_{1}$ the intensity is zero.

Suppose that the tube continues some distance from $S_{1}$ and

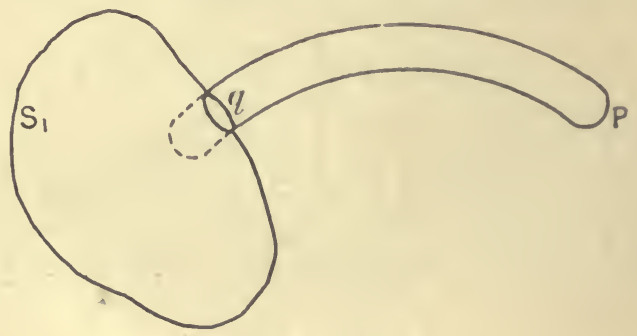

FIG. 26.

that it ends at $P$, as represented in the figure, without reaching any other charge. Prolong the tube within $\mathrm{S}_{1}$, make another closed end, and to the closed surface thus formed apply Gauss's theorem. Over the whole of this surface $\mathbf{N}=\mathbf{0}$, for along the 
sides of the tube the intensity is parallel to the surface of the tube and has no component perpendicular to it, and therefore $\int \mathbf{N} d \mathbf{S}=0$. But $\int \mathbf{N} d \mathbf{S}=4 \pi q$, since $q$ is the charge within. Then $4 \pi q=0$ and $q=0$.

'Then the supposition that the tube, starting from any real charge $q$, can end at a point in the field where there is no charge, is inadmissible.

Now suppose that the tube begins at $\mathrm{S}_{1}$, Fig. 27, on a charge $q_{1}$ and ends at $S_{2}$ on a charge $q_{2}$. By ending we mean that within $S_{2}$

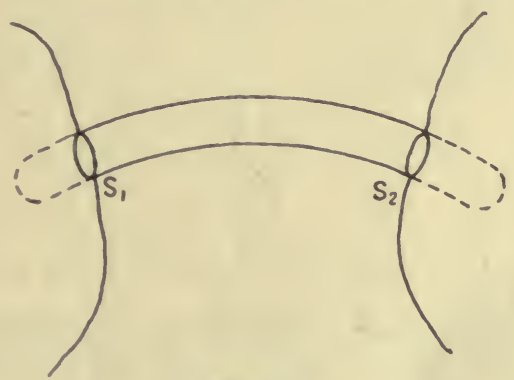

Fra. 27.

the intensity is zero. Prolong the tube as dotted within $s_{1}$ and $s_{2}$ and thus make two ends. Apply Gauss's theorem to the closed surface thus formed. Then $\int \mathbf{N} d \mathbf{S}=\mathbf{0}$, since $\mathbf{N}=\mathbf{0}$ at every point, and $4 \pi\left(q_{1}+q_{2}\right)=0$.

Hence $\eta_{1}=-\tau_{2}$.

The product intensity $\times$ cross-section is constant along a tube of force which contains no charge. Let $S_{1} S_{2}$, Fig. 28 , be two cross-sections of such a tube, and let $I_{1} I_{2}$ be the intensities at those sections. Let us apply Gauss's theorem to the closed

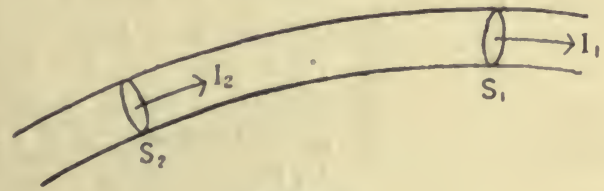

Fir. 28.

surface formed by the tube between $S_{1}$ and $S_{2}$ and by the ends $S_{1} S_{2}$. $\mathbf{N}$ has no value over the sides of the tube since the intensity is along the tube. Over the end $S_{1}, \mathbf{N} d \mathbf{S}=I_{1} S_{1}$. Over the end $S_{2}, \mathbf{N} d \mathbf{S}=$ $-I_{2} S_{2}$, negative as $I_{2}$ is inwards. Then $\int N d S=I_{1} S_{1}-I_{2} S_{2}$.

But since there is no charge within the tube between $S_{1}$ and $s_{2}, \int \mathrm{N} d \mathrm{~S}=0$, and therefore $\mathrm{I}_{1} \mathrm{~S}_{1}=\mathrm{I}_{2} \mathrm{~S}_{2}$. 
If a tube of force passes through a charge and $\eta$ is the charge within the tube, the product intensity $\times$ cross-section changes by $4 \pi \%$. Let $\mathrm{S}_{1} \mathrm{~S}_{2}$, Fig. 29, be such at tube having charge $q$ within it between $S_{1}$ and $S_{2}$. Applying

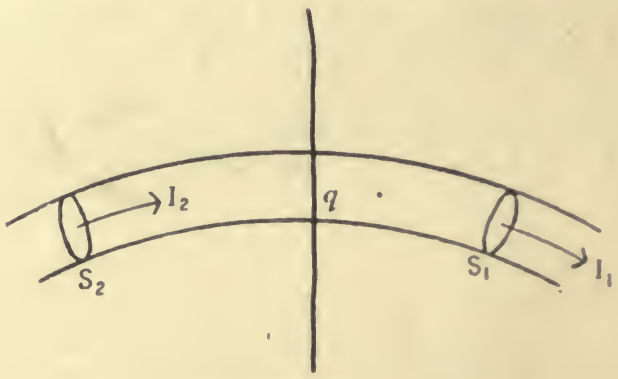

Fı. 29.

Gauss's theorem to the tube and its ends $\mathrm{S}_{1} \mathrm{~S}_{2}$, it is evident that $\mathrm{I}_{1} \mathrm{~S}_{1}-\mathrm{I}_{2} \mathrm{~S}_{2}=4 \pi \%$.

The intensity just outside a conducting surface is normal to the surface. Let $u$, Fig. 30, represent the section of a small circular area of a conducting surface charged with $\sigma$ per unit area, and let $a$ be so small that it may be regarded as plane and the surface density of its charge as uniform. I et it be circular. I e.t $\mathbf{P}_{1} \mathbf{P}_{2}$ be two points on the axis of a respectively at equal distances just outside and just inside the surface and so near that $P_{1} P_{2}$ is indefinitely small compared with the radius of $u$.

Now the intensity at any point may be regarded as the resultant of the intensity due to a, and the intensity due to the rest of the

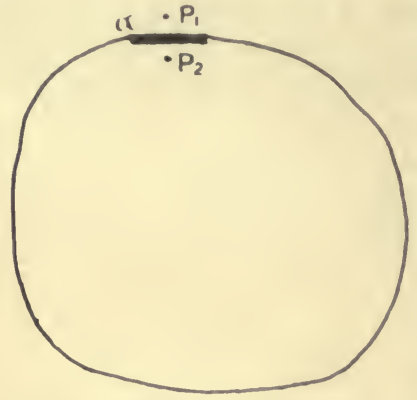

Fig. 30. surface and to other bodies in the system which we may denote collectively by $\mathrm{s}$. At $\mathrm{P}_{2}$ the intensity is zero, as it is a point within a conductor, so that the intensity there due to $\mathbf{S}$ is equal and opposite to that due to $a$. From symmetry, that due to $u$ is normal. So also must that due to $\mathrm{S}$ be normal. At $P_{1}$ the intensity due to $S$ is the same as at $\mathrm{P}_{2}$, since the distance between the points is negligible compared with the distance of the nearest parts of $\mathrm{S}$. 'The intensity at $\mathrm{P}_{1}$ due to a is equal and opposite to its value at $\mathbf{P}_{2}$ and is therefore equal to and in the same direction as the intensity due to $s$, and both are normal to the surface.

The intensity just outside a conductor is $4 \pi \sigma$, where $\sigma$ is the charge per unit area or the surface density. For 
suppose that the tube in Fig. 99 starts from unit area on a conducting surface at $\%$. 'Then the charge at $q$ is $\sigma$. Let the tube be prolonged within the conductor and be made to end at $S_{2}$. 'Then $I_{2}=0$, since there is no field within the conductor. Then $I_{1} S_{1}=4 \pi \sigma$. If $S_{1}$ is close to the surface, by the last proposition it is parallel to the surface, and also has unit area, and therefore $I_{1}=4 \pi \sigma$.

Representation of intensity by the number of lines of force through unit area perpendicular to the lines. Along a tube of force intensity $\times$ cross-section is everywhere constant if there is no charge within the tube. Draw a cross-section $S_{1}$ where the intensity is $I_{1}$, and imagine that through each unit area at $S_{1}, I_{2}$ lines of force pass along the tube. There will be in all $\mathrm{I}_{1} \mathrm{~S}_{1}$ lines in the tube. I.et $S_{2}$ be a section further on where the intensity is $I_{2}$. All the $I_{1} S_{1}$ lines pass through $S_{2}$, and the number through unit area of it will be $\frac{I_{1} S_{1}}{S_{2}}=\frac{I_{2} S_{2}}{S_{2}}=I_{2}$. or will be equal to the intensity at $\mathbf{S}_{2}$. Hence if we draw lines of force from any surface at the rate of I lines per unit area (the area being perpendicular to the intensity I), the number passing through unit area at any point in their course (the area being perpendicular to the lines) will be equal to the intensity at that area.

If the area of the surface from which we draw the lines is S, the total number through it, f IIS, is termed the flux of force through S. 'The magnitude of the intensity, as well as its direction, is therefore indicated by the lines of force when drawn on this scale. Where they are closer togrether the field is stronger: where they open out the field is weaker.

Number of lines of force starting from unit quantity. If a charge $\eta$ is at a point, the intensity over the surface of a sphere: radius $r$ drawn with the point as centre is $q / r^{2}$. We must then allow $\eta / r^{2}$ lines per unit area, or $4 \pi r^{2} \frac{q}{r^{2}}=4 \pi \eta$ in all. That is, from quantity $q, 4 \pi q$ lines start, and from unit quantity $4 \pi$ lines start.

If a tube of force contains $a$ charge $q$ at any point the flux of force, as we have seen, changes by $4 \pi \eta$, that is, the charge $q$ adds its lines to those already going along the tube.

The normal component of the intensity $\mathbf{N}$ at any surface is equal to the number of lines of force passing through unit area of the surface. I,t AB, Fig. 31, represent an element $a$ of the surface, and let I, the intensity, make an angle $\theta$ with the normal to $\mathrm{AB}$. I et $\mathrm{AC}$ represent the projection of $\mathrm{AB}$ perpendicular to I. 'Then $\mathrm{AC}=\alpha \cos \theta$. 'The number of lines through $A C=I_{a} \cos \theta=I \cos \theta a .=N_{a}$. But the same number of lines pass through $\mathrm{AC}$ and $\mathrm{AB}$. Hence the number through unit aren of $A B$ is $\mathbf{N}$. 
The total number of lines of force through a surface is equal to the sum of the numbers proceeding from each element of charge considered separately. For if $q$ is one element the number which it sends through an area $a$ at distance $r$ is $\frac{q a \cos \theta}{r^{2}}$, where $\theta$ is the angle between the normal to $a$ and $r$. The total number sent by all the elements is

$$
\Sigma \frac{q \alpha \cos \theta}{r^{2}}=\alpha \Sigma \frac{q \cos \theta}{r^{2}} \text {. }
$$

But $\Sigma \frac{q \cos \theta}{r^{2}}=$ sum of components of intensities along the normal $=\mathrm{N}$, which proves the proposition.

We call now state Gauss's theorem thus: The total flux of force

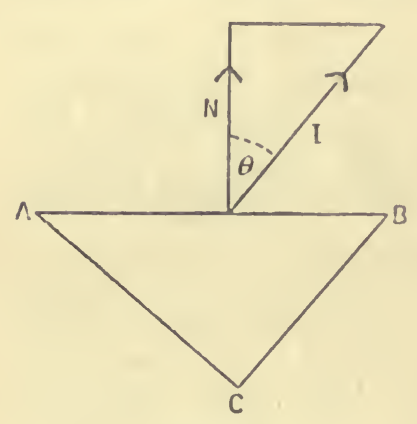

Fra. 31 .

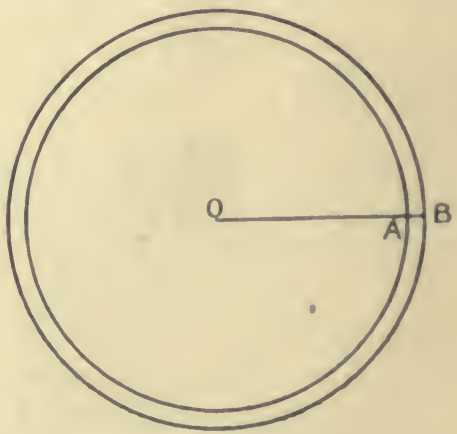

Fio. 32.

through a closed surface is cqual to $4 \pi \times$ charge zeithin, or is squal to the number of lines of force sent ont from that charge.

Fluid displacement tubes used to prove the properties of tubes of force. The properties of tubes of force may be deduced from the properties of "tubes of displacement" in an incompressible fluid, and as these tubes of displacement form a valuable symbolic representation of the tubes of force in magnetic -and to some extent in electric-systems, we shall here add this mode of proof.

Imagine space to be filled with an incompressible fluid with "sources" at various points at which fresh fluid can be introduced, and "sinks" at other points at which fluid can be withdrawn. If a small volume $v$ of fluid be introduced at any point $O$, then to make room for it an equal volume $v$ must be pushed out through every surface surrounding $O$. Draw a. sphere round $O$, Fig. 32, with radius $\mathrm{OA}=r$, and let the fluid originally lying in its surface be pushed out to the concentric spherical surface with radius $\mathrm{OB}=r+d$. The volume which has passed through the inner surface is equal to that contained between the two surfaces. Since 
it is small, $d$ also is small, and the volume between the surfaces is $4 \pi r^{2} d$. Hence

or

$$
\begin{aligned}
& v=4 \pi r^{2} d \\
& d=\frac{v}{4 \pi} \cdot \frac{1}{r^{2}}
\end{aligned}
$$

That is, the displacement of any particle varies inversely as the square of its distance from the source.

Now imagine an electrical system in which a charge $q$ is put at $\mathrm{O}$. The intensity will be numerically equal to the fluid displacement if we make $q=\frac{z}{4 \pi}$. If the point $O$ is $\Omega$ sink instead of a source, and if volume $v$ of fluid is removed, the displacement is reversed and is equal to the intensity due to a quantity of negative electricity $-q=-\frac{v}{4 \pi}$ placed at the sink.

Evidently if the quantities of fluid introduced or removed at the various points of a system are exceedingly small, we may compound the displacements due to each separately, according to the vector law, in order to obtain the resultant displacement. We may, therefore, imagine a fluid system corresponding to any extended gravitative, magnetic, or electric system, the matter in a gravitative system being replaced by a series of sinks, while Northseeking magnetism and positive electricity are replaced by sources, and South-seeking magnetism and negative electricity are replaced by sinks. The quantities of fluid introduced or withdrawn are proportional to the quantities of matter, magnetism, or electricity which they represent. Lines and tubes of flow in the fluid system will then follow the same course as lines and tubes of force in the corresponding force systems. Drawing a displacement tube in the fluid system, since no fluid passes out through its walls, equal quantities must pass across every section in any part of the tube not containing sources or sinks. If, then, $d_{1} d_{2}$ be the displacements at two sections $\mathrm{S}_{1} \mathrm{~S}_{2}, d_{1} \mathrm{~S}_{1}=d_{2} \mathrm{~S}_{2}$, or displacement $\times$ cross-section is constant. Replacing displacements by the corresponding and proportional intensities, we obtain for a tube of force the corresponding property that intensity $\times$ cross-section is constant.

We may also prove easily the other properties of tubes of force, but these we leave to the reader.

We shall now find the value of the intensity in some simple cases.

Spherical shell uniformly charged. Intensity outside the shell. Let $\mathrm{S}$ be a sphere, Fig. 33, over which a charge $Q$ is uniformly spread. Let $S_{1}$ be a concentric sphere outside $S$ and of radius $r_{1}$. The intensity over $S_{1}$ is, from the symmetry of the system, everywhere perpendicular to $S_{1}$ and everywhere of the same magnitude. I et it be equal to I. 'Then 
by Gauss's theorem $4 \pi r_{1}{ }^{2} I=4 \pi Q$ or $I=\frac{Q}{r_{1}{ }^{2}}$, that is, it is the same as if $Q$ were all concentrated at the centre.

It is obvious that for a gravitating sphere arranged in concentric shells, each of uniform density, the same result holds, and

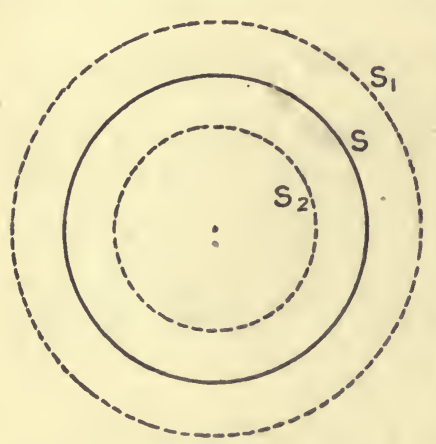

Fua. 33. the attractive intensity at a point outside the sphere is the same as if the whole mass were collected at the centre.

Intensity inside the shell. Now draw a concentric sphere $S_{2}$ of radius $r_{2}$ inside the shell $\mathrm{S}$. 'I'the intensity $\mathrm{I}$ over $\mathrm{S}_{2}$ is from symmetry everywhere normal to $S_{2}$ and everywhere the same in magnitude. Then $4 \pi r_{2}^{2} \mathrm{I}=4 \pi \times 0=0$, since there is no charge within $S_{2}$. Hence $I=0$ every. where within $S_{1}$.

It is obvious that if $\mathbf{S}$ is the surface of a gravitating sphere arranged in concentric shells, each of uniform density, the shells outside $\mathrm{S}_{2}$ produce no intensity at $\mathrm{S}_{2}$, while the shells within act as if all collected at the centre.

Intensity at any point in the axis of a uniformly charged circular disc. Let AB, Fig. 34, be a diameter of the disc, and CP its axis. The intensity at $\mathrm{P}$ will, from symmetry, be along CP. Let the charge per unit area of the disc be $\sigma$. 'This is

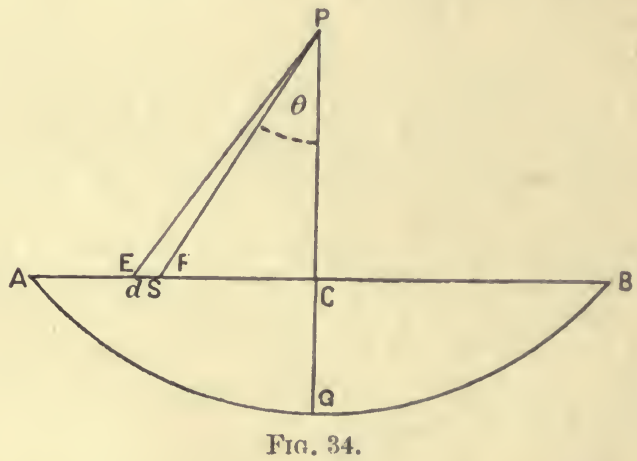

termed the surface density of charge. 'To find the contribution of any element to the intensity we need only consider the resolved part along CP. Take an element of surface $d S$ with section $\mathbf{E F}$ and let $\mathbf{F P C}=\theta$. The intensity due to it along $\mathbf{C P}$ is $\frac{\sigma d \mathrm{~S} \cos \theta}{\mathrm{PF}^{2}}$. But if we draw a cone with $\mathbf{P}$ as vertex and $d \mathbf{S}$ as 
base, $\theta$ is the angle between $d \mathbf{S}$ and the cross-section perpendicular to PF. The solid angle of the cone is therefore $d \omega=\frac{d \mathrm{~S} \cos \theta}{\mathrm{PF}^{2}}$. Hence $d \mathbf{S}$ contributes $\sigma d \omega$.

'The whole disc then gives intensity $\sigma \times$.solid angle subtended by the disc. If we draw a sphere radius PA the area of this sphere cut off by $\mathrm{AB}$ is $2 \pi$.PA.CG, where $\mathrm{PG}$ is the radius through $\mathrm{C}$, and the solid angle which it subtends at $\mathrm{P}$ is $2 \pi \mathrm{PA} . \mathrm{CG} / \mathrm{PA}^{2}$ $=2 \pi \mathrm{PA}(\mathrm{P} A-\mathrm{PC}) / \mathrm{PA}^{2}=2 \pi(1-\cos a)$, where $\alpha=\mathrm{APC}$.

The intensity at $\mathrm{P}$ is therefore $2 \pi \sigma(1-\cos \alpha)$. If the radius of the dise is very large compared with $\mathrm{PC}, \alpha$ is very nearly $90^{\circ}$, and the intensity is very nearly $2 \pi \sigma$.

Whatever the form of the disc may be, so long as it is plane the intensity will still be $2 \pi \sigma$ if the radius to the nearest point of the edge makes an angle with P'C indistinguishable from 90 '. Fol the whole of the area outside that distance subtends a vanishing solid angle at $P$.

Intensity due to a very long uniformly charged cylinder near the middle. In the plane perpendicular to the axis of the cylinder and bisecting it the intensity is evidently radial. It will be radial, too, at a distance from this plane small compared with the distance from either end, if the distance from the axis is also small compared with the distance from the ends. For if EE', Fig. 35, are the ends, and $C^{\prime}$ is the central point, take a

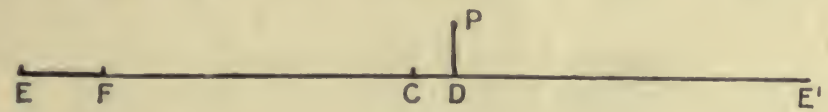

Fig. 35.

point $\mathrm{P}$, not quite in the plane through $\mathrm{C}$. Draw PD perpendicular to the axis and make $\mathrm{DF}=\mathrm{DEE}:$. 'The intensity due to $\mathrm{FE}^{\prime}$ is evidently radial. That due to $\mathrm{EF}$ is in comparison negligible, since EF is by supposition not large, and it is very distant from $\mathbf{P}$ as compared with the part of the cylinder immediately under $\mathbf{P}$. Hence the intensity at $\mathbf{P}$ is radial. Further, if the position of $\mathbf{P}$ changes by a small amount parallel to the axis the intensity is only changed by a removal of a small length from one end of the cylinder to the other with negligible effect. Draw a cylinder radius $r$ and length $\mathbf{P Q}=1$, Fig. 36, co-axial with the charged cylinder, and apply Gauss's theorem to the surface thus formed. 'The intensity I, as we have just seen, is normal over the circular surface and everywhere the same, and it has no normal component over the flat ends of the cylinder $P Q$. The quantity of charge within is $2 \pi a \sigma$, where $a$ is the radius of the charged surface.

Then

$$
\begin{aligned}
\int \mathrm{N} d \mathbf{S} & =2 \pi r \mathrm{I}=4 \pi \times 2 \pi u \sigma \\
\mathrm{I} & =4 \pi \| \sigma / r .
\end{aligned}
$$

and 
If $e$ is the charge per unit length $=2 \pi a \sigma$

$$
\mathrm{I}=2 e / r .
$$

If $\mathbf{P}$ is inside the charged surface, the cylinder drawn through it now contains no charge, and we see at once that $I=0$.

Potential. The description of inverse square systems is very much assisted by the use of a quantity termed the potential, which we shall define as follows:

The potential at a point. If $q$ is an element of charge and $r$ is its distance from the point, the sum of all the terms $q / r$,

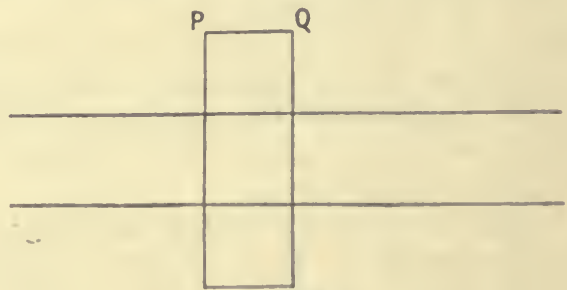

Fı. 36.

where each element of charge in the system is divided by its distance from the point, is termed the potential at the point. The potential is usually denoted by $\mathrm{V}$. We have then $\mathrm{V}=\Sigma q / r$.

We shall show that the potential at $a$ point is equal to the work done in bringing a small body charged with unit quantity from an infinite distance or from outside the field up to the point against the forces due to the system.

Let an element of charge $q$ be at the point O, Fig. 3\%, and let the small body with unit charge be moved from a point $\mathrm{A}$ by

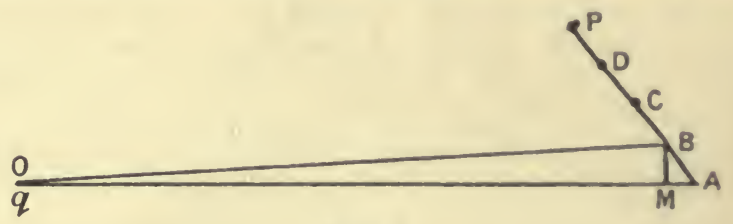

Fic. 37.

any path $\mathrm{ABC}$ to $\mathrm{P}$. Let $\mathrm{B}$ be a point very near to $\mathrm{A}$ and let BM be perpendicular to OA. 'The work done in moving the unit from $\mathrm{A}$ to $\mathrm{B}$ is $\frac{q}{\mathrm{OA}^{2}}$. AM, since $\mathrm{AM}$ is the distance moved in the direction of the force. But since $A B$ is very small, we may put $\mathrm{AM}=\mathrm{OA}-\mathrm{OB}$ and $\mathrm{OA}^{2}=\mathrm{OA} . \mathrm{OB}$.

The work done is therefore $q \frac{\mathrm{OA}-\mathrm{OB}}{\mathrm{OA} \cdot \mathrm{OB}}=q\left(\frac{1}{\mathrm{OB}}-\frac{1}{\mathrm{OA}}\right)$. 
Similarly the work done along $\quad \mathrm{BC}=q\left(\frac{1}{\mathrm{OC}}-\frac{1}{\mathrm{OB}}\right)$,

$$
\text { and } \quad, \quad, \quad, \quad \quad \quad \quad \quad \mathrm{CD}=q\left(\frac{1}{\mathrm{OD}}-\frac{1}{\mathrm{OC}}\right) \text {, }
$$

and so on.

When we add up, evidently all the terms but the first and last occur twice and with opposite signs, and the total work done $=q\left(\frac{1}{\mathrm{OP}}-\frac{1}{\mathrm{OA}}\right)$.

The work done from $\mathbf{A}$ to $\mathbf{P}$ is, therefore, the same, whatever the path from A to $\mathbf{P}$.

If $\mathrm{A}$ is at an infinite distance the work done to $\mathrm{P}$ is $q / \mathrm{OP}$. 'That is, it is equal to the potential at $\mathbf{P}$ due to $\mathbf{Q}$.

'The work done from $\mathrm{A}$ to $\mathrm{P}$ is equal to the difference of the potentials due to $q$ at $\mathrm{P}$ and $\mathrm{A}$.

In any extended system we may divide the charges into elements so small that each may be regarded as at a definite point. The intensity at any point in the field is the resultant of the intensities due to the separate elements, and the resolute of the intensity in any direction is the algebraic sum of the resolutes of all the separate intensities in that direction. The work done in moving unit quantity along any path is, therefore, the sum of the works done against the forces due to the separate elements.

Hence the work done in moving the unit from an infinite distance to the point $\mathbf{P}$ in the field is $\Sigma \frac{q}{O P}$, or is equal to the potential at the point. The work done in moving from $P$ to $Q$ is evidently $\Sigma \frac{q}{O()}-\Sigma \frac{q}{O P}$, or is equal to the difference of potentials at $Q$ and $P$.

The resolute of the intensity in any direction in terms of potential variation. I Let $P Q$, Fig. 38 , be a given direction, and let $\mathbf{X}$ be the resolute of the intensity in that direction.

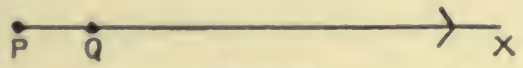

F10. 38.

Iet $V_{p} V_{2}$ denote the potentials at $P$ and $Q$, two neighbouring points. The work done agninst $\mathbf{X}$ in going from $\mathbf{P}$ to $Q$ is $-\mathbf{X} . \mathbf{P Q}$, since we have supposed $\mathbf{X}$ to act from $\mathbf{P}$ to $\mathbf{Q}$. Then $V_{2}-V_{P}=-X \cdot P Q$

or

$$
X=-\frac{V_{Q}-V_{P}}{P Q} .
$$

If we denote $P Q$ by $d x$, then in the limit

$$
\mathrm{X}=-\frac{d V}{d \cdot x} \text {. }
$$


Equipotential surfaces. We have seen that we may assign to each point in a field a definite number expressing its potential. I.et us suppose a surface drawn through all points having the same number indicating their potential. Such a surface is termed an equipotential surface or a level surface. Since it is all at one potential, no work is done in moving unit quantity in a path lying on the surface. 'The intensity must, therefore, be normal to the surface. In other words, it cuts the lines of force everywhere at right angles. If we draw a series of equipotential surfaces with unit difference of potential between successive members of the series, their distances apart show the magnitude of the intensity everywhere. For if $I$ is the intensity at any point on one surface and $d$ the distance of the point from the next surface, $\mathrm{I} d$ is the work done on the unit in going from one surface to the next. But this is by supposition unit work, then $\mathbf{I} d=1$ or $\mathrm{I}=1 / d$.

The energy of a system in terms of the charges and potentials. If there is a charge $q_{1}$ at a point $\Lambda$, the work done in bringing up unit charge to a point $B$ against the force due to $q_{1}$ is $q_{1} / \mathrm{AB}$. If instead of unit charge we bring up charge $\eta_{2}$, the work done is $q_{1} q_{2} / \mathrm{AB}$.

Let us suppose that we have a systen consisting of elementary charges $q_{1}$ at $\mathrm{A}, q_{2}$ at $\mathrm{B}, q_{3}$ at $\mathrm{C}$, and so on, and let us put $\mathrm{AB}=r_{12}$. $\mathrm{AC}=r_{13}, \mathrm{BC}=r_{23}$, and so on. Imagine that initially $q_{1}$ only is in position, and that all the other charges are at infinite distances away and apart from each other. Bring $q_{2}$ up to B. The work done is $\frac{q_{1} q_{2}}{r_{12}}$. Now bring $q_{3}$ up to $\mathrm{C}$ and the work done is

$$
\frac{q_{1} q_{3}}{r_{13}}+\frac{q_{2} q_{3}}{r_{23}}
$$

Continue this process till the system is built up, and evidently we shall have the product of each pair of charges divided by their distance apart occurring once and once only. The total work done, or the potential energy of the system, is therefore $\Sigma \frac{q_{m} q_{n}}{r_{m a}}$, where we sum for all pairs of elementary charges.

Now suppose that all the charges are in position except $q_{1}$, which is at an infinite distance. The work done in bringing $q_{1}$ into position is

$$
\begin{aligned}
& \frac{q_{1} q_{2}}{r_{12}}+\frac{q_{1} q_{3}}{r_{13}}+\ldots \\
& =q_{1}\left(\frac{q_{2}}{r_{12}}+\frac{q_{3}}{r_{13}}+\ldots\right) \\
& =q_{1} V_{1}
\end{aligned}
$$

where $V_{1}$ is the potential at $A$ due to the rest of the system. 
Next suppose that all the charges are in position except $q_{2}$, which is at an infinite distance. 'The work done in bringing $q_{2}$ into position is

$$
\begin{aligned}
& \frac{\eta_{2} \eta_{1}}{r_{12}}+\frac{\eta_{2} \eta_{3}}{r_{23}}+\ldots . \\
& =\eta_{2}\left(\frac{q}{r_{12}}+\frac{\eta_{3}}{r_{23}}+\ldots\right) \\
& =\eta_{2} V_{2}
\end{aligned}
$$

where $\mathrm{V}_{2}$ is the potential at $\mathbf{B}$ due to the rest of the system.

Proceeding thus with each element in turn, the total work is $\eta_{1} V_{1}+q_{2} V_{2}+q_{3} V_{3}+\& c .=\Sigma_{q} V$. But in the work thus done each product such as $\frac{\eta_{\mathrm{m}} \eta_{\mathrm{n}}}{r_{\min }}$ occurs twice, and twice only, once when $\eta_{\mathrm{m}}$ is brought up, and once when $\eta_{n}$ is brought up, and therefore the total work done is twice the potential energy of the system. We have therefore

$$
\text { Potential energy of the system }=\frac{1}{2} \Sigma_{\mathcal{I}} \mathrm{V},
$$

where each element of charge is multiplied by its potential.

We may illustrate these results by considering

The potential due to a uniformly charged sphere at points without and within its surface. I et a charge $Q$ be distributed uniformly over a splere radius $\mathrm{OA}=a$, Fig. 39. We

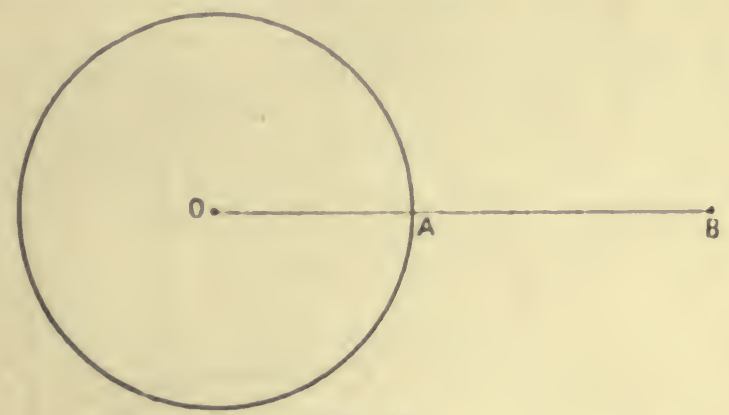

Fiv. 39.

have shown (p. 39) that the intensity due to the charge at any outside point $B$ is the same as if the charge were concentrated at $O$. Hence the work done in bringing up unit charge from an infinite distance to $B$ is the same as if $Q$ were concentrated at $O$ and $V_{B}=\frac{Q}{r}$, where $r=0 B$. Within the sphere, as we have shown, the intensity is everywhere \%ero, so that the potential is constant, 
since no work is done in carrying the unit from point to point. It is, therefore, the same as the value at $O$. As all the charge is at the same distance from $\mathrm{O}$,

$$
\mathrm{V}_{\mathrm{o}}=\Sigma \frac{q}{r}=\frac{Q}{\mathrm{OA}}=\frac{\mathrm{Q}}{\mathrm{a}} \text {. }
$$

The same result is obtained by putting the potential of the sphere equal to the value at $\mathrm{A}$, which is obtained by supposing $\mathrm{B}$ to move up to $A$, whence

$$
\mathrm{V}_{\mathrm{A}}=\frac{\mathrm{Q}}{\mathrm{a}} \text {. }
$$

The energy of the charge, since it is all at the same potential, is

$$
\frac{1}{2} Q V=\frac{1}{2} Q^{2} / a \text {. }
$$




\title{
CHAPTER IV
}

\section{THE FIELD CONSIDERED WITH REGARD TO THE INDUCTION OR ELECTRIC STRAIN PRODUCED IN IT}

\begin{abstract}
The two kinds of charge always present and facing each other-Electric action in the medium-Electric strain-No strain in conductors when the charges are at rest-Direction of electric strain-The electric strain just outside a conductor is normal to the surface of the conductorMagnitude of electric strain-Variation of electric strain with distance from the charged bodies: The inverse square law-Lines and tubes of strain- Cnit tube-Results deduced from the inverse square law - The transference of tubes of strain from one charge to another when the clanged bodies more-Molecular bypothesis of electric strain.
\end{abstract}

The two kinds of charge always present and facing each other. Experiments of the kind described in Chapter II show that the two kinds of electrification are always produced together in equal quantities, that they accompany each other while they continue in existence, and that on ceasing to exist they disappear in equal quantities. Sometimes, in experimenting, we devote all our attention to a charge of one kind alone and speak as if it existed independently; but it is of the greatest importance to remember that the opposite or complementary charge is really in existence, perhaps on the neighbouring surface of a non-conductor user to develop the electrification which we are studying, or perhaps on the conducting table, floor, or walls of the room, so that not only is it in existence, but is in the presence of the inducing charge. If we have a charge in one room we cannot have the complementary charge in another. If we attempt thus to separate the two kinds each will at once induce its own opposite on the inner surface of the surrounding conducting walls. Again, when we put a charged conductor "to earth" we sometimes speak of the disappearance of the charge as if it merely spread away to the infinite conducting earth. But in fact we are making a conducting bridge by means of which the charge and its opposite may come together and neutralise each other. The two kinds of charge, then, are always present together in the electric field, each, as it were, inducing the other. As Faraday * put it, "Bodies 
cannot be charged absolutely," i.e. with one kind of electrification alone in existence, "but only relatively" to other bodies somewhere in their presence which take up the opposite charge "and by a principle which is the same with that of induction. All charge is sustained by induction."

We may arrange our experimental knowledge of induction more clearly by the aid of a hypothesis, originally due to Faraday and subsequently developed and extended by Maxwell. We shall here give a general account of this hypothesis, filling in the details as they become necessary for the explanation of the various points in the theory of electric action.

Electric action in the medium. Let us suppose that a charged insulated body is placed within a hollow conductor. We may think, for instance, of a charged brass sphere hanging by a silk thread in the middle of a room. Then there will be an opposite charge on the walls. In describing the two opposing surfaces as electrified the most important experimental fact which we counote is that the two surfaces are being pulled each towards the other. 'This action was formerly supposed to be direct, each little bit of wall surface, for instance, pulling at each bit of brass surface at a distance, the intervening matter playing no part in the action and being unaffected by the existence of the two electrified surfaces between which it lay. But Faraday succeeded in showing that the action varies with the nature of the intervening insulator, and we may describe the variation by saying that the pull on the electrified surfaces with given charges is greater with some insulators than with others. 'I'his fact is entirely unexplained, unless we adopt the view taken by Faraday that the insulator plays an essential part in the action. He supposed that it is altered in some way during the process of electrification, so that it exerts pulls on the conducting surfaces with which it is in contact. In fact, he regarded electrical actions as similar to the drawing towards each other of two masses connected by a stretched indiarubber cord. In the case of a cord the pulls on the masses depend on the nature, quality, and strain of the cord, and can be expressed in terms of the strain. The energy which appears in the motion of the masses is regarded as having been previously stored up as strain energy in the cord during its stretching. So the electrical pulls are supposed to be accompanied by a condition in the in. sulating medium analogous to strain and to be expressible in terms of this condition, which we may call electrical strain. 'The electrical energy is supposed to be stored in the medium during the electrification and to pass out of it again on the motion of the charged bodies towards each other or on the neutralisation of their charges. The electrification of a conducting surface may therefore be regarded as a surface manifestation of an alteration in the state of the contiguous insulator, somewhat as the pressure on the ram of a hydraulic press may be regarded as a surface manifestation of 
the compressed state of the water which connects it with the plunger. When Faraday first gave this account of electrical action it was only supported by the variation of the effect of a given charge with variation of the medium, and no independent evidence of the state of "electrical strain" was in existence. But it has been discovered that some insulating media when between charged surfaces are affected in regard to the transmission of light. Unless we make the further and very far-fetched hypothesis that the light itself is acted on at a distance by the charged surfaces, Faraday's supposition becomes for these media a proved fact. We have also good evidence of electrical strain in the electro-magnetic waves discovered by Hertz and used now in wireless telegraphy. We know only one way of explaining the phenomena discovered by Hertz, viz. by the supposition that the disturbances are waves travelling through the air with a definite velocity. This implies the existence of both electric and magnetic energy in the air. The electric energy implies the existence of that which we have called electric strain. It is also called electric polarisation, electric displacement (Maxwell) and electric induction (Faraday).

No strain within conductors when the charges are at rest. When the charges and the charged bodies in a system are at rest this condition of electric strain is confined to the air or other insulator between the charged snrfaces and does not occur within the substance of the conductors. 'This is evident if we consider the experiments already described with closed conductors. These show that a hollow closed conductor may be regarded as entirely screening the space within from the space without. No electrification is induced on bodies within by external charges; no mutual pull is exerted on an electrified surface inside by an electrified surface outside. Interpreting these facts by Faraday's hypothesis, we must suppose that the substance of the conductor is not itself in a state of electric strain. If it can acquire that condition it rapidly loses it, being incapable of permanently storing electric energy. The electric strain which exists in the insulator and which is manifested by a pull on the charged surface of a conductor ceases at that surface. 'I'he pull outwards on the conductor, of course, strains it, and corresponding to this strain there is a stress between the external lnyer and the layer beneath it which neutralises the outward electrical pull. But the strain in the conductor is an ordinary elastic strain, not an "electric strain," a change of shape of a visible kind which can be calculated from the elastic properties of the conductor, though it is too small to be seen and there are no recognised electric phenomena connected with it. 'The nature of the electric strain in the insulating medium is at present unknown, though we may make guesses as to its nature, and we only term it "strain " from analogy, since there are forces accompanying it somewhat resembling those accompanying elastic strain. Taking this view of the nature and function of 
the insulator, it is usual to call it the Dielectric. The region round a system in which electric strain is manifested is termed the Field of the system.

Even though at present we can give no complete explanation of electric strain, we can still compare the magnitude of the strain at different points of the field by means of some chosen effect due to it, and we may also assign direction to it. For instance, the magnitude may be taken as proportional to the force on a very small charged body, the direction coinciding with that of the force exerted. Or some other effect may be taken, such as the charge which gathers on either side of a proof plane when held perpendicularly to the direction of the force.

Similarly, without attempting to explain the nature of a beam of light when traversing a transparent medium, we may assign direction to it at any point-say that of the normal to a surface held in the position of maximum illumination-and we may compare the magnitude at different points by the illumination of an interposed surface.

The direction of electric strain. Iet us suppose that a small insulated conducting sphere is placed at any point in the field. Then one hemisphere will be positively, the other negratively electrified. 'This we know from direct experiment. There will be one diameter about which each charge is symmetrically arranged if the sphere is sufficiently small, its ends being at the two points of maximum electrification. We may define the direction of electric strain as that of the dinmeter so drawn, and we shall consider it as drawn from the negative towards the positive electrification.

But we shall have similar symmetrical electrification at the two ends of any small conductor itself symmetrical about an axis and

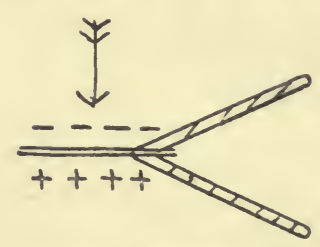

Fig. 40. an equatorial plane, when the axis is placed parallel to the direction of strain as above defined. For instance, if two proof planes be held together in the position shown in Fig. 40, the arrow-head denoting the direction of strain, the one is entirely positively, the other entirely negatively electrified, and on separating the two while in this position the charges may be shown to be equal and opposite by the electroscope. Further, the charge on ench plane is a maximum when so held. For on holding the planes at some other angle to the strain, and then separating and testing the charges, these will be found appreciably less than when held as in Fig. 40. When the planes are as in Fig. 41 the electrification is as indicated, and on separating and testing the planes they are found to be unelectrified. From this effect of induction, combined with the tendency of a positively electrified body to move with, and a negatively electrified body to move against, the strain, we may obtain a 
very easy mode of indicating the direction of electric strain. Suspending a small elongated conductor, say a piece of a match, a straight cotton fibre, or a short piece of wire, by a cocoon fibre passing as nearly as possible through its centre of gravity, the two ends will be oppositely electrified unless the conductor is at right angles to the direction of strain. When so electrified the ends will be acted on by forces constituting a couple, which will set the conductor with its axis along the direction of strain as a position of stable equilibrium. The position at right angles is evidently unstable. Such a conductor may be termed an "electric pointer."

Finally, we may indicate the direction of strain by the direction of the force on a very small positively charged body, say a pith ball hung by a silk fibre. Its charge should be so small that surrounding charges are not sensibly affected by its presence.

Direct experiments in the field round a charged globe or round the knob of a charged Leyden jar

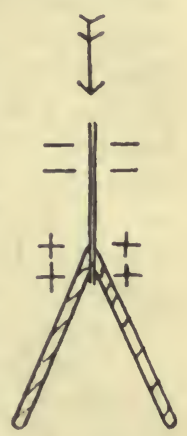

Fia. 41. show that the three methods-(1) by two proof planes, (2) by an electric pointer, (3) by a charged pith ball-give the same direction for the strain.

We may use any of these tests for the direction to prove that

The electric strain just outside a conductor is normal to the surface of the conductor. Perhaps the simplest proof of this is attained by suspending a small electric pointer from the end of a thin ebonite or shellac rod and bringing it near the surface of an insulated conductor, when it will always take the direction of the normal. A very striking verification is obtained by fixing the pointer at some little distance from the conductor and then introducing another conductor, say the hand, into the field and gradually bringing it up to the pointer. When the second conductor is brought quite close to the pointer it will be normal to this second surface. If pith balls be fixed by short silk fibres to the sides of a conductor, when the conductor receires a charge they will all hang in positions agreeing with the supposition that the strain is normal to the surface. Or if two proof planes be used, they give the naximum charge on separation after being held parallel to the surface and close to it, and no charge when they have been held perpendicular to the surface. 'This last method enables us also to show that the direction of the strain is from a positively electrified surface and towards one negatively electrified. The law thus proved may be regarded as a case of the more general law that positive electrification tends to move in the direction of strain and negative electrification against it. Had the strain a component parallel to the surface of the conductor, the electrification would move either with or agrainst that component. Such motion of the charge does actually occur while a body is receiving its electrification. The 
strain is not then altogether normal to the surface, and the electrification moves about until a distribution is attained such that the strain is normal to the surface. Then there is equilibrium, for, the conductor being surrounded by an insulator, the electrification can move no further. In the case of a conducting circuit carrying a current produced, we may suppose, by a voltaic cell, equilibrium does not exist, and the strain has a component along the surface of the wire from the copper terminal towards the zinc terminal, and therefore either positive electrification moves with it or negative electrification moves against it, this motion of electrification being one of the chief features of the current. While the motion goes on there is also strain in the conductor itself, but such strain can only be maintained by perpetual renewal, the energy for the renewal being supplied by the cell.

The magnitude of electric strain just outside a conductor. Usually we are practically concermed with the magnitude of the strain only where it ceases at the surface of a conductor. We shall take the amount of charge gathering per unit area on the conductor as the measure of the strain there. For instance, if on a given conductor there is at one time twice the charge per unit area that there is at another time, the strain just outside the conductor in the former case is twice as much as in the latter.*

Definition of surface density. The charge per unit area on a conducting surface is termed the surface density. The surface density, then, may be taken as measuring the strain in the insulator just outside the conductor. If we wish to measure the strain at a point not close to a conducting surface we may bring a small conductor there and note the charge gathering per unit area on one side or the other. But in general the introduction of a conductor into a system alters the strain in the part of the field immediately around it, so that the charge per unit area does not in general ineasure the original strain, though it is probably proportionate to it. But there is one case in which the introduction of a conductor does not appreciably affect the strain in its neighbourhood, viz. when the conductor is a small exceedingly thin conducting plane held perpendicular to the direction of strain, and this case may be more or less nearly approached in practice by the proof plane. By using a pair of exceedingly thin equal proof planes of known area we may measure, at least in imagination, the strain at a point by holding the planes in contact at the point and normal to the strain, then separating them and measuring the charge of either. The strain equals the charge per unit area. We shall therefure take as a definition of the magnitude of strain the following:

\section{Measurement of strain at any point. We measure the}

* In taking this definition, it may be noted that the analogy with elactic strain fails, for elastic strain has zero dimensions, while electric straln has dimensions charge /length 2 . 
electric strain at any point in a medium by the charge per unit area gathering on a conducting surface which is introduced so as to pass through the point, but which does not alter the strain in the region immediately outside itself.

We shall usually denote the strain by $\mathrm{D}$. The quantity which is here termed electric strain is, as already stated, that which Maxwell termed "electric displacement." We have thought the former term preferable as having no meaning beyond that which we put into it. The latter term almost irresistibly carries with it the idea that electric strain is a displacement of something in the direction of induction, an idea which may prove a hindrance rather than a help.

The variation of electric strain with distance from the charged surfaces or bodies. The inverse square law. If a conducting sphere A, Fig. 42 , with a

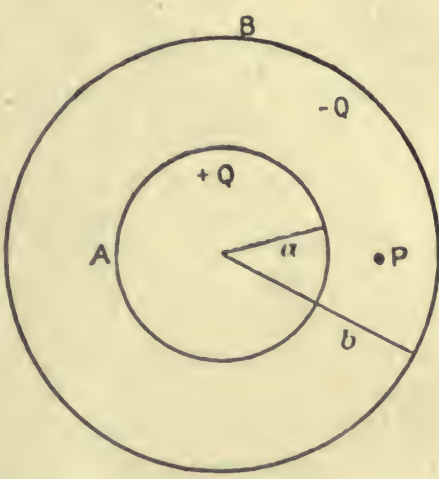

FIG. 42. charge $+Q$ is hung by an insulating thread within a hollow spherical cavity in a conductor B, the two spherical surfaces being concentric, $-\mathbf{Q}$ will be induced on the inner surface of $\mathrm{B}$. If the insulating medium is homogeneous, then from symmetry each charge is spread uniformly so that if A and B are the radii and $\sigma_{A} \sigma_{B}$ the charges per unit area or surface densities,

$$
Q=4 \pi a^{2} \sigma_{\mathrm{A}}:-Q=4 \pi l^{2} \sigma_{\mathrm{B}}
$$

whence

$$
\sigma_{A}=\frac{Q}{4 \pi a^{2}} \quad \sigma_{B}=-\frac{Q}{4 \pi b^{2}} \text {. }
$$

'Then the strain in the medium just outside each surface is from the centre and inversely as the square of the distance from the centre. If we suppose the radius of the outer surface reduced till it passes through a point close to $\mathrm{P}$ distant $r$ from the centre, the strain at $P$ is now mensured by $\frac{Q}{4 \pi r^{2}}$. But there is no reason to suppose that the approach of the conductor towards the point has altered the strain in the region between the sphere through $\mathbf{P}$ and the inner fixed sphere, for the strain just outside $A$ still remains the same, viz. $\frac{Q}{4 \pi a^{2}}$, and it is difficult to imagine this constancy existing with a variation occurring in the medium a little way off.

We may therefore consider that the strain at $\mathbf{P}$ is always $\frac{Q}{4 \pi r^{2}}$ so long as $r$ is intermediate between $a$ and $b$.

'This is exactly the value for the strain which we should obtain 
by calculating it on the supposition that it is due to the direct action of the charged surfaces, each little bit of charge $q$ acting directly at its distance $d$ without regard to the nature, conducting or insulating, of the intervening matter, and producing strain $\frac{q}{4 \pi d^{2}}$ always from or towards itself according as it is positive or negative, all the strains due to the various elements being compounded as vectors. For with this law of action, as we proved in Chapter III, the charge on B will have on the whole no effect on a point within its surface, while the charge on A will have the same effect as if collected at its centre, giving therefore $\frac{Q}{4 \pi r^{2}}$ as the resultant. Within $\mathbf{A}$ and without $\mathbf{B}$ there is no strain, a result again agreeing with that obtained on the supposition of direct action. For within A the point is within both spherical surfaces, and neither will on the whole have any effect. Without B each charge will have the same effect as if collected at the centre of the sphere on which it lies. Being equal and opposite, the two charges will evidently produce equal and opposite strains neutralising each other. These results suggest the general law that -

The strain at any point of any electrical system with "homogeneous insulator may becalculated by supposing ihat it is the resultant of the strains due to separate clements of the charge, cuch acting. according to the laze charge $\div 4 \pi$ (distance) $)^{2}$, these clementary strains being compounded according to the rector luse.

Assuning this, we may pass to the general case in which we have any assigned arrangement of conducting electrified bodies of known shape possessing known charges, and separated by a homogeneous insulator. Whatever the distribution, we know (1) that there is no strain within the conductors, and (2) that the strain just outside each surface is at every point perpendicular to the surface. Assuming that the strain is everywhere, both in the conductors and in the medium, the same as if each element acted separately according to the inverse square law, it is possible to find a general mathematical expression for a distribution which will satisfy the conditions stated above, and it may be shown that there is in each case only one possible distribution. 'The mathematical expression can be interpreted numerically only in a few cases. Among these cases are an ellipsoid with a given charge, a sphere acted on inductively by a charged point, and two charged spheres in contact. In some cases the results of calculation have been verified by experiment, and we may therefore consider that the employment of the inverse square law is justified.*

* A proof of the inverse square law is frequently given which depends only on the fact that there is no strain within a closed charged conducting sphere. It is usually assigned to Cavendish, but it appears to lave been given first by Priestley in his "Flectricity," 1st ed. 1767, p. 732. Objections to this proof will le found in the Report of the British Assuciation, 1856, p. 523. 
Lines and tubes of strain. A line, straight or curved, drawn in the electric field so that at every point of its course the tangent to it coincides in direction with the strain at the point is termed a line of strain. We may suppose such a line traced out by an " electric pointer" moved always in the direction of its length, and with its positive end forward. Imagining the lines drawn closely together and in all parts of the field, they map out the field for us in regard to the direction of strain. There is no method like that of the use of iron filings in magnetism which will show the whole course of the lines, though sawdust scattered on a thin ebonite plate gives some indication of the electric field. We may, however, have a number of pointers in the field to show the direction at different points. It is interesting to study in this way the field due to the knobs of two or more charged Leyden jars. We may show, for instance, that the field due to two similar jars charged equally and oppositely from the terminals of an influence machine resembles the magnetic field of a bar magnet.

A bundle of lines of strain forms a tube of strain.

Results deduced from the inverse square law. We may at once apprly to electric strain the results proved in Chapter III for systems in which the inverse square law holds. It is important to notice that the quantity which we have there called "intensity" must be divided by $4 \pi$ to give what we here call strain, for the intensity just outside a conductor is $4 \pi \sigma$, while the strain is $\sigma$. We have

The product strain $X$ cross-section is constant for every cross-section of a given tube. Calling strain $\times$ cross-section the total strain at the section, we may say that the total strain is the same throughout the tube.

Unit tube. If a tube starts from +1 of electrification it is termed a unit tube. If the area on which that +1 is spread is $\alpha$, and the surface density is $\sigma$, then $\sigma a=1$. But if $\mathrm{D}$ be the strain iust outside the surface, $D=\sigma=\frac{1}{a}$. Then just outside the surface $\mathrm{I}_{a}=1$, and this product is constant along the tube. 'The total strain in a unit tube is, therefore, equal to unity.

A tube starting from a given quantity of positive electrification either continues indefinitely or ends on an equal quantity of negative electrification.

For example, each unit begins on +1 and ends on -1 . 'Then the statement in Chapter II, p. 25, that equal quantities of the two electrifications always induce each other is true not only of the whole charge, but also of the separate elements. We may connect each element of the one with an equal element of the other by a tube of strain.

Applying Gauss's theorem to any closed surface and remembering that we have to divide intensity by $4 \pi$ to give strain, 
'The total normal strain over any closed surface drawn in the medium is equal to the total quantity of the electrifica. tion within.

'The total quantity is, of course, the algebraic sum of the positive and negative electrifications.

If the surface is unclosed we still

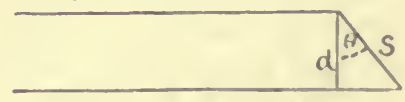

Fig. 43. have a useful expression for the total normal strain over it.

If Fig. 43 represents a unit tube with cross-section $a$ and strain D, $D_{u=1}$. Let $S$ be a cross-section making any angle $\theta$ with $a$. Then $S=\alpha / \cos \theta$. If $D_{N}$ be the component of strain normal to $\mathrm{S}, \mathrm{D}_{\mathrm{N}}=\mathrm{D} \cos \theta$. Then $\mathrm{D}_{\mathrm{N}} \mathrm{S}=\mathrm{D} \cos \theta \times \frac{\alpha}{\cos \theta}=\mathrm{D} a=1$. Or the total normal strain over any section of a unit tube is unity.

Now suppose that we have any surface through which $n$ unit tubes pass. 'The total normal strain over their ends cut off by the surface will be $n$. Or

'The total normal strain over any surface is equal to the number of unit tubes passing through the surface.

The transference of tubes of strain from one charge to another when the charged bodies move. We have seen that it follows from the inverse square law that in an electric system occupying a finite region each element of positive charge is comnected with an equal elenent of negative charge by a tube of strain. But if the charged bodies move, the pairs of elements comnected may change and we can sce in a general way how this may occur. As an illustration let us take the case of a charged body originally near a conducting table, Fig. 44, with nearly all its tubes going down to the surface.

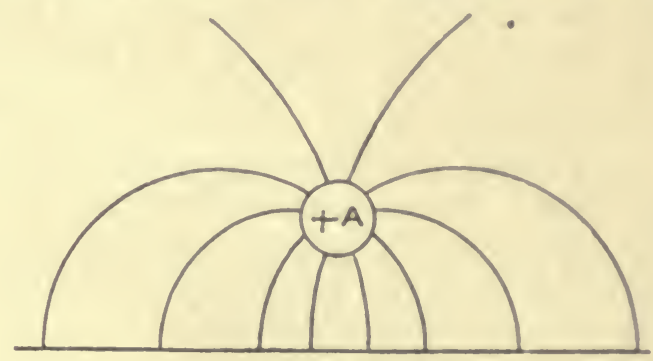

Fio. 41.

Suppose that then an insulated conductor B, Fig. 45 , is brought between $A$ and the table. If $\mathbf{B}$ were an insulator the tubes would pas through it. But the parts of the tubes within $\mathbf{B}$ will disappear, since a conductor camnot maintain any electric strain ; and we have the arrangement more or less like that in Fig. 45 , where most of the 
positive elements of charge on $A$ are now joined up to negative elements of charge on B, and positive elements on $\mathbf{B}$ are joined up to negative elements on the table.

If $\mathbf{A}$ and $\mathbf{B}$ are commected by an insulated conductor, most of

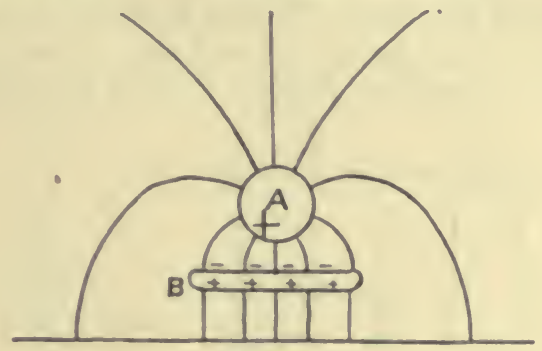

Fro. 45.

the tubes from A to B will move sideways into that conductor and disappear. Others of the tuhes from A to the table will move

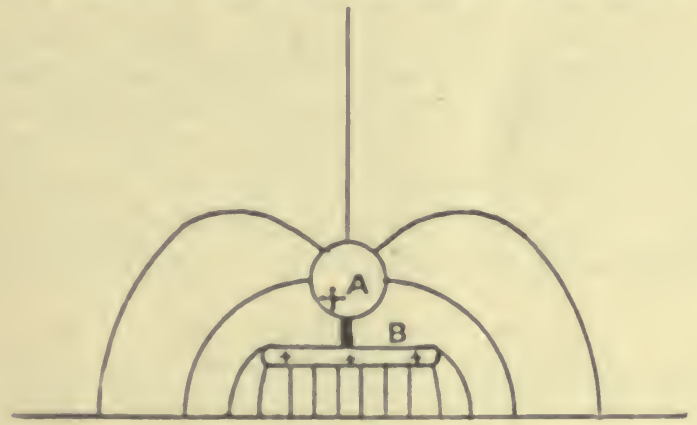

Fis. 4 \%

inwards, and the upper parts will melt away in the connecting piece, so that their positive ends will lie on $\mathbf{B}$, and we shall have
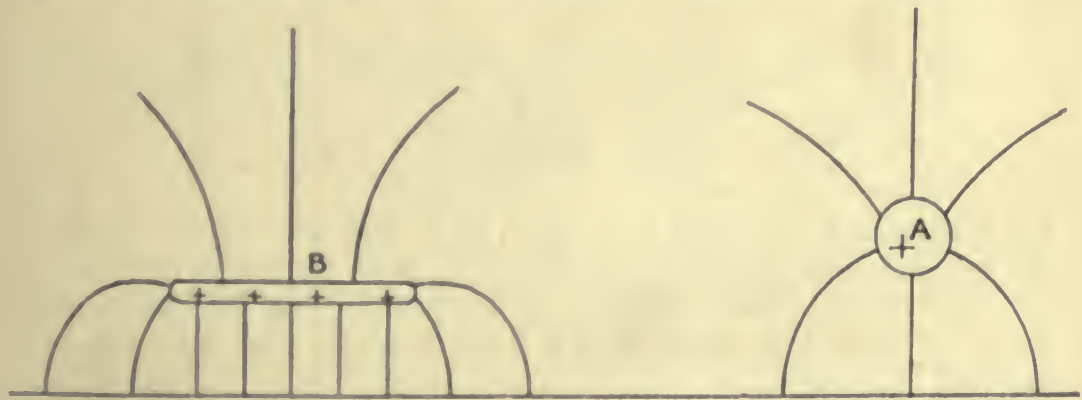

F16. 47.

more positive charge on $\mathrm{B}$ connected with the table, as in Fig. 46. If $\mathrm{A}$ is removed to a distance we shall have something like Fig. 47. 
As another illustration, suppose we have two insulated spheres charged with equal +5 and -5 respectively and near together, so that nearly all the lines of strain go from one to the other. Let us further suppose that we have two plates charged with +5 and -5 , and with five lines of strain between them, as in Fig. 48 .

Let us suppose that in the spheres the + charge is below and in the plates the + charge is above. Then the lines of strain are
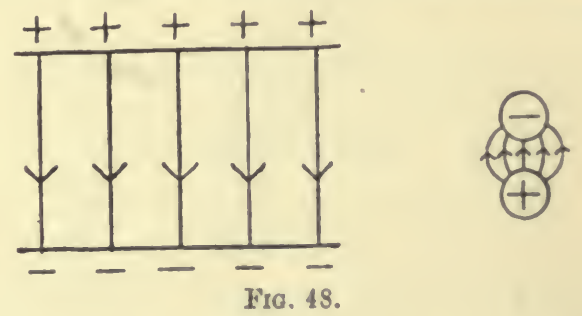

in opposite directions, as indicated by the arrows. Now move the two spheres towards and finally into the space between the plates. When the nearest lines of strain of the two systems come together the strains are in opposite directions, and where they overlap they will neutralise each other and disappear, and we may trace the successive steps in what happens to one line of strain somewhat as in Fig. 49. Repeating this process, we may inagine that in this way, say three out of the five lines of strain between the spheres

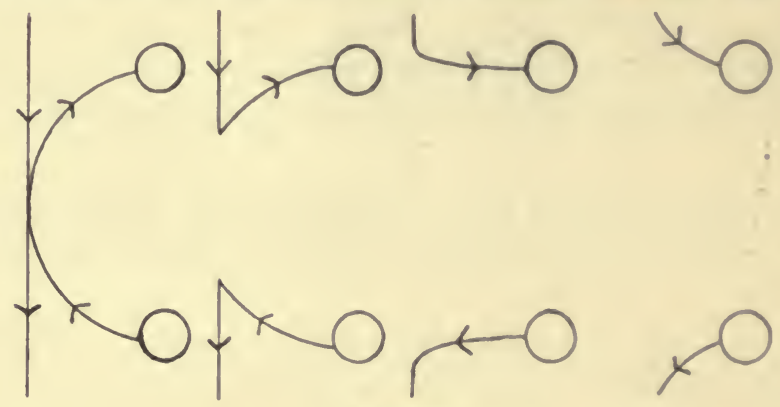

Fig. 49.

are neutralised one after the other, and that ultimately we have the arrangement shown in Fig. 50. We still have five lines from each sphere, but three come to the upper from the top plate, and three go from the lower to the bottom plate, and only two go between the spheres.

Molecular hypothesis of electric strain. It may be useful to give here a hypothetical representation of the condition of a dielectric when it is the seat of electric strain. We begin by assuming that the molecules of the dielectric are really made up 
each of two portions, one positively, the other negatively electrified, and that the two portions are connected by tubes, or, as more easily drawn, lines, of strain, and that they are pulled towards each other by the forces accompanying the strain. In making this supposition we are really identifying chemical with clectric attraction.

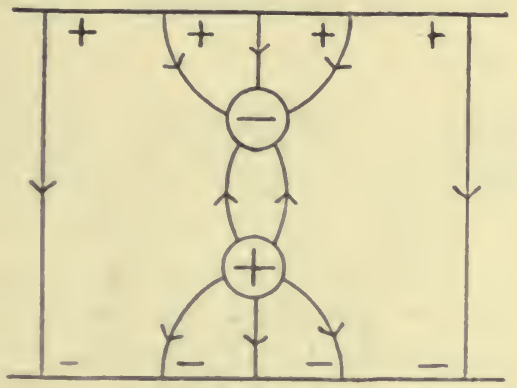

Fic. 50.

We must also have motion of the two parts of the molecule round each other, otherwise the two would be pulled into contact and the tubes would disappear. But we may for our present purpose leave the motion out of account and conventionally represent a molecule as in Fig. 51, where we suppose, for definiteness, that there are five lines of strain connecting the two parts. If the dielectric is in a neutral condition we must suppose that the molecular axes are distributed equally in all directions, so that there are equal numbers of positive and negative ends of axes facing in any direction, and there is no resultant electric action outside, and therefore no external indication of the molecular charges.

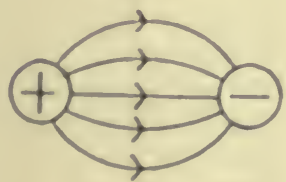

Fio. 51.

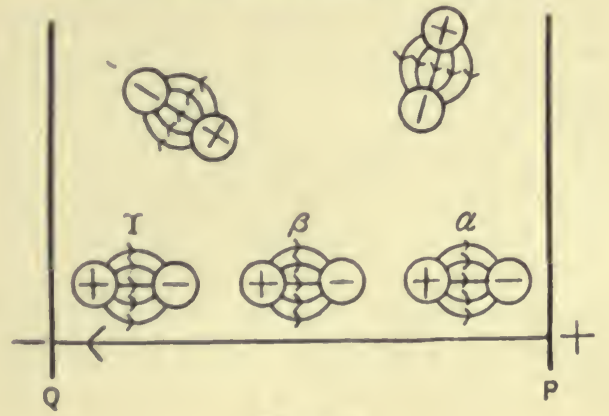

Fro. 52.

Let us now suppose that the medium is betwcen two conducting plates, Fig. 52, and that these become charged. We may suppose that, in the charging, + and - are moving along the surfaces, each pair of elements being connected by a tube of strain as $P Q$, and we may think of such a tube as picking out, as it were, the molecules 
suited for its purpose-those which will help it to stretch from plate to plate. Let $\alpha \beta \gamma$ be three such molecules. The line of strain PQ coming against these will neutralise one of the lines of strain of each in the way followed out in the illustration last considered, and we shall get the arrangement of Fig. 53 changing to that of Fig. 54, where now one line of strain goes across the spaces between

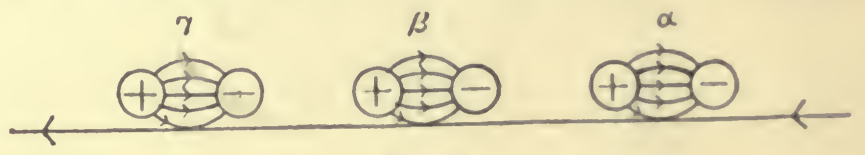

Fio. 53.

the molecules in the row forming a series of extended links in the chain. The strain between the two parts of each molecule is not only lessened, but the + of each is also now comnected up to the - of the next, and so they will tend to move apart. There is thus separation of the constituents of the molecules, the beginning of chemical dissociation, und this beginning constitutes the

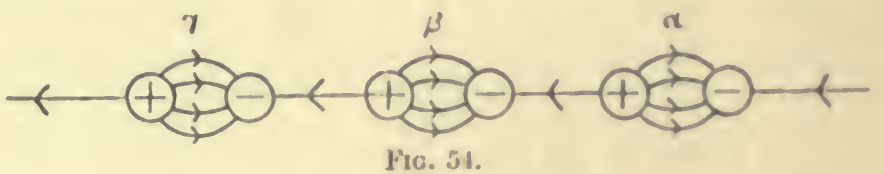

electric strain in the medium on the hypothesis considered. If a second tube moves into the same chain of molecules it will leave three tubes between each portion, and there will now be two outside, and the separation is still greater. If a third tube moves in there will be only two tubes between each portion of the original molecules, and three tubes connecting each with the opposite portion of the next, and still greater separation. If five tubes move in, then, there will be a total neutralisation of the strain between the parts of the original molecules, and each will be connected with the opposite of the adjacent molecule. There will be re-pairing all along the line and no continuity of strain. The electrification will, in fact, have been destroyed. We may imagine that something of this kind occurs when a spark dischange begins. 


\title{
CHAPTER $V$
}

\section{THE FORCE ON A SMALL CHARGED BODY IN THE FIELD, AND THE PULL OUT. WARDS PER UNIT AREA ON A CHARGED CONDUCTING SURFACE}

\begin{abstract}
The inverse square law for the force deduced from that for electric strain-Coulomb's direct measurements-General statement of the inverse square law-Electric intensity-Unit quantity-Relation between electric intensity and electric strain-Intensity just outside the surface of a conductor-Lines and tubes of force-Outward pull per vait area on a charged conducting surface-Note on the method of in restigating the fold in Chapters III-V.
\end{abstract}

The force on a small body in the fleld. Electric intensity. In the foregoing investignations of the mode in which electric charges are distributed and of the uature of electric induction, we have not considered the magnitude of the forces acting on electrified bodies or the connection between these forces and the strain existing where they are manifested. It is true that our practical method of fixing the direction of strain depends on the direction of the forces on an electric pointer, and our measure of the magnitude of strain depends ultimately on forces exerted in an electroscope either directly or indirectly by a body charged by induction. But these forces tell us nothing as to the forces acting on the body when in the electric field before it was brought into the electroscope. We shall now investigate the field with regard to the forces exerted in it. We may suppose that we explore the field by the aid of a small insulated conductor carrying a charge which is, however, so small that it does not appreciably alter the distribution of the charges on the surrounding conductors.

The inverse square law for the force deduced from the law for electric strain. We may without further experiment show that the force on a small charged body may be calculated in a similar way to the strain, i.e. by the application of the inverse square law to each element of charge. For from the fact that the strnin and the force on the small charged body everywhere coincide in direction, we can show that they must be proportional to each other. 
Let a charge $Q_{1}$ be placed at a point $A$, Fig. 5is. I.et the force on a small charged body at $P$, due to $Q_{1}$, be $F_{1}$ and let the strain be $\mathrm{PC}$. Both $\mathrm{F}_{1}$ and $\mathrm{PC}$ are along $\mathrm{AP}$, produced. If the charge $Q_{1}$ is removed and a charge $Q_{2}$ is placed at $\mathbf{B}$, let the force at $\mathrm{P}$ be $\mathrm{F}_{2}$ and let the strain be $\mathrm{PD}$, both along $\mathrm{BP}$ produced. If the two charges coexist, the direction of the force coincides still with that of the strain.

Now we know that the resulting strain represented by $P E$ is the resultant according to the vector law of the two strains repre-

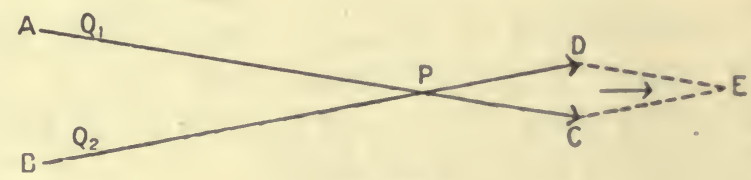

FIG. 55.

sented by $\mathrm{PC}$ and $\mathrm{P} \mathrm{D}$, and in order that the resultant of $\mathrm{F}_{1} \mathrm{~F}_{2}$ should also be in the direction PE we must have

$$
\frac{F_{1}}{F_{2}}=\frac{P C}{P I)}=\frac{\frac{Q_{1}}{4 \pi A P^{2}}}{\frac{Q_{2}}{4 \pi B P^{2}}}
$$

or the force on a given small charged body due to an element of another charged body is proportional to charge $\div$ (distance $)^{2}$.

Further, assuming that action and reaction are equal and opposite, the force is proportional to the charge on the body at P. For if that charge be $Q$ the force exerted on the body at $\Lambda$ by the body at $\mathbf{P}$ is proportional to $\frac{Q}{\mathrm{PA}^{2}}$, i.e. is proportional to $\mathbf{Q}$. Hence the reaction of the body at $\mathbf{A}$ on that at $\mathbf{P}$ which is equal and opposite is also proportional to $\mathbf{Q}$. 'Then the force exerted by the body at $\mathrm{A}$ on the body at $\mathrm{P}$ is proportional to $\frac{\mathrm{Q} Q_{1}}{\mathrm{~A} \mathrm{P}^{2}}$.

Coulomb's direct measurement of the forces between electrified bodies. Having regard to the importance of the law just stated, it is worth while to consider the direct method of obtaining it employed by its discoverer, Coulomb. To measure the force, he used the torsion balance of which the general arrangement is represented in Fig. 56. bc represents a "torsion rod" of shellac with a pith ball $b$ at one extremity, $c$ and $d$ being small loads to keep the rod horizontal. It is suspended by a fine metal wire from the torsion head $t$. 'This can be rotated and the angle through which the upper end of the wire is thus turned can be read by means of a pointer $p$ moving over a scale $s$ 
fixed at the top of the tube through which the suspending wire passes. 'The position of the torsion rod is read by means of a scale rumning round the side of the glass ease containing it. A ball $a$ is at the end of a thin vertical shellac rod which can be passed through a hole in the top of the case and fixed so that the ball is in the horizontal plane containing the torsion rod and at the same distance from the centre as the ball $b$. 'The method of using the balance will be understood from the following account of one of Coulomb's experiments.

Both $a$ and $b$ being without charge, the torsion head was turned so that $b$ was just touching $a$ when the wire was free from twist. A charge was then imparted to $a$; it was at once shared with $b$, which was then repelled through $36^{\circ}$, so that the torsion on the wire was $36^{\circ}$. The torsion head was then moved round till the angle between $a$ and $b$ was $18^{\circ}$, this requiring a motion at the head of $126^{\circ}$. 'The torsion of the wire was not, however, increased to $126+36$, as the lower end had turned in the same direction as the upper through $18^{\circ}$. The total torsion was

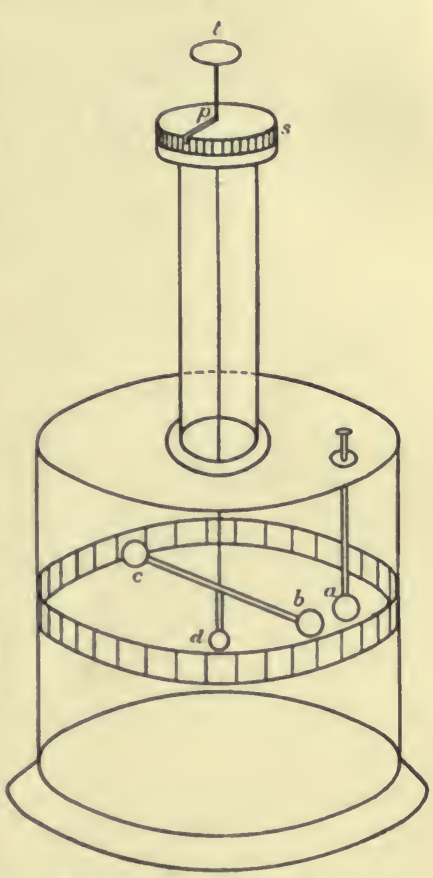

FIG. 56. therefore only $126^{\circ}+18^{\circ}=144^{\circ}$. The head was then tumed round until the angle between $a$ and $b$ was $81^{\circ}$; the additional turn of the torsion head was $441^{\circ}$, giving a total torsion of $441^{\circ}+126^{\circ}+81^{\circ}=575 \frac{1}{2}^{\circ}$.

Assuming as a first approximation that up to $36^{\circ}$ the repulsive force acts always at the same arm and that the distance between $a$ and $b$ is proportional to the angle between them, then, if the first observation is correct at a distance 36, the force is proportional to the torsion due to $36^{\circ}$.

According to the inverse square law, the torsion at $18^{\circ}$ should be $36 \times 4=144^{\circ}$, and at $8 \frac{1}{2}^{\circ}$ it should be $36 \times\left(\frac{36}{8 \frac{1}{2}}\right)^{2}=645^{\circ}$.

The last result gives a torsion very considerably in excess of that actually

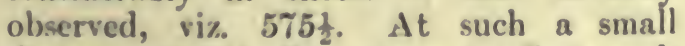
distance as $8 \frac{1}{2}$ the charges affect each other and, being like in sign, collect on the

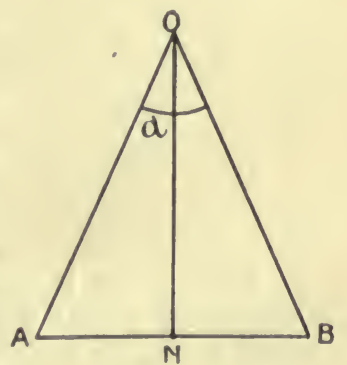

Fil. 67. 
remote sides of the two balls, so that the distaice is really greater than $82^{\circ}$.

Let us now take into account the true distance from centre to centre, i.e. the chords instead of the ares, and also the true values of the arm at which the force on the ball acts. Let (Fig. 5\%) $\mathrm{AOB}=a$, the angle between $a$ and $b$. Let $\theta$ be the torsion, and $\mu \theta$ the couple due to it. Let $F$ be the force on $b$ due to $a$, and let $\mathrm{ON}$ be perpendicular to $\mathrm{AB}$.

$$
\text { Then } \begin{aligned}
\text { Tho } & \text { FoN } & =\mu \theta \\
\text { and } & \theta & =\frac{F \cdot O N}{\mu} \\
\text { But } & O N & =\left(O A \cos \frac{a}{2}\right.
\end{aligned}
$$

and assuming the inverse square law,

$$
\begin{aligned}
& F x \frac{1}{d 1^{2}} \\
& x\left(\frac{1}{2.1 N}\right)^{2} \\
& \quad x \frac{1}{4\left(1 \Lambda^{2} \sin ^{2} \frac{a}{2}\right.}
\end{aligned}
$$

Then to verify the law we ought to find that

$$
\theta x \frac{\cos \frac{a}{2}}{\sin ^{2} \frac{a}{2}}
$$

Or that $\theta \tan \frac{\alpha}{2} \sin \frac{\alpha}{2}$ is constant. 'The values of this product for the experiments we have discussed are the following:

$\begin{array}{ccc}\alpha & \theta & \theta \tan \frac{a}{2} \sin \frac{a}{2} \\ 36^{\circ} & 36^{\circ} & 3 \cdot 615 \\ 18^{\circ} & 144^{\circ} & 3 \cdot 568 \\ 8 \frac{1}{2}^{\circ} & 575 \frac{1}{2}^{\circ} & 3 \cdot 169\end{array}$

The numbers in the third column are fairly near together.

The torsion balance may be used also for the case in which the charges on $a$ and $b$ are opposite in sign, though the experiment is not so easy when the repulsion is replaced by attraction. For within a certain distance equilibrium is unstable, a small dis- 
placement making a greater change in the attraction than in the torsion.

Coulomb also tested the inverse square law by a method which possesses interest since it corresponds to the vibrating-needle method which he employed in his magnetic experiments. An insulating shellac needle was suspended horizontally by a cocoon fibre, a small charged disc being fixed at one end of it. It was placed in the field of an insulated globe charged in the opposite way, and the time of oscillation was observed to vary directly as the distance from the centre of the globe, the result which we should expect, assuming the inverse square law.

Coulomb showed in the following manner that the force on a given charged body is proportional to the charge acting. Supposing $a$ and $b$ in the torsion balance to be exactly equal spheres, on contact they will be equally charged and the torsion rod will be repelled through a certain angle. If $a$ is now withdrawn and its charge shared with a third equal sphere, only half the charge remains on it. Replacing it in the balance, it is found that only half the torsion is needed to keep the ball $b$ at its original distance from $a$. Hence it follows that if two small bodies a distance $d$ apart contain charges $\mathbf{Q}$ and $\mathbf{Q}^{\prime}$ the force on either is proportional to $\mathbf{Q Q}^{\prime} / d^{2}$.

'Two other researches of historical interest made by Coulomb may be here referred to - that on the distribution of electrification on conductors of various forms by means of the proof plane which he invented for the purpose, and that on the rate of loss of cliarge and on the best methods of insulation. He showed that with a given conductor insulated in a given way the rate of loss was proportional to the total charge, though the rate varied on different days, being, in accordance with common experience, more considerable on damp than on dry days. Coulomb ascribed the loss partly to the air and partly to the supports, supposing that the air was a conductor when containing much water-vapour. We now know that his supposition as to water-vapour was a mistake for it, like air, is an excellent insulator. It is true that if air is nearly saturated with water-vapour the rate of loss of charge is frequently great, but this is almost certainly due to the condensation of a thin layer of water on the surface of the supports, which latter may be hygroscopic and may so condense the vapour into water before normal saturation is reached. We now know that neither dry air nor water-vapour is a perfect insulator, for there is a loss of charge due to the presence of electrified "ions" in the air, and the electrified surface draws to itself the ions with opposite charges. But this loss is exceedingly slow under ordinary conditions.

'The inverse square law may be illustrated by the following experiment. 'Two metre rules are fixed horizontally, one over the other (Fig. 58). A conducting sphere A is mounted on a pillar, 
which must be a very good insulator, with a base having an index mark which slides along the lower rule. On the upper rule is a slider with an index mark, and from the slider depends a pith ball $\mathrm{B}$ hung by a cocoon fibre, the ball being on a level with the centre of A. A telescope sights B when it is uncharged, and the position of the slider is read. Then $\mathbf{A}$ is brought into the middle of the field of view, and the position of its base is read. $A$ is then charged, $\mathbf{B}$ touches it, is charged and repelled. $A$ is then removed say, $10 \mathrm{~cm}$. to the left, and the slider is moved to the left till B is in the middle of the telescope field. If the slider has been moved $d$ the force on B is for small angles proportional to $d$. Now move B to, say, $20 \mathrm{~cm}$. from the central position and move the slider till $\mathbf{B}$ is in the middle of the field; let its displacement be $d$. It is

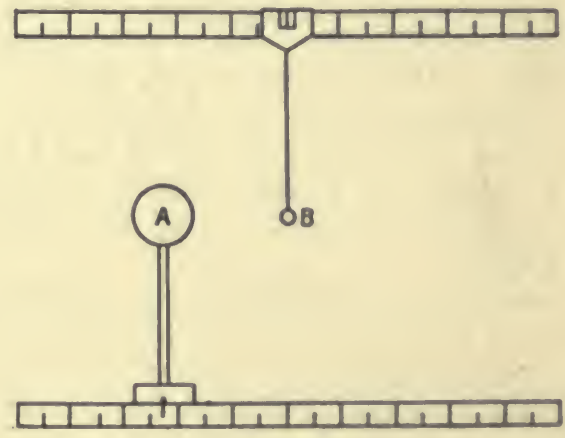

Fia. 58.

found that with fair accuracy $d: d^{\prime}=4: 1$. If $\mathrm{A}$ be now touched to another equal insulated sphere, its charge is halved and the forces at the same distances are halved.

General statement of the inverse square law. Both directly and indirectly, then, it has been proved that the force on a small electrified body placed at any point in a field containing charged surfaces separated by air may be calculated by supposing that each element of charged surface acts directly on the body with a force proportional to the product of the element of charge and the charge on the small body, and inversely as the square of the distance between them, the actual force being the resultant of all such elementary forces. Since the force is thus proportional to the charge on the small body, it is the same per unit of charge on that body whatever the total charge and whatever the size of the body, both being very small. We have, therefore, a definite and consistent measure by which to describe the field, in the force acting per unit charge on a small body. This method of measuring has already been employed in Chapter III, but we shall here repeat some of the definitions and propositions of that chapter, with 
some modifications suggested by the experiments which we have described.

Definition of electric intensity. If a small positively charged body be placed at any point in a field, the body and its charge both being so small as not to affect the previous charges sensibly, the force on the body per unit charge is termed the electric intensity at the point. We shall usually denote the intensity by $\mathrm{E}$.

Definition of the unit quantity. If two small bodies charged with equal quantities and placed $1 \mathrm{~cm}$. apart act each on the other with unit force of 1 dyne, each is said to have unit charge on it. Then unit charge produces unit intensity at unit distance. At distance $d$ a quantity $Q$ has intensity $Q / d^{2}$. If at the point we place a body charged with $Q^{\prime}$, the force on the body is $Q Q^{\prime} / d^{2}$.

Relation between electric intensity and electric strain. If a small quantity of electricity $Q$ is placed at a point the values of the electric strain $\mathrm{D}$ and of the electric intensity $\mathrm{E}$ at a distance $d$ from it are given by

$$
\begin{aligned}
& \mathrm{D}=\frac{\mathrm{Q}}{4 \pi d^{2}}(\text { p. 54) } \\
& \mathbf{E}=\frac{\mathbf{Q}}{d^{2}} \text {. } \\
& \mathrm{E}=4 \pi \mathrm{D} \text {. }
\end{aligned}
$$

This relation holding for each element of the electrification to which the field is due, it nust hold for the resultant of all the elements. It is to be noted that it is only true when the medium is air, for only then is the intensity $Q / d^{2}$ for each element. As a particular case we have another proof of the proposition already proved in Chapter III. vi\%: :

The intensity just outside the surface of a conductor is $4 \pi \times$ surface density. For the strain is by definition measured by $\sigma$. Hence the intensity is $4 \pi \sigma$.

Lines and tubes of force. The lines and tubes of strain may also be regarded in non-crystalline media as lines and tubes of force or intensity, and the various strain properties of such tubes may be stated in terms of intensity. The most important of these, viz. that area $\times$ strain is constant and equal to unity along a unit tube, becomes: area of cross-section $x$ intensity $=4 \pi$ along a unit tube. The relation between strain and intensity is not to be regarded merely as a numerical definition of one in terms of the other. It expresses a physical fact, viz. that whichever method we adopt to explore the field, the force on a small electrified body or the charge gathering on one side of a properly held proof plane, the field varies in the same proportion from point to point by either 
system of measurement. 'The numerical relation obtained depends on the units employed. Whatever be the unit of charge, if we define strain as on p. 52, the strain is $q / 4 \pi d^{2}$, but the intensity is only $q / d^{2}$ when a particular unit is chosen. We shall see hereafter that with this particular unit, but with a medium other than air, the relation is $\mathrm{KE}=4 \pi \mathrm{D}$, where $\mathrm{K}$ is a constant for each medium. When we discuss the relation between intensity and strain in media other than air we shall be able to show that we may interpret the intensity as analogous to elastic stress and the strain as analogous to elastic strain.

The outward pull per unit area on a charged conducting surface. Since the intensity is always outwards from a

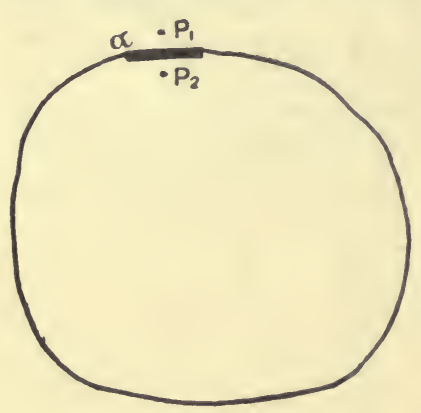

FiG. 59. positively electrified surface and inwards to a negatively electrified one, it is evident that in each case an actual element of the surface, if loose, would tend to move outwards. We shall now find the amount of the pull outwards per unit area. We have seen in Chapter III that the intensity at a point $P_{1}$, Fig. 59, just outside a conductor is normal to the surface and is equal to $4 \pi \sigma$. Also we found that it may be regarded as made up of two equal parts, one due to the charge on the area $a$ immediately under it, and the other due to the rest of the charges of the system. Each is therefore $2 \pi \sigma$.

Now consider an element $d a$ of a just under $P_{\mathrm{f}}$. The force outwards on this element is not due to the rest of $a$, since $a$ is practically all in the same plane as dar. It must therefore be due to $\mathrm{S}$ alone, and its value is: intensity due to $\mathrm{S} \times$ charge on da

$$
\begin{aligned}
& =2 \pi \sigma \times \sigma d a \\
& =2 \pi \sigma^{2} d a .
\end{aligned}
$$

The pull outwards per unit area is therefore

$$
\mathbf{F}=2 \pi \sigma^{2} .
$$

If, then, we know the distribution of charge on a conducting body, we know all the forces acting upon it, and the resultant force is the resultant of all the elementary pulls outwards.

Note on the method of investigating the field in Chapters III-V. The mode of analysing the total strain at a point into the actions of the separate elements each supposed to act directly, and without regard to the intervening medium, is that 
suggested by, and most appropriate to, the old "action at a distance "view of electric action, and it might appear out of place with our new conception of action through the medium. But if, as in the text, we only say that the strain is the same, however produced, as if the direct action occurred, the method is perfectly justifiable for purposes of calculation, and it is the best in the first treatment of the subject owing to the ease with which it admits of mathematical representation. As an illustration of this method, consider the somewhat similar case of the equilibrium of a body pulled by a number of strings, with given forces applied at the free ends. IVe consider these forces as applied directly at the points of attachment of the strings, and do not trouble to consider how the strings behave or to take them into account, so long as we are dealing only with the equilibrium of the body. For experiment has shown us that if a force is applied at one end of a string an equal force is exerted by the string at the other, and we therefore are justified in a kind of "direct action" treatment, though obviously the string has an all-important part to play, and must be considered if we are to have a complete investigation of all the phenomena accompanying the equilibrium of the body.

There is a method of treating electrical actions in which the mathematical representation fits in perfectly with the supposition of action by and through the medium. It is fully set forth in Maxwell's Electricity and Magnetism, vol. i, chap. iv, and we shall here only point out very briefly the principle of the method for the sake of advanced readers who may wish to compare it with the easier, though much less general, method which starts from the inverse square law.

Let us suppose that we wish to find how electrification will be distributed under given conditions on any given system of conductors in air. Starting with the idea of the electric field as a space in which electric strain and intensity may be manifested, we must deduce from experiment that in air the strain has everywhere the same direction as, and is proportional to, the intensity, and that we have chosen units so that $\mathrm{E}=4 \pi \mathrm{D}$. 'Then introducing the ider of the energy possessed by the system, we must show from our experiments that its amount depends solely on the configuration. From this it follows that a potential $\mathrm{V}$ exists, and the intensity at any point may be expressed in terms of $\mathrm{V}$.

'The total energy of the system may be shown to be

$$
\mathbf{W}=\frac{1}{2} \iint \sigma \mathrm{V} d \mathbf{S}
$$

where $\sigma$ is the surface density on the element of surface $d S$, the integral being taken over all the conducting surfaces. 
But $\sigma=$ strain just outside $d \mathbf{S}$

$$
\begin{aligned}
& =\frac{1}{4 \pi} \text { intensity } \\
& =\frac{1}{4 \pi} \frac{d V}{d n}
\end{aligned}
$$

where $d n$ is an element of the normal.

Then

$$
\mathrm{W}=\frac{1}{8 \pi} \iint \mathrm{V} \frac{d \mathrm{~V}}{d x} d \mathbf{S} .
$$

But by Green's theorem this is equal to

$$
\begin{gathered}
\frac{1}{8 \pi} \iiint\left\{\left(\frac{d V}{d x}\right)_{1}^{2}+\left(\frac{d V}{d y}\right)^{2}+\left(\frac{d V}{d x}\right)\right\} d z d y d z \\
+\frac{1}{8 \pi} \iiint V \nabla^{2} V d x d y d z
\end{gathered}
$$

where the integrations are taken throughout the air.

Now going back to the experimental basis, we must suppose that we are entitled to assume that the total strain throughout a tube of strain is constant. It is ensy to think of experimental illustrations of this, such as the equal and opposite charges on concentric spheres, and the equality always existing between the two mutually inducing charges whatever the conductors, though it is not very easy to arrange any general proof. From this it follows that the total strain through any closed surface containing no electrification is zero. 'The total normal intensity is therefore also zero, and applying this to the case of the element $d x d y d x$, it can be shown that $\vec{\nabla}^{2} \mathrm{~V}=0$.

$$
\text { Hence } \mathrm{W}=\frac{1}{8 \pi} \iiint\left\{\left(\frac{d V}{d x}\right)^{2}+\left(\frac{d V}{d y}\right)^{2}+\left(\frac{d V}{d z}\right)\right\} d x d y d z \text {. }
$$

Now by 'Thomson's theorem there is one, and only one, value of $\mathrm{V}$ satisfying $\nabla^{2} \mathrm{~V}=0$ in the air, and fulfilling the conditions given on the conductors, and this value makes the integral which is equal to $\mathrm{W}$ a minimum. In other words, it is the value of $\mathrm{V}$ which corresponds to a distribution of electrification making the energy a minimum, and therefore it is a distribution in equilibrium. Hence we require to find a function $\mathrm{V}$ satisfying the conditions. 
(1) $\nabla^{2} \mathrm{~V}=0$; (2) $\mathrm{V}=\mathrm{a}$ constant for each conductor; (3) $\iint \frac{d V}{d n} d \mathbf{S}=4 \pi \times$ given charge on each conductor. This function is the only one satisfying the conditions, and tells us all about the field.

Green's theorem states that if $U$ and $V$ are two functions,

$$
\begin{aligned}
& \iint \mathrm{U} \frac{d \mathrm{~V}}{d n} d \mathrm{~S}-\iiint \mathrm{U} \nabla^{2} \mathrm{~V} d x d y d z \\
= & \iint \mathrm{V} \frac{d \mathrm{U}}{d n} d \mathrm{~S}-\iiint \mathrm{V} \nabla^{2} \mathrm{U} d x d y d z
\end{aligned}
$$

where the surface integrals are taken over the conductors and the volume integrals are taken through the intervening air dielectric. Make $\mathbf{V}$ the function considered above, and make $\mathrm{U}$ the potential according to the inverse square law of a positive unit of charge distributed uniformly with density $\rho$ through a very small sphere of radius $a$ and centre at any point $P$ in the field. Then at a distance $r$ from $\mathrm{P}$, greater than $a, \mathrm{U}=1 / r$, and at a distance $r$ less than $\alpha, U=\frac{2}{3} \pi \rho r^{2}$. Then within the small sphere $\nabla^{2} U=4 \pi \rho$ and without it $\nabla^{2} U=0$.

On the conducting surfaces $\frac{d \mathrm{~V}}{d n}+4 \pi \sigma=0$, and in the dielectric $\nabla^{2} \mathrm{~V}=0$. Since $\mathrm{V}$ is constant over each conductor $\iint \mathrm{V} \frac{d \mathrm{U}}{d n} d \mathrm{~S}=$ $\mathrm{V} \iint \frac{d \mathrm{U}}{d n} d \mathrm{~S}=0$ by Gauss's theorem. Also $\iiint \mathrm{V} \nabla^{2} \mathrm{U} d x d y d z$ only differs from zero in the small sphere round $P$ and there it may be put $V_{\mathrm{P}} \iiint 4 \pi \rho d x d y d z=4 \pi \mathrm{V}_{\mathrm{P}}$, since the total charge round $\mathrm{P}=1$. Then

$$
\mathrm{V}_{\mathrm{P}}=\iint \frac{\sigma}{r} d \mathrm{~S}
$$

or the potential is that which would be found by calculation on the inverse square law with direct action. In other words, the inverse square law is the only one which would give the strain along a tube constant, and a constant potential in the conductors. 


\title{
CHAPTER VI
}

\section{ELECTRICAL LEVEL OR POTENTIAL. THE ENERGY IN ELECTRIFIED SYSTEMS}

\begin{abstract}
Electrical level-Potential at a point-Equipotential surfaces-Intensity expressed in terms of rate of change of potential-General nature of level surf ices-Energy of an electrified system in terms of charges and potentials-Unit cells-Number of unit cells in a system double the number of units of energy-Distribution of energy in a system.
\end{abstract}

The existence of forces on charged bodies in an electrified system implies that these bodies may be set in motion, or that the system contains energy which may appear in the kinetic form. We must suppose that before its appearance as kinetic energy it was stored in some way as electric energy, and in that form was the equivalent of the work done in charging the system. Regarding the system from this "work" point of view, we are led to the idea of electrical level or potential, which affords most valuable aid in describing the system in respect to the forces exerted and to the energy stored. We have already discussed the potential in inverse square systems in Chapter III, but we shall now approach the subject from another point of view.

Electrical level. If a positively charged conductor is suspended in the middle of a room a small body brought against it will receive a little of the charge and will then tend to move to the wall, on which is the negative charge. Let us imagine that the small carrier has no weight so that we need not think of any but the electric forces. At the wall we may think of it as going into a hole and touching the side of the hole, when it will be entirely discharged. It can then be moved back to the large body, where it will receive a little more charge, go again to the wall, and so on. On each journey from the body to the wall it can be made to do work, and the system will gradually yield up its energy. Now compare this with what happens in a system containing gravitative energy, such as a reservoir of water on a high level. We may let the water run down to a lower level and gradually transform the potential energy into some other form in the process. Or, to make the cases more alike, we may let the water.down by a bucket which corresponds to the electrical carrier. The water gives up its energy by falling in level.

Using this analogy, we may describe the electrified body in the 
middle of the room as being at a higher electrical level than the walls and think of the level as gradually falling from the body to the walls in every direction. 'The conductor is all at the same level, for the charged carrier will not move along its surface, but will be pulled straight out from it at every point. The walls, if conducting, are also at one level, since the intensity is everywhere perpendicular to the surface, and the carrier will not tend to move along the surface at all.

The gravitative analogy, if followed out, also suggests a definite method of measuring electrical level. We usually measure difference of level above the earth's surface in vertical feet or metres. But this measurement will not give us consistent results in extended systems. For think of two canals at different levels reaching from the latitude of London to the Equator. Suppose the surface of the upper one is $978 \mathrm{~cm}$. above that of the lower at London. At the Equator the difference in level will be, not 978 , but $981 \mathrm{~cm}$. For the work done in letting a given mass of water down from one canal to the other must be the same at each end. Otherwise it would be possible to get an endless store of energy out of the water by allowing it to trickle down at the end where the most work was given out, and to do work as it fell. We might use some of this work to raise an equal mass of water up again at the other end and we might transform or store the balance. This is contrary to all experience, so that we are certain that the work done in lifting a gramme of water from one level to the other is the same at each end and indeed at every intermediate point. Since then $g$ at London : $g$ at the Equator $=981: 978$, if one canal is $978 \mathrm{~cm}$. higher than the other at London, it must be $981 \mathrm{~cm}$. higher at the Equator. It is worth noting that if we were concerned with exact measurements of work done in raising masses, our Ordnance maps should be marked, not in feet above sea-level, but in ergs or foot-poundals.

Now, turning to an electrical system, let us imagine a surface $\mathrm{S}_{1}$, Fig. 60 , drawn in the dielectric all at one level, drawn, that is, so that at every point of the surface the force on a small charged carrier is perpendicular

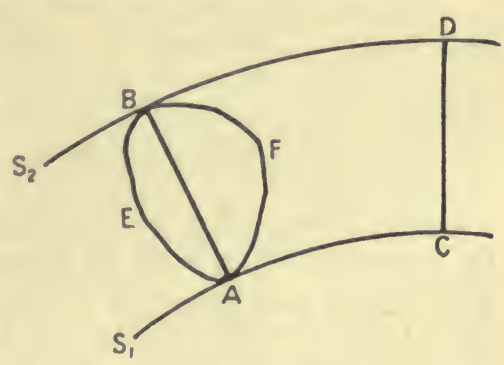

FIG. 60. to the surface, and no work is done in moving the carrier along that surface. Imagine another surface $S_{2}$ in the dielectric, also level, but at a higher level than the first. 'Then the work done in taking a small charged carrier from the first surface to the second is the same by all paths. For imagine that it is greater by one path, AB, than by another path, CD, from the first surface, $S_{1}$, to the second surface, $S_{2}$. Taking the carrier round 
the circuit $\mathrm{BACDB}$, we shall get a supply of energy each time we go round. This could only be accounted for by a gradual discharge of the system due to the motion, and experiment shows that no such discharge occurs. Hence the work along $\mathrm{AB}$ equals the work along CD. As a particular case the work from $\mathbf{A}$ to $\mathbf{B}$ is the same by all paths, such as AEB or AFB. If then we choose some convenient surface as having zero-level, corresponding to sea-level with gravity, the level of any other point, measured by the work done in carrying the unit positive charge from zerolevel to that point, is definite, whatever be the path pursued. Level as thus estimated is termed Potential, and we have the following definition :

The potential at a point is the work done in carrying a small body with unit positive charge from zero-level to the point.

The potential at a point is usually denoted by $\mathrm{V}$.

It is evident that the work done in carrying unit charge from a point $A$ at potential $V_{A}$ to a point $B$ at potential $V_{B}$ is $V_{B}-V_{A}$. For if $\mathrm{O}$ is a point in the zero-level, $\mathrm{V}_{\mathrm{A}}$ is the work done in going from $\mathrm{O}$ to $\mathrm{A}, \mathrm{V}_{\mathrm{B}}$ that in going from $\mathrm{O}$ to $\mathrm{B}$, and we may go to $\mathrm{B}$ through $A$. Hence the work along $A B$ is $V_{B}-V_{A}$.

Alteration of surface chosen as zero-level or zeropotential. If we alter our starting-point or zero-level to one at potential $U$ referred to the old starting-point, the potential at every other point is evidently decreased by $U$.

Equipotential surfaces. The surfaces which we have hitherto termed level, surfaces everywhere cutting the lines of force at right angles, are also equipotential surfaces. Drawing a series of such surfaces corresponding to potentials $0,1,2,3, \&$ c., we may map out the variations of level in the system.

A conducting surface is an equipotential surface, and any closed conductor containing no electrification within it is all at one potential, for there is no component of the intensity along the surface, and if there is no charge within there is no intensity within, and no work is done in taking unit charge from one point to another.

The intensity at a point in any direction expressed in terms of the rate of change of potential. Let $A$ be the given point and $\mathrm{B}$ a point very near it such that $\mathrm{AB}$ is the direction in which the component of the intensity is to be expressed. 'Then the work done in carrying the unit charge from $A$ to $B$ is $V_{B}-V_{A}$; but if $\mathrm{X}$ is the component of the intensity along $\mathrm{AB}$, the component along $\mathbf{B A}$ is $-\mathbf{X}$, and this is the force against which the unit charge is carried.

$$
\text { Then }-\mathrm{X} \cdot \mathrm{AB}=\mathrm{V}-\mathrm{V}_{\mathrm{A}}
$$

or, using the notation of the differential calculus and putting $\mathrm{AB}=d x$ and $\mathrm{V}_{\mathrm{A}}-\mathrm{V}_{\mathrm{B}}=d \mathrm{~V}$,

$$
-\mathrm{X} \cdot d x=d \mathrm{~V}
$$


and ultimately $\quad \mathrm{X}=\cdots \frac{d \mathrm{~V}}{d x}$

or the intensity in any direction is the rate of fall of potential per unit length in that direction.

General nature of level surfaces. If the surfaces are drawn at intervals such that the successive potentials differ by unity, it is evident that the surfaces crowd most closely together where the intensity is greatest, for there the shortest distance will be traversed with the unit charge in doing unit work. On the other hand, where the intensity is less the surfaces open out. Indeed, we can at once obtain a relation between the distance from surface to surface and the cross-section of a unit tube of force.

If $\mathrm{E}$ be the intensity at a point and $d$ the distance between two consecutive level surfaces there.

$$
\therefore \mathrm{Ed}=1 \text {. }
$$

But if $\alpha$ be the area of the cross-section of the unit tube at the point, $\mathrm{E}_{\alpha}=4 \pi$ (p. 67),

$$
\therefore \quad \frac{a}{d}=4 \pi
$$

or the distance $d$ is proportional to the cross-section $\alpha$ of the tube.

When a conducting surface is charged entirely with one kind of electricity, the intensity is everywhere outwards if the charge be

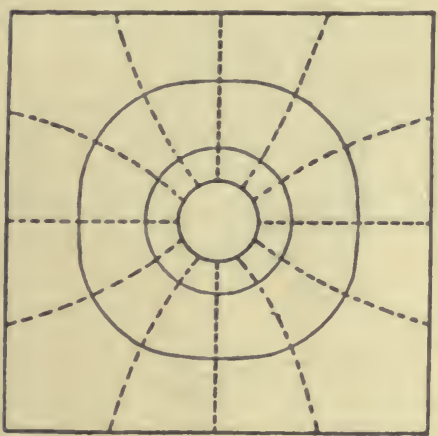

FIG. 61. Charged sphere in the middle of a cubical room.

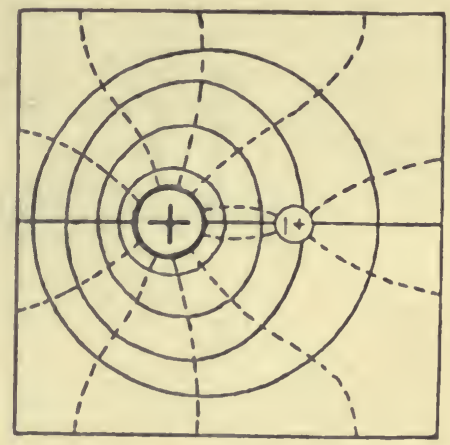

Fic. 62. Charged sphere inducing opposite charge on a neighbouring insulated sphere in the middle of a cubical room.

positive, and everywhere inwards if it be negative. Then in the former case the potential falls and in the latter rises as we go outwards from the surface in all directions. In other words, 
the conductor is entirely surrounded by level surfaces lower in the former case, higher in the latter, than the surface itself.

If the surface is partly positively and partly negatively charged, the surfaces are lower near the one part and higher near the other part.

We may illustrate these statements diagrammatically by Figs. 61 and 62 , which are sections through systems showing iu each case lines of force and level lines in the section. There is no attempt to draw to scale in these figures.

Energy of an electrified system in terms of charges and potentials. Let the charges be $Q_{1}, Q_{2}, Q_{3}, \& c$., respectively on conductors at potentials $V_{1}, V_{2}, V_{3}$, \&c., the zero-level being any conveniently chosen conductor. If all the charges be altered in any given ratio the same for all, say to $\lambda Q_{1}, \lambda Q_{2}, \lambda Q_{3}, \& c_{\text {. }}$, evidently the intensity at every point will still remain the same in direction, but will have $\lambda$ times the original magnitude. Hence the original level surfaces will still be level, and the charges in the new system will still be in equilibrium. The potentials will evidently be $\lambda V_{1}, \lambda V_{2}, \lambda V_{3}$, \&c.

Let us represent the charges and potentials of the conductors in the original system by points $A, B, C, D, \& c$, on a chargepotential diagram, Fig. 63, the abscissa representing charges, the ordinates potentials. Thus $A E=Q_{1}, O E=V_{1}, B F^{\circ}=Q_{2}$, $\mathrm{OF}=\mathrm{V}_{2}+\mathrm{C}$.

Taking into account the whole system, the sum of the charges, i.e. the sum of the abscissa, must be zero. Now let us bring to the

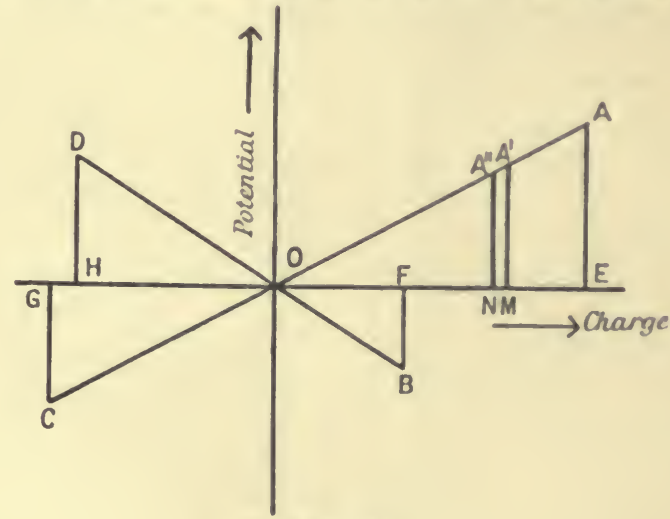

FIG. 63.

zero-level the same very small fraction of each charge. Then the elementary charges will on their arrival exactly neutralise each other. During the convection some small fraction of the energy of the system will be given up. Repeating the operation a sufficient number of times, we shall gradually exliaust the system, and it is evident that as we remove the same fraction of each charge in 
an operation, the charges and potentials of the conductors will be all reduced in the same ratio, so that the points representing them on the diagram will all travel in straight lines towards $O$. The figure may be regarded as merely shrinking in towards $O$.

Now consider the work done by the small charge removed from the first conductor while its state changes from $\mathbf{A}^{\prime}$ to $\mathbf{A}$." The quantity removed is evidently MN. The potential at the beginning of the removal is $\mathbf{A}^{\prime} \mathbf{M}$, and at the end it is $\mathbf{A}^{\prime \prime} \mathbf{N}$. 'Then the work done lies between

\section{MN. A'M and MN. A"N.}

But one of these is greater and the other less than the area of the slip $\mathrm{A}^{\prime} \mathrm{MNA}^{\prime \prime}$, and the less the charge MN, the more nearly are they equal to each other, ultimately differing negligibly. 'Then the work done must ultimately be represented by $\mathrm{A}^{\prime} \mathrm{MNA}$ ".

'The total work clone in discharging the first conductor is the sum of all such slips or is represented by the area of the triangle $\mathrm{AEO}$, which equals

$$
\frac{\mathrm{AE} \times \mathrm{EO}}{2}=\frac{Q_{1} \mathrm{~V}_{1}}{2}
$$

Similarly for the other conductors. Whence

$$
\text { the total energy }=\frac{Q_{1} V_{1}}{2}+\frac{Q_{2} V_{2}}{2}+\ldots=\Sigma \frac{Q V}{2} \text {. }
$$

When all the charges have been thus brought to the same level all the electrical energy has been transformed and the system is exhausted.

It is perhaps sufficiently evident that the quantity of energy obtained must be independent of the arbitrary zero of potential chosen, but we may also deduce this from the expression for the total. For if we had chosen another zero at potential $U$ relatively to the first, then the potentials would have been $V_{1}-U, V_{2}-U, \& c$, and the total energy would have been expressed by

$$
\begin{gathered}
\frac{Q_{1}\left(V_{1}-U\right)}{2}+\frac{Q_{2}\left(V_{2}-U\right)}{2}+\ldots \\
=\frac{Q_{1} V_{1}}{2}+\frac{Q_{2} V_{2}}{2}+\ldots-\left(Q_{1}+Q_{2}+\ldots\right) \frac{U}{2} \\
=\frac{Q_{1} V_{1}}{2}+\frac{Q_{2} V_{2}}{2}
\end{gathered}
$$

for $Q_{1}+Q_{2}+\ldots=0$.

It is to be noted that if any charge is on the original zero-level surface, it must be taken into account in expressing the energy in terms of the levels referred to the new zero. 
Unit cells. Drawing all the unit tubes of force and all the level surfaces at unit differences of potential, all the space between the conductors of a system is divided up into cells. Maxwell has termed these unit cells.

The number of unit cells in a system is $\Sigma Q V$. In the case of one conductor at potential $V$ entirely surrounded by another. at zero-potential, it is evident that each tube is cut into $\mathrm{V}$ cells, and as there are $\mathbf{Q}$ tubes there are in all $\mathbf{Q V}$ cells. When there are several conductors each tube does not necessarily pass from the highest to the lowest level, but some may begin or end at intermediate levels.

Take any one tube passing from a conductor at level $V_{M}$ to one at level $V_{N}$. Evidently it contains $V_{M}-V_{N}$ cells, or, since it has +1 of electricity at one end and -1 at the other, we may write this

$$
1 \times \mathrm{V}_{\mathrm{M}}+(-1) \times \mathrm{V}_{\mathrm{N}} .
$$

Doing this for every tube in the system, we have

$$
\begin{aligned}
& \text { Total number of cells }=\Sigma\left\{1 \times V_{M}+(-1) V_{N}\right\} \\
& =\Sigma \text { each element of charge } \times \text { its potential. }
\end{aligned}
$$

If on any conductor at potential $\mathrm{V}$ there be both positive and negative charges, say $Q_{P}$ and $Q_{N}$, both charges will enter into this expression and will contribute

$$
\left(Q_{P}-Q_{N}\right) V
$$

to the result. But if $Q_{P}-Q_{N}=Q$, this becomes $Q V$. Hence the total number of cells $=\Sigma Q V$.

Distribution of the energy in the system. From the preceding result we see that the number of unit cells is always double the number of units of energy in the system. We have already seen that we must suppose the energy to be in the dielectric, accompanying the strain there. Bearing in mind that where the cells are larger the electric strain is less, so that the energy is presumably less densely stored, this relation between the quantity of energy and the number of cells suggests that the energy is distributed at the rate of half a unit to each cell. Adopting the suggestion, let us find the energy per unit volume.

\begin{tabular}{|c|c|}
\hline volume of cell & $=a d$ \\
\hline but & $\mathrm{E} \alpha=4 \pi \quad($ p. 67) \\
\hline and & $\mathrm{E} d=1 \quad($ p. 75) \\
\hline
\end{tabular}

The area of the cross-section of a tube at any point being $a$, and the distance between the two consecutive level surfaces being $d$, we have 
If this volume contains half a unit of energy, the quantity per unit volume

$$
=\frac{\frac{1}{2}}{\frac{4 \pi}{\mathrm{E}^{2}}}=\frac{\mathrm{E}^{2}}{8 \pi}
$$

or, substituting for $\mathrm{E}$ its equivalent $4 \pi \mathrm{D}$,

$$
=2 \pi \mathrm{D}^{2} \text {. }
$$

Now this result is of the kind which we should expect from Faraday's view of the nature of electric action. For if electric strain be analogous to elastic strain, we should expect that the energy stored up per unit volume would be proportional to the square of the electric strain, just as the energy stored by an elastic strain for which Hooke's law holds is proportional to the square of the strain. Since then the distribution at the rate of half a unit to each cell or of $\frac{\mathrm{E}^{2}}{8 \pi}=2 \pi \mathrm{D}^{2}$ to each unit volume just accounts for the energy of the system, and also carries further the analogy between electric and elastic strain, it is accepted as the true law of distribution.

It is noteworthy that the energy per unit volume $\frac{\mathrm{E}^{2}}{8 \pi}$ may be expressed in the form $\frac{E I}{2}$. This supports the suggestion on p. 101 that the intensity $\mathrm{E}$ is analogous to elastic stress. For we know that in elastic strain

$$
\text { energy per unit volume }=\frac{\text { stress } \times \text { strain }}{2} .
$$




\title{
CHAPTER VII
}

\section{POTENTIAL AND CAPACITY IN CERTAIN ELECTRIFIED SYSTEMS. SOME METHODS OF MEASURING POTENTIAL AND CAPACITY}

\begin{abstract}
Definition of capacity-Sphere in the middle of large room-Two concentric spheres-'Two parallel plane conducting plates-Two long co-axial cylinders-Two long thin equal and parallel cylinders-Long thin cylinder parallel to a conducting plane-Instruments to measure poten. tial difference-Quadrant electrometer-Attracted diso electrometer -Practical methods of measuring potential-Reduction of the potential of a conductor to that of a given point in the air-Simple methods of measuring capacity-Capacity of a Leyden jar-Capacity of a gold-leaf electroscope.
\end{abstract}

WE have shown in Chapter III that if we define the potential at a point as the sum of each element of electrification divided by its distance from the point, or $\Sigma q$, , it is the work done in bringing unit charge from a zero-level at a distance so great that the system has no appreciable action there. We may fiequently bring the zero-level quite close to the system. Thus, if a room has conducting walls, an electrified system within it has no intensity outside, and the walls may be regarded as having zero-level. We shall now consider some special systems which will illustrate the use of the formula for the potential.

Infinitely thin small metal plate held normally to the lines of force or strain. The plate is to be so small that the lines of force may be regarded as straight and parallel in the region just about the point at which it is introduced before that introduction takes place. Then, when it is introduced, since it is perpendicular to the previous course of the lines, the positive charge on one side will evidently be equal, element by element, to the negative charge on the other side. 'The potential due to the plate at any point outside it will be made up of equal and opposite terms, since for every element of positive charge there is an equal element of negative charge practically at the same distance from the point, as the plate is infinitely thin. Then the plate does not affect the potential in its neighbourhood, and therefore does not alter 
the course of the lines of force. This justifies the use of such a plate in the measurement of strain at a point.

In practice no plate is infinitely thin. A real proof plane will therefore produce some slight modification of the surrounding field, especially near the edges, where the lines must turn inwards, somewhat as in Fig. 64, to enable some of them to meet the edge normally.

Capacity of a conductor. If the potential of one conductor of a system is raised while all the rest are kept at zero-potential, as the charge rises in value the induced opposite charges rise in proportion. Hence $\Sigma_{r}^{q}$, or the potential at any point, rises in the same proportion. 'Thus the ratio of

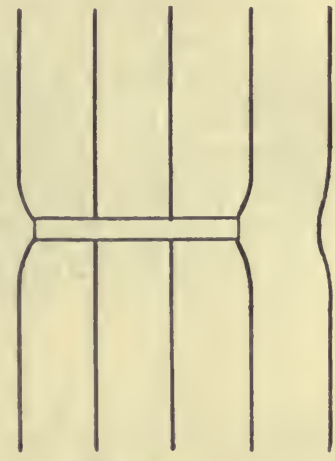

F1G. 64 . the charge on the conductor to its potential is constant and is termed the capacity of the conductor. We have then this definition:

If a conductor receives a charge $\mathbf{Q}$ and is raised thereby to potential $\mathrm{V}$, while the surrounding conductors are at zero-potential, $\frac{Q}{V}$ is constant, and is termed the capacity of the conductor. It is usually denoted by $\mathrm{C}$.

Sphere in the middle of a large room. Suppose a sphere radius $a$ to receive a charge $+Q$. Let the sphere be placed insulated in a room and let its distance from the walls be great compared with its radius. The potential of the sphere will be uniform throughout, and therefore we may find it by calculating the potential at the centre. In the formula $\mathrm{V}=\Sigma_{r} \underline{q}$ the positive elenients are all at the same distance from the centre, and therefore contribute $\mathrm{Q} / \boldsymbol{a}$. The negative elements are all at a great distance, and the sum of the terms $\frac{q}{r}$ is negligible in comparison with $Q / a$. We have then

The capacity is

$$
\mathrm{V}=\frac{\mathrm{Q}}{a}
$$

$$
\mathrm{C}=\frac{\mathrm{Q}}{\mathrm{V}}=\boldsymbol{a}
$$

Since the potential at the surface of the sphere has everywhere the same value as that at the centre, viz.: $\frac{Q}{a}$, and is due to the distribution on the surface, the wall charges having negligible effect, 
that distribution must be uniform. Hence the potential at a distance $r$ from the centre outside the sphere is, by Chapter III,

$$
\mathbf{V}=\frac{\mathbf{Q}}{r}
$$

provided that $r$ is small compared with the distance to the walls.

Two concentric spheres with equal and opposite charges $\mathbf{Q}$. Let the radii be $a$ and $b$. 'The potential of the inner sphere is uniform and equal to that at the centre. Since the whole of the positive charge is distant $a$ from the centre and the whole of the negative charge is distant $b$, we have

$$
\mathrm{V}=\Sigma \frac{?}{r}=\frac{Q}{a}-\frac{Q}{b} .
$$

Between the spheres, at $r$ from the centre, the potential due to the positive charge is $\frac{Q}{r}$ (Chapter III), while the potential due to the negative charge is still $\frac{Q}{b}$. 'Then

$$
\mathbf{V}=\frac{\mathbf{Q}}{r}-\frac{\mathbf{Q}}{\mathrm{b}}
$$

'The outer sphere has potential

$$
V=\frac{Q}{b}-\frac{Q}{b}=0 \text {. }
$$

The capacity of the inner sphere is

$$
C=\frac{Q}{\frac{Q}{a}-\frac{Q}{b}}=\frac{a b}{b-a} .
$$

'These two cases show, as is indeed obvious from the definition, that a capacity has the dimension of a length.

Two oppositely charged parallel conducting plane plates, the plates being a distance apart very small compared with their linear dimensions.

'The distribution must satisfy the two conditions found to hold in all electrified systems when the charges are at rest, viz. (1) the intensity close to each surface in the space between the plates must be normal to the surface, and (2) the intensity within the substance of the conductors must be zero. These conditions will be practically fulfilled if the distributions are equal and opposite and uniform over the two surfaces except near the edges, and the lines of force and strain will then go straight across from plate to plate except near the edges. For let P, Fig. 65, be a point between the 
plates. Take two circular areas of which $\mathrm{AB}, \mathrm{A}^{\prime} \mathrm{B}^{\prime}$ are sections with equal radii, and with centres in the common normal to the plates through $\mathbf{P}$.

Let the radii of these areas be large compared with the distance $d$ of the plates from each other. The surface densities being uniform and equal to $\pm \sigma$, the intensity at $\mathbf{P}$ due to the circular area on the positive plate will be $2 \pi \sigma$, and away from it; and that due to the circular area on the negative plate will be $2 \pi \sigma$, and towards it (Chapter III, p. 36), or $4 \pi \sigma$ in all. Outside these circular areas the charges can be divided into equal positive and negative elements, each pair lying close together and at practically equal distances from $P$. These, acting practically in the same line,

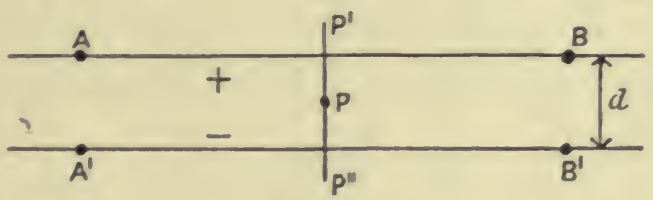

FIG. 65.

neutralise each other and do not contribute towards the intensity. The inteusity between the plates is therefore $4 \pi \sigma$ and normal to each.

Within either conductor, say, for instance, at $\mathrm{P}^{\prime}$ or $\mathrm{P}^{\prime \prime}$, the two circular areas neutralise each other, and, as above, the outlying areas also neutralise each other and the intensity is zero. The want of uniformity round the edges may be neglected (if these are sufficiently distant) in calculating the intensity at points well within the boundary.

Since the intensity is $4 \pi \sigma$ and the distance apart is $d$, the work done in carrying unit charge from one plate to the other is $4 \pi \sigma d$. Hence the difference of potential $\mathrm{V}=4 \pi \sigma d$.

If $\mathbf{A}$ is the area of either charged face, the total charge on the positive plate is approximately $\mathbf{A} \sigma$. The capacity then is

$$
\mathrm{C}=\frac{\mathrm{Q}}{\mathrm{V}}=\frac{\mathrm{A} \sigma}{4 \pi \sigma d}=\frac{\mathrm{A}}{4 \pi d}
$$

Two long co-axial circular cylinders. The cylinders are to be supposed of length great compared with the radius of either. The inner cylinder is charged to potential $\mathbf{V}$ above the outer. 'The radii are $a$ and $b$.

The lines of force will evidently be radial at a distance from the ends, and the tubes of force will be wedge-shaped as in Fig. $65 \mathrm{~A}$, the apex of each wedge lying along the axis of the cylinders. Then the area of the cross-section of a tube at distance $r$ from the axis 
is proportional to $r$, and since cross-section $\times$ inteusity is constant, the intensity is inversely proportional to $r$, and may be written as $\lambda / r$. Now if $\sigma$ is the surface density on the inner cylinder the intensity just outside it is $4 \pi \sigma$, and as this is distant $a$ from the

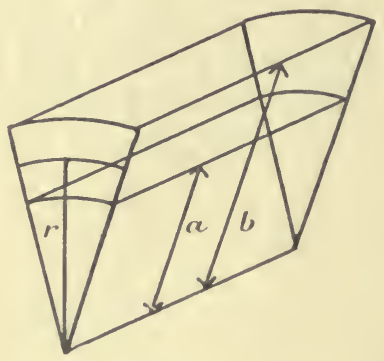

FIG. 65A.

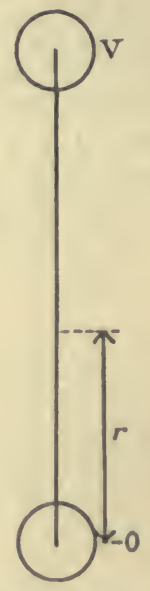

FIG. 65B.

axis we have $4 \pi \sigma=\lambda / a$ or $\lambda=4 \pi \sigma a$. Then between the surfaces

$$
\begin{aligned}
& \mathbf{E}=4 \pi \sigma_{r}^{a} \\
& \mathrm{E}=-\frac{d \mathrm{~V}}{d r}=4 \pi \sigma \frac{a}{r} .
\end{aligned}
$$

But

Integrating, we have

$$
\mathrm{V}=-4 \pi \sigma a \log r+\text { const }
$$

and putting $\mathrm{V}=0$ for the value of $\mathrm{V}$ when $r=b$,

$$
\mathrm{V}=4 \pi a \sigma \log \frac{b}{r}
$$

If $\mathrm{C}$ is the capacity per unit length measured along the axis the area of the inner surface of that length is $2 \pi a$ and its charge $2 \pi a \sigma$. Its potential is $4 \pi a \sigma \log \frac{b}{a}$. Then

$$
\mathrm{C}=\frac{\mathrm{Q}}{\mathrm{V}}=\frac{2 \pi a \sigma}{4 \pi a \sigma \log \frac{b}{a}}=\frac{1}{2 \log \frac{b}{a}} .
$$

Two very long thin equal and parallel cylinders. Let the two cylinders, Fig. 65 s, have, each, radius $a$ small compared with 
the distance $d$ apart. Now if one cylinder only were charged with surface density $\sigma$, the corresponding negative charge being infinitely distant, the intensity due to it at distance $r$ would be $4 \pi \sigma_{r}^{a}$ by the preceding investigation. Then the intensity at one cylinder due to the other is practically $4 \pi \sigma \frac{a}{d}$ and is very small compared with the intensity due to its own charge if $\frac{a}{d}$ is very small. Hence the uniformity of distribution on each cylinder will hardly be disturbed by the presence of the other, and we shall obtain a nearly correct result by supposing the charges uniformly distributed on the two cylinders with densities $\pm \sigma$. Let us take the potential of one cylinder as 0 and that of the other as $V$, and let us calculate $V$ by the work done in going straight from one cylinder to the other. At a distance $r$ from the axis of the negative cylinder the intensity is

$$
\begin{gathered}
\mathrm{E}=-\frac{4 \pi \sigma a}{r}-\frac{4 \pi \sigma a}{d-r} . \\
\text { and } \quad \mathrm{V}=-\int_{r=a}^{r=d-a} \mathrm{E} d r \quad=\left[4 \pi \sigma a \log \frac{r}{d-r}\right]_{a}^{d-a} \\
=8 \pi \sigma a \log \frac{d-a}{a}=8 \pi \sigma a \log \frac{d}{a} \text { nearly. }
\end{gathered}
$$

'The capacity per unit length is

$$
\begin{gathered}
\mathrm{C}=\frac{2 \pi a \sigma}{\mathrm{V}}=\frac{1}{4 \log \frac{d-a}{a}} \\
=\frac{1}{4 \log \frac{d}{a}} \text { nearly. }
\end{gathered}
$$

This case occurs in practice in two telegraph wires running parallel to each other at a distance from the ground, and equally charged respectively positively and negatively.

Long thin cylinder parallel to a conducting plane. We may at once deduce the case of a single cylinder running parallel to an indefinitely extended conducting plane - a case occurring in practice in a single telegraph wire at a constant height above the ground.*

If we draw a plane midway between the two cylinders considered above, the lines of force, from symmetry, everywhere cut f. 42 .

* For a more exact investigation see Heaviside's Electrical Papers, vol. i. 
this plane at right angles and it will be a level surface. Now consider a unit tube passing as in Fig. 65c from one cylinder to the other and cutting the plane in the area $\alpha$. If $\alpha$ be made into an infinitely thin conductor, it then has charges \pm 1 induced on it, but these will not disturb the course of the lines of force, since $a$ is infinitely thin. We may imagine each unit tube treated in the same way and the corresponding \pm units formed on the two sides of the median plane. The charges thus imagined are all at one

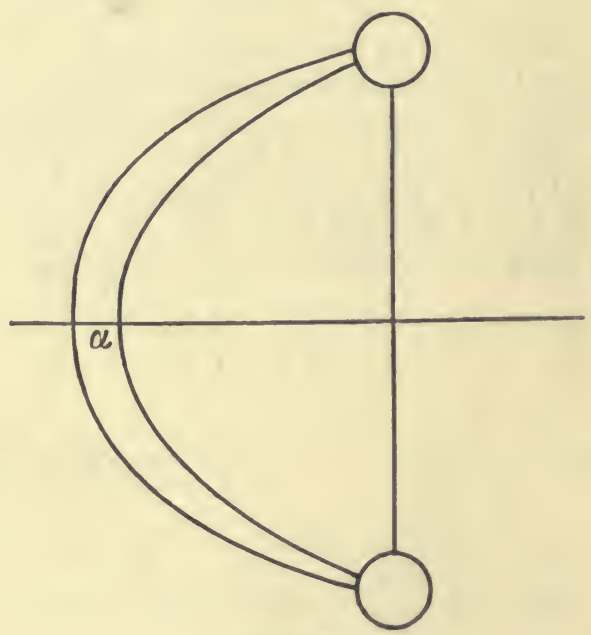

Fig. $65 \mathrm{c}$.

potential and will not tend to move, so that the system thus formed is in equilibrium. We shall then have two systems really independent of each other, since there is a conducting screen entirely separating one from the other. The upper cylinder positively electrified will have the corresponding negative on the upper face of the median plane, and the lower cylinder negatively electrified will have the corresponding positive on the lower face of the median plane. Either gives the case of a cylinder with axis running parallel to a plane. Evidently the difference of potential between the plane and the cylinder is half that between the two cylinders, and we have approximately, if we put $d=2 h$,

$$
\text { and } \quad \mathrm{C}=\frac{1}{2 \log _{\epsilon} \frac{2 h}{a}}=\frac{1}{0.87 \log _{10} \frac{2 h}{a}} .
$$

The result shows that wires used in laboratory experiments may have quite considerable capacities. If, for instance, a wire 
with radius $0.01 \mathrm{~cm}$. (about $36 \mathrm{~S} . W . G$.) runs parallel to and $10 \mathrm{~cm}$. above a conducting table, the formula shows that the capacity per $\mathrm{cm}$. length is more than $1 / 3$. $1 / 15$

Condenser of any form. A condenser consists essentially of two conducting plates everywhere the same distance apart, this distance being usually very small compared with the linear dimensions of the plates, and the curvature being everywhere small. We may then apply the treatment already used for two parallel plane plates, and we have, when air is the insulator,

$$
\begin{aligned}
\mathrm{V} & =4 \pi \sigma d \\
\text { and } \quad \mathrm{C} & =\frac{\mathrm{A}}{4 \pi d}
\end{aligned}
$$

where $\mathrm{A}$ is the area of the inner surface of either plate and $d$ is their distance apart.

\section{INSTRUMEN'TS USED TO MEASURE DIFFERENCE OF PO'TENTIAL}

The quadrant electrometer. The quadrant electrometer devised by Lord Kelvin is the most sensitive instrument for the measurement of differences of potential. We shall describe first a simple form of the original instrument represented in Fig. 66.*

Four hollow brass quadrants, like that shown in Fig. $66(a)$, are mounted on insulating pillars fixed on a metal base so as to form a horizontal circular box, cut across two diameters at right angles, since the quadrants are insulated from one another by narrow air spaces. 'The opposite quadrants are connected in pairs by very fine wires. Under the quadrants and between the supporting pillars is an open Leyden jar lined outside with tinfoil and containing inside strong sulphuric acid, the surface of contact of the acid with the glass serving as the inner coating of the jar. The acid also serves to dry the air within the case of the instrument and so maintains the insulation of the various parts. Within the hollow space made by the quadrants is a " needle," really a "figureof-eight" shaped piece of sheet aluminium, Fig. $66(b)$, the sectors being each about a quadrant. The needle is supported from the top of the case by an insulating suspension which also introduces torsion when the needle is displaced from its "zero " position. The torsion may be obtained from a bifilar suspension, a quartz fibre suspension, or from a small magnet fastened on to the needle system. The position of the needle is indicated by a beam of light reflected on to a scale from a mirror above the quadrants and rigidly attached to the needle.

From two adjacent quadrants two brass rods pass vertically

- In Lord Kelvin's Papers on Elcctrostatics and Electro-magnetism a form of the instrument suited for exact work is described in the Report on Electrometers. 
downwards through holes in the brass plate so large that the rods do not touch the sides. The rods turn outwards, ending in binding screws. A third vertical brass rod passes through an insulating block in the brass plate, and can turn about its own axis so as to bring a horizontal wire at its upper end in contact with a platinum wire which is attached to the needle, and passes vertically down
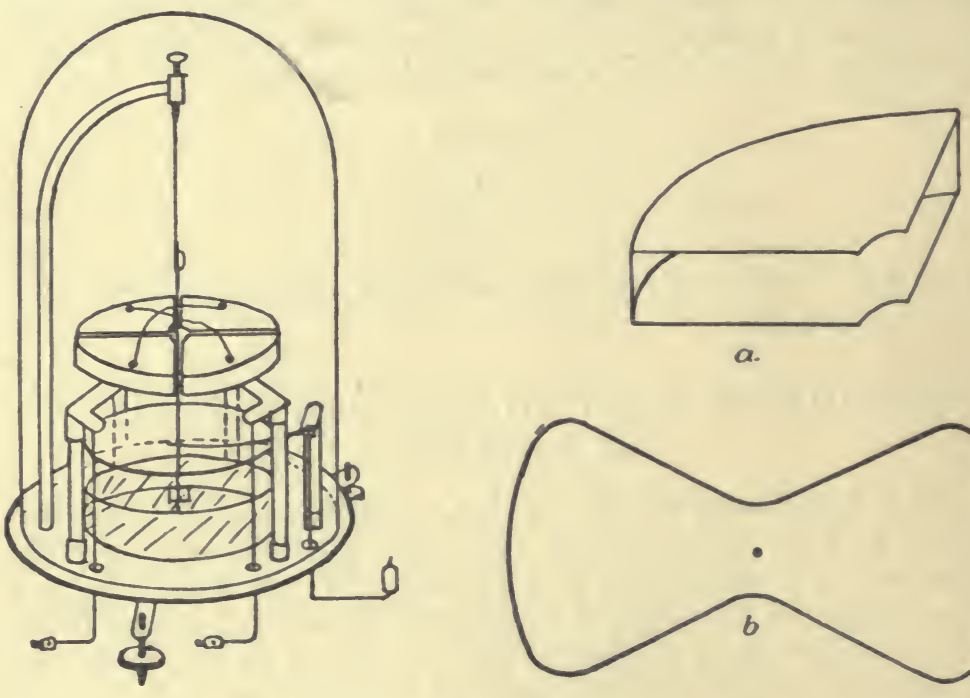

a.

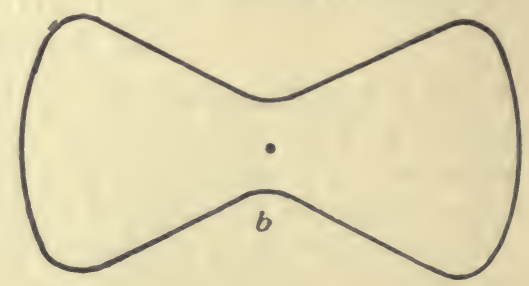

FiG. 66.

into the acid in the jar. When the jar is to be charged the contact is made and the jar may be charged by two or three sparks from an electrophorus cover. The contact is then broken and the horizontal wire turned well out of the way so that the needle is free to move.

The instrument is contained in a glass case resting on the brass plate, which is itself supported on levelling screws. 'The glass should be lined with tinfoil except where the indicating beam of light passes, and the case should be put to earth by a wire going to the gas or water pipes. 'The instrument is thus completely secured from external electrifications.

The general principle of its action is simple. 'To begin with, the needle should be adjusted in its zero position so that its median line is parallel to one of the lines of separation of the quadrants. If now one opposite pair of quadrants is connected by the outside terminal to earth, and the other pair to a body of a potential differing from that of the earth, the needle, being at the potential of the jar, which we may suppose positive, is itself positively charged and tends to move so as to carry its charge to the region of lowest potential. If the outside body 
is at positive potential the needle will tend to set under the earth-connected pair of quadrants, while if the outside body is at negative potential the needle will tend to set under the pair of quadrants connected to it. If the torsion couple introduced by the displacement is so great that the displacement is always small, then the angle of displacement is, as we shall show below, nearly proportional to the difference of potential between the pairs of quadrants. It is also, for a certain range, nearly proportional to the potential of the needle, so that the sensitiveness of the instrument may be adjusted by altering the charge in the jar. It is usual to calibrate the scale of the electrometer by putting on known differences of potential from the terminals of cells of known voltage.

Dolazalek electrometer. A modification of the instrument introduced by Dolazalek, which is more sensitive than the original type, is much used. In this the quadrants are smaller and are mounted often on amber pillars. The needle is made of silvered paper and so is lighter, and it is preferably suspended by a quartz fibre made conducting or by a very fine metallic wire. The Leyden jar can then be dispensed with and the needle can be charged directly from a battery of, say, 100 volts. There is a potential of maximum sensitiveness, however, which can be found by trial for the particular instrument used. The instrument is contained in a brass case with a window for the beam of light to pass to and from the mirror.

Elementary theory of the quadrant electrometer. We shall take the case in which the two pairs of quadrants, which we denote respectively by $1,1^{\prime}$ and $2,2^{\prime}$, are maintained at constant potentials $V_{1} V_{2}$. 'The needle is connected to the jar, which has very great capacity compared with its own. 'I'he potential of both jar and needle therefore remains sensibly constant even if the charge on the needle varies. We denote this potential by $\mathrm{V}_{\mathrm{n}}$. If $\mathrm{V}_{\mathrm{z}}$ is greater than $\mathrm{V}_{1}$ the needle tends to move towards $1,1^{\prime}$, and it will only be in equilibrium when the electrical couple is balanced by the torsional couple. Now had the quadrants and needle, after charging, been insulated, the motion of the needle would have diminished the electrical energy of the system, the differences of potential of the fixed charges decreasing, and the position of equilibrium would have been that in which the decrease in electrical energy on small displacement would have just supplied the work necessary for the increase in torsion. But the potentials remaining constant, there is an actual increase in the energy of the system. 'The area of the needle under 1,1 ', where the difference of potential is greatest, increases, and therefore its charge increases and consequently the electrical energy increases. The energy is supplied by the sources which maintain the constant potentials, the supply being sufficient both for the increase in electrical energy and for that used in increasing the torsion of the wire. We shall make use of the following important theorem : 
When a system of conductors is maintained each at a constant potential by connection with a source of electricity, the total energy supplied by the source in any displacement of the system is double the increase in the electrical energy of the system. This may be proved as follows:

Let $Q_{1}, Q_{2}, Q_{3}$ be the charges at potentials $V_{1}, V_{2}, V_{3}$ : then the total energy is

$$
W=\frac{Q_{1} V_{1}}{2}+\frac{Q_{2} V_{2}}{2}+\frac{Q_{3} V_{3}}{2}+\cdots
$$

Let a displacement be given to the system so that the charges become $\mathbf{Q}_{1}+q_{1}, \mathbf{Q}_{2}+q_{2}, \mathbf{Q}_{3}+q_{3}, \& \mathbf{c}$. : the energy is now

$$
\mathrm{W}+w=\frac{\left(\mathbf{Q}_{1}+q_{1}\right)}{2} \mathrm{~V}_{1}+\frac{\left(\mathbf{Q}_{2}+q_{2}\right)}{2} \mathrm{~V}_{2}+\ldots
$$

whence the increase in energy is

$$
v=\frac{q_{1} \mathrm{~V}_{1}}{2}+\frac{q_{2} \mathrm{~V}_{2}}{2}+\ldots
$$

But the quantities $q_{1}, q_{2}, q_{3}$, \&c., have been drawn from sources of energy at constant potentials $V_{1}, V_{2}, \& c$., and therefore the energy given up by these sources is

$$
q_{1} \mathrm{~V}_{1}+q_{2} \mathrm{~V}_{2}+\cdots=2 v
$$

which proves the theorem.

Applying this to the quadrant electrometer, the sources supply double the energy required for the electrical system. At first, then, while the torsion couple is small, an excess of energy is supplied. But the torsion couple increases, and ultimately a position of equilibrium is attained when on further small displacement the increase of torsional energy equals the increase in electrical energy.

To calculate the latter we assume that the motion does not affect the distribution on the outer edges of the needle and under the line of separation of the quadrants; this implies that the linear displacement of an edge is small compared with its distance from a quadrant other than that under which it is moving. Hence the motion through a small angle $d \theta$ may be regarded as merely transferring a part of the needle distant from both the edge and the median line from the $2,2^{\prime}$ pair to the $1,1^{\prime}$ pair. 'The capacity of such a part may be taken as proportional to the angle it subtends. Let it be represented by $\operatorname{Cd} \theta$. Hence the charge under $1,1^{\prime}$ is increased by $\operatorname{Cd} \theta\left(V_{n}-V_{1}\right)$, while that under $2,2^{\prime}$ is decreased by $\operatorname{Cd} \theta\left(V_{n}-V_{2}\right)$. The potential differences of these charges from that on the needle are $V_{n}-V_{1}$ and $V_{n}-V_{2}$ respectively. Then the total gain of energy is 
PO'TENTIAL AND CAPACITY

$$
\begin{aligned}
& \frac{C d \theta}{2}\left\{\left(V_{n}-V_{1}\right)^{2}-\left(V_{n}-V_{2}\right)^{2}\right\} \\
= & C d \theta\left(V_{2}-V_{1}\right)\left(V_{n}-\frac{V_{1}+V_{2}}{2}\right) .
\end{aligned}
$$

The increase of torsional energy in the same displacement is

$\lambda \theta d \theta$

where $\theta$ is the total angle of displacement from zero and $\lambda \theta$ is the corresponding couple.

Then for equilibrium we may equate these and

$$
\begin{gathered}
\lambda \theta d \theta=\mathrm{Cd} \theta\left(\mathbf{V}_{2}-\mathbf{V}_{1}\right)\left(\mathbf{V}_{\mathrm{n}}-\frac{\mathbf{V}_{1}+\mathbf{V}_{2}}{2}\right) \\
\operatorname{or} \theta=\frac{\mathrm{C}}{\lambda}\left(\mathbf{V}_{2}-\mathbf{V}_{1}\right)\left(\mathbf{V}_{\mathrm{n}}-\frac{\mathbf{V}_{1}+\mathbf{V}_{2}}{2}\right) .
\end{gathered}
$$

If $V_{n}$ be great compared with $V_{1}$ and $V_{2}$ we may take as an approximation

$$
\theta=\frac{\mathrm{C}}{\lambda}-\mathrm{V}_{\mathrm{n}}\left(\mathrm{V}_{2}-\mathrm{V}_{1}\right)
$$

If the needle and one pair of quadrants be both connected to the same source at potential $V_{n}$ and the other pair be earthed, $\mathrm{V}_{2}=\mathrm{V}_{\mathrm{n}}, \mathrm{V}_{\mathrm{1}}=0$

$$
\text { and } \quad \theta=\frac{\mathrm{CV}_{\mathrm{n}}^{2}}{2 \lambda} \text {. }
$$

This is independent of the sign of $\mathrm{V}_{\mathrm{n}}$, and the electrometer may then be used to measure an alternating potential, giving the mean square of $V_{n}$.

By taking into account the increase or decrease of charge induced on the two pairs of quadrants as well as the increase on the needle it is easy to verify directly for this particular case the fact that the sources supply double the energy added in the field within the quadrants.*

The attracted disc, or the trap-door electrometer. In Thomson's trap-door electrometer two parallel plates are arranged as a condenser, one being connected to the source of which the potential is required, the other being, say, to earth. We might measure the pull of one plate on the whole of the other, and in the first instrument of the kind-Harris's attracted disc electrometer-this was actually done. If we could express the potential

- The formula found above is quite sufficiently exact for many of the experiments in which the quadrant electrometer is used. The explanation of the voltage for maximum sensitiveness will be found in 'Thomson's Elements of Electricity and Magnetism, \& 61 . 
difference in terms of this pull the instrument would be a satisfactory electroneter. But the density in a condenser is not uniform near the edges, and when the plates are some distance apart the edge effect may extend some distance inwards. We cannot therefore apply the results of p. 83 to the whole plate. In the central part of each plate, however, the density is practically uniform ; the lines of strain go straight from plate to plate and these results apply. 'Taking the surface density as $\sigma$, the difference of potential as $\mathrm{V}$, and the distance of the plates apart as $d$,

$$
\mathrm{V}=4 \pi \sigma d \text {. }
$$

Since the pull per square centimetre is $2 \pi \sigma^{2}$, the total pull $\mathbf{P}$ on a central area $\mathbf{A}$ is

$$
\mathrm{P}=2 \pi \mathrm{A} \sigma^{2}=2 \pi \mathrm{A}\left(\frac{\mathrm{V}}{4 \pi d}\right)^{2}=\frac{\mathrm{AV}}{8 \pi d^{2}}
$$

In practice the pull is measured on a "trap-door," i.e. on a movable plate nearly filling a hole in one of the plates of the condenser, but with free edges. 'The surrounding plate is termed the guard-ring, its function being to guard the density on the trapdoor from variation. 'The trap-door and guard-ring are electrically connected and the force measured is that which is required to keep the two in the same plane against the electrical pull of the opposite plate. The effective area $\mathrm{A}$ is approximately (Maxwell, Electricity and Magnetism, 3rd ed. vol. i. p. 333) the mean between the aperture of the guard-ring and the area of the disc or trap-door. Fig. 67 is one form of the instrument, ${ }^{*}$ showing only the essential parts. 'The guard-ring is fixed, while the movable disc or trapdoor is hung by metal wires from the end of a metal lever having a counterpoise at the other. 'The lever is supported by a wire stretched horizontally between two insulated metal pillars connected to the guard-ring so that disc and ring are in connection. The position of the trap-door is indicated by a hair stretched across the forked end of the lever. This is viewed by a lens, and is so adjusted that when it is midway between two black dots on a white upright passing up through the fork behind the hair, the trap-door and guard-ring are coplanar. Suppose that a known weight $\mathbf{P}$ is placed on the disc and that a movable rider is adjusted on the lever until the hair is in the central position when there is no charge on the plates. Now remove $\mathrm{P}$ and connect the plates to bodies at different potentials, adjusting the distance $d$ of the lower plate until the hair is again central. Evidently the electrical pull is equal to $\mathrm{P}$, and if $\mathrm{V}$ is the difference of potential we have from (1)

- Thomson's Papers on Electrostatics and Magnetism, p. 281. 


$$
\mathrm{V}=d \sqrt{\frac{8 \pi \mathrm{P}}{\mathrm{A}}}
$$

It is very difficult to measure $d$ accurately, and it is better to make the results depend on the differences of the distance in two successive experiments, that is, on the distance the lower plate is moved. To do this let the lower plate be connected to a source of

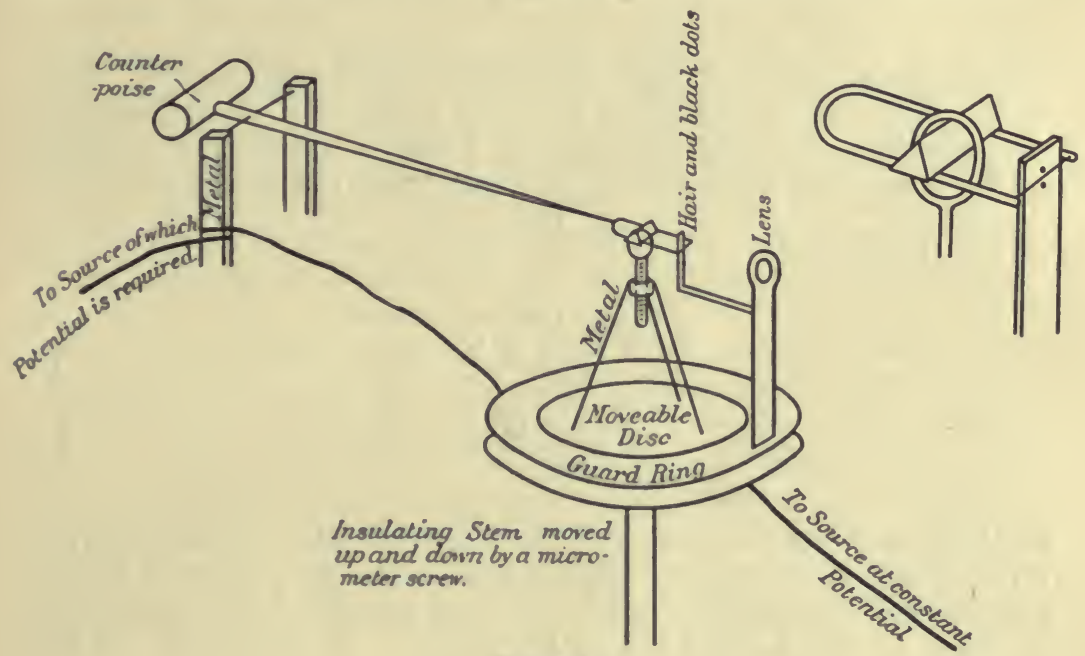

FIG. 67.

potential $\mathrm{V}$, the upper plate being connected to earth; then equation (2) holds.

Now connect the upper plate to a source at potential $V^{\prime}$, the lower plate being connected as before. If the distance, after adjustment, is now $d$,

$$
\mathrm{V}-\mathrm{V}^{\prime}=d^{\prime} \sqrt{\frac{8 \pi \mathrm{P}}{\mathrm{A}}}
$$

Subtracting (3) from (2),

$$
\mathrm{V}^{\prime}=\left(d-d^{\prime}\right) \sqrt{\frac{8 \pi \mathrm{P}}{\mathrm{A}}}
$$

which gives $V^{\prime}$ in terms of measurable quantities. It may be noted that in obtaining (4) we suppose $V^{\prime}$ either less than $V$ or opposite in sign.

In another form of the instrument the attracted disc is hung from one arm of a delicate balance and counterpoised so that when there is no electrification it hangs exactly in the plane of the guard-ring. A stop is then arranged so that the disc cannot move upwards and an extra weight $\mathbf{P}$ is put on the other arm. The subsequent working is as with the arrangement just described. 
Practical methods of measuring potential. If the centre of a conducting sphere is brought to the point at which the potential $\mathrm{V}$ is required and is then connected to the zero-level surface, say the earth, by a wire, its potential becomes zero. Let $\mathbf{Q}$ be the charge induced upon it. The potential at the centre of the sphere being zero, we have

$$
\Sigma \frac{q}{r}=0
$$

But, if the wire is exceedingly fine and if the sphere is sufficiently small, this may be split up into the terms due to the original electrification practically undisturbed by the introduction of the sphere and giving value $V$, and the terms due to the charge gathering on the sphere and giving value $\frac{Q}{a}$. Then

$$
\begin{aligned}
& \mathrm{V}+\frac{\mathrm{Q}}{a}=0 \\
& \text { or } \quad \mathrm{V}=-\frac{\mathrm{Q}}{a} .
\end{aligned}
$$

On breaking the earth connection and taking the sphere away, if we measure its charge, we obtain a number proportional to the potential at the point, though opposite in sign. A very obvious weakness in this method lies in the neglect of the charge on the connecting wire. It is not easy to make the capacity of the wire negligible in comparison with that of the sphere.

Reduction of the potential of a conductor to that of a given point in air. Suppose, for example, that we wish to equalise the potential of a pair of the quadrants of a quadrant electrometer to the potential of a given point P, Fig. 68 , in the air.

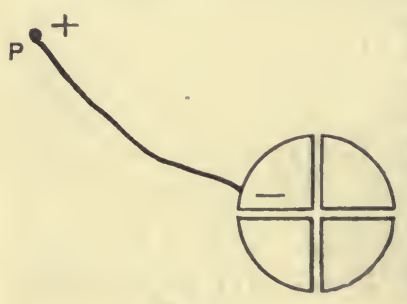

FIG. 68 .

Suppose, to begin with, that they are at higher potential. Take an insulated wire from the electrometer to the point $\mathbf{P}$ : the end of the wire is evidently at higher potential than its surroundings. Then it is positively electrified, while the quadrants are negatively electrified. Imagine now that the outer layer of the wire is loose, so that it can be drawn off into the air with the charge on it. 'I'he potential of the wire at $\mathbf{P}$ is thereby lowered more nearly to that of the air near it. A new positive charge will now gather at $\mathrm{P}$ and more negative will go into the quadrants. If the new surface is also loose, the new charge will also be drawn off into the air, and if we so imagine successive layers to be removed they will carry with then positive charges until the end 
of the wire $\mathbf{P}$ is brought to the same level as its surroundings, or at least to the mean level so that each layer takes with it equal and opposite quantities.

If the electrometer is at lower potential to begin with than the neighbourhood of $\mathrm{P}$, it is evident that negative will go off, while the electrometer will be positively electrified.

This process has been realised practically by various methods. In the method first used a burning match was fixed at one end of an insulated conducting rod, which was brought to the given point, the other end being connected to the electrometer. The hot mass of the flame is a conductor, and as it is continually being thrown off it carries electrification with it as long as there is any difference of potential between the end of the rod and the surrounding air.

In a second method, still sometimes used, an insulated can of water discharges through a fine nozzle drop by drop. In Lord Kelvin's original description of this instrument as applied to find the potential outside a window he says: "With only about ten inches head of water and a discharge so slow as to give no trouble in replenishing the can of water, the atmospheric effect is collected so quickly that any difference of potentials between the insulated conductor and the air at the place where the stream from the nozzle breaks into drops is done away with at the rate of 5 per cent. per half second, or even faster. Hence a very moderate degree of insulation is sensibly as good as perfect so far as observing the atmospheric effects is concerned." (Electrostatics and Magnetism, p. 200).

It is usual now to employ a wire tipped with radium. The air is ionised by the radium, and the charge on the end of the wire is neutralised by the ions of opposite sign.

Atmospheric electricity. Either of these instruments may be employed to determine the potential at any point in the air with regard to the earth. It is found in general that the potential rises upwards from the surface, especially in clear weather. This of course implies negative electrification of the earth's surface, the corresponding positive electrification being scattered through the air above the surface. When clouds are formed they act as conducting masses and become electrified on their surfaces. If the weather is not stormy the potential in the air is still usually positive with regard to the earth, the under surface of a cloud being probably positively charged and its upper surface negatively charged. But in stormy weather it frequently happens that the earth is positively charged under a cloud. This may possibly be explained on the supposition that the cloud was electrified by induction by the ground under it and that the positive charge on the cloud has been removed by the rain falling from it; the negative then spreading over the whole might produce a negative potential in the air under it. We cannot here go into details of the very puzzling subject of Atmospheric Electricity, a subject in 
which the facts are only beginning to fall into order. We refer the reader to the Encyclopoedia Britannica, 11 th ed. vol. ii. p. 860, or to Gockel Die Luftelektrizität.

Simple methods of measuring capacity. We shall describe two methods of measuring capacity merely in order to make the idea of capacity more definite. Other methods will be described later or may be found in laboratory manuals.

The definition of capacity shows that it is in dimension a length. Thus the capacity of a sphere, far from surrounding conductors, is equal to its radius, and the capacity of two parallel plates in air is equal to the area of either $/ 4 \pi$ distance apart, when the distance apart is so small that the edge effect may be neglected. We may then determine the capacity in either of these cases by actual measurement of the dimensions of the system. But when the system is of such form or with such a dielectric that we cannot determine the capacity by direct measurement of dimensions, then we may use a method of comparison.

Capacity of a Leyden jar by comparison with a sliding condenser with parallel plates. Let AB, Fig. 69,

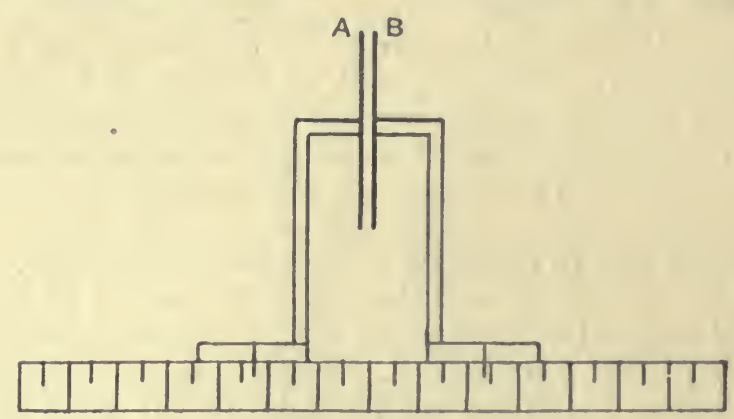

FIG. 69.

represent the sections of two round plates mounted on insulating supports with bases sliding along a divided scale, so that the plates are always parallel to each other and so that their distance apart can be observed. The capacity of this "sliding condenser" can therefore be varied at will. The method consists in charging this sliding condenser and the system of which the capacity is required with electrification at the same difference of potential, and then varying the distance apart of the plates of the sliding condenser till the charges on the two are equal.

Suppose AB, Fig. 70, are the plates of the sliding condenser, and let $J$ be the jar on an insulating stand. Let $A$ and the outside coating of the jar be connected to earth. Let $\mathbf{B}$ and the inside coating of the jar be connected to the positive terminal of a battery whose negative terminal is earthed. Then disconnect the battery from both and insulate $\mathrm{A}$ and the outside coating of the 
jar. Connect $\mathrm{A}$ to the inside coating of $\mathrm{J}$, and $\mathrm{B}$ to the outside coating of $J$. If the charges are equal these connections result in complete discharge, and if $\mathrm{A}$ is now connected to a quadrant electrometer no charge is shown. But if $J$ has the larger capacity some positive charge is left unneutralised and is indicated by the electrometer. If $J$ is the smaller, some negative charge is indicated. The distance apart of the two plates A B must be varied till no charge is indicated and the capacities are then equal. The

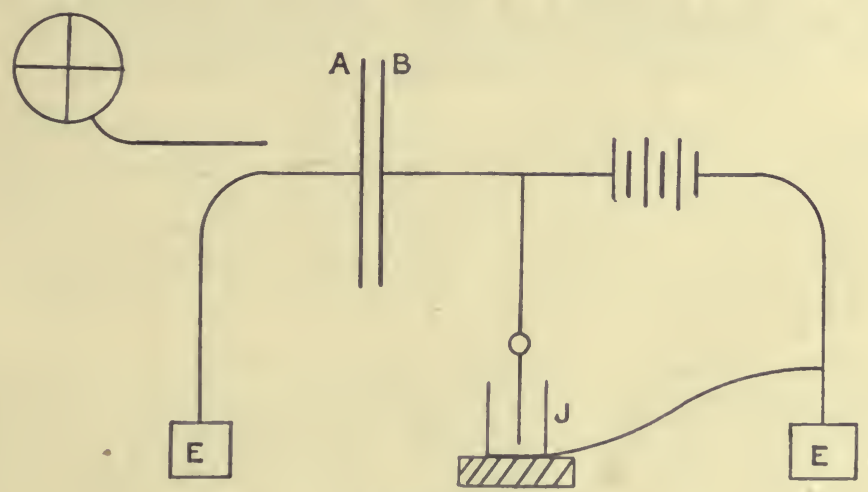

FIG. 70.

difficulty in such measurement arises from " residual charge," which will be discussed in Chapter VIII. This method was used by Cavendish (Electrical Researches, p. 144).

Capacity of a gold-leaf electroscope by comparison with a sphere. We shall describe the method used by C. 'T. R. Wilson (P.R.S. lxviii. p. 157). The electroscope was charged by a battery of small accumulators to different potentials so that the relative values of the deflections of the gold leaf were known. A brass sphere, radius $2 \cdot 13 \mathrm{~cm}$., was suspended by a silk thread at a distance great compared with its radius from all other conductors except two fine wires, one earth-connected with which it rested in contact, and another leading from the electroscope and with its free end near the sphere. The electroscope was charged to some potential $\mathrm{V}$ given by the deflection of the gold leaf. Then the charge on it was $\mathrm{CV}$, where $\mathrm{C}$ was its capacity. The sphere was then drawn aside by a silk thread so that it momentarily broke contact with the earth and came into contact with the wire from the electroscope. Suppose the new value of the potential indicated by the electroscope was $V^{\prime}$. 'The charge on it was now $C^{\prime} V^{\prime}$. But the charge lost, viz. $\mathrm{CV}-\mathrm{CV}^{\prime}$, was given up to the sphere with capacity $2 \cdot 13$, and raised its potential to $V^{\prime}$ so that its amount was $2 \cdot 13 \mathrm{~V}^{\prime}$. Equating

$$
C\left(V-V^{\prime}\right)=2 \cdot 13 V^{\prime}
$$




$$
\text { or } \quad \mathrm{C}=2 \cdot 13 \frac{\mathrm{V}^{\prime}}{\mathrm{V}-\mathrm{V}^{\prime}} \text {. }
$$

By this method Wilson found the capacity of an electroscope which he was using to be $1 \cdot 1 \mathrm{~cm}$., and this was practically constant for different positions of the gold leaf.

In carrying out this experiment the wires, in order to reduce their capacity, should be made as fine as possible and not longer than is necessary to keep the sphere sufficiently distant from the electroscope. 


\title{
CHAPTER VIII
}

\section{THE DIELECTRIC. SPECIFIC INDUCTIVE CAPACITY. RESIDUAL EFFECTS}

\begin{abstract}
Specific inductive capacity-Faraday's experiments-Effect of specific inductive capacity on the relations between electric quantities-Conditions to be satisfied where tubes of strain pass from one dielectric to another-Law of refraction-Capacity of a condenser with a plate of dielectric inserted-The effect of placing a dielectric sphere in a previously uniform field-Residual charge and discharge-Mechanical model.
\end{abstract}

Faraday, as we have seen in Chapter IV, abandoned the old method of regarding electric forces as due to direct action of the charges at a distance, and sought to explain electric induction by the action of contiguous particles on each other in the dielectric, an action which he supposed to be "the first step in the process of electrolysation." Taking this view of electric induction, " there seemed reason to expect some particular relation of it to the different kinds of matter through which it would be exerted, or something. equivalent to a specific electric induction for different bodies, which, if it existed, would unequivocally prove the dependence of induction on the particles." $*$ He was thus led to the great discovery that the quantity of electricity which a condenser will receive when charged to a given potential-that is, its capacity - depends on the nature of the dielectric. This implies that the force which a given electric charge will exert depends on the medium through and by which it acts.

The uature of Faraday's discovery may be illustrated by supposing that we have two exactly equal condensers of the same dimensions, the plates in one being separated by air and those in the other by, say, ebonite. If each is charged to the same potential difference, that with ebonite will have about two and a half times as great a charge as that with air us the dielectric, and the ebonite is said to have two and a half times the specific inductive capacity of air.

A very simple experiment with a gold-leaf electroscope suffices to show the greater inductive capacity of ebonite. Let the electroscope have a table on the top of the rod to which the gold leaves are attached, and let there be a cover the size of the table provided with an insulating handle. Put a thin plate of ebonite

- Exp. Res. Ser. XI. i. p. 373 (November 1837). 
on the table and put the cover on it, the cover and table thus forming the plates of a condenser. Then connect the table with the negative terminal, and the cover with the positive terminal, of a voltaic battery of several cells, when charges will gather proportional to the potential difference of the terminals. Disconnect the wires, first from the table, and then from the cover, and lift the cover. The charge on the table is then shared with the leaves, which diverge by an amount to be noted. Now remove the ebonite plate and put three very small pieces of ebonite of the same thickness as the ebonite plate on to the table merely to serve as spacing pieces, and place the cover on them. We now have an air condenser with the plates the same distance apart as before, for the spacing pieces occupy a very small fraction of the volume and may be neglected in a rough experiment. On connecting and then disconnecting the terminals of the battery as before and lifting the cover we note that the leaves diverge much less than before, or the table has received a much smaller charge when air is the dielectric than it received when ebonite was the dielectric. The experiment is not suitable for exact measurement, for the capacity of the table when the cover is removed will not be quite the same in the two cases, so that the gold leaves will not get quite the same fraction of the charge. If a plate of indiarubber or a plate of sulphur be used, similar effects are noticed; the induced charge is always greater than with air, or, as Faraday expressed it, the specific inductive capacity is greater. We may give exact signification to this term in the following definition:

Specific inductive capacity. Let two condensers $A$ and $B$ have exactly equal dimensions, and let the dielectric in $\mathbf{A}$ be air, while in B it is some other substance. Then the ratio

$$
\text { capacity of } B
$$

is termed the specific inductive capacity of the dielectric in $\mathbf{B}$. It is usually denoted by $\mathbf{K}$.

The specific inductive capacity of a given specimen is probably constant over a wide range of electric intensity, and assuming this constancy, it is frequently termed the dielectric constant. It is also termed the electric or electrostatic capacity of the material.

If we consider the ease or difficulty of producing electric strain in the dielectric, we obtain an analogy with elastic strain which has some value. Suppose two equal condensers, $\mathbf{A}$ with air as dielectric, $\mathrm{B}$ with a dielectric with constant $\mathbf{K}$. If we charge $\mathbf{A}$ to potential difference $\mathrm{V}$, producing surface density $\sigma$ and strain $\mathrm{D}=\sigma$, an equal potential difference in $\mathrm{B}$ will produce surface density $K_{\sigma}$ and strain KD. To produce in B surface density $\sigma$ and strain $\mathrm{D}$, we only require potential difference $\frac{\mathrm{V}}{\mathrm{K}}$, and the 
energy per charge on unit area in $B$ will only be $\frac{V_{\sigma}}{2 K}$, while in $A$ it is $\frac{V_{\sigma}}{2} \cdot$ Or, to produce a given strain $D$ in $B$, requires only $\frac{1}{\mathbf{K}}$ of the work needed to produce an equal strain $\mathrm{D}$ in $\mathrm{A}$. The "electric modulus" of $\mathrm{B}$, then, is only $\frac{1}{\mathrm{~K}}$ that of air.

We may put this more precisely if we consider the intensities in the two condensers with equal charges. When the dielectric $B$ is solid, we cannot directly measure the intensity within it, but in order to account for the difference of potential $\frac{\mathrm{V}}{\mathrm{K}}$ we must suppose that the intensity has $\frac{1}{\mathrm{~K}}$ of the value which it has in $\mathrm{A}$. If in the air condenser it is $\mathrm{E}=4 \pi \mathrm{l}$ ), then in the other it is $\mathrm{E}^{\prime}=4 \pi \mathrm{D} / \mathrm{K}$. If we regard intensity as electric stress and $\mathrm{D}$ as electric strain, then, using the analogy to ordinary elastic stress, we should define the electric modulus as electric stress $\div$ electric strain. In air, then, it is $\mathrm{E} \div \mathrm{D}=4 \pi$. In the dielectric with constant $\mathrm{K}$ it is $\mathrm{E}^{\prime} \div \mathrm{D}=\frac{4 \pi}{\mathrm{K}}$.

'The account of Faraday's discovery is given in Series XI, vol. i, of the Experimental Researches in Electricity, and to the paper we refer the reader for details. It is well worth study not only for the importance of the results but also as a splendid example of Faraday's mode of thought and work. It will suffice here to say that Faraday prepared two equal condensers, each consisting of an outer hollow brass sphere on a stand and an inner concentric brass sphere supported by a metal rod passing up through a neck at the top of the outer sphere, and fastened in position by a plug of shellac. 'The outer sphere was made of two hemispheres like the Magdeburg hemispheres. The rod terminated in a knob. Each condenser was thus virtually a Leyden jar. Initially air was the dielectric in each. One of the jars was charged and its knob was then touched by the carrier ball of a Coulomb electrometer (see p. 63). 'Ihe charge received by the ball was measured and gave, as we should now express it, the difference of potential between the coatings. Then the knobs of the two jars were touched together and the charge was shared. The carrier ball now showed that the charge was equally shared, or the capacities of the jars were equal. 'The lower half of the space between the spheres of one condenser, which we will call $B$, was then filled with a hemispherical cup of shellac, while the other condenser, A, still contained air only. A was charged, and its potential in terms of the electrometer reading was found, after certain corrections, to be 289. 'The charge was shared with $\mathbf{B}$ by 
touching the knobs together, and the potential of each was found to be 113. A had given to $\mathrm{B} 176$ and retained 113. But 176 in $B$ only produced the same potential difference as 113 in $A$, or the capacity of $\mathrm{B} \div$ the capacity of $\mathrm{A}=173 \div 113=1 \cdot 55$. Both were now discharged and $\mathrm{B}$ was charged afresh to potential 204. Its charge was shared with A and each indicated a potential 118. The charge which produced a potential 118 when given to A produced a fall only of $204-118=86$ when taken from B. Since the capacity is inversely as the potential produced by given charge, the capacity of $\mathrm{B} \div$ the capacity of $\mathrm{A}=118 \div 86=1.3 \%$.

Making corrections for loss of charge by "residual effect" to be discussed below, Faraday found that the two corrected values were 1.5 and $1 \cdot 47$, say $1 \cdot 5$. He assumed that the excess of capacity for the $\mathbf{B}$ condenser was only half what it would have been had the whole space been filled with shellac, and thus he found that the capacity of $\mathbf{B}$ was twice that of $\mathbf{A}$, or the specific inductive capacity of shellac was 2. He pointed out that this was an underestimate, for the hemispherical cup did not change quite half the capacity of the jar, since the rod passing through the neck had some capacity, which was the same in both conditions of $\mathbf{B}$. With a flint-glass cup in place of the shellac he found $K=1 \cdot \tau 6$, and with sulphur $K=2 \cdot 24$.

When liquids were introduced into the condenser no certain measurements could be made owing to conduction, and when different gases replaced air no difference could be detected, for the apparatus used was not sufficiently sensitive. 'The difficulties of experiment with liquids and gases have only been overcome since Faraday's time.

Faraday also used two condensers consisting of three parallel equal circular plates, the middle plate forming a condenser with each side plate.* He charged the middle plate, and then showed that by introducing a slab of shellac or sulphur between the middle and one side plate the capacity on that side was increased. He saw that this arrangement might be used for exact measurement, as indeed it has been used later in a modified form.

Though our knowledge of the existence of specific inductive capacity is entirely due to Faraday, it is not a little remarkable that it was discovered by Cavendish some time between $17 \tau 1$ and 1781. But he communicated his results to no one, and they remained buried in his MS. notes till these were edited and published by Maxwell in 1879 as The Electrical Researches of the Hon. Henry Cavendish. His discovery was thus entirely without influence on the progress of electrical knowledge.

For many years after the publication of Faraday's original paper little experimental work was done on the subject, but the publication of Maxwell's Electro-magnetic 'Theory of Light, $\dagger$

* Exp. Res. i. p. 413.

† Phil.Trans., 1865, p. 459; Electricity and Magnetism, vol. ii. chap. xx. (1873). 
according to which the dielectric constant should be equal to the square of the refractive index, led to a renewal of interest in the matter, and this was no doubt increased on the practical side by the necessity of knowing something about the electric capacity of the insulating material of telegraph cables. Since then a great amount of work has been done, and the specific inductive capacities of a great number of solid, liquid, and gaseous dielectrics have been deternined in a great variety of ways. 'The phenomena termed residual charge and discharge, which so much complicate the measurements, have also been investigated.

Before proceeding to an account of the work we shall examine the effect of the existence of specific inductive capacity on the various electrical relations, assuming that for a given material it has a constant value.

The effect of specific inductive capacity on the relations between electrical quantities. Let us suppose that we have two condensers (Fig. 71), A with air as the dielectric

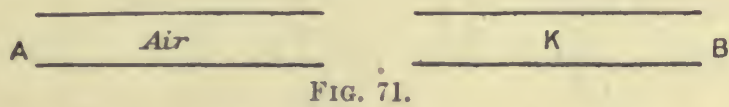

and $K=1,13$ with a substance as dielectric which has constant $K$, and let the two be charged to the same surface density $\sigma$.

Electric strain. Since the electric strain is measured by the quantity induced per unit area on a conductor bounding the medium, and this quantity is $\sigma$ in both $A$ and $B$, the strain $D$ is the same in each.

Difference of potential. To produce the same difference of potential we should require $K \sigma$ in $B$. Then $\sigma$ in $B$ will only produce $\frac{1}{K}$ of the potential difference that the same charge produces in $A$.

Electric intensity. If we imagine it possible to move unit charge through the medium $\mathrm{B}$ we shall require an intensity $\frac{1}{\mathrm{~K}}$ of that in A to produce the observed difference of potential. Or, since this is usually an imaginary and impracticable way of regarding intensity in B, we must now define it as the slope of potential, and again, of course, we get it as $\frac{1}{\mathrm{~K}}$ of the value in air. If then in $\mathrm{A}$ we put $\mathrm{E}=4 \pi \sigma=4 \pi \mathrm{D}$, in $\mathrm{B}$ we must put

$$
\mathbf{E}^{\prime}=\frac{4 \pi \sigma}{\mathrm{K}}=\frac{4 \pi \mathrm{D}}{\mathrm{K}} \text {. }
$$

Energy of charge. If $Q$ is the charge in each, and if $V$ is the potential in $A$, and $V^{\prime}=\frac{V}{K}$ is that in $B$, the energy of charge will 
still be $\frac{1}{2}$ charge $\times$ potential, or $\frac{Q V}{2}$ in $A$ and $\frac{Q V^{\prime}}{2}$ in $B$. For the work done in raising element $q$ through potential $v$ will still be $q v$ whatever the medium between the conductors.

Energy per cubic centimetre. Let us consider a tube of unit cross-section going from plate to plate and let $d$ be the distance between the plates. The energy to be assigned to this tube in $\mathrm{B}$ is $\frac{1}{2} \sigma \mathrm{V}^{\prime}=\frac{2 \pi \sigma^{2} d}{\mathrm{~K}}$, since $\mathrm{V}^{\prime}=\frac{4 \pi \sigma d}{\mathrm{~K}}$.

Since there are $d$ cubic centimetres in the tube the energy per cubic centimetre is $\frac{2 \pi \sigma^{2}}{K}=\frac{2 \pi D^{2}}{K}=\frac{K^{\prime 2}}{8 \pi}$, sirice $\mathrm{D}=\frac{\mathrm{KE}^{\prime}}{4 \pi}$.

The pull per unit area of charged surface. 'This can only be measured directly in liquid and gaseous dielectric. Let us suppose that, keeping the charges the same, the distance between the plates in B is increased by $1 \mathrm{~cm}$. 'The energy stored in each cubic centimetre added is $\frac{2 \pi \mathrm{D}^{2}}{\mathrm{~K}}$, and as this energy is imparted by the work done in separating the plates we require a pull per square centimetre of $\frac{2 \pi \mathrm{D}^{2}}{\mathrm{~K}}$ to give the required energy. Hence the pull on the charged surface is $\frac{2 \pi \mathrm{D}^{2}}{K}=\frac{2 \pi \sigma^{2}}{K}$.

We shall assume that there is the same pull when the dielectric is solid.

Force between charged bodies. 'To account for the pull $\frac{2 \pi \sigma^{2}}{K}$ on the surface by action at a distance according to the inverse square law, we must suppose that each element of charge acts with force $\frac{1}{\mathbf{K}} \frac{q}{\text { distance }^{2}}$ in the medium with dielectric constant $\mathbf{K}$, on the element of charged surface having $\sigma$ on it. Hence charge $q$ acts on charge $q^{\prime}$ at distance $d$ with force $\frac{1}{\mathbf{K}} \frac{q q^{\prime}}{d^{2}}$.

The energy in the field. In Chapter VI we showed that if we assign energy at the rate $\frac{\mathrm{E}^{2}}{8 \pi}=2 \pi \mathrm{D}^{2}$ per cubic centimetre to each element of volume in a field where $K=1$, we just account for the total energy of the system.

Let us take two electrical systems identical as regards conductors and the charges upon them, but one having air as the dielectric with specific inductive capacity 1 , and the other having a dielectric with specific inductive capacity $\mathbf{K}$. 'The work done in charging the two systenıs respectively will be $\frac{1}{2} Q V$ and $\frac{1}{2} Q V / K$. The number of unit cells will be respectively $Q V$ and $Q V / K$, so that we may assign half a unit of energy to each unit cell in each case. But the cells will be larger in the second case in the ratio 
$\mathbf{K}: 1$, and so the energy assigned to unit volume will be less in the ratio $1: K$, or, instead of energy $2 \pi \mathrm{D}^{2}$ per cubic centimetre, we have $2 \pi \mathrm{D}^{2} / \mathrm{K}$ per cubic centimetre, $\left.\mathrm{D}\right)$ being the same in each system since the charges are the same.

But this is only the distribution of the energy which is added by the work done in charging the system. If $\mathbf{K}$ varies with the temperature heat has to be added or subtracted as well, if the temperature is to be kept constant. Just the same consideration comes in with a wire undergoing stretch. The energy put in per unit volume by the stretching force is $\frac{1}{2}$ stress $\times$ strain, but heat must be added or subtracted to keep the temperature constant, and the total energy added is $\frac{1}{2}$ stress $\times$ strain $+Q$, where $Q$ is the heat given to unit volume.

We can calculate the heat added in the electrical case by taking a charged condenser with a fluid dielectric through a thermodynamic cycle, represented in Fig. 72, where abscissa represent distance apart and ordinates represent pull per unit area. Let the condenser be charged with $\pm \sigma$ per unit area. 'The pull per unit area is $2 \pi \sigma^{2} / \mathrm{K}$.

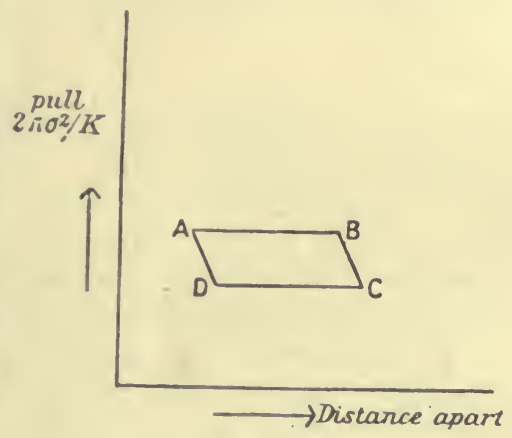

FIG. 72.

Let the distance of the plates apart be increased by a small quantity $l=\mathrm{AB}$, so slowly that there is no change in the temperature $\theta$, and let heat $d Q . l$ be added to each volume $l \times 1^{2}$ in order to keep the temperature constant. Now make a further adiabatic increase in the distance, the change being represented by $\mathrm{BC}$, the temperature in the dielectric which flows in through the changes represented by $\mathrm{AB}$ and $\mathrm{BC}$ falling to $\theta-d \theta$, and the specific inductive capacity falling to $\mathbf{K}-\frac{d \mathbf{K}}{d \theta} d \theta$.

Now make an isothermal decrease $C D$ in the distance, at $\theta-d \theta$, carrying it so far that a further adiabatic decrease along DA con1pletes the cycle. Then if the process is exceedingly slow it is reversible, and the work done is $d Q . l . \frac{d \theta}{\theta}$ by the second law. But the work done is also equal to the area $-\mathrm{ABCD}$, which is

$$
-l .2 \pi \sigma^{2}\left(\frac{1}{\mathrm{~K}}-\frac{1}{\mathrm{~K}-\frac{d \mathrm{~K}}{d \theta}} d \theta\right)=+l \frac{2 \pi \sigma^{2}}{\mathrm{~K}^{2}} \frac{d \mathrm{~K}}{d \theta} d \theta
$$

the negative sign being given to $\mathrm{ABCD}$ because it is work done on the system. Equating the two expressions 
and

$$
\begin{aligned}
d \mathbf{Q} . l \cdot \frac{d \theta}{\theta} & =l \frac{2 \pi \sigma^{2}}{\mathbf{K}^{2}} \frac{d \mathbf{K}}{d \theta} \cdot d \theta \\
d \mathbf{Q} & =\frac{2 \pi \sigma^{2}}{\mathbf{K}} \frac{\theta}{\mathbf{K}} \frac{d \mathbf{K}}{d \theta}
\end{aligned}
$$

or the heat energy added per unit volume is $+\frac{\theta}{\mathbf{K}} \frac{d \mathbf{K}}{d \theta}$ of the energy added by the work done in charging the system.

In some experiments by Cassie* the following values of $\frac{1}{\mathbf{K}} \frac{d \mathbf{K}}{d \theta}$ and $\frac{\theta}{\mathrm{K}} \frac{d \mathrm{~K}}{d \theta}$ were obtained at about $30^{\circ} \mathrm{C}$., say $300^{\circ} \mathrm{A}$

$\begin{array}{lll} & \frac{1}{K} \frac{d K}{d \theta} & \frac{\theta}{K} \frac{d K}{d \theta} \\ \text { Glass } & 0.002 & 0 \cdot 6 \\ \text { Mica } & 0.0004 & 0.12 \\ \text { Ebonite } & 0.0007 & 0.21\end{array}$

We see that the heat supplied to keep the temperature constant when glass of the kind used by Cassie is electrically strained is 0.6 of the energy supplied by the work done.

If this heat is not supplied and the charging takes place under adiabatic conditions the temperature falls, $K$ is decreased, and the medium becomes as it were electrically stronger, and we have the analogue to the increased elasticity of solids under adiabatic elastic strain. But it can be shown that the adiabatic capacity of a condenser bears to its isothermal capacity a ratio which differs from unity by a quantity quite negligible in practical measurements, even though the excess over unity is proportional, as investigation shows, to the square of the potential difference. $\dagger$

Conditions to be satisfied where tubes of strain pass from one dielectric to another. Law of refraction. When tubes of strain pass from one medium to another with different dielectric constant, no charge being on the surface, they change their direction unless they are normal to the separating surface, and they are said to be refracted.

There are two conditions to be satisfied. We may describe the first as (1) continuity of potential on passing through the surface. This continuity of potential implies that the potential is the same at two points indefinitely near to each other, one on each side of the separating surface; and this implies that the intensity in any direction parallel to the surface, and close to it, is the same in each

* Thomson's Applications of Dynamics to Physics and Chemistry, p. 102.

$\dagger$ If $\mathrm{C}_{\phi}$ and $\mathrm{C}_{\theta}$ are the two capacities,

$$
\frac{\mathrm{C}_{\phi}}{\mathrm{C}_{\theta}}=1+\frac{\mathrm{V}^{2}}{4 \pi} \frac{1}{\mathrm{~K}} \frac{d \mathrm{~K}}{d \theta} \frac{\theta}{\mathrm{J} \rho \sigma}
$$

where $V$ is the potential difference, $\rho$ is the density, and $\sigma$ the specific beat of the medium. 
medium. We may see the necessity for this by supposing two fluid dielectrics in contact along a surface AB, Fig. 73. Now imagine a small charge taken along $\mathrm{CD}$ in one medium close to and parallel to $\mathrm{AB}$, and then across to $\mathrm{E}$, back along $\mathrm{EF}$ in the other medium, close to and parallel to DC, and than across to C. We may neglect the work done along $\mathrm{DE}$ and $\mathrm{FC}$ by making them small enough. If the intensity along $C D$ were greater than that along EF, then, on the whole, work would be obtained from the cycle, and repetition of the cycle would lead to discharge of the

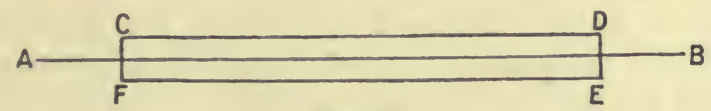

Frg. 73.

energy of the system without altering the charges, and such discharge is contradicted by experience. Hence the intensity parallel to $\mathrm{AB}$ and close to it is the same in each medium, or the potential is continuous.

We may describe the second condition as (2) continuity of strain. The equality of the opposite charges in an electrified system is not affected by the presence of dielectrics of different capacities, and we have every reason to suppose that if we draw unit tubes, each starting from +1 , they will end each on -1 , whatever dielectrics they pass through. If then $\mathrm{ABCD}$, Fig. 74, represents a unit

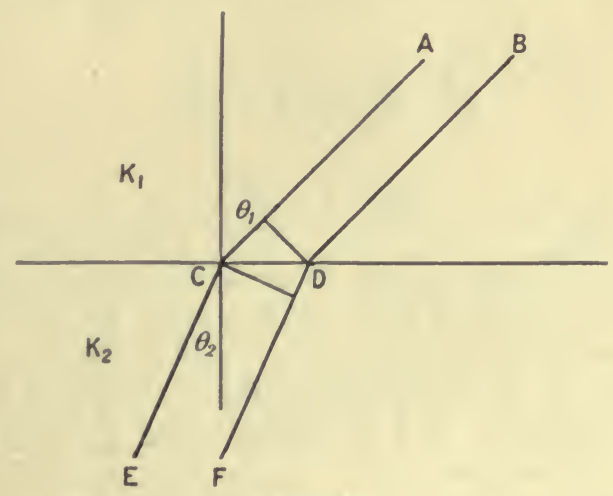

FIG. 74.

tube in a medium with dielectric constant $K_{1}$, continued as CDEF in another medium with constant $\mathbf{K}_{2}, \mathrm{CDEF}$ is also a unit tube. If the area of the surface cut out by each tube is $\alpha$, and if the two tubes make angles $\theta_{1} \theta_{2}$ with the normal, the cross-section of $\mathrm{ABCD}$ is $a \cos \theta_{1}$, while the cross-section of CDEF is $\alpha \cos \theta_{2}$. If the strains on the two sides of $C D$ are $\mathrm{D}_{1}$ and $\mathrm{D}_{2}$, the equality of the total strain in the two tubes gives 


$$
\text { or } \quad \begin{aligned}
\mathrm{D}_{1} & \alpha \cos \theta_{1}=\mathrm{D}_{2} a \cos \theta_{2} \\
\mathrm{D}_{1} \cos \theta_{1} & =\mathrm{D}_{2} \cos \theta_{2}
\end{aligned}
$$

or the normal component of the strain is the same in each medium.

If we are considering non-crystalline media, in which the directions of strain and intensity coincide, let the plane of Fig. 74 pass through $\mathrm{AC}$ and the normal; the tube in the second medium must also be in that plane. For the components of the intensities at $\mathrm{C}$ in any direction parallel to the surface are the same in both media. 'The intensity in the first medium has zero component perpendicular to the plane of the figure. Therefore the intensity in the second medium has zero component in that direction. 'This implies that CE lies in the plane of the figure or the plane through $\mathrm{AC}$ and the normal. Then the incident and refracted tubes are in the same plane with the normal.

'The continuity of potential gives us

$$
\mathrm{E}_{1} \sin \theta_{1}=\mathrm{E}_{2} \sin \theta_{2}
$$

'The continuity of strain gives us

whence

$$
\mathrm{D}_{1} \cos \theta_{1}=\mathrm{I}_{2} \cos \theta_{2}
$$

or, since

$$
\frac{\mathbf{E}_{1}}{D_{1}} \tan \theta_{1}=\frac{\mathbf{E}_{2}}{D_{2}} \tan \theta_{2}
$$

$$
\begin{gathered}
\frac{\mathrm{E}_{1}}{\mathrm{D}_{1}}=\frac{4 \pi}{\mathrm{K}_{1}} \text { and } \frac{\mathrm{E}_{2}}{\mathrm{D}_{2}}=\frac{4 \pi}{\mathrm{K}_{2}} \\
\frac{\tan \theta_{1}}{\mathrm{~K}_{1}}=\frac{\tan \theta_{2}}{\mathrm{~K}_{2}}
\end{gathered}
$$

We shall now consider some effects of the presence of a dielectric other than air, which will be useful when we come to describe methods of measuring specific inductive capacity.

Capacity of a condenser with a slab or plate of dielectric inserted. Let us suppose that the distance between

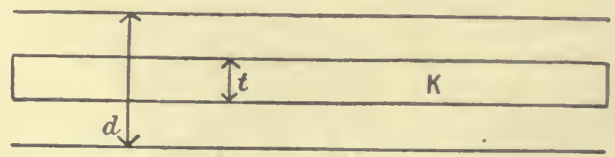

Frg. 75.

the charged conducting plates is $d$, and that a slab of dielectric with constant $K$ occupies a thickness $t$ of the space between the plates and that its surfaces are parallel to the plates as in Fig. 75 . Let the plates and slab extend indefinitely. Let the surface density 
of charge be $\sigma$. We must suppose that the tubes of strain go normally from plate to plate, and that the strain $\mathrm{D}=\sigma$ is the same in air and in the slab. The intensity in the air is $4 \pi \sigma$, while that in the dielectric is $\frac{4 \pi \sigma}{\mathrm{K}}$. Then the potential difference between the plates is

$$
\begin{aligned}
\mathbf{V} & =4 \pi \sigma(d-t)+\frac{4 \pi \sigma}{\mathrm{K}} t \\
& =4 \pi \sigma\left(d-\frac{\mathrm{K}-1}{\mathrm{~K}} t\right) .
\end{aligned}
$$

The capacity per unit area is

$$
\frac{\sigma}{\mathrm{V}}=\frac{1}{\left(4 \pi d-\frac{\mathrm{K}-1}{\mathrm{~K}} t\right)}
$$

or is equal to that of an air condenser in which the plates are nearer together by $\frac{\mathrm{K}-1}{\mathrm{~K}} t$.

If such a slab of dielectric is inserted between the plates of an attracted disc electrometer maintained at a given potential, a layer of air intervening -between the slab and the attracted disc, the charge $\sigma$ is increased in proportion to the capacity. If then $\mathbf{P}$ is the pull per unit area when air alone is between the plates, it becomes with the insertion of the slab

$$
\mathrm{P}\left(\frac{d}{d-\frac{K-1}{K} t}\right)^{2}
$$

The effect of placing a dielectric sphere in a previously uniform field deduced from the effect of placing a conducting sphere in the same field. If a sphere of dielectric constant $\mathrm{K}$ is placed in a field in air, previously uniform, the lines of strain crowd in upon the sphere, for it is more easily strained than the air. 'The effect of a sphere with $\mathrm{K}$ about 1.35 is shown in Fig. 76 , where it will be seen that the strain within the sphere is uniform and parallel to the original direction.

We shall not give a strict proof that this is the distribution, but shall merely show that it will satisfy the conditions required at the surface of separation.

First, let us suppose that a conducting sphere occupies the position. Then on one hemisphere a + charge is induced, and on the other a-charge. These two charges must be so distributed that they will produce a field within the sphere, just neutralising the field $\mathrm{E}$, which previously existed, for there is no field within the conductor. We may find this distribution by the following device. 
Let electrification, density $\rho$, be distributed uniformly within the sphere centre $\mathrm{O}$, radius $a$, Fig. 7\%. At any point $\mathrm{P}$ within the sphere the intensity due to this is along $\mathrm{OP}$ and since the shell

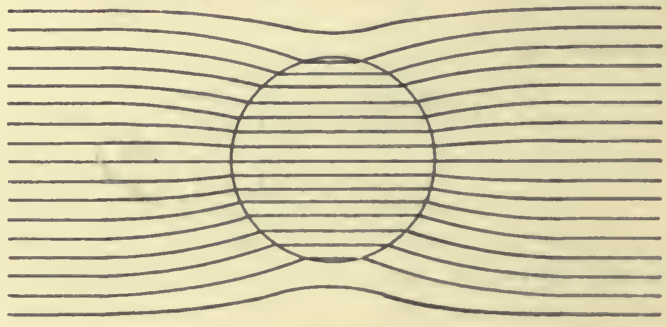

Fia. 76.

external to $\mathrm{P}$ has no effect and the sphere within $\mathrm{P}$ may be regarded as collected at its centre it is equal to $\frac{4}{3} \pi \rho \frac{O P^{3}}{O P^{22}}=\frac{4}{3} \pi \rho$ OP. Now let electrification density $-\rho$ be uniformly distributed within

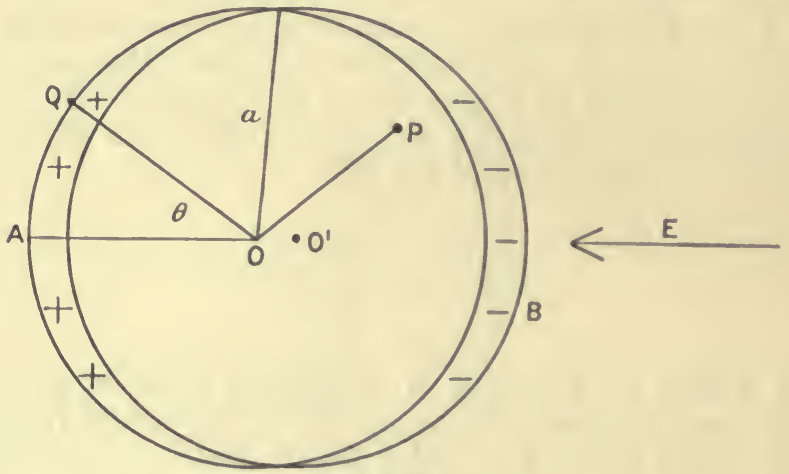

Fra. 77.

a sphere, radius $a$, centre $\mathrm{O}^{\prime}$ close to $\mathrm{O}, \mathrm{O}^{\prime} \mathrm{O}$ being in the direction of $\mathrm{E}$. The intensity at $\mathrm{P}$ due to this second sphere is $\frac{4}{3} \pi \rho \mathrm{PO}^{\prime}$ along $\mathrm{PO}^{\prime}$, since the electrification is negative. 'The two distributions superposed will have resultant $\frac{4}{3} \pi \rho \mathrm{OO}^{\prime}$ through $\mathrm{P}$ parallel to $\mathrm{OO}^{\prime}$. The result of this superposition is zero density, or no charge in the overlapping part of the spheres, a + layer on the hemisphere towards $\mathrm{A}$, and $\mathrm{a}$ - layer on the hemisphere towards $\mathrm{B}$. The thickness of the layer at any point $Q$ in a direction $\theta$ with $\mathrm{O}^{\prime} \mathrm{OA}$ is $\mathrm{OO}^{\prime} \cos \theta$, and therefore the surface density is $\rho O \mathrm{OO}^{\prime} \cos \theta$. Now adjust $\rho$ and $\mathrm{OO}^{\prime}$ so that 


$$
\frac{4}{3} \pi \rho \mathrm{OO}^{\prime}=\mathrm{E} \text {. }
$$

'Then the two layers will give a uniform intensity $-\mathrm{E}$ in the overlapping region, which will neutralise the external field $\mathrm{E}$ in that region, and the surface density of the layers is

$$
\frac{3}{4} \frac{\mathrm{E} \cos \theta}{\pi} \text {. }
$$

Externally, and only externally, to the spherical space the two distributions will act as if each were collected at its centre, and therefore as if we had electrification $\frac{4}{3} \pi \rho a^{3}$ at $\mathrm{O}$ and $-\frac{4}{3} \pi \rho a^{3}$ at $\mathrm{O}^{\prime}$. These two constitute what is termed an electric doublet, and they correspond to the two poles of a magnet. We define their moment to be $\mathrm{M}=\frac{4}{3} \pi \rho a^{3} \mathrm{OO}^{\prime}=\mathrm{E} a^{3}$. Just as with a magnet, the intensity at distance $d$ making $\theta$ with $\mathrm{OO}^{\prime}$ is $\frac{2 \mathrm{E} a^{3} \cos \theta}{d^{3}}$ along $d$, and $\frac{\mathbf{E} a^{3} \sin \theta}{d^{3}}$ perpendicular to $d$ (see Magnetism), ${ }^{*}$ and this field is superposed on the uniform field E. It may be noted that just

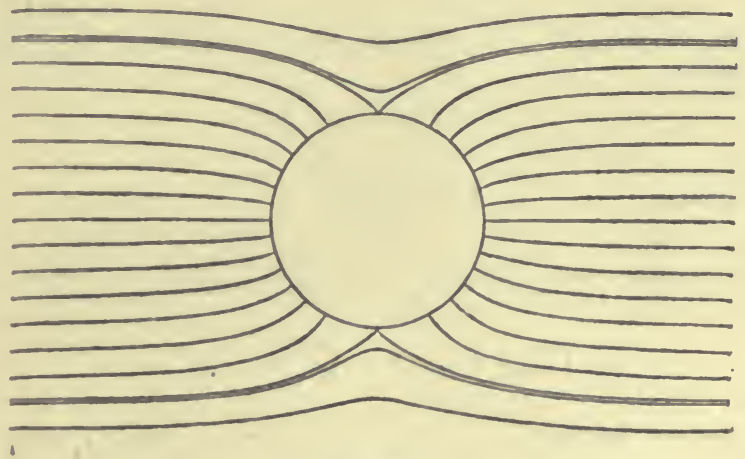

Fig. 78.

outside the sphere at the ends of the diameter parallel to $\mathrm{E}$ where $d=a$ and $\theta=0$, the total field is $\mathbf{E}+2 \mathrm{E}=3 \mathrm{E}$.

The field in the neighbourhood of the sphere is shown in Fig. 78. Since the field without is modified by the presence of the

* These components may be obtained very easily by noting that the two nearly equal forces $\frac{4}{3} \pi \rho a^{3} / d^{2}$ and $\frac{4}{3} \pi \rho a^{3} /(d+\delta)^{2}$ acting at an angle differing from $\pi$ by the small angle $\phi$ have resolutes along the two bisectors of the angle respectively equal to the difference $\frac{8}{3} \pi \rho a^{3} \delta / d^{3}$ and to $\frac{4}{3} \pi \rho \alpha^{3} \phi / d^{2}$. Here $\delta$ is $\mathrm{O}^{\prime} \mathrm{O} \cos \theta$ and $\phi$ is $00^{\prime} \sin \theta^{\prime} d$. 
conductor as if it were a doublet, the forces on the external charges producing the field will be the same as those of the doublet.

We have just seen that two almost overlapping spheres with equal radii $\alpha$ and equal and opposite densities of charge, will change the internal intensity from $\mathbf{E}$ to 0 , i.e. by an amount $\mathbf{E}$ if they are equivalent externally to a doublet of moment $\mathbf{E} \mathbf{a}^{3}$. 'Then in order that the internal intensity may be changed from $\mathbf{E}$ to $\mathbf{E}$ ', i.e. by an amount $\mathbf{E}-\mathrm{E}^{\prime}$, the pair of spheres must be equivalent externally to a doublet $\left(\mathbf{E}-\mathbf{E}^{\prime}\right) a^{3}$.

Let us assume that when a dielectric sphere, radius $a$, and with dielectric constant $K$ is substituted, the field is uniform and equal to $\mathbf{E}^{\prime}$ within, and without is $\mathbf{E}$ with the field due to the doublet $\left(\mathbf{E}-\mathbf{E}^{\prime}\right) a^{3}$ superposed on it. In order that this may be the actual arrangement of intensity it has to satisfy the two conditions:(1) equality of intensity in the two media tangential to and close to the surface of the sphere; and (2) equality of strain in the two media normal to and close to the surface.

'The tangential intensities at a point on the surface distant $a$ in the field assumed are:-just outside, $\mathbf{E} \sin \theta-\left(\mathbf{E}-\mathbf{E}^{\prime}\right) \sin \theta=$ $\mathbf{E}^{\prime} \sin \theta$; just inside, $\mathbf{E}^{\prime} \sin \theta$.

So that any uniform value of $\mathrm{E}^{\prime}$ will satisfy this condition.

The normal intensities at the same point are:-just outside, $2\left(\mathbf{E}-\mathbf{E}^{\prime}\right) \cos \theta+\mathbf{E} \cos \theta=\left(3 \mathbf{E}-2 \mathbf{E}^{\prime}\right) \cos \theta$; just inside, $\mathbf{E}^{\prime} \cos \theta$.

Since the strains are respectively intensity $/ 4 \pi$ in air and $K \times$ intensity $/ 4 \pi$ in the sphere, we must have for equality of normal strain

$$
\begin{array}{ll} 
& 3 E^{\prime}-2 E^{\prime}=K^{\prime} E^{\prime} \\
\text { whence } & E^{\prime}=\frac{3 E}{K+2} .
\end{array}
$$

The moment of the doublet equivalent for the outside is

$$
\left(\mathrm{E}-\mathrm{E}^{\prime}\right) a^{3}=\frac{\mathbf{K}-1}{\mathbf{K}+2} \mathbf{E} a^{3}
$$

and the values of the internal and external fields thus obtained satisfy the conditions, and so constitute a solution. We shall assume that it is the only solution.

Since the field without is modified as if the dielectric were replaced by the doublet, the action on any external charge will be the same as that of the doublet, or will be $\frac{K-1}{K+2}$ times the action of the equal conducting sphere. Similarly, the reaction of the charge on the dielectric will be $\frac{K-1}{K+2}$ times the action on the equal conducting sphere. We shall see how Boltzmann used this result to obtain $\mathbf{K}$. 


\section{RESIDUAL CHARGE AND DISCHARGE}

The investigation of specific inductive capacity is very much complicated by what are termed residual effects. If a Leyden jar is charged and, after standing for a short time, is discharged by a spark in the ordinary way, it appears to be completely discharged and the two coatings are at the same potential. But if the jar is allowed to stand for a short time, with the inner coating insulated, a new charge gathers of the same sign as the original charge, and a second much smaller spark may be obtained on discharging it. 'This process may be repeated, and with some jars three, four, five, or more visible sparks may be obtained in succession, the jar being allowed to rest insulated after each discharge. If, immediately after the first discharge, the inmer coating is connected to a goldleaf electroscope, the charge can be seen to gather, for the leaves diverge till they touch the side plates and so discharge the jar. They will diverge and discharge many times in succession. The electroscope may be used to show that all these charges are of the same sign as the original charge. 'They are known as Residual Charges, and the discharges as Residual Discharges.

If a jar which shows very conspicuous residual effect is charged to some ineasured potential and left insulated, it is found that the potential gradually falls, and the discharge obtained on connecting the coatings for a moment will be less than the original charge. 'This fact suggests that the phenomenon is in some way ronnected with conduction, and Faraday sought to explain it by supposing that the two charges left the plates to some extent and penetrated the dielectric towards each other, some of the + charge on AB, Fig. 79, for instance, reaching $a b$, while some of the - charge on CD reached $c d, a b$ and $c d$ being probably further in

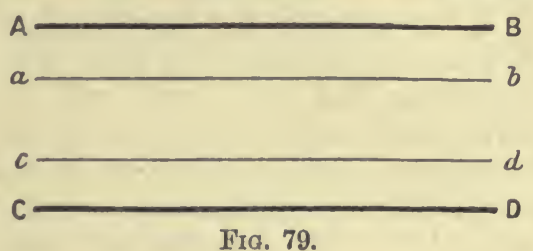

the longer the time.. On discharging $\mathrm{AB}$ and $\mathrm{CD}$ the charges $a b$ and $c d$ would no longer be pushed from behind by charges on the plates, and some part of them would return to $\mathrm{AB}$ and $\mathrm{CD}$ and be ready for a second discharge. But when we come to examine the process of conduction we shall see that this account, though probably containing a good deal of truth, hardly gives the correct view as it stands. ${ }^{*}$

R. Kohlrausch $\dagger$ showed that if a given jar is charged to a certain

* Exp. Res., vol, i. § 1245.

† Pogg. Ann. xcì. (1854). 
potential and is then insulated, the fall of potential in a given time is proportional to the initial potential, and, further, that if at any instant the jar is discharged, the instantaneous discharge is proportional to the potential just before the discharge was made. Kohlrausch formed a theory of the action going on which apparently involved the idea of conduction, though he did not express it in that form. But he pointed out the resemblance of the residual phenomena to those of "clastic after-action" in strained wires, which gives a very valuable analogy. If a wire is twisted-for instance, if a glass fibre is fixed vertically in a clamp at its upper end, and if the lower end is twisted and held, it will return on release towards its original position, but not the whole way. If it is now held in its new position for a time and is then released, it will return another portion towards its original position, and so on. We may explain this after-action, as it is termed, by supposing that some parts of the glass retain strain energy as long as the strain is retained, while in other parts, though the strain remains, the energy is dissipated, or the stress diminishes. Imagine, for instance, that the outer shell of the fibre is "true " in its elasticity, i.e. that the stress is always proportional to the strain, but that the imner core gradually loses its strain energy, even though its strain is maintained. Now twist the compound fibre. If it is instantly released before the energy in the core has had time to become dissipated it will return to the original position. But if the fibre is held twisted for a time the stress in the core gradually decreases and the effective strain decreases. When the fibre is released the effective strain in the core will be entirely removed before the fibre is entirely untwisted. But some stress still remains in the outer shell when this point is reached, and the outer shell still tends to untwist, and will continue to untwist till the core is strained in the opposite direction so much that the negative stress in it just balances the positive stress still remaining in the shell On again holding the fibre this negative stress decreases, and on release the outer shell will be able to impart some more negative strain to the core, and so on. Gradually the fibre will return to its original position if the outer shell is perfectly "true" in its elasticity. But if it too exhibits some dissipation of energy the return will not be complete.

The most probable explanation of residual effects is analogous to this, and was given by Maxwell* somewhat in the following form. It takes account of effects which must certainly exist, and so far it must be a true explanation, though it may not be complete. In it the dielectric is regarded as heterogeneous, of which parts are slightly conducting, while other parts remain completely insulating. We may imagine, as in Chapter IV, that the strain in the

* Electricity and Magnetism, vol. i. chap. x. An account of Maxwell's theory on somewhat simpler lines is given in the Plil. Mag., Series V. vol. xxi. (1856), p. 419 . 
dielectric consists in the formation of chains of molecules. If each molecule consists of a positively charged part $a$ and a negatively charged part $b$, in the entirely unelectrified condition these molecules $\iota b$ will be turned indifferently in all directions. When the dielectric begins to be strained we may suppose that some, at any rate, arrange themselves in chains between $\mathrm{AB}$ and $\mathrm{EF}$, and if $\mathrm{AB}$ is positively charged we shall have the negative elements towards $\mathrm{AB}$ thus : $\mathrm{AB}|a b a b a b a b| \mathrm{EF}$. As the strain increases we may suppose the links of the chain, as it were, stretched out thus :

$$
\begin{array}{llllllllllll}
\mathrm{AB} & \mid & a & b & a & b & a & b & a & b & \mathrm{EF}
\end{array}
$$

and conduction implies a breakdown of the chain, the first $a$ going to $\mathrm{AB}$ and neutralising part of its positive charge, while its $b$ unites with the $a$ of the second molecule, and so on along the line, the last $b$ going to $\mathrm{EF}$ and neutralising part of the negative charge there, thus :

$$
\mathrm{AB}|a \quad b a \quad b a \quad b a \quad b| \mathbf{E F}
$$

We may suppose that, in the slightly conducting dielectric, a very small fraction of the whole number of chains breaks down per second, the fraction being proportional to the slope of potential.

'To illustrate Maxwell's theory, let us imagine a condenser, $\mathrm{ABCD}$, Fig. 80, with a dielectric of specific inductive capacity $K$ throughout. Let the upper half above EF be a slight conductor, while $A-B$ the lower half is a perfect insulator. Let the condenser be charged till the strain is, say, 32 throughout, and then let the upper plate be insulated. Then strain 32 remains

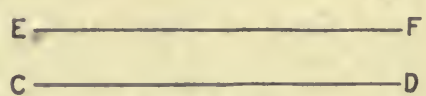
in the lower half, EFCD. But conduction in the upper half means that the effective strain in it is gradually diminishing.

Now let us suppose that the condenser remains insulated till strain 16 only remains in the upper half. 'There will then only be charge +16 on $\mathrm{AB}$. On EF there will be charge $32-16=$ +16 , and on $\mathrm{CD}$ there will be -32 . If we now discharge the condenser by connecting $\mathrm{AB}$ and $\mathrm{CD}$ we shall get in the first place +16 of $\mathrm{AB}$. neutralising -16 of $\mathrm{CD}$. $\mathrm{AB}$ is now discharged. But there is still - 16 remaining on $\mathrm{CD}$, which will be equally shared between $A B$ and $C D$. This implies a transfer of -8 from $\mathrm{CD}$ to $\mathrm{AB}$, which is equivalent to a further discharge of +8 from $\mathrm{AB}$, or a discharge of $24 \mathrm{in}$ all. The potential of $\mathrm{AB}$ is made equal to that of $\mathrm{CD}$, and the strain is -8 in $\mathrm{ABEF}$ and +8 in EFCD, while there is a charge +16 on EF.

If $\mathrm{AB}$ is now insulated, the negative strain -8 between $\mathrm{EF}$ and AB gradually breaks down, while the positive strain between EF 
and $\mathrm{CD}$ remains, and the potential difference between $\mathrm{AB}$ and $\mathrm{CD}$ becomes positive again. Let us suppose that $\mathrm{AB}$ remains insulated till the strain between it and $\mathrm{EF}$ has decreased from -8 to -4 . There will then be charge +12 on EF. On connecting $\mathrm{AB}$ and $\mathrm{CD}$ the two charges -4 and -8 will be equally shared, which implies a discharge of -2 from $C D$ to $A B$, equivalent to a discharge of +2 from $A B$. There will then be -6 on each of $A B$ and $\mathrm{CD}$, with the corresponding +12 on EF. Again insulate $\mathrm{AB}$ till the strain -6 between $\mathrm{EF}$ and $\mathrm{AB}$ has fallen to -3 . On connecting $\mathrm{AB}$ and $\mathrm{CD}$ there will be a passage of -1.5 from $\mathrm{CD}$ equivalent to +1.5 from $\mathrm{AB}$, and 9 remains on $\mathrm{EF}$. If we suppose the strain between $\mathrm{AB}$ and $\mathbf{E F}$ to be halred in this way each time, the successive discharges from the first will be

$$
24, \quad 2, \quad 1 \cdot 5, \quad 1 \cdot 125, \quad \& \text { c. }
$$

Evidently discharges will be obtained till the space between $\mathrm{EF}$ and $\mathrm{CD}$ is completely free from strain-that is, until all the charge on $\mathrm{CD}$ is gone, Hence, if we have a completely insulating layer, the sum of all the discharges must equal the original charge. If, however, the layer between EF and CD is not completely insulating, but is a worse conductor than the upper layer, though we shall have residual discharges their sum will be less than the original charge, owing to the decay of strain terminating on CD.

It is obvious that if the dielectric is homogeneous and slightly conducting throughout there will be no residual phenomena according to this theory. For the strain will break down simultaneously from plate to plate, and the first discharge will be complete, as there will be no charges left within the medium as we have supposed those left on EF in the above explanation.

We may note here how this explanation differs from that of Faraday. He appears to have thought of the charge from $A B$ as gradually moving towards EF, the first links of the chain, as it were, breaking down first. 'Then after discharge he thought of the charge as gradually moving back again towards AB. It is evident, however, that Faraday had the essential features of the present theory.

If a jar is charged, allowed to rest, discharged, and then charged in the opposite direction to a less extent than at first, it is found that it may ultimately show a residual charge of the same sign as the first charge. The theory gives an explanation of this phenomenon. For suppose that after the first discharge in the case we have considered, where -8 is the strain in the upper half and +8 is that in the lower, we give a negative charge -4 on $A B$ and +4 on $\mathrm{CD}$, we shall begin with strain -12 in the upper half and +4 in the lower, or with $\mathrm{AB}$ at lower potential than CD. But if we leave the jar long enough the strain in the upper half will decay to less than -4 , and the potential of $A B$ will then 
rise above that of $\mathrm{CD}$, or we shall again be able to get a positive discharge from $\mathrm{AB}$.

It is easy to work out a more general theory where the dielectric consists of any number of layers of different thicknesses with different dielectric constants and different conductivities or rates of decay of strain, if we assume that Ohm's law holds-that is, that the rate of decay is proportional to the intensity. If $\mathrm{D}$ is the strain and $\mathbf{E}$ the intensity, we have

whence

$$
\begin{gathered}
-\frac{d \mathrm{D}}{d t}=c \mathrm{E}=\frac{4 \pi c \mathrm{I})}{\mathrm{K}} \\
\mathrm{D}=\mathrm{D}_{0} e^{-\frac{4 \pi r \cdot t}{\mathrm{~K}}}
\end{gathered}
$$

where $\mathrm{D}_{\mathrm{o}}$ is the initial strain and $c$ is the specfic conductivity of the material. If then at any instant the strains are $\mathrm{D}_{1} \mathrm{D}_{2} \ldots$ in layers having conductivities $c_{1} c_{2} \ldots$ dielectric constants $K_{1} K_{2} \ldots$ and thicknesses $d_{1} d_{2} \ldots$ at any future time $t$ they will be

$$
\mathrm{D}_{1} e^{-\frac{4 \pi c_{1} t}{\mathrm{~K}_{1}}}, \mathrm{D}_{2} e^{-\frac{4 \pi c_{2} t}{\mathrm{~K}_{2}}}, \& c .
$$

and the potential will alter from

$$
\begin{aligned}
& \frac{4 \pi \mathrm{D}_{1} d_{1}}{\mathrm{~K}_{1}}+\frac{4 \pi \mathrm{D}_{2} d_{2}}{\mathrm{~K}_{2}}+\ldots \\
& \text { to } \\
& \frac{4 \pi \mathrm{D}_{1} d_{1} e^{-\frac{4 \pi r_{1} t}{K_{1}}}}{K_{1}}+\frac{4 \pi \mathrm{D}_{2} d_{2} e^{-\frac{4 \pi c_{2} t}{K_{2}}}}{\mathrm{~K}_{2}}+\cdots
\end{aligned}
$$

But this general investigation has little value, for in the first place the dielectric heterogeneity does not consist in a parallel arrangement of layers, each homogeneous, but much more probably in an irregular granular arrangement. That it is complex was shown by Hopkinson (Original Papers, ii. p. 2). He found that the potential of a jar charged and then insulated could not be expressed as a function of the time by two exponentials only. If it could be expressed by a series of the above form, certainly more than two terms would be required, or the heterogeneity is more than twofold.

In the second place, even if Ohm's law holds, we cannot assume that $K$ and $c$ are constant for each element of the structure while it is breaking down. If the breakdown is, as we have supposed, electrolytic, the products of decomposition may alter the values of $K$ and $c$. It is even possible that they may alter the values of $d$ if the heterogeneous portions are of molecular dimensions.

Hopkinson (loc. cit. ii. pp. 10-43) investigated the rate of fall of potential of a Leyden jar, and though he could not obtain a mathematical expression for the rate, he found that it was not very different in some cases from $\frac{\mathrm{A}}{t}$, where $t$ is the time from 
insulation. Boltzmann had found that the strain in a twisted wire decays at this rate.

But though we cannot as yet give a full quantitative explanation of residual phenomena, there can be no doubt that they are, at any rate, largely due to conductivity in parts, and that Maxwell's theory contrins a large element of truth. On the one hand exceedingly good insulators, such as air and other gases, show no trace of residual charge, while on the other hand poor insulators, with structure probably heterogeneous, show residual phenomena in a marked degree.

Rowland and Nichols * showed that a plate of Iceland spar exhibited no residual effect whatever, as might be expected if heterogeneity of structure is a necessary condition for its existence.

Mechanical model illustrating the theory. $\dagger$ The model consists of a trough of semicircular cross-section (Fig. 81), say 24 in. long, 6 in. diameter, and divided into eight equal compart-

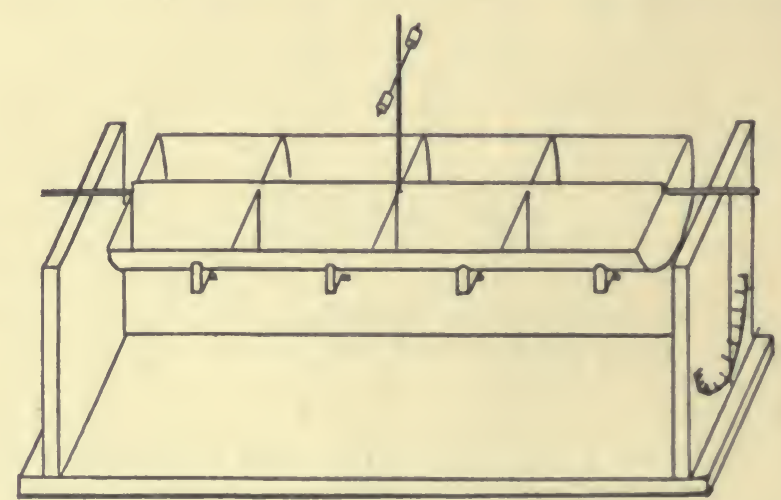

Fro. 81.

ments by a middle partition along the axis and three crosspartitions.

It is supported at the two ends, so that it can rock about its axis, and a pointer attached to one end moves in front of a scale. Four pipes, with taps, connect the opposite compartments when the taps are turned on. The trough is balanced by weights on an upright, so that when empty it is in neutral equilibrium. On turning the taps off, and on pouring in water to the same depth in all the compartments, the equilibrium at once becomes stable, and the trough, if displaced round the axis, stores up energy. It may be considered as analogous to a tube of strain connecting charges $\pm q$ on the surfaces of two opposite conductors, the angle of displacement representing the charge at either end, or the strain along the

* Rowland's Pliysical Papers, p. 204.

† Proc. Birmingham Phil. Soc., vol. vi. p. 314. 
tube. As long as the taps are turned off, the trough represents a perfect insulator, for the energy is undissipated. Release of the trough corresponds to discharge, and we have oscillations corresponding to the electric oscillations in the discharge of a condenser. If the taps are all turned on equally the trough represents a leaky dielectric of the same conductivity throughout, and holding it in the displaced position for a time and then releasing it, it returns to and remains, after the oscillations have ceased, in a position short of the original position. But if the taps are turned on unequally-if, say, the two end taps are turned off and the two middle ones are turned on-it is easily seen that the phenomena of residual discharge are exactly imitated. For on turning the trough through a given angle and holding it there, the energy in the middle compartments decreases, and on release the trough only moves part way back, groing to the point at which the mean level is the same on the two sides. There is now a negative difference of level in the middle compartments if the original difference is called positive. If the trough is held in its new position for a short time the negative difference is reduced, and on release the trough returns by another amount towards its original position, and this may be repeated several times until finally the original position is practically regained. 


\title{
CHAPTER IX
}

\section{RELATION OF SPECIFIC INDUCTIVE CAPACITY TO REFRACTIVE INDEX. THE MEASURE- MENT OF SPECIFIC INDUCTIVE CAPACITY}

\begin{abstract}
The relation between specific inductive capacity and refractire index in the electro-magnetic theory of light-Determinations of specific inductive capacity-Boltzimann's condenser method for solids-His experiinents with crystalline sulphur-Hopkinson's experiments-Boltzmann's experiments on gases-Specific inductive capacity of water, alcohol, and other electrolytes-Experiments of Colın and Arons, Rosa, Heerwagen, and Nernst-Experiments of Dewar and Fleming at low temperaturesDrude's experiments with electric wares.
\end{abstract}

The relation between specific inductive capacity and refractive index in the electro-magnetic theory of light. Maxwell's electro-magnetic theory of light supposes that light consists of waves of electric strain transverse to the direction of propagation accompanied by magnetic induction perpendicular to the electric strain, and also transverse to the direction of propagation. If, for instance, the light is plane polarised, we must suppose the electric strain always in one plane, say that of the paper as represented by the vertical lines in Fig. 82, and alternately up and down in successive half wave-lengths. The accompanying magnetic

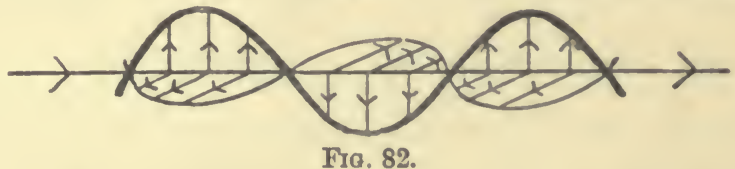

induction will be in a plane perpendicular to that of the paper, and alternately in and out in successive half wave-lengths. An analogy with sound waves will suggest to us the ratio of the velocities of propagation in different media. The full investigation belongs to Optics.

In sound waves there are the two types of energy, elastic strain and kinetic, and the velocity of propagation is given by

$$
\mathrm{V}=\sqrt{\frac{\text { modulus of bulk elasticity }}{\text { density }}} \text {. }
$$

'The numerator is proportional to the energy stored per unit 120 
strain, and the denominator is proportional to the energy possessed per unit velocity.

If we have two media with different elasticities $\mathrm{E}_{1} \mathrm{E}_{2}$, but with equal densities, the ratio of the velocities of propagation or the refractive index of sound waves from one to the other will be

$$
\mu=\frac{V_{1}}{V_{2}}=\sqrt{\frac{E_{1}}{E_{2}}}
$$

Guided by this analogy, we may regard the energy of electric strain as corresponding to the energy of elastic strain, and the energy of magnetic induction as corresponding to kinetic energy. Since in all transparent media the magnetic permeability is practically the same, the energy due to unit induction in the two media is the same, or the media for electric waves correspond to media of equal density for sound waves. The electric modulus is, as we have already seen, $\frac{4 \pi}{K}$, so that if for two media the dielectric constants are $K_{1}$ and $K_{2}$, the analogy suggests that the refractive index should be given by

$$
\mu=\frac{V_{1}}{V_{2}}=\sqrt{\frac{K_{2}}{K_{1}}}
$$

If one of the media is air, for which $K_{1}=1$, and $K$ is the dielectric constant of the other medium with respect to air,

$$
\mu=\sqrt{\bar{K}} \text {. }
$$

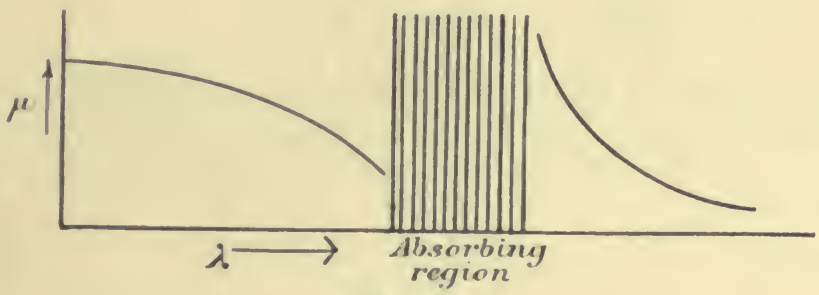

Fra. 83.

But the analogy is obviously incomplete. In sound waves in gases the elasticity is definite and independent of the periodicity of the waves. All waves travel with the same velocity, and the refractive index from one to another is a definite constant. But in light the velocity varies with the periodicity, and we have the phenomena of dispersion. 'The refractive index $\mu$ for transparent substances decreases in general as the wave-length increases. If, however, a substunce absorbs a particular wave-length or a group of wave-lengths, the refractive index in that neighbourhood varies in a manner which is termed anomalous, and the general nature of the connection between $\mu$, the refractive index, and $\lambda$, the wave-length in air, is shown in Fig. 83. 
In ordinary methods of determining $\mathbf{K}$ by experiments on capacity, we charge and discharge a condenser in times enormously long compared with the period of a visible light vibration, and we should only, therefore, expect to find $\sqrt{\mathbb{K}}=\mu$ if the value of $\mu$ is that for very long waves with period long, and if that value is unaffected by absorption-that is, if the substance is transparent to very long waves.

We shall now give an account of some of the determinations of the dielectric constant for solids, liquids, and gases, selecting typical methods, and not attempting to give any complete account of the subject.*

At the time when Maxwell published his treatise on Electricity and Magnetism the only substance of which the dielectric constant was at all accurately known was paraflin. Gibson and Barclay (Phil. Trans., 1871, p. 573) had shortly before found $k$ to be 1.975. From the refractive index of melted parafin for the $\mathrm{A}, \mathrm{D}$, and $\mathrm{H}$ lines Maxwell calculated the refractive index for light of infinite wave-length to be $1 \cdot 422$, whence $\mu^{2}=2 \cdot 02$.

Boltzmann's condenser method for solids. + Boltzmann used an air condenser (Fig. 84) with parallel plates so arranged

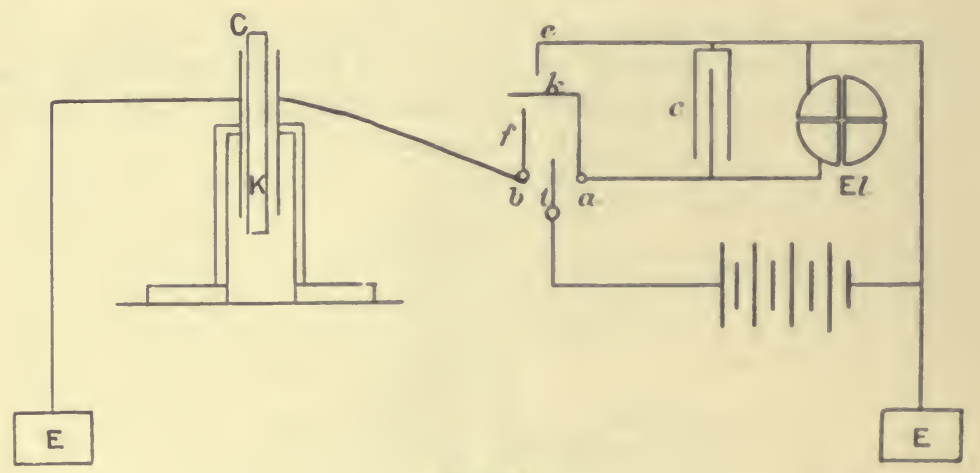

Fra. 84.

that a slab of the dielectric to be experimented on could be inserted. He compared the capacities of the condenser with and without the slab by charging it in each case to a definite potential, and then sharing the charge with a quadrant electrometer with a small air condenser added in order to increase the capacity. 'This was done so that any change in the capacity of the electrometer due to the motion of the needle should be negligible. 'The fall of potential due to the division of the charge gave the ratio of the capacity of the

* An account of the earlier work on specific inductive capacity will be fount in Gordon's Electricity and Magnetism, vol. i. chap. xi. ; or Gray's Absulute Men. surements, vo!. i. Later work is deseribed in Winklemann's Hamabuch, vol. iv.

$\dagger$ Carl's Repertorium, x. p. 109. See Gordon's Electricity, vol. i. 
large condenser to that of the electrometer + the small condenser. In order to eliminate the distance of the plates apart, a quantity not easily measured, Boltzmann moved one plate and took observations at different distances, so that the distance the one plate was moved alone came into consideration. The principle of the experiment may be represented as follows :

Let $C_{E}$ be the capacity of the electrometer + small condenser $c$, and let $V$ be the potential of the battery. When the key $k$ is open and $t$ is connected to $a$, the electrometer will indicate $\mathrm{V}$. Now let $k$ be connected to $e$, the electrometer being thus discharged. Let $t$ be connected to $b$, the large condenser $\mathrm{C}$ being thus charged to $V$. Let its capacity be $C_{1}$ when the plates are distance $d_{1}$ apart. Let $t$ be disconnected from $a$ and $b$, and let $k$ be connected to $f$. Thus the charge on $\mathrm{C}$ is shared with $\mathrm{C}_{\mathrm{E}}$, and we have the potential falling to $V_{1}$, where

whence

$$
\begin{aligned}
& C_{1} V=C_{1} V_{1}+C_{E} V_{1} \\
& C_{1}=C_{E} \frac{V_{1}}{V-V_{1}}
\end{aligned}
$$

Since the two readings of the electrometer give us $V_{1} / V$, we have $\mathrm{C}_{\mathbf{1}}$ in terms of $\mathrm{C}_{\mathbf{E}}$.

Repeat these operations when the distance of the plates in $\mathbf{C}$ is altered to $d_{2}$ and the capacity to $C_{2}$, and let the potential after sharing be $V_{2}$. Then we have

$$
C_{2}=C_{r} \frac{i V_{2}}{V-V_{2}}
$$

Then insert the slab with dielectric constant $\mathrm{K}$ and with thickness $d$, the distance of the plates apart being $d_{3}$. Let the capacity now be $C_{3}$, and the potential after sharing be $V_{3}$, and

$$
\mathrm{C}_{3}=\mathrm{C}_{\mathrm{E}} \frac{\mathrm{V}_{\mathrm{3}}}{\mathrm{V}-\mathrm{V}_{3}}
$$

(1), (2), (3) give $C_{1}: C_{2}: C_{3}$.

Since the dielectric is equivalent to $\frac{d}{\mathrm{~K}}$ of air, we have

$$
\frac{1}{\mathrm{C}_{1}}: \frac{1}{\mathrm{C}_{2}}: \frac{1}{\mathrm{C}_{3}}=d_{1}: d_{2}: d_{3}-d+\frac{d}{\mathrm{~K}}
$$

whence $\frac{1}{\mathrm{C}_{2}}-\frac{1}{\mathrm{C}_{1}}: \frac{1}{\mathrm{C}_{3}}-\frac{1}{\mathrm{C}_{1}}=d_{2}-d_{1}: d_{3}-d \frac{\mathrm{K}-1}{\mathrm{~K}}-d_{1}$

and

$$
\mathbf{K}=\frac{d}{\left(d_{2}-d_{1}\right) \frac{\mathrm{C}_{1}-\mathrm{C}_{3}}{\mathrm{C}_{1}-\mathrm{C}_{2}} \frac{\mathrm{C}_{2}}{\mathrm{C}_{3}}+d_{1}-d_{3}+d} .
$$


By this method Boltzmann obtained the following results :

$\begin{array}{lr}\text { Ebonite } & \mathbf{K}=3.15 \\ \text { Paraffin } & 2.32 \\ \text { Sulphur (non-crystalline) } & 3.84 \\ \text { Resin } & 2.55\end{array}$

The residual effect in these cases was practically negligible, for the effect was the same whether the contacts only lasted for a fraction of a second or whether the operation lasted from one to two minutes. With glass, gutta-percha, and other less perfect insulators, however, the residual effects were so great that the method was inapplicable.

'The square of the refractive index for paraflin for an infinite wave-length is calculated to be 2.022. 'That for sulphur for the $\mathrm{D}$ line is a little over 4 . 'That for ebonite * for extreme red rays is about 2.76. That for resin is given by Boltzmann as 2.38. The dielectric constant and the square of the refractive index, then, are not very different in these cases.

Boltzmann's investigations with crystalline sulphur. $\dagger$ We have seen that if a conducting sphere radius $a$ is placed in a uniform ficld $\mathbf{E}$ the external field is charged as if there were an electric doublet at the centre of moment Ea ${ }^{3}$. Suppose that a splere $\mathbf{M}$ is charged with $\mathbf{Q}$, and that a conducting sphere $\mathbf{N}$, radius $a$, is placed at a distance $d$ from its ceutre, and is so small that the field round $\mathrm{N}$ due to $\mathrm{M}$ may be regarded as uniform and of intensity $\mathbf{E}=\frac{\mathbf{Q}}{d^{2}}$. The distribution on $\mathbf{N}$ is externally equivalent to a doublet $\frac{\mathrm{Q} a^{3}}{d^{2}}$. The pull by it on $\mathrm{M}$ will therefore be $\frac{2 Q^{2} a^{3}}{d^{5}}$ if we neglect the effect of $\mathrm{N}$ in disturbing the charge on $\mathrm{M}$. 'The reaction of $\mathrm{M}$ on $\mathrm{N}$ will be equal to this.

Now replace $\mathbf{N}$ by a dielectric sphere of the same size with constant $\mathbf{K}$. The dielectric sphere is equivalent to a doublet $\frac{\mathbf{K}-1}{\mathbf{K}+2}$ of that representing the conducting sphere, and the pull on the dielectric due to $M$ will only be $\frac{K-1}{K+2}$ of that on the conducting sphere.

Boltzmann arranged a small conducting sphere suspended by silk threeds from one end of a torsion arm, and measured the deflection when an electrified sphere was in its neighbourhood. 'The conducting sphere was then replaced by an equal crystalline sulphur sphere, and the pull was again measured when the three axes of the crystal were in succession directed towards the attracting ephere. 'The ratio of the pulls on the conducting sphere and

* Ayrton and Perry, Phil. Mag., xii. (1-81), p. 196.

+ Wiener Sitzungaberichte, Ixx. part ii. p. 342; Gordon, loc. cil. p. 100. 
on the sulphur sphere gave the following values of $K$ parallel to the three axes. "Ihe values of $\mu^{2}$ along the axes as assigned by Boltzmann are also given.

\begin{tabular}{|c|c|c|c|}
\hline & $=$ & $4 \cdot 773$ & $4: 596$ \\
\hline$-m$ & $=$ & $3 \cdot 9 \% 0$ & $3 \cdot 886$ \\
\hline $\mathbf{K}_{1}$ & $=$ & $3 \cdot 811$ & $3 \cdot 591$ \\
\hline
\end{tabular}

Hopkinson's experiments. Hopkinson * made an extensive series of researches on the specific inductive capacities of solids and liquids, using for exact measurement a parallel plate condenser in which the distance between the plates could be varied till the capacity was equal to that of another condenser. The principle of the method may be gathered from the diagrammatic representation in Fig. 85.

Let $B$ be a battery of cells earthed at its middle point, so that the potential of one terminal is as far above that of the earth as the

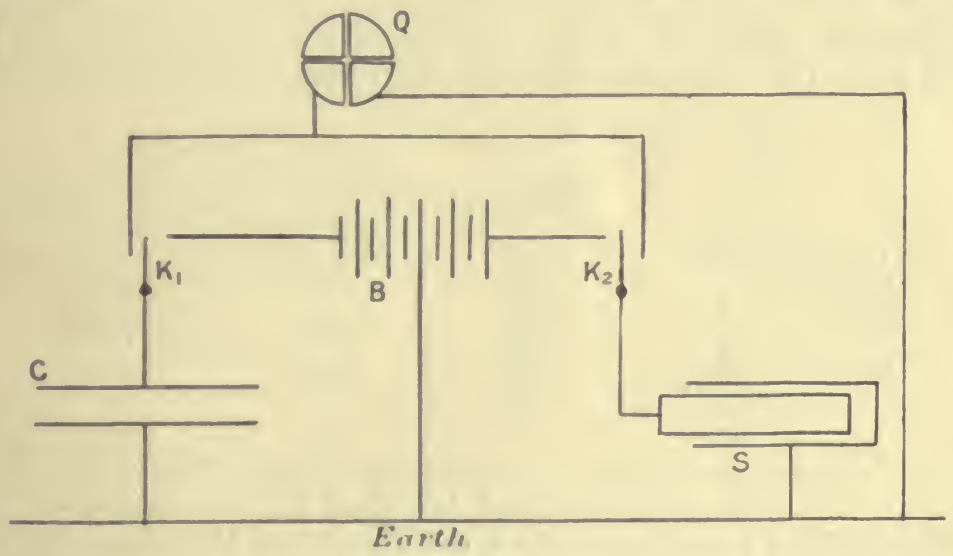

F16. 85.

potential of the other terminal is below it. Let $\mathrm{C}$ be the variable condenser with parallel adjustable plates, and let $\mathrm{S}$ be the condenser with which it must be equalised, in practice a sliding condenser consisting of two co-axial cylinders. $Q$ is a quadrant electrometer. Iet the lower plate of $\mathrm{C}$ and the outer cylinder of $\mathrm{S}$ be earthed. First connect the upper plate of $\mathrm{C}$ and the inner cylinder of $\mathrm{S}$ respectively with the + and - terminals of the battery by means of the keys $\mathbf{K}_{1} \mathbf{K}_{2}$. 'Then move $K_{1}$ and $\mathbf{K}_{2}$ over so that the two condensers are connected together and to the quadrant electrometer. If the capacities are equal the two charges are equal and opposite, and will neutralise each other, but if there is an excess of one kind of charge indicated by the electro- 
meter the condenser with that kind has the greater capacity. $\mathrm{C}$ must be adjusted till the electrometer shows no charge and the equality is obtained. Now let a layer of dielectric be interposed in C, of thickness $t$. If the distance of the plates apart is $d$, the capacity is increased in the ratio $d-t \frac{\mathrm{K}-1}{\mathrm{~K}}: d$.

'To obtain equality with $\mathrm{S}$ the distance $d$ must be increased by $t \frac{\mathbf{K}-1}{\mathbf{K}}=\delta$. This distance $\delta$ can be measured exactly and so $\mathbf{K}$ can be found.

In the apparatus actually used $\mathrm{C}$ was a "guard-ring" condenser of which the inner plate, sharing its charge with $\mathrm{S}$, was $15 \mathrm{~cm}$. in diameter. It was surrounded with a guard-ring connected to the battery when charging, so as to be at the same potential as the disc. The lines of force thus went straight across from plate to plate, and the edge effect which would depend on distance and dielectric was eliminated. Before the disc was connected to $\mathrm{S}$ the guard-ring was earthed, and remained enrthed during the connection to $\mathrm{S}$. 'This method was used for plates of flint glass, for which the following values were found:

\begin{tabular}{|c|c|c|c|c|}
\hline \multirow{2}{*}{\multicolumn{2}{|c|}{ Very light flint }} & Density & $\mathbf{K}$ & $\mu$ for $D$ line \\
\hline & & $2 \cdot 87$ & $6 \cdot 61$ & $1 \cdot 5+1$ \\
\hline Light & , & $3 \cdot 2$ & $6 \cdot 72$ & $1 \cdot 5 \% 4$ \\
\hline Dense & , & $3 \cdot 66$ & $7 \cdot 37$ & $1 \cdot 622$ \\
\hline Extra & , & $4 \cdot 5$ & $9 \cdot 90$ & 1.710 \\
\hline
\end{tabular}

It is evident that $\mu^{2}$ is very much less than $K$.

Hopkinson also showed that the result was the same whether $\mathrm{C}$ and $\mathrm{S}$ were connected to each other. and to the electrometer, for a time comparable with a second or for a minute fraction of a second. Residual phenomena, then, did not come appreciably into play. With plate glass, however, it was necessary to have only an instantaneous comnection, for with long connection the method failed owing to residual charge.

To show that $K$ is really constant, i.e. independent of the potential difference, for a given specimen of glass, a flask of extra dense flint glass containing sulphuric acid was placed in water, the contacts of the liquids with the glass forming the coatings. The capacity of this flask was found to be the same, comparing it with the sliding condenser $\mathrm{S}$, when charged with 20 elements as when charged with 1800 elements of a chloride of silver battery.

'To find the dielectric constant of liquids a fluid condenser 
was used, consisting of a double cylinder in which an insulated cylinder could hang, the vertical cross-section being as represented in Fig. 86.

'The capacity of this condenser was compared with that of a sliding condenser, first with air and second with the liquid to be tested as the dielectric. The sliding condenser was brought to equality in each case. 'The sliding condenser was graduated, and its capacity for so many divisions of the inner cylinder within the outer was known.

The hydrocarbon oils agree in giving $\mathbf{K}=\mu^{2}$ nearly, while their values for vegetable and animal oils differ widely. 'The 'Table below is extracted from Hopkinson's paper (Original Papers, ii. p. 85). The square of

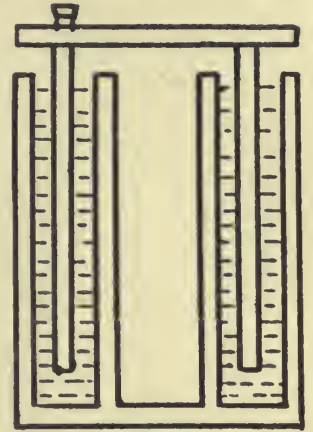

FIG. 86. the refractive index for infinitely long waves, which is given as well as $K$, is calculated from the dispersion in the visible spectrum by the formula $\mu=a+b / \lambda$, using the sodium and hydrogen lines.

$\begin{array}{lcc} & \mathbf{K} & \mu^{2} \infty \\ \text { Petroleum spirit } & 1 \cdot 92 & 1 \cdot 922 \\ \text { Petroleum oil (Field's) } & 2 \cdot 07 . & 2 \cdot 075 \\ \text { Turpentine } & 2 \cdot 23 & 2 \cdot 128 \\ \text { Castor oil } & 4 \cdot 78 & 2 \cdot 153 \\ \text { Sperm oil } & 3 \cdot 02 & 2 \cdot 135 \\ \text { Olive oil } & 3 \cdot 16 & 2 \cdot 131\end{array}$

Boltzmann's experiments on gases. Boltzmann made determinations of $\mathbf{K}$ for different gases by a simple method, using a condenser within a closed metallic earth-connected vessel which could be exhausted or filled with any gas. The connections with the plates of the condenser were made by wires passing through the sides of the ressel, but hermetically sealed in, as represented diagrammatically in Fig. 87. The ressel was first exhausted, and one plate, A, was connected to a battery of about 300 Daniell's cells, the other plate, $\mathbf{B}$, being connected to an electrometer and to earth. After A was thus raised to a potential which we will call $V_{1}$ it was insulated, $B$ and the connected pair of quadrants of the electrometer were insulated, and the gas to be experimented on was admitted. 'The potential of $\mathbf{B}$ still remained \%ero, for, as there was no leakage from $\mathbf{A}$, all the electrification on $\mathbf{B}$ was connected with that on $\mathrm{A}$, and there were therefore no lines of force between $\mathrm{B}$ and the case, and no change of its potential. 'The potential of $\mathbf{A}$ fell by the admission of the gas to, say, $V_{2}$, where $V_{1}=K V_{2}, K$ being the dielectric constant of the gas, that of vacuum being 1 . 
Connecting $\mathrm{A}$ again to the battery, its potential was once more raised to $V_{1}$. But $B$ being insulated, its potential was raised by an amount proportional to the additional charge on $\mathbf{A}$, for this additional charge induced on $\mathbf{B}$ an equal and opposite charge con-

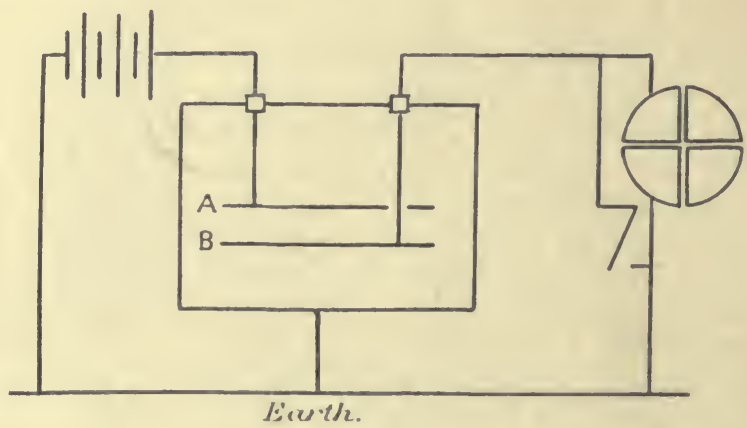

FIG. 87.

nected with itself and an equal like charge connected with the sides of the vessel. We may therefore put the potential of $\mathrm{B}$ as equal to

where $m$ is a constant,

$$
m\left(V_{1}-V_{2}\right)
$$

or

$$
m V_{1}\left(1-\frac{1}{K}\right) \text {. }
$$

Suppose the charge observed in the electrometer to be denoted by $\delta$. 'The number of cells was now increased from $n$ to $n+1$, and the potential of $A$ was consequently raised to $V_{1}+\frac{V_{1}}{u}$, and that of $\mathrm{B}$ was raised by $m \frac{\mathrm{V}_{1}}{n}$.

If the change observed in the electrometer was $\delta^{\prime}$, we have

$$
\frac{\delta}{\delta^{\prime}}=\frac{m \mathrm{~V}_{1} \frac{\mathrm{K}-1}{\mathrm{~K}}}{m \frac{V_{1}}{n}}=n \frac{\mathrm{K}-1}{\mathrm{~K}}
$$

whence $K=\frac{n \delta^{\prime}}{n \delta^{\prime}-\delta}$.

The following Table gives Boltzmann's results at $0^{\circ} \mathrm{C}$. and $760 \mathrm{~mm}$., with the values of $\mu$ as determined by Dulong for white light. As the difference between this and $\mu \infty$ is probably small for gases, these values may fairly be taken. 


\begin{tabular}{|c|c|c|c|c|}
\hline & & K & $\sqrt{\mathrm{K}}$ & $\mu$ \\
\hline Air & & $1 \cdot 000590$ & $1 \cdot 000295$ & $1 \cdot 000294$ \\
\hline Carbonic acid & & $1 \cdot 000946$ & $1 \cdot 000473$ & $1 \cdot 000449$ \\
\hline Hydrogen . & & $1 \cdot 000264$ & $1 \cdot 000132$ & $1 \cdot 000138$ \\
\hline Carbonic oxide & & $1 \cdot 000690$ & $1 \cdot 000345$ & $1 \cdot 000340$ \\
\hline Nitrous oxide & & $1 \cdot 000994$ & $1 \cdot 000497$ & $1 \cdot 000503$ \\
\hline Olefiant gas . & & 1.001312 & $1 \cdot 000656$ & 1.000678 \\
\hline Marsh gas & & $1.00094 \mathrm{t}$ & $1 \cdot 0004 \% 2$ & $1 \cdot 000443$ \\
\hline
\end{tabular}

Specific inductive capacity of water, alcohol, and other electrolytes. The ordinary condenser method, with comparatively slow charge and discharge, is quite inapplicable to such substances as water, in which the conduction is very appreciable. The difficulty introduced by conduction was first overcome by Cohn and Arons, ${ }^{*}$ who used a modification of a method previously applied to insulating liquids by Silow. If a quadrant electrometer has one pair of quadrants to earth and the other pair is connected to the needle and to a source giving potential $\vec{V}_{n}$, then the deflection (see p. 91) is approximately

$$
\theta=\frac{C}{\lambda} \frac{V_{n}{ }^{2}}{2}
$$

where $\mathrm{C}$ is the capacity of the needle per radian and $\lambda$ is the torsion couple per radian - that is, it is independent of the sign of $V_{n}$. If $V_{n}$ alternates rapidly $\theta$ is proportional to the mean square of $\mathrm{V}_{\mathrm{n}}$.

If the medium between the needle and the plates is not air, but a liquid with dielectric constant $K$, then

$$
\theta=\frac{\mathrm{KC}}{\lambda} \frac{\mathrm{V}_{\mathrm{n}}{ }^{2}}{2} \text {. }
$$

Cohn and Arons used two electrometers and the alternating potential supplied by one terminal of a Helmholtz induction coil. When both contained air the deflections were, say, $\delta_{1}$ and $\delta_{2}$. 'The second was then filled with the liquid to be experimented on, the first still containing air. The deflections were now, say, $\delta_{1}{ }^{\prime}$ and $\delta_{2}{ }^{\prime}$.

In the first case we have

$$
\frac{\delta_{2}}{\delta_{1}}=\frac{\mathrm{C}_{2}}{\lambda_{2}} \frac{\lambda_{1}}{\mathrm{C}_{1}}
$$


In the second case we have

Diviảing, we get

$$
\frac{\delta_{2}^{\prime}}{\delta_{1}^{\prime}}=\frac{\mathrm{KC}_{2}}{\lambda_{2}} \frac{\lambda_{1}}{\mathrm{C}_{1}}
$$

$$
K=\frac{\delta_{0}^{\prime}}{\delta_{1}^{\prime}} \frac{\delta_{1}}{\delta_{2}}
$$

'They obtained the following values:

Distilled water
Ethyl alcohol
Aınyl alcohol
Petroleum

K

76

26.5

15

$2 \cdot 04$

The remarkably high values for water and alcohol were confirmed by Rosa, * who measured the attraction between two plates immersed in the liquid, connected through a commutator respectively to the two terminals of a battery supplying any desired potential difference up to 60 volts. 'The commutator was reversed from 2000 to 4000 times per minute, so that the charges of the plates alternated rapidly. One of the plates was fixed, and the other was suspended at the end of a torsion arm, the small torsion measuring the force. The specific inductive capacity was determined by finding the ratio of the attraction for the same difference of potential, with the liquid as medium and with air. For the $\mathbf{K}$ medium the charges are $\mathbf{K}$ times as great, and, therefore, the forces which are (p. 104) proportional to $\frac{\sigma^{2}}{\mathbf{K}}$ are also $\mathbf{K}$ times as great. Rosa obtained for water at $25^{\circ} K=75 \%$, and for alcohol at $25^{\circ} K=25 \%$. He found that the conductivity of water might be increased many times by adding minute quantities of acid without much change in the attracting force. The force was, however, slightly lessened by the addition.

Heerwagen, $\dagger$ using a method somewhat like that of cohn and Arons, found the value of $\mathbf{K}$ for water and its variation with temperature, the results agreeing very closely with the formula

$$
K_{\mathbf{t}}=80.8 \% 8-0.362\left(t-17^{\circ}\right) .
$$

Nernst $\ddagger$ put the liquid to be tested in a condenser and determined the capacity by a Wheatstone bridge method, using

* Phil. Mag., xxxi. (1891), p. 188.

+ Wied. Ann. (1893), xlviii. p. 35, and xlix. p. 272.

$\ddagger$ Zeit. Phys. Chem.(1894), vol. xiv. 1. 622. 
an alternating current. For water at $17^{\circ} \mathrm{C}$. he obtained $\mathrm{K}=80.00$, and other observers* by various methods have found nearly the same value. For alcohol he found $\mathrm{K}$ to be about 26 .

Experiments of Dewar and Fleming at low temperatures. $\dagger$ Dewar and Fleming made experiments on the dielectric constant of ice and other substances from a temperature of $-200^{\circ} \mathrm{C}$. upwards, using a condenser consisting of two co-axial brass cones, about $15 \mathrm{~cm}$. long, the outer tapering from an inside diameter of $5 \cdot 1 \mathrm{~cm}$. to $2.6 \mathrm{~cm}$., and the space between the two being $3 \mathrm{~mm}$. The condenser was charged and discharged about 120 times per second by an interrupting tuning-fork and the circuit was arranged so that either the charging or the discharging current alone should go through a galvanometer. The equality of the two was taken to show that conduction was not coming into play. 'I'he space between the cones was filled with the substance to be examined, and cooled to the temperature of liquid air, and the galvanometer deflection was observed on charging at given potential or on discharging. 'Ihen the substance was melted out and replaced by gaseous air at the same temperature, and the galvanometer deflection was again observed. 'I'he ratio of the deflections, after certain corrections, gave the specific inductive capacity of the substance. Observations were also made at higher temperatures by allowing the temperature of the condenser to rise gradually.

The dielectric constant of pure ice at $-200^{\circ} \mathrm{C}$. was $2 \cdot 43$, rising with rise of temperature to 70.8 at $-7.5^{\circ} \mathrm{C}$., though here conduction had set in and the measurement was not so trustworthy. A large number of solutions and compounds were thus examined, and the general result was that at $-200^{\circ} \mathrm{C}$. the dielectric constant was not much greater than the square of the refractive index for exceedingly long waves, as calculated firom dispersion formulæ. For castor oil, olive oil, and bisulphide of carbon they were nearly coincident.

Drude's experiments with electric waves. As a type of an entirely different method of research, in which electric waves are used, we shall take Drude's experiments on water and on other liquids. $\neq$

If $U_{1}$ is the velocity of electric waves in air and $U_{2}$ is their velocity in another medium, the refractive index for the waves is $\mu=U_{1} / U_{2}$. But if $\lambda_{1} \lambda_{2}$ be the lengths in the two media of waves starting from a source of the same frequency $\lambda_{1} / \lambda_{2}=U_{1} / U_{2}$, so that $\mu=\lambda_{1} / \lambda_{2}$. According to the electro-magnetic theory $K=\mu^{2}=\lambda_{1}{ }^{2} / \lambda_{2}{ }^{2}$. To find the ratio $\lambda_{1} / \lambda_{2}$, Drude used a Lecher system in which wares were transmitted between two parallel wires, first through air and then through a trough containing the

* For a summary see Dewar and Fleming, Proc. Roy. Soc., lxi. r. 2.

† Proc. Roy. Soc., lxi. (several papers).

I Ann, der Phys, 1896, lyiii. p. 1, lix. p, 17, 
liquid to be examined. The principle of the method may be gathered from Fig. 88.

$\mathrm{J}$ was an induction coil connected by wires A A to the terminals of the Blondlot exciter $\mathbf{E ~} \mathbf{E}$, which consisted of two semicircles with diameter in some cases $5 \mathrm{~cm}$. and in other cases $15 \mathrm{~cm}$. The brass terminals of $\mathrm{E} \mathrm{E}$ were $5 \mathrm{~mm}$. diameter, and the spark gap between them could be varied by a micrometer. Round E E was a circular wire continued by the parallel wires D D about $2 \mathrm{~cm}$. apart. These might be continued, when desired, into a trough containing the liquid to be tested and from $30 \mathrm{~cm}$. to $60 \mathrm{~cm}$. long. When sparks took place across the gap in E E waves were propagated in the space between D D with the velocity of free waves. $\mathrm{H}_{1} \mathrm{H}_{2}$ were holders to keep the wires adjusted and $B_{1}$ and $B_{2}$ were two wire bridges. Between these, stationary waves were formed. $B_{1}$ was adjusted to be at the first node; the second node was where the wires entered the trough of liquid,

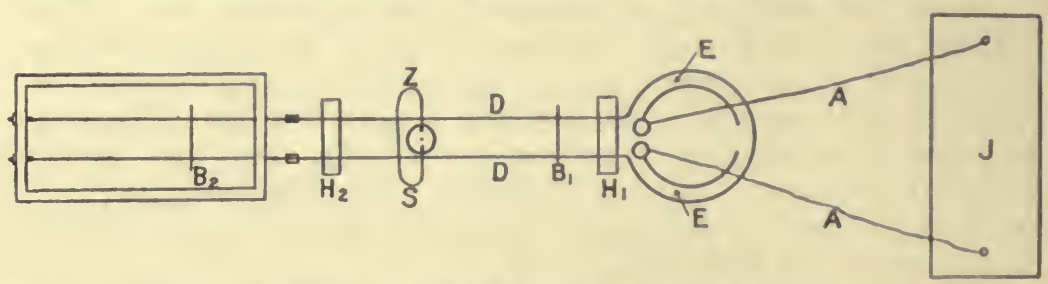

Fig. 88. J induction coil ; A A wires from its terminals to the exciting circuit $\mathrm{E} \mathrm{E}$, round which is a circuit prolonged by the two wires $\mathrm{DD} ; \mathrm{B}_{1} \mathrm{~B}_{2}$ bridges, $B_{1}$ fixed, $B_{2}$ in the liquid trough movable; $Z$ \%ehnder vacuun tube lighting up at a loop.

and $B_{2}$ was moved about till it was at a third or further node. In the original arrangement $Z$ was a vacuum tube with terminals connected by a wire $s$ of such length that the electrical period of the tube coincided with that of the exciter, but a neon tube laid across the wires suffices. When $B_{2}$ was at a node and $Z$ at a loop, $Z$ lighted up with maximum brilliance. Several successive nodal positions were observed by means of this maximum brilliance, and these were, of course, at intervals of $\lambda_{2} / 2$. 'Then the trough was detached and replaced by a continuation of the wires in air. Nodal positions of $\mathrm{B}_{2}$ were again observed, and thus $\lambda_{1} / 2$ in air was found. Thence $\mu=\lambda_{1} / \lambda_{2}$ was known.

With this apparatus Drude was able to show the existence of dispersion-that is, a difference of velocity with difference of frequency. He used two frequencies, respectively $150 \times 10^{6}$ and $400 \times 10^{6}$. The velocity for water was practically the same for both frequencies, but for glycerine $\mu^{2}=39 \cdot 1$ for the former and $\mu^{2}=25.4$ for the latter, and for other liquids there were considerable differences. He found that for water at $t^{\circ} \mathrm{C}$. the value

$$
\mu^{2}=88.23-0.4044 t+0.001035 t^{2}
$$


sufficiently expressed the dependence on temperature, though there were slight variations with variation of frequency.

With solutions of small conductivity the refractive index was nearly the same as for watcr, but as the conductivity increased, the value of the refractive index decidedly decreased. For details the reader may consult the original papers. 


\section{CHAP'TER X}

\section{STRESSES IN THE DIELECTRIC}

Tension along the lines of strain-Pressure transverse to the lines of strain-Value of the pressure in a simple case-This value will maintain equilibrium in any case-There may be other solutions of the problemThese stresses will not produce equilibrium if $\mathrm{K}$ is not uniform-Quincke's experiments-General expression for the force on a surface due to the electric tension and pressure-The electric stress system is not an elastic stress system and is not accompanied by ordinary elastic strains.

WE have shown that a charged conducting surface in air is pulled out by a normal force $2 \pi \sigma^{2}$ per unit area, and that in a dielectric of specific inductive capacity $\mathrm{K}$ the pull becomes $2 \pi \sigma^{2} / \mathrm{K}$ per unit area.

Tension along the lines of strain. In accordance with the dielectric theory of electric action we must suppose that this pull is exerted on the conductor by the insulating medium in contact with it. Further, assuming that reaction is equal and opposite to action, the surface is pulling on the medium with an equal and opposite force.

Let $\mathrm{AB}$, Fig. 89, be a small area $\alpha$ of a charged surface; AC BD the tube of strain or force starting normally from it.

The conductor is pulling on the medium in the tube with force $2 \pi \sigma^{2} \alpha / \mathrm{K}=2 \pi \mathrm{D}^{2} \alpha / \mathrm{K}$, since $\mathrm{D}=\sigma$. Now consider the equilibrium

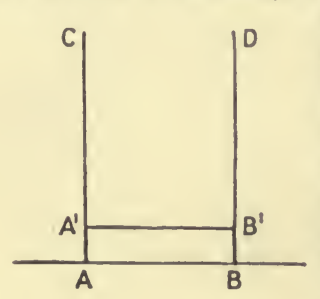
of a lamina of the medium between $\mathrm{AB}$ and a parallel cross-section $A^{\prime} B^{\prime}$ very near to it. 'The area of $\mathbf{A}^{\prime} \mathbf{B}^{\prime}$ will also be $\alpha$ if $\mathbf{A A}^{\prime}$ is small. 'The forces on the sides of this lamina are negligible compared with those on the ends, since the area of the sides is vanishingly small compared with the area of either end. Then for equilibrium the part of the medium above $A^{\prime} B^{\prime}$ must pull on $A^{\prime} A^{\prime} B^{\prime} B$ with a force

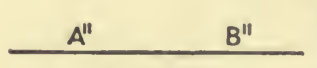

FIG. 89. equal and opposite to that across $\mathrm{AB}$, viz. $2 \pi \mathrm{I})^{2} \alpha / \mathrm{K}$. There is therefore a tension $2 \pi \mathrm{D}^{2} / \mathrm{K}$ across $\mathrm{A}^{\prime} \mathrm{B}^{\prime}$.

Now imagine the charged surface to be removed to $\mathrm{A}^{\prime \prime} \mathrm{B}$ " some distance back, but so adjusted as to position and charge that strain in the neighbourhood of $A^{\prime} B^{\prime}$ remains the same in direction and in magnitude. We can hardly suppose that the stress across $\mathbf{A}^{\prime} \mathbf{B}^{\prime}$ is altered, and so we obtain the result that a 
any point in the medium there is a stress along the lines of force, a tension $2 \pi \mathrm{D}^{2} / \mathrm{K}$, where $\mathrm{D}$ is the electric strain. Since $\mathrm{E}=4 \pi \mathrm{D} / \mathrm{K}$, we may put the tension equal to $\mathrm{KE}^{2} / 8 \pi$.

Pressure transverse to the lines of strain. A portion of the medium, say the portion between two cross-sections $\alpha_{1} \alpha_{2}$ of a tube of strain, could not be in equilibrium under these forces unless the lines of force were parallel and the field uniform. Equilibrium under the end pulls is obviously impossible unless $\alpha_{1}$ is parallel to $a_{2}$. To show that the field must also be uniform, let $\mathrm{D}_{1}$ and $\mathrm{D}_{2}$ be the strains at $\alpha_{1}$ and $\alpha_{2}$; the difference of the pulls on the two areas is

$$
2 \pi \mathrm{D}_{1}{ }^{2} \alpha / \mathrm{K}-2 \pi \mathrm{D}_{2}{ }^{2} \alpha_{2} / \mathrm{K},
$$

and since $\mathrm{D}_{1} \alpha_{1}=\mathrm{D}_{2} \alpha_{2}$, this may be put

$$
\frac{2 \pi \mathrm{D}_{1} \alpha_{1}}{\mathrm{~K}}\left(\mathrm{D}_{1}-\mathrm{D}_{2}\right),
$$

which only vanishes if $\mathrm{D}_{1}=\mathrm{D}_{2}$ or $\alpha_{1}=\alpha_{2}$, and this is the condition for a uniform field. When the field is not uniform there must be forces across the sides of the tube to make equilibrium possible.

Value of the pressure in a simple case. A value for the side forces is suggested by considering a special case. Let a particle charged with $Q$ be placed at $O$, Fig. 90, and let the

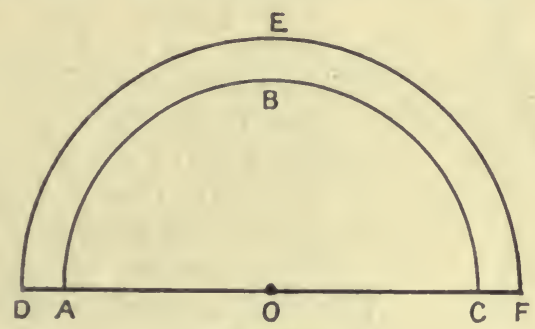

Fis. 90.

opposite charge be so far away that the lines of force radiate straight away from $\mathrm{O}$. Consider the equilibrium of the shell between two hemispheres, $\mathrm{ABC}$ radius $r$ and DEF radius $r+d r$, drawn with $\mathrm{O}$ as centre. We have a tension normal to the surface inwards across $\mathrm{ABC}$ equal to $2 \pi \mathrm{D}^{2} / \mathrm{K}=\frac{\mathrm{Q}^{2}}{8 \pi \mathrm{K}^{4}}$, since $\mathrm{D}=\frac{\mathrm{Q}}{4 \pi r^{2}}$.

Now a closed surface is in equilibrium under a uniform tension or pressure, so that if we put an equal tension, $Q^{2} / 8 \pi K r^{4}$, on the diametral plane $\mathrm{AC}$ we have equilibrium for the hemisphere OABCO. Or the tensions over the curved surface $\mathrm{ABC}$ have a resultant inwards equal to the total tension $\frac{\mathrm{Q}^{2} \pi r^{2}}{8 \pi \mathrm{K} r^{4}}=\frac{\mathrm{Q}^{2}}{8 \mathrm{~K} r^{2}}$ across the diametral plane. 
Dealing in the same way with the tensions across DEF, they have a resultant outwards equal to $\frac{Q^{2}}{8 \mathrm{~K}(r+d r)^{2}}$.

When $d r$ is very small, the resultant of these two is a pull inwards

$$
\frac{Q^{2}}{8 \mathrm{~K}}\left(\frac{1}{r^{2}}-\frac{1}{(r+d r)^{2}}\right)=\frac{Q^{2} d r}{4 \mathrm{~K} r^{-3}} \text {. }
$$

'This inward pull can only be neutralised by forces applied round the rim of the shell.

Let us assume at the rim a uniform pressure $\mathbf{P}$ upwards, i.c. perpendicular to the lines of force. 'The total area of the rim is $2 \pi r d r$, so that for equilibrium

$$
2 \pi r d r \mathrm{P}=\frac{\mathbf{Q}^{2} d r}{4 \mathrm{~K} r^{3}} \quad \text { and } \quad \mathbf{P}=\frac{\mathbf{Q}^{2}}{8 \pi \mathrm{K} r^{4}}=\frac{2 \pi \mathrm{I}^{2}}{\mathrm{~K}}
$$

or a pressure normal to the lines of force equal to the tension along the lines of force would maintain equilibrium.

These equal values will maintain equilibrium in any case. It is easy to show that when we have a field in which the level

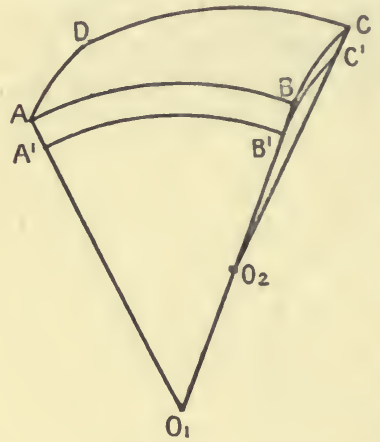

FIG. 91. surfaces have double curvature these equal values of the tension along the lines and of the pressure transverse to the lines will suffice for equilibrium.

Let $\mathrm{ABCD}$, Fig. 91, be a small rectangle on a level surface with its sides in the planes of principal curvature.

Let $\mathrm{O}_{1} \mathrm{O}_{2}$ be the centres of curvature and let $\mathrm{O}_{1} \mathrm{~A}=\mathrm{R}_{1}, \mathrm{O}_{2} \mathrm{~B}=\mathrm{R}_{2}$.

Let $\mathrm{AO}_{1} \mathrm{~B}=\phi_{1}$ and $\mathrm{BO}_{2} \mathrm{C}=\phi_{2}$. 'The area of $\mathrm{ABCD}$ is $\mathbf{R}_{1} \mathbf{R}_{2} \phi_{1} \phi_{2}$.

The normals through ABCD form the tube of strain. Now draw a section of the tube nearer to the centres of curvature by $\delta$, where $\delta$ is very small, and let

$\mathrm{A}^{\prime} \mathrm{B}^{\prime} \mathrm{C}^{\prime} \mathrm{D}^{\prime}$ be the corners of this section.

Its area is

$$
\begin{gathered}
\left(\mathbf{R}_{1}-\delta\right)\left(\mathbf{R}_{2}-\delta\right) \phi_{1} \phi_{2} \\
=\mathbf{R}_{1} \mathbf{R}_{2} \phi_{1} \phi_{2}\left(1-\frac{\delta}{\mathbf{R}_{1}}-\frac{\delta}{\mathbf{R}_{2}}\right) \text {, neglecting } \frac{\delta^{2}}{\mathbf{R}_{1} \mathbf{R}_{2}} .
\end{gathered}
$$

If $\mathrm{D}_{1} \mathrm{D}_{2}$ are the strains over the two surfaces $\mathrm{ABCD}, \mathrm{A}^{\prime} \mathrm{B}^{\prime} \mathrm{C}^{\prime} \mathrm{D}$ respectively,

$$
\text { or } \quad \begin{aligned}
\mathrm{D}_{1} \mathrm{R}_{1} \mathrm{R}_{2} \phi_{1} \phi_{2} & =\mathrm{D}_{2} \mathrm{R}_{1} \mathrm{R}_{2} \phi_{1} \phi_{2}\left(1-\frac{\delta}{\mathrm{R}_{1}}-\frac{\delta}{\mathrm{R}_{2}}\right) \\
\mathrm{D}_{1} & =\mathrm{D}_{2}\left(1-\frac{\delta}{\mathrm{R}_{1}}-\frac{\delta}{\mathrm{R}_{2}}\right) .
\end{aligned}
$$


The tensions being $2 \pi \mathrm{D}_{1}{ }^{2} / \mathrm{K}$ and $2 \pi \mathrm{D}_{2}{ }^{2} / \mathrm{K}$, the resultant pull inwards is

$$
\frac{2 \pi \mathrm{D}_{2}^{2}}{\mathrm{~K}} \mathbf{R}_{1} \mathbf{R}_{2} \phi_{1} \phi_{2}\left(1-\frac{\delta}{\mathrm{R}_{1}}-\frac{\delta}{\mathbf{R}_{2}}\right)-\frac{2 \pi \mathrm{D}_{1}^{2}}{\mathrm{~K}} \mathbf{R}_{1} \mathbf{R}_{2} \dot{\phi}_{1} \phi_{2}
$$

And substituting from the previous equation the total pull inwards is

$$
\frac{2 \pi}{\mathbf{K}} \mathrm{I}_{1}^{2} \mathbf{R}_{1} \mathbf{R}_{2} \phi_{1} \phi_{2}\left(\frac{\delta}{\mathbf{R}_{1}}+\frac{\delta}{\mathbf{R}_{2}}\right) \text {. }
$$

But now suppose there is a transverse pressure $\mathrm{P}$ normal to the surfaces of the rim of the lamina between $A B C D$ and $A^{\prime} B^{\prime} C^{\prime} D^{\prime}$. The area of $\mathrm{ABB}^{\prime} \mathrm{A}^{\prime}$ is $\mathbf{R}_{1} \phi_{1} \delta$. 'That of $\mathrm{BCC}^{\prime} \mathbf{B}^{\prime}$ is $\mathbf{R}_{2} \phi_{2} \delta$.

Resolving $\mathrm{P}$ along the normal to $\mathrm{ABCD}$ and perpendicular to it, the latter gives resolutes neutralising each other in pairs. The former gives

$$
\mathbf{P}^{\prime}\left(\mathbf{R}_{1} \phi_{1} \cdot \delta \cdot \phi_{2}+\mathbf{R}_{2} \phi_{2} \delta \phi_{1}\right)=\mathrm{PR}_{1} \mathbf{R}_{2} \phi_{1} \phi_{2}\left(\frac{\delta}{\mathbf{R}_{1}}+\frac{\delta}{\mathbf{R}_{2}}\right)
$$

so that there is equilibrium if

$$
\mathrm{P}=\frac{2 \pi \mathrm{D}^{2}}{\mathrm{~K}}
$$

There may be other solutions of the problem. Faraday was led to the idea of longitudinal tension and lateral pressure by considering the nature of electric induction. He says : * "The attractive force which exists amongst the particles of the dielectric in the direction of the induction is accompanied by a repulsive or a diverging force in the transverse direction." Clerk Maxwell showed that equilibrium would be maintained if the tension were equal to the pressure. But it is to be remembered that the system thus obtained is only a possible solution of the problem of the stresses in the medium. It is $a$ solution, but there may be others. Meanwhile its simplicity recommends it as worthy of trial, and we shall assume henceforth that it is the solution.

These stresses will not produce equilibrium if $\mathbf{K}$ is not uniform. It is important to observe that if we assume the existence of these stresses the medium is not necessarily in equilibrium unless $\mathbf{K}$ is constant. 'Thus a solid dielectric body suspended in air behaves like a magnetic body in a magnetic field, and Boltzmann's method of determining $K$ by the force on a small sphere hung up in a field radiating from a centre depends upon this fact. Even in a system in equilibrium stresses other than the electrical stresses must intervene if there is a change of value of $K$. 
If, for example, the lines of force pass normally from air into a dielectric of specific inductive capacity $K$ the electrical tension in air is $2 \pi !)^{2}$, while that in the other medium is $\frac{2 \pi 1^{2}}{K}$, so that for equilibrium a force $2 \pi \mathrm{D}^{2} \frac{\mathrm{K}-1}{\mathrm{~K}}$ must be supplied to keep the surface layer in equilibrium. The medium is no doubt elastically strained, and the corresponding stress supplies the required force.

If the lines of force are parallel to the surface separating one dielectric from another, the level surfaces are normal to that surface, and the electric intensity is the same in each medium. If the electric pressure in air is $\mathrm{E}^{2} / 8 \pi$, that in the other medium is $\frac{\mathrm{KE}^{2}}{8 \pi}$. Hence $(\mathrm{K}-1) \frac{\mathrm{E}^{2}}{8 \pi}$ is the pressure needed on the surface of the other medium in addition to the electric pressure to maintain equilibrium.

Quincke's experiments. Quincke made a series of experiments of historical interest to test the existence of these electrical tensions and pressures in dielectric liquids. He used a condenser * with horizontal plates, the upper being hung insulated from a balance beam, while the lower was insulated and so arranged that it could be connected to a battery of Leyden jars charged to given potential. 'The condenser being charged, the pull $G_{1}$ on the upper plate was first determined in air. Then, the condenser being surrounded with the liquid to be experimented on, the pull $\mathbf{G}_{2}$ was determined, when the potential difference, and, therefore, the electric intensity, was the same as before, the plates being the same distance apart. 'Taking the area as $\mathrm{A}$ and neglecting the edge effect, we have by the foregoing theory:

$$
\frac{\mathbf{G}_{2}}{\mathrm{G}_{1}}=\frac{\mathrm{AKE^{2 }}}{8 \pi} / \frac{\mathrm{AE}^{2}}{8 \pi}=\mathrm{K}
$$

or the pulls are in the ratio of the specific inductive capacity.

In each case the condenser was discharged through a ballistic galvanometer. The quantity flowing through the galvanometer and indicated by the kick-off was proportional to the capacity of the condenser, and, therefore, to the spccific inductive capacity of the medium. This, of course, gave another determination of $\mathbf{K}$.

At first the values by the two methods were somewhat widely apart, owing to the neglect of the capacity of the connecting wire and key. When this was taken into account the determinations were fairly in accord. $\dagger$

The existence of the pressure at right angles to the lines of force was verified as follows: The upper suspended plate was replaced by a fixed plate with a short vertical tube passing up

* Plil. Mag., vol. xvi. (1853).

† Nature, vol. xxxv. (1887), p. 33 . 
from a hole in the centre. This was connected to an india-rubber tube provided with a stop-cock and to a bisulphide of carbon manometer. The plates being immersed in the liquid near together, and to begin with to earth, air was blown into the space between them through the india-rubber tube until a large central space extending from plate to plate was cleared of liquid. The cock was then turned off and the manometer read. 'The lower plate was then brought to the same potential as in the experiment above described, and at once the manometer showed an increase of pressure, this increase being needed to balance the difference between the electrical pressures at right angles to the lines of force in the air and in the liquid. If $h$ is the increase of height in the manometer and $\sigma$ the specific gravity of the liquid used, we have, since $\mathbf{E}$ is the same in the liquid and in the air,

$$
(\mathrm{K}-1) \frac{\mathrm{E}^{2}}{8 \pi}=g h \sigma .
$$

If the difference of potential and distance apart are the same as in the previous experiment we may eliminate $\mathbf{E}$ by the result of that experiment. If we write $K_{p}$ for the value of $K$ as involved in the pressure at right angles, and $K_{\mathrm{T}}$ for its value as involved in the tension along the lines of force, the two experiments give us respectively :

$$
\left(\mathbf{K}_{\mathrm{p}}-1\right) \frac{\mathrm{E}^{2}}{8 \pi}=g h \sigma
$$

and

$$
\frac{K_{\mathrm{T}} \mathrm{E}^{2}}{8 \pi}=\frac{\mathrm{G}_{2}}{\mathrm{~A}}
$$

whence

$$
\mathbf{K}_{\mathrm{p}}=1+\frac{g h_{\sigma} \mathrm{A}}{\mathbf{G}_{2}} \mathbf{K}_{\mathrm{T}} \text {. }
$$

'The ralues obtained for $K_{\mathrm{p}}$ and $\mathrm{K}_{\mathrm{T}}$ were nearly equal to each other and to the value obtained by the ballistic galvanometer, which we may denote by $K$.

'The following 'Table shows the results in a few cases obtained in two series of experiments, the earlier comparing $K_{\mathrm{p}}$ and $\mathrm{K}_{\mathrm{T}}$, the later comparing $K_{\mathbf{T}}$ and $K$.

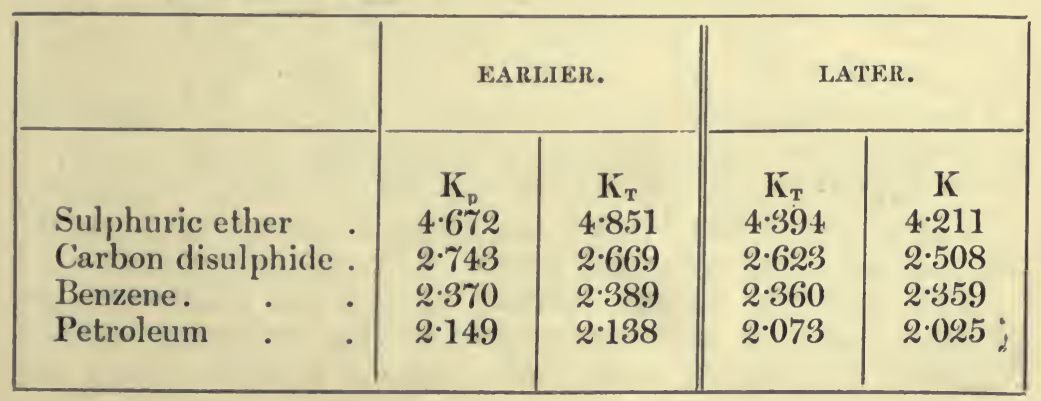


The difference in the values for $K_{\mathrm{T}}$ in the earlier and later experiments is probably to be ascribed to slight differences in the constitution of the specimens.

General expression for the resultant force on a surface due to the electric tension and pressure. Let a rectangular tube of strain meet the surface in $\mathrm{AB}$, Fig. 92, making $\theta$ with the normal, and let the area of $\mathrm{AB}$ be $a$. Consider the equilibrium of the wedge with section $\mathrm{ABC}$.

Perpendicular to $\mathrm{BC}$ we have a pull $\frac{2 \pi \mathrm{I}^{2}}{\mathrm{~K}} a \cos \theta$, since the area of $\mathrm{BC}$ is $\alpha \cos \theta$.

Perpendicular to AC we have a push $\frac{2 \pi \mathrm{D}^{2}}{\mathrm{~K}} a \sin \theta$.

'The forces on the sides parallel to the paper neutralise each other. Resolving along the normal and tangent, we have

Along the normal outwards

$$
\frac{2 \pi)^{2}}{\mathrm{~K}} \alpha\left(\cos ^{2} \theta-\sin ^{2} \theta\right)=\frac{2 \pi \mathrm{D}^{2}}{\mathrm{~K}} a \cos 2 \theta .
$$

'Tangential along AB,

$$
\frac{2 \pi \mathrm{D}^{2}}{\mathrm{~K}} a(\cos \theta \sin \theta+\sin \theta \cos \theta)=\frac{2 \pi)^{2}}{K} a \sin 2 \theta \text {. }
$$

'The force on $\mathrm{AB}$ must be equal to the resultant of these, since
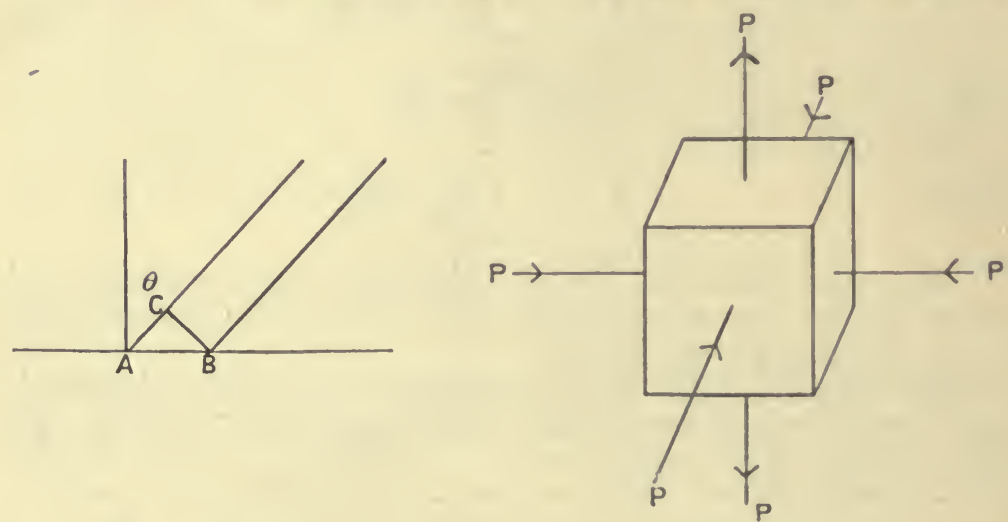

FIG. 92.

FIG. 93.

the wedge considered is in equilibrium. 'Thus there is a force outwards $\frac{2 \pi \mathrm{D}^{2}}{\mathrm{~K}}$ per unit area, and at an angle $2 \theta$ with the normal.

The system of forces thus made up should give as resultant the force actually observed in any case.

The electric stress system is not an elastic stress system, and is not accompanied by ordinary elastic strains. The electric stress system applies equally whether the 
dielectric be solid or fluid. Consider a small cube with four vertical edges parallel to the lines of force, Fig 93. The system of stresses is obviously equivalent to a shear stress in the plane of the figure and to pressures on the two faces parallel to that plane. Now, in a fluid in equilibrium ordinary elastic shear stresses cannot exist and cannot contribute to maintenance of equilibrium. Indeed, hydrostatic equilibrium is only possible in a fluid when the pressures about a point are equal in all directions. Further, even in a solid the elastic system of a shear stress and a perpendicular pressure would produce on the whole a decrease in volume. For the shear would not affect the volume, while it is easy to show that the pressure $\mathrm{P}$ would decrease it by $\mathrm{P} \frac{1-2 \sigma}{\mathrm{Y}}$, where $\sigma$ is Poisson's ratio and $\mathrm{Y}$ is Young's modulus. But experiments to be described in the next chapter show that, at any rate in the case of glass, the presence of electric strain is accompanied by a uniform dilatation.

We must therefore suppose that the electric stresses are not elastic forces accompanied by elastic changes of shape, but that they are called into play in some other way. Where the medium is material we may probably account for the stresses by molecular arrangement, on the supposition that the molecules are electric doublets.

We may illustrate the idea by considering the corresponding case of a magnetic system. Suppose that we have a number of little magnets pivoted on points arranged in rows and columns, as in Fig. 94. If the magnetic axes are equally distributed in all directions there will be no continuous lines of force going in any one direction. But if the axes are arranged as in the figure the opposite poles in the columns will attract each other, forming tensions along the lines of axes. The like poles in the neighbouring columns will repel each other, forming pressures perpendicular to the lines of axes. Some such arrangement of electric doublets in a material medium may account for the electric stresses accompanying electric strain. We cannot say, a priori, what effect such a rearrangement should have on the dimensions of the system. We only

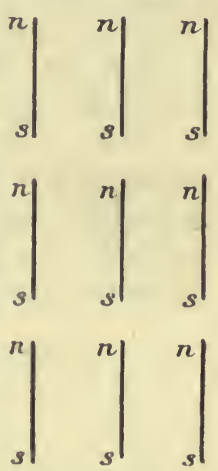

Fir. 94. know by experiment that it appears to lead to a uniform dilatation.

When the matter is exceedingly attenuated it may be doubted whether we can account for the stresses by the molecules present, and we may have to imagine some structure in the ether before we can frame a hypothesis to supply the forces. 


\title{
CHAP'TER XI
}

\section{ALTERATIONS OBSERVED IN THE DIELEC- TRIC WHEN IT IS SUBJECTED TO ELECTRIC STRAIN}

\begin{abstract}
Electric expansion in glass-Maxwell's electric stresses do not explain the effect-Electric expansion of liquids-Electric double refraction: The Kerr effect.
\end{abstract}

Whev a dielectric is subjected to electric strain it shows in some cases a change in volume and in other cases it becomes doubly refracting.

Electric expansion in glass. The dielectric in a glass condenser generally expands when the condenser is charged, and the dielectric becomes the seat of electric strain. 'This expansion was known to Volta and has since been studied by Govi, Duter, Righi, and especially by Quincke.* It may be conveniently observed and measured by using a common thermometer as a Leyden jar. The bulb is immersed in melting ice and water, the water serving as the outer coating, while the liquid contained in the bulb serves as the immer coating. On charging the inside by connecting it with a large Leyden jar the level is observed to fall. Quincke found that if $\Delta v$ is the change in the internal volume $v$ of the bulb, if $e$ is the thickness of the glass and $\mathrm{V}$ is the potential difference, then

$$
\frac{\Delta v}{v} \text { is proportional to } \frac{V^{2}}{e^{2}} \text { nearly. }
$$

With thicknesses between $0.1 \mathrm{~mm}$. and $0.5 \mathrm{~mm}$. and with a potential difference sparking at $2 \mathrm{~mm}$. between knobs $2 \mathrm{~cm}$. in diameter, Quincke found that the change in volume lay between 1 in $10^{5}$ and 1 in $10^{7}$. In general, when it rose above 1 in $10^{5}$ sparking occurred and the glass was perforated.

The intensity and strain are proportional to $\mathrm{V} / e$ so that the change in volume is proportional to the square of the electric strain in the glass. 'The expansion occurs equally in all directions, for simultaneous measurements of increase in length $l$ and of volume $v$

* Phil. May. [5], yol. X. p. 30, where references to eariier work will be found. 
of a condenser like a thermometer with a long cylindrical bulb showed that

$$
\frac{\Delta v}{v}=3 \frac{\Delta l}{l}
$$

Maxwell's electric stresses do not explain the effect. 'The result just given is important as showing at once that no such system of stresses as that considered in the last chapter will account for the effect. 'I'hat system would give extension parallel to the lines of force, and compression perpendicular to them. But it fails to account for the effect in that it would give on the whole a contraction in volume. If, as in the last chapter (Fig. 93), we take a cube of the dielectric, with its vertical edges parallel to the lines of force, it is acted on by its surroundings with the set of forces represented in the figure.

'The vertical tensions and one pair of side forces will give a shear with no change of volume, while the other pair of pressures will produce a decrease in unit volume $(1-2 \sigma) \mathbf{P} / \mathbf{Y}$, where $\sigma$ is Poisson's ratio and $Y$ is Young's modulus. We can perhaps estimate this effect as compared with the increase measured by Quincke. In one case of flint glass with a thickness $0.014 \mathrm{~cm}$. the change in volume was 1 in $10^{5}$ when the potential difference would spark over $2 \mathrm{~mm}$. between knobs $2 \mathrm{~cm}$. in diameter. We may perhaps take this as a potential difference about equal to 25 E.S. units, and it gives intensity within the glass $\mathbb{E}=\frac{25}{14} \times 10^{3}$. For flint glass $K$ is probably about 7 , so that $\mathrm{P}=\frac{\mathrm{KE}^{2}}{8 \pi}=10^{6}$ nearly.

We may take $1-2 \sigma=\frac{i}{2}$ and $Y=5 \times 10^{11}$, so that the decrease in unit volume should be of the order $\frac{10^{6}}{10^{12}}=10^{-6}$ or 1 in $1,000,000$, as against the observed increase of 1 in 100,000 . 'The Maxwell system of stresses then would only lessen the electric expansion slightly, even if we conld suppose them to produce elastic strain.

Electric expansion of liquids. Quincke also experimented with liquids. A condenser was formed in the liquid by a pair of vertical platinum plates. The containing ressel was entirely filled with the liquid and closed except that a capillary tube rose from it to show any change in volume of the liquid. On charging the plates as a condenser the level in the tube was observed to alter. The results indicated that the change in volume is probably proportional to the square of the intensity. In general there is expansion, but with fatty oils, such is colza and almond oils, there is a clecrease in volume. 
Quincke also observed a decrease in the torsional rigidity of glass, mica, and india-rubber when electrically strained.

Electric double refraction: The Kerr effect. Kerr discovered* that a liquid dielectric is in general doubly refracting when it is the seat of electric strain. It behaves as a uniaxial crystal with the optic axis along the lines of force. 'There is therefore a difference in the velocity of rays which traverse the liquid perpendicular to the lines of force, according as their plane of polarisation is parallel to or perpendicular to the lines of force. 'The effect may be shown by the arrangement of which Fig. 95 is a plan.

A ray of light is sent through a polariser $\mathbf{P}$ set so that its plane of polarisation is at $45^{\circ}$ to the vertical. It then passes into a trough

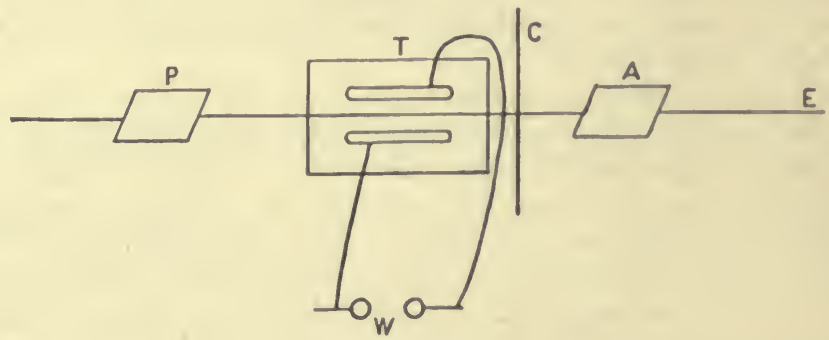

FIG. 95.

' 1 with glass ends containing the liquid, which may be carbon bisulphide very pure and dry. In the trough are two brass cylinders $3 \mathrm{in}$ or $4 \mathrm{in}$. long, with their axes parallel and in the same horizontal plane. 'These are comnected by rods rising out of the trough to the terminals of a Wimshurst machine. After emerging fiom the trough the ray passes through the analyser $\mathrm{A}$, set at $90^{\circ}$ to the polariser, so that when the liquid is not electrically strained there is complete extinction. 'The ray then passes on to the eye of an observer $\mathbf{E}$, or by using lenses we may project an image of the ends of the conductors in the trough on to a screen. On working the Wimshurst the field between the two conductors transmits the light to some extent, and the more the stronger the field. 'The vibration of the incident beam may be resolved into equal components vibrating respectively vertically and horizontally, and therefore respectively along and perpendicular to the lines of force. 'These travel at different speeds through the strained medium so that when they emerge from the electric field the phases differ, and instead of uniting to form a plane polarised beam, with plane parallel to that of the original, they unite to form an elliptically polarised beam which cannot be entirely extinguished by the analyser. By inserting a Jamin compensator C, Kerr determined

*Phil.Mag. [4], 1875, vol. 1. pp. 337 and 446; [5], 1879, vol. viii. pp. 85 and 229. 
the delay in phase of one ray relative to the other and showed* that the delay is proportional to the square of the strength of field as measured by a Thomson electrometer.

Kerr investigated the effect for a large number of liquids + and found that some, which he ternicd positive liquids, behave like quartz with its axis parallel to the lines of force, or like glass extended in a direction parallel to the lines of force. Of the liquids in which he was able to measure the effect, carbon disulphide shows it in the greatest degree. Paraffin oil, toluene, and benzene are other positive liquids. Others, which Kerr termed negative liquids, behave like Iceland spar with its axis parallel to the lines of force, or like glass compressed in the direction of the lines of force. Of these colza oil gives a strong effect; others are olive oil and seal oil.

The effect may be expressed conveniently by the number of wave-lengths difference between the two components in a length $l$ of the dielectric when the transverse electric field has intensity E. Experiment shows that if $\delta$ is the difference of path pursued in the same time, and if $\lambda$ is the wave-length of the light used, the number of wave lengths difference or $\delta / \lambda$ is given by

$$
\delta / \lambda=B l E^{2}
$$

where $\mathrm{B}$ is a constant for a given substance and wave-length-the Kerr electric constant. The difference of phase is $2 \pi \delta / \lambda$, and $\delta$ and $\lambda$ must both be measured in the liquid or both in air. It is easily shown that if $\mu_{0}$ and $\mu_{e}$ are the refractive indices for the two rays and $\lambda$ is the wave-length in air

$$
\mu_{0}-\mu_{e}=\mathrm{B} \lambda l \mathrm{E}^{2} \text {. }
$$

Quincke $\ddagger$ was the first to obtain a value of $B$, working with carbon disulphide. Since then various measurements for this liquid have been made $\S$ and the value of $\mathbf{B}$ for sodium light appears to be very near to $3 \cdot 1 \times 10^{-7}$.

But the value changes with the wave-length, increasing as $\lambda$ diminishes. 'This was observed by Kerr, and he thought that $\mu_{0}-\mu_{e}$ varied inversely as the wave-length. Subsequent observers $\|$ have not verified the simplicity of the relation though confirming the general nature of Kerr's observation. Cotton and Mouton have investigated the effect of change of temperature and have found that $\mathrm{B}$ decreases rapidly as the temperature rises (loc. cit.).

Kerr observed in 1879 that though nitrobenzene is not a

* Phil. May. [5], 1880, vol. ix. p. $15 \%$.

† Phil. May. [5], vol. xiii. pp. 153 and 248.

† Wied. Ann., XIX, 1883, p. 729 .

$\S$ A careful determination by McComb is given in Phys. Rev., 29, 1909, p. 534. References are given to earlier result.s.

II Blackwell, Proc. Amer. Acad. of A rts and Sciences, XLI, 1906, p. 147. McComb, loc. cit. Cotton and Mouton, Journal de Physique, 5th. ser., vol. I, 1911, p. 5. 
sufficiently good insulator to allow a persistent field to be established in it, yet on making a spark-gap in one of the connecting wires, at each spark the field lighted up, due no doubt to the oscillations producing a large oscillating field between the conductors.

Schmidt* succeeded in measuring the effect in nitrobenzene. He used a compensation method in which the ray of light passed through the liquid in one cell and then through another cell containing a liquid for which $B$ was known, and arranged so as to have an effect of the opposite kind on the phase relation of the two components. It was adjustable so that the effect of the two cells exactly neutralised each other when the same rapidly alternating potential difference was applied to each. He found that the Kerr constant is sixty times as great for nitrobenzene as for carbon disulphide, and that it is about the same for the latter and for water. McComb found that the effect is large for other aromatic compounds. $\dagger$

Cotton and Mouton (loc. cit.) discovered that nitrobenzene exhibits magnetic double refraction, that is that a polarised ray transmitted perpendicularly to a magnetic field established in the liquid travels at different speeds according as its plane of polarisation is parallel or perpendicular to the lines of force. 'They found that the effect could be expressed by a formula exactly corresponding to that for the Kerr electric effect, viz. :

$$
\delta / \lambda=\mathrm{C} \mathrm{H}^{2}
$$

where $\mathrm{H}$ is the maynetic intensity and $\mathrm{C}$ is the constant of magnetic double refraction for the substance for the wave-length used. For nitrobenzene they found that $\mathrm{C} / \mathrm{B}$ is nearly the same for different wave-lengths. 'This has been confirmed for several other aromatic compounds by McComb and Skinner. $\ddagger$

In Cotton and Mouton's paper there is some reference to theories of the subject by Voigt, Havelock, and themselves. 'These, however, belong to optics rather than to electricity.

We may state the Kerr effect in terms of the electro-magnetic theory of light by saying that $K$ is slightly different in the electric field. Since the change in velocity is given by

$$
\frac{d v}{v}=-\frac{1}{2} \frac{d K}{K}
$$

and Dr. Kerr's observations show that $\frac{d v}{v}$ is proportional to $\mathbf{E}^{2}$, where $\mathbf{E}$ is the intensity, then $\frac{d \mathbf{K}}{\mathbf{K}}$ is proportional to $\mathbf{E}^{2}$, and

\footnotetext{
* Ann. der Phys. [4], 7, 1902, p. 142.

$\dagger \Lambda$ number of values will be found in Tables Annuelles Interrationales de $C$ nstantes $I$.

$\ddagger$ Pliys. Rev., 29, pp. 525 and 541.
} 
$\mathbf{K}$ is not constant, but decreases as $\mathbf{E}$ increases, and by an amount proportional to $\mathrm{E}^{2}$. 'I'his is on the supposition that the speed of the ray with its electric vibrations along the lines of force is alone affected. Some experiments by Kerr * appeared to establish this, but some experiments by Aeckerlein $\dagger$ showed that in nitrobenzene the ray with its electric vibrations along the lines of force was retarded and that with its ribrations perpendicular was advanced and about in the ratio 2:1. It is to be noted that the change in $K$ is exceedingly minute, the change in phase being only a fraction of a period in a path of several centimetres through a field of perhaps 100.000 rolts per centimetre. The Kerr effect in glass is exceedingly small, so small that it cannot always be cer tainly distinguished from the effect due to the elastic stresses present.

* Phii. Mag. [5], 37, 189t, p. 350.

+ Phys Zeit., VII, 1906, p. 594. 


\section{CHAP'IER XII}

\section{PYROELECTRICITY AND PIEZOELECTRICITY}

Pyroelectricity-Historical notes-Analogous and antilogous polesSome methods of investigating pyroelectricity-Gaugain's researchesLord Kelvin's theory of pyroelectricity-Voigt's experiment on a broken crystal-Piezoelectricity-The discovery br the brothers Curie -The piezoelectric electrometer-Voigt's theory connecting pyroelectricity and piezoelectricity - Electric deformation of crystals Lippmann's theory-Verification by the Curies-Dielectric subjected to uriform pressure-Change of temperature of a pyroelectric crystai on changing the potential.

Certain crystals initially showing no electrification develop, if heated uniformly, opposite surface electrifications on opposite surfaces. If they are cooled from a neutral condition the polarity is reversed. The phenomenon is termed Pyroelectricity, and it was observed first in the eighteenth century. If these crystals, without being heated, are subjected to pressure along the axis of electrification which was observed when the temperature was changed, then opposite electrifications develop at the ends of the axis. If they are subjected to tensions the polarity is reversed. 'The phenomenon is termed Piezoelectricity. It was discovered by J. and P. Curie

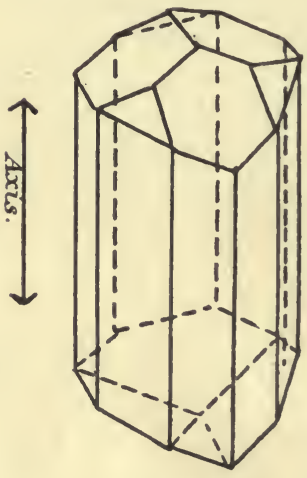

FIG. 96. in 1880. They found that the electrification under pressure was the same in sign as that due to cooling, while that under tension was the same in sign as that due to heating. The two phenomena are evidently related to each other.

The crystals in which they are observed are those known as "hemihedral with inclined faces." 'They have axes with the faces at the two ends unlike, so that they are unsymmetrical with regard to a plane at right angles to the axis through its middle point. As an example we may take tourmaline, of which Fig. 96 represents one form, the axis of electrification being as shown by the arrow. We shall describe first the phenomenon of pyroelectricity. 
Pyroelectricity: Historical notes. It was discovered about 1700 that a tourmaline crystal placed in hot ashes attracted the ashes. In 1756 Epinus showed that the effect was electrical and that the charges were opposite in sign at the two ends of the crystal. Soon after, Canton* showed that the charges were equal as well as opposite. He connected an insulated tin cup filled with boiling water to a pith-ball electroscope. A tourmaline crystal was dropped into the water and the pith ball showed no trace of electrification, either then or during the subsequent cooling of the water. Canton found also that the development of electrification depended only on change of temperature from the neutral condition and not on the absolute temperature. For if the crystal was in a neutral condition at any temperature it showed polarity of one kind if raised above that temperature, and polarity of the opposite kind if cooled below that temperature. He broke a tourmaline prism into three pieces and found that each piece exhibited the same kind of action along the same axis as the whole crystal. He found that Brazilian topaz acted like tourmaline, and his contemporary Watson discovered other pyroelectric gems.

Haiiy about 1800 discovered that only crystals hemihedral with inclined faces developed pyroelectricity, and that the end forming the most acute angles with the axis was positively electrified on cooling. Other crystals may show electrification when not heated uniformly, but this is due to local strains set up by the non-uniform heating and a consequent local alteration, as it were, of the crystalline form.

Analogous and antilogous poles. Riess termed the pole on which + electricity appears on heating the analogous pole, and the other the antilogous pole.

A crystal may have as many as four pyroelectric axes. Quartz has three axes, bisecting the three angles of $120^{\circ}$ between the faces of the prism. The axis of the prism is not a pyroelectric axis.

Some methods of investigating pyroelectricity. The earliest method consisted in merely heating a crystal and then bringing one part of its surface near an electroscope. Hankel heated the crystal in copper filings, passed it through a spirit flame by which it was entirely reduced to the neutral condition, and then allowed it to cool, when its electrification could be tested by an electroscope.

Kundt $\uparrow$ heated the crystal in an air bath and after neutralisation allowed it to cool. He then blew on to it, through muslin, a powder consisting of a mixture of red lead and sulphur. 'The red lead was positively electrified by the friction with the muslin and the sulphur was negatively electrified. The positive region of the crystal attracted the sulphur and the negative the red lead, and the two regions became differently coloured. If a tourmaline prism 
is immersed in liquid air and is then lifted out and dusted with red lead and sulphur before it has time to condense ice on its surface, it shows the colours excellently.

Gaugain's researches. Gaugain* employed an air bath in which a tourmaline crystal was suspended. One end of the crystal was earthed and the other end was connected to a self-discharging goldleaf electroscope, and the total charge developed was measured by the number of discharges of the gold leaf. He found that

1. The charge is proportional to the change of temperature from neutrality.

2. With different tourmalines of the same kind the charge is independent of the length and is proportional to the crosssection.

Riecke's researches. Riecke $†$ made important contributions to our knowledge of the subject. He investigated the loss of charge through leakage and showed that it was very much diminished if the crystal was suspended in a diy vacuum. He showed that if $\Omega$ is the change of temperature from neutrality, the charge is not exactly proportional to 2 , but may be represented more nearly by

$$
\mathbf{E}=a \vartheta+b \Im^{2} \text {. }
$$

He worked in a manner similar to that of Gangain, and graduating the electrometer by known charges, he was able to determine the charges developed in electrostatic units. Thus for a certain tourmaline which gave the largest effect he found that 180 E.S. units per square centimetre cross-section were developed per $100^{\circ} \mathrm{C}$. rise of temperature.

Lord Kelvin's theory of pyroelectricity. 'To Lord Kelvinł we owe the theory of the subject which is generally accepted. Let us take the simple case of a crystal like tourmaline with one pyroelectric axis. We suppose that each molecule of the crystal is an electric doublet with lines of force or strain passing between the pair and into and out of the ends. The molecules set in the crystal with their electric axis parallel to a certain direction, and there will therefore be a definite amount of strain passing from each positive element of a doublet to the negative element of the doublet next to it. 'The crystal as a whole will be the seat of electric strain along the direction of the axis. 'The constitution corresponds to that of a suturated permanent magnet on the molecular theory of magnetism. The electric strain will manifest itself almost entirely at the ends of the crystal if the doublets are near enough together, and the crystal will produce a field outside equal to that which would be produced by charges respectively

* Ann. de Chim. [3], 57, p. 5 (1859).

+ Wied. Ann., 28, p. 43 (1886); 31,p. 889 (1887); id.p. 902 ; 40, p. 264 (1890) ; id. p. 305 .

$\ddagger$ Nicol's Cyclopcedia, 2nd ed. (1860), or Math. and Phys. Papers, vol. i. p. 315 . The fullest account is in the Baltimore Lectures, p. 559. 
positive and negative at the two ends, and giving out the same number of tubes of strain as those actually issuing. But through the positive and negative ions which always exist to some small extent in the air, through conducting films on the crystal and sometimes by conduction in the body of the crystal itself, charges will in time gather on the surfaces at the ends of the axis, negative on the end from which tubes of strain issue, and positive on the other end into which they enter, entirely masking the existence of the strain so that there is no external field. This is the ordinary neutral condition of a pyroelectric crystal.

We have now to suppose that the electric strain passing forward from molecule to molecule depends on the temperature and that it.alters nearly in proportion to the change of temperature. Then when the temperature changes there will be an unbalanced strain proportional to the change, producing the same effect as a positive charge at one end and a negative charge at the other until conduction again does its work in bringing up masking charges. The apparent charges are proportional evidently to the cross-section and independent of the length.

We may picture a molecular model which might give the effect, and though it suffers from the common defect of such models in being statical, whereas in reality everything must be in motion, it will at least serve as a working hypothesis. We suppose the molecules to be doublets set with their axes all parallel to a given direction and with the positive part of each to the right, as in Fig. 97 , and we suppose that there is some connection between the positive of each molecule and the negative in the next. For instance, in Fig. 97 there are five internal lines of force in each

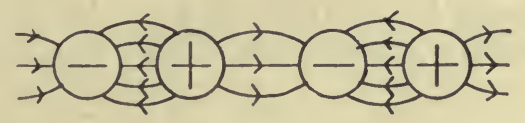

FIG. 97.

molecule, and three extermal lines connecting it to the next on each side, and these lines run in opposite directions from the same charge. The external lines to the extreme molecule on the left begin on the positive masking charge, and those from the extreme molecule on the right end on the negative masking charge.

Let us further suppose that through a rise in temperature the molecules are more separated. 'Then the external lines of force will bulge out, as it were, and this bulging out may take place to such an extent that one between each pair of molecules may touch one between each of the next pairs on either side, as represented in Fig. 98, where only the external lines concerned are drawn. At the points of contact $\mathrm{P}$ and $\mathrm{Q}$ the strains are in opposite directions in the two coalescing lines and neutralise each other. 
Then more or less straightening occurs and we get something of the arrangement represented in Fig. 99, which shows that one of the external connecting lines disappears, there is one more internal connecting line, and there is one extruded line passing from end to end of the crystal, not through the chains of molecules, and not

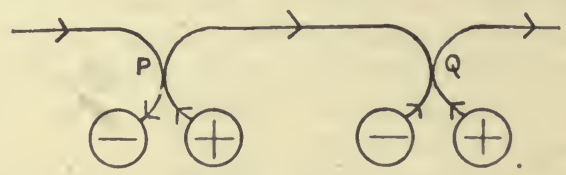

FiG. 98.

even necessarily through the crystal. At each end of this line there will be an element of what was previously the masking charge, but is now what we may term free charge, and the condition is represented in Fig. 100. This model would explain pyroelectricity as due merely to molecular separations, and we ought

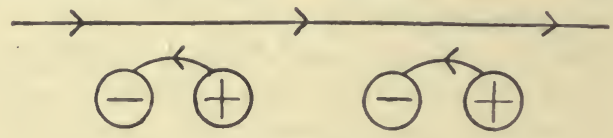

FIG. 99.

to get a similar effect if the molecular separation is produced by tension, and, as we shall see, this is just what the Curies found.

But we may imagine another molecular model in which pyroelectric effects should occur if rise of temperature produces separation of the constituent charges in each molecule without extension as a whole. It will easily be seen that then the internal lines of force bulge out and finally extrude a line of force rumning in the opposite

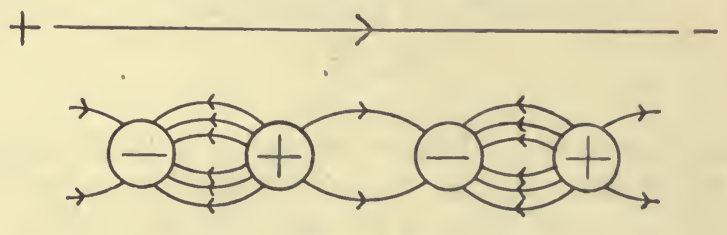

FIG. 100.

direction to that in Fig. 100, and finally we have one more external line and one less internal line, as represented in Fig. 101. In this case the charges developed at the ends will be opposite to those developed in the last case. It is possible that both effects occur.

We may describe the electrical condition within the crystal on this molecular chain theory by the number of tubes of strain issuing per square centimetre from the end of an axis. We may term this the "Intensity of Electrification" along that axis, and it 
corresponds to the intensity of magnetisation of a magnet. We should expect, on the theory, that if a pyroelectric crystal is broken across an electric axis, then the two broken axes would show apparent electrifications equal and opposite. Canton is sometimes supposed to have shown this, ${ }^{*}$ but he does not appear to have gone further than a demonstration that each fragment

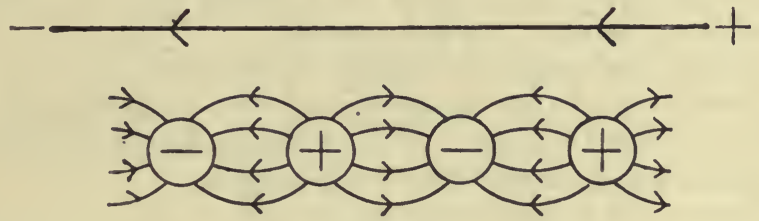

FIG. 101.

behaved with change of temperature like the whole crystal. 'The experiment was first successfully made by Voigt. $\dagger$

Voigt's experiment on a broken crystal. He fastened the two ends of a small tourmaline rod to two brass rods which were connected electrically. He then broke the tourmaline in the middle and dipped the two broken surfaces into mercury cups connected to a Nernst-Dolazalek electrometer which showed charges on the ends. He found as a mean result that there was an intensity of electrification at $24^{\circ} \mathrm{C}$. of -33.4 E.S. units, or the electrical moment of 1 c.c. at $24^{\circ} \mathrm{C}$. was $-33 \cdot 4$, the sign being negative because the electrification was opposite to that which would have appeared on the same surface with rise of temperature.

Now for this kind of crystal the electrification gathering per $1^{\circ} \mathrm{C}$. rise of temperature was found to be about $+1 \cdot 2$ E.S. units per square centimetre. Hence at $24^{\circ}+\frac{33 \cdot 4^{\circ}}{1 \cdot 2}=52^{\circ} \mathrm{C}$., the electrical moment of the crystal should be zero. Or, if we return to the molecular models, it may be that both effects, dilatation and intermolecular separation, exist, and that at $52^{\circ} \mathrm{C}$. they balance each other.

Piezoelectricity: The discovery by the brothers Curie. 'The discovery of piezoelectricity was made by the brothers Curie by an experiment represented diagrammatically in Fig. 102. $\mathrm{C}$ is a crystal hemihedral with inclined faces cut into the form of a rectangular block. It was placed between the jaws $\mathrm{J} J \mathrm{~J} J$ of a vice. Between the jaws and the crystal were ebonite plates $e e$ and tinfoil $t t$, the tinfoil being against the ends of the crystal. The tinfoil was connected to the quadrants of an electrometer, and one tinfoil and the connected quadrants could be earthed.

* Kelvin's Baltimore Lectures, p. 560. Cf. Priestley on Electricity, 4th ed. p. 300 .

† Wied. Ann., 60, p. 368 (1896). 
When the vice was screwed up, a charge was shown in the electrometer. On discharging this and then releasing the crystal from pressure the polarity was reversed.

A number of crystals were thus tested, and it was found that always compression gave the same sign of charge at one end as cooling, and extension the same sign as heating.* Working with tourmaline and applying pressure by a loaded lever by which the force could be measured, it was found that

1. The positive and negative charges were equal.

2. 'The change in charge de developed was proportional to the change in the total force $d \mathrm{~F}$ and changed sign with $d \mathrm{~F}$.

3. 'The charge was independent of the length.

4. 'The charge was independent of the cross-section for a griven total force. Hence $d e x d \mathrm{~F}$.

We may use the first molecular model described on p. 151 to give us an idea of a possible way in which the effect is produced,

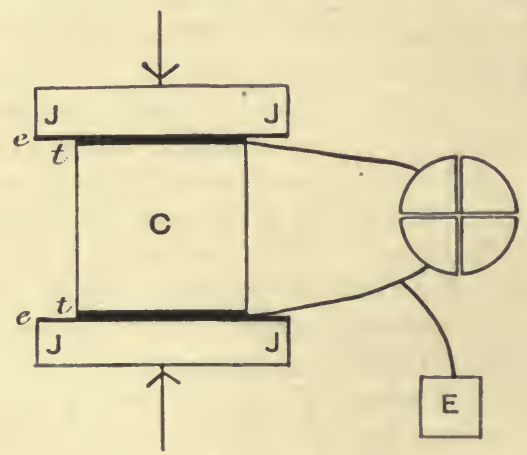

FIG. 102.

the changes in dimensions being produced now by tension and pressure instead of by heating and cooling.

To find the amount of charge produced, an arrangement represented in Fig. 103 was adopted. A block of the crystal with its ends lined with tinfoil was placed between insulating ebonite plates, and a known force $\mathbf{F}$ could be applied by a lever, not shown. 'The upper tinfoil was comnected to one plate of a small condenser of known capacity $\mathrm{C}$, and also to one pair of quadrants of an electrometer QE. 'The lower tinfoil and the other plate of the condenser were earthed. 'I'he other pair of quadrants of the electrometer was charged by a Daniell's cell $\mathrm{D}$ so connected that the sign of the charge was the same as that given by the crystal when a force $\mathrm{F}$ was put on to it. 'This force was then so adjusted that the electrometer needle came to the zero reading found when the two pairs of quadrants were earthed.

* The account of the experimerts mace by $J$. and P. Curie is given in a series of papers in Euvres de Pierre Curie. 
Let $\mathrm{E}$ be the capacity of the electrometer and tinfoil, though the latter is probably negligible. Let $q$ be the charge due to $\mathbf{F}$. 'This charges capacity $\mathrm{C}+\mathrm{E}$ to $\mathrm{D}$ volts, where $\mathrm{D}$ is the E.M.F. of the Daniell's cell, or to D/300 E.S. units.

Then

$$
q=(\mathrm{C}+\mathrm{E}) \mathrm{D} / 300 \text {. }
$$

Now $\mathrm{C}$ was removed and the force was adjusted to $\mathrm{F}^{\prime}$, again giving zero reading. Let $q^{\prime}$ be the charge.

$$
\begin{array}{cc}
\text { Then } & q^{\prime}=\mathrm{ED} / 300 \\
\text { and } & q-q^{\prime}=\mathrm{CD} / 300
\end{array}
$$

which gives us $q-q^{\prime}$ in terms of known quantities. we find

But $q$ is proportional to $\mathbf{F}$. Put, then, $q-q^{\prime}=\lambda\left(\mathbf{F}-\mathbf{F}^{\prime}\right)$ and

$$
\lambda=\frac{q-q^{\prime}}{\mathrm{F}-\mathrm{F}^{\prime}}
$$

which determines $\lambda$.

'The Curies found that a load of $1 \mathrm{kgm}$. on a certain tourmaline

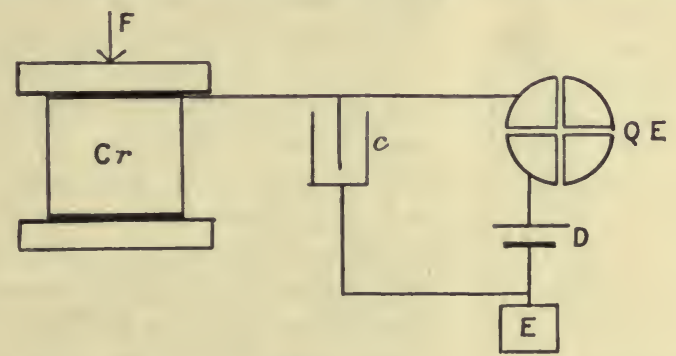

FIG. 103.

gave $q$, charging a capacity of $14.2 \mathrm{~cm}$. to $1 \cdot 12$ volts, the E.M.F. of the Daniell's cell.

$$
\text { Then } q=\frac{14.2 \times 1 \cdot 12}{300}=\cdot 053
$$

so that 1 dyne would give a charge $5.41 \times 10^{-8}$. On a quart\% plate cut perpendicular to one of the three axes which are perpendicular to the axis of the prism a load of $1 \mathrm{kgm}$. gave

$$
q=\cdot 062 .
$$

'I'hen 1 dyne would give a charge $6.32 \times 10^{-8}$.

The authors pointed out that the capacities of small condensers might be compared by piezoelectricity. For suppose that, as above, we have found with a condenser capacity $\mathrm{C}_{1}$

$$
q_{1}-q^{\prime}=\lambda\left(\mathrm{F}_{1}-\mathrm{F}^{\prime}\right)=\mathrm{C}_{1} \mathrm{D} / 300 \text {. }
$$


Now substitute a second condenser capacity $\mathrm{C}_{2}$. If the first charge is now $q_{2}$ and the force is $\mathrm{F}_{2}$, the second will still be $q^{\prime}$ with force $\mathrm{F}^{\prime}$, and we have

$$
\begin{gathered}
\left.q_{2}-q^{\prime}=\lambda\left(\mathrm{F}_{2}-\mathrm{F}^{\prime}\right)=\mathrm{C}_{2} \mathrm{D}\right) / 300 \\
\text { and } \\
\frac{\mathrm{C}_{1}}{\mathrm{C}_{2}}=\frac{\mathrm{F}_{1}-\mathrm{F}^{\prime}}{\mathrm{F}_{2}^{\prime}-\mathrm{F}^{\prime \prime}}
\end{gathered}
$$

The piezoelectric electrometer. The Curies were lad from this to the invention of a very convenient instrument for producing definite small quantities of electricity.

Let Fig. 104 represent a rectangular block of quart\% with the optic axis-the axis of the prism-parallel to the edge AC. Let one of the pyro- and piezoelectric axes be parallel to the edge BF. They found that load $\mathrm{F}$ on the face $\mathrm{ACDE}$ gave charge on the face DEFG

$$
q=-\frac{{ }_{k} \mathrm{LF}}{e}
$$

where $\mathrm{L}$ is the length $\mathrm{AB}, e$ is the thickness $\mathrm{Al}$, and $\kappa$ has the

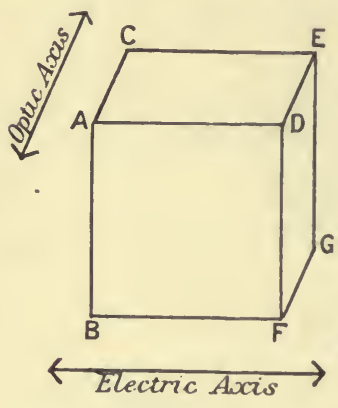

FIG. 104. value already found, viz. $6.32 \times 10^{-8}$ E.S. unit per dyne.

In the instrument a quart\% plate is cut with length $\mathrm{L}$, several centimetres, with breadth $\mathrm{AC}$ two or three centimetres, and with thickness $e$ as small as is consistent with strength sufficient to carry loads of the order of $1 \mathrm{kgm}$. 'This plate is fixed in brass pieces at the top and bottom and hung vertically from a support above, with a scale pan below, as shown diagrammatically in Fig. 105 , to carry a load.

Tinfoil $t t$ is pasted on to the front and back of the plate, and wires pass from the tinfoils to the system to be charged. 'The charge is proportional to the total load and if the load be increased the additional charge is proportional to the additional load. 'The instrument may be graduated by the use of an electrometer and condenser in the manner already explained.

Voigt's theory connecting pyroelectricity and piezoelectricity.* 'The Curies found that pressure produces electrification at the ends of an axis the same in sign as that produced by cooling, and tension the same in sign as heating. 'This suggests that the change in dimensions of the crystal may alone be concerned in the two classes of phenomena, and Voigt has investigated the question. The following is a mere indication of the method. 
The deformation of an element of a body is completely expressed in terms of six quantities* which we may denote by $\mathrm{ABC} a b c$. If the intensities of electrification due to the strains are $\mathbf{E}_{1} \mathbf{E}_{2} \mathbf{E}_{3}$ in three directions at right angles, we may suppose that each term in the strain contributes to the electrification, and so we get

$$
\mathrm{E}_{1}=\lambda_{1} \mathrm{~A}+\lambda_{2} \mathrm{~B}+\lambda_{3} \mathrm{C}+\lambda_{4} a+\lambda_{5} b+\lambda_{6} c
$$

with similar values for $\mathrm{E}_{2}$ and $\mathrm{E}_{3}$, and for the most general case with different values for the coefficients $\lambda$. Thus for this most general case- a hemihedral crystal with inclined faces in the triclinic systemwe shall have eighteen independent coefficients of the type $\lambda$. In all but this general case there are relations holding between the coefficients owing to the symmetry of the crystal about certain planes or directions, and their number may be greatly reduced. 'Thus we get definite values of the intensities of electrification in terms of the change of shape whether it is produced by heat or by stress.

Riecke and Voigt $†$ have tested the theory for tourmaline and quart\%. Knowing the piezoelectricity produced by given deformations, they were able to determine the coefficients. 'Then, measuring the dilatations in different directions with rise of temperature, they were able to determine the charges which should be produced merely by the change of shape on heating. Voigt $\ddagger$ found that in a

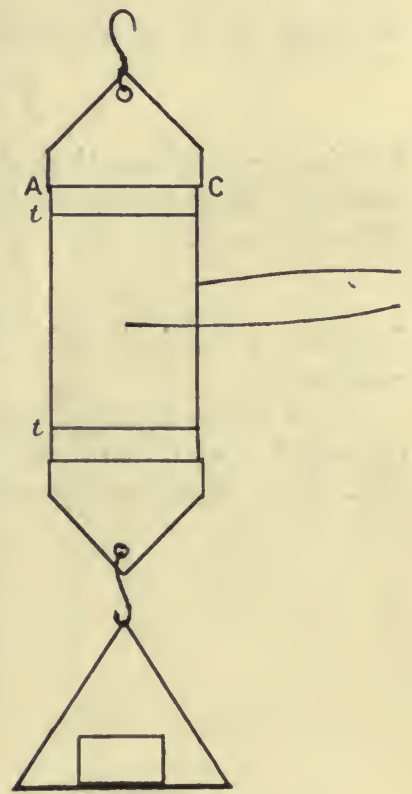

Fig. 105. tourmaline crystal about four-fifths of the pyroelectric charge could be accounted for by dilatation.

Pockels $§$ found a fairly close agreement with Voigt's theory in the case of sodium chlorate.

Electric deformation of crystals: Lippmann's theory. Soon after the discovery of piezoelectricity Lippmann \| pointed out that there should be a converse effect, vi\%. a change in the dimensions of a piezoelectric crystal when it was subjected to electric strain. We may present Lippmann's theory as follows :

* Thomson and Tait, Natural Philosoplıy, vol. ii. p. 461.

+ Wied. Ann. 45, p. 523 (1892).

Wied. Ann. 66, p. 1030 (1895).

$\S$ Winkelmann, Handbuch der Physik, 2nd. ed. iv. p. 783.

II Ann. de. Chim. et de Phys. (5), vol. xxiv. p. 145 (1881); Principe de la Canservition de l'Electricité. 
Let us take such a system as a condenser with one surface earthed and with the other connected to a source at potential $x$ where $x$ can be varied at will. Let the charge at $x$ be $m$. Let the plates of the condenser be $l$ apart and let them be subjected to force $\mathrm{F}$, compressing the dielectric. 'Then in changing the length $l$, work $\mathrm{Fdl}$ is done. Let the temperature be kept constant so that in any cycle $\int d \mathbf{Q}=0$, where $d \mathbf{Q}$ is an element of heat imparted. Then, on the whole, no heat is converted into work or vice versa during a cycle, and we have only to consider the interchange of energy between the electrical form and that represented by the work done.

If $\mathrm{E}$ is the electrical energy, the principle of the conservation of energy gives in any cycle

$$
\int(d \mathrm{E}-\mathrm{F} d l)=0 .
$$

Further, we assume that the charge $m$ is a definite function of the condition of the system, there being no leakage, and so when we go through a cycle

$$
\int d m=0 \text {. }
$$

Lippmann termed this the principle of the conservation of electricity.

Now let us represent the system on a diagram in which abscissae represent $l$ and ordinates $\mathbf{F}$, so that areas represent work done, Fig. 106. The dependence of $m$ on the physical condition implies

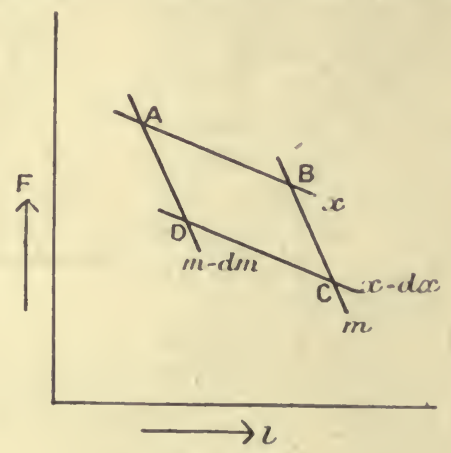

FIG. 106.

that we can draw a series of equal charge lines on the diagram, such as $\mathrm{AD}$ at charge $m-d m$ and $\mathrm{BC}$ at charge $m$. Further, the potential will be a definite function of the physical condition, for it is equal to charge/capacity, each term of which is such a function. Hence we can draw a series of equipotential lines, such as $A B$ at $x$, and $\mathrm{DC}$ at $x-d x$. 'These lines are analogous to isothermals, and the equal charge lines to isentropics on the ordinary indicator diagram. 
Now let us take the condenser round the cycle ABCD, starting from $\mathrm{A}$, one plate being earthed throughout. Along $\mathrm{AB}$ the other plate is connected to a source at $x$, and $\mathrm{F}$ and $l$ are varied as represented by the slope of $\mathrm{AB}$, and charge $d m$ is taken in. At B the plate is insulated and $\mathrm{F}$ and $l$ varied as represented by the slope of $\mathrm{BC}$. At $\mathrm{C}$ the plate is connected to a source at $x-\dot{d} x$, and we move back along $\mathrm{CD}$ till $d m$ is given back to the source. 'Then the plate is insulated and we return along DA to the initial condition.

The net electrical energy received is

$$
x d m-(x-d x) d m=d x d m .
$$

The net work done is represented by the area $A B C D$, which is easily seen to be

$$
\left(\frac{d \mathrm{~F}}{d x}\right)_{l} d x d l
$$

where $d l$ is the increase of $l$ as we move along $\mathrm{AB}$ at constant $x$.

Equating the two expressions for the energy,

$$
\begin{aligned}
d x d m & =\left(\frac{d \mathrm{~F}}{d x}\right)_{l} d x d l \\
\text { or } \quad\left(\frac{d m}{d l}\right)_{x} & =\left(\frac{d \mathrm{~F}}{d x}\right)_{l} .
\end{aligned}
$$

'Taking $x$ and $\mathrm{F}$ as the independent variables, let us put

$$
\begin{aligned}
d m & =c d x+h d \mathbf{F} \\
d l & =a d x+b d \mathbf{F}
\end{aligned}
$$

If $d x=0$ in (2) and (3),

$$
\left(\frac{d m}{d l}\right)_{x}=\frac{h}{b}
$$

If $d l=0$, we get from (3)

$$
\left(\frac{d \mathrm{~F}}{d x}\right)_{l}=-\left(\frac{a}{b}\right)
$$

Substituting from (4) and (5) in (1),

$$
h=-a
$$

so that (2) and (3) become

$$
\begin{aligned}
d m & =c d x-a d \mathrm{~F} \\
d l & =a d x+b d \mathrm{~F} .
\end{aligned}
$$

Applying these formula to the case of a piezoelectric crystal compressed along an electric axis, let us suppose that the potential is kept constant, and that a force of $d \mathbf{F}$ dynes is added 'Then 


$$
\frac{d m}{d \mathrm{~F}^{\prime}}=-a \text {. }
$$

The Curies found that for tourmaline (p. 155)

$$
a=5.41 \times 10^{-8} \text {. }
$$

Now let us suppose that $\mathrm{F}$ is kept constant and that the potential is altered. Then $\frac{d l}{d x}=+a$, and for tourmaline this should be equal to $5.41 \times 10^{-8}$.

Similarly for quartz $\frac{d l}{d x}$ should be equal to $6.32 \times 10^{-8}$.

If the quartz plate is cut and loaded as in the piezoelectric electrometer, it is evident that a similar investigation would give

$$
\frac{d m}{d 1^{\prime}}=-\frac{d l}{d x^{0}}
$$

Verification by the Curies. This result was rerified at once by J. and P. Curie." It will be sufficient to describe one experiment.

A thin quartz plate cut perpendicular to an electric axis, like that in the piezoelectric electrometer, was covered with tinfoil on its two faces and fixed so that any variation in its length could be measured by means of a magnifying lever. When a traction of $258 \mathrm{grm}$. or $258 \times 981$ dynes was put on, the charge produced was found to be $0 \cdot 186$, so that one dyne would give $7.39 \times 10^{-7}$.

A difference of potential estimated at 65.2 E.S. units was then put on between the tinfoil plates. 'This should give

$$
d l=7.39 \times 65.2 \times 10^{-7}=480 \times 10^{-7} \mathrm{~cm} .
$$

Direct measurement gave

$$
d l=500 \times 10^{-7} .
$$

Dielectric subjected to uniform pressure. If a dielectric is subjected to uniform pressure $p$ instead of to end thrust, a precisely similar investigation to that above gives

$$
\begin{aligned}
d m & =c d x-a d p \\
d v & =a d x-b d p
\end{aligned}
$$

where $d v$ is the volume change. 'The diagram of Fig. 106 may be taken to represent the relation between $p$ and $v$ for values of $m$ and $x$. Quincke (ante, p. 142) found that the total change of volume of a jar for a rise of potential $x$ was nearly proportional to $x^{2} / t^{2}$, where $t$ was the thickness of the dielectric. We may therefore put

$$
\int d v=\frac{\kappa x^{2}}{2 t^{2}}
$$

* Euvres de Pierre Curie, p. 26 and p. 30, where another form of $\epsilon$ xperiment is described as well as that in the text. 
PYROELEC'TRICI'TY AND PIEZOELEC'TRICITY 161

whence

$$
\left(\frac{d v}{d x}\right)_{p}=a=\frac{\kappa x}{t^{2}} \text {. }
$$

He found that for a particular case the total volume change $\int d v$ was equal to about $10^{-5}$, the original volume, when the potential was such as to spark across $2 \mathrm{~mm}$., say, 20 E.S. units, and the thickness was $014 \mathrm{~cm}$. ; whence, if we deal with unit volume of dielectric, $\kappa$ is of the order $10^{-11}$.

If we keep $x$ constant,

$$
d m=-a d p,
$$

which shows that there will be a change in $m$ depending on the pressure but, for any moderate values of $x$ and $d p$, exceedingly minute.

Change of temperature of a pyroelectric crystal on changing the potential. Lippmann, in the paper already cited, pointed out that there should be a change of temperature in a pyroelectric crystal when the electric strain in it is changed, and the investigation applies to any condenser.

We assume that the pressure and volume remain constant, so that the only interchanges of energy are between the electrical and the heat forms. We further assume reversibility.

It is convenient to employ the entropy temperature diagram. Let us draw on it equal charge lines, $\mathrm{AD}$ at $m-d m$ and $\mathrm{BC}$ at $m$, and

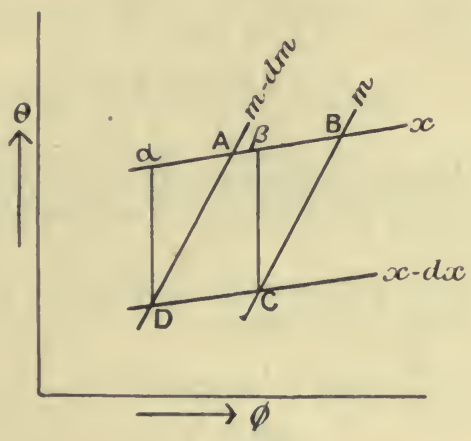

FIG. 107.

equipotential lines $\mathrm{AB}$ at $x$ and $\mathrm{DC}$ at $x-d x$. Let us take a dielectric with charges on its two faces through the cycle ABCD.

'The net electrical energy given out in the cycle is $d m d x$. The heat put in is the area $\mathrm{ABCD}=\alpha \beta \mathrm{CD}=\left(\frac{d \theta}{d x}\right)_{\phi} \cdot d x \cdot d \phi$, and equating the two quantities,

$$
\left(\frac{d m}{d \phi}\right)_{x}=\left(\frac{d \theta}{d x}\right)_{\phi}
$$


Taking $x$ and $\theta$ as the independent variables, put

$$
\begin{aligned}
& d m=c d x+h d \theta \\
& d \phi=a d x+b d \theta
\end{aligned}
$$

and it follows, as in the investigation on p. 159, that $h=-a$.

Let us apply the formulæ to the case of a tourmaline $1 \mathrm{sq} . \mathrm{cm}$. cross-section and thickness $t$.

According to Riecke (p. 150)

$$
h=\left(\frac{d m}{d \theta}\right)_{x}=1 \cdot 8
$$

If $\phi$ is constant, i.e. if we allow no heat to pass in or out,

$$
\left(\frac{d \theta}{d x}\right)_{\phi}=-\frac{a}{b}=\frac{h}{b}
$$

But $\quad b=+\left(\frac{d \phi}{d \theta}\right)_{x}=\frac{1}{\theta}\left(\frac{d Q}{d \theta}\right)_{\phi}$ since $\frac{d Q}{\theta}=d \phi$

$\frac{d Q}{d \theta}$ is the heat capacity, which we may put as $\rho \sigma t$, where $\rho$ is the density, $\sigma$ the specific heat in work measure.

Then

$$
\left(\frac{d \theta}{d x}\right)_{\phi}=\frac{1 \cdot 8 \theta}{\rho \sigma t} .
$$

Integrating from $x=0$ to $x=x$, the rise of temperature is

$$
\Delta \theta=\frac{1.8 \theta}{\rho \sigma} \frac{x}{t}=\frac{1.8 \theta}{\rho \sigma} \mathrm{E}
$$

where $\mathbf{E}$ is the electric intensity. In practice this is small. If, for instance, $\mathbf{E}$ is $100, \theta$ is 300 , and $\rho \sigma$ is of the order $10^{7}, \Delta \theta$ is of the order $0.005^{\circ} \mathrm{C}$.

Let us now apply the formulæ to find the temperature change on charging an ordinary condenser.

We are to find $\frac{d \theta}{d x}$ when $\phi$ is constant.

We have

$$
\left(\frac{d \theta}{d x}\right)_{\phi}=\frac{h}{b}
$$

where

$$
h=\left(\frac{d m}{d \theta}\right)_{x} \text { and } b=\left(\frac{d \phi}{d \theta}\right)_{x} .
$$

Now

$$
m=\frac{\mathbf{K} x}{4 \pi t}
$$

Then

$$
\left(\frac{d m}{d \theta}\right)_{x}=\frac{x}{4 \pi t} \frac{d \mathbf{K}}{d \theta}
$$

neglecting the change in $t$; 
and

$$
\left(\frac{d \phi}{d \theta}\right)_{x}=\frac{1}{\theta}\left(\frac{d Q}{d \theta}\right)_{x}=\frac{\rho \sigma t}{\theta} \text {. }
$$

Then

$$
\left(\frac{d \theta}{d x}\right)_{\phi}=\frac{x}{4 \pi t} \frac{d K}{d \theta} \frac{\theta}{\rho \sigma t} .
$$

If we integrate from $x=0$ to $x=x$ the total change in $\theta$ is small, so that we may put $\theta$ constant on the right hand and the change is

$$
\begin{aligned}
\Delta \theta & =\frac{x^{2}}{8 \pi t^{2}} \cdot \frac{d \mathrm{~K}}{d \theta} \cdot \frac{\theta}{\rho \sigma} \\
& =\frac{\mathrm{KE}^{2}}{8 \pi} \frac{1}{\mathrm{~K}} \frac{d \mathrm{~K}}{d \theta} \cdot \frac{\theta}{\rho \sigma}
\end{aligned}
$$

where $\mathbf{E}$ is the intensity.

'This is much smaller than the value just found for a pyroelectric crystal, unless $\mathrm{E}$ is very much greater than 100 .

It is easily found that the isentropic capacity

$$
\left(\frac{d m}{d x}\right)_{\phi}=c\left(1+\frac{h^{2}}{b c}\right)
$$

and $\frac{h^{2}}{b c}$ is found to be

$$
\frac{\mathrm{KE}^{2}}{4 \pi}\left(\frac{1}{\mathrm{~K}} \frac{d \mathrm{~K}}{d \theta}\right)^{2} \frac{\theta}{\rho \sigma}
$$

which is quite negligibly small.*

- Pockels has generalised the theory of Lippmann. The reader is referred to Winkelmann's.Handbuch, vol. iv., where a full account of pyro-and piezoelectricity will be found, with references to the original memoirs. 



\title{
PAR'T II : MAGNE'TISM
}

\section{CHAP'IER XIII \\ GENERAL ACCOUNT OF MAGNETIC ACTIONS}

\begin{abstract}
Natural and artificial magnets-Poles-Mechanical action of poles on each other-The two poles have opposite mechanical actions-In a wellmagnetised bar the poles are near the ends-The two poles always accompany each other in a magnet-The two poles are equal in strengthThe magnetic action takes place equally well through most media-Induced magnetisation-Magnetisation can take place without contactRetentivity and permanent magnetism-The earth is a great magnetMagnetisation induced by the earth-Methods of magnetisation-Single touch-Divided touch-Double touch-The electro-magnetic methodElectro-magnets-Ball-ended magnets-Horseshoe magnets-Compound magnets-Distribution of magnetisation-Consequent poles-Magnetisation chiefly near the surface-Saturation and supersaturation-Preservation of magnetisation-Armatures-Portative force-Nickel and cobalt magnets-The magnetic field-Lines of force-Mapped by iron filings.
\end{abstract}

Natural and Artificial magnets. Magnetite, a mineral found in various parts of the world, having the composition $\mathrm{Fe}_{3} \mathrm{O}_{4}$, has been known for ages to possess the property of attracting small pieces of iron. It was found by the ancients near Magnesia, in Lydia, and from the locality, probably, the Greeks gave it the name $\mu a \dot{\gamma} \nu \eta \eta$, whence we derive the name magnet. A piece of the mineral exhibiting the attractive property is now called a "natural magnet," to distinguish it from the steel magnets which are made by processes described hereafter.

If a natural magnet is dipped in iron filings it is usually found that the filings cling in tufts, chiefly about two points at opposite ends of the specimen, and if the magnet is suspended so that the line joining these two points is horizontal, this line will in this part of the world set nearly North and South, and from this directive property the mineral acrquired the name "lode-stone," that is, way-stone.

If a bar of stecl is stroked in one direction with one end of a natural magnet, the bar itself becomes a magnet, acquiring the property of attracting iron filings, and the tendency to set North 
and South when freely suspended horizontally. Such a bar was formerly termed an artificial magnet.

In describing the fundamental phenomena we shall suppose that we are dealing with steel magnets. Natural magnets are now mere curiosities and have not been usefully employed since the eighteenth century. The prefix "artificial " has long ceased to be applied to steel magnets.

Poles. 'The two ends of a magnetised bar round which the attractive properties are exhibited are termed its poles. The end setting towards the North is termed the North-seeking pole, the other the South-seeking pole, or more shortly the North and the South poles respectively. They may be designated by NSP and $\mathrm{SSP}$, or more shortly by $\mathrm{N}$ and $\mathrm{S}$. 'The NSI' is frequently marked on the magnet either by a cut across it, or by $\mathbf{N}$ cut in the metal.

Mechanical actions of poles on each other. If we suspend one magnet so that it is free to move round on a pivot, forming in fact a compass needle, and then present to its poles in succession each of the poles of a second magnet, we find that two unlike poles attract each other, while two like poles repel each other, or :

NSP attracts SSP and repels NSP and

SSP $"$ NSP $, \quad, \quad$ SSP.

The troo poles have opposite mechanical actions. Where one attracts the other repels, and the actions of the two poles tend to neutralise each other. We may therefore call them respectively positive and negative, and if we consider a NSP to be positive, we must consider a SSP to be negative.

Position of poles. In a well-magnetised bar the poles are near the ends. For, on dipping the bar into iron filings, we find

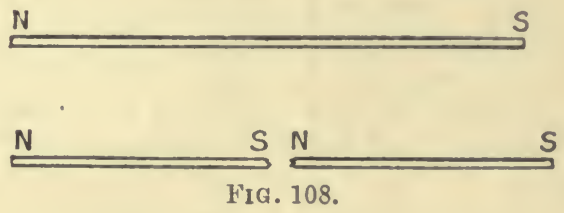

that the filings cluster chiefly about the ends, and hardly adhere at all near the middle. But though we speak of the poles of a magnet we must remember that they are not definite points, but rather regions, in general near the ends of the magnet.

The two opposite poles always accompany each other on a magnet. If we break a magnetised knitting needle, for instance, we do not isolate the two poles, for two new poles are at once developed at the broken ends, as in Fig. 108, so that each fraginent becomes a complete magnet with its opposite poles. All experi. 
GENERAI، ACCOUNT OF MAGNETIC ACTIONS 167 ments go to show that it is impossible to obtain one kind of pole alone on a magnet.

The two poles are equal in strength-that is, they exert at equal distances equal forces respectively of attraction and repulsion. This may be proved by bringing up a magnet to a compass needle, the magnet being held vertically with its centre on a level with the needle, Fig. 109, and its poles $\mathrm{N}$ and S equidistant from the pole $n$. The two forces then have a vertical resultant, as will be seen from Fig. 110, and the compass needle is not deflected in the horizontal plane, but only tends to dip.

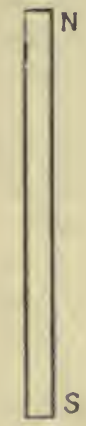

FrG. 109.

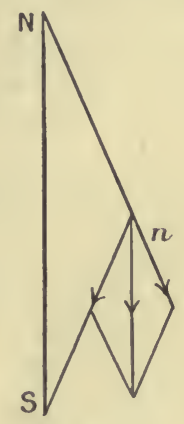

FIG. 110.

Another proof of this equality is given by the fact that a bar of steel weighs the same before and after magnetisation. In the latter case it is acted on by the earth's magnetisation, but with forces at the two ends equal and opposite, and therefore at most only forming a directive couple. 'The forces being equal, we conclude that the poles are equal.

The magnetic action takes place equally well through most media within the limits of our powers of observation. If, for instance, a magnetised needle is suspended so as to set North and South and a magnet is brought near it as in Fig. 111, the needle is

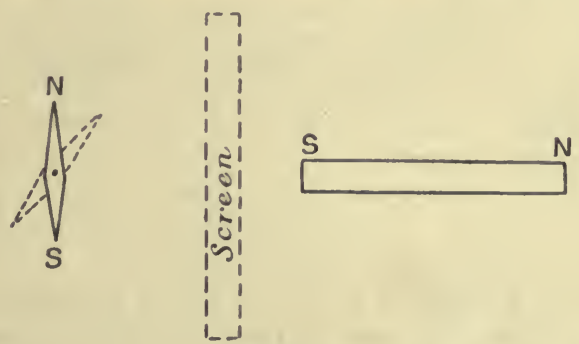

FIG. 111.

deflected with the North-seeking pole towards the East. 'The deflection is still the same if a screen of wood or cardboard or brass be 
interposed. There is, however, one striking exception. If the screen is iron and is of sufficient extent the deflection diminishes, the action of the magnet being screened off by the iron from the space beyond.

Induced Magnetisation. A piece of iron brought into contact with a magnet becomes itself a magnet by induction, the iron being magnetised in such a manner that the end of it in contact with the magnet is a pole of the kind opposite to that with which

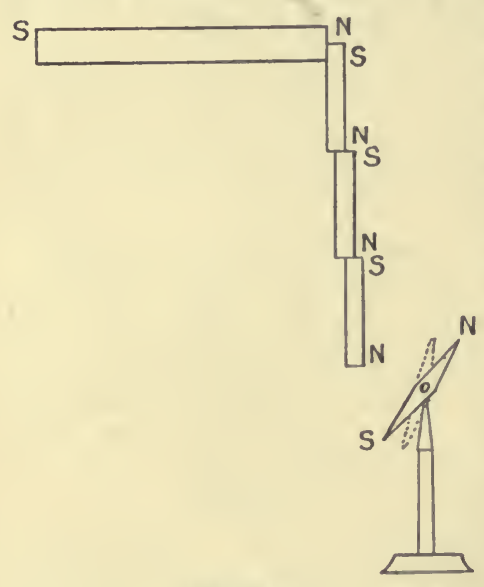

it is in contact, while the other end of the iron is a pole of the same kind. 'The adherence of the iron to the magnet is explained by the formation of the unlike pole, while the existence of the like pole at the further end may easily be tested by means of a suspended compass needle.

FIG. 112.

Several pieces of iron may thus be hung one below the other from a magnet pole, each magnetising the next below it, unlike poles being together and the last pole being like the inducing pole of the magnet, as in Fig. 112. 'The NSP of the last piece of iron repels the NSP of a needle. This magnctisution can take place without contuct, a given pole inducing a pole of the opposite kind to itself in the nearer part of the piece of iron in which the induction occurs, the further part having a pole of the same kind. 'This may' be shown by placing a compass needle near one end of a bar of iron and then bringing a magnet up near the other end, as in Fig. 113, say with the NSP towards the bar: the NSP of the needle is repelled, showing that a NSP is induced in the part of the bar more

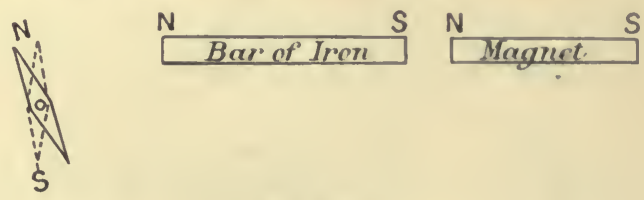

FIG. 113.

distant from the magnet. If the compass be moved along a line parallel and near to the bar it will be found that the NSP is attracted by the part of the bar nearer to the inducing magnet, which is therefore a SSP.

If we bring a piece of unmagnetised iron near a compass needle, either end of, the needle is attracted. 'This is an example of 


\section{GENERAI, ACCOUNT OF MAGNETIC ACTIONS 169}

magnetisation by induction, the needle pole inducing in the near part of the iron an unlike pole by which it is attracted, the like pole by which it is repelled being at a greater distance and therefore not having so much effect.

Retentivity and permanent magnetism. With a soft iron bar the induced magnetisation usually disappears on withdrawal of the inducing magnet, but if the bar is of steel more or less magnetisation remains permanently. This quality of retaining magnetisation after the removal of the inducing magnet is termed retentivity, and the magnetisation so retained is termed permanent magnetism. In general the harder the steel the greater is its retentivity, while the more nearly it approaches soft, pure iron the less is its retentivity. 'The highest degree of retentivity appears to be possessed by steel containing a certain proportion of tungsten, and called magnet steel.

If we wish to determine whether a given bar possesses permanent, magnetism, i.e. whether it is a magnet or not, we may bring up the two ends in succession near to one pole of a suspended compass needle. If the bar is magnetised in the usual way, one end or the other will be a pole like that of the needle to which it is presented, so that in one case repulsion will ensue. If, however, it is unmagnetised both ends will attract the same pole of the compass needle, for by induction an unlike pole will in each case be formed in the nearer part of the bar, and by this it will be attracted. Frequently with a weakly magnetised bar we may observe repulsion of a pole by one end of the bar when at some distance from the pole, and this changes to attraction at a smaller distance. We may ascribe this change to induction. At a small distance the pole of the compass needle acts by induction, forming in the nearer part of the bar a pole unlike itself and so strong as to mask the effect of the like pole by which it was previously repelled.

The earth is a great magnet. The tendency of a horizontally suspended magnet to point towards the North led to the invention of the compass, which was introduced into Europe about the twelfth century, probably from China, where it had been used for many hundreds of years. At first it was supposed to point towards the Pole Star, but as early as 1269 Adsiger discovered that the needle did not point exactly North.* Columbus in voyaging across the Atlantic in 1492 again discovered that the compass needle was deviated from the geographical North, and by different amounts in different longitudes. 'This deviation is termed the "variation" or "declination." In 1576 Norman discovered the "dip." He found that if a needle was suspended through its centre of gravity it dipped with its North end down wards through an angle of $71^{\circ} 50^{\prime}$ with the horizontal at London. $\dagger$

* Encyc. Met.-Maynetism, p. 737.

$\dagger$ Hartmann had previously discovered the tendency to dip, but did not publish the discovery. See Dove's Tepertorium der Physik, ii. (1838). 
In 1600 Dr. Gilbert, of Colchester, published his great book De Magnete, containing an account of experiments which founded the science of Magnetism and Electricity, and in this work he pointed out that the behaviour of the compass needle could be explained on the supposition that the earth is a great magnet, somewhat irregularly magnetised so that it cannot be supposed to have poles merely coincident with the earth's poles. He used a spherical lode-stone as a model, and showed that near the surface a small needle exhibited the tendency to point towards the pole. He showed further that it dipped in one way in one hemisphere and in the other way in the other hemisphere, that it was perpendicular to the surface at the two poles and parallel to the surface round the equator.

It must not be supposed that there are definite magnetic poles in the earth, but rather that there is a region of South-seeking polarity in the Northern Hemisphere towards which the NSP of a compass points, and a region of North-secking polarity in the Southern Hemisphere. 'These regions are not round but near the geographical poles. There is one point in each hemisphere at which a magnetised needle free to move about its centre of gravity dips vertically downwards. 'That in the Northern Hemisphere has been visited several times and is situated about $\tau 0^{\circ} \mathrm{N}$ latitude and $97^{\circ} \mathrm{W}$ longitude, and there the NSP' is downwards. 'That in the Southern Hemisphere was visited in 1909 and is situated about $72^{\circ}$ $\mathrm{S}$ latitude and $155^{\circ} \mathrm{E}$ longitude. The positions of these points show that the earth is not magnetised symmetrically.

It is very important to observe that the earth's action on magnets is directive only. 'The two poles of any ordinary magnet may be regarded as practically at the same distance from a pole of the earth, and therefore they will be acted on by equal and opposite forces by that pole. 'The resultant action of the two poles of the earth thus being a couple is directive and does not tend to more the centre of gravity of a magnet.

Magnetisation induced by the earth. Not only is the magnetisation of the earth sufficient to direct magnetised needles, but it produces quite considerable induced magnetisation. If in this part of the world a poker be held upright and struck sharply the lower end becomes a NSP and the upper a SSP, the polarity acting strongly on a compass needle. If the poker be reversed and again struck, the magnetisation is quickly reversed, and what is now the lower end becomes a NSP. Any iron lying more or less in the general direction of the dipping needle is thus magnetised by induction, as, for instance, the front plates of iron fire-grates or iron gas-pendants. Iron ships are magnetised by the earth and their compasses are affected seriously by this magnetisation. The deviation of the compass thus produced must be allowed for.

Methods of magnetisation. Various methods have been devised to magnetise bars of steel by stroking them with magnets. 


\section{GENERAL ACCOUNT OF MAGNETIC ACTIONS 171}

They are all modifications of three, called respectively the methods of single, double, and divided touch. In our description we shall assume that the object is to make flat bar magnets, and it appears that good dimensions for such magnets are on the scale-length 10 to 15 , breadth 1 , depth $\frac{1}{2}$.

Single touch. The bar to be magnetised is rubbed several times in the same direction by one pole of the magnetising magnet, which is moved parallel to itself either at right angles to the bar or inclined as shown in Fig. 114, the course of the magnet being

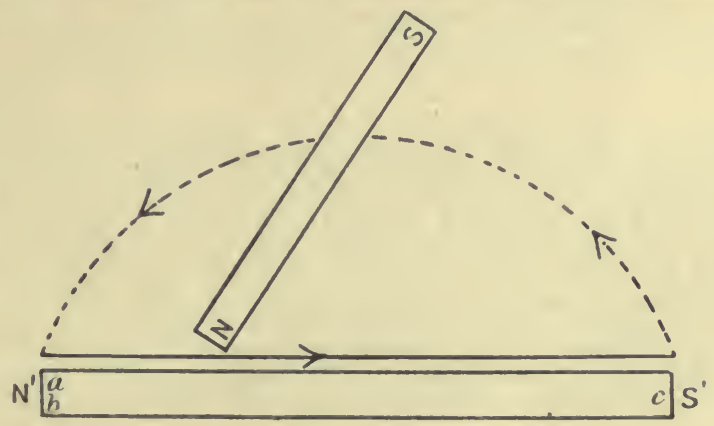

FIG. 114.

indicated by the solid line, the dotted line indicating the return journey at such a distance from the bar that the magnet has no appreciable influence on it.

'The probable action of the process is that when the $\mathbf{N}$ pole of the magnetising magnet is put on the end of the bar to be magnetised it induces a SSP at $a$ immediately under it and Northseeking polarity chiefly on the other side, $b$, but also at the more distant parts of the bar and at the other end, $c$. But as $\mathbf{N}$ moves along the bar it carries along with it the SSP originally at $a$, while the NSP' at $b$ moves round to occupy the whole of the end $\mathbf{N}^{\prime}$, and the SSP carried to the other end neutralises the weak North-seeking polarity already there. Repetition with the same magnet somewhat increases the effect, but if a magnet weaker than that employed at first be substituted in the repetition it partially demagnetises the bar. 'The process is unsatisfactory, inasmuch as it does not lead to strong magnetisation or to symmetrical distribution. It may be somewhat improved by beginning with, say, the $\mathbf{N}$ magnetising pole in the middle of the bar and drawing it along to the end. 'The magnetising magnet is then turned over and the $\mathrm{S}$ magnetising pole is drawn from the middle to the other end. It is best in this last process to slide the one over the other as indicated in Fig. 115. But practically the same process may be carried out much more effectually by the method of

Divided touch. This method was devised by Dr. Knight about the middle of the eighteenth century, and he made by it 
small magnets of very great power. One, for example, weighing $1 \mathrm{oz}$. troy, when armed with iron at the two ends so that the weight was still under $2 \mathrm{oz}$, lifted forty-four times its own weight. 'The method is especially applicable to compass needles or short bar's. Two magnets are used and are placed with their unlike poles initially as close together as possible, and the needle or bar is laid

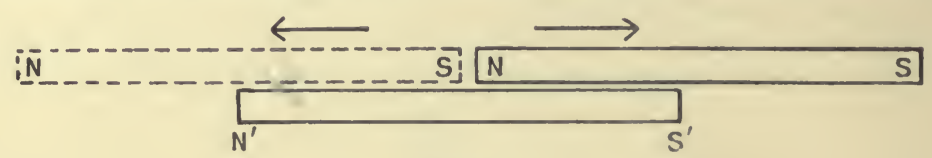

FIG. 115.

under them as in Fig. 116, and fixed by a button. 'The two magnets are then gradually drawn apart till their ends slide off the bar, leaving the bar magnetised as indicated in the figure. The two magnetising poles are again brought together or near each other, and then brought down on the middle of the magnet, and the process is repeated three or four times. 'The bar is then turned over and the same number of operations repeated on the other face. 'The explanation of the magnetisation is obvious. 'The

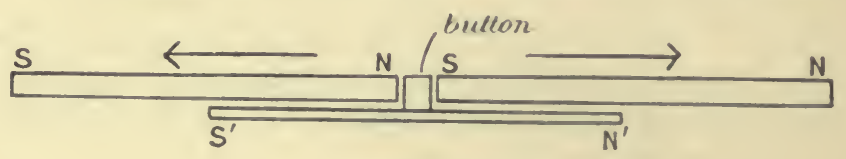

FIG. 116.

method of divided touch requires in general more powerful magnets than the following method, known as that of

Double touch. In this method the mannetising system consists of two unlike poles, NS, which are kept a constant distance apart. A horseshoe magnet may be used, or its equivalent, two bar magnets with a piece of wood between their lower poles, their

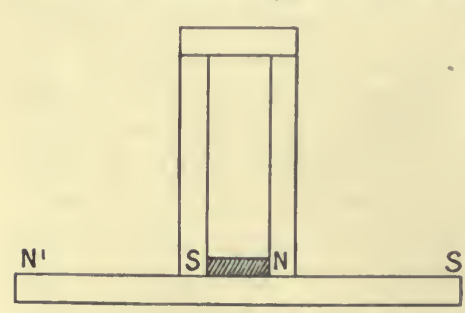

FIG. 117. upper poles being united by soft iron, Fig. 117. If one bar is to be magnetised, the magnetising poles are brought down on to the middle of it and moved to and fro several times, each half being traversed the same number of times. 'They are then withdrawn from the centre. If several bars are to be magnetised they may be laid end to end and treated as one bar. After several journeys of the magnetising poles the end bar's are brought into the middle and the process is continued until all the bars have thus been in the middle. 


\section{GENERAL ACCOUNT OF MAGNETIC ACTIONS 173}

These methods of making magnets give interesting illustrations of magnetisation by induction, but they are now little used except sometimes for compass needles and for the needles used in the determination of the dip of the earth's magnetic force, in which case the divided touch is employed.* They are for general purposes entirely superseded by

The electro-magnetic method. In this method a coil of wire is wrapped round a hollow cylinder, forming what is termed a solenoid, the bar to be magnetised is inserted in the cylinder, and a strong electric current is passed through the coil. When the current is broken and the bar is withdrawn it is found to be magnetised.

Electro-magnets. The electro-magnetic method is not only useful for thus making permanent magnets by the insertion of bars and their withdrawal when magnetised. If a coil of wire carrying a current surrounds an iron core that core is a powerful magnet while the current is rumning, though on the stoppage of the current the magnetisation in general falls off very greatly. By far the strongest magnets are made by some such arrangement as that represented in Fig. 118, where the two limbs

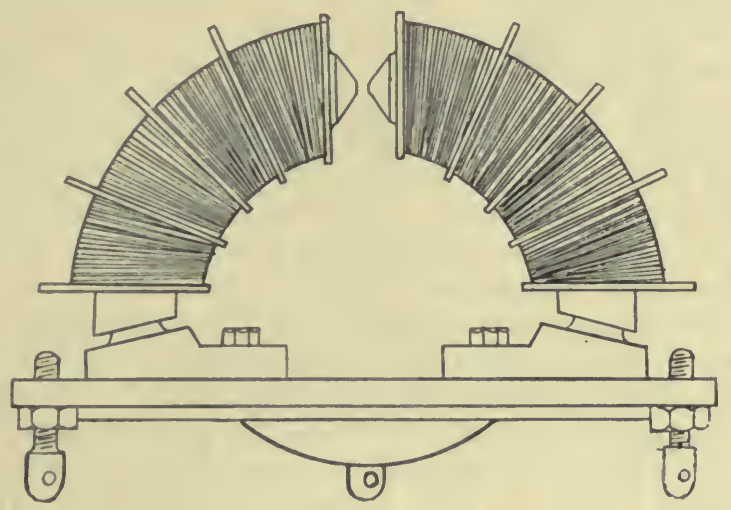

FIG. 118.

consist of soft iron surrounded by coils through which a current may be passed, the limbs being joined by a soft iron cross-piece as the base. With a strong current passing round the two limbs from inside to outside in both limbs as seen from the front, or in the opposite direction in both, the ends of the soft iron become exceedingly strong poles, far stronger than can be obtained in permanent magnets of steel. 'It is to be observed, however, that the magnetism only maintains its great strength while the current is passing.

* A full account of the methods used before the introduction of the electromagnetic method will be found in the Library of Useful Knoulcdge, article Magnetism, chap. v. (1832). 
Horseshoe magnets. For purposes in which the two poles are required close together, steel magnets of the horseshoe form are often convenient. Bars of this shape may have their length greater in proportion to breadth and thickness than straight bars, and they may be conveniently magnetised either by the electromagnetic method, a coil being placed on each limb, or by the method of double touch after connecting the two ends by a soft iron cross-piece.

Compound magnets. Very powerful "compound " magnets are often formed by building together a number of single bars or horseshoes magnetised separately. This method is not so much used now as formerly, when it was difficult to get thick bars of homogeneous steel.

Distribution of magnetisation. If a straight bar magnet is very carefully magnetised the magnetic action appears to radiate from very near the ends, and at some distance from the bar the resultant action is very nearly the same as if the poles were actual points within the bar and near the ends. The thinner the bar in comparison to its length, the nearer are the poles to the ends. If the bar has dimensions, say, $10 \times 1 \times \frac{1}{2}$, then the poles may perhaps be considered as an eighth or a tenth of the length from the ends. 'This is, however, only an approximation. If we imagine an ideal magnet as consisting of a very fine wire or fibre, with its poles actually at the two ends, then any real magnet may be regarded as a bundle of such fibres of unequal lengths. If the magnet is well made the fibres will have their middle points at the middle of the bar so that the magnetisation is symmetrically distributed, and the greater number will be of nearly the same length as the bar.

Ball-ended magnets. Balls of steel or soft iron are bored and are put one on each end of a steel rod which is magnetised. 'The force is directed very much more nearly to a definite pole at the centre of the ball than in other magnets.*

Consequent poles. If there is any irregularity in the stroking of a bar in the process of magnetisation-as, for example, magnetising it first in one direction and then in the otherconsequent poles may be developed, i.e. poles of the same kind may be formed together within the bar. We may easily obtain a knitting needle magnetised as in Fig. 119, each end being North-

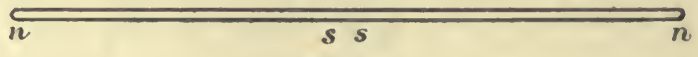

FIG. 119.

seeking, two consequent South-seeking poles occurring somewhere between them.

Magnetisation chiefly near the surface. The per-

* Searle, Camb. Phil. Soc. Proc., 12, p. 27, 1903. Ball-ended magnets were devised by Robison in the eighteenth century, but bad been entirely forgotten till they were re-invented by Searle. 
manent magnetisation is usually near the surface of a bar, for if the surface layers be dissolved away by nitric acid the inner core will be found to be nearly unmagnetised. $\mathrm{Or}_{\text {r }}$ if a steel tube with a steel core be magnetised the core on removal is only slightly magnetised.

Saturation and supersaturation. It is found that there is a limit to the magnetisation of a given bar, depending on its composition, temper, and shape. When the limit is reached the bar is said to be saturated. Just after being magnetised the bar is frequently found to have attained a degree of magnetisation greater than it can permanently retain, and it is then said to be supersaturated. The supersaturation disappears in time if the bar is left to itself, and rapidly if it is shaken.

Preservation of magnetisation. Armatures. A single magnet falls below even the normal saturation in course of time, especially if subjected to rough usage. To preserve the magnetisation it is usual to keep bar magnets in pairs with their like poles turned in opposite ways, cross-pieces of soft iron termed armatures connecting the unlike poles. Horseshoe magnets are provided with a single armature connecting the poles. The armatures have poles developed by induction as marked in Fig. 120, and each pole

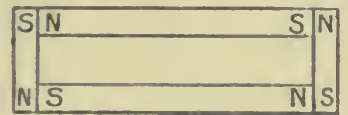

FIG. 120.

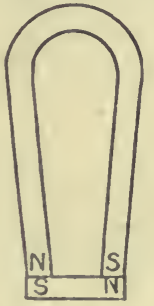

being faced by an unlike pole, they are supposed to tend to strengthen rather than to weaken each other.

Mr. Hookham * has found that if a bundle of bar magnets has large soft iron "pole pieces," Fig. 121, fixed on the ends, the surfaces of the pole pieces being separated only by a very small air space, the horseshoe magnet which is thus virtually formed retains its magnetisation indefinitely without an armature.

Long thin bars retain their magnetisation much better than short thick ones.

Portative force. Experiments have been made to find a general formula for the weight which a horseshoe magnet

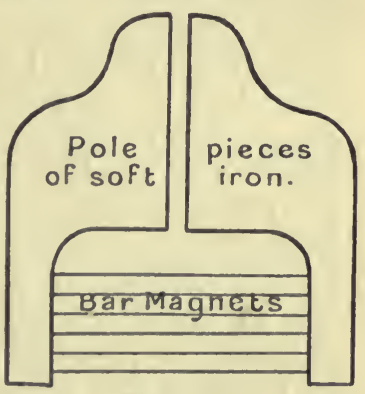

FIG. 121. can sustain, or rather the force which is required to pull away its armature. Häcker gives 


$$
\mathrm{W}=a m^{\frac{2}{3}}
$$

where $m$ is the weight of the magnet $\mathbf{W}$, the weight just sufficient to detach the armature, and $a$ a constant for a given quality of steel, the magnet in each case being in the state of normal saturation.

Inasmuch as $a$ differs very widely with the quality of steel employed, the formula has little general value, but it may be pointed out that for magnets of similar form and of the same quality it is equivalent to

$$
\mathrm{W} \propto l^{3 \times \frac{2}{3}} \propto l^{2},
$$

where $l$ is a linear dimension, say the breadth ; in othel words, $\mathrm{W}$ is proportional to the cross-section.

Nickel and cobalt magnets. While iron is pre-eminent in its magnetic properties, all bodies, as we shall see in Chapter 15, give signs of polarity under magnetic induction, though in general so slight that the polarity can only be observed easily in exceedingly strong fields such as are produced by powerful electro-magnets. 'There are, however, two other metals, nickel and cobalt, which, though far less magnetic than iron, still show very appreciable polarity under induction. 'P'ieces of these metals will fly to, and be held on by, a strong pole.

'J'hey may be very appreciably macrnetised by insertion within a magnetising coil and they exhibit some retentivity, nickel, especially when hardened, more than cobalt. It has been found possible to make a compass needle of hardened nickel.

The magnetic field. When we consider the space round a magnet in connection with the magnetic actions which may be manifested in it, we term the space the "field" of the magnet.

If we place a magnet on a table, a small compass needle also placed on the table tends to set in a definite direction in the field. Its NSP is acted on by a force which we may regard as the resultant of the repulsion of the NSP and the attraction of the SSP of the magnet. We shall suppose that the needle is very small, so small that its poles may be taken as equidistant from the magnet poles; then its SSP is acted on by the resultant of the attraction of the NSP and the repulsion of the SSP of the magnet, and these two forces are respectively equal and opposite to those on the NSP. Then the two resultants are equal and opposite, and a couple acts on the needle. It therefore sets with its axis in the direction of the resultant forces on the poles. If it is disturbed from this equilibrium position the couple tends to restore it, and if left to itself it will vibrate more or less rapidly according to the magnitude of the force on either pole. In fact, the needle is a kind of double magnetic pendulum with opposite pulls on the two ends, and just as the time of vibration of a given gravity pendulum is inversely as the square root of the force of 
GENERAL ACCOUNT OF MAGNETIC ACTIONS $17 \%$

gravity, so the time of vibration of the compass needle is inversely proportional to the square root of the force due to the magnet acting on either pole. Hence by moving the needle about we may investigate the direction of the force on a pole at any point by the direction of the axis, and we may compare the value of the force at different points by observing the time of vibration.

Lines of force. If a curve is drawn in the magnetic field so that at every point of the curve its direction coincides with that of the magnetic force on a North-seeking pole placed at the point, the curve is called a "line of force." It is to be regarded as having for its positive direction that in which a North-seeking pole would tend to move, and the opposite direction is negative.

If, for instance, the line of force is in a horizontal plane and a small compass needle is laid at a point in it, the needle will be a tangent to the curve, and we must think of the line of force as coming in to the SSP of the needle and going out of the NSP.

We may trace a line of force in a horizontal plane by laying a magnet on a table and then moving a small compass needle in its field so that its centre is always travelling in the direction of its length.

What the compass needle shows in the horizontal plane would be shown all round the magnet in other planes if we could suspend a needle so that it was free to move in any direction round its centre of gravity.

Lines of force mapped by iron filings. There is a very simple and beautiful method of exhibiting these lines of force in the horizontal plane through the magnet by means of iron filings.

The magnet is laid on or under a sheet of smooth cardboard, and the card is sprinkled with iron filings. 'The filings are elongated fragments of iron, and owing to the inductive action of the magnet each filing becomes a little magnet and tends to set with its longer axis in a line of force. 'The friction, however, prevents immediate setting. If the card is gently tapped the filings are thrown up a little from the card, are momentarily relieved from friction, and so are pulled into the lines of force. They thus map out the direction of the lines.

Fig. 122 shows the general course of the lines of force round a single bar magnet.

It is important to notice how the lines of force starting from the NSP curve round and enter the SSP. They start mostly from near the end, and where they are most crowded the frequency of vibration of a compass needle shows that the force there is strongest. Near the middle of the magnet few lines issue or enter, and there the force is less. As the lines issuing from the pole pass outwards they diverge, and a needle shows by its diminishing frequency that the force diminishes as the lines diverge.

Fig. 123 shows the field due to two magnets arranged parallel, with their unlike poles near each other. It should be noticed in 
this case how many of the lines issuing from the NSP of one magnet enter the SSP of the other.

Fig. 124 shows the field due to two bar magnets arrainged in one

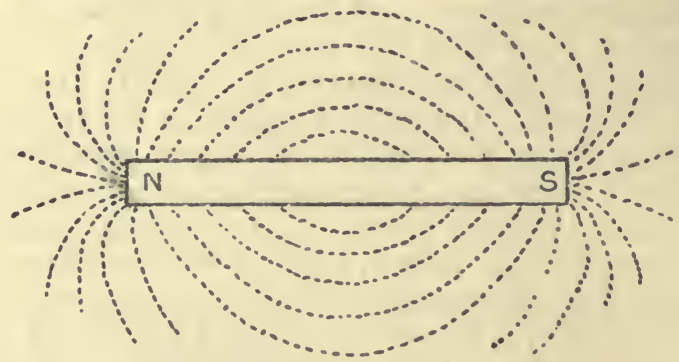

FIG. 122.

line with unlike poles towards each other, and it may be noted that the mutual induction pulls the two neighbouring poles towards each other.

Fig. 125 is the field due to two bar magnets arranged in one line with like poles towards each other, and here we notice that

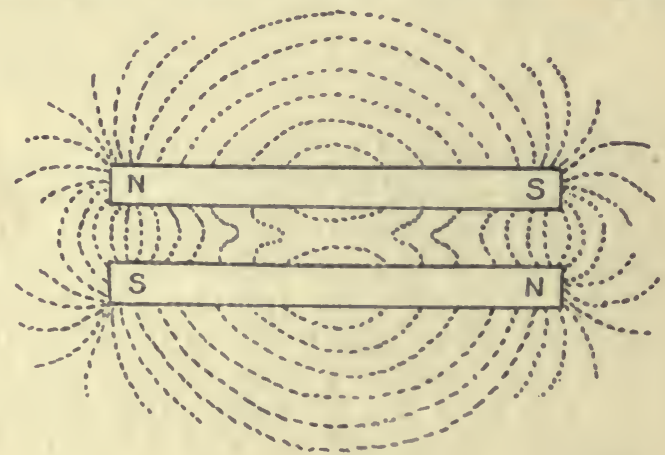

FIG. 123.

the lines of force from one NSP do not enter another NSP, but as it were the two sets repel each other and travel out sideways, each set bending round and entering the other end of its own magnet. We notice, too, that there is a point midway between the adjacent

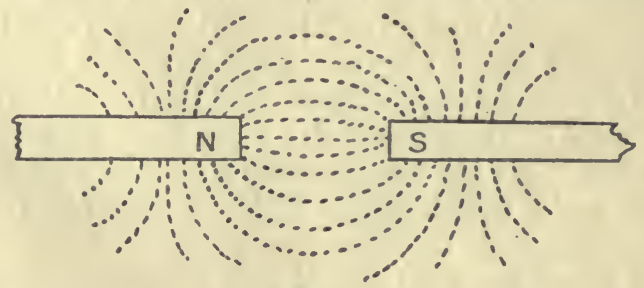

Frg. $1::$ 
poles where there are no lines, and a compass needle at that point shows no directive force.

If two bar magnets are placed in line with unlike poles in contact, then they act very nearly as one magnet with poles near the two distant ends, and very little polarity is shown at the junction. But if they are separated by a small gap a strong field is exhibited in the gap.

When the course of the lines of force in a field has been traced, as in any of the above figures, a compass needle shows us by its

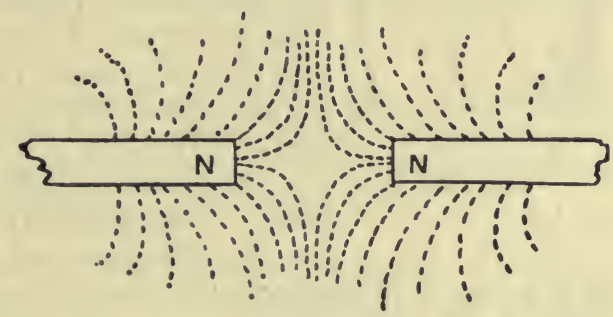

FIG. 125.

frequency of vibration that, where the lines crowd together, there the action is stronger; where the lines open out from each other, there the action is weaker. We shall see later that the lines may be drawn according to a plan such that the number passing through a unit area, held so that they pass through it perpendicularly, serves as a measure of the action.

We regard the lines of force as symbolising some change which has taken place in the field, some action which is going on there, an action which is indicated by the setting of a needle and by the magnitude of the pulls on its poles. Now we have seen that we always have the two poles on the same bar and that the lines of force leaving one end of the bar in general bend round and enter the other, and above all we have seen that if we break a magnet in two and slightly separate the broken ends, or if, as equivalent to this, we slightly separate two magnets as in Fig. 124, then there is a strong field between the two ends. These facts lead to the irresistible conclusion that the lines of force pass through the magnet, that the inside of the magnet is as it were the origin of the action, and that the polarity over its ends is merely the manifestation at the surface of what is going on within ; or, putting the same idea in another form, the polarity is due to the passage of the lines of force from the steel into the air. 'The lines of force $*$ are to be regarded as continuous closed curves issuing from

* In a later chapter we shall see that it is convenient to introduce a term to denote the alteration due to magnetic action taking place in the field, and the term used is "induction." The lines which are continuous, and which form closed curves, are really lines of induction. But in attempting to get a general idea of the subject it is sufficient to use the idea of the lines of force which are exhibited so directly by experiment. 
the NSP, bending round through the air, entering the SSP, travelling through the steel body of the magnet, and joining on at the point of issue. The condition symbolised by the lines of force is

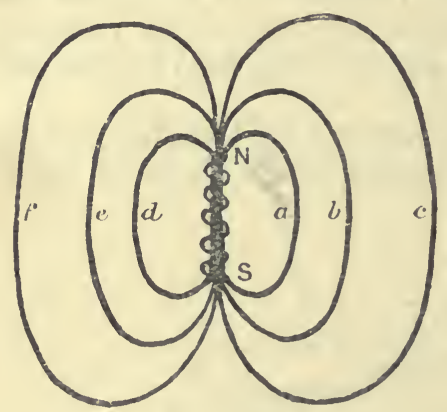

FIG. 126. somehow maintained by what goes on in the body of the magnet.

We may roughly illustrate the idea of continuity by imagining a number of steel flexible rings of different sizes $a, b, c, d, e, f$, bound together along NS as in Fig. 126. The part where they are bound together represents the magnet, the region where they bend round represents the field in air.

Magnetisation by induction. Permeability. We may use the filing method to show something of what is going on in magnetisation by induction. If a piece of soft iron is placed in a field-for instance, in the field between two unlike poles as in Fig. $127(a)$-and the field thus produced is compared with that when the soft iron is removed, Fig. $127(b)$, it is seen that the lines from $N$ bend round and enter the iron as if they

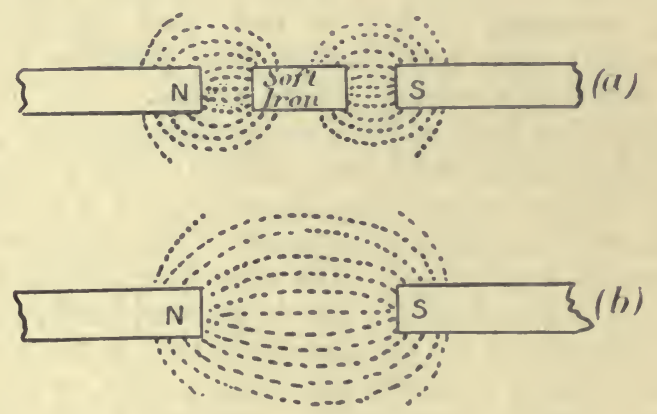

FIG. 127.

found it easier to go through the iron than through the air. Where they enter the soft iron the passage from air to iron is manifested by a SSP; where they leave the iron at the other end the passage into air is manifested by a NSP. But the idea to be dwelt upon is that the iron forms an easier passage for the magnetic action-is, as Faraday put it, a better conductor-than the air, or, as we now say, using terms introduced by Lord Kelvin, is more "permeable," or has greater "permeability." 


\title{
CHAPTER XIV
}

\section{GENERAL ACCOUNT OF MAGNETIC ACTIONS-continued}

\begin{abstract}
Relation between magnetisation and the magnetising force producing it-The hysteresis loop-Susceptibility and permeability-Magnetisation and temperature-Permeability and temperature-Change of length on magnetisation-Magnetisation and strain.
\end{abstract}

Is this chapter we shall give some account of certain less obvious phenomena of magnetism, which for their complete discussion require a knowledge of the methods of magnetic measurement. But without quantitative details a general idea of the phenomena may be obtained, and this general idea will be valuable when we come to the more exact investigations of magnetic quantities in later chapters.

Relation between magnetisation and the magnetising force producing it. Without entering into detail we shall now give a short account of the relation between the

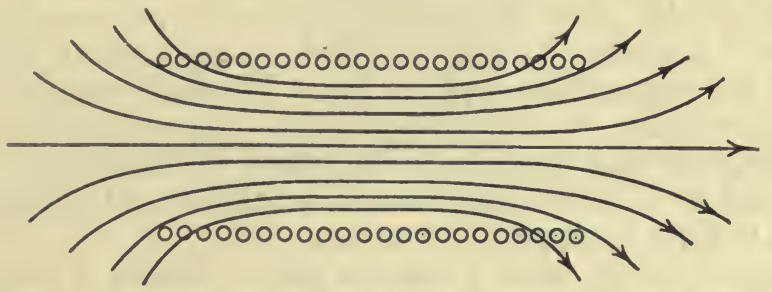

FIG. 128.

magnetisation in iron and the magnetic force by which the magnetisation is produced, and we shall suppose that we use the electro-magnetic coil method of producing it. We have already seen that a coil carrying a current is itself a magnet. If Fig. 128 represents the section of such a coil by a plane through its axis, the course of the lines of force may be investigated by inserting a card with iron filings on it, and they are somewhat as represented in the figure. The lines of force are practically parallel within the coil except near the ends. They diverge as they pass out fiom one end and converge as they come in at the other. If we turn a screw round so as to travel along the lines of force inside the coil 
in their positive direction, the direction of flow of the current in the coil must be that in which the head is turned round. If the current be reversed, the magnetisation is reversed. The force on a pole inside the coil is proportional to the current.

Now let us imagine that two equal coils, or solenoids, $\mathbf{A}$ and $\mathbf{B}$, are arranged as in Fig. 129 so that the same current flows in opposite directions round them. Let a small needle be placed midway between the near ends. It will be at a neutral point - that is, a point where the two coil magnets neutralise each other-and if

N

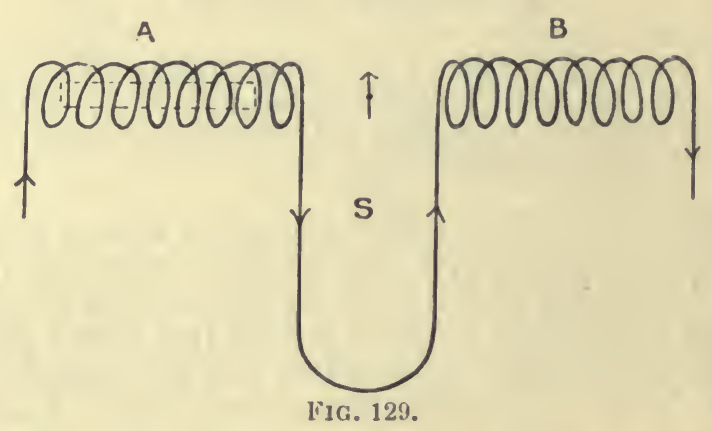

the coils are placed East and West the needle will set $\mathbf{N}$ and $\mathbf{S}$ under the earth's force which is perpendicular to the common axis.

Now let a bar of iron be placed within $\mathrm{A}$. When the current is put on, the bar at once becomes a magnet and there is nothing on the other side, $\mathbf{B}$, to counteract its magnetic action on the needle, which will therefore be deflected and by an amount which will indicate the magnetisation of the bar. We shall here assume that the magnetisation is proportional to the tangent of the angle of deflection, and that the magnetising force producing it is due to the current in $\mathbf{A}$ and is proportional to that current, $\mathbf{B}$ being so far away as to have no appreciable action.

Imagine that we make a series of measurements of magnetising force as given by the current and of the accompanying magnetisation as given by the deflection of the needle, gradually increasing the current from zero to a large value, and let the magnetisation be plotted against magnetising force on a diagram. The general nature of the result is indicated in Fig. 130.

'The curve of magnetisation, though varying greatly with different kinds of iron, shows in general three characteristic parts: a part $\mathrm{O} \mathrm{A}$ for very small magnetising forces, at a very small slope, a part A B sloping very steeply, and a part B C tending to become a nearly horizontal line for very great forces. 'These three parts change from one to another more or less gradually.

If, instead of merely increasing the magnetising force indefinitely, we take it up to a certain point and then gradually decrease and 
GENERAL ACCOLNT OF MAGNETIC AC'TIONS 183

ultimately reverse it, we find that the magnetisation will in general decrease in different ways according to the position of the point $\mathrm{P}$,

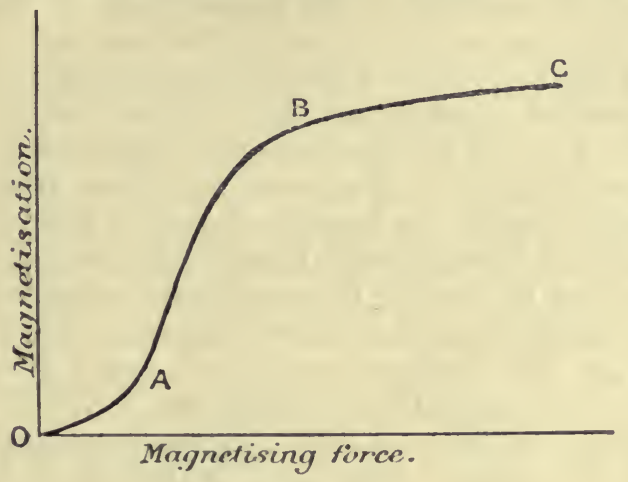

FIG. 130.

Fig. 131, on the curve representing the condition arrived at before decrease. If $\mathrm{P}$ is in the straight initial part $\mathrm{O} \mathrm{A}$, the magnetisation will decrease in proportion to the decrease in the magnetising force. But if $\mathrm{P}$ is on the upper part of the curve when we begin

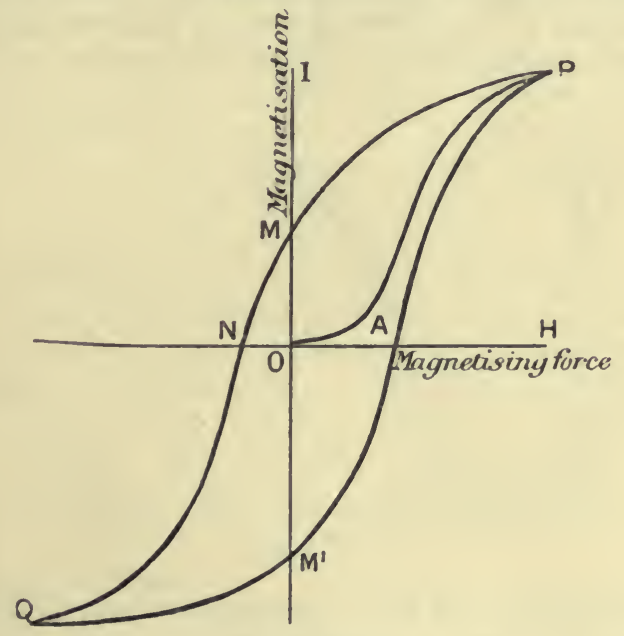

FIG. 131.

to diminish the magnetising force, the magnetisation for a given current on the return journey is greater than it was on the outgoing journey, and the magnetisation curve $\mathbf{P}$ M now lies above $\mathrm{O}$ A P. 'The magnetic condition tends then to persist, and this tendency for a preceding magnetic condition to persist is termed magnetic hysteresis, a term introduced by Ewing. If the current is brought down to zero so that there is no magnetising force, there is still 
magnetisation retained, represented by $\mathrm{O} \mathrm{M}$ in the figure, and this retained magnetisation is the residual magnetism. It measures the retentivity of the specimen. If the current is now gradually increased from zero in the opposite direction the magnetisation decreases and will be zero for a certain magnetising force $\mathrm{O} N$ in the direction opposite to that of the original force. 'This is termed the coercive force. On still further increasing the reversed current the magnetisation is reversed. If we cease to increase it at $\mathbf{Q}$ and then begin to diminish again, hysteresis is shown, and when the current is again zero there will be residual magnetisation $O \mathbf{M}^{\prime}$ now in the opposite direction to $\mathrm{O} \mathbf{M}$. Increasing the current from zero in the original direction, we shall travel to a point near $\mathbf{P}$, but a little below it, when the original maximum current is reached, and repeating the cycle we shall get another curve. After a number of cycles the curves obtained for each practically coincide, as we have supposed in the figure. 'The area $\mathbf{P} \mathbf{M} \mathbf{M}^{\prime} \mathbf{P}$ is termed the hysteresis loop.

The hysteresis curve varies for different specimens of iron and steel. Thus for magnet steel $\mathrm{OM}$ is not very great, but $\mathrm{ON}$ is considerable; while for soft iron $\mathrm{O}$ M may be very great and () $\mathrm{N}$ may be small.

Susceptibility and permeability. The greater the poles developed in a bar of iron or steel under a given magnetising force, the greater is the susceptibility of the bar to magnetisation. When

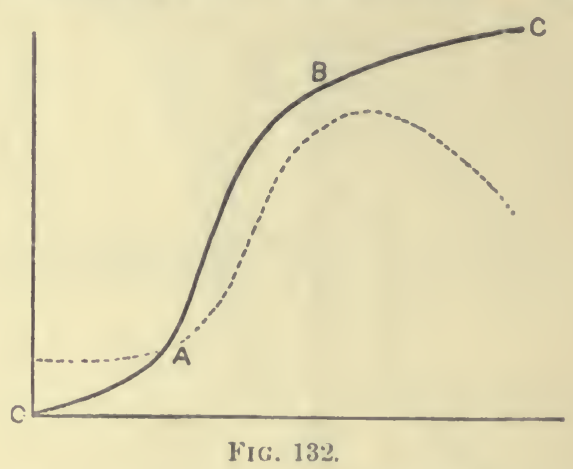

the magnetising force and the magnetisation are suitably measured the ratio magnetisation/magnetising force is termed the magnetic susceptibility (see p. 240). It is usually denoted by $\kappa$. Without attending to the scale of measurement we can see that if $\mathrm{O} \mathrm{A} \mathrm{B} \mathrm{C}$, Fig. 132, represents the curve of magnetisation, then the curve representing the susceptibility will have a form somewhat like that of the dotted line. In the first straight line stage $\mathrm{O} A$, the susceptibility will be constant. 'Then in the early part of $\mathrm{A} \mathrm{B}$ as the curve bends round it will rise rapidly, increasing to somewhere near the second bend about $\mathbf{B}$ and then falling off again. 


\section{GENERAL ACCOUNT OF MAGNETIC ACTIONS 185}

In the last chapter we introduced the idea of magnetic permeability as describing the power of conducting the lines of magnetic force possessed by a magnetic body. Though permeability is connected with susceptibility, it implies a somewhat different way of looking at magnetic actions and is not measured in the same way. We may realise the difference, perhaps, by considering the case of a bar inserted in a magnetising solenoid A B, Fig. 133, and placed some distance from a needle $\mathrm{N}$, which it deflects. Before the bar is inserted the space A B contains air, and the lines of magnetic force due to the electric current in the coil are passing along this space and ultimately out into the field, and are deflecting the needle $\mathbf{N}$. Let us suppose that $\mathbf{N}$ is brought back to its original
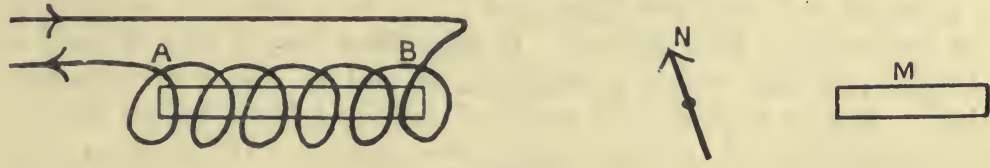

FIG. 133 .

position by a magnet M placed to the right. Now let the bar be inserted. Many more lines of force pass through it than through the air which it displaces, and we say that poles are developed on the bar. 'The needle $\mathbf{N}$ is again cleflected, and we measure the strengith of the poles or the magnetisation of the bar by the increase in the lines of force, that is, by the deflection which is due to the substitution of iron for air. We do not consider the magnetisation of the space A B before the bar was inserted, but only the increase when the iron displaces the air. If we inserted an equal bar of wood or of glass instead of the iron, the lines of force would pass through it practically as through the air and the needle would show no appreciable change in deflection or development of polarity in the wood or glass. Hence, though they are the seat of magnetic action, yet as they only have the same action as air, or as a vacuous space, we say that they have no susceptibility-their $\kappa=0$. We shall see later that this is not strictly true, for $\kappa$ has some small value for so-called non-magnetic substance, but so small that we could not detect the poles developed in the experiment which we are imagining.

Susceptibility then depends on the excess of lines of force over and above those passing through air.

Permeability depeads on the total number passing through, not on the excess. Before the iron is inserted the air carries or conducts lines of force, and its permeability is taken as the standard and as having value unity. When the iron is inserted many more lines of force pass through, and when the number is measured in a way to be described later, the permeability, which is denoted by $\mu$, is measured by number of lines through iron/number of lines through air, when the magnetising current is the same. 'The susceptibility 
is measured by the excess and the permeability by the multiplication of lines of force. We shall see later that it is convenient to adopt such units of permeability $\mu$ and of susceptibility $\kappa$ that $\mu=1+4 \pi \kappa$.

We see then that the permeability curve is like the susceptibility curve, but we must multiply the ordinates of the latter by $4 \pi$ and raise the whole curve by 1 to give the former.

"Permeability" is the more fundamental idea, for it takes account of the whole magnetic action going on. "Susceptibility" is somewhat artificial, but it is convenient to use the term, as $\kappa$ is more directly measured in certain methods of experiment.

Magnetisation and temperature. If we heat a magnet to a bright red heat and then cool it, we find that the magnetisation has entirely disappeared. If we place a bar of iron or steel at a bright red heat within a magnetising coil it shows no susceptibility. But as it cools to a dull red usually a little below $800^{\circ} \mathrm{C}$, its susceptibility is almost suddenly regained. If an iron ball heated to a bright red is hung by a spiral spring over a strong pole it is not attracted, but as it cools a point is reached when it is suddenly pulled down to the pole.

Hopkinson* found that with a certain specimen of iron the permeability increased with rise of temperature up to $775^{\circ} \mathrm{C}$., when it was many times the value at ordinary temperatures. $A$ slight further rise to $786^{\circ}$ sufficed to render it non-magnetic. He called the temperature at which the magnetic permeability ceases to be more than that of air the critical temperature. 'The critical temperature varies, with different specimens of iron and steel, from $690^{\circ}$ to $870^{\circ} \mathrm{C}$. It may be taken usually as not very far from $750^{\circ} \mathrm{C}$. 'This sudden change in property is related to other phenomena. Gore $\dagger$ observed that if an iron wire was heated to redness and then allowed to cool, at a certain point a slight elongation occurred. Barrett $\ddagger$ observed that if a piece of iron, or, better, hard steel, is heated to bright redness and then allowed to cool, at a certain point it suddenly glows more brightly. This can easily be observed in a dark room. The phenomenon was termed by Barrett "recalescence," as implying a sudden rise of temperature. Hopkinson § measured this rise. He found that a certain piece of hard steel, after cooling to $680^{\circ}$, suddenly rose to $712^{\circ} \mathrm{C}$. and then began to cool again, and he showed that the magnetic condition was assumed at the same time. No doubt the lengthening observed by Gore occurs at the same point. There is then some sudden change in the molecular condition of the iron when it passes from the magnetic to the non-magnetic condition, or rather when its susceptibility disappears and its permeability falls to that of air.

Permanent magnets and temperature change. If a permanent magnet has already been subjected to several varia-

* Phil. Trans. (1889), A, p. 443.

† Phil. Mag., Jan. 1874.

† Proc. R.S., xvii. (1S69), p. 260.

§ Proc. R.S., xlv. (1889), p. 455. 
tions of temperature, to get rid of a sort of temperature hysteresis which occurs at first, then subsequent rise of temperature within ordinary range results in diminution of magnetisation, but on the fall of temperature to the original value the magnetisation increases to its original value. 'This may be easily veritied by an experiment represented in plan in Fig. 134. A needle, N S, with an attached mirror is suspended so as to throw an image on a scale. 'Two
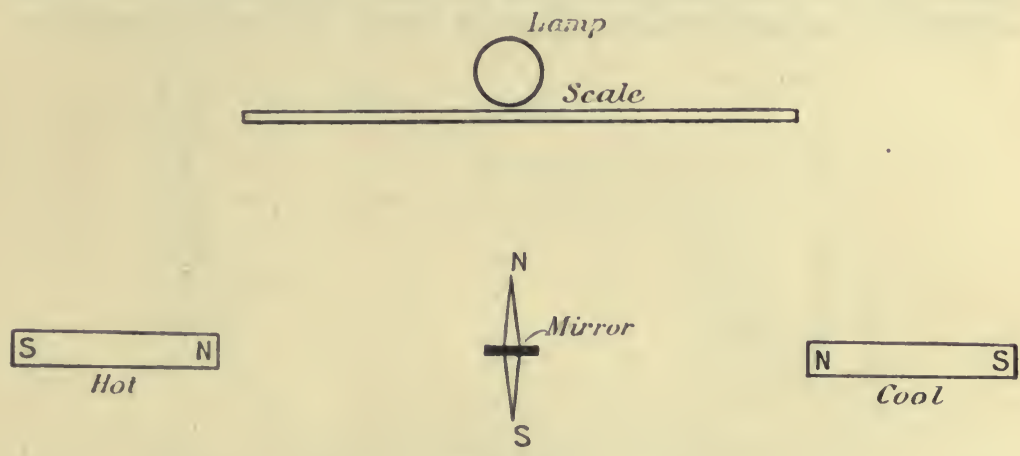

FIG. 134.

magnets are brought up, one on either side, at such distances that they neutralise each other's action on the needle. 'They are fixed in position. Then one magnet is warmed, say, by bringing up from below a beaker of warm water to surround it. At once its magnetisation is diminished, and the needle shows by its deflection that the cool magnet is the stronger. When the temperature is reduced once more to its original value the needle returns to its original position. By this arrangement the magnet whose temperature is varied is brought quite near to the needle, so that a small change in its magnetisation may produce a considerable deflection.

Ewing* found that if a bar is only very weakly magnetised, then rise of temperature produces increase of magnetisation. As the bar is more strongly magnetised this increase falls off and ultimately changes sign. In ordinary steel bar magnets the effect is always decrease in strength with rise of temperature.

Permeability and temperature. A large number of experiments have been made with different qualities of iron and steel to investigate the change of permeability with change of temperature. $\dagger$ We shall here only describe the results of some experiments of Hopkinson on a specimen of Whitworth mild steel.

* Magnetic Induction in Iron and other Metals, 3rd ed. p. 181.

+ Ewing, loc. cit. chap. viii, gives an account of the fundamental work on the effects of change of temperature. A paper by S. P. Thompson on The Magnetism of Permanent Magnets (Journ. Inst. Elect. E'ng., vol. 1. p. 80, 1913) gives an excellent account of the properties of permanent magnets and their dependence on the nature of the steel from which they are made. The effect of temperature on magnetisation is described. A bibliography is given. 
There are two cases to be distinguished, that when the iron is in a very weak field comparable with that of the earth, and that when the field is very much stronger.

With the weak field it will be observed that the permeability increases enormously up to nearly $735^{\circ} \mathrm{C}$. and then suddenly drops apparently to that of air. With a field about ten times as great it is at first more considerable, remains nearly constant for a time, but drops gradually after $600^{\circ}$ to the air value at $735^{\circ} \mathrm{C}$. With a field one hundred times as great it begins at the same value as

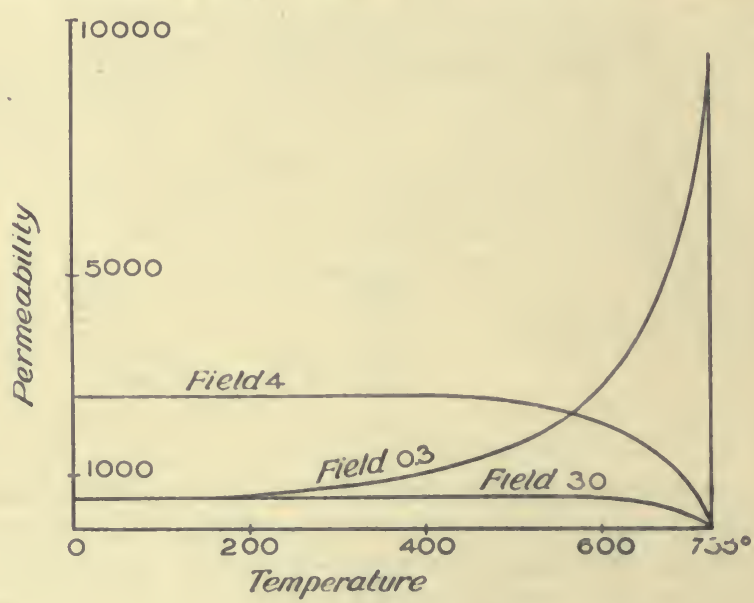

FIG. 135.*

with the weak force, remains practically constant to $500^{\circ}$, and then falls off to the condition of, apparently, no susceptibility at $735^{\circ} \mathrm{C}$. Subsequent researches, which will be referred to in Chapter XXII, have shown that the permeability does not fall quite to that of air.

Change of length on magnetisation. Joule $\dagger$ was the first to observe that a bar of iron changes its length on magnetisation. He placed the bar or rod to be experimented on within a magnetising coil with its lower end fixed and its upper end pressing against a lever. 'This in turn pressed against a second lever, of which the free end moved in the field of a microscope. One division of the microscope scale corresponded to about $1 / 140000$ of an inch. The rod was 36 inches long, and he observed lengthening, increasing with the magnetisation, up to twenty-eight divisions, or $1 / 180000$ of the length. He used only comparatively small magnetising forces, and as far as he went the lengthening was as the square of the magnetising force. Joule further sought to measure any change in the total volume of the iron by immersing the rod in a tube filled with water and provided with a stopper

* Hopkinson, Phil. Trans. A. (1889), Plate 16.

† Scientific Papers, i. [1. 235. 
GENERAL ACCOUNT OF MAGNETIC ACTIONS 189

through which a capillary tube passed. A change in volume of 1 in 4,500,000 would have been shown, but no motion of the end of the liquid in the capillary was observed.

Many others have worked on this subject, but we owe our most exact knowledge to Bidwell, who showed that as the magnetising force increases, the lengthening reaches a maximum, decreases to zero, and is followed by a shortening. Bidwell worked with both rods* and rings. His method of carrying out the rod experiment will be seen from Fig. 136. The solenoid was fixed on the rod to

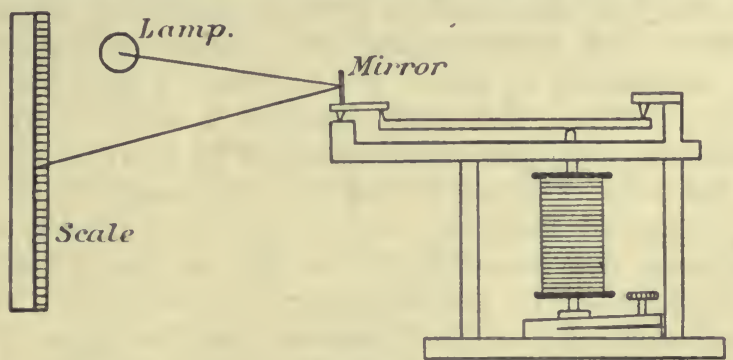

FIG 136.

avoid alteration in the pressure of the rod against the base due to the attraction between the solenoid and the rod-a source of considerable error when such small changes of length are to be observed. 'The apparatus for multiplying the change of length

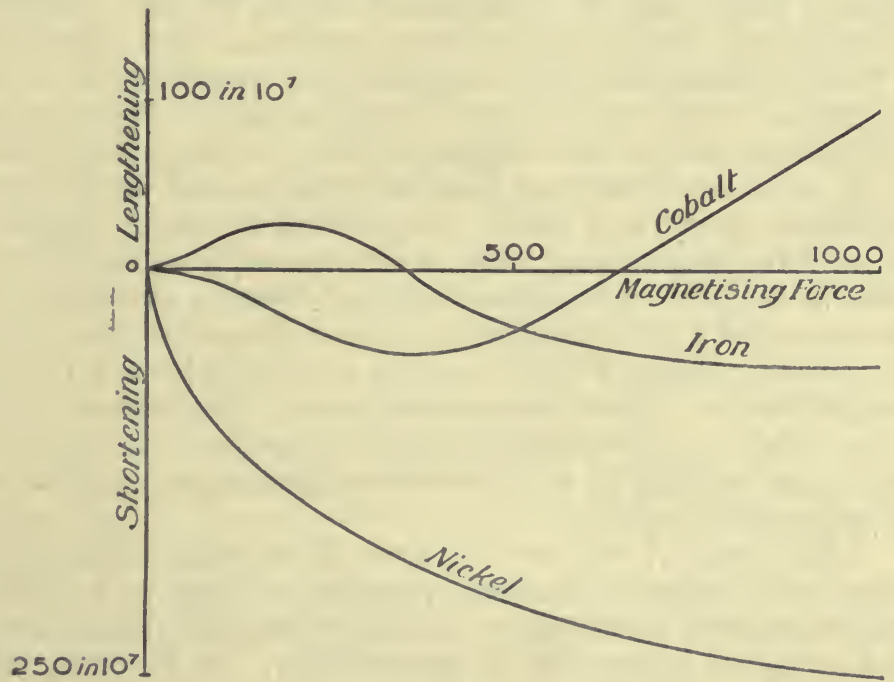

FiG. 137.

into an observable quantity will be understood from the figure. 'The results obtained are given in Fig. 137, taken from the paper in the Philosophical Transactions, loc. cit. p. 228.

* Proc. R.S., xl. (1856), p. 109 ; Phil. Trans. A. (1888), p. 205. 
'The results for cobalt and nickel rods are also given. It will be observed that the greatest lengthening in iron is about two in a million, while the ultimate shortening approaches seven in a million. 'The behaviour' of cobalt is just the opposite to that of iron, while nickel contracts always and is much more affected than either of the other metals. In Rapports ảu Congrès International de Physique, vol. ii. p. 536, there is an account of the work done on this subject. It has been found that there is an increase in volume on magnetisation of the order of 1 in $10^{6}$ for a field of 1000 in iron and steel, and an increase somewhat less in nickel.

Stress and magnetisation. When a body either previously magnetised or under the action of a magnetising force is subjected to stress, the magnetisation is affected by the stress. We shall here only give a short account of two cases, referring the reader for a fuller treatment of this very complicated subject to Ewing's Magnetic Induction in Iron and other Metals, chap. ix.*

Matteucci discovered that if a bar of magnetised iron "is pulled lengthwise" its magnetisation was increased. Villari found that the nature of this effect depends upon the degree of magnetisation. If the bar is weakly magnetised, then the effect of a pull is to increase the magnetisation as Matteucci observed, but if the bar is strongly magnetised the pull decreases the magnetisation. 'That is, after a certain point in the magnetisation the effect is reversed. This is known as the Villari reversal. Comparing this with the effect of magnetisation on change of length, we note that there is a reciprocity. Weak magnetisation lengthens a bar. Lengthening a weakly magnetised bar increases the magnetisation. Strong magnetisation shortens a bar. Lengthening a strongly magnetised bar decreases, while shortening increases, the magnetisation.

It may be noted here that with nickel and cobalt bars the reciprocal effects also hold. Thus nickel shortens, whatever the value of the magnetising force, and reciprocally a longitudinal pull leads to diminution of inagnetisation. Weakly magnetised cobalt shortens, and a pull diminishes the weak magnetisation. Strongly magnetised cobalt lengthens, and a pull increases the strong magnetisation. 'These reciprocal effects are illustrations of reciprocities which are continually met with in physical phenomena. They are dealt with in J. J. 'Thomson's Applications of Dynamics to Physics and Chemistry.

Lord Kelvin, $\uparrow$ who was the first to investigate these effects of stress with requisite exactness, pointed out that we may regard a pull on a bar in a weak magnetic field as increasing its permeability, and a push as decreasing its permeability. In a strong field the change of permeability is reversed.

* For earlier work see Wiedemann's Galvanismus, epitomised in Encyc. Brit., 9th ed., Magnetism, p. 253. Later work and references will be found in Ewing (loc. cit.), who has himself largely contributed to the exact study of the subject.

$\dagger$ Math. and Phys. Papers, vol. ii. p. 332. 


\section{GENERAL ACCOUNT OF MAGNETIC ACTIONS 191}

'The second case which we shall consider is one discovered by G. Wiedemann. If an iron wire is carrying an electric current, then the lines of force are circles round the axis of the wire and the wire is circularly magnetised, but as the lines of force are complete within the wire, the iron does not produce an external field. But Wiedemann found that if the wire is twisted it becomes longitudinally magnetised. If the current is flowing down the wire and the lower end is twisted so that the front part moves from right to left, that lower end becomes a NSP. Suppose that the square A B C D represents the unsheared form of a piece of the metal in the front part of the wire, A D being parallel to the axis of the
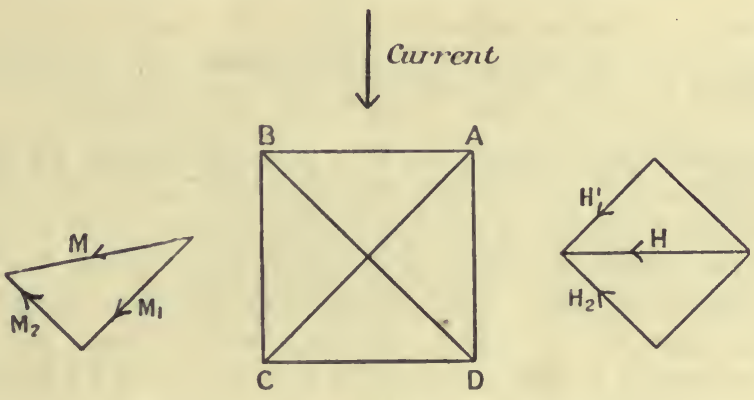

IIIG. 138

wire and the direction of the current. The magnetising force $\mathrm{H}$ due to the current will be perpendicular as to A D. Now let the wire be twisted so that $\mathrm{CD}$ moves to the left relatively to A B. This shear may be represented by a lengthening of the diagonal A C and of all lines parallel to it, and a shortening of the diagonal B D and all lines parallel to it. With weak magnetisation the permeability is increased along A C, and is decreased along $\mathbf{B} \mathbf{D}$. The magnetising force due to the current is still $\mathrm{H}$. Resolve it into $\mathrm{H}_{1}$ parallel to $\mathrm{AC}$, and $\mathrm{H}_{2}$ parallel to $\mathrm{D} \mathrm{B}$. These are each inclined at $45^{\circ}$ to $\mathrm{H}$, and are equal. But $\mathrm{H}_{1}$, acting in a direction of greater permeability, produces stronger magnetisation along A C than $\mathrm{H}_{2}$ produces along $\mathrm{D} \mathrm{B}$. 'The resultant magnetisation, therefore, has a component downwards. For if $\mathbf{M}_{1} \mathbf{M}_{2}$ represent these magnetisations, their resultant $M$ slants down. Hence the lower end of the wire has a NSP.

Again we have a reciprocal effect. If the wire be magnetised longitudinally and a current be passed along it, then the lower end twists round.

We might expect that with strong magnetising current these effects would be reversed owing to the Villari reversal, but so far they have always been found to be in the direction described, perhaps because the intensity of magnetisation has not reached the critical point. 


\title{
CHAP'TER XV
}

\section{MOLECULAR HYPOTHESIS OF THE CONSTITUTION OF MAGNETS}

\begin{abstract}
Molecular bypothesis-Ewing's theory and model-Dissipation of energy in a hysteresis cycle-Temperature and magnetisationAttempts to explain the constitution of molecular magnets.
\end{abstract}

Molecular hypothesis of the constitution of magnets. The fundamental phenomena of magnetisation receive an explanation on the hypothesis that the molecules of iron and steel are themselves small permanent magnets, capable of being turned round their centres. 'This hypothesis was first propounded in definite form by Weber:* It has since been dereloped by G. Wiedemann, Hughes, Maxwell, and above all by Ewing. $†$

In the eighteenth century Aepinus supposed that magnetism consisted of two fluids with opposite properties, uniformly mixed in an unmagnetised bar, and that one was pulled to one end and the other pushed to the other end under a magnetising force. Coulomb and others supposed that the separation went on in each molecule separately, and this was the first step in the molecular hypothesis. Poisson developed it in this form and investigated its mathenatical theory. But Weber gave it a new form, now universally adopted, in supposing that each molecule of iron or steel, or at least each molecule which contributes to the magnetic condition, is itself a permanent magnet, with definite polarity, and capable of rotation about its centre. No attempt was made to account for the magnetisation of the molecules, so that the hypothesis attempts to give an account of the structure of a magnet as built up of snialler magnets in a particular way, and does not in any way explain magnetism.

According to Weber, the axes of these molecular magnets in an unmagnetised bar are pointing equally in all directions, as represented in Fig. 139, so that in any space small compared with the whole magnet, but large compared with that occupied by a single molecule, an equal fraction of the whole number of molecules will point in every given direction, and so neutralise each other's external

* Pogg, Ann. Ixxxvii. (1852), p. 145.

+ Ewing's paper describing his magnetic model is in Proc. R.S., xlviii. (1890), p. 342. He gives an account of the molecular hypothesis in chap. xi. of Magnetic Induction in Iron and other Metals. 
field. When a magnetising force acts, it is supposed to turn the magnetic molecules round with their axes more or less in the direction of the force, though there is some constraint (not accounted for by Weber) tending to restore them to their original position. But the stronger the magnetising force the more the constraint is overcome, and in a very strongly magnetised bar we

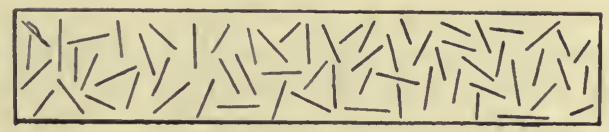

FIG. 139.

may suppose that all the molecular axes are turned nearly into line, with their north ends towards one end of the bar, and their south ends towards the other end, forming molecular magnetic chains as in Fig. 140. 'The poles are to be supposed to be arranged on all the chains as shown on the central chain. The unlike poles of successive members of a chain are close together so as to neutralise each other, but obviously each chain will have an unneutralised

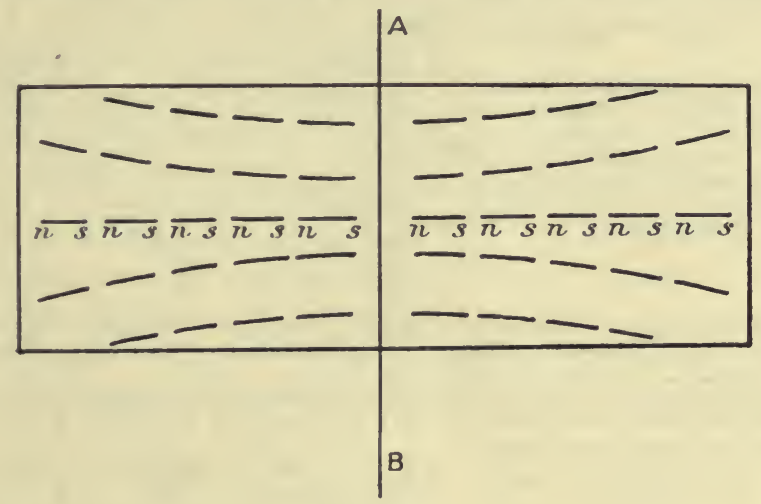

FIG. 140 .

pole at each of its ends so that there will be a number of unnentralised NSP's on the surface near one end of the bar, and an equal number of unneutralised SSP's near the other end. And the molecules all having equal poles, the total polarity is the same at the two ends. Further, if the bar be cut through at A B, on the new surface to the left a row of unneutralised SSP's will be exposed, while on the new surface to the right a row of NSP's will be exposed. Obviously, as we do not cut through the molecules we cannot obtain polarity of one kind alone.

On this hypothesis there is a limit to the magnetisation which a given bar will undergo, reached when all the molecular magnets 
are turned round so as to be parallel to the magnetising force. The existence of such a limit is borne out by experiment. The curve B C in Fig. 130, p. 183, tends to become nearly parallel to the axis when the magnetising force is increased to a very great value. An interesting experiment by Beetr* also shows that there is probably a limit. A rarnished silver wire with a very narrow scratch through the varnish was made the cathode in an electrolytic cell containing a salt of iron in solution. 'This was arranged in a weak magnetic field parallel to the scratch, and the iron molecules as they were laid down on the silver one by one were free to turn in the direction of the field. 'The magnetisation of the iron deposited was very great for so small a mass, and was found to be only very slightly increased when the wire was placed in a very powerful field.

When a bar undergoing magnetisation is vibrated, say, by hammering, it is magnetised more strongly than if quiet, and we may perhaps assume that the vibration frees the molecules from their constraint so that they yield more easily to the magnetising force.

A modification of the theory was made by Wiedemann $t$ and by Hughes.t If a number of bars of iron are made up into a bundle, magnetised along their lengths, and then partially

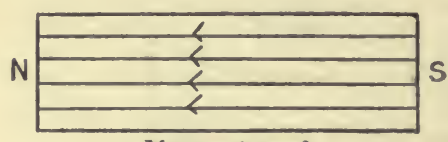

Magnelised

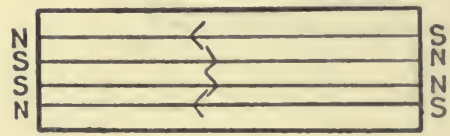

FIG. 141. demagnetised by, say, hammering, on taking the bundle apart it is found that some of the bars have their polarity reversed. The apparent demagnetisation is partly due to the completion of the magnetic circuit of the stronger bars through the weaker ones, which they reverse as represented in Fig. 141. Carrying this idea down to molecular regions, Wiedemann and Hughes suggested that in an unmagnetised bar the molecular magnets are arranged in neutralising circuits of two or more molecules, as in Fig. 142.

As originally described, Weber's theory gives no account of hysteresis. Wiedemann sought to explain it by supposing that there is some sort of friction to be overcome in turning the molecules round, friction which has to be overcome again when the molecules seek to return after the magnetising force is removed. Maxwell $\S$ introduced the idea that the constraining force on each molecule is analogous to an elastic force, that for small displace-

* Pogg. Ann. cxi. (1860), p. 107.

+ Galvanizmus, 2nd ed. vol. ii. [1], p. 379.

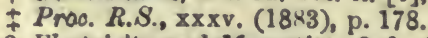

$\$$ Electricity and-Magnetism, 3rd ed. vol. 11. p. 85. 
ments it is proportional to the displacement, and on removal of the magnetising force there is complete return. With large displacements, however, there is something analogous to permanent

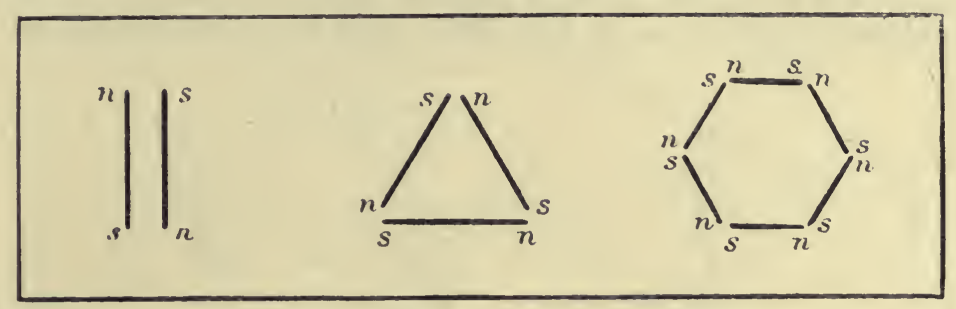

FIG. 142.

set and therefore not complete return. He did not attempt to account for the permanent set, but simply assumed it.

Ewing's theory and model. Eiving brought a great simplification into the hypothesis by showing that the mutual action of the molecular magnets would account for the controlling or constraining forces, tending to restore the molecules exactly after small displacements, and for the permanent set, that is, the residual magnetisation, after large displacements. Thus no sort of friction need be assumed, and the hypothesis in its simple form accounts for the facts. To illustrate the ideas he constructed what is known as Ezving's model of a magnet, consisting of a number of compass needles placed together as in Fig. 143 (in plan), each needle representing a molecule. Such a model, if inserted within a

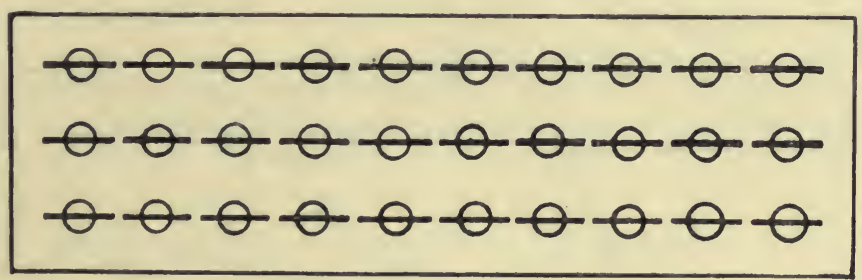

FIG. 143.

magnetising solenoid in place of the bar in Fig. 129 shows all the phenomena of magnetic induction and gives a curve of magnetisation repeating all the features of the hysteresis curve in Fig. 131.*

'To understand how these mutual molecular actions account for the phenomena let us follow Ewing in supposing that we have four needles arranged at the corners of a square. With no external magnetising force and under their own mutual actions only, they will set as in Fig. 144, where the arrow-heads are north-seeking

* Induction in Iron and other Metals, 3rd ed. p. 351. 
poles. The magnetic circuit is completed within the group and there is practically no external field exhibited at a distance.

If a small magnetising force is put on, represented by $\mathrm{H}$, each magnet will be turned round a little, its $\mathbf{N}$ pole tending to move in the direction of $\mathrm{H}$, as in Fig. 145. But the displacement of a

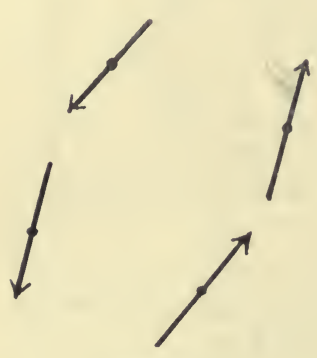

FJG, 144.

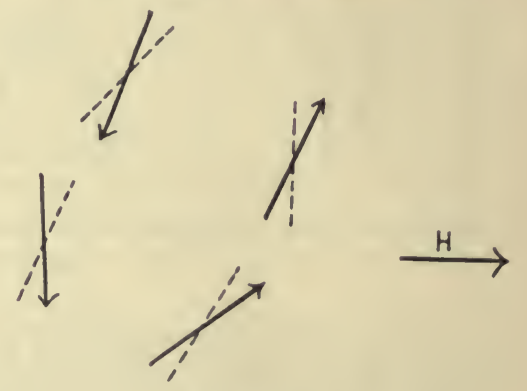

Fis: 145 .

magnet fiom a position NS to a position $N^{\prime} S^{\prime}$, Fig. 146, may be represented by the superposition of two other magnets with equal poles, for superpose on $\mathrm{NS}$, Fig. 146, a magnet $\mathrm{S}_{1} \mathbf{N}^{\prime}$ and a magnet $\mathrm{N}_{1} \mathrm{~S}^{\prime}$, then $\mathbf{N}$ and $\mathrm{S}_{1}$ neutralise each other, and $\mathrm{S}_{\mathrm{and}} \mathrm{N}_{1}$ neutralise each other, and we have $N^{\prime} S^{\prime}$ left.

Thus the effect of the rotation of the four magnets in Fig. 145 may be represented by superposing eight little magnets as in Fig. $14 \%$, and these will have a field generally in the direction of $H$.

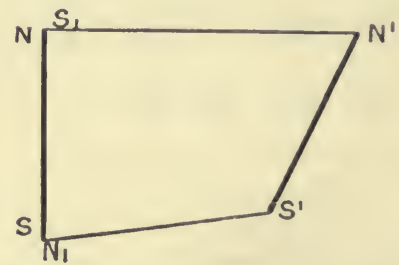

FIG. 146.

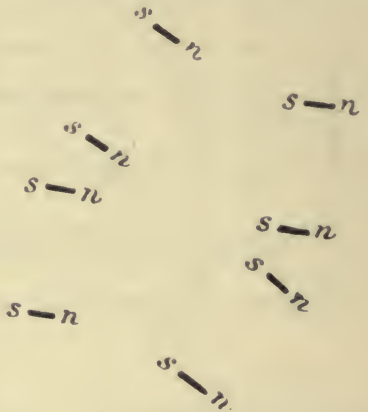

FIG. 147 .

While the magnetising force $\mathrm{H}$ is small, these deflections, and therefore the lengths of the small magnets in Fig. 147, will be proportional to the magnetising force, and the net magnetisation will also be proportional to it. But when a certain point is reached in the value of the magnetising force the configuration becomes unstable and the needles swing round into some such position as that in Fig. 148, and their external field suddenly and greatly increases. 
On still further increasing $\mathrm{H}$ the magnets tend to set more and more nearly parallel to $\mathrm{H}$, as in Fig. 149, and then the limit of magnetisation is practically reached.

'The curve of magnetisation is represented in Fig. 150, where O A represents the initial stage with no hysteresis, A B the sudden turning round, and $\mathrm{BC}$ the subsequent approach to parallelism with $\mathrm{H}$.

If $\mathrm{H}$ be removed the return will be from Fig. 149 to Fig. 148 - that is, a very large anount of residual magnetism remains in the direction of $\mathrm{H}$. Reversal of $\mathrm{H}$ will ultimately send the magnets round in the
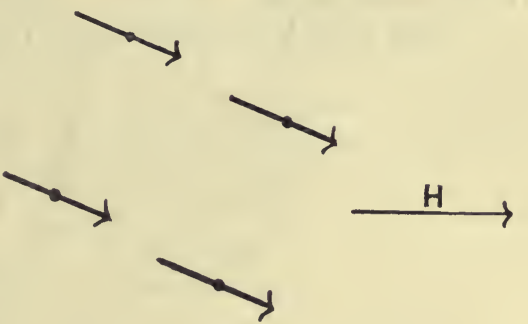

Fic. 148. opposite direction and we shall have a sudden reversal of magnetisation. 'The behaviour of such a group may be studied by placing four compass needles in a square. 'The earth's field must be as nearly as possible neutralised by means of a large magnet, placed so that its field is equal and opposite to that of the earth. On gradually approaching a second magnet towards the group the phenomena will be observed.

If there are many such square

Fisi. 149 . groups near together, and with the sides of the squares at different inclinations to $H$, instability will set in at different values of $\boldsymbol{H}$ for the different squares, and " the transition from one stage

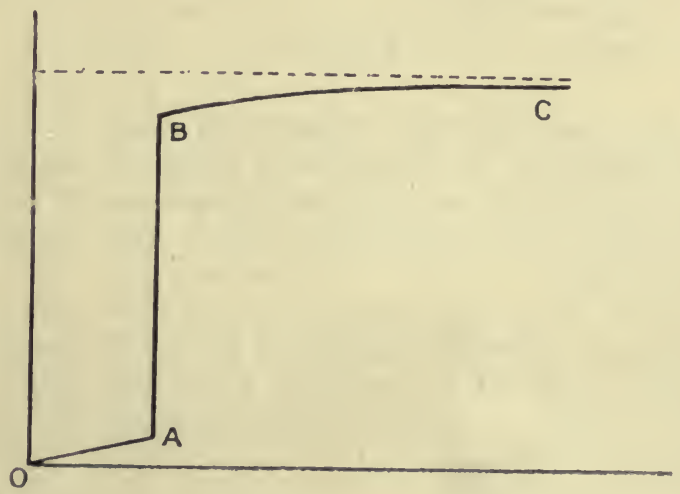

Flli. 150 . 
to another will be gradual in the aggregate, for it will happen at different values of $\mathbf{H}$ in different groups. Hence the curve will assume a rounded outline in place of the sharp comers " of Fig. 150. As each group separately shows hysteresis the aggregate will also show it.

Figs. $151,152,153 *$ show the imagined behaviour of a large

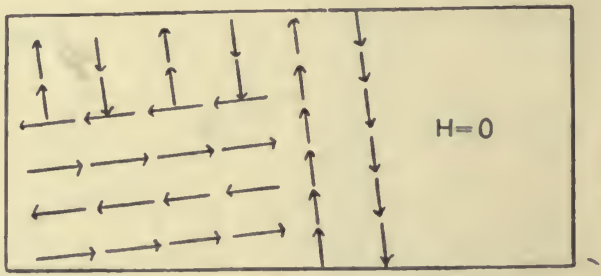

FIG. 151.

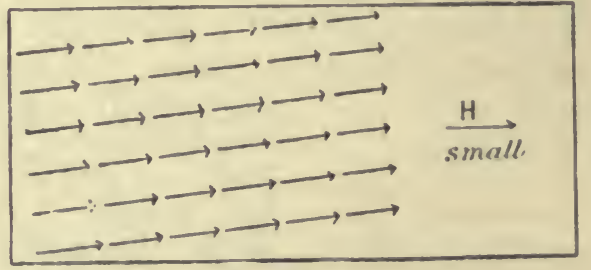

FIG. 152.

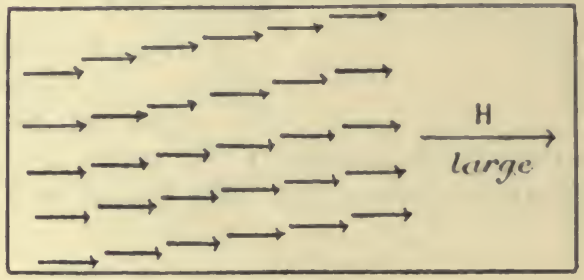

FIG. 153.

group of square set molecules respectively under no force, a force which has pulled them nearly parallel, and a very large force.

From Ewing's model we see how retentiveness is accounted for by the molecular poles holding each other more or less in their displaced positions.

We see, too, that as $\mathrm{H}$ increases in the later stage the mutual action tending to hold the magnets in the nearly parallel position increases. Hence if $\mathbf{H}$ is increased to a large value and then decreased to a smaller value the magnets, being under a larger controlling force due to their mutual action, do not return to the position

\footnotetext{
* Ewn:r, lnidnetion in Iron und other Mleluls, 3rd ed. p. 3.5I.
} 
which they had under that smaller value when the value of $\mathrm{H}$ was rising through it.

Fig. 154, taken from Ewing (loc. cit. p. 351), shows the magnetisation curve of a group of twenty-four pivoted magnets when the magnetising force is taken through a complete cycle.

Dissipation of energy in a hysteresis cycle. A study of the behaviour of the members of a model at once suggests that there is dissipation of energy when a piece of iron or steel is taken through a cycle. For work is done on the magnets in putting

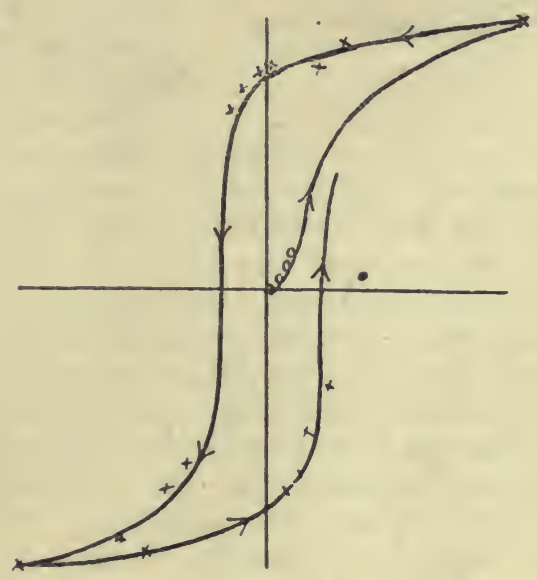

Fig. 154.

them into line, and when on the diminution and reversal of the magnetising force a point of instability is reached, the magnets fly round and vibrate about their new position through large amplitude. This means that some of the work done appears as energy of vibration. In the molecular magnets we should expect similar behaviour, and the molecular vibration would imply the development of heat and a rise in temperature of the iron. In fact, it can be shown that the area of the hysteresis loop is proportional to the energy dissipated in a cycle.

'The cycle may be performed in another way than that which we have supposed, viz. by the rotation of the specimen in a constant field. 'This is what takes place in a dynamı armature when the core is a cylinder of iron rotated between strong poles. Besides changing the magnetisation, the rotation will tend to produce electric currents in the iron and to prevent these currents the iron is laminated transversely to the direction in which the currents would flow, so that they are practically destroyed. 'The work done in a revolution is then due to the hysteresis. 'The molecular groups as they move round from $a$ or $b$ in Fig. 155 tend to keep in their original line, given to them by the magnetising force, so that at $c$ or $d$ they should be perpendicular to the magnetising force; but 
before they reach these points they become unstable and swing round, thus dissipating energy. A remarkable conclusion was predicted by Swinburne as a consequence of Ewing's theory, and was subsequently verified by experiment, viz. that when the field

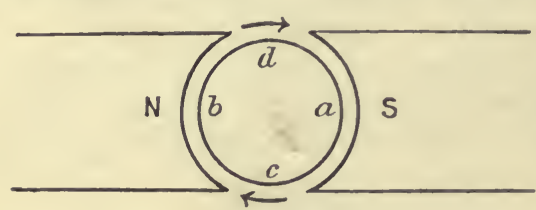

FiG. 155 . in such a case as that represented in Fig. 155 is exceedingly strong, hysteresis should disappear. If the field is so strong that the molecular magnets are held all the time in the line of the magnetising force and rotate round their centres, as it were, as the iron travels round, there is no instability, no conversion of magnetic energy into energy of vibration, and no hysteresis.

Temperature and magnetisation. We have secn that in a permanent magnet surall rise of temperature is accompanicd by diminution of magnetisation, the magnetisation returning to its initial value when the temperature falls again. We may perhaps explain this by supposing that rise of temperature is accompanied by increased vibration of the molecular magnets about their centres, so that they do not point so entirely in the direction of the magnetising force. For instance, let N S, Fig. 156, be the equilibrium position of a molecule under a force $H$ and let it vibrate between $n_{1} s_{1}$ and $n_{2} s_{2}$. When at $n_{1} s_{1}$, we may represent the effect of the displacement of $\boldsymbol{N}$ by superposing a small magnet $s n_{1}$; when at $n_{2} s_{2}$, by superposing a small magnet $s u_{2}$. $s u_{2}$ has more effect in destroying the magnetisation in the direction of $\mathrm{H}$ than $s n_{1}$ has in increasing it, since it is more nearly parallel to it. 'There is another possibility which may be mentioned. 'The permeability of iron increases with rise of temperature. 'The magnetic circuit of a molecule or of a group of molecules is partly completed through the

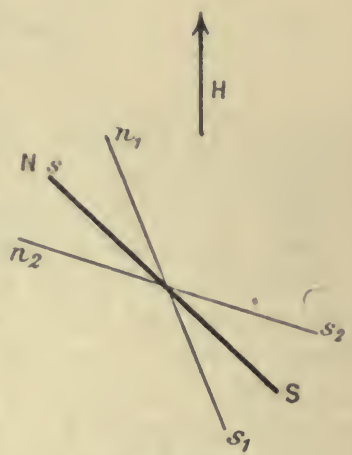

Fig. 156. iron, and it is only part which issues into the air. As the permeability rises with rise of temperature the groups may find it easier to complete more of the circuit within the iron, and some of the lines of force may be withdrawn fiom the air.

'I'he increase of permeability with rise of temperature which occurs in weak fields may probably be accounted for by supposing that initially the iron is in the first stage of magnetisation, where the molecular magnets are only slightly deflected, is in Fig. 145. But as the temperature rises molecular vibration increases and 
some of the groups become unstable, and for those groups we pass to the second stage, represented in Fig. 148. As the temperature rises, more and more groups pass to this stage, and between $700^{\circ}$ and $800^{\circ}$ all the groups have become unstable and have then come more or less into line with the magnetising force. 'This is represented by the great rise in permeability. The sudden disappearance of magnetic quality at the critical temperature, about $785^{\circ}$, may perhaps be explained by supposing that after that temperature the vibration has become so violent as to change into molecular rotation.

With strong fields the iron is already in the second or third stage. Vibration due to heating has two opposite and neutralising effects. On the cne hand it eases the breaking up of groups and alignment with the force and so tends to increase the magnetisation, while on the other it tends to reduce the magnetisation by the movements out of the line of the force in the way illustrated in Fig. 156. We may suppose that these opposite effects keep the curve of permeability nearly level, till at a temperature near $800^{\circ}$ the diminution due to vibration overpowers the increase due to facility of group breaking and the permeability decreases.

'The change in length of a bar on magnetisation is yet to be explained on the molecular hypothesis. It would obviously require some further assumptions as to molecular grouping or as to molecular dimensions in different directions.

Attempts to explain the constitution of the molecular magnets. We have already pointed out that the molecular liypothesis only carries down the magnetisation from the visible niass of the bar as a whole to its molecular constituents, and does not account for their magnetisation. Attempts have been made to explain the magnetisation of the molecules in terms of electricity. 'The earliest was Ampère's hypothesis. He started from the experimental fact that a current flowing in a circular wire forms a magnet of which the axis is perpendicular to the plane of the wire. He supposed that there was a channel round a magnetic molecule through which an electric current could flow, and he further supposed that this channel was perfectly conducting, so that the energy of the current was not dissipated, and therefore, if once started, the current should persist unless an equal and opposite current were in any way superposed. But a continuous closed current, such as that flowing in a circular wire, is essentially a locus into which electric lines of force are continually converging and are there dissipated. As these lines sweep through the surrounding space they are accompanied by magnetic action, and so in a sense produce the magnetic field. We know of no such current as that which Ampere supposed.

But if we could imagine a bundle of lines of magnetic force in the form of those of a small magnet, bound round as it were with a perfectly conducting channel, then no lines of force could be 
added to, or subtracted from, the bundle. For an addition of lines of force through the channel would mean an inverse current in the channel which would just suffice to neutralise the lines added. Thus Ampère's hypothesis really gives no explanation in terms of known facts, but when analysed is a hypothetical device for securing permanent magnets.

Another hypothesis much more in touch with experimental knowledge has lately gained acceptance. It is founded on the discovery that a moving charge of electricity produces a magnetic field. If, adopting astronomical language, we suppose that a molecule consists of a large positively electrified primary and a small negatively electrified satellite revolving round a centre of mass much nearer the large primary, then it can be shown that the system would be magnetically equivalent to a small magnet with axis through the centre of mass perpendicular to the plane of the orbit.* Thus the magnetism of iron and steel would be explained in terms of the magnetism which accompanies moving electricity. Still, that magnetism remains to be explained.

We shall refer to the molecular hypotheses and to an attempt to determine the strength of the molecular magnets in Chapter XXII.

"This "electron" hypothesis has been most fully developed by Largevin, Ann. de Chim. et de Phys., 8th series [5], 1905, p. 70. A brief account is given at p. 297 infra. 


\title{
CHAP'IER XVI
}

\section{GENERAI ACCOUNT OF MAGNETIC QUALITIES OF SUBSTANCES OTHER THAN IRON, AND STEEL}

\begin{abstract}
Faraday's classification of all bodies as either paramagnetics or diamagnetics-General law-Explanation of the action of the medium -by an extensicn of Archimedes' principle-Ferromagnetics, paramagnetics, and diamagnetics.
\end{abstract}

Paramagnetics and diamagnetics.-We have in the previous chapters only described the magnetisation of iron and steel and have mentioned that nickel and cobalt show sensible magnetisation by induction, though in a far less degree than iron. They attract and are attracted by the poles of a magnet and possess retentivity in some conditions. Biot found that a certain needle of carefully purified nickel-which stands next to iron in its capacity for magnetisation-retained its magnetism and possessed about one-third the directive force towards the North of that of a certain steel needle of exactly the same dimensions. Faraday experimented with cobalt, which could easily be made to lift more than its own weight, though losing all its magnetism on withdrawal of the inducing magnet.

Magnetisation was eften apparently detected by early experimenter's in other metals and alloys, but we may probably ascribe the effects they observed to the presence of small quantities of iron.

'Towards the end of the eighteenth century it was observed that antimony and bismuth were repelled from the pole of a strong magnet.

On the one hand, thell, iron, nickel, and cobalt are attracted, and on the other hand bismuth and antimony are repelled, by a strong magnetic pole, and it was at one time supposed that other substances possessed no magnetic properties at all. Faraday, however, showed that these two groups are really only at the two extremes and that all bodies are either attracted or repelled, though the forces are usually so slight that they can only be detected with very powerful magnets.

The mode of experiment by which he discovered this consisted in suspending a small bar of the substance to be tested between the poles of a strong electro-magnet by a tine thread. If 
the substance resembled iron, the bar set itself "axially," i.c. with its longer axis along the line NS, Fig. 15\%, joining the two poles, and he classed all such substances together as paramagnetics. If it resembled bismuth or antimony the bar set itself "equa-

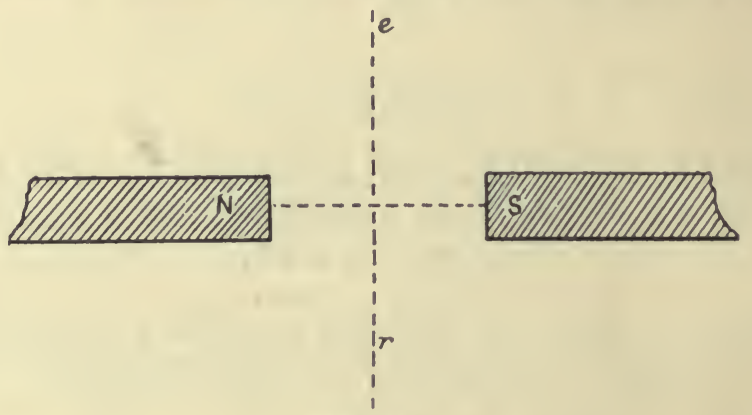

FIG. 157.

torially," i.e. along the line $c r$ at right angles to $\mathrm{NS}$, and all such substances le designated diamagnctics.

Liquids. By enclosing liquids in glass ressels laraday was enabled to determine to which class they belonged. 'Thus he found that solutions of salts of iron are paramagnetic, while alcohol and water are diamagnetic.

Gases. He also succeeded in showing the magnetic character of gases in several ways. For instance, if a stream of gas rendered evident by traces of ammonia and hydrochloric acid was allowed to ascend between the poles, if dimnagnetic it divided into two streams pushed out from the central region. Bubbles filled with the various gases were attracted into or repelled from the central space between the poles. He found from his experiments that oxygen in air is strongly magnetic, while nitrogen and hydrogen are diamagnetic. 'The following 'Table gives a few of the more important substances, arranged under the two heads, air being the medium surrounding the poles (Exp. Res. vol. iii. p. 71):

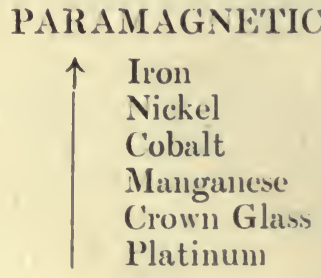

DIAMAGNE'TIC

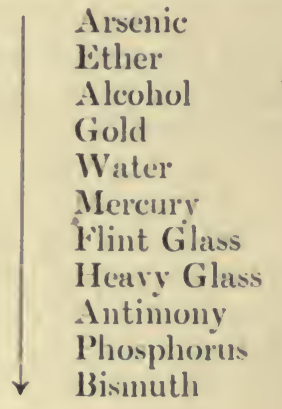


The arrows show the direction in which the magnetic action increases.

Faraday was able to state the following Gencral Law: Paramagnetic substances tend to move from positions where the magnetic action is weaker to where it is stronger, i.e. from weaker to stronger parts of the field; while diamagnetic substances tend to move from stronger to weaker parts of the field. The axial setting of paramagnetic bars and the equatorial setting of diamagnetic bars at once come under this general law. The field in Fig. 157 is strongest along and close around the axis N S, and a paramagnetic body tends to stay there. A bar is in the strongest part of the field when it lies along the axis. The field rapidly weakens as we travel along the line $e r$ either way from the axis. A diamagnetic bar therefore gets, on the whole, into a weaker part of the field when it sets equatorially.

'The law is further illustrated by an experiment devised by Pliicker, which shows whether a liquid is paramagnetic or diamagnetic. A watch glass containing some liquid is placed on the poles ; the liquid if paramagnetic is attracted to, and if diamagnetic

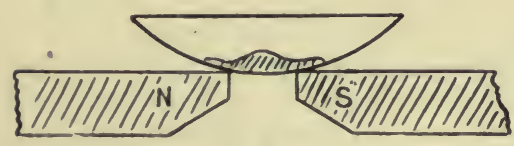

a

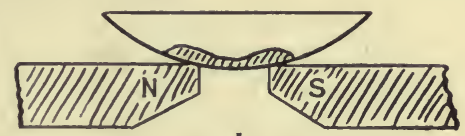

$b$

Fic. 158.

is repelled from, points where the intensity of the field is greatest and so the level of the surface is disturbed, its shape depending on the nature and distance apart of the poles. 'Thus when the poles are very close together, say $\frac{1}{10}$ inch apart, a paramagnetic liquid has the section shown in Fig. 158 (a), while a diamagnetic liquid has the section shown in Fig. $158(b)$, the liquid retreating from the central strongest field. When the poles are further apart the strongest parts of the field are near to the pole pieces and the centre is weaker and the effects are nearly reversed.

Effect of the medium. Faraday also made experiments on the effect of the medium, and he showed that a paramagnetic solution enclosed in a glass tube behaves as a paramagnetic if suspended in a weaker solution of the same kind, and as a diamagnetic if suspended in a stronger solution. For example (Exp. Res. vol. iii. p. 58), a clear solution of protosulphate of iron was prepared containing 74 grains of the hydrated crystals to 1 ounce of water. Another weaker solution was prepared by adding to one volume of this three volumes of water. Suspended in air in tubes both were paramagnetic, the former the more strongly so. If the tube containing the first solution was suspended between the poles in a vessel containing the same solution no directive force acted upon 
it. Suspended in the second weaker solution it behaved again as a paramagnetic. If a tube containing the weaker solution was suspended in a vessel containing the stronger, it pointed equatorially, behaving as a diamagnetic. Surrounded by a solution of its own strength it was neutral, and in a still weaker solution it was paramagnetic. He also prepared a solution of protosulphate of iron in water which was neither paramagnetic nor diamagnetic, the iron salt being neutralised by the water.

Explanation of the action of the medium by an extension of Archimedes' principle. In Chapter XX we shall investigate the action of the medium in detail. For the present we may regard the action as being in accordance with an extension of Archimedes' principle, the resultant force on a body in a magnetic field being equal to that on the body minus that on the medium which it displaces. We may put this extension of the principle in the following form : Let $\mathbf{N}$, Fig. 159 , be a pole with a
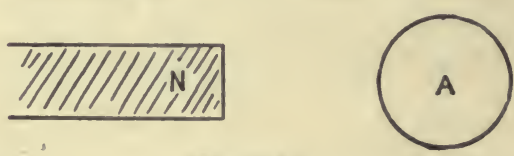

FIG. 159.

magnetic field round it. We may regard all parts of the medium as acted on by the pole, and as coming, under that action, into a state of equilibrium in which the medium is in a condition of strain. We may resolve the total action on any part of the medium A into the magnetic action of the pole on it, and the actions of its surroundings due to their state of strain, the resultant of the latter being equal and opposite to the former. If now A be removed and replaced by some other body, $\mathbf{A}^{\prime}$, of exactly the same shape, the magnetic action on $A^{\prime}$ differs from that on $A$, while, unless $\mathrm{A}^{\prime}$ is so strongly magnetic as appreciably to alter the field, the magnetic action on the rest of the medium remains unchanged and its strain is the same as before. Since the action of the medium on $A^{\prime}$ depends on this strain it is unchanged. Hence there is no longer equilibrium, and $A^{\prime}$ will be attracted to or repelled from the pole according as it is more or less paramagnetic than the medium. A difficulty in making this explanation perfectly general so as to account for all diamagnetic actions is that we have to suppose a vacuum magnetic, for the diamagnetics in the list given above are diamagnetic in a vacuum, and we should have to suppose that their repulsion from a pole is due to the stronger attraction on the surrounding vacuum. This is only another way of saying that what we term a vacuum is not empty space, but contains something capable of acting on and being acted on by material bodies, a supposition to which we are forced by the transmission of light through it. In a beam of light there 
is energy and there is momentum, whether it is passing through ordinary matter or through the highest vacuum of which we have experience.

Ferromagnetics, paramagnetics, and diamagnetics. Though Faraday only used the two classes, paramagnetics and diamagnetics, and to one or other of these referred all bodies, it is usual now to separate out the three metals iron, nickel, and cobalt, and to these may probably be added manganese, and to class them as Ferromagnetics. 'Their magnetisation does not increase in linear fashion with magnetising force and they show hysteresis and retentivity. The term paramagnetic is reserved for the bodies with far feebler magnetic qualities. In these the magnetisation is proportional to the magnetising force. Curie* showed that with given magnetising force the magnetisation for paramagnetics is inversely as the absolute temperature over a very wide range, thus extending a result which had been found to hold in certain cases over a smaller range by G. Wiedemann. This is now known as Curie's Law.

Ferromagnetics may probably be regarded as passing into paramagnetics when above their critical temperature.

Diamagnetics, if we describe them in terms of polarity, may be regarded as having a like, instead of an opposite, pole formed by an inducing pole, and Curie found that their magnetisation, except in the case of bismuth, is very nearly independent of temperature. 'The diamagnetisation of bismuth decreases with rise of temperature between $-182^{\circ}$ and the point of fusion very nearly linearly, and by about $\frac{1}{1000}$ for $1^{\circ}$ rise. $\dagger$

This has led to the suggestion that diamagnetism is not to be regarded merely as a negative paramagnetism. The temperature effect on paramagnetics would appear to show that paramagnetism is concerned with molecular structure, which changes with change of temperature, while the absence or smallness of the effect of change of temperature on diamagnetics suggests that diamagnetism is concerned rather with the atomic structure, which is, we may suppose, independent of temperature.

We shall return to the forces on paramagnetic and diamagnetic bodies in Chapters XX and XXII.

* Anr. de Chim. et de Phys., $7^{\circ}$ série, t. V. (1895). An account of the subject is given by H. du Bois in the Congres International de Physique, vol. ii. p. 460.

$\dagger$ Congres International, loc. cit. p. 503. 


\section{CHAPTER XVII}

\section{THE INVERSE SQUARE LAW}

Variation of the force due to a pole as the distance from the pole is varied-The inverse square law-Magnetic measurements founded on the law-Coulomb's experiments-Unit magnetic pole-Strength of pole $m$ - Magnetic intensity-Geometrical construction for the direction of the intensity in the field of a bar magnet-Gauss's proof of the inverse square law-Deflection method of verification-Moment of a magnetComparison of moments-Some consequences of the inverse square law -Application of Gauss's theorem-Flux of force-Unit tubes- $\Lambda$ pole $m$ sends out $4 \pi m$ unit tubes-Potential.

Variation of the force due to a pole as the distance from the pole is varied. The inverse square law. If a NSP is placed at any point in the field of a magnet, the force on it is due to the action of the magnet as a whole. We cannot isolate one of the two polarities. Always the two come into play and the action is the resultant of the North-seeking repulsion and the Southseeking attraction. 'The best approach we can make to isolation of one polarity consists in so arranging the acting magnet that one pole has practically very little action as compared with the other.

Coulomb's experiments. 'This method was used by Coulomb, to whom we owe the decisive experimental proofs which finally established the inverse square law.* In one experiment he used a steel wire magnet 25 inches long. He first found that the poles might be considered an inch from each end. 'Then, placing the wire vertically, he noted the time of vibration of a needle opposite the lower pole and at different small distances from it in the plane of the magnetic meridian. 'The upper pole was not only at a much greater distance, but its action was nearly vertical, so that for two reasons the horizontal component of the force due to it was sufficiently small to be negligible.

It was necessary to take into account the action of the earth which was added to that of the magnet in all cases. Let us suppose that the horizontal force on each pole of the needle due to the earth is $\mathrm{H}_{1}$, and that the force due to the pole of the magnet at distance $d_{1}$ is $\mathbf{F}_{1}$ and at distance $d_{2}$ is $\mathbf{F}_{2}$, and let the times of

* For a history of the experiments made by Coulomb's predecessors, see Enry. Brit. 9th ed., Magnetism, p. 236. 
vibration be $t$ when the earth alone acts, and $t_{1}$ and $t_{2}$ when the pole is at the distances $d_{1}$ and $d_{2}$ from the needle. Regarding the needle as a sort of double pendulum with equal and opposite forces acting at the two ends, it is seen that the times of vibration are inversely as the square roots of the forces acting:

$$
\mathrm{H}: \mathrm{H}+\mathrm{F}_{1}: \mathrm{H}+\mathrm{F}_{2}=\frac{1}{t^{2}}: \frac{1}{t_{1}^{2}}: \frac{1}{t_{2}^{2}} \text {. }
$$

whence the forces due to the magnet alone are given by

$$
\mathrm{F}_{1}: \mathrm{F}_{2}=\frac{1}{t_{1}^{2}}-\frac{1}{t^{2}}: \frac{1}{t_{2}^{2}}-\frac{1}{t^{2}}
$$

By observing the times of vibration Coulomb found that the forces were approximately proportional to the inverse square of the distances $d_{1}$ and $d_{2}$, or that the force due to a given pole is inversely as the square of the distance from that pole.

He also verified the law by means of the torsion balance (see ante, p. 62), using two long wire magnets about the same length as the one described above, and about $\frac{1}{8}$ inch in diameter, one being suspended horizontally in the torsion balance, the other being fixed vertically so that if the suspended one had remained in the magnetic meridian, their two like poles, taken as being one inch from the end of each, would have been in contact. 'The suspended needle was repelled from the magnetic meridian, and the torsions were then measured which were required to bring the two poles to observed distances from each other. In each case the action of the earth aided the torsion, and a preliminary experiment was necessary to determine the torsion equivalent to the earth's action. 'Thus in one experiment, before the vertical magnet was put in position, two turns of the torsion head, i.e. $720^{\circ}$, pulled the magnet round $20^{\circ}$, or $700^{\circ}$ torsion were required to balance the earth's couple due to $20^{\circ}$ deflection from the meridian, giving $35^{\circ}$ of torsion per $1^{\circ}$ of deflection. Putting the vertical magnet in position and bringing the torsion head back to its original position, the suspended magnet was deflected $24^{\circ}$, the torsion being therefore $24^{\circ}$. 'To this we must add $24 \times 35=840^{\circ}$ for the torsion equivalent to the effect of the earth, making a total of $864^{\circ}$ at $24^{\circ}$. 'To halve the deflection, the torsion head had to be turned eight times round, giving a torsion of $8 \times 360+12=2892^{\circ}$. 'To this must be added $12 \times 35=420$ for the torsion equivalent of the earth's effect, making a total of $3312^{\circ}$ at $12^{\circ}$. According to the inverse square law, with this value at $12^{\circ}$, taking distances as equal to arcs, we ought to have at $24^{\circ}$ a torsion of $\frac{3312}{4}=828^{\circ}$, which is not very far from the observed value $864^{\circ}$.

Magnetic measurements founded on the law. We shall describe below a still more accurate method of verifying 
the inverse square law, but it is convenient here to show how the law enables us to arrange a definite and practical system of measurement for the strength of magnetic poles. Were it possible to obtain perfectly constant long magnets, it might be convenient to use one pole of such a magnet as the standard, and copies of this might be used just as we use copies of the standard unit of mass. But no magnet retains its magnetisation unchanged, and we are obliged to fix on a standard depending on the magnetic force exerted. We therefore take the following definition :

A unit magnetic pole is that which at a distance of $1 \mathrm{~cm}$. would exert a force of 1 dyne on another equal pole in air.

In practice it would not be advisable to bring two poles so near as $1 \mathrm{~cm}$. At so small a distance they might weaken each other appreciably by induction. Also in actual magnets we camnot fix on any definite point as the pole. 'The region of North-or South-secking polarity might have dimensions quite comparable with $1 \mathrm{~cm}$., but the inverse square law at once enables us to get over this difficulty. Arranging the two poles at a distance $d$ apart, where $d$ is so great that these objections do not hold, the force will be $\frac{1}{d^{2}}$ dynes.

Having thus a definite standard, if magnetic poles were constant in strength we might measure the strength of any other pole by finding the number of unit poles which, placed together, would produce at an equal distance an effect equal to that produced by the pole to be measured. But it is easy to show that if we put a number of equal poles together the total effect is not the sum of the separate effects, since they weaken each other. If one of two exactly similar equal bar magnets is placed with its axis Last and West, and a compass needle is placed at some distance from it at a point in the axial line, and the deflection noted, if the second nagnet is now superposed on the first with like poles together the deffection is by no means doubled. But we may still measure the strength of a pole in terms of the unit by the ratio which its action bears to that of the unit at the sane distance. We say that

The strength of a magnetic pole is $\mathrm{m}$ if it exerts on a unit pole at a distance $d$ a force of $\frac{m}{d^{2}}$ dynes.

We have now to investigate the force with which a pole $m$ will act on a pole $m^{\prime}$ at a distance $d, m$ and $m^{\prime}$ being measured by their actions on the unit pole.

Let us arrange two poles of strength $m$ and 1 respectively at two points, A and B, Fig. 160, on opposite sides of a pole 1 at C at such distances $d$ and $d^{\prime}$ that the actions on the pole at $C$ are equal and opposite. 'Then we have

$$
\frac{m}{d^{2}}=\begin{gathered}
1 \\
d^{\prime 2}
\end{gathered}
$$


Now we can show by experiment that any other pole can be placed at $\mathrm{C}$ instead of the unit pole and there will still be equality of the two actions on it. This may easily be carried out in a slightly modified form by bringing two vertical long bar magnets up $\mathbf{E}$ and $W$ of a given compass needle with their like poles towards it and on the same level. If the magnets are so

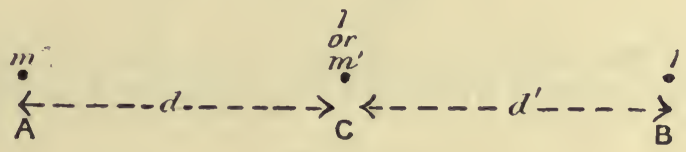

FIG. 160 .

adjusted that the compass remains in the meridian, any other compass needle placed in the same position will also lie in the meridian.

If then we place a pole or strength $m^{\prime}$ at $\mathrm{C}$ it acts on $\mathbf{1}$ at $\mathrm{B}$ with a force $\frac{m \prime^{\prime}}{d^{\prime 2}}$ and is reacted on by an equal force which balances the action of $m$ upon it. Hence the force exerted by $m$ on $m^{\prime}=\frac{m}{d}$ or $\left(\right.$ since $\left.\frac{1}{d^{2}}=\frac{m}{l^{2}}\right)=\frac{m m \prime^{\prime}}{d^{2}}$.

We have already pointed out that we may assign the algebraic + and - to the two kinds of poles inasmuch as they have opposite actions tending to neutralise each other when acting under similar circumstances on the same pole. A NSP is always considered to be +, a SSP to be -. 'The force between two poles is therefore $\frac{m m i^{\prime}}{d^{2}}$ and is a repulsion or an attraction according as their product is + or - .

Magnetic intensity. 'The force which would act on a unit pole placed at any point in a magnetic field is the niagnetic intensity at that point. The unit intensity is termed one gauss. We may conveniently denote the magnetic intensity by the letter $H$. If a pole $m$ be placed at a point where the intensity is $H$ the force acting upon it is $m \mathrm{H}$.

Geometrical construction for the direction of the intensity in the field of a bar magnet. On the supposition that a magnet consists of two poles concentrated at two points a given distance apart, the equations to the lines of force may easily be determined. A construction for drawing the lines will be found in the article Magnetism (Ency. Brit. 9th ed. p. 230). 'The following construction will give the direction of the intensity at any point. Let NS, Fig. 161, be the two poles, $\mathrm{P}$ a point in the field, $\mathrm{C}$ the middle point of $\mathrm{NS}$. From $\mathrm{S}$, the nearer pole to $\mathrm{P}$, draw SO perpendicular to PS, meeting $\mathrm{CO}$, the perpendicular to 
Ns through $C$, in $O$. Let the circle with $\mathrm{O}$ as centre and radius ON or OS meet PN in Q. Since the circle touches PS at $S$

$$
\begin{aligned}
& P\left(\cdot P N=P S^{2}\right. \\
\therefore \quad & \frac{P Q}{P S^{2}}=\frac{P N}{P N^{2}}
\end{aligned}
$$

or $\mathbf{P Q}$ and $\mathbf{P N}$ are inversely as the squares of $\mathbf{P N}$ and $P S$ respectively. Produce $N P$ to $Q^{\prime}$ so that $P Q=P^{\prime} Q^{\prime}$, and $P$ s to $N^{\prime}$ so

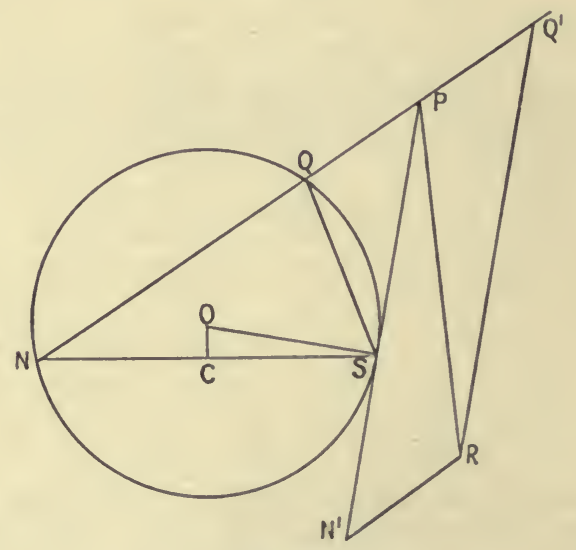

Fig. 161 .

that $\mathbf{P N}=\mathbf{P N}^{\prime} ; \mathbf{P Q}^{\prime}, \mathbf{P N}^{\prime}$ are proportional to and in the direction of the intensities due to the two poles, and PR, the diagonal of the parallelogram $\mathbf{P Q}^{\prime} \mathbf{R} \mathbf{N}^{\prime}$, is the line of their resultant.

'To verify this construction for a given magnet, the magnet should be laid on a sheet of paper and the direction of PR determined. 'The sheet should be turned round till PR is in the line of the magnetic meridian. On placing a compass needle at $\mathrm{P}$, the needle will remain in the line PR if the construction has been correctly made, since the force due to the magnet is in the same line as the earth's force. 'The agreement of experiment with calculation of course verifies the inverse square law.

Gauss's proof of the inverse square law. 'The most exact proof of the law is due to Gauss.* 'The general nature of his method is as follows: If two points $\mathrm{D}$ and $\mathrm{E}$ be taken at equal distances from the centre $\mathrm{C}$ of a small magnet NS, D in the axis and $\mathrm{E}$ in the perpendicular to the axis through $\mathrm{C}$, then the intensity at $\mathrm{D}$ will be approximately double that at $\mathrm{E}$ if, and only if, the inverse square law is true. Without entering into the full

* Intensitus Vis Maynetice Terrestris (Werke, Bd. V). 
proof that the inverse square is the only law satisfying this condition we may easily show, as follows, that it does satisfy it.

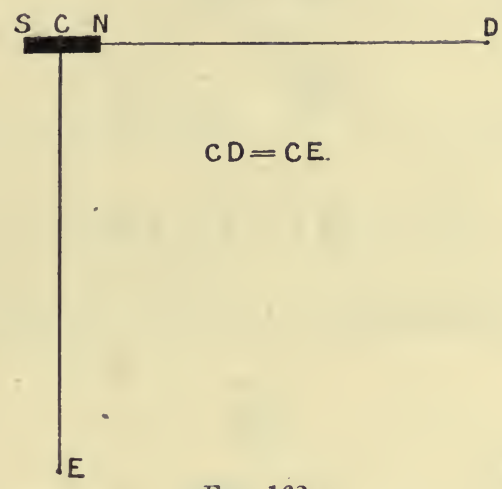

FIG. 162.

Let $m$ be the strength of each pole and $l$ the distance between them. Let $\mathrm{CD}=d$.

'The intensity due to $\mathrm{N}$ is

$$
\frac{m}{\left(d-\frac{l}{2}\right)^{2}}
$$

and that due to $\mathbf{S}$ is

$$
\frac{m}{\left(d+\frac{l}{2}\right)^{2}}
$$

The resultant is

$$
\begin{aligned}
& \frac{m}{\left(d-\frac{l}{2}\right)^{2}}-\frac{m}{\left(d+\frac{l}{2}\right)^{2}}={ }^{m} \frac{2 l d}{\left(d^{2}-\frac{l^{2}}{4}\right)^{2}} \\
= & \frac{2 m l d}{d^{4}\left(1-\frac{l^{2}}{4 d^{2}}\right)^{2}}=\frac{2 m l}{d^{3}}\left(1+\frac{l^{2}}{2 d^{2}}+\& c .\right)
\end{aligned}
$$

If $l$ is so small compared with $d$ that we may neglect $\frac{l^{2}}{2 d^{2}}$, and higher powers, the intensity at $\mathrm{D}$ is

$$
\frac{2 m l}{d^{3}}
$$

The intensity at $\mathrm{E}$ is the resultant of $\frac{m}{\mathrm{NL}^{2}}$, along $\mathrm{NE}$, and $\frac{m}{\mathrm{SE}^{2}}$ along ES. By the triangle of forces we may represent these two 
equal forces and their resultant by NE, ES, and NS respectively 'Then the intensity at $\mathbf{E}=\frac{\mathbf{N S}}{\mathrm{NE}} \times \frac{m}{\mathrm{NE}^{2}}$

$$
\begin{aligned}
& =\frac{m l}{\mathrm{NE}^{3}}=\frac{m l}{\left(d^{2}+\frac{l^{2}}{4}\right)^{\frac{3}{2}}} \\
& =\frac{m l}{d^{3}}\left(1-\frac{3}{8} \frac{l^{2}}{d^{2}}+\& \mathrm{c} .\right)
\end{aligned}
$$

'To a similar approximation as before the intensity at $\mathrm{E}$ is

or half that at $\mathrm{D}$.

$$
\frac{m l}{d^{3}}
$$

It may easily be shown that if the force were inversely as the $p$ th power of the distance the intensity at $\mathrm{D}$ would be $p$ times that at E.

Deflection method of verification. 'This result may be verified by the method of deflection.

A small needle $u s$, Fig. 163, is suspended so that it lies in the
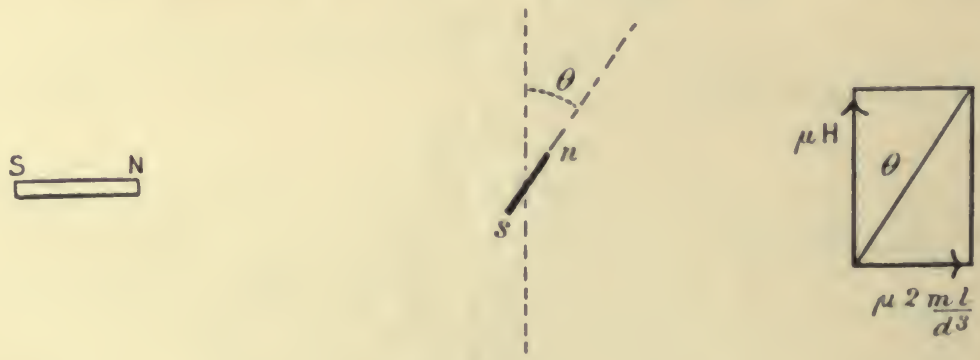

FIG. 163.

magnetıc meridian. A magnet NS is then brought up, say, to the west of it in the line through its centre perpendicular to the meridian. 'The magnet is said to be in the "end-on position." Considering the horizontal action on the pole $n$ of the needle, if its strength is $\mu$ it will be acted on by a force $\mu \mathrm{H}$ in the meridian, where $\mathrm{H}$ is the horizontal intensity of the earth's action; it will be acted on by a force $\mu \frac{2 m l}{d^{3}}$ at right angles to the meridian, and the needle will set with its axis in the line of the resultant of these two, since there are equal and opposite forces on the other pole, s. From the parallelogram of forces on the right hand of Fig. 163 it will be seen that if $\theta$ be the angle of deflection, 


$$
\begin{aligned}
\tan \theta & =\frac{\mu \frac{2 m l}{d^{3}}}{\mu \mathrm{H}} \\
& =\frac{2 m l}{\overline{\mathbf{H}} d^{3}}
\end{aligned}
$$

or the deflection is the same whatever the strength of the suspended needle.

Now move the magnet NS till it is north or south of the suspended needle, with its centre in the meridian and at the same distance $d$ away, and with its axis perpendicular to the meridian : the magnet is now said to be in the "broadside-on position." "The deflection $\theta^{\prime}$ will be given by

$$
\begin{aligned}
\tan \theta^{\prime} & =\frac{m l}{H d^{3}} \\
\therefore \tan \theta & =2 \tan \theta^{\prime} .
\end{aligned}
$$

If the angles are small we shall have

$$
\theta=2 \theta^{\prime} .
$$

Gauss* verified the law in this manner with very great accuracy. He used a more general formula for the action, carrying the approximation a step further than in the investigation just given.

Moment of a magnet. It will be observed that in the above values for the intensity the strength of pole does not occur alone, but in the product, strength of pole $\times$ distance between the poles.

When we may consider the poles as points, this product is termed the "moment of the magnet," and we may denote it by $\mathbf{M}$.

In actual magnets we cannot consider that the poles are two points at a definite distance apart, but we may still give a meaning to the term moment. Whatever the nature of the magnets there are always equal amounts of the opposite polarities. Let us treat each kind of polarity as if it were mass, and find the centre $\mathbf{N}$ of North-seeking polarity at one end, and the centre S of South-seeking polarity at the other, just as we should find the centres of mass. Let those two points be denoted by $\mathbf{N}$ and $\mathbf{S}$. If the total quantity of either kind of magnetisation is $m$, then the moment of the magnet is given by $\mathbf{M}=m \times \mathrm{NS}$.

Axis and centre. 'The line NS is termed the axis of the magnet, and the point bisecting $\mathrm{NS}$ is the centre of the magnet.

We may give a physical interpretation to the moment thus: It the magnet is placed in a field in which the lines of force are parallel and the intensity everywhere of the same value, $\mathrm{H}$, i.e. in a uniform

* Encyc. Brit., 9th ed., Magnetism, p. 237. 
field, and if the magnet is turned round so that its axis is perpendicular to the lines of force, the couple acting on it is MH.

Though we thus imagine two "centres of polarity," it is to be remembered that a distribution of matter, or of electricity, or of magnetism, cannot be replaced by a concentration at a single point in calculating the field which is produced.

Comparison of moments. We may compare the moments of two magnets of small dimensions to a first approximation by using the value for the field at a distance $d$ from the centre along the axis,

$$
\frac{2 m l}{d^{3}} \text { or } \frac{2 \mathrm{M}}{d^{3}} \text {. }
$$

For this purpose we may bring up the two magnets, one enst and the other west of a suspended needle, and placed so that they tend to deflect the needle in opposite directions; then adjusting them at distances $d_{1}$ and $d_{2}$ so that the needle is not deflected :

$$
\text { or } \begin{aligned}
\frac{2 M_{1}}{d_{1}{ }^{3}} & =\frac{2 M_{2}}{d_{2}{ }^{3}} \\
M_{1} & =\frac{d_{1}{ }^{3}}{d_{2}{ }^{3}} .
\end{aligned}
$$

Or we may use each in succession to deflect the needle at the same distance. If the deflections be $\theta_{1}$ and $\theta_{2}$ respectively, the earth's horizontal intensity being $\mathrm{H}$,

$$
\begin{aligned}
\tan \theta_{1} & =\frac{2 M_{1}}{H d^{3}} \\
\tan \theta_{2} & =\frac{2 M_{2}}{H d^{3}} \\
\text { or } \quad M_{1} & =\frac{\tan \theta_{1}}{\tan \theta_{2}} .
\end{aligned}
$$

Some consequences of the inverse square law. Application of Gauss's theorem. Flux of force. As all experiments go to show that the intensity at any point of a magnetic field in air or in any other non-magnetic medium may be regarded as due to each element of polarity $d m$ acting according to the law $d m / r^{2}$, we may apply Gauss's theorem (p. 32) to a magnetic system. If we define the flux of force through an element of surface $d \mathbf{S}$ as $\mathbf{N} d \mathbf{S}$, where $\mathbf{N}$ is the component of the intensity perpendicular to $d S$, the theorem tells us that the total flux of force through any closed surface, which is entirely in air or other non-magnetic medium, is zero. For the total polarity within the surface, even if it encloses magnets, is zero. 
Tube of force. If a small closed curve is drawn at any place in a field, and if a line of force is drawn through every point of it, the lines so drawn will enclose a tubular space termed a tube of force.

Let us apply Gauss's theorem to the closed surface in air or other non-magnetised medium, formed by a portion of a tube of force, and any two cross-sections of it at right angles to the lines of force. Thus, let $S_{1}, S_{2}$, Fig. 164, be the two cross-sections, and let $\mathrm{H}_{1}, \mathrm{H}_{2}$ be the intensities at $\mathrm{S}_{1}, \mathrm{~S}_{2}$. The normal intensity over the curved sides of the tube is everywhere zero. At $S_{1}$ it is $\mathrm{H}_{1}$ and at $\mathrm{S}_{2}$ it is $-\mathrm{H}_{2}$, negative because $\mathrm{H}_{2}$ is inwards. We have, therefore,

\section{$\mathrm{H}_{1} \times \mathrm{S}_{1}-\mathrm{H}_{\triangleleft} \times \mathrm{S}_{2}=4 \pi \times$ magnetisation} within the tube $=0$

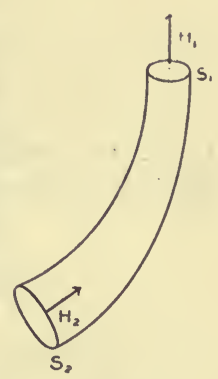

FIG. 164.

'That is, the product $\mathrm{H} \times \mathrm{S}$, or the flux of force, is constant throughout the tube so long as it does not contrin magnetisation.

Unit tubes. If we draw tubes so that for each, intensity $x$ cross-section $=1$, that is, so that there is unit flux of force along it, each is termed a unit tube.

A pole $m$ sends out $4 \pi m$ unit tubes, for if we draw a sphere of radius $r$ round $m$ as centre the intensity on the surface of the sphere is $m / r^{2}$, and the total flux of force through the sphere is $4 \pi r^{2} m / r^{2}=4 \pi m$. There are, therefore, $4 \pi m$ tubes passing through the surface.

If we imagine one line of force along each unit tube the number of lines of force passing through any area will be equal to the total flux of force through the area.

The number of unit tubes or of lines of force passing through unit area perpendicular to them is equal to 1 /cross-section of unit tube or is equal to the intensity of the field.

Potential. If we define the potential at any point in the field as the work done in bringing a unit positive pole to the point from a distance so great that the field is negligible, we can show (see Electricity, p. 4\%) that

$$
V=\int \frac{d m}{r}
$$

where every element of magnetisation $d m$ is to be divided by its distance $r$ from the point.

If the unit pole is moved a distance $d x$ in any direction $x$ to a point where the potential is $\mathrm{V}+\frac{d \mathrm{~V}}{d x} d x$, the work done is $\frac{d \mathrm{~V}}{d x} d x$. So that the force on the unit pole opposing the motion is 
$\frac{d V}{d x}$. Then the force on the unit pole, in the direction of motion or the component of the intensity in the direction $x$ is

$$
\mathbf{H}_{x}=-\frac{d \mathbf{V}}{d_{x}} .
$$

Level or equipotential surfaces can be drawn in magnetic as in electric systems. 


\section{CHAPTER XVIII}

\section{SOME MAGNETIC FIELDS}

Small magnets-Magnetic shells-Uniform sphere.

The potential of a small magnet at any point in its field. Let ACB represent a small magnet of length $l$, poles $\pm m$, moment $\mathbf{M}=m l$. Let $\mathbf{P}$ be a point at a distance $r$ from its centre $\mathrm{C}$ in a direction CP making $\theta$ with $\mathrm{CA}$. The potential at $\mathbf{P}$ is

$$
\mathrm{V}=\frac{m}{\mathrm{AP}}-\frac{m}{\mathrm{BP}} .
$$

Draw MCN perpendicular to CP, and draw PAM, PNB. We

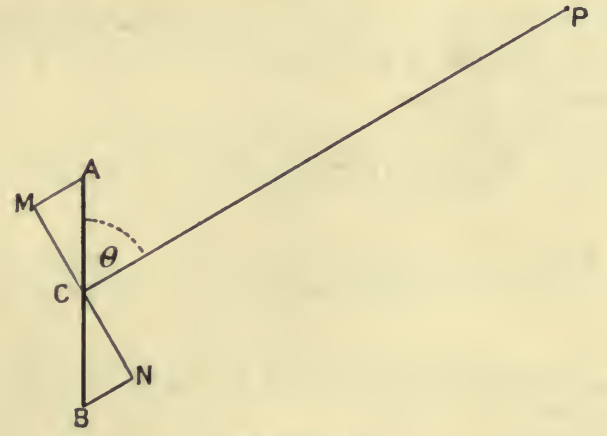

FIG. 165.

suppose $\mathrm{CP}$ so large compared with $l$ that $\mathrm{AM}$ and $\mathrm{BN}$ may be taken as perpendicular to $\mathbf{M N}$, and $\mathbf{P M}=\mathbf{P N}=\mathbf{P C}$.

$$
\begin{aligned}
& \text { Then } \mathrm{PA}=\mathrm{PM}-\mathrm{AM}=\mathrm{PC}-\mathrm{AM}=r-\frac{l}{2} \cos \theta \\
& \text { and } \mathrm{PB}=\mathrm{PN}+\mathrm{NB}=\mathrm{PC}+\mathrm{NB}=r+\frac{l}{2} \cos \theta \\
& \therefore \quad \mathrm{V}=\frac{m}{r-\frac{l}{2} \cos \theta}-\frac{m}{r+\frac{l}{2} \cos \theta}=\frac{m l \cos \theta}{r^{2}-\frac{l^{2}}{4} \cos ^{2} \theta} \\
& =\frac{\mathrm{M} \cos \theta}{r^{2}} \text { when we neglect } \frac{l^{2}}{r^{2}} .
\end{aligned}
$$


If $\theta=0$ the point $\mathbf{P}$ is in the axis of the magnet and $\mathbf{V}=\frac{\mathrm{MI}}{r^{2}}$. The intensity along the axis is

$$
-\frac{d V}{d r}=\frac{2 \mathrm{M}}{r^{3}}
$$

If $\theta=90^{\circ}$, i.e. if $\mathrm{P}$ is in a line through $\mathrm{C}$ perpendicular to the axis, the intensity at $\mathrm{P}$ parallel to the axis is

$$
-\frac{1}{r} \frac{d \mathbf{V}}{d \theta}=\frac{\mathrm{M} \sin \theta}{r^{3}}=\frac{\mathrm{M}}{r^{3}}
$$

and is in the direction in which $\theta$ increases. The component $\frac{d V}{d r}$ along $r$ vanishes, since $\cos \theta=0$.

'These are the values which we obtained in the last chapter for the end-on and broadside-on positions, the values on which Gauss founded his proof of the inverse syuare law.

Vector resolution of a small magnet. If $M$ be regarded as a vector, drawn in the direction of the axis, the potential $\frac{M \cos \theta}{r^{2}}$ at a point $\mathrm{P}$ is the potential of the resolute of $\mathrm{M}$ in the direction of $r$, and if $\mathrm{M}$ be resolved like a force into any number of components, $\mathbf{M}_{1}, \mathbf{M}_{2}, \& c$, making $\theta_{1}, \theta_{2} \& c$, with $r$, the potential of the components will be

$$
\frac{M_{1} \cos \theta_{1}+M_{2} \cos \theta_{2}+\ldots}{r^{3}}
$$

But $\frac{M_{1} \cos \theta_{1}+M_{2} \cos \theta_{2}+\ldots}{r^{3}}=\frac{M \cos \theta}{r^{-3}}$

since the resolved part of $\mathrm{M}$ equals the sum of the resolved parts of its components.

Hence the potential of $\mathbf{M}$ equals the sum of the potentials of its vector components.

'This result follows also from the consideration that a magnet AB (Fig. 166) may be replaced by two magnets AC, CBB, with equal poles. For the two magnets $\mathrm{BC}, \mathrm{CA}$ have equal and opposite poles at C, nentralising each other, and are equivalent therefore to $m$ at $\mathrm{A}$ and $-m$ at $\mathbf{B}$.

The direction and magnitude of the intensity at any point in the field of a small magnet. I et AB (Fig. 16\%) be the magnet with moment $\mathrm{M}$; resolve it into DE along CP' with moment $\mathbf{M} \cos \theta$, and $\mathbf{F G}$ perpendicular to $\mathbf{C P}$ with moment $\mathbf{M} \sin \theta$.

'The intensity due to DE is along $\mathrm{CP}$ and is $\frac{2 \mathrm{M} \cos \theta}{r^{3}}$ where $\mathrm{ACP}=\theta$ and $\mathrm{CP}=r$. 
'The intensity due to $\mathrm{FG}$ is perpendicular to $r$ and is $\frac{M \sin \theta}{r^{3}}$.

'The resultant is $\frac{M}{r^{3}} \sqrt{4 \cos ^{2} \theta+\sin ^{2} \theta}$.

'The direction makes an angle $\phi$ with $\mathrm{CP}$ given by

$$
\tan \phi=\frac{\sin \theta}{2 \cos \theta}=\frac{1}{2} \tan \theta .
$$

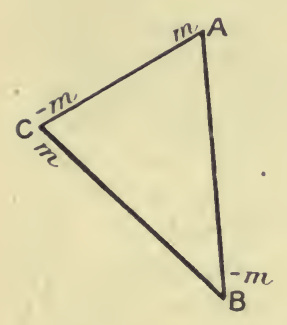

Fici. 166.

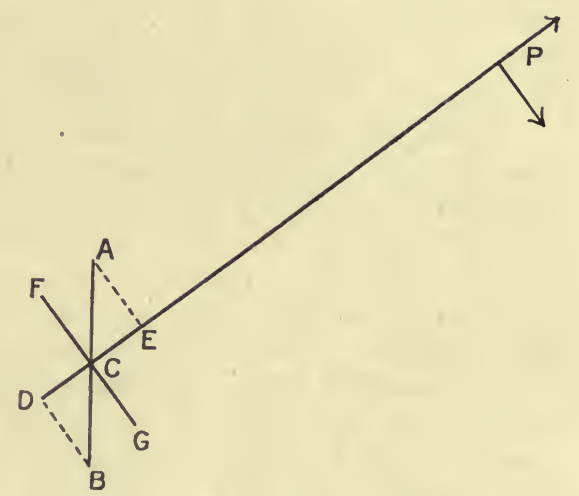

FLG. 167.

'The following construction gires the direction of the intensity. Draw PN, Fig. 168, perpendicular to $\mathrm{Cl}^{\prime}$, meeting the axis produced

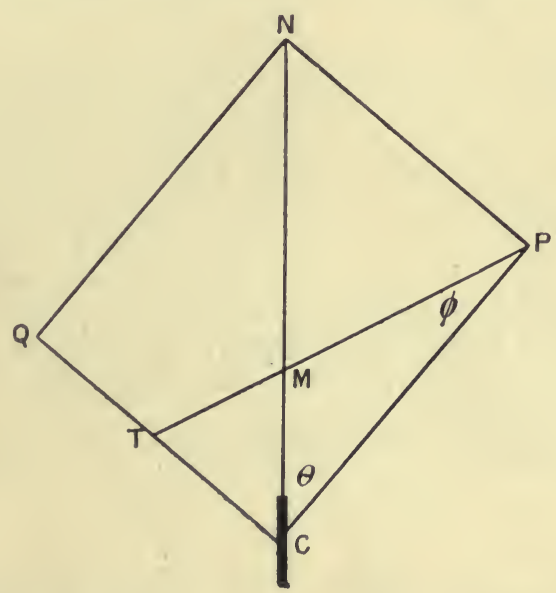

FIG. 168.

in $\mathbf{N}$; complete the rectangle CPNQ. Bisect CQ in ' $\mathrm{I}$.' 'Then ' $P P$ ' is the direction of the intensity at $P$. For 


$$
\tan \mathrm{Cl}^{\prime} \mathrm{T}=\frac{\mathrm{C}^{\prime \prime} \mathrm{I}^{\prime}}{\mathrm{CP}^{\mathrm{P}}}=\frac{1}{2} \frac{\mathrm{CQ}^{\prime}}{\mathrm{CP}^{\prime}}=\frac{1}{2} \frac{\mathrm{NP}}{\mathrm{CP}}=\frac{1}{2} \tan \theta .
$$

Either from an easy geometrical construction or from the trigonometry of the two triangles CPM CPN it can be seen that CM $=\frac{1}{3} \mathrm{CN}$. Hence the following somewhat simpler construction: Draw PN perpendicular to CP, meeting the axis in $\mathbf{N}$; trisect $\mathrm{CN}$ in M. 'Then MP is the direction of the intensity at P.

We may easily work out the force on one small magnet due, to another, for particular cases. For these we refer the reader to J. J. 'Thomson's Elements of Electricity and Magnetism, chap. vi, as we shall not need them in what follows.

Uniform magnetic shell. Imagine a very thin steel or iron plate of uniform thickness bent into a surface of any form. The plate will of course be bounded by a closed curve. Let the plate be magnetised uniformly at each point and normal to the surface. This is equivalent to the supposition that it is built up of equal small magnets, placed side by side, the axis of each perpendicular to the surface of the plate; or it is cquivalent to the supposition that a layer of North nagnetism of uniform density is spread over one face of the plate and that a layer of South magnetism of equal uniform density is spread over the other face. 'Ihis system is termed a uniform magnetic shell. If $\sigma$ is the uniform surface density and $t$ the uniform thickness, $\sigma t$ is the moment of the plate per unit area.

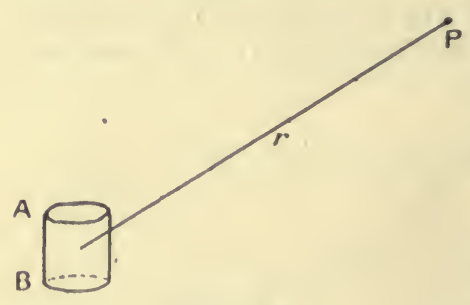

F1G. 169. 'The product $\sigma t$ is termed the "strength" of the shell. We shall denote this strength by $\phi$.

Though we never meet with a uniform magnetic shell in practice, the conception is very useful, since the mannetic field, for which we can find a simple expression, is the same everywhere outside the shell as that of an electric current of a certain strength circulating round the rim of the shell.

Potential of a magnetic shell. Let $\triangle \mathrm{B}$, Fig. 169, represent a cylindrical element of the shell with cross-section $\alpha$. Its poles are $\pm \alpha \sigma$, and its moment is $\alpha \sigma t=\alpha \phi$.

'The potential at $\mathbf{P}$ of the element is :

$$
\frac{u \sigma t \cos \theta}{r^{2}}=\phi \frac{a \cos \theta}{r^{2}} \text {. }
$$

If $d \omega$ is the solid angle subtended by $a$ at $P, d \omega=\frac{1}{r^{2}}$. projection of a on a plane perpendicular to $\mathrm{AP}=\frac{a \cos \theta}{r^{2}}$. Then the potential 
due to the element is $\phi d \omega$. Now sum up for all the elements of the shell and $V=\Sigma \phi d \omega=\phi \Sigma d \omega=\phi \omega$, where $\omega$ is the solid angle subtended at $\mathbf{P}$ by the rim of the shell.

At an infinite distance the angle subtended is zero and the potential is therefore rero.

If we bring a unit NSP from an infinite distance to the point on the positive side of the shell where the angle subtended is $\omega$, then we do more work against the near side than is done for us by the more remote side and the potential is $+\phi \omega$.

If we approach from an infinite distance to a point on the negative side where $-\omega$ is subtended the potential is evidently $-\phi \omega$. 'The potential depends only on the strength $\phi$ and the angle subtended by the rim. Hence all shells of the same strength and the same contour have the same external magnetic field.

Difference of potential of two neighbouring points, one on each side of the shell. Let PQ, Fig. 170a, be two

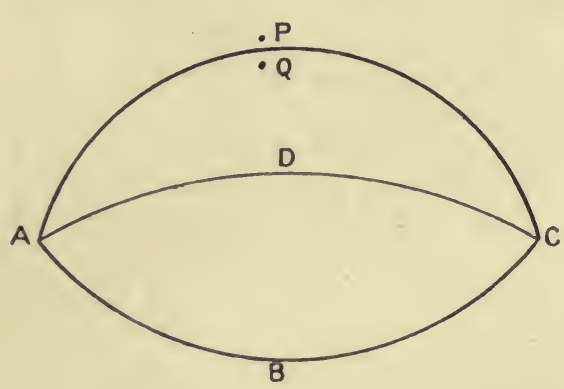

Fil. $170 \mathrm{k}$.

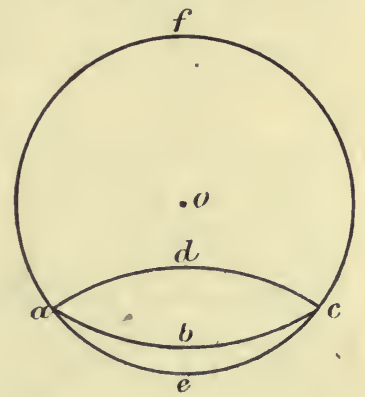

Fic. $170 b$.

neighbouring points: $P$ on the + side, $Q$ on the - side. Let us suppose them so near that they subtend the same solid angle $\omega$ at the rim. In reckoning the potential, if $\mathbf{P}$ subtends $\omega, \mathbf{Q}$ must be considered to subtend $4 \pi-\omega$. For take a point $o$, Fig. $170 b$, and draw a sphere of unit radius with $o$ as centre. Let a line from P, Fig. 170a, trace out the rim $\mathrm{ABCD}$ of the shell and let a parallel line from $o$, Fig. $170 b$, trace out the curve $a b c d$ on the sphere. 'The area $a b c d e$ represents $\omega$. And as the pole moves from an infinite distance on the + side to $P$ the angle increases from zero to $\omega$ and the potential at $\mathrm{P}$ is $\phi \omega$. Now let the pole move from an infinite distance on the - side to P. 'The angle subtended increases from zero to $a b c d f=$ whole area of sphere-abcle :

$$
=4 \pi-\omega
$$

and the potential at $Q$ is $-\phi(4 \pi-\omega)$,

Hence potential at, $\mathbf{P}$ - potential at $\mathbf{Q}$

$$
\begin{aligned}
& =\phi \omega+\phi(4 \pi-\omega) \\
& =4 \pi \phi
\end{aligned}
$$


or the difference is the same at all pairs of points one on each side of the shell.

Potential of pole $m$ due to a shell. Since the potential at any point of the unit pole is $\phi \omega$, the potential of pole $m$ is $\phi m \omega$. We have seen that pole $m$ sends out $4 \pi m$ lines of force or unit tubes through the whole solid angle $4 \pi$ surrounding it ; therefore through solid angle $\omega$ it sends out $\phi m \omega$, or its potential is $\phi \times$ number of lines of force it sends through the closed curve forming the rim of the shell. Conversely the potential of the shell with respect to the

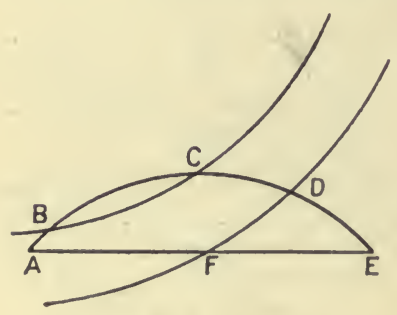

FIG. 171 . pole is $\psi \times$ number of lines from the pole passing through the shell. It is easy to see that if lines of force which enter the shell on the positive side are reckoned positive, and if those which enter on the negative side are reckoned negative, the number will be the same, whatever the form of the shell, so long as it has the same bounding curve. For let $\mathrm{ABCDE}$ and $\mathrm{AFE}$ (Fig. 171) represent two forms of the shell. A line $C B$ which passes through the first shell at $\mathbf{C}$ in one direction and at $\mathbf{B}$ in the other direction will not count in the sum; while a line 1)F which passes only once through the first shell must pass through the second shell also, and will count equally for each.

Potential of a shell in any field. If we regard the field as made by a distribution of polarity, the potential of a shell will be equal to $\phi \times \Sigma$ lines sent through, summed up for every element of polarity, or $\phi \times \mathbf{N}$, where $\mathbf{N}$ is the total number of lines sent through.

Force on a shell in any direction. I.et a shell be displaced parallel to itself through suall distance $\delta x$ in the given direction. Let the rate at which $\mathbf{N}$, the total number of lines of force through the shell, increases in the given direction be $\frac{d \mathrm{~N}}{d x^{\circ}}$. 'Then the number passing through in the displaced position is $\mathbf{N}+\frac{d \mathbf{N}}{d x} \delta x$ and the potential has been increased by

$$
{ }_{d}^{d x} \delta .
$$

'This is the work which must be done in the displacenent. There is therefore a force resisting the displacement eyual to $\phi \frac{d N}{d x}$ or the force tends to decrease $\mathbf{N}$, and in the positive direction of $\delta x$ is

$$
-\phi \stackrel{d N}{d x}
$$


The shell, therefore, tends to move into the position in which as many lines of force as possible pass through in the negative direction. This can easily be remembered if it is considered that it will tend to set with its negative face towards a positive pole.

The resultant force on the shell evidently acts in the direction in which a given motion produces the maximum decrease in $\mathbf{N}$.

Representation of the force by elementary forces each acting on an element of the rim of the shell. The value of $\mathbf{N}$ can alter only by lines moving in or out across the boundary curve of the shell as it is displaced. This is seen at once if we imagine the lines of force to be materialised into wires. The number can only be altered by the rim cutting through the wires. We may expect then that the force on the whole shell can be represented by forces acting at the rim where the cutting takes place.

Let us suppose that the force on an element $\delta s$ of the boundary in direction $\delta x$ is $-\phi n$, where $n$ is the number of lines cut by $\delta s$ per unit displacement in the direction $\delta x$. Then the total force is $-\phi \Sigma n=-\phi \frac{d \mathrm{~N}}{d x}$, since $\Sigma n$ must be equal to $\frac{d \mathrm{~N}}{d x}$. Then we may regard the total force $-\phi \frac{d \mathbf{N}}{d x}$ as the resultant of forces such as $-\phi n$ on the elements of the boundary. The direction in which the force on each element acts is that in which a given motion will make $n$ the greatest.

If $\mathrm{H}$, Fig. 172, represents the field at an element $\delta s$, it is evident that $n$ is the greatest when $\delta x$ is perpendicular to $\mathrm{H}$ and to $\delta s$. 'Then

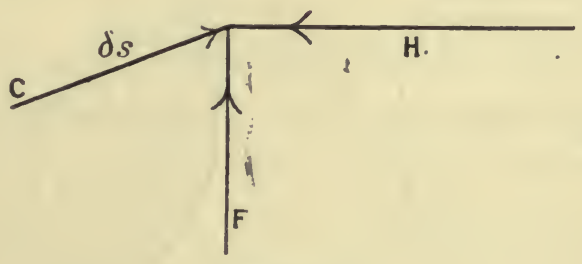

FIG. 172.

the direction of the resultant force $\mathrm{F}$ on the element is along the normal to the plane through $\delta s$ and $\mathrm{H}$. 'The number of lines of force cut per unit motion of $\delta s$ along $\mathrm{F}$ is $\mathrm{H} \sin \theta \delta s$, where $\theta$ is the angle between $\mathrm{H}$ and $\delta s$. 'Then $\mathrm{F}=\phi \mathrm{H} \sin \theta \delta s$.

We shall see when we study the magnetic field of a current that the current equivalent to a shell circulates counter-clockwise round the rim when seen from the side towards which the positive layer faces. Hence in the figure we may assign to $\delta s$, considered as an element of the current, the direction in wards through the paper wheri the shell lies above $\delta s$ with the positive layer to the right. The force 
on $\delta s$ tends to lessen the positive lines of force through the shell, that is, $\delta s$ tends to move upwards when $\mathrm{H}$ is as drawn. If we represent current by $\mathrm{C}$, field by $\mathrm{H}$, then force is in the direction in which a screw would move if its head were turned round from $\mathrm{C}$ to $\mathrm{H}$, where $\mathrm{C}$ and $\mathrm{H}$ both act towards or both form the point of intersection. The rule may be remembered by noting the alphabetical order of C to $\mathrm{H}$.

Field due to a uniformly magnetised sphere. Let us suppose that a sphere consists of small parallel bars or fibres of equal cross-section $\alpha$ and with equal poles $\pm I \alpha$. 'Then it is said to be uniformly magnetised with intensity $I$ in the direction of the fibres.

Let PQ, Fig. 173, represent the end of one of the fibres, and let the radius $\mathrm{OP}$ make $\theta$ with the direction of magnetisation.

The polarity at $P Q$ is $\mathbf{I} \alpha$. 'The area of the end of the fibre on the sphere is $\frac{\alpha}{\cos \theta}$, and if the surface density on that area is $\sigma$

$$
\frac{\sigma \alpha}{\cos \theta}=\mathrm{I} \alpha \text { or } \sigma=\mathrm{I} \cos \theta .
$$

We may therefore represent the action of the magnetisation outside the sphere by a layer

$$
\sigma=\mathrm{I} \cos \theta,
$$

which will be positive on one hemisphere through which $\theta$ increases

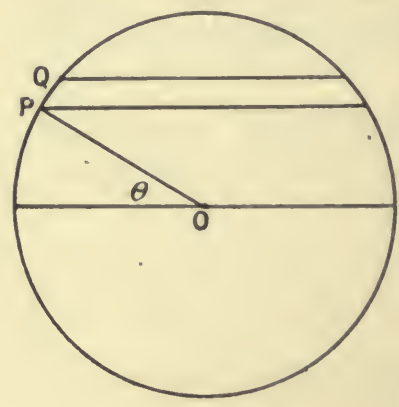

FIG. 173.

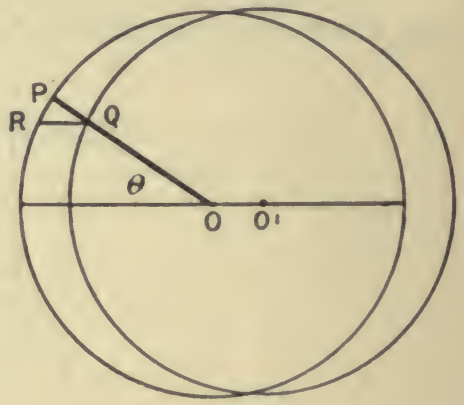

FIG. 174 .

from $0^{\circ}$ to $90^{\circ}$, and negative on the other hemisphere through which $\theta$ increases from $90^{\circ}$ to $180^{\circ}$.

We may obtain the potential of this distribution by a simple device due to Poisson.

Imagine a sphere equal to the magnetised sphere, but consisting of positive polarity of density $\rho$, uniformly distributed through its volume. Let its centre be at $O$. Superpose on this an equal sphere consisting of uniformly distributed negative polarity density - $\rho$ 
but with its centre at $\mathrm{O}^{\prime}$, a sinall distance from $\mathrm{O}, \mathrm{O}^{\prime} \mathrm{O}$ being in the direction of magnetisation. Then the two spheres will neutralise each other everywhere where they overlap. But on one hemisphere there is left a positive layer unneutralised, and on the other a negative layer unneutralised. The total quantity thus left acting at $\mathbf{P}$ on area $d \mathbf{S}$, Fig. 174, will be $\rho \mathbf{P Q} d \mathbf{S}=\rho \mathbf{R Q} \cos \theta d \mathbf{S}=\rho \mathbf{O O}^{\prime} \cos \theta d \mathbf{S}$. If we make $\rho \mathrm{OO}^{\prime}=\mathrm{I}$, this quantity is $\sigma d \mathbf{S}$, where $\sigma=\mathrm{I} \cos \theta$.

That is, the two superposed spheres give the actual distribution which exists on a uniformly magnetised sphere. Now the potential of a sphere at an external point is the same as if it were collected at its centre. Hence the potential of the two at an external point, distant $r$ in direction $\theta$ with $\mathrm{OO}^{\prime}$, is the same as that of a pole $\frac{4}{3} \pi a^{3} \rho$ at $\mathrm{O}$, and a pole $-\frac{4}{3} \pi u^{3} \rho$ at $\mathrm{O}^{\prime}$, where $a$ is the radius of the sphere, or is

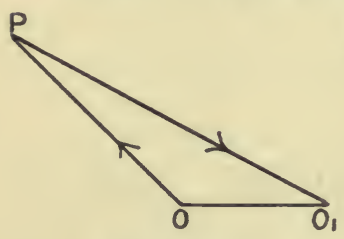

FIG. 175 .

the same as that of a short magnet length $\mathrm{OO}^{\prime}$ with moment

$$
\frac{4}{3} \pi a^{3} \rho \mathrm{OO}^{\prime}=\frac{4}{3} \pi a^{3} \mathrm{I}
$$

We can calculate the field within the sphere on the supposition that the two surface layers act as if the space within were air. The force due to a uniform sphere at a point within it is due to the part of the sphere included within a concentric sphere drawn through the point. Hence at a point P, Fig. 175, it will be $\frac{4}{3} \pi \rho \frac{\mathrm{OP}^{3}}{\mathrm{PU}^{2}}=\frac{4}{3} \pi \rho \mathrm{OP}$ along $\mathrm{OP}$, due to the positive sphere, and $\frac{4}{3} \pi \rho \frac{\mathrm{PO}^{\prime 3}}{\mathrm{PO}^{\prime 2}}=\frac{4}{3} \pi \rho \mathrm{PO}^{\prime}$ along $\mathrm{PO}^{\prime}$, due to the negative sphere.

The resultant of these two is by the triangle of forces equal to $\frac{4}{3} \pi \rho \mathrm{OO}^{\prime}=\frac{4}{3} \pi \mathrm{I}$ parallel to $\mathrm{OO}^{\prime}$, or the field within the sphere is everywhere uniform and in the direction parallel to $\mathrm{OO}^{\prime}$.

Force on a small magnet placed at a point in a nonuniform field. If a small magnet, length $l$, pole strength $m$, and therefore with moment $\mathbf{M}=m l$, is placed in a field which is not uniform, the magnet will set in the direction of the line of force through its centre, but the forces on the two poles will not be quite equal and opposite, and the magnet will move in the direction of the resultant of the two forces.

If, for instance, it is placed in the field radiating from the NS pole of a long magnet, it will ultimately set in a line of force and its SSP will be nearer the pole producing the field than its NPS. It will therefore tend to move inwards, that is, to the stronger part of the field.

Or if it is placed in the circular field round a straight current. 
the intensity of which is $\frac{2 \mathrm{C}}{r}$, where $\mathrm{C}$ is the current and $r$ the distance from the axis of the wire, the forces on the pole, though equal, will not be opposite. Let

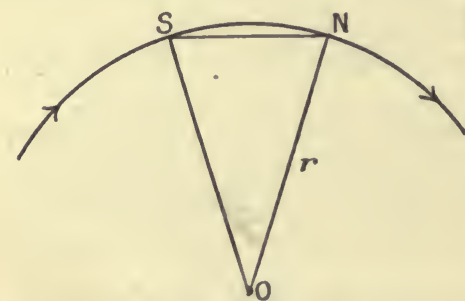

FIG. 176. $\mathrm{O}$, Fig. 176 , be the centre of the wire, NS the small magnet. The forces on $\mathrm{N}$ and $\mathrm{S}$ are $\frac{2 \mathrm{Cm}}{r}$, tangential to the circular line of force through NS. We inay take the sides of the triangle ONS to represent the two forces and their resultant, each side being perpendicular to the force which it

represents. Hence the resultant $\mathrm{F}$ is given by

$$
\mathbf{F}: \frac{2 \mathrm{C} m}{r}=\mathrm{NS}: \mathrm{ON}=l: r \text {. }
$$

Then

$$
F=\frac{2 C M}{r^{2}}
$$

and it is directed inwards towards $O$ and again towards the stronger part of the field.

We can obtain an expression for the force acting in any field by finding the potential energy of the magnet with respect to the field. Suppose that the magnet is brought from a great distance, where the field is zero, to its actual position, and let it move always so that the South pole follows in the track of the North pole. The total work done in moving the South pole is equal and opposite to the work done in moving the North pole up to the point finally occupied by the South pole, and the potential energy will therefore be the work done in moving the North pole the extra distance equal to the length of the magnet. Let $\mathbf{H}$ be the intensity of the field, then the potential energy will be $\mathrm{V}=-m l \mathrm{H}=-\mathrm{MH}$.

The force in any direction $x$ will be

$$
-\frac{d \mathrm{~V}}{d x}=\mathrm{M} \frac{d \mathrm{H}}{14 i^{\circ}}
$$

It is therefore the greatest in the direction in which $\mathrm{H}$ increases most rapidly, or the magnet tends to move to the strongest part of the field.

If the magnet be turned end for end, and be constrained to keep in that direction, the forces on the poles are just reversed, and the magnet tends to move in the direction of most rapid decrease of $\mathrm{H}$ or into the weakest part of the field. 


\title{
CHAPTER XIX
}

\section{INDUCED MAGNETISATION}

\begin{abstract}
Magnetic induction-Induction and intensity in air-Lines and tubes of induction in air-Induction within a magnetised body-Hydraulic illustration-Equations expressing continuity of potential and continuity of induction tubes-Representation of induced magnetisation by an imaginary distribution of magnetic poles acting according to the inverse square law-Magnetic susceptibility-Imagined investigation of $\mathrm{B}$ and $\mathrm{H}$ within iron-Permeability and the molecular theory-Induction in a sphere of uniform permeability $\mu$ placed in a field in air uniform before its introduction-Long straight wire placed in a field in air uniform before its introduction-Circular iron wire in a circular field-Energy per cubic centimetre in a magnetised body with constant permeability-The energy supplied during a cycle when the permeability varies-Calculation of induced magnetisation is only practicable when the permeability is constant-Diamagnetic bodies-Magnetic induction in a body when the surrounding medium has permeability differing from unity.
\end{abstract}

Magnetic induction. We have seen that when a magnetisable body, such as a piece of iron or steel, is introduced into a magnetic field it becomes a magnet by induction. We shall investigate in this chapter methods of representing this induced magnetisation.

'The magnetic force on a pole placed at any point in the air surrounding the magnetised body may be calculated as if it were due to the direct action of polarity scattered over the surface or through the volume of the body, each element of the polarity acting directly on the pole with intensity inversely as the square of the distance. But it must be remembered that this is a mere working representation, adopted for the purpose of calculation. The magnetic condition must be regarded as existing at every point in the field, whether a test pole is placed there to reveal it or not. The force on a pole in the field is due to the altered-the magnetic-condition of the medium immediately round it.

Faraday, who introduced the idea of action through and by the medium into magnetism as well as into electricity, introduced also the use of lines of force, or, as we shall here term them, lines of induction, to describe the condition of the field, and he showed that these lines gave not only the direction of the intensity but also that by their crowding or sparseness they gave its magnitude. $\mathrm{He}$ was led by his idea of an altered condition of the medium to the 
great discovery that when polarised light is passed through certain media along the lines of magnetic induction, the plane of polarisation is rotated, a discovery clearly proving that in these media the altered condition is no mere hypothesis, but a certain fact. He also laid emphasis on the idea, which, howerer, was not a new one, that the magnetic condition of a magnet is not merely a condition on its surface or at its poles, but that there is something going on inside, something symbolised by the passage of lines of induction through it. We are driven to this conclusion by observing that when we break a magnet in two, new poles are developed on the broken ends. The change in length of iron bars on magnetisation, a change proportional to the length, is perhaps further evidence of some action or some change of condition within the metal.

It may appear to be a difficulty in the way of accepting the action by and through the medium that the action of a magnet is practically the same through or in most media surrounding it. It is very nearly the same whether the magnet is surrounded by water or by air, or whether it is placed in a vacuum. We must then suppose that there is some medium eren in a racuum which is altered by the presence of the magnet, that there is something which can transmit momentum and which can possess energy. But this difficulty has to be faced in considering the phenomena of radiation. Radiant energy passes at very nearly the same speed through air and through the highest vacuum which we can create, and it passes through the vacuum, as far as we yet know, without any loss of energy. 'There is something, then, in the vacuum which can take up and propagate radiant energy and its accompanying momentum, and nearly in the same way as if air were present. When we speak of a field in air, we must not, then, imply that the air is the seat of all the action, the storer of all the energy. Doubtless it takes some share in the action, but the nedium which pervades a so-called vacuum is no doubt present, and in all probability takes its share in the action.

Induction and intensity in air. When magnetisation of iron takes place by induction, we symbolise it by supposing that lines of induction pass through the iron, and we say that induction goes on within it. We may think of the induction within as implying a condition in the iron similar to that which exists in the neighbouring air outside, but in general the action, whatever it is, will be greater. The term induction is therefore extended to denote the alteration existing in the air, as well as that in iron.

In the air, or in any non-magnetic mediun, we take the intensity $\mathrm{H}$, the force on a unit pole, to represent the induction, both in magnitude and direction. In doing this we make no hypothesis as to the nature of the induction. All that we imply is that the inductions at two points $P$ and $Q$ in air will differ from each other in magnitude and direction in the same way as the intensities at $\mathbf{P}$ and $\mathbf{Q}$, and we so choose the unit of induction 
that if the intensity at a point in the non-magnetic medium is $\mathrm{H}$ and the induction at the same point is $\mathrm{B}$, then $\mathrm{B}=\mathrm{H}$.

Since the induction and the intensity are thus made to coincide in air, the only medium in which we directly measure $\mathrm{H}$, the employment of the two terms appears at first sight needless. But we shall see directly that we are led to assign to them different values in magnetic media, and in permanent magnets and in crystalline media they may even have different directions. They imply, too, even in air, different modes of viewing the action. "Intensity" is the result of experiment. We suppose that we place a unit test pole at the point considered and measure the mechanical force upon it. It is the measure of the action of the medium on something, as it were, outside itself, viz. the test pole. It corresponds in fact to stress in ordinary elasticity. "Induction" implies that there is an alteration in the medium and corresponds to strain in elasticity. In elasticity we have

$$
\text { stress }=\text { modulus } \times \text { strain, }
$$

and when we know the modulus we can determine the strain by measuring the stress. The magnetic equation $\mathrm{B}=\mathrm{H}$ implies units so arranged that the magnetic quantity corresponding to the modulus is unity in air.

We may here follow the analogy with elasticity one step further. The energy per unit volume in a strained body is proportional to stress $x$ strain. In the magnetic field, then, we might expect it to be proportional to the product $\mathrm{HB}$, or, since we make $\mathrm{B}=\mathrm{H}$, proportional to $\mathrm{H}^{2}$. We shall see later in the chapter that there is some reason to suppose that it is $\mathrm{H}^{2} / 8 \pi$.

Lines and tubes of induction in air. Lines and tubes of force in air may now be described as lines and tubes of induction, and along a given tube

$$
\text { induction } \times \text { cross-section }=\text { constant. }
$$

The total flux of induction through any closed surface, the surface being entirely in air, will be zero whether it encloses magnetised matter or not. For if it does enclose it the two polarities are equal and opposite, and the total polarity within the surface is zero.

Induction within a magnetised body. Let us suppose that an iron sphere without permanent magnetism is placed in a field in air which before its introduction was uniform, i.e. was such that the lines of induction were straight and parallel. We take the case of a sphere, since on a certain supposition, as we shall see later (p. 242), the course of the lines of force within the sphere can be determined, but the general ideas we gain from the sphere will apply for any form. The course of the lines of induction in the air will be altered by the introduction of the iron as indicated in 
Fig. 17\%, the lines being drawn in towards the sphere. 'The figure represents those on a diametrical plane parallel to the original direction of the field. The total polarity over one hemisphere is equal and opposite to the total polarity over the other henisphere.

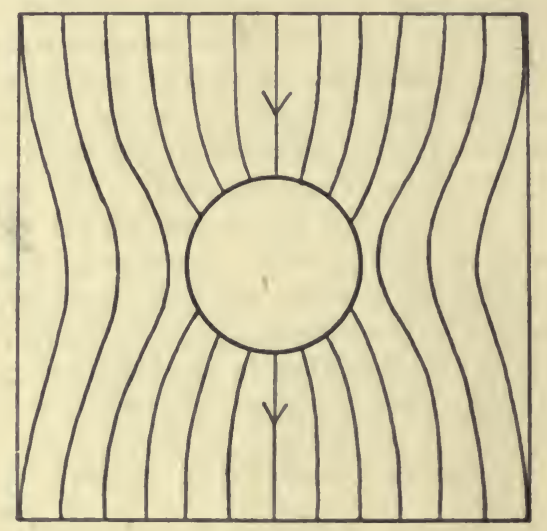

FIG. 177.

so that as many lines of induction converge on to one hemisphere as diverge from the other.

Now imagine that the sphere is cut through in a plane perpendicular to the central line of induction and that the two halves are

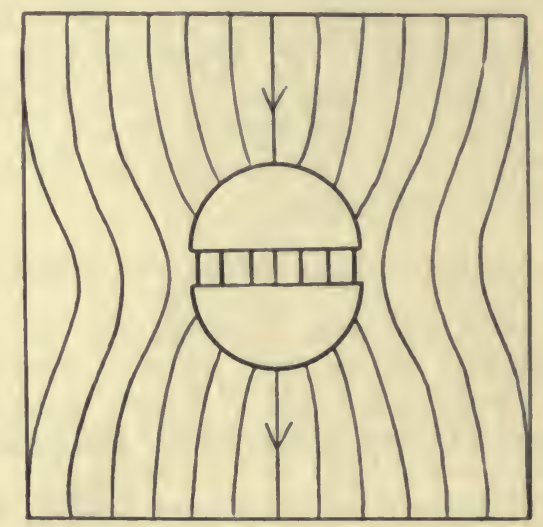

FIG. 178 .

drawn a very small distance apart. Then we shall have tubes of induction crossing the air gap thus formed, and as many tubes will cross the gap as enter the curved surface of one hemisphere and leave that of the other. For we can draw a closed surface entirely in air closely surrounding either hemisphere, and the total flux through such a surface is, as we have seen, zero, or the 
flux across the gap must be equal and opposite to the flux over the curved hemispherical surface.

All this may be regarded as experimental. We now make the fundamental supposition that the lines and tubes of induction are continued through the iron, that the tubes crossing the air gap are the continuations of those in the air outside, and that along each tube, induction $\times$ cross-section is constant, and has the same value

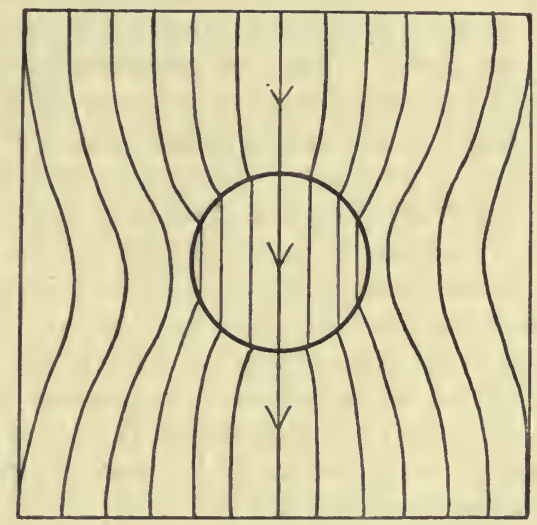

FIG. 179.

as the same product for the same tube outside the iron. We shall see later that on a certain supposition the tubes will go straight through the iron, or that the induction within it is uniform.

We may now close up the gap, having only used it to emphasise the idea that the induction runs through the iron, and we have the lines of induction as in Fig. 179, and along any tube induction $\times$ crosssection $=$ constant whether it is in iron or air. Let us now, Fig. 180,

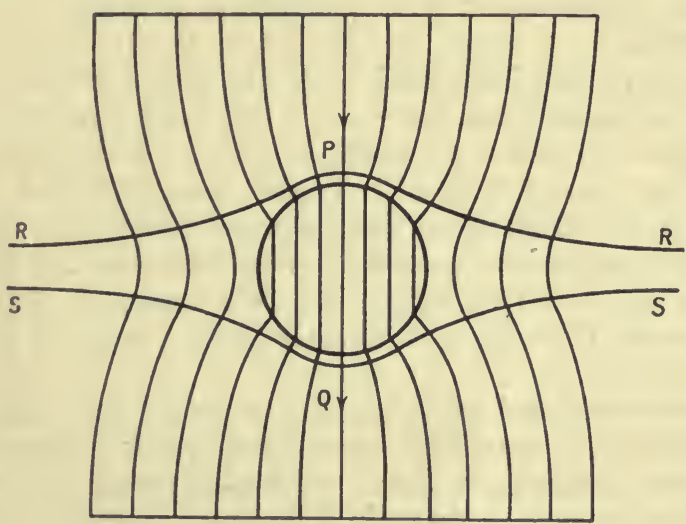

FIG. 180. 
draw two level surfaces, or surfaces cutting the lines of induction at right angles, one through Pclose to the point at which the central line enters the iron, and the other through $Q$ close to the point at which it leaves the iron. It is evident that these surfaces must bend, the one down wards, the other upwards, as indicated in the figure, coming nearer together far out at $\mathrm{RS}$, where the field is practically unaffected by the iron, than they are at $P Q$, close to the iron. Now, we have no way of experimentally measuring the intensity $\mathbf{H}$ within the iron. But we make the supposition that it is such that in passing from one level surface to another, intensity $\times$ path is constant for all paths whether in air or iron; or, if $\mathrm{H}$ is not uniform, then if $d s$ is an element of path, $\int \mathrm{H} d s$ is the same for all paths from one level surface to another. Since, then, the level surfaces are further apart at $P Q$ than they are in distant parts of the field, $H$ is less within the iron than in those distant parts, i.e. less than it was before the iron was introduced. But just outside the sphere at $\mathbf{P}$ or $\mathbf{Q}$ the tubes of force or induction have narrowed, in converging on to the iron, and so $\mathrm{H}$ is greater there than it was before the iron was introduced. 'Then $\mathrm{H}$ in air at $\mathbf{P}$ is greater than $\mathrm{H}$ in iron close to $\mathrm{P}$. But $\mathrm{B}$ in air at $\mathrm{P}$ is equal to $\mathrm{B}$ in iron close to $\mathrm{P}$, and since $\mathrm{B}$ in air is equal to $\mathrm{H}$ in air, $\mathrm{B}$ in iron close to $\mathrm{P}$ is greater than $\mathrm{H}$ in iron at the same point.

Let us put in the iron

$$
\mathbf{B}=\mu \mathbf{H} .
$$

$\mu$ is termed the permeability of the iron.

$$
\text { If we put } \mathrm{H}=\frac{1}{\mu} \mathrm{B} \text { and compare with the elasticity relation }
$$

$$
\text { stress }=\text { modulus } \times \text { strain }
$$

we see that $1 / \mu$ corresponds to the modulus of elasticity. It represents as it were the difficulty, while $\mu$ represents the ease, of magnetisation.

To sum up. We have in air $B=H$, where $H$ can be experimentally determined, and therefore $\mathrm{B}$ can be determined. In iron we can suppose that $B$ is determinate from the continuity of the induction flux along a tube as it passes from air into iron. We can also suppose that $\mathrm{H}$ is determinate from the constancy of potential difference as we pass from one level surface to another, whether through air or through iron. We have seen that in iron B must be greater than $\mathrm{H}$, and its ratio to $\mathrm{H}$ is termed the permeability of the iron.

We have considered only the case in which the magnetisation is produced by induction, and have disregarded the possibility of permanent magnetism in the iron. If there is permanent magnetism we can no longer assume that $\int \mathrm{H} d s$ between two level surfaces is the same through air as through iron. 
Hydraulic illustration. A hydraulic illustration may help us to realise the meaning of magnetic permeability.

Imagine that $\mathrm{ABCD}$, Fig. 181, is the cross-section of a slab of spongy or porous material indefinitely extended to right and left, and that a liquid which entirely fills the pores is being forced through it by a pressure across $A B$ in excess of that across $C D$. Then the liquid will flow with a velocity from $A B$ to $C D$ such that the viscous resistance balances the difference of pressure. On the average the flow will be straight across from $\mathrm{AB}$ to $\mathrm{CD}$ and the pressure will fall uniformly from one face to the other. This corresponds to a uniform magnetic field. 'The pressure corresponds to the potential, and its slope, or fall per centimetre, to the intensity. The velocity will be

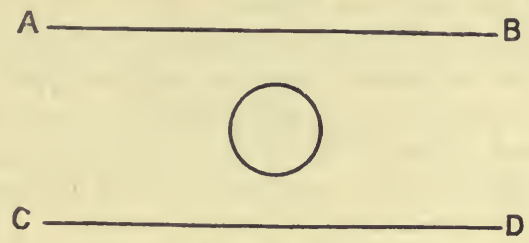

FIG. 181.

proportional to the pressure-slope and will correspond to induction. Evidently, if we draw a tube of flow, velocity $\times$ cross-section, or total flow, will be the same throughout its length.

Now imagine a portion of the slab-represented as spherical in the figure-made more porous, and therefore less resisting, than the rest. Evidently the liquid will flow more rapidly through this portion. 'I'he lines of flow will converge on to the end nearer' $A B$, and diverge from that nearer $C D$. 'The surfaces of equal pressure will be deformed just as are the magnetic level surfaces in Fig. 180. 'The total flow along a tube will be the same whether it is within or without the larger-pored space occupied by the sphere, and the pressure-slope will be less within than without that space.

We could replace the sphere with its larger pores by one having pores equal to those outside it, if we supposed that where the tubes of flow enter the sphere, there are "sinks" or points at which fluid is removed somehow from the system, the amount removed being the excess of fluid coming up over that which the given pressureslope will drive through the sphere on the supposition of pores equal in size to those outside. On the surface of the other hemisphere, where the fluid emerges, we must suppose that there are "sources" or points at which fluid is somehow introduced into the system to make up the excess of outward flow over the flow through the sphere. 'The "sources" must introduce just as much as the "sinks" remove. 'This mode of representation corresponds to the representation of the magnetic induction by polarity distributed over the surface and it brings to the front the artificial nature of that representation.

'Though we are not here considering permanent magnetism, we 
may point out what would correspond to a permanent magnet in the fluid illustration.

A steel magnet in air would correspond to a larger pored region, and in the pores we should have to imagine small turbines fixed, driving the liquid in a given direction. The turbines would represent the magnetic molecules, and each turbine would have to produce a flow proportional to the strength of the molecular pole. As much fluid would be drawn in at one end of the region as was driven out at the other, and the fluid would circulate back through the surrounding space, the lines of flow representing the lines of force.

Equations expressing continuity of potential and continuity of induction tubes. We may conveniently term the condition that the difference of potential between two points is the same, whether we pass from one to the other through air or through iron, the condition of continuity of potential, for it implies that the potential is the same at two points quite close together, one in air, the other in iron, and we may put the condition into the following form :

Let $P Q$ be two neighbouring points on the surface of the iron. Let $H_{2}$ be the intensity in the air close to $P Q$ and let its direction make $\theta_{1}$ with the normal to the surface at $\mathrm{PQ}$. Then $H_{1} \sin \theta_{1}$ is the component of the intensity parallel to the surface in the air. Let $\mathrm{H}_{2}$ be the intensity in the iron close to $P Q$ and let its

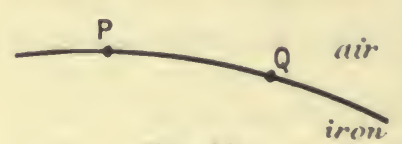

FıG. 182. direction make $\theta_{2}$ with the normal. Then $\mathrm{H}_{2} \sin \theta_{2}$ is the component of the intensity parallel to the surface in the iron. In order that the difference of potential between $\mathbf{P}$ and $\mathbf{Q}$ shall be the same in air and in iron, these components must be the same in direction and equal in magnitude. 'The first condition implies that $\mathrm{H}_{1}$ and $\mathrm{H}_{2}$ are in one plane with the normal, while the second requires that

$$
\mathbf{H}_{\mathbf{1}} \sin \theta_{\mathbf{1}}=\mathbf{H}_{2} \sin \theta_{2} \text {. }
$$

We may conveniently term the condition that the flux of induction across a tube is constant, the condition of contiuuity of induction tubes, and we may put it in the following form:

Let Fig. 183 represent the section of a tube of induction entering the iron at $\mathbf{P Q}$ by the plane through the normal QM and the line of induction in the air through $Q$. It passes through the line of induction in the iron also, if we assume that the directions of induction and intensity in the iron coincide, as they do in air. Let $\theta_{1} \theta_{2}$ be the angles which the direction of the induction makes with the normal in the two media. Let QR PS represent crosssections of the tube in the two media, the breadth of the tube perpendicular to the paper being the same in each. 'The con- 
tinuity of induction gives $B_{1} \cdot Q R=B_{2}$. PS, where $B_{1}$ is the induction in air, $\mathrm{B}_{2}$ that in iron;

$$
\text { or }
$$

whence

$$
\begin{gathered}
\mathrm{B}_{1} \cdot \mathrm{PQ} \cos \theta_{1}=\mathrm{B}_{2} \cdot \mathrm{PQ} \cos \theta_{2} \\
\mathrm{~B}_{1} \cos \theta_{1}=\mathrm{B}_{2} \cos \theta_{2} .
\end{gathered}
$$

Thus while the components of intensity parallel to the surface are

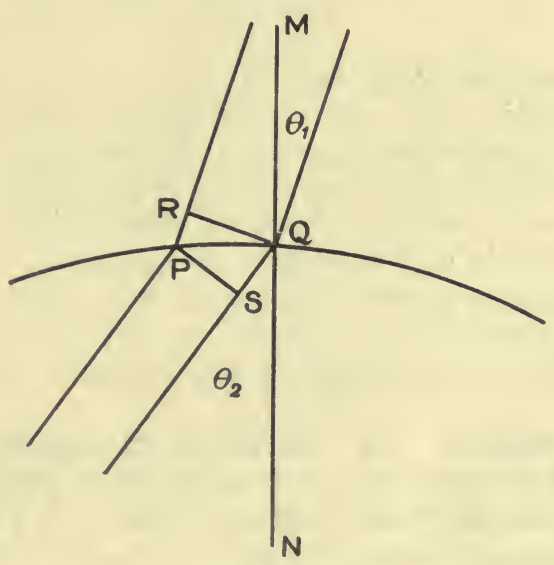

FiG: 183.

equal, the components of induction perpendicular to the surface are equal.

If instead of air and iron we have any two media with permeabilities $\mu_{1}$ and $\mu_{2}$, intensities $H_{1} H_{2}$, and inductions $B_{1} B_{2}$, we still have

$$
\begin{aligned}
& \mathrm{H}_{1} \sin \theta_{1}=\mathrm{H}_{2} \sin \theta_{2} \\
& \mathrm{~B}_{1} \cos \theta_{1}=\mathrm{B}_{2} \cos \theta_{2} .
\end{aligned}
$$

Putting $\mathrm{B}_{1}=\mu_{1} \mathrm{H}_{1}$, and $\mathrm{B}_{2}=\mu_{2} \mathrm{H}_{2}$, the equation of continuity of induction becomes

$$
\mu_{1} \mathrm{H}_{1} \cos \theta_{1}=\mu_{2} \mathrm{H}_{2} \cos \theta_{2}
$$

and dividing the two sides of the equation of continuity of potential by these we get

$$
\frac{\tan \theta_{1}}{\mu_{1}}=\frac{\tan \theta_{2}}{\mu_{2}}
$$

We may term this the lnw of refraction of magnetic tubes. It npplies of course only to cases in which the conditions of continuity hold. 
When we compare the foregoing investigations with the corresponding investigations in electricity we see that magnetic intensity corresponds to electric intensity, and magnetic induction corresponds to electric strain. 'The magnetic equation

$$
\mathrm{B}=\mu \mathrm{H}
$$

corresponds to the electric equation

$$
\mathrm{D}=\frac{\mathrm{KE}}{4 \pi}
$$

so that $\mu$ corresponds to $K / 4 \pi$.

The introduction of $4 \pi$ in one of these is very unfortunate, for it destroys the exact correspondence of formula in the two cases. It arises from the fact that the units are so chosen that unit quantity of electricity sends out one tube of strain, while unit quantity of magnetism sends out $4 \pi$ tubes of induction. Heaviside* has introduced a system of "rational units" in which unit magnetic pole, like unit quantity of electricity, sends nut one tube. But the difficulty in the way of change in the practical units has not yet been faced.

Repres'ntation of induced magnetism by an imaginary distribution of magnetic poles acting according to the inverse square law. We have seen that the inteusity changes when we pass from air into iron, or more generally when we pass across a surface at which the permeability changes. For purposes of calculation we can imagine the change of field to be produced by a layer of magnetism, a layer of poles distributed over any surface where $\mu$ changes from one con-

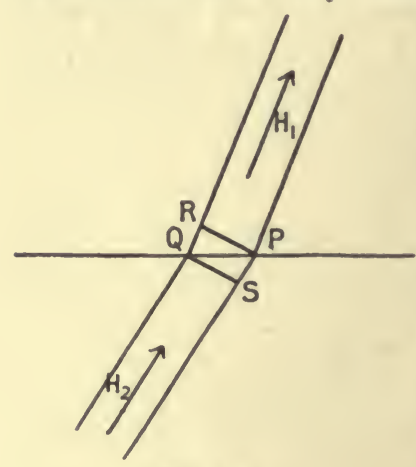

FiG. 184. stant value to another, or distributed through the volume if the change in $\mu$ is gradual. These poles are supposed to act according to the inverse square law and as if the whole space were of unit permeability.

Let us consider a change of permeability at a surface PQ, Fig. 184, from 1 to $\mu$, and let the figure represent the section of a tube of induction by a plane containing $\mathrm{H}_{1}$ and $\mathrm{H}_{2}$, and let the tube stamp out an area $a$ on the surface, represented in section by $\mathbf{P Q}$. Let $\sigma$ be the surface density of the imagined magnetic poles. Since our supposition is that the surface magnetism would, in combination with the external poles, produce the observed intensities if it acted in air on both sides, we may apply Gauss's theorem to the surface PRQS. The theorem states 
that if $\mathrm{N}$ is the component of the intensity normal to the surface,

$$
\int \mathbf{N} d \mathbf{S}=4 \pi \times \text { quantity within. }
$$

For the surface chosen $N$ has value $H_{1}$ over $P R$ and $H_{2}$ over QS, and is zero on the sides. If $\theta_{1} \theta_{2}$ are the angles with the normal made by $\mathrm{H}_{1} \mathrm{H}_{2}$, the cross-sections at PK and QS are $\alpha \cos \theta_{1}$ and $\alpha \cos \theta_{2}$, so that we get

$$
\begin{aligned}
& \mathrm{H}_{1} \alpha \cos \theta_{1}-\mathrm{H}_{2} \alpha \cos \theta_{2}=4 \pi \sigma \alpha \\
& \text { and } \sigma=\frac{\mathrm{H}_{1} \cos \theta_{1}-\mathrm{H}_{2} \cos \theta_{2}}{4 \pi} .
\end{aligned}
$$

We may express $\sigma$ in terms of either $\mathrm{H}_{1}$ or $\mathrm{H}_{2}$, since $\mathrm{H}_{1} \cos \theta_{1}$ $=\mathrm{B}_{1} \cos \theta_{1}=\mathrm{B}_{2} \cos \theta_{2}=\mu \mathrm{H}_{2} \cos \theta_{2}$, whence

$$
\begin{aligned}
\sigma & =\frac{\mu-1}{4 \pi \mu} \mathrm{H}_{1} \cos \theta_{1} \\
& =\frac{\mu-1}{4 \pi} \mathrm{H}_{2} \cos \theta_{2} .
\end{aligned}
$$

It is easily seen that if $\mu$ is constant within the surface the total magnetism on one end of a tube where it enters the surface is equal and opposite to that on the other end where it leaves. For $\sigma \cdot \mathbf{P Q}=\sigma \mathrm{QS} / \cos \theta_{2}=\frac{\mu-1}{4 \pi} \mathrm{H}_{2} \cdot \mathrm{QS}$, which is constant along the tube within the body.

Magnetic susceptibility. In another way of viewing the magnetisation of iron in air, the distribution of polarity imagined as above is regarded as the surface manifestation of the internal magnetisation of the iron, and we may then consider the surface layer $\sigma$ as describing a real physical condition of the iron beneath it. I et PQRS, Fig. 185, represent a tube of induction entering the iron at $\mathrm{PQ}$, and leaving it at RS. We shall suppose that $\mu$ is constant within the iron, so that magnetism need be imagined only at the ends $P Q$ and RS. We may represent this magnetism by supposing that the tube is divided into magnetised

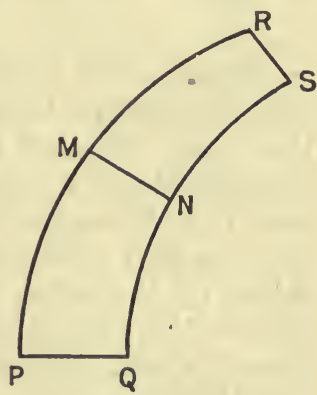

FIG. 185. fibres, each with poles only at $\mathbf{P Q}$ and RS. If we could cut through these fibres at any intermediate section MN, perpendicular to the axis of the tube, opposite polarities would be developed at the two cut faces, each equal in total quantity to the polarity at either end. If the surface density of magnetism on $\mathbf{M N}$ is $\mathrm{I}, \mathbf{M N} \times \mathbf{I}$ is constant along the tube and is equal to $\mathbf{P Q} \times \sigma$. If $\mathbf{M N}$ is close 
to $\mathrm{PQ}$, then if $\theta_{2}$ is the angle which the axis of the tube makes with the normal to $P Q$,

$$
\begin{array}{rlrl}
\mathrm{MN} & =\mathrm{PQ} \cos \theta_{2} \\
\text { and } \quad \mathrm{PQ} \times \sigma & =\mathrm{MN} \times \mathrm{I}=\mathrm{PQ} \times \mathrm{I} \cos \theta_{2} \\
\text { or } \quad & \quad \sigma & =\mathrm{I} \cos \theta_{2} .
\end{array}
$$

I is termed the intensity of magnetisation. It must not be confused with $\mathrm{H}$, the field intensity, or the force per unit pole. Since $\mathrm{I} \times \mathrm{MN}$ is constant along the tube and since $\mathrm{B} \times \mathrm{MN}=$ $\mu \mathrm{H} \times \mathrm{MN}$ is also constant, $\mathrm{I}$ is proportional to $\mu \mathrm{H}$, or to $\mathrm{H}$ since we are taking $\mu$ as constant.

Let us put $\quad \mathrm{I}={ }_{\kappa} \mathrm{H}$.

$\kappa$ is termed the magnetic susceptibility of the iron, or, sometimes, the coefficient of magnetisation.

Let us take $M N$ close to $P Q$ :

We have

$$
\begin{gathered}
\sigma=\mathrm{I} \cos \theta_{2}=\kappa \mathrm{H} \cos \theta_{2} . \\
\sigma=\frac{\mu-1}{4 \pi} \mathrm{H} \cos \theta_{2} . \\
\frac{\mu-1}{4 \pi}=\kappa \\
\mu=1+4 \pi \kappa .
\end{gathered}
$$

But also

Then

and

Imagined experimental investigation of $B$ and $H$ within iron. Lord Kelvin imagined a method of investigating the values of $\mathbf{B}$ and $\mathrm{H}$ within iron which, though experimentally unrealisable, is a valuable aid to understanding the difference between the two quantities, induction and intensity of field. Let us suppose that a long and exceedingly narrow tunnel is made along a line of induction in the iron. If narrow enough it will not disturb the magnetisation in the iron round it except very close to the ends, and at a distance from the ends the intensity within the tunnel will be the same as in the iron close to its sides, or as in the iron before the tunnel was made. We may regard this as a consequence of the condition of continuity of potential, or if we do not wish to assume that condition we must regard this as a definition of $\mathrm{H}$ within the iron. Since the tunnel is free from iron we can imagine $\mathrm{H}$ to be measured within it by means of the vibration of a minute needle of known moment. Since the needle is in iron-free space we can calculate the intensity of the field in which 
it is placed from the distribution of poles throughout the system, these poles acting according to the inverse law.

Now suppose that instead of making the tunnel we make a cut through the iron perpendicular to the lines of force and remove a very thin wafer-shaped portion of the iron. The lines of induction go straight across the crevasse thus formed and the tubes of induction have the same cross-section in the crevasse as in the iron on either side. But in the iron-free space in the crevasse the induction is equal to the intensity. 'The intensity will be that previously existing + that due to the new surface layers formed on the two faces of the crevasse, say $\pm I$, and these will produce intensity $4 \pi I$. Hence within the cavity the intensity is

$$
\mathrm{B}=\mathrm{H}+4 \pi \mathrm{I}
$$

where $\mathbf{H}$ is the intensity before the crevasse was made, or putting $\mathrm{I}={ }_{\kappa} \mathrm{H}$, the intensity in the crevasse which is equal to the induction in the iron before the crevasse was made is

$$
\mathrm{B}=\mathrm{H}+4 \pi \kappa \mathrm{H} \text {. }
$$

Putting $\quad \mathrm{B}=\mu \mathrm{H}$

we get $\quad \mu=1+4 \pi \kappa$.

The quantities $\mathrm{B}$ and $\mathrm{H}$ are to be regarded as the two fundamental quantities. The quantity $\mathrm{I}$, which is equal to $(\mathrm{B}-\mathrm{H}) / 4 \pi$, is a convenient quantity to introduce in that it enables us to express simply the polarity which we may suppose to be distributed over the system where $\mu$ changes in order to calculate the intensity of the field, using the inverse square law. This supposed polarity is somewhat artificial if we accept the principle of continuity of flux of induction. In electricity the tubes actually end on conductors and the charges at their ends may be regarded as having a real existence. But in magnetism the tubes of induction never end. Either they come from an infinite distance and go off to another infinite distance or they are re-entrant and form closed rings. The polarity $\sigma$ which we suppose to exist where a tube passes from one medium to another with different $\mu$ is merely a device introduced to enable us to calculate the effect of change of medium.

Permeability and the molecular theory. We can give some account of the greater permeability of iron as compared with air on the molecular theory in the following way, in which we only attempt to give a general idea.

I et NS, Fig. 186, represent a magnet and consider one of its lines of force as drawn through P. Now introduce just outside this line a. small magnet xs, Fig. 187, turned so that its lines run in the opposite direction through $\mathrm{P}$, and let us suppose for simplicity that it is of such strength and in such a position that it just neutralises 
the field of the other at P. Then the lines will destroy each other at $\mathbf{P}$ and the line from $\mathbf{N}$ will run through the small magnet somewhat as drawn in Fig. 188, or the line has been pulled out of the air into the iron of xs.

If a piece of previously unmagnetised iron is put into the position of ws its molecular magnets first tend to set along the lines

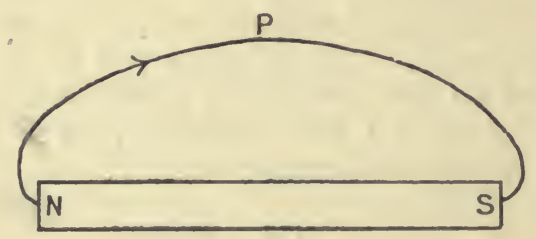

Fig. 186.

of force of NS, and then each molecular magnet so setting has an action like that of ss as above considered. It draws out of the

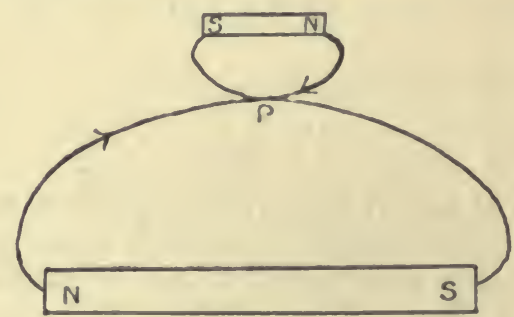

F1G. 187.

field round it lines of force due to NS, concentrating them on itselt. Regarding induction rather than force, we may say that each molecular magnet setting along the lines in the field draws into

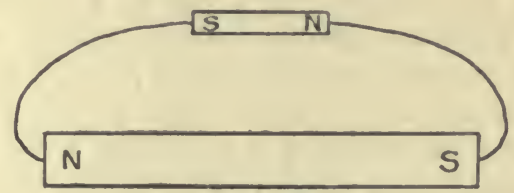

FIG. 188.

itself some of the induction due to NS and makes it continuous with its own induction.

We shall now consider some cases of induction.

Induction in a sphere of uniform permeability $\mu$ placed in a field in air uniform before its introduction. Let the uniform field into which the sphere is introduced be $\mathrm{H}$. Let us assume that the sphere is uniformly magnetised by induction 
and that its field is superposed on H. We have shown (p. 226) that a sphere radius $a$, uniformly magnetised with intensity of magnetisation I, has a field without it the same as that of a small magnet with moment $\frac{4}{3} \pi a^{3} \mathrm{I}$ placed at its centre, and with axis in the direction of $I$, and that it has a field within the sphere uniform and equal to $-\frac{4}{3} \pi \mathrm{I}$. We are to find the value of $\mathrm{I}$ which will satisfy the conditions of continuity of potential and

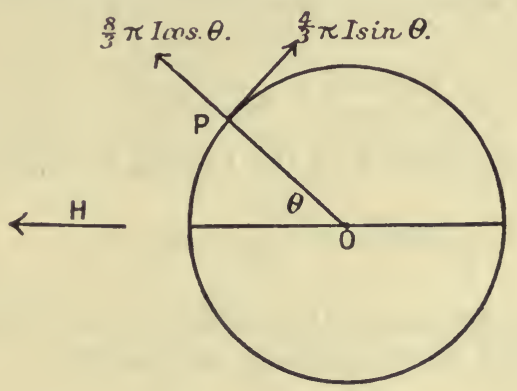

FIG. 189.

flux of induction. The supposition to which we have already referred (p. 231) is that this gives the actual magnetisation.

Consider a point $\mathrm{l}$ ' at the end of a radius OP making $\theta$ with the direction of $\mathrm{H}$. We may resolve the small magnet equivalent to the sphere into two, one along OP with moment $\frac{4}{3} \pi a^{3} \mathrm{I} \cos \theta$, and the other perpendicular to $\mathrm{OP}$ and with moment $\frac{4}{3} \pi a^{3} I \sin \theta$ respectively.

The intensity of the field just outside $\mathrm{P}$ due to the former will be along $\mathrm{OP}$ and equal to $\frac{8}{3} \pi \mathrm{I} \cos \theta$. That due to the latter will be perpendicular to OP and equal to $\frac{4}{3} \pi \mathrm{I} \sin \theta$. The ficld intensity parallel to the surface is obtained by combining this with the component of $\mathrm{H}$ and is

$$
\mathrm{H} \sin \theta-\frac{4}{3} \pi \mathrm{I} \sin \theta
$$

Just within the magnet sed sphere its own field is $\frac{4}{3} \pi \mathrm{I}$ parallel to $\mathrm{H}$, so that, adding $\mathrm{H}$, the total intensity of field is $\mathrm{H}-\frac{4}{3} \pi \mathrm{I}$. 
The component parallel to the surface is

$$
\mathrm{H} \sin \theta-\frac{4}{3} \pi \mathrm{I} \sin \theta \text {. }
$$

Thus the condition for continuity of potential is satisfied.

The normal intensity just outside the surface is

$$
\mathrm{H} \cos \theta+\frac{8}{3} \pi \mathrm{I} \cos \theta
$$

and as the medium is air this is equal to the normal induction.

The normal intensity just inside the surface is

$$
\mathrm{H} \cos \theta-\frac{4}{3} \pi \mathrm{I} \cos \theta
$$

and since the permeability is $\mu$ the normal induction is

$$
\mu\left(H \cos \theta-\frac{4}{3} \pi I \cos \theta\right) \text {. }
$$

Equating the normal induction without and within, the condition of continuity of flux of induction is satisfied by

$$
\mathrm{H}+\frac{8}{3} \pi \mathrm{I}=\mu\left(\mathrm{H}-\frac{4}{3} \pi \mathrm{I}\right)
$$

whence

$$
\mathrm{I}=\frac{3}{4 \pi} \frac{\mu-1}{\mu+2} \mathrm{H} .
$$

The intensity of field within the sphere is

$$
\mathrm{H}-\frac{4}{3} \pi \mathrm{I}=\frac{3}{\mu+2} \mathrm{H} .
$$

If $\mu$ is large, as it may be for iron when $H$ is not very small, this approaches the value

$$
\frac{3 \mathrm{H}}{\mu}
$$

while I approaches the value

$$
\frac{3 \mathrm{H}}{4 \pi}
$$

or approaches the same value for all large values of $\mu$. The normal intensity just outside the sphere $\left(H+\frac{8}{3} \pi I\right) \cos \theta$ approaches the value

\section{$3 \mathrm{H} \cos \theta$}

and the tangential intensity $\left(\mathrm{H}-\frac{4}{3} \pi \mathrm{I}\right) \sin \theta$ approaches zero unless 
$\theta$ is very near $90^{\circ}$. That is, the lines of force enter the sphere very nearly normally when $\mu$ is large except near the equatorial plane.

If we use $\kappa$ instead of $\mu$ we obtain

$$
I=\frac{3 \kappa}{3+4 \pi \kappa} \mathbf{H}
$$

Long straight wire placed in a field in air uniform before its introduction. Experiment shows that with a long straight wire placed in a uniform field the polarity is developed chiefly near the ends.* Let us suppose that the induced poles are $\pm m$, that the radius of the wire is $r$, and that its length is $l$. Let us as an admittedly rough approximation suppose that $\pm m$ are at the ends If the original field is $\mathbf{H}$, the field at the centre within the wire $\mathrm{i}$

$$
\mathrm{H}-2 m /\left(\frac{l}{\overline{2}}\right)^{2}=\mathrm{H}-8 m / l^{2} \text {. }
$$

If the magnetic susceptibility is $\kappa$ the intensity of magnetisation is

$$
\mathbf{I}=\kappa\left(\mathrm{H}-\frac{8 ı n}{\iota^{2}}\right)
$$

and the total polarity on a section imagined to be made through the centre will be

$$
\pi r^{2} \kappa\left(\mathrm{H}-\frac{8 m}{l^{2}}\right)
$$

The assumption that the poles are at the ends gives

$$
\begin{aligned}
\text { or } \quad m=\pi r^{2} \kappa\left(\mathrm{H}-\frac{8 m}{l^{2}}\right) \\
\quad m=\frac{\pi r^{2} \kappa \mathrm{H}}{1+\frac{8 \pi \kappa r^{2}}{l^{2}}} .
\end{aligned}
$$

If $\kappa r^{2} / l^{2}$ is so small that we may put the denominator $=1$, that is, if we may neglect the effect of the ends in calculating the induction in the middle parts of the wire:

$$
m=\pi r^{2} \kappa \mathrm{H}=\frac{\mu-1}{4} r^{2} \mathrm{H} .
$$

This is nearly true if $l$ is greater than $800 r$.

Circular ir on wire in a circular field. If a solenoid be bent round into a circle, the intensity $\mathbf{H}$ of the field within it, at a

* Ewing, Magnetic Induction, chap. ii ; Searle and Bedford, Phil. Trans., A, 198, p. 98 . 
distance $r$ from the axis through the centre of the circle, is given by

$$
2 \pi r \mathrm{H}=4 \pi n \mathrm{C}
$$

where $n$ is the number of turns of the solenoid and $\mathrm{C}$ is the current in it.

Then

$$
\mathrm{H}=\frac{2 n \mathrm{C}}{r} \text {. }
$$

If a wire of permeability $\mu$ and of uniform cross-section forms a closed ring of mean radius $r$ within the solenoid, the iron is magnetised; but there are no poles formed, for the tubes of induction go round through the iron and do not pass into or out of it anywhere. The intensity in the iron must be the same as that in the air close to it and is therefore given by the above equation. The induction and the intensity of magnetisation are

$$
\begin{gathered}
\mathrm{B}=\mu \mathrm{H}=\frac{2 \mu n \mathrm{C}}{r} \\
\mathrm{I}={ }_{\kappa} \mathrm{H}=\frac{2 \kappa n \mathrm{C}}{r}=\frac{(\mu-1) n \mathrm{C}}{2 \pi r} .
\end{gathered}
$$

Energy per cubic centimetre in a magnetised body with constant permeability. We may use the last case to obtain an expression for the distribution of energy in a magnetised body when the permeability is constant as, for instance, in iron in the first stage of magnetisation represented by O A Fig. 130 .

We shall suppose that a thin iron circular ring forms the core of a circular solenoid as above and that the field intensity within the solenoid, due to the current in the solenoid, is $\mathrm{H}$. Let $a$ be the

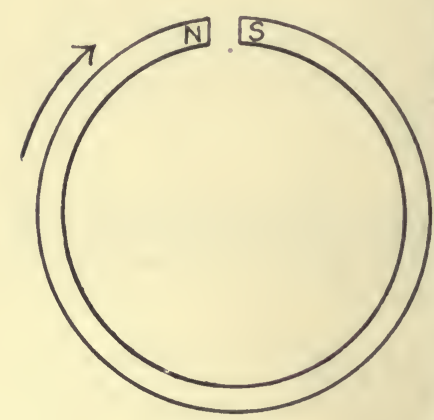

FIG. 190. cross-section of the iron. Let $\mathrm{B}=\mu \mathrm{H}$ be the induction in it. Then the total flux of induction through the iron is $a \mathrm{~B}$. Now imagine a cut made through the iron and a gap formed as in Fig. 190. If $\mathrm{H}$ due to the solenoid is in the direction of the arrow, the poles at the gap will be as indicated by $\mathbf{N}$ and $\mathrm{S}$. Let the gap formed be so narrow that the magnetisation of the iron remains the same as before it was made. 'The induction in the gap, then, is $\mathbf{B}$, and this would be produced by polarity of surface density $\pm B / 4 \pi$ or total polarity $\pm a \mathrm{~B} / 4 \pi$.

It is to be noted that the intensity of magnetisation $I$ in the iron does not give us this polarity. From it we should get $\sigma=\mathrm{I}={ }_{\kappa} \mathrm{H}$, and the intensity of field in the gap due to $\sigma$ would 
be $4 \pi \kappa \mathrm{H}$. But $\sigma$ is only imagined in order to account for the excess of intensity of field in the air over and above that which was existing before the iron was introduced. We must, therefore, add $\mathrm{H}$, and the total intensity in the gap is $4 \pi \kappa \mathrm{H}+\mathrm{H}=\mu \mathrm{H}=\mathrm{B}$ as above. In calculating the energy in the iron, we have to deal with the total induction, not the excess over that previously in the air displaced.

We observe that $\mathrm{H}$ is due to an external force applied to the iron. In elastic stress and strain an external stress strains a body until an internal stress is brought into play equilibrating the external stress. Work is done against this internal stress in increasing the strain, and energy is put into the body proportional to internal stress and increase in strain. So in the magnetisation of the iron, induction - "magnetic strain "-is produced by the external magnetising stress $\mathrm{H}$ until an equal internal stress is called into play by the iron, and the energy put into the iron is the work done against this internal stress, equal and opposite to $\mathrm{H}$ at each step, as $\mathbf{H}$ increases from zero to its final value.

Let the induction be increased by $d \mathbf{B}$, by a small increase of current in the solenoid. We may represent the process of increase by imagining that North-seeking magnetism $a d \mathrm{~B} / 4 \pi$ is separated from an equal quantity of South-seeking on the South side of the gap and that it is carried round through the iron against the intensity $\mathrm{H}$ exerted by the iron, this intensity being equal and opposite to the external intensity due to the coil.

If the length of the iron is $l$ the work spent in the increase is

$$
l \mathrm{H} a d \mathrm{~B} / 4 \pi
$$

and the total work spent in magnetising the iron will be

$$
\frac{l_{a}}{4 \pi} \int_{0}^{\mathrm{H}} \mathrm{H} d \mathrm{~B}
$$

Since $\mu$ is constant we may put $d \mathbf{B}=\mu d \mathbf{H}$ and we get total work spent, or the energy supplied

$$
l_{\alpha} \frac{\mu \mathrm{H}^{2}}{8 \pi}
$$

or, since $l_{\alpha}$ is the volume of the iron, the energy supplied per cubic centimetre is

$$
\frac{\mu \mathrm{H}^{2}}{8 \pi}=\frac{\mathrm{B}^{2}}{8 \pi \mu}
$$

and if we assume that this energy is stored as magnetic energy in the iron, these expressions give the energy per cubic centimetre. 
It is to be noted that the energy supplied only gives us the energy stored, when the process of magnetisation is "reversible," so that a given field produces a given induction, as it does when $\mu$ is constant. When the magnetisation may be the same for quite different fields the energy spent is different for different paths from one condition to another, and our investigation does not lead to a value for the energy stored in the iron. But we may

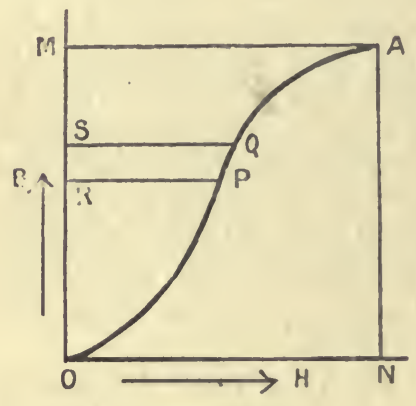

FIG. 191. find an expression for the energy supplied in any change.

The energy supplied during a cycle when the permeability varies. If $\mu$ varies we can represent the energy supplied by an area on the diagram, Fig. 191, which gives the relation between $\mathbf{H}$ and $B$. Let the curve OPQA represent the relation between $\mathrm{B}$ and $\mathrm{H}$ when $\mathrm{H}$ is increased from zero to $\mathrm{ON}$ in a piece of iron previously unmagnetised.

Let $\mathbf{P Q}$ represent a small step in the process; then if we deal with 1 c.c., the work done in the step $\mathrm{PQ}$ is $\mathrm{H} d \mathrm{~B} / 4 \pi=\mathrm{PR} \times \mathrm{RS} / 4 \pi$ $=\frac{1}{4 \pi} \times$ area of strip PS. The total work done as $H$ changes from 0 to $\mathrm{ON}$ is $\frac{1}{4 \pi} \times$ the sum of such strips, or $\frac{1}{4 \pi} \times$ area of OPAMO.

If when $H$ has reached the value $O N$ it is diminished again, we know that $B$ does not fall off as would be represented by the curve AQPO, but somewhat as represented by the curve A'TCD in Fig. 192.

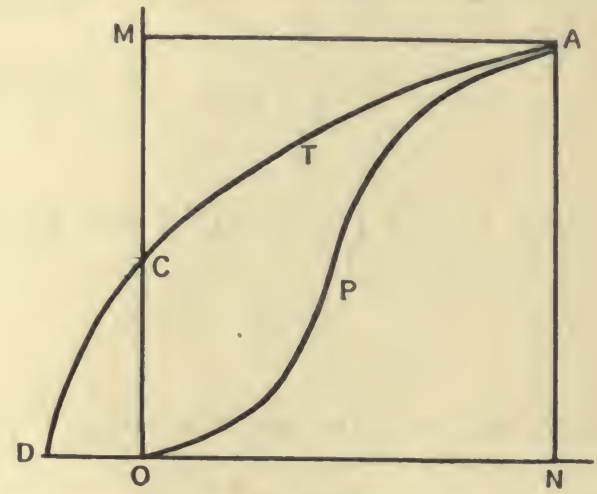

FIG. 192.

When $\mathrm{H}$ is reduced to zero, induction $\mathrm{OC}$ still remains in the iron and it is necessary to reverse $\mathrm{H}$ and give it a value, such as $\mathrm{OD}$, before the induction is destroyed. The work done in increasing 
$\mathrm{H}$ to $\mathrm{ON}$ and then reducing it to zero is $\frac{1}{4 \pi}$ (OPAMO-CTAMC) $=\frac{1}{4 \pi}$ OPATCO. The work done in increasing the negative value of $\mathrm{H}$ to OD is $\frac{1}{4 \pi}$ OCDO, or the total work done is $\frac{1}{4 \pi}$ OPACDO.

If we take the iron through several hundred cycles of $\mathrm{H}$ between values $+\mathrm{ON}$ and $-\mathrm{ON}$, then in the later cycles its cycle of magnetisation is represented very nearly by a closed loop as in Fig. 193, the hysteresis loop. In each cycle the work done on the

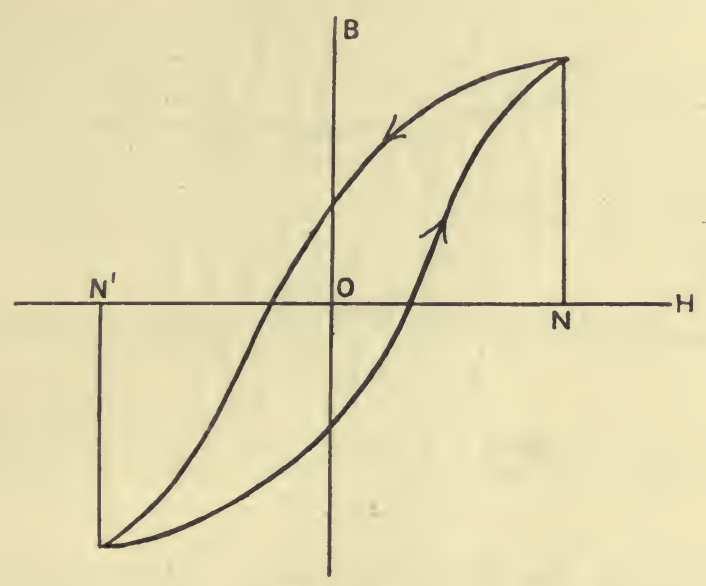

FIG. 193.

iron piece is represented by $\frac{1}{4 \pi} \times$ area of loop, and in each cycle the energy represented by this work is dissipated and appears as heat in the iron.

We shall return to this subject in Chapter XXI.

Calculation of induced magnetisation is only practicablewhen the permeability is constant. When $\mu$ is constant we may calculate the magnetisation induced in a given field in certain cases, of which we have given easy examples in the sphere and the ring. But when $\mu$ varies, as it does with ferromagnetic bodies as soon as $\mathrm{H}$ rises above a small value, hysteresis comes into play and the equations $\mathrm{B}=\mu \mathrm{H}$ and $\mathrm{I}={ }_{\kappa} \mathrm{H}$ may cease to have meaning. For example, suppose that, as in Fig. 192, $\mathrm{H}$ is taken to the value $\mathrm{ON}$, and is then reduced again to zero, $\mathrm{B}$ and I still have positive values, that of $\mathrm{B}$ being represented by $\mathrm{OC}$, and there is no meaning in saying that $\mathrm{B}=\mu \mathrm{H}$ at this stage.

Diamagnetic bodies. If a material, instead of concentrating the lines of force upon itself, widened them out-that is, if, taking the fluid illustration of p. 235, it were like a hydraulic system in 
which the pores were smaller and more resisting than in the surrounding medium - then a sphere placed in a previously uniform field would produce a distribution of lines of force like that given in Fig. 194.

Let us assume as before that the lines of force are parallel within the sphere. In order that the level surfaces may cut the lines of force at right angles, they must be nearer together within the surface than they are at a distance outside. The intensity within the body is therefore greater than it was before the body was

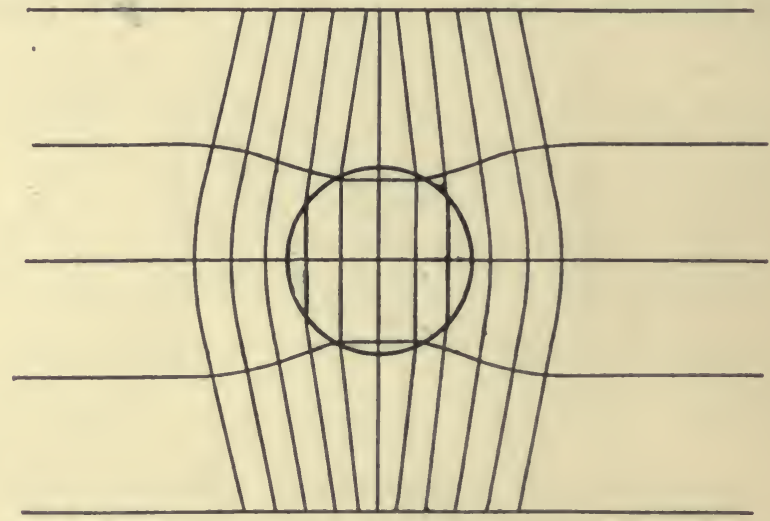

FIG. 194.

introduced, while the flux of induction, which we suppose to be continuous, is less. If then we take $\mathbf{B}=\mu \mathrm{H}, \mu$ must be less than 1. If we represent the alteration of field by induced polarity $\sigma$ scattered over the surface, as on p. 239,

$$
\sigma=\frac{\mu-1}{4 \pi \mu} \mathbf{H}_{1} \cos \theta_{1}
$$

and, as $\mu<1, \sigma$ becomes negative, or the polarity is reversed and the forces are reversed. This is just what we observe with diamagnetic bodies. A small diamagnetic body is repelled from the pole of a magnet.

Hence we interpret the distinction between paramagnetic and diamagnetic bodies by saying that for the former $\mu$ is greater than 1, while for the latter it is less than 1 . If we use the magnetic susceptibility $\kappa=\frac{\mu-1}{4 \pi}, \kappa$ is positive for the former, negative for the latter. If we exclude the ferromagnetic bodies, $\mu-1$ and $\kappa$ are always very small and for a given substance practically constant, and the value of $\sigma$ is very small.

Magnetic induction in a body when the surrounding medium has permeability differing from unity. In the 
foregoing investigations we have supposed that air is the medium surrounding the body in which magnetisation is induced, and that for air $\mu=1$ and $\kappa=0$. Now we shall consider the case in which the medium has permeability differing from 1 . Let us suppose that it has permeability $\mu_{1}$, and that the body which it surrounds has permeability $\mu_{2}$, and let us find the value of the surface density of magnetism or polarity which we should have to suppose spread over the surface of the body to account for the change of field when the permeability is everywhere reduced to the same value $\mu_{1}$ as it has in the medium. This corresponds to the investigations on p. 238, where the imagined surface layer acts as if it were in air on all sides.

We shall first show that the intensity due to a pole $m$ at distance $r$ in a medium of permeability $\mu_{1}$ is no longer $m / r^{2}$, but $m / \mu_{1} r^{2}$. Suppose two poles each $m$, i.e. each a source of the same total induction, to be placed one in air and the other in the $\mu_{1}$ medium. At a distance $r$ from the first the induction is

$$
\mathbf{B}=m / r^{2}=\mathbf{H} \text {. }
$$

At a distance $r$ from the second the induction is also

$$
\dot{\mathbf{B}}=m / r^{2} \text {. }
$$

But if $\mathrm{H}_{1}$ is the intensity in the second medium

$$
\begin{aligned}
& \mathbf{B}=\mu_{1} \mathrm{H}_{1} \\
\therefore \mathrm{H}_{1} & =m / \mu_{1}{ }^{2} .
\end{aligned}
$$

Then if we apply Gauss's theorem in a system where the permeability is everywhere $\mu_{1}$, and regard the field as due to poles acting according to the law $m / \mu_{1} r^{2}$,

$$
\int \mathbf{N} d \mathbf{S}=4 \pi m / \mu_{1}
$$

where $m$ is the total polarity within the surface $\mathbf{S}$.

- Now suppose that a body of permeability $\mu_{2}$ is placed in a magnetic field and surrounded by a medium of permeability $\mu_{1}$. We still assume the principles of continuity of flux of induction and continuity of potential, so that if $\mathrm{H}_{1} \mathbf{H}_{2}$ are the intensities just without and just within the surface, and if they make $\theta_{1}$ and $\theta_{2}$ with the normal,

$$
\mu_{1} \mathrm{H}_{1} \cos \theta_{1}=\mu_{2} \mathrm{H}_{2} \cos \theta_{2} \text { and } \mathrm{H}_{1} \sin \theta_{1}=\mathrm{H}_{2} \sin \theta_{2} \text {. }
$$

Let Fig. 195 represent the section of a tube of induction passing out of the body and cutting unit area on the surface at $\mathrm{AB}$, and let AC, BD be cross-sections perpendicular to the tube. Apply Gauss's theorem to the surface of which ACBD is a section, and find the value $\sigma$ of the surface density which would account for the change in intensity at the surface on the supposition that the permeability is 
everywhere $\mu_{1}$. 'T'he normal intensity $\mathbf{N}$ only has value over $\mathrm{AC}$ where it is $\mathbf{H}_{1}$ and over $\mathrm{BD}$ where it is $-\mathrm{H}_{2}$, and the theorem gives

$$
\mathrm{H}_{1} \cdot \mathrm{AC}-\mathrm{H}_{2} \cdot \mathrm{BD}=4 \pi \sigma / \mu_{1}
$$

or, since $\mathrm{AC}=\mathrm{AB} \cos \theta_{1}$ and $\mathrm{BD}=\mathrm{AB} \cos \theta_{2}$ and $\mathrm{AB}=1$,

$$
\sigma=\frac{\mu_{1}}{4 \pi}\left(\mathrm{H}_{1} \cos \theta_{1}-\mathrm{H}_{2} \cos \theta_{2}\right) \text {. }
$$

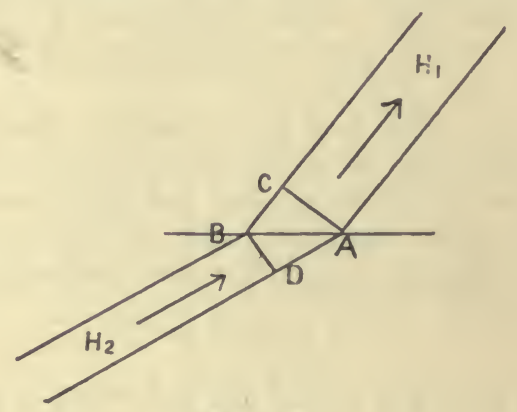

FiG. 195.

Bu $\mu_{1} \mathrm{H}_{1} \cos \theta_{1}=\mu_{2} \mathrm{H}_{2} \cos \theta_{2}$, and hence

$$
\begin{aligned}
\sigma & =\frac{\mu_{1}\left(\mu_{2}-\mu_{1}\right)}{4 \pi \mu_{2}} H_{1} \cos \theta_{1} \\
& =\frac{\mu_{2}-\mu_{1}}{4 \pi} \mathrm{H}_{2} \cos \theta_{2} \\
& \left.=\kappa_{2}-\kappa_{1}\right) \mathrm{H}_{2} \cos \theta_{2} .
\end{aligned}
$$




\title{
CHAPTER XX
}

\section{FORCES ON MAGNETISED BODIES. STRESSES IN THE MEDIUM}

\begin{abstract}
The forces on a body of constant permeability $\mu$ placed in a magnetic field in air-Illustrations-The forces on a body of permeability $\mu_{2}$ placed in a field in a medium of permeability $\mu_{1}$-Forces on an elongated bar in a uniform field in air when its permeability differs very slightly from 1The magnetic moment of a small paramagnetic or diamagnetic body placed in a magnetic field-Time of vibration of a small needle with $\mu-1$ very small suspended in the centre of the field between the poles of a magnet-Stresses in the medium which will account for the forces on magnetic bodies-The stresses on an element of surface separating media of permeabilities $\mu_{1}$ and $\mu_{2}$.
\end{abstract}

The forces on a body of constant permeability $\mu$ placed in a magnetic fleld in air. We can obtain useful expressions for the forces on a body in a magnetic field when the permeability is constant, that is, for a body which is paramagnetic or diamagnetic with permeability very near to unity.

We have seen (p. 239) that the field outside the body is altered by the introduction of the body just as it would be altered by the introduction of a layer of magnetism occupying the position of the surface of the body and of density

$$
\sigma=\frac{\mu-1}{4 \pi \mu} \mathbf{H}_{1} \cos \theta_{1}=\frac{\mu-1}{4 \pi} \mathrm{H}_{2} \cos \theta_{2}={ }_{\kappa} \mathrm{H}_{2} \cos \theta_{2}
$$

and acting everywhere according to the law $m / r^{2}$.

The forces on the poles or bodies producing the field can be calculated by replacing the magnetised body by this layer, and,

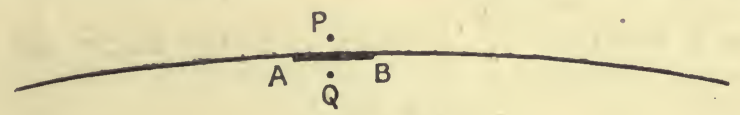

FIG. 196.

equally, the reactions on the body can be calculated by replacing it by the layer and finding the force on each element of the surface. Let AB, Fig. 196, be an element of the surface so small that it may be regarded as plane and as having a layer of magnetism of uniform density $\sigma$ all over it. 
Let $\mathbf{P}$ be a point in air and $Q$ a point in the body each very close to $A B$ and so near together that $P Q$ is very small compared with the diameter of $\mathrm{AB}$. We may divide the intensity of the field at $\mathrm{P}$ into two parts, the one $\mathrm{H}$ due to the rest of the system when $\mathrm{AB}$ is excluded, the other due to the layer on AB. The latter will be $2 \pi \sigma$ along the normal upwards in the figure if $\sigma$ is positive. The intensity at $Q$ may similarly be divided into two parts, the one due to the rest of the system when $\mathrm{AB}$ is excluded, and this will also be $H$, of the same value and in the same direction as at $P$, since $P Q$ is very small compared with the distance of either from the nearest parts of the system to which $H$ is due. The other will be $2 \pi \sigma$ along the normal downwards if $\sigma$ is positive.

Let us draw OH, Fig. 197, from a point $\mathrm{O}$ in $\mathrm{AB}$ to represent $\mathrm{H}$, $\mathrm{HC}$ parallel to the normal to AB to represent $2 \pi \sigma$ upwards, and HD parallel to the normal to represent $2 \pi \sigma$ downwards. 'Then $\mathrm{OC}$ will represent $\mathrm{H}_{1}$ at $\mathrm{P}$ and $\mathrm{OD}$ will represent $\mathrm{H}_{2}$ at $\mathrm{Q}$.

The force on $A B$ is due neither to $H_{1}$ nor to $H_{2}$, but to $H$, since $\mathrm{AB}$ as a whole exerts no force on itself. 'The force per unit area of $\mathrm{AB}$, then, is $\mathrm{H}_{\sigma}$ in magnitude and direction. We may regard $\mathrm{OH}$ in Fig. 197 as the resultant of $\mathrm{OD}$ and $\mathrm{DH}$, i.e. of $\mathrm{H}_{2}$ and $2 \pi \sigma$.

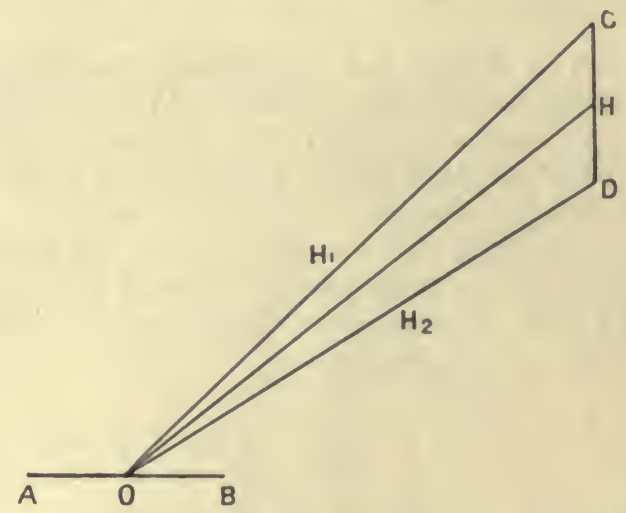

FIG. 197.

'Then we have two forces per unit area, $\mathrm{H}_{2} \sigma$ along $\mathrm{H}_{2}$ and $2 \pi \sigma^{2}$ along the normal outwards.

Putting $\sigma={ }_{\kappa} \mathrm{H}_{2} \cos \theta_{2}$ (p. 253), the pull along the normal outwards may be written

$$
2 \pi \kappa^{2} \mathrm{H}_{2}^{2} \cos ^{2} \theta_{2}
$$

where $\mathrm{H}_{2}$ makes $\theta_{2}$ with the normal.

'The system of forces $\mathrm{H}_{2} \sigma$ per unit area on each element and parallel to $\mathrm{H}_{2}$ may be conveniently represented as the resultant of a system of forces acting throughout the volume in the following way: 
Draw a narrow tube bounded by lines of induction through the body from AB to CD, Fig.198. We may describe the body as magnetised throughout with intensity of magnetisation given at any point by $\mathrm{I}={ } \mathrm{H}$, where $\mathrm{H}$ is the actual intensity of field at the point. If $\alpha$ is the cross-section at any point of the tube, $I \alpha$ is constant and is equal to the total surface polarity at $\mathrm{AB}$ or CD. Let us then cut the tube into short lengths and on each section suppose poles $\pm \mathrm{I} \alpha$. 'These poles may be supposed to be acted on with intensity $\mathrm{H}$, the actual intensity at the cross-section. 'I'hus at each section we have a pair of forces in equilibrium, and the whole system of pairs may be superposed on the system consisting of the two end forces on $\mathrm{AB}$ and $\mathrm{CD}$.

But now we have a series of short magnets each placed in a magnetic

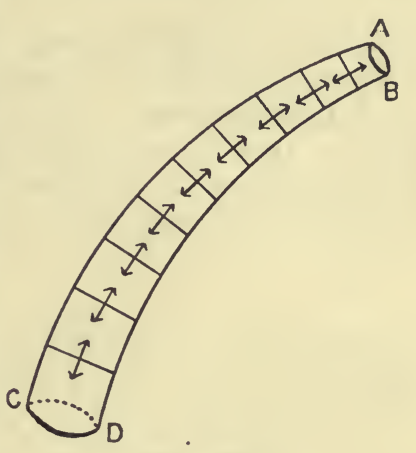

Fig. 198. field of intensity $\mathrm{H}_{2}$, the actually existing field where each is situated. If $l$ is the length of one of these and $\alpha$ its cross-section, its moment is $\mathrm{I} \alpha l={ }_{\kappa} \mathrm{H} \alpha l={ }_{\kappa} \mathrm{H} \times$ volume.

But the force on a small magnet in any direction $x$ placed in a field $H$ is (p. 228, Chapter XVIII)

$$
\text { moment } \times \frac{d \mathrm{H}}{d x}={ }_{\kappa} \mathrm{H} \frac{d \mathrm{H}}{d x} \times \text { volume, }
$$

or is $\quad \frac{1}{2} \kappa \frac{d \mathrm{H}^{2}}{d x}$ per unit volume.

We may therefore replace the surface system $\mathrm{H}_{\sigma}$ by a volume system $\frac{1}{2} \kappa \frac{d \mathrm{H}^{2}}{d x}$ per unit volume in any direction $x$.

We may further represent the volume system, if we choose, as due to a pressure within the body:

$$
p=\mathrm{C}-\frac{1}{2} \kappa \mathrm{H}^{2}
$$

where $C^{\prime}$ is some unknown constant. For this pressure will give the force on any element in direction $x$.

The forces, then, on a magnetised body in air may be calculated by supposing that we have

1. A tension $2 \pi \kappa^{2} \mathrm{H}_{2}^{2} \cos ^{2} \theta_{2}$ outwards along the normal.

2. A volume force $\frac{1}{2} \kappa \frac{d \mathrm{H}^{2}}{d x}$ per unit volume in any direction $x$ at each point in the interior.

For all but the ferromagnetic bodies $\kappa$ is very small, and the 
system represented by $2 \pi \kappa^{2} \mathrm{H}_{2}{ }^{2} \cos ^{2} \theta_{2}$ is negligible, since it is proportional to $\kappa^{2}$. Any field into which the body is introduced is sensibly the same after the introduction, so that in the calculation of the volume force the value of $\frac{d \mathrm{H}^{2}}{d x}$ maybe taken as being the same as before its introduction. We shall see how this is used in making measurements of $\kappa$ in Chapter XXII.

Illustrations. As an illustration of the foregoing let us suppose that a long bar of material with very small susceptibility $\kappa$ is hanging with its lower end in the horizontal field $\mathrm{H}$ between the poles of a magnet, Fig. 199. Let the upper end $\mathrm{O}$ be so far

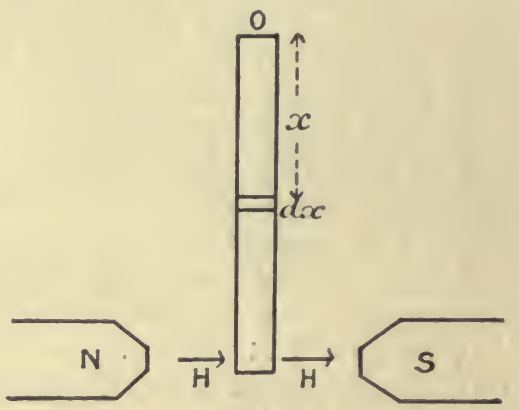

FIIG. 199.

away that the intensity of the field there may be neglected in comparison with $\mathbf{H}$.

Let us measure $x$ downwards from 0 . Let $a$ be the crosssection of the bar, .l its length. The force pulling the bar down is

$$
\begin{gathered}
\int_{0}^{1} \kappa \int_{\frac{d \mathrm{H}^{2}}{d x}}^{l} a d x=\frac{1}{2} \kappa a \int_{0}^{\mathrm{H}} d \mathrm{H}^{2} \\
=\frac{1}{2} \kappa \alpha \mathrm{H}^{2} .
\end{gathered}
$$

'The surface force $2 \pi \sigma^{2}$ per area 1 may be neglected since $\kappa$ is small.

As an illustration of a case in which this surface force is not
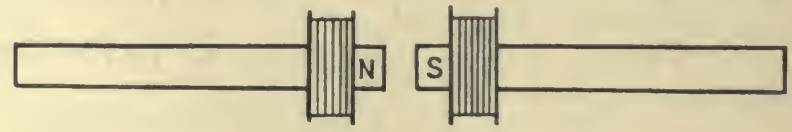

FIG. 200.

negligible, let us suppose that two iron bars of constant permeability are magnetised by coils near their neighbouring ends and placed as in Fig. 200, with unlike poles near each other. Let the bars be so 
long that the intensities at the remote ends are negligible. We shall assume that the intensity near the neighbouring ends is parallel to the axis. As above, the volume force gives us a pull $\frac{1}{2} \kappa \mathrm{H}_{2}{ }^{2}$ where $\mathrm{H}_{2}$ is the intensity just inside the iron. 'The surface pull $2 \pi \sigma^{2}$ gives us $2 \pi \kappa^{2} \mathrm{H}_{2}{ }^{2}$. 'The total pull is therefore

$$
\begin{aligned}
\frac{1}{2}{ }^{\kappa \mathrm{H}_{2}{ }^{2}+2 \pi \kappa^{2} \mathrm{H}_{2}{ }^{2}=} & \frac{1}{2}{ }^{\kappa \mathrm{H}_{2}{ }^{2}(1+4 \pi \kappa)=}=\frac{(\mu-1) \mu \mathrm{H}_{2}{ }^{2}}{8 \pi} \\
& =\frac{\mu-1}{8 \pi \mu} \mathrm{H}_{1}{ }^{2}
\end{aligned}
$$

where $\mathrm{H}_{1}$ is the intensity in the air in the gap.

The assumptions of constant permeability and of field parallel to the axis in the bar near the facing ends are only very rough approximations to the truth at the best, so that the result obtained has no real value except as an illustration of the formulx.

Forces on a body of permeability $\mu_{2}$ placed in a field in a medium of permeability $\mu_{1}$. The field is altered by the introduction of the body just as it would be by the introduction of a layer of magnetism of surface density

$$
\sigma=\left(\kappa_{2}-\kappa_{1}\right) \mathrm{H}_{2} \cos \theta_{2}
$$

and acting everywhere according to the law $m / \mu_{1} r^{2}$ (p. 251). The forces due to the body, and therefore the reactions on the body, can be calculated by replacing the body by this layer and finding the forces upon it. The forces on the surface layer $\sigma$ may be obtained as on p. 254, remembering that now the intensity due to $\sigma$ is $2 \pi \sigma / \mu_{1}$, and that this is now the value of $\mathrm{DH}$ or $\mathrm{HC}$ in Fig. 197. 'T'he intensity $\mathrm{H}$ acting on the surface layer may be regarded as the resultant of $2 \pi \sigma / \mu_{1}$ along the normal and of $\mathbf{H}_{2}$. The former gives a tension along the normal

$$
2 \pi \sigma^{2} / \mu_{1}=2 \pi\left(\kappa_{2}-\kappa_{1}\right)^{2} \mathrm{H}_{2}^{2} \cos ^{2} \theta_{2} / \mu_{1} \text {. }
$$

'The latter may be treated as on p. 255, remembering that now $\mathrm{I}=\left(\kappa_{2}-\kappa_{1}\right) \mathrm{H}$. The volume force is then found to be

$$
\frac{1}{2}\left(\kappa_{2}-\kappa_{1}\right) \frac{d \mathrm{H}^{2}}{d x}
$$

which may be represcnted as due to a pressure system

$$
\text { C }-\frac{1}{2}\left(\kappa_{2}-\kappa_{1}\right) \mathrm{H}^{2} \text {. }
$$

In para- and diamagnetic bodies in which $\kappa$ is very small the tension on the surface is negligible. Faraday's experimental 
observations that a paramagnetic body in a more paramagnetic medium behaves as if it were diamagnetic is at once explained by the expression for the volume force, $\frac{\kappa_{2}-\kappa_{1}}{2} \frac{d \mathrm{H}^{2}}{d x}$. For suppose that the body is first in air : the force on unit volume in direction $x$ is $\frac{\kappa_{2}}{2} \frac{d \mathrm{H}^{2}}{d x}$, where $\frac{d \mathrm{H}^{2}}{d x}$ may be determined from the value of the field before the introduction of the body, since when $\kappa$ is small the intensity of the field is not sensibly altered by its introduction.

Now surround the body by a medium with susceptibility $\kappa_{1}$, and the force changes to

$$
\frac{\kappa_{2}-\kappa_{1}}{2} \frac{d \mathrm{H}^{2}}{d x}
$$

and if $\kappa_{1}$ is greater than $\kappa_{2}$ the force changes sign.

Forces on an elongated bar in a uniform field in air when its permeability differs very slightly from 1 . If we neglect the outward tension proportional to $\kappa^{2}$ the other term in the surface force is $\mathrm{H}_{2} \sigma$ or ${ }_{k} \mathrm{H}_{2}{ }^{2} \cos \theta_{2}$ per unit area of surface in the direction $\mathrm{H}_{2}$. This is a force ${ }_{\kappa} \mathrm{H}_{2}{ }^{2}$ per unit area of the tube of induction. Then to the extent to which we can adopt the supposition that the tubes go straight through the body, that is, that their course is unaltered by its presence, these forces will neutralise each other in pairs at the entrance and exit of the tube. The body will to this approximation rest in any position. But Lord Kelvin showed* that when we take into account the alteration of field due to the induced magnetism, it follows that both paramagnetic and diamagnetic bar's tend to set with their longer axes along the lines of force. 'This result is important, for it implies that the equatorial setting of small diamagnetic bars placed midway between the poles of a magnet is not due to a tendency to set transverse to the lines of force, but to a tendency of the material to get into a weaker field. By setting transversely, the two ends of the bar get into the weakest fields available, for the field diminishes in the equatorial plane as we move out from the centre.

'To understand the longitudinal setting in a uniform field let us imagine a bar of square section to be made of separate cubes placed end to end. First let the body be paramagnetic and let it be held longitudinally in the field. Lach cube will tend to increase the magnetisation of its neighbours, just as each of a series of magnets tends to increase the magnetisation when they are placed end to end. Thus the polarity at the ends is increased. Next let the bar be placed transversely to the field; then each cube will tend to weaken the magnetisation of its neignbours, just as each of a inumber of magnets set side by side tends to weaken its neighbours.

* Papers on Electrcstatics and Magnetism, Sect. 691. 
Now let the bar be inclined at any angle to the field, as in Fig. 201. The mutual action of the cubes tends to increase the end magnetisation and to weaken the side magnetisation. Neglecting this mutual action, that is, neglecting the effect of the surface distribution on the intensity, the bar, as we have seen, will be in equilibrium - that is, the "centre of polarity" of the end $\mathrm{A}$ and of the side $\mathrm{B}$ must be at $c$ in the line of force $\mathrm{OH}$ through the centre $O$, Fig. 201. But taking into account the increase of polarity at $\mathrm{A}$ and the decrease at $\mathrm{B}$, the magnetic axis will be thrown on to the $\mathrm{A}$ side of $o c$ to $c_{1}$, say, and the force, acting through $c$ will tend to pull the bar into the direction of the field.

Now take a diamagnetic bar made up in the same way of cubes. If it is held longitudinally in the field, we represent its polarity by supposing that a NSP induces a NSP, while a SSP induces a SSP. At the plane of contact of two cubes where there are opposite polarities each therefore tends to weaken the other, and the net result is that the end magnetisation is decreased. Now put the bar transversely.

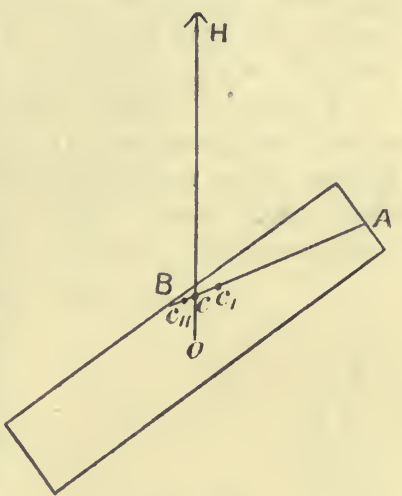

FIG. 201. Since like induces like, each cube will tend to strengthen its neighbours and the side magnetisation is increased.

If the bar is inclined at an angle to the field the mutual action of the cubes tends to increase the side magnetisation and to decrease the end magnetisation, and the centre of polarity is thrown towards $B$, say to $c_{11}$. 'This is a SSP, and the force due to the intensity $H$ will push it. The moment round the centre $O$ will tend to make the bar turn round parallel to the lines of force.

The magnetic moment of a small paramagnetic or diamagnetic body placed in a magnetic field. If the field intensity is $\mathrm{H}$ before the body is introduced, that value is almost unaltered by its introduction when $\mu-1$ is exceedingly small. 'The magnetisation at any point may therefore be taken as $\mathrm{I}={ }_{\kappa} \mathrm{H}$. Any small cylinder of length $l$ and cross-section $\alpha$ and with axis along $\mathrm{H}$ may be regarded as a magnet with poles $\pm \mathrm{I}_{\alpha}={ }_{\kappa} \mathrm{H}_{\alpha}$, and with moment $\kappa \mathrm{H} \alpha l=\kappa \mathrm{H} \times$ volume. The total magnetic moment will be the resultant of all such moments, and if the body is so small that $\mathrm{H}$ may be regarded as uniform in it, its moment will be $\kappa \mathrm{H} \times$ volume.

Force on the small body. We showed in Chapter XVIII that the force on a small magnet of moment $\mathbf{M}$ in direction $x$ is $\frac{\mathbf{M} d \mathbf{H}}{d x}$. If its volume is $d v$, this is equal to 


$$
{ }_{\kappa} \mathbf{H} \frac{d \mathbf{H}}{d x} d v=\frac{1}{2} \kappa \frac{d \mathbf{H}^{2}}{d x} d v .
$$

If the body is large, the total force in direction $x$ is

$$
{ }_{2}^{\kappa} \frac{d}{d x} \int \mathrm{H}^{2} d v
$$

from which we may again draw the conclusion that if $\kappa$ is positive the body tends to move towards the position where $\int \mathrm{H}^{2} d v$ is a maximum, and that if $\kappa$ is negative it tends to move towards the position where $\int \mathrm{H}^{2} d v$ is a minimum.

Time of vibration of a small needle with $\mu-1$ very small suspended in the centre of a field between the poles of a magnet, the field being symmetrical about the axis and the equatorial plane. Lord Kelvin has shown * that the time of vibration of a needle-shaped body

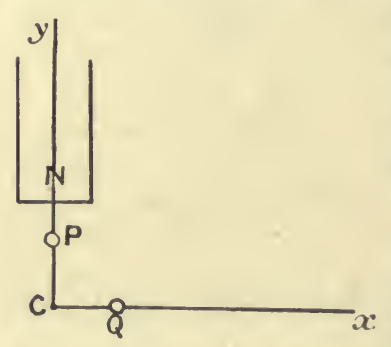

depends only on its density and magnetic susceptibility, and not on the particular shape or size of the needle so long as it is a needle and small. 'This very remarkable result has led to a method of determining susceptibilities.

First imagine a particle of volume $v$, density $\rho$, and susceptibility $\kappa$ to be somehow freed from weight and to be suspended, under the action of magnetic forces only, by a very fine fibre from the central point $\mathbf{C}$ of the field represented in Fig. 202.

If paramagnetic, it will set at $\mathbf{P}$ with the fibre along $\mathbf{C P}$; if diamagnetic, at $\mathbf{Q}$, with the fibre along $\mathrm{CQ}$; and if dis-

FIG. 202. turbed it will vibrate, forming as it were a magnetic pendulum. Let us take axes as in the figure. We may put the intensity near $C$ in the form

$$
\mathrm{H}^{2}=\mathrm{H}_{\mathrm{c}}{ }^{2}-\mathrm{A} x^{2}+\mathrm{B} y^{2} .
$$

For putting $\mathrm{H}$ as a function of $x$ and $y$ and expanding, first powers must disappear to satisfy symmetry. Powers higher than the second may be neglected if we are near enough to $C$. The signs of the terms with $\mathrm{A}$ and $\mathrm{B}$ as coefficients are determined by the fact that $\mathbf{H}$ increases from $\mathrm{C}$ towards $\mathrm{P}$, while it decreases from $\mathrm{C}$ towards $\mathbf{Q}$.

'The magnetic force on the particle in any direction $s$ is $\frac{1}{2} \kappa v \frac{d \mathrm{H}^{2}}{d s}$. Let us suppose it to be diamagnetic, so that its undisturbed

* Electrostatic's and Magnetism, Sect. 670. 
position is $Q$. Let it be disturbed through a small angle $\theta$ from CQ. The force along the arc $s$, the restoring force, is

$$
\frac{1}{2} \kappa v \frac{d \mathbf{H}^{2}}{a d \theta}
$$

putting $d s=a d \theta$, where $a$ is the length CQ of the fibre. But in the disturbed position $x=a \cos \theta$ and $y=a \sin \theta$, so that

since $\theta$ is small.

$$
\mathrm{H}^{2}=\mathrm{H}_{\mathrm{c}}{ }^{2}-\mathrm{A} a^{2} \cos ^{2} \theta+\mathrm{B} a^{2} \sin ^{2} \theta
$$

and

$$
\begin{aligned}
\frac{d \mathrm{H}^{2}}{d \theta} & =2 a^{2}(\mathrm{~A}+\mathrm{B}) \sin \theta \cos \theta \\
& =a^{2}(\mathrm{~A}+\mathrm{B}) \sin 2 \theta \\
& =2 a^{2}(\mathrm{~A}+\mathrm{B}) \theta
\end{aligned}
$$

'I'he acceleration on the particle is therefore

$$
\begin{aligned}
& \frac{1}{2} \frac{\kappa v}{\rho v a} \frac{d \mathrm{H}^{2}}{d \theta} \\
= & \frac{\kappa}{\rho} a(\mathrm{~A}+\mathrm{B}) \theta \\
= & \frac{\kappa}{\rho}(\mathrm{A}+\mathrm{B}) s
\end{aligned}
$$

and as we suppose $\kappa$ negative, this is towards $\mathbf{Q}$. The vibration is therefore harmonic of period

This is independent of $a$.

$$
\mathrm{T}=2 \pi \sqrt{\frac{\rho}{-\kappa(\mathrm{A}+\mathrm{B})}} .
$$

If, then, a thin bar or needle is pivoted at $\mathrm{C}$ so that its weight need not be considered, every particle of it tends to vibrate in the same time, and it vibrates as a whole with the above period.

If the bar is paramagnetic, it is easily seen that the sign of the denominator must be changed, and that the time is

$$
\mathrm{T}=2 \pi \sqrt{\frac{\rho}{\kappa(\mathrm{A}+\mathrm{B})}}
$$

The experiments made by Rowland and Jacques, using this formula, are referred to in Chapter XXII.

Stresses in the medium which will account for the forces on magnetic bodies. It is not so easy to work out a system of stresses in the surrounding medium to account for the observed forces on magnetised bodies as it is to find a system which will account for the forces on electrified bodies or for 
dielectrics in the electric field. In the electric field, for instance, between two parallel metal plates charged with $\pm \sigma$ the tension along the lines of force must be $2 \pi \sigma^{2}$ in order to account for the work done in separating the plates. But there is no exactly corresponding magnetic system, nothing like a charged conductor. Since the magnetic induction tubes are continuous, there are magnetic stresses on each side of the surface separating two media, and energy is distributed throughout the tube. We cannot make a real magnetic experiment like that with an attracted disc electrometer, where we weigh the pull by the dielectric on the surface. Even the electric system is incleterminate, for we can hardly argue conclusively from the pulls in a uniform field to those in a field in which the level surfaces are curved. The tensions and the pressures keeping an elenent in equilibrium might depend on the curvature of the lines of force. But we can at least show that the electric stress system devised by Maxwell holds with a uniform field, and can further show that it gives forces which agree with the actual forces on bodies. We have no satisfactory starting-pointfor a magnetic system of stresses, and it is probably best to be guided by analogy with the electric field, remembering again that the problem is really indeterminate and that the system assumed is only one of an infinite number of possible solutions. Maxwell* proposed a magnetic stress system which in a medium of permeability 1 gives a tension $\frac{\mathrm{H}^{2}}{8 \pi}$ along the lines of force and an equal pressure $\frac{\mathrm{H}^{2}}{8 \pi}$ perpendicular to them. In a medium of permeability $\mu$, homogeneous and isotropic, so that induction and intensity are in the same direction, his system consists of a tension $\mathrm{H}^{2} / 8 \pi$ along, and a pressure $\mathrm{H}^{2} / 8 \pi$ perpendicular to, the lines of force, and in addition a tension $(\mu-1) \frac{\mathrm{H}^{2}}{4 \pi}={ }_{\kappa} \mathrm{H}^{2}$ along the lines of force. This stress system is not satisfactory. The system of tension and pressure, each equal to $\mathrm{H}^{2} / 8 \pi$, would keep each element of a homogeneous medium in equilibrium, and it can be shown that it would account for the surface forces on a magnetised body in air, that is, for the forces on the surfaces of discontinuity. But the additional tension $\kappa \mathrm{H}^{2}$ within the body would only form a system in equilibrium in a uniform field. We shall consider in place of Maxwell's system one analogous to the electric system, and shall therefore assume that there is in a homogeneous isotropic body a tension $\frac{\mu \mathrm{H}^{2}}{8 \pi}$ along the lines of force and an equal pressure $\frac{\mu \mathrm{H}^{2}}{8 \pi}$ perpendicular to them.

* Elestricity and Magnetism, ii. Sect. 642. The stresses in the medium are discussed at length in Walker's Aberrution and the Electro-mugnetic Field, and in Heaviside's Electrical Papers, vol. ii. 
This system, like the electric system, will maintain each element of a body in which $\mu$ is constant in equilibrium. Tne forces acting on the two sides of an element of surface where $\mu$ changes will not be in equilibrium, and their resultant ought to be equal to the mechanical force acting on the surface, equal, in fact, to the forces deduced by supposing that the imaginary surface layer is acted on by the field existing about it. We shall find that the two systems are not equivalent, element by element, but that they give the same resultant force on the body as a whole. Perhaps this is as much as could be expected.

The tensions and pressures $\frac{\mu \mathrm{H}^{2}}{8 \pi}$ are in equilibrium on any element of a medium in which $\mu$ is constant, exactly as the tensions and pressures $\frac{\mathrm{KE}^{2}}{8 \pi}$ are in equilibrium on an element of dielectric. Any portion of a medium with $\mu$ constant is therefore in equilibrium, and if we draw a closed surface $S$ wholly within the medium, the tensions and pressures acting on $\mathrm{S}$ from outside form a system in equilibrium. Equally the reactions from inside on the medium outside $\mathrm{S}$ are in equilibrium. If we imagine the medium within $\mathrm{S}$ to have the samedistribution of induction, but some other constant value of $\mu$, we can superpose the stresses due to this on the actual system without destroying equilibrium. Obviously we need not even have the distribution of induction the same in the superposed system so long as it is a possible distribution obeying the tube law, but we shall only require the more limited case.

The stresses on an element of surface separating media of permeabilities $\mu_{1}$ and $\mu_{2^{\circ}}$ Let the plane of Fig. 203 be that through the normal to the surface and the axis of a tube in the $\mu_{1}$ medium, meeting the surface in an element $d$ S represented by its trace $A B$. Let the tube make $\theta_{1}$ with the normal. Let the tube be rectangular in section with two sides parallel to the plane of the figure, and consider the equilibrium of the wedge $\mathrm{ABC}$. The forces across $\mathrm{AC}$

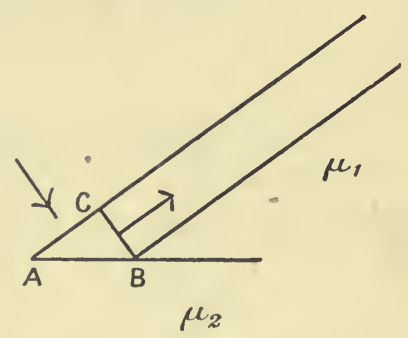

FIG. 203. and $\mathrm{BC}$ must give the force across $\mathrm{AB}$.

Let $\mathrm{N}$ be the normal force outwards across $\mathrm{AB}$ and $\mathrm{T}$ the tangential force in the direction $\mathrm{AB}$. Since $\mathrm{BC}=d \mathrm{~S} \cos \theta$ and $\mathrm{AC}=d \mathrm{~S} \sin \theta$, we have, on resolving the assumed tension and pressure,

or

$$
\begin{aligned}
\mathbf{N} d \mathbf{S} & =\frac{\mu_{1} \mathrm{H}_{1}^{2}}{8 \pi} \cdot d \mathbf{S} \cos ^{2} \theta_{1}-\frac{\mu_{1} \mathrm{H}_{1}^{2}}{8 \pi} d \mathbf{S} \sin ^{2} \theta_{1} \\
\mathbf{N} & =\frac{\mu_{1} \mathrm{H}_{1}^{2}}{8 \pi}\left(\cos ^{2} \theta_{1}-\sin ^{2} \theta_{1}\right)=\frac{\mu_{1} \mathrm{H}_{1}^{2}}{8 \pi} \cos 2 \theta_{1} .
\end{aligned}
$$


Similarly

$$
\begin{gathered}
\mathrm{I}^{\prime}=\frac{\mu_{1} H_{1}{ }^{2}}{8 \pi} \cos \theta_{1} \sin \theta_{1}+\frac{\mu_{1} H_{1}^{2}}{8 \pi} \sin \theta_{1} \cos \theta_{1} \\
=\frac{\mu_{1} H_{1}^{2}}{8 \pi} \sin 2 \theta_{1} .
\end{gathered}
$$

'The resultant of $\mathbf{N}$ and ' $\mathrm{T}$ is a tension on unit area of the surface in a direction making an angle $2 \theta_{1}$ with the normal. 'The resultant of the system $\mathbf{N}$ and ' $\mathrm{I}$ over the body should give the actual force.

But we may add to this system the system due to the internal stresses, for these form a system in equilibrium. We may take these internal stresses either of their actual value, $\frac{\mu_{2} \mathrm{H}_{2}{ }^{2}}{8 \pi}$, or, as is more convenient, of value $\frac{\mu_{1} \mathrm{H}_{2}{ }^{2}}{8 \pi}, i$.e. we superpose on the actua systein $\frac{\mu_{2} \mathrm{H}_{2}{ }^{2}}{8 \pi}$ a system $\left(\mu_{1}-\mu_{2}\right) \frac{\mathrm{H}_{2}{ }^{2}}{8 \pi}$.

When the internal system is superposed we get

$$
\begin{aligned}
\mathbf{N} & =\frac{\mu_{1} H_{1}{ }^{2}}{8 \pi}\left(\cos ^{2} \theta_{1}-\sin ^{2} \theta_{1}\right)-\frac{\mu_{1} H_{2}^{2}}{8 \pi}\left(\cos ^{2} \theta_{2}-\sin ^{2} \theta_{8}\right) \\
& =\frac{\mu_{1} H_{1}^{2} \cos ^{2} \theta_{1}-\mu_{1} H_{2}{ }^{2} \cos ^{2} \theta_{2}}{8 \pi}, \text { since } H_{1} \sin \theta_{1}=H_{2} \sin \theta_{y} \\
& =\frac{H_{1} \cos \theta_{1}+H_{2} \cos \theta_{2}}{2} \cdot \mu_{1} \frac{H_{1} \cos \theta_{1}-H_{2} \cos \theta_{2}}{4 \pi} \\
& =\text { Normal component of } \frac{\check{H}_{1}+\check{H}_{2}}{2} \times \text { surface density } \sigma,
\end{aligned}
$$

where the accents are put to denote the vector values.

$$
\begin{array}{r}
\mathrm{T}=\frac{\mu_{1} \mathrm{H}_{1}^{2}}{4 \pi} \sin \theta_{1} \cos \theta_{1}-\frac{\mu_{1} \mathrm{H}_{2}^{2} \sin \theta_{2} \cos \theta_{2}}{4 \pi} \\
=\mathrm{H}_{1} \sin \theta_{1} \cdot \mu_{1}\left(\frac{\mathrm{H}_{1} \cos \theta_{1}-\mathrm{H}_{2} \cos \theta_{2}}{4 \pi}\right)
\end{array}
$$

since $H_{1} \sin \theta_{1}=H_{2} \sin \theta_{2}$

$$
=\frac{H_{1} \sin \theta_{1}+H_{2} \sin \theta_{2}}{2} \cdot \mu_{1} \frac{H_{1} \cos \theta_{1}-H_{2} \cos \theta_{2}}{4 \pi}
$$

$=$ 'langential component of $\frac{\check{\mathrm{H}}_{1}+\check{\mathrm{H}}_{2}}{2} \times$ surface density $\sigma$. 
Hence the assumed system gives the same force on a whole body as the system obtained by considering the imaginary surface layer of magnetism.

It is easy to show that the system of pressures and tensions in air will account for the force on a straight wire carrying a current $\mathrm{C}$ due to a field $\mathrm{H}$ at right angles to the wire or in the field due to another parallel current. 


\title{
CHAPTER XXI
}

\section{THE MEASUREMENT OF PERMEABILITY AND THE ALLIED QUALITIES IN FERRO- MAGNETIC BODIES}

\begin{abstract}
Magnetometer method-Ballistic method-Magnetisntion in very weak fields-Magnetisation in very strong fields-Ewing's isthuus methodNickel and cobalt-The hysteresis loop and the energy dissipated in a cycle-Mechanical model to illustrate hysteresis.
\end{abstract}

A KNowledge of the permeability of iron, and of the way in which $B$ varies as $H$ is changed, is of the highest importance as a guide to dynamo construction. In this chapter we shall give an account of the principles upon which are based some of the methods used to investigate the relation between $\mathrm{B}$ and $\mathrm{H}$ for iron and steel, and we shall indicate the general nature of the results.* It will be necessary to assume some knowledge of electro-magnetism.

Magnetometer method. In this method, applicable to a long thin rod or wire, the rod is placed within a solenoid some-

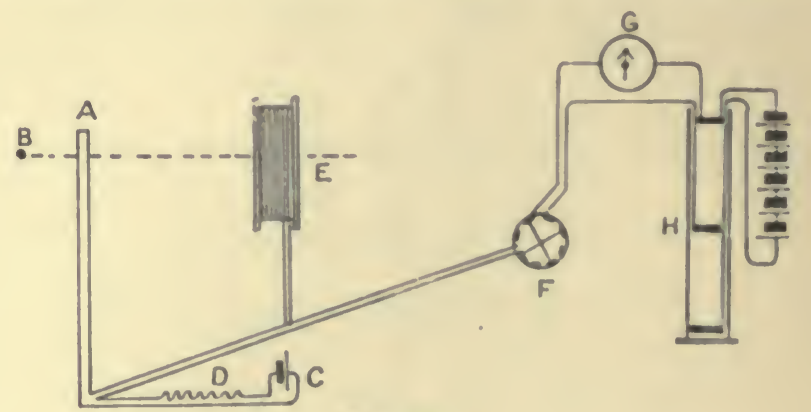

FIG. 204.

what longer than itself and is magnetised by a known current through the solenoid. One end of the wire is near a magnetic needle - the magnetometer-and from the deflection of this needle the magnetisation of the rod is calculated. A very convenient arrangement, due to Ewing, is represented in Fig. 204.

* The reader will find the details of the methods and their adaptation to technical needs in Ewing's. Mngnetic Induction in Iron and other Metals. 
A represents the solenoid placed vertical. B is the magnetometer nearly level with the upper end of $A$, and at a distance from it so short that the action on the needle is almost entirely due to the upper pole. The rod must be adjusted to the height at which the action is a maximum. The magnetising current through the solenoid is supplied by the cells on the right, and on its way to the amperemeter $\mathbf{G}$ and the commutator $\mathbf{F}$ it passes through the adjustable liquid resistance $\mathrm{H}$, which consists of a tall glass jar with three discs of zinc immersed in a dilute solution of zinc sulphate.

It will be seen from the figure that the circuit from one terminal of the battery passes through the top disc on its way to $\mathrm{G}$ and the solenoid, while the other terminal is connected by an insulated wire to the bottom disc. The other end of the solenoid is connected to the middle disc, which is movable up and down. If the middle disc touches the top one, the current flows through the jar and none goes through the solenoid. As the middle disc is lowered the current through the solenoid increases and is a maximum when the middle disc touches the bottom one. On its way to the solenoid the current passes through the "compensating coil " $\mathbf{E}$, which is adjusted in such a position that when there is no rod within the solenoid the action of $\mathbf{E}$ on the magnetometer is just equal and opposite to that of the solenoid. The magnetic action of the solenoid itself may therefore be left out of account.

In order that the earth's field may be eliminated a second solenoid is wound round the first and a constant current from a cell $\mathrm{C}$ is passed through it and through a resistance $\mathrm{D}$ so adjusted that the field due to the constant current within this second solenoid is equal and opposite to the earth's vertical field.

When a specimen is to be tested it is necessary in the first place to demagnetise it. This is effected by the "method of reversals." The rod is placed within the solenoid and the full current is put on by lowering the middle plate in the liquid resistance to contact with that at the bottom. The commutator $\mathbf{F}$ is then rapidly revolved, so as to alternate the field within the solenoid. While the rapid alternation is going on the middle plate is slowly raised through the liquid to the top so that the amplitude of the alternating current slowly decreases from its largest value to zero, and the rod is found to be completely demagnetised by the process. 'This method serves also to adjust the current through the outer solenoid to the value required to neutralise the earth's field, for the reversals only completely demagnetise the rod when the field is finally reduced to zero. It is useful to employ for this adjustment a special rod of annealed soft iron.

'The specimen having been demagnetised, let a current of A amperes be established in the solenoid. If there are $n$ turns per centimetre the field due to it is $\mathrm{H}^{\prime}=4 \pi u \mathrm{~A} / 10$. 
If $\mathrm{I}$ is the intensity of magnetisation of the rod and $\pi \alpha^{2}$ is its cross-section, its pole strength is $\pi a^{2} I$. Let $\mathbf{Q Q}^{\prime}$, Fig. 205, represent the two poles of the rod, $o$ being the magnetometer, the horizontai component of the field in which it is placed being perpendicular to the plane of the figure, and being of intensity $\mathbf{F}$.

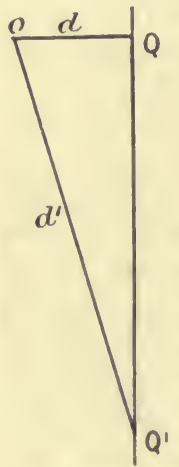

Let $o \mathbf{Q}=d, o \mathbf{Q}^{\prime}=d^{\prime}$.

If $\theta$ is the deflection of the needle, then

$$
\mathrm{F} \tan \theta=\pi a^{2} \mathrm{I}\left(\frac{1}{d^{2}}-\frac{1}{d^{\prime 2}} \frac{d}{d^{\prime}}\right)
$$

and

$$
\mathrm{I}=d^{2} \mathrm{~F} \tan \theta / \pi a^{2}\left(1-\frac{d^{3}}{d^{3}}\right) \text {. }
$$

This gives $\mathbf{I}$ in terms of measurable quantities. Since $B=\mu H=(1+4 \pi \kappa) H=H+4 \pi I$, if we know $H$ and $I$ we find $\mathrm{B}$ and $\mu$. The value of $\mathrm{H}^{\prime}$ due to the magnetising current is not, however, the actual value of the field, for the poles formed in the specimen produce

FIG. 20\%. an opposing field, and some assumption is needed to allow for the reduction due to this. If the specimen is very thin with diameter, say, 1/400 of the length, the reduction

E

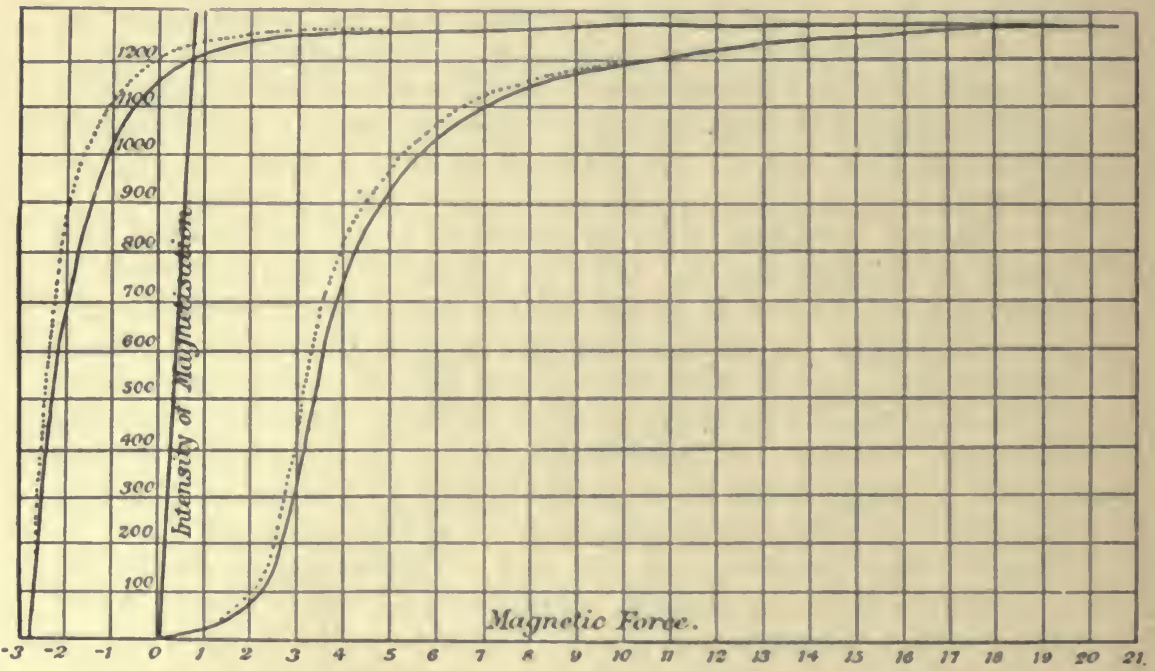

FIG. 206.

is very small. Ewing supposes that such a specimen may be treated as an elongated ellipsoid and finds that the actual field is

$$
\mathrm{H}=\mathrm{H}^{\prime}-0.00045 \mathrm{I} \text {. }
$$


The following Table gives particulars of a test made by Ewing on an annealed wrought-iron wire of diameter $0.077 \mathrm{~cm}$. and length $30.5 \mathrm{~cm}$. or 400 diameters. The current was increased step by step from 0 to a value giving a field of 22.27 gausses and was then reduced by steps through 0 to $-2 \cdot 87$ gausses. 'The continuous curves in Fig. 206 (taken from Ewing) show the values of $I$ as ordinates plotted against $\mathrm{H}^{\prime}$ as abscissæ, the lower curve for increasing current and the upper one for decreasing current.

\begin{tabular}{|c|c|c|c|c|c|}
\hline $\begin{array}{l}\text { Vield due to mag- } \\
\text { netising current } \\
\mathrm{H}^{\prime}=4 \pi n \mathrm{~A} / 10\end{array}$ & $\begin{array}{c}\text { Intensity of } \\
\text { magnetisation } \\
\text { I }\end{array}$ & $\begin{array}{c}\text { Corrected } \\
\text { field } \\
\mathbf{H}=\mathbf{H}^{\prime}-\mathbf{4 5} \mathrm{I} / 10^{5}\end{array}$ & $\begin{array}{c}\text { Magnetic sus. } \\
\text { ceptibility } \\
\kappa\end{array}$ & $\begin{array}{c}\text { Magnetic } \\
\text { induction } \\
\mathrm{B}=\mathrm{H}+4 \pi \mathrm{I}\end{array}$ & $\begin{array}{c}\text { Magnetic } \\
\text { permeability } \\
\mu=-\mathrm{B} / \mathrm{H}\end{array}$ \\
\hline $0 \cdot 0$ & 0 & 0.0 & - & 0 & - \\
\hline $0 \cdot 32$ & 3 & 0.32 & 9 & 40 & 120 \\
\hline 0.85 & 13 & 0.84 & 15 & 170 & 200 \\
\hline $1 \cdot 38$ & 33 & $1 \cdot 37$ & 24 & 420 & 310 \\
\hline $2 \cdot 18$ & 93 & $2 \cdot 14$ & 43 & 1,170 & 550 \\
\hline $2 \cdot 80$ & 295 & $2 \cdot 67$ & 110 & 3,710 & 1390 \\
\hline $3 \cdot 50$ & 581 & $3 \cdot 24$ & 179 & 7,300 & 2250 \\
\hline $4 \cdot 21$ & 793 & $3 \cdot 89$ & 204 & 9,970 & 2560 \\
\hline $4 \cdot 92$ & 926 & $4 \cdot 50$ & 206 & 11,640 & 2590 \\
\hline $5 \cdot 63$ & 1009 & $5 \cdot 17$ & 195 & 12,680 & 2450 \\
\hline $6 \cdot 69$ & 1086 & $6 \cdot 20$ & 175 & $13,6+0$ & 2200 \\
\hline $8 \cdot 16$ & 115. & $7 \cdot 94$ & 145 & 14,510 & 1830 \\
\hline $10 \cdot 23$ & 1192 & $9 \cdot 79$ & 122 & 14,980 & 1530 \\
\hline $12 \cdot 11$ & 1212 & $11 \cdot 57$ & 105 & 15,230 & 1320 \\
\hline $15 \cdot 61$ & 1238 & $15 \cdot 06$ & 82 & 15,570 & 1030 \\
\hline $20 \cdot 32$ & 1255 & $19 \cdot 76$ & 64 & 15,780 & 800 \\
\hline $22 \cdot 27$ & 1262 & $21 \cdot 70$ & 58 & 15,870 & 730 \\
\hline $16 \cdot 42$ & $125 \mathrm{~S}$ & - & - & - & - \\
\hline $8 \cdot 46$ & 1245 & 一 & - & - & - \\
\hline $4 \cdot 92$ & 1235 & - & - & - & - \\
\hline $3 \cdot 15$ & 1225 & - & - & - & - \\
\hline $1 \cdot 38$ & 1205 & - & - & - & - \\
\hline $0 \cdot 0$ & 1162 & - & - & - & - \\
\hline-0.41 & 1135 & - & - & - & - \\
\hline-0.81 & 1092 & - & - & - & - \\
\hline$-1 \cdot 10$ & 1056 & - & - & - & - \\
\hline-1.45 & 979 & - & - & - & - \\
\hline$-1 \cdot 80$ & 840 & - & - & - & - \\
\hline$-2 \cdot 20$ & 551 & - & - & - & 二 \\
\hline $\begin{array}{l}-2.51 \\
-2.87\end{array}$ & $\begin{array}{r}232 \\
-40\end{array}$ & - & - & $=$ & - \\
\hline & & & & & \\
\hline
\end{tabular}

'The figure shows how the correction from $\mathrm{H}^{\prime}$ to $\mathrm{H}$ may be inade graphically. Since the correction is $-0.00045 \mathrm{I}$, draw a straight line $O E$ with abscissa 0.45 at $I=1000$. 'The abscissa of $\mathrm{OE}$ for any value of $\mathrm{I}$ will give the correction to $\mathrm{H}^{\prime}$ at that value. 'Then draw a new curve at each point a distance to the left of the continuous one equal to the abscissa of OE at that level, and this new curve will show the relation between $\mathbf{I}$ and $\mathbf{H}$. 'The dotted curves in the figure are thus obtained. 'The 'I'ables show that with a long thin wire the correction is not great. The 
maximum value of $\kappa$ is raised from 185 to 206 and $\mu$ is increased practically in the same proportion.

'The magnetometer method is not the best for accurate absolute measurements. The polarity is not all concentrated at or close to the ends of the wire and its distribution cannot be calculated. 'The value obtained for I is therefore inexact. The correction to $\mathrm{H}$, too, is only an approximation to the true correction. More exact results are obtained by the ballistic method described below. But the magnetometer method is specially well adapted for investigations on the effects of stresses on magnetisation, since the wire tested can be subjected to end pulls or to twists while within the solenoid. It serves well, too, to give a general idea of the results obtained on subjecting a specimen to a magnetising force and on carrying the magnetising force through a cycle, inasmuch as it is easy to work and the theory is simple and gives fairly good results when the specimen tested is very thin compared with its length. By it we may obtain the curve already briefly described in Chapter XIV, p. 183, and represented in Fig. 207.

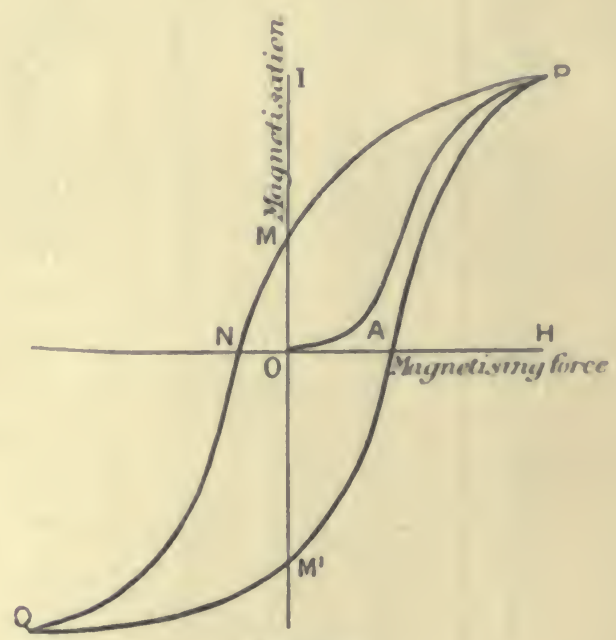

FIG. 207.

It is interesting to note that in the test represented by Fig. 206 the residual magnetisation when the current is reduced to 0 is still 1162, or 92 per cent. of the value it attained with the increasing current. When the current is reversed the magnetisation does not disappear till the field $\mathrm{H}=-2 \cdot 75$ gausses, and this is the value of the "coercive force" for the specimen. It is to be remembered that though the external effect of the magnetisation disappears under this coercive force, the wire is not in the demagnetised condition from which it started. It is still affected by 
the process through which it has been carried. For if the current in the reverse direction is further increased the curve does not descend as it originally ascended from $\mathrm{O}$, but is much steeper.

The Ballistic method. This was first devised by Weber and has since been used in many important researches. We shall describe its application to a ring-shaped specimen of the metal to be tested. Round the ring is wrapped a coil of wire $\mathbf{A}$, Fig. 208, and in the circuit is a battery, an amperemeter $\mathrm{G}_{1}$, an adjustable resistance $B_{1}$, and a commutator $K$. If $r$ is the mean

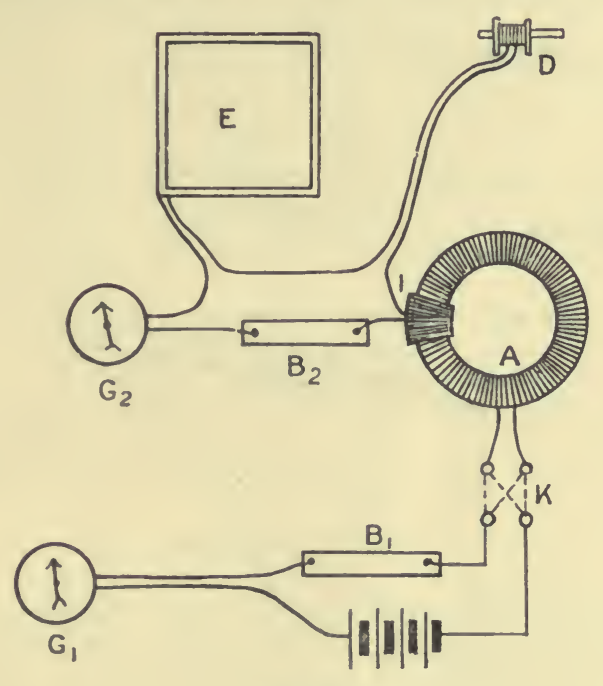

Fig. 208.

radius of the ring and if the number of turns of the coil is $\mathbf{N}$ per $\mathrm{cm}$. length of the circle of radius $r$, a current $\mathrm{A}$ in the coil gives a field of intensity $\mathrm{H}=4 \pi \mathrm{NA} / 10$ at the distance $r$. We shall suppose the ring so thin that $\mathrm{H}$ is constant across a section, and of this value. Round one part of the ring a secondary coil I of $n$ turns is wrapped. In the circuit of this coil is a ballistic galvanometer $G_{2}$, an adjustable resistance $B_{2}$, an " earth coil" E, and a small coil $\mathrm{D}$, which can be used to reduce the swings of the needle $G_{2}$. 'The earth coil is used to find the total quantity of electricity passing through $G_{2}$ for a given throw. suppose that $\mathrm{E}$ is laid on a horizontal table. If the sum of the areas of its turns is $\mathrm{S}$ and the intensity of the earth's vertical ficld is $\mathbf{V}$, the total flux of induction through it is VS. If $\mathbf{E}$ is suddenly turned over through $180^{\circ}$, the change in induction is $2 \mathrm{VS}$, and if $\mathrm{R}$ is the total resistance of the circuit the total flow of electricity is $\frac{2 \mathrm{VS}}{10 \mathrm{R}}$ coulombs. 
If the throw due to this is $\theta$ any other throw $\theta$ will indicate a flow of

$$
\frac{2 \mathrm{VS}}{10 \mathrm{R}} \frac{\theta}{\theta} \text { coulombs. }
$$

In carrying out a test on a ring it is first denagnetised by reversals, as already described in the magnetometer method. 'Then a small current $A_{1}$ is established in the coil $A$,producing within it a field $H_{1}=4 \pi \mathrm{NA}_{1} / 10$. If this creates induction $B_{1}$ through the secondary coil the flow of electricity in the ballistic galvanomcter will be $\frac{n \mathrm{~B}_{1}}{\mathbf{R}}$, and if the throw is $\theta_{1}$

$$
\text { whence } \quad \begin{aligned}
\frac{n B_{1}}{\mathrm{R}} & =\frac{2 V \operatorname{VS} \theta_{1}}{10 \operatorname{R} \theta} \\
\mathrm{B}_{1} & =\frac{2 \mathrm{VS} \theta_{1}}{10 n \theta}
\end{aligned}
$$

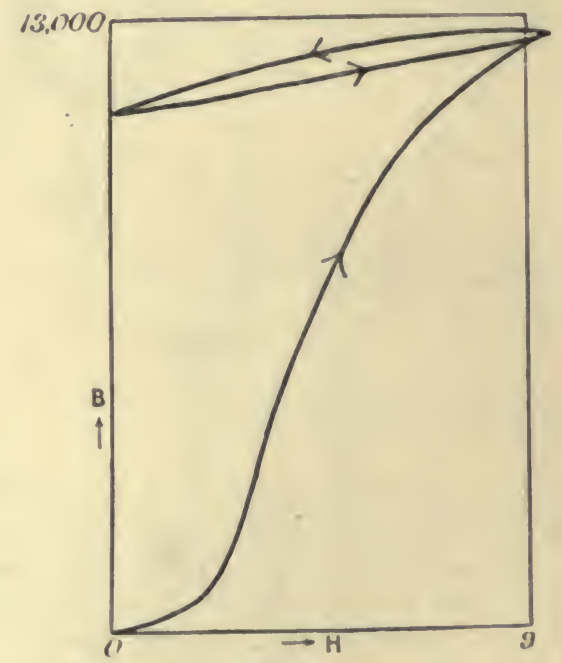

FIc. 209.

Unless the primary coil A is very close in every turn to the iron a correction must be subtracted from $B_{1}$ for the induction through the space between wire and iron. If the mean area of the coil is $\mathrm{S}^{\prime}$ and the cross-section of the iron is $\mathrm{S}$, the amount to be subtracted from $B_{1}$ to give the induction in the iron is $\left(S^{\prime}-S\right) H_{1}$. But the correction is very small.

The current in $\mathrm{A}$ is then increased by an addition of $\boldsymbol{A}_{2}$ amperes. 'This increases the field by $\mathrm{H}_{2}$, and the induction by $\mathrm{B}_{2}$ measured by the new throw of the ballistic galvanometer. The 
total field will be $\mathrm{H}_{1}+\mathrm{H}_{2}$ and the total induction $\mathrm{B}_{1}+\mathrm{B}_{2}$. To find the induction per sq. $\mathrm{cm}$. in the iron, we must divide the total induction by the cross-section $\mathrm{S}$. The current may be increased step by step to any desired amount and then taken round a cycle in any desired way, the values of $\mathrm{H}$ and $\mathrm{B}$ being determinate at every point.

Fig. 209 represents a test carried out by Ewing (loc. cit. pp. 70-72) on a wrought-iron ring, mean radius $5 \mathrm{~cm}$. and cross-section $0.0483 \mathrm{sq} . \mathrm{cm}$. The magnetising coil had 474 turns and the secondary had 167 turns. H was gradually raised from 0 to $9 \cdot 14$, then taken back to 0 and again raised to $9 \cdot 14$.

The induction per sq. $\mathrm{cm}$. in the iron rose to 12,440 at the maximum. The maximum value of $\mu=\mathrm{B} / \mathrm{H}$ was 1740 when the value of $H$ was 4.95 .* $^{*}$

Magnetisation in very weak fields. Baur, $\uparrow$ experimenting by the ballistic method on a ring of soft iron with values of $\mathrm{H}$ rising to 0.384 , found that $\mathrm{I}$ and $\mathrm{B}$ could be represented by the parabolic formulæ

$$
\begin{aligned}
& I=14 \cdot 5 \mathrm{H}+110 \mathrm{H}^{2} \\
& \mathrm{~B}=183 \mathrm{H}+1382 \mathrm{H}^{2}
\end{aligned}
$$

whence

and

$$
\kappa=14 \cdot 5+110 \mathrm{H}
$$

$$
\mu=183+1382 \mathrm{H} \text {. }
$$

'These results suggest that for extremely small values of $H, \kappa$ and $\mu$ are constant. This point was investigated by Lord Rayleigh $\ddagger$ and the suggestion was confirmed. He used the magnetometer method with a second compensating coil through which the magnetising current passed, adjusted in such a position that it neutralised the effect of the iron wire tested (Swedish iron unannealed) on the magnetometer needle when the intensity of the magnetic field within the solenoid did not exceed 0.04 . When the intensity of the field was still further reduced the compensation remained perfect and the reduction was carried down to an intensity about one two-thousandth of the initial value. It might therefore be concluded that, between $\mathrm{H}=0$ and $\mathrm{H}=0.04, \kappa$ and $\mu$ are constant and that within this range there is no retentivity. The value of $\mu$ was about 100 . Above 0.04 the constancy no longer held.

Lord Rayleigh also found that if a specimen was already magnetised by a moderate force, and there was then made a small change in the magnetising force opposite in direction to that

* For other arrangements in which the ballistic method is used, especially adapted for technical requirements, the reader should consult Ewing on Magnetic Induction in Iron and other Metals, chap. xii.

† Wied. Ann., xi. p. 399 (1880).

$\ddagger$ Scientific Papers, vol. ii. p. 579. 
already employed the susceptibility for that change was nearly the same as when the specimen was initially unmagnetised.

Thus if OAP, Fig. 210, represents the magnetisation curve with an increasing current, $\mathrm{OA}$ is the part under small forces with constant

- $\mu$ and $\kappa$ and no retentivity. If the magnetisation is carried up to

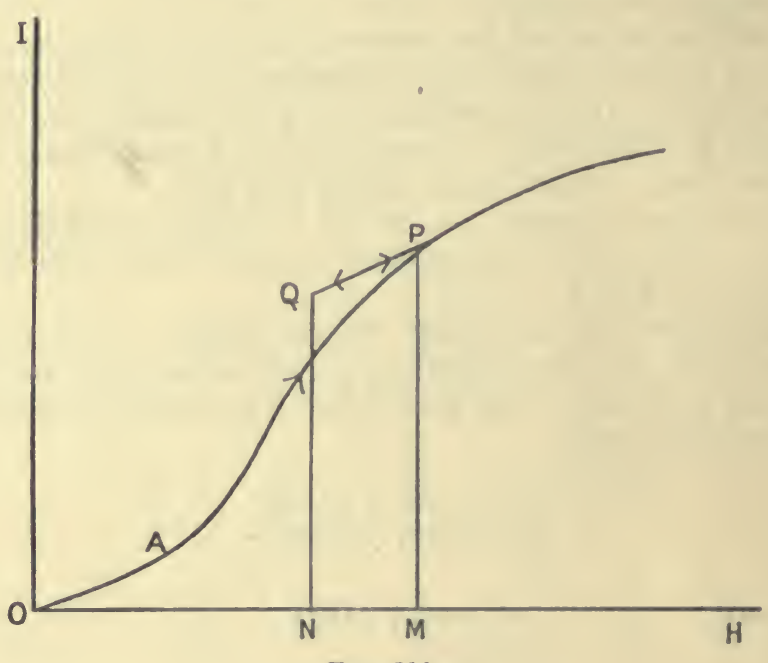

FIG. 210.

$\mathrm{P}$ and then a small decrease $\mathrm{MN}$ is made in $\mathrm{H}, \mathrm{PQ}$ parallel to $\mathrm{OA}$ is the magnetisation curve. If $H$ is again increased by N.M we arrive again at $P$.

But when the initial magnetisation is much increased and the region of saturation is approached, $P Q$ has a less slope, or the susceptibility for the small change, which we may denote by $\frac{d \mathrm{I}}{d \mathrm{H}}$, decreases.

With annealed iron there is a time lag in the magnetisation. This effect has been examined by Ewing, and we refer the reader to his work, p. 129, for an account of the experiments.

Magnetisation in very strong fields. Ewing's isthmus method. In Fig. 211 NS are the pole pieces of a very powerful electro-magnet, the tips being bored out cylindrically about an axis through o perpendicular to the plane of the figure. A bobbin B just fits in between the poles. It has a coil of one turn wrapped close about the central neck and with its ends connected to a ballistic galvanometer. It can be rotated through $180^{\circ}$ about the axis through $o$ so that the induction through it is reversed. The throw of the galvamometer on this rotation gives the value of the induction through the neck. Since there is no free magnetism on the surface of the iron about the neck the field 
is continuous within and without the iron there, and Ewing calculated that if the pole pieces had an angle of $78^{\circ} 28^{\prime}$ the field would be nearly uniform as well as continuous. In order to measure the intensity $\mathrm{H}$ of the field a second coil of somewhat larger area was wound round the first, the difference in areas being

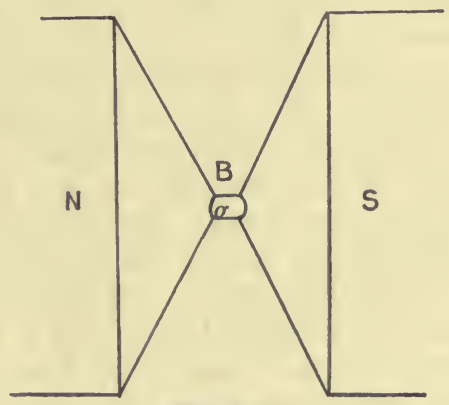

FIG. 211.

known. The difference in the galvanometer throw when the second coil was used and when the first coil was used gave the value of $\mathrm{H}$ in the air, and therefore in the iron.

'The following 'Table (Ewing, loc. cit. p. 150) gives the results obtained with a specimen of Swedish iron, and will serve as a type :

\begin{tabular}{|c|c|c|c|}
\hline H & B & I & $\mu$ \\
\hline 1,490 & 22,650 & 1680 & $15 \cdot 20$ \\
3,600 & 24,650 & 1680 & $6 \cdot 85$ \\
6,070 & 27,130 & 1680 & $4 \cdot 47$ \\
8,600 & 30,270 & 1720 & $3 \cdot 52$ \\
18,310 & 38,960 & 1640 & $2 \cdot 13$ \\
19,450 & 40,820 & 1700 & $2 \cdot 10$ \\
19,880 & 40,140 & 1700 & $2 \cdot 07$ \\
\hline
\end{tabular}

All the specimens examined showed the same tendency towards a limiting value of $I$, that is, towards saturation, and a consequent tendency in $\mu$ to diminish towards unity. 'These are merely two aspects of the same fact, for

$$
\mu=\frac{B}{H}=\frac{H+4 \pi I}{H}=1+\frac{4 \pi I}{H} .
$$

If $\mathrm{I}$ has a finite limiting value and $\mathrm{H}$ is indefinitely increased $\mu$ must ultimately equal 1 .

These results have been confirmed by du Bois, using an entirely different method depending on the Kerr effect of rotation of the plane of polarisation of light reflected from a magnet pole.*

* Phil. Mag., vol. xxix. (1890), pp. 253 and 293. 
The relations between permeability and magnetising force vary greatly with the quality of the iron or steel tested. 'The softer the iron the greater the maximum permeability. With steel the permeability is in general lower the harder the steel. Alloys of iron with manganese, "manganese steel," have been made with a permeability less than $1 \cdot 5$, and Hopkinson found that a certain specimen of nickel steel containing 25 per cent. of nickel had practically a constant permeability of $1 \cdot 5$.

Nickel and cobalt. 'The methods which we have described for iron may be used also for nickel and cobalt, and similar results are obtained, though the magnetisation and permeability for a given magnetising force are much less than for iron. For an annealed nickel wire Ewing found that $\kappa$ attained a maximum value of 23.5 when the field was $9 \cdot 5$. 'This corresponds to $\mu=296$. In general the saturation value of I for nickel is a third or a fourth the value for wrought iron. Cobalt has less susceptibility than nickel in weak fields, but greater in strong fields.

The hysteresis loop and the energy dissipated in a cycle. We have seen that if we start with demagnetised iron and apply a magnetising force gradually increasing to a maximum of considerable value $+H_{m}$, and if then we carry $H$ through a complete cycle $+\mathrm{H}_{\mathrm{m}}, \mathrm{O},-\mathrm{H}_{\mathrm{m}}, \mathrm{O},+\mathrm{H}_{\mathrm{m}}$, the magnetisation I and the induction $\mathrm{B}$ return to very nearly the same values, and the magnetisation curve forms a very nearly closed loop. Probably I and B do not return to exactly the same values. But successive repetitions of the cycle give curves very nearly overlying the first, and the longer the cycle is repeated the more nearly do the successive curves coincicle. We shall suppose that the stage is reached in which we may take the magnetisation curve as a closed loop-the liysteresis loop.

In each cycle a certain amount of energy is dissipated and appears as heat in the iron. We may calculate the energy dissipated in a way somewhat different from that already given in Chapter XIX. We shall suppose that we are using the ring method and that the magnetising coil is wound so closely on the iron that the induction may be considered as all within the iron.

Let $\mathrm{E}$ be the E.M.F. put on to the magnetising coil from outside. Let $\mathrm{B}$ be the number of induction tubes through unit area of the iron, and let $\alpha$ be the area of its cross-section. Then $a \mathrm{~B}$ is the total induction. If there are $u$ turns of the coil the virtual number of tubes through it is $n_{a} \mathbf{B}$. If $\mathbf{C}$ is the current through the coil and $\mathbf{R}$ its resistance the current equation is

$$
\mathrm{E}-n a \frac{d \mathrm{~B}}{d t}=\mathrm{CR}
$$

or

$$
\mathrm{E} d t=n a d \mathrm{~B}+\mathrm{CR} d t .
$$

Multiplying by C,

$$
\mathrm{CE} d t=n_{a} \mathrm{C} d \mathrm{~B}+\mathrm{C}^{2} \mathrm{R} d t .
$$


The left-hand side, CEdt, is the energy supplied to the coil by the external source of current in time $d t .{ }^{\mathbf{2}} \mathbf{R} d t$ is the energy dissipated as heat in the wire according to Joule's law. Then $n_{a} \mathrm{C} d \mathrm{~B}$ is the energy supplied to the iron in increasing the induction by $d \mathrm{~B}$.

If $l$ is the mean length of the iron its volume is $l_{\alpha}$, so that the energy supplied to unit volume is $\frac{n \mathrm{C} d \mathrm{~B}}{l}$

If $\mathrm{H}$ is the intensity of the field within the coil, $\mathrm{H} l=4 \pi n \mathrm{C}$, so that $\frac{n \mathrm{C}}{l}=\frac{\mathrm{H}}{4 \pi}$.

Hence the energy supplied per unit volume in the increase of magnetic induction $d \mathrm{~B}$ is

$$
\frac{\mathrm{H} d \mathrm{~B}}{4 \pi} \text {. }
$$

Let CD, Fig. 212, represent the hysteresis loop when the curve is drawn with abscissa representing $\mathbf{H}$ and ordinate representing $\mathrm{B}$. Let $\mathrm{PQ}$ be an element in the ascending branch. The strip $\mathrm{PQNM}$ is $\mathrm{H} d \mathrm{~B}$, so that the energy supplied is $\frac{\mathrm{PQNM}}{4 \pi}$. In the descending branch there will be an element $\mathbf{Q}^{\prime} \mathbf{P}^{\prime}$, with both $\mathbf{H}$

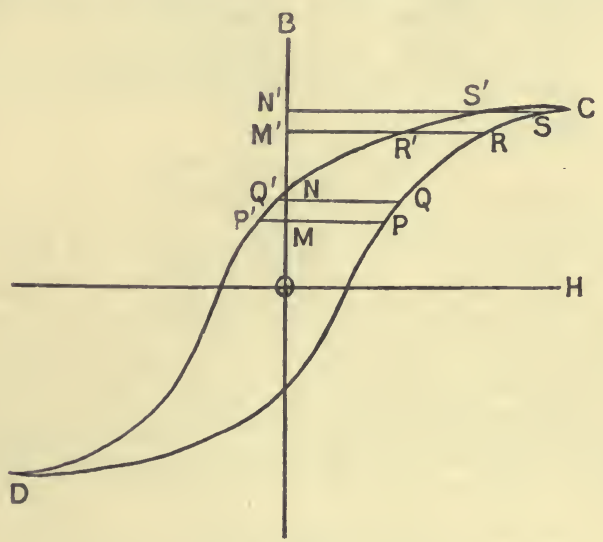

FIG. 212.

and $d \mathrm{~B}$ negative, so that $\mathrm{H} d \mathrm{~B}$ will be positive and the energy supplied there is $+\frac{\mathrm{P}^{\prime} Q^{\prime} N M}{4 \pi}$. 'The total for the two elements is

$$
+\frac{P Q Q^{\prime} \mathrm{P}}{4 \pi} \text {. }
$$

For an element RS higher up the curve on the ascending branch and for the corresponding element $\mathbf{R}^{\prime} \mathbf{S}^{\prime}$ on the descending 
branch $\mathrm{H}$ is + for both, while $d \mathrm{~B}$ is + in one, - in the other, and the total energy supplied is the difference $\frac{\mathbf{R S S}^{\prime} \mathbf{R}}{4 \pi}$. It is easily seen, then, that in the complete cycle the energy supplied is $\int \frac{\mathrm{H} d \mathbf{B}}{4 \pi}=\frac{1}{4 \pi} \times$ sum of all such strips as $\mathbf{P Q Q}^{\prime} \mathbf{P}^{\prime}=\frac{1}{4 \pi} \times$ area of hysteresis loop.

If we put $\mathrm{B}=\mathrm{H}+4 \pi \mathrm{I}$, then $d \mathrm{~B}=d \mathrm{H}+4 \pi d \mathrm{I}$, and

$$
\int \frac{\mathrm{H} d \mathrm{~B}}{4 \pi}=\int \frac{\mathrm{H} d \mathrm{H}}{4 \pi}+\int \mathrm{H} d \mathrm{I}=\left[\frac{\mathrm{H}^{2}}{\pi}\right]+\int \mathrm{H} d \mathrm{I} .
$$

Now $\left[\frac{\mathrm{H}^{2}}{2 \pi}\right]$ is 0 round a cycle, since $\mathrm{H}$ returns to its initial value. Then the energy supplied is $\int \mathbf{H} \mathbf{d} \mathbf{I}$. Or if the hysteresis curve is drawn for $\mathrm{H}$ and $\mathrm{I}$, the energy supplied is represented by the area of the loop. Since the iron returns to its original condition after a complete cycle, and mean while there is an addition of energy, we may conclude that this energy is dissipated and is the equivalent of the heat which appears.

It can be shown by a thermodynamic eycle that if $\mu$ is constant for a given temperature so that the iron can be magnetised by a reversible process, and if $\mu$ varies with change of temperature, then energy must be supplied to a magnetised body to keep the temperature constant if the magnetisation is increased. The amount thus supplied * is

$$
\begin{aligned}
d Q & =\theta \mathrm{I} d \mathrm{I} \cdot \frac{1}{\kappa^{2}} \frac{d \kappa}{d \theta} \\
& =\frac{\mathrm{H} d \mathrm{~B}}{4 \pi} \frac{\theta}{\mu} \frac{d \mu}{d \theta} .
\end{aligned}
$$

We can only apply this formula to real iron in the initial stage investigated by Lord Rayleigh, Fig. 210, and in the small reversals from subsequent stages where the process of magnetisation is practically reversible. We camnot deduce the heat required to keep the temperature constant in a non-reversible process, nor the heat appearing in a non-reversible cycle. It is possible that if we could account for the heat required in the reversible process by some physical explanation, the same explanation would account for the heat dissipated in hysteresis. But the mechanical illustration given (p. 279) would suggest that they are distinct, that the magnetisation consists of two parts, one strictly reversible and with the heating also reversible, another part non-reversible, dissipating energy by something analogous to friction.

In connection with this subject some very interesting experi-

* Thomson, Applications of Dynamics to Physics and Chemistry, p. 103. 
ments by Baily are to be noted.* Swinburne had pointed out that if Ewing's model, described in Chapter $\mathrm{XV}$, gives a right idea of the physical nature of the magnetisation of iron, then the hysteresis of iron rotating in a constant magnetic field should show a falling off as saturation is approached. For if all the molecular magnets are parallel to the imposed force they will simply rotate as the field rotates relative to the iron, without dissipating energy. Baily found that up to a value of $B$ in the neighbourhoou of 15,000 the hysteresis increased, and for both soft iron and hard steel it is not very different in a rotating field from its value in a field reversed in the ordinary way. If the rotating field is further increased, then hysteresis falls off and apparently would vanish for a value of B about 21,000, though that value was not actually reached.

Mechanical model to illustrate hysteresis. It may be interesting to describe a mechanical model which, if it could be constructed, would give relations between force $\mathbf{P}$ and displacement $d$ very nearly corresponding to the relations between magnetic intensity $\mathrm{H}$ and magnetisation $\mathrm{H}$. Let A B, Fig. 213, be a cylinder of air with unit-area cross-section and of length 2D. Let CDEF be a short cylinder moving within it with friction $\mathrm{F}$. Let $\mathrm{G}$ be a piston

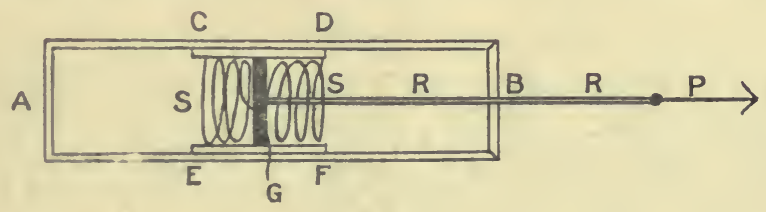

FIG. 213.

moving without friction in CDEF, but against springs SS attached to CDEF. Let the piston-rod $\mathbf{R}$ pass out through an air-tight hole at $\mathbf{B}$, and let any desired force $\mathbf{P}$ be applied at the end of $\mathbf{R}$. I cet us suppose that $\mathrm{G}$ has area practically equal to the unit-area of cross-section of $\mathrm{AB}$. Let us start with the piston in the centre and with equal pressures of air $p$ on the two sides. It is easily seen that if the piston is displaced $d$ to the right, then, as far as the air pressure alone is concerned, $\mathbf{R}$ must be pulled with a force

$$
\mathrm{P}_{1}=2 p \frac{d / \mathrm{D}}{1-d^{2} / \mathrm{D}^{2}}
$$

and the curve giving the relation between $P_{a}$ and $d$ will be somewhat as in Fig. 214.

But the springs will also introduce a force $P_{2}$, which may be considered separately, on the supposition that the ends of $\mathrm{A}$ and $\mathrm{B}$ are open. At first the spring force, and therefore $\mathbf{P}_{\mathbf{2}}$, is proportional to the displacenient of $G$, and will continue so till that displacement has come to a certain value $d_{1}$, at which the spring

* Plil. Trans., A, vol. 77 (1896), p. 715. 
force can just overcome the friction $\mathrm{F}$. 'Thereafter the pull $\mathrm{P}_{2}$ will be constant and equal to $\mathrm{F}$ as long as the motion is from right to left.

To reverse the motion the force inust be reversed from

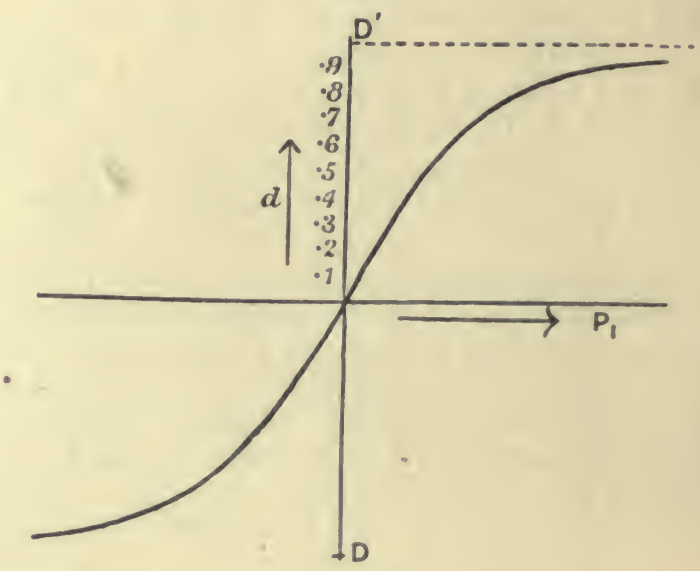

Fio. 214.

$P_{2}=+F$ to $P_{2}=-F$, giving a displacement of $\mathrm{G}=2 d_{1}$ before $\mathrm{CF}$ begins to move. Then $\mathrm{P}_{2}$ remains constant at $-\mathrm{F}$ till another reversal. Again there is a change of $2 \mathrm{~F}$ and a displacement meanwhile of $2 d_{1}$; then motion with constant $\mathrm{F}$. The diagram

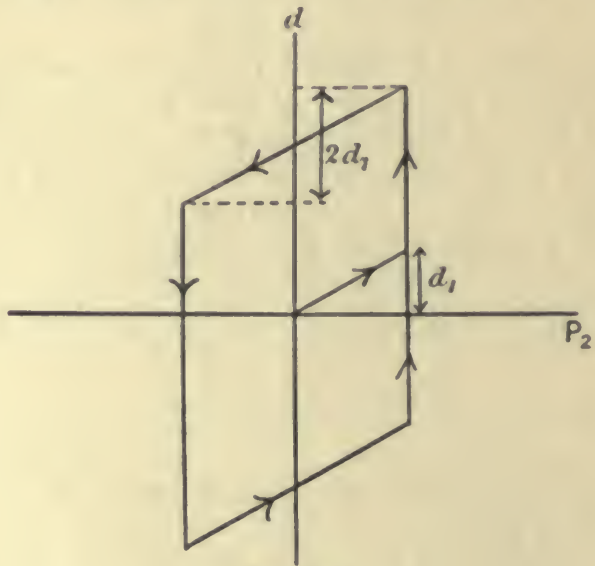

Fio. 215.

giving the relation between $\mathrm{P}_{2}$ and $d$ will be as in Fig. 215. After the first motion from the centre it is a parallelogram.

Now let us add the two forces $P_{1}+P_{2}=P$ for a given displacement and we get Fig. 216, a hysteresis loop very much 
resembling the magnetic hysteresis loop. 'The reader may easily follow out the close analogies between the two systems.

We have only attempted in this chapter to give such an account of the magnetisation of the ferromagnetic metals that the reader may gain a general idea of the methods of experiments and a

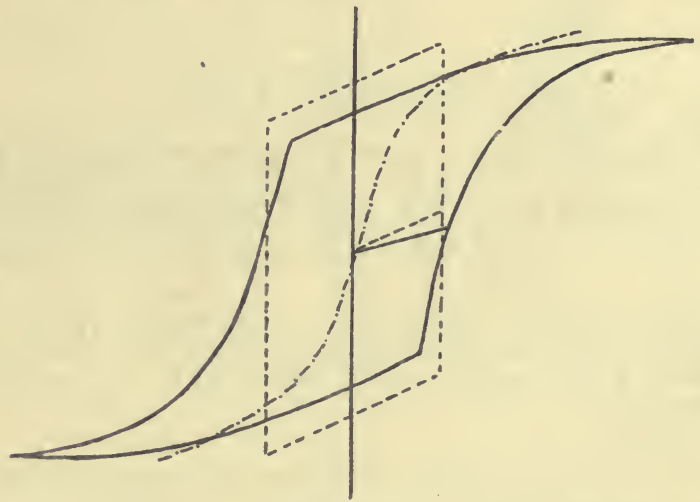

FIG. 216.

general idea of the results obtained. For details of the methods and results, for effects of temperature and stress, and for modifications of the methods for technical purposes, the reader should consult Ewing's Magnetic Induction in Iron and Other Metals on which this chapter is based. That work is a very clear account by one who has largely contributed to our knowledge of magnetic phenomena. Additional information will be found in Rapports au Congrès International, 1900, vol. ii, in articles by $\mathrm{H}$. du Bois and E. Warburg. 'The latter contains some account of the various changes in iron as the temperature rises, investigated by F. Osmond. Another and a very full account of the present state of the subject will be found in Winkelmann's Handbuch der Physik, vol. v. Further information will be found in the paper by S. P. Thompson referred to on p. 187. 


\title{
MEASUREMENTS OF SUSCEPTIBILITY AND PERMEABIIITY OF PARAMAGNETIC AND DIAMAGNETIC SUBSTANCES
}

\author{
Faraday's experiments - Rowland's experiment - Experiments of \\ v. Ettingshausen-Curie's experiments-Curie's law-Wills' experiment \\ -Townsend's experiment-Pascal's experiments-The electron theory \\ - The magneton.
}

'THE earliest measurements of the magnetic qualities of substances other than ferromagnetic were made, soon after Faraday's discovery of diamagnetism, by Pliicker,* by E. Becquerel, $\uparrow$ and by Faraday. $\neq$ 'Though excelled in accuracy by later work, these early experiments are worthy of attention, since the methods used are simple in principle and easily understood. 'They consisted in suspending a body at a given point in the field between the poles of a strong magnet near to, but not quite in, the axis, and in measuring either by a common balance or by a torsion balance the furce acting on the body. 'That force in any direction $x$ is $\frac{\kappa_{2}-\kappa_{1}}{2} \frac{d H^{2}}{d x}$ per unit volume, where $\kappa_{2}$ is the susceptibility of the body and $\kappa_{1}$ that of the surrounding medium, and $\mathrm{H}$ is the intensity of the field (p. 258). If bodies of the same volume are used in succession at the same point in the same field, the forces are proportional to $\alpha_{2}-\kappa_{1}$ or to $\mu_{2}-\mu_{1}$.

We shall select for description here the experiments of Faraday. It is to be noted that he gave an account of them only in a lecture at the Royal Institution, and the account was evidently intended as provisional. But the work was apparently never continued.

Faraday's experiments. Faraday used a large horse-shoe permanent magnet to give the field. 'The plan is shown in Fig. 217. 'The body, shaped into a cylinder, was hung at $m$, usually $0.5 \mathrm{in}$. from the strongest part of the field $c$. To measure the force on it, it was hung by a fine glass fibre $5 \mathrm{in}$. long from one end of a torsion rod parallel to ae, but with the end exactly over $m$. A counterpoise was attached to the other arm of the torsion rod. The

* Pogg. Ann., lxxiv. (1849), p. 321.

† Ann. de Chem. et de Phys., xxxii. (1851), p. 68.

¥ Exp. Res., iii. p. 497. 


\section{PARAMAGNETIC \& DIAMAGNETIC SUBSTANCES 283}

fibre supporting the torsion rod was attached to a graduated head, and the position of the rod was read by a mirror and scale. Its zero position was determined by removing the cylinder and placing an equal load on the rod itself, where any force due to the field was negligible. This zero position was so arranged that the vertical through the end of the rod passed through the point $m, 0.5 \mathrm{in}$. from $c$. Then the body was suspended, and it was forced inwards or outwards according as it was more or less paramagnetic than the surrounding medium, the torsion rod

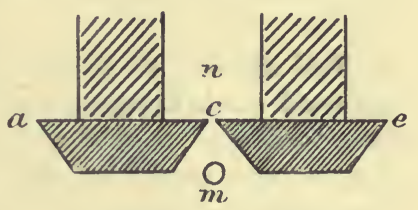

FIG. 217. being pulled round one way or the other. The torsion head was then turned round till the rod was ugain in the zero position and the angle of torsion measured the force acting. Since the force due to the field was exceedingly small compared with the weight of the body, the suspending fibre was so near the vertical that the body could be regarded as at the point vertically under the end of the arm.

'The standard force selected was that on water in air, and was taken as 100. 'To determine it a cylinder of glass was suspended in air. It was repelled from the zero position, and required $15^{\circ}$ of torsion to restore it. It was then suspended in water. Now it was attracted and required $54.5^{\circ}$ of torsion to restore it. If $\kappa_{\mathrm{g}}, \kappa_{\mathrm{a}}, \kappa_{\mathrm{w}}$ are the susceptibilities of glass, air, and water respectively,

$$
\begin{gathered}
\kappa_{\mathrm{d}}-\kappa_{\mathrm{d}} \propto-15 \\
\kappa_{\mathrm{g}}-\kappa_{\mathrm{w}} \propto+54.5 \\
\text { whence } \quad \kappa_{\mathrm{w}}-\kappa_{\mathrm{n}} \propto-69.5 .
\end{gathered}
$$
chosen.

Then $69.5^{\circ}$ of torsion are represented by 100 on the scale

Now, suppose that the same cylinder is hung in any other liquid with susceptibility $\kappa_{1}$, and that $\theta^{\circ}$ of torsion are required to restore it.

$$
\begin{aligned}
& \text { Then } \kappa_{\mathrm{g}}-\kappa_{1} \propto \theta . \\
& \text { But } \quad \kappa_{\mathrm{g}}-\kappa_{\mathrm{a}} \propto-15 . \\
& \text { Subtracting } \kappa_{1}-\kappa_{\mathrm{a}} \nsim-(15+\theta)
\end{aligned}
$$

and this is expressed on the air-water scale by

$$
-(15+\theta) \frac{100}{69 \cdot 5} \text {. }
$$

If the susceptibility $\kappa_{\mathrm{s}}$ of a solid was required, taking a cylinder 
of it of the same shape and size as the glass, if the restoring force in air was $\phi$,

$$
\kappa_{\mathrm{s}}-\kappa_{\mathrm{a}} \propto \phi
$$

whence $\kappa_{\mathrm{s}}-\kappa_{\mathrm{a}}$ on the air-water scale was $\phi \times \frac{100}{69 \cdot 5}$.

Full details of the work are not given, but apparently a bulb was used containing in succession various liquids and gases, and the same bulb was used when exhausted so that the values of $x$ relative to a vacuum could be deduced. 'Taking a vacuum as having zero susceptibility and $\kappa_{n}-\kappa^{w}=100$, Faraday found for $\kappa_{n}$ the value 3.5 , and therefore for water $\kappa_{w}=-96.5$. The following 'Table gives some of his measurements on this scale. Becquerel's values for some of the same substances are given, adapted to Faraday's scale. Becquerel took $\kappa_{\mathrm{B}}-\kappa^{w}=-10$ and $\kappa_{\mathrm{A}}=0$. To compare them his numbers have been multiplied by 10 , and have been added to 3.4. The numbers for air and water must of course agree :

$\begin{array}{lcr} & \text { FARADAY } & \text { BECQUERE. } \\ \text { Oxygen } & 17 \cdot 5 & 21.4 \\ \text { Air } & 3 \cdot 4 & 3.4 \\ \text { Vacuum } & 0 & \\ \text { Carbon dioxide } & 0 & \\ \text { Hydrogen } & -0 \cdot 1 & \\ \text { Zinc } & -74 \cdot 6 & -21.6 \\ \text { Alcohol } & -78.7 & -75.5 \\ \text { Water } & -96.6 & -96.6 \\ \text { Carbon disulphide } & -99 \cdot 6 & -129 \cdot 6 \\ \text { Sulphur } & -118 & -110.3 \\ \text { Bismuth } & -1967 \cdot 6 . & -2142\end{array}$

The closeness of the values in many cases is evidence of the excellence of the work of the two experimenters.

Faraday made experiments at different distances and found different results. The value for bismuth, for instance, rose quite considerably as the distance increased. This has not been confirmed by later work, and there was no doubt some undetected error important at the greater distances, but apparently not so important at the half-inch distance, since the results there obtained are in very fair accordance with the best measurements.

We shall now describe some of the later experiments, selecting typical methods.

Rowland's experiment. Rowland and Jacques* used the method of a vibrating needle, of which the simple theory is given on p. 260. According to that theory the time of vibration of a needle-shaped body in the field is

- Rowland's Physical Papers, pp. 75 and 184. 


$$
\mathrm{T}=2 \pi \sqrt{\frac{\rho}{-\kappa(\mathrm{A}+\mathrm{B})}},
$$

where $A$ and $B$ are known if we know the rates of change of field along and perpendicular to the axis. These rates were determined by means of an "exploring coil" which was connected to a ballistic galvanometer and placed, to begin with, at the centre of the field and with its plane perpendicular to the field. It was then moved short measured distances and the galvanometer throws gave the changes to be measured. The coil was also moved from the centre right out of the field and thus the field at the centre was found.

The method was applied to crystals of bismuth and of calc spar, and it was found that the value of $\kappa$ in each case was different along different axes. The values of $10^{9} \mathrm{~K}$ obtained along two axes were

$\begin{array}{ccc} & \text { BISMUTH } & \text { CAIC SPAR } \\ 10^{9} \kappa_{1} & -12554 & -38 \\ 10^{9} \kappa_{2} & -14324 & -40\end{array}$

These appear to be the earliest determinations in absolute measure.

Experiments of von Ettingshausen. Four different methods were used by von Ettingshausen * to determine the susceptibility of bismuth. In the first method two primary coils were placed in series and an intermittent current was sent through them. Round the primary coils were wound secondary coils connected in series through a galvanometer but in opposition-that is, so that the simultaneous currents induced in them by the primaries went in opposite directions through the galvanometer. There was a commutator in the circuit worked at such a rate that both make and break currents went through the galvanometer in the same direction. First the coils were so adjusted that the secondary currents just neutralised each other. Then a bismuth cylinder was inserted in one of the primaries. Since the permeability of bismuth is less than unity, the mutual induction between that primary and its secondary was decreased, the current induced in it was less than the current in the other secondary, and the galvanometer was deflected. The bismuth was then withdrawn, and in its place was inserted a solenoid through which a known current was passed, made and broken as frequently as the primary current in the previous experiment. This induced an alternating current in the secondary, rectified as before through the galvanometer. The magnetisation of the bismuth under the original known primary could thus be compared with the magnetisation-if we may so term it - of the inserted solenoid with its known current, and the susceptibility of the bismuth could be determined. 
A precaution is necessary in this method. Foucault currents are induced in the bismuth at both make and break in the primary. If each Foucault current has time to be formed and die away before the next change in the primary, it will induce equal and opposite currents in the secondary and may be left out of account. But if the interval between make and break is too short, the Foucault current formed in one interval extends partly over the next interval, where its effect on the secondary is commuted, and so a balance is left over to affect the galvanometer. Experiments showed that the frequency must not exceed more than 8 or 9 per second.

In a second method a horizontal bismuth cylinder was suspended from one end of a torsion rod so as to be partly within, partly without a horizontal solenoid through which a known current was passed and the force on the bismuth was measured. That force is, as we have seen, $\int \frac{\kappa}{2} \frac{d \mathrm{H}^{2}}{d x} d v$. H could be calculated for each element of the cylinder and hence $\kappa$ was determinate.

A third method resembled that of Rowland and Jacques, and need not be described.

In a fourth method a bismuth cylinder was brought between the poles of a strong magnet. At a distance from the poles either 9 or $17 \mathrm{~cm}$. was a magnetometer needle. Before the bismuth was inserted the effect of the magnet on the needle was compensated by a second magnet. When the bismuth was inserted it behaved as a feeble reversed magnet and deflected the magneto. meter needle through

$$
\theta=\frac{2 M}{H d^{3}}
$$

where $\mathrm{M}$ is the magnetic moment of the bismuth and $\mathrm{H}$ is the field at the magnetometer. 'The deflection was too minute for direct measurement. The bismuth was inserted and withdrawn with a periodicity the same as that of the needle, producing ultimately a swing $g$. If $\kappa$ is the damping coeflicient of the swings the direct deflection $A$ is then given by

$$
\Lambda=g_{\kappa+1}^{\kappa-1}
$$

The values obtained by the successive methods for $-10^{6} \mathrm{~K}$ were : I, 13.57 ; II, $14 \cdot 11$; III, $15 \cdot 3$; IV, 13.6 . The second method is probably the most exact.

Curie's experiments. P. Curie * determined the ralue of $\kappa$ for a number of substances and investigated the effect of change of temperature, using a method like that of Becquerel and Faraday. The body to be tested was on a torsion arm, and the torsion was measured which was needed to keep it at $o$ in the field

* Inn. de Chim.et Dhys., 7 ser. V. (1895), p. 289 ; or Eiuercs de P. Curie, p. 232. 
PARAMAGNETIC \& DIAMAGNETIC SUBSTANCES 287 of an electro-magnet EEEE, Fig: 218. The figure represents the horizontal plane through the magnetic axis. 'The torsion rod was arranged so that the body could move along $o x$. The force upon it was $\mathrm{F}=\frac{\kappa}{2} \frac{d \mathrm{H}_{\mathrm{y}}^{2}}{d x} v$, where $\mathrm{H}_{\mathrm{y}}$ is the intensity, which from symmetry must be perpendicular to $o x, \kappa$ is the susceptibility, and

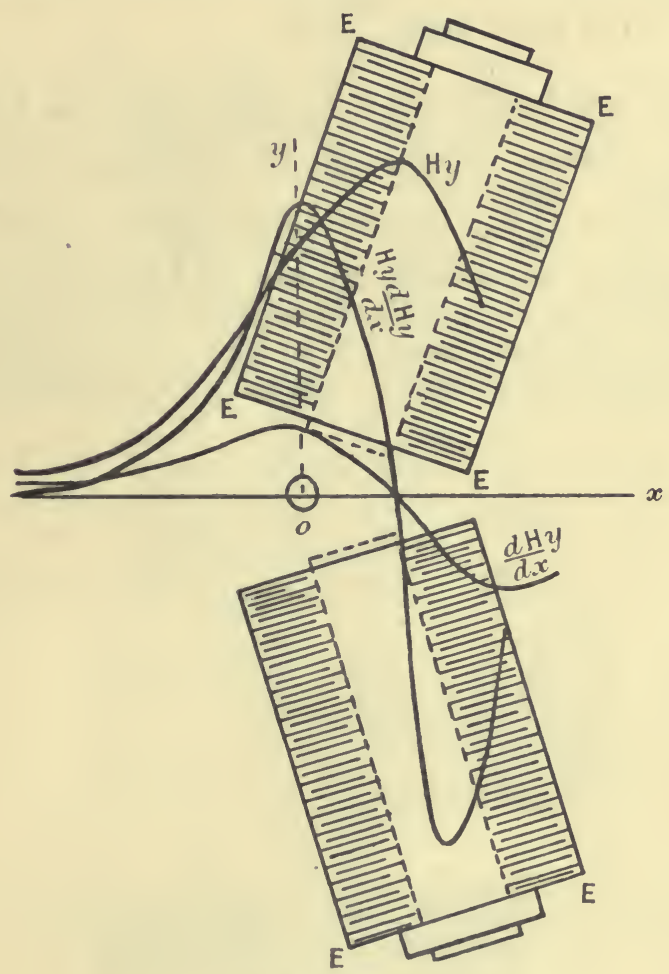

F1G. 218.

$v$ is the volume. If $p$ is the density and $m$ is the mass, we may put

$$
\begin{aligned}
\mathbf{F} & =\frac{1}{2} \frac{\kappa}{\rho}-m \frac{d \mathbf{H}_{\mathbf{y}}{ }^{2}}{d x} \\
& =\chi^{m \mathrm{H} y} y \frac{d \mathbf{H}}{d x}
\end{aligned}
$$

where $\chi=\frac{\kappa}{\rho}$. Curie investigated the value of $\chi$, which he termed "the specific coefficient of magnetisation."

'To find $\mathrm{H}_{y}$ a small coil connected to a ballistic galvanometer was placed at $o$ perpendicular to $\mathrm{H}_{\mathrm{y}}$ and then turned through $180^{\circ}$. 
The galvanometer throw gave $H_{y}$. To find $\frac{d \mathbf{H}_{y}}{d x}$ use was made of the fact that $\frac{d \mathrm{H}_{\mathrm{y}}}{d x}=\frac{d \mathrm{H}_{\mathrm{x}}}{d y}$, since each is equal to $-\frac{d^{2} \mathrm{~V}}{d x d y}$, where $\mathrm{V}$ is the magnetic potential. 'The coil was placed at $o$ with its plane perpendicular to $\mathbf{H}_{x}$, and was moved a given small distance $d y$ along oy, and the throw gave $\frac{d \mathrm{H}_{x}}{d y} d y$.

By these experiments, then, $\mathrm{H}_{y} \frac{d \mathbf{H}_{y}}{d x}$ could be found. Preliminary experiments were made to determine the values of $\mathrm{H}_{y}$ and $\frac{d \mathrm{H}_{x}}{d_{y}}$ or $\frac{d \mathrm{H}_{y}}{d x}$ at various points along $o x$, and the point where the product was a maximum was fixed as at $o$. Since, thereabouts, it varied slowly, exact adjustment of the body at $o$ was not necessary. Further, the force along ox was practically constant for a small displacement along ox. In the experiments the

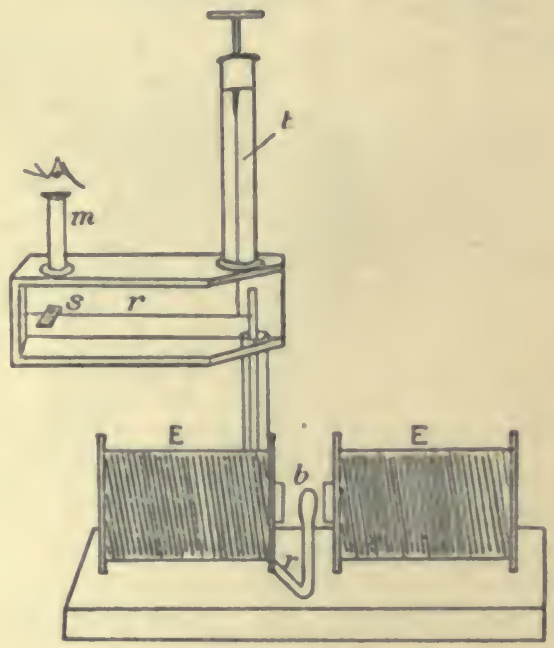

F1G. 219.

$t$ torsion fibre, $r$ torsion rod, $b$ body, EE electro-magnet, $s$ scale, $p$ pointer, $m$ microscope

displacement due to magnetic action was never more than $0.15 \mathrm{~cm}$. It was not necessary, then, to restore the body to its original position, when the deflecting force acted. It was sufficient to read the deflection. This was done by viewing through a microscope a pointer on the other end of the torsion arm which moved over a scale. The arrangement is represented diagrammatically in Fig. 219, where certain details for measuring the moment of inertia are 


\section{PARAMAGNETIC \& DIAMAGNETIC SUBSTANCES 289}

omitted. The force for a given deflection was obviously determinate from the moment of inertia and the time of vibration.

'The body was contained in a small bulb. When the effect of temperature was to be investigated the bulb could be surrounded by a jacket electrically heated, as represented diagrammatically in Fig. 220, the heating coil being surrounded by porcelain. Outside

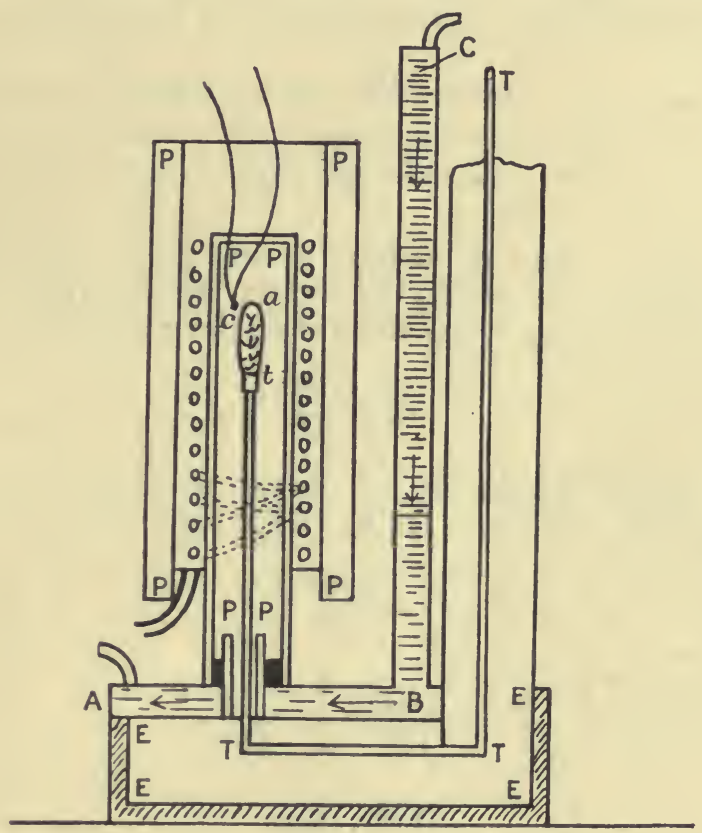

FIG. 220.

$a$ bulb containing the body, $t \mathrm{~T}$ porcelain rod, 'TT' metal tube from end of torsion rod, PP porcelain electrically heated oven, $c$ thermo junction, $\mathrm{ABC}$ water jacket, $\mathrm{EE}$ case.

this was a screen through which water circulated. Below, the apparatus was boxed in by wood to lessen convection currents.

'To correct for the action on the bulb and the supporting rod the force was measured on the bulb when containing the body and again when it was empty. 'To correct for the air displaced by the body it was assumed that the value of $\chi$ for nitrogen is negligible, so that its value for air is due to the oxygen. To determine $\chi$ for oxygen the bulb was filled with oxygen at a known considerable pressure, and the force was measured. 'Then the point of the bulb was opened and oxygen escaped till the pressure was that of the atmosphere. 'The force was again measured, and the difference gave the force upon the oxygen which had escaped if that amount occupied the volume of the bulb. 
A series of determinations of $\chi$ for oxygen was made at temperatures rising from $20^{\circ} \mathrm{C}$. to $450^{\circ} \mathrm{C}$, and it was found that if $\mathbf{T}$ is the absolute temperature,

$$
x=\frac{33700}{10^{6}} \cdot \frac{1}{T} .
$$

Hence $\chi$ is independent of the density, and is constant at constant temperature.

If $\kappa_{0}$ and $\rho_{0}$ are the susceptibility and density of oxygen,

$$
\kappa_{0}=\frac{33700}{10^{6}} \cdot \frac{\rho_{0}}{T} \text {. }
$$

Since the pressure of oxygen in air is 0.2 of the whole, its density is 0.2 of the density of oxygen at atmospheric pressure. Neglecting the nitrogen, the value of $\kappa_{\alpha}$, the susceptibility of air, is

$$
\kappa_{\mathrm{s}}=\frac{6740}{10^{6}} \frac{\rho_{\mathrm{o}}}{\mathrm{T}^{\prime}}
$$

where $\rho_{0}$ is now the density of oxygen at atmospheric pressure. Dividing by the density of air $\rho_{n}$, we have $\chi$ for air

$$
\begin{aligned}
& =\frac{\kappa_{\mathrm{a}}}{\rho_{\mathrm{a}}}=\frac{6740}{10^{6}} \frac{1}{\mathrm{~T}} \frac{\rho_{\mathrm{o}}}{\rho_{\mathrm{a}}} \\
& =\frac{6740}{10^{6}} \cdot \frac{1}{\mathrm{~T}} \cdot \frac{16}{14 \cdot 43} \\
& =\frac{7470}{10^{6}} \cdot \frac{1}{\mathrm{~T}} .
\end{aligned}
$$

In correcting for air displaced we require $\kappa_{\mathrm{a}}$. Assuming the pressure to be $760 \mathrm{~mm}$., then, for oxygen at T,

$$
\rho_{0}=\frac{.00143 \times 273}{\top}
$$

whence

$$
\kappa_{\mathrm{a}}=\frac{2640}{10^{6}} \cdot \frac{1}{1^{2}} *
$$

The value of $\chi$ was first determined for a number of dianagnetic bodies, and it was found to be constant for each with fields varying from 50 to 1350 . With the exception of bismuth and antimony the value did not vary when the temperature was raised-in some cases - up to $500^{\circ} \mathrm{C}$.

With bismuth and antimony there was a great decrease as

* Curie gives 7830 instead of 7470 , and 2760 instead of 2640 . 


\section{PARAMAGNETIC \& DIAMAGNE'TIC SUBSTANCES 291}

the temperature rose. Bismuth was submitted to special examination, and it was found that between $20^{\circ} \mathrm{C}$. and the melting-point $273^{\circ} \mathrm{C}$. the value of $\chi$ was given by

$$
10^{6} \chi=1.35[1-0.00115(t-20)] \text {. }
$$

At $273^{\circ}$ C. $10^{6} \chi$ fell from 0.957 in the solid to 0.38 in the liquid state, and then remained constant as the liquid was raised to $400^{\circ} \mathrm{C}$.

The value of $10^{6} \chi$ for water was -0.79 .

An easy calculation shows that Curie's relative values of $\kappa$ for air, water, and bismuth at $20^{\circ}$ agree closely with those found by Becquerel and Faraday; Curie's values, reduced to Faraday's scale, being $3 \cdot 74,-96 \cdot 3$, and -1830 respectively.

Certain paramagnetic substances were next investigated, especially as to the effect of rise of temperature. G. Wiedemann* had already shown that for solutions of certain salts, over the range from $15^{\circ} \mathrm{C}$. to $80^{\circ} \mathrm{C}$., the value of $\chi$ agreed nearly with $\chi_{\mathrm{t}}=\chi_{0}(1-a t)$ and he found $\alpha=0.00325$ the same for all. Plessner $\dagger$ also found a constant temperature coefficient for other solutions, obtaining $\alpha=0.00355$. These values, so near to $0.0036 \%$, suggest that $\chi$ varies inversely as the absolute temperature. Curie re-examined the results of Wiedemann and Plessner and showed that they agreed very nearly with $\chi^{\prime} \mathrm{T}=$ constant, the law which he had already obtained for oxygen. He showed also that Plessner's measurements with the salts in the solid state agreed with the law. He then showed that the product was constant for protosulphate of iron over a range from $12^{\circ} \mathrm{C}$. to $108^{\circ} \mathrm{C}$., and for palladium over the range from $22^{\circ}$ to $1370^{\circ}$.

Curie's law. 'These results may be summed up in what is known as Curie's law, which states that for a paramagnetic body the product of the specific coefficient of magnetisation $\chi$ and the absolute temperature $\mathrm{T}$ is constant, each body having its own constant.

Glass and porcelain, if paramagnetic at ordinary temperatures, became diamagnetic at high temperatures, and if diamagnetic to begin with showed stronger diamagnetism at higher temperatures. In each case the diamagnetism appears to approach a limit. 'This is easily explained if we suppose that there are two constituents present, one paramagnetic, the other diamagnetic. We shall then have

$$
\chi=\frac{\mathrm{A}}{\mathrm{T}}-\mathbf{B},
$$

$\frac{\mathrm{A}}{\mathrm{T}}$ representing the paramagnetism of one constituent varying inversely as the absolute temperature, and therefore diminishing 
as ' $\mathrm{T}$ rises; and $\mathrm{B}$ the constant diamagnetism of the other constituent. As ' $\mathrm{I}$ increases $\chi$ approaches -13 .

Curie also studied the behaviour of iron, magnetite, and nickel when carried to high temperatures. In the case of iron he experimented in succession on three wires, each about $1 \mathrm{~cm}$. long, and of respective diameters $0.002,0.014$, and $0.035 \mathrm{~cm}$. These were enclosed in glass or platinum tubes to protect them from oxidation and arranged on the torsion rod along oy, rig. 218. The temperature was raised from $20^{\circ} \mathrm{C}$. to $1360^{\circ} \mathrm{C}$., and the field from 25 to 1300.

About $750^{\circ} \mathrm{C}$. the value of $\mathrm{I}$, the intensity of magnetisation, began to be nearly independent of the field and fell rapidly, continuing to fall to $950^{\circ} \mathrm{C}$. Then it was nearly constrnt, though falling slightly to $1280^{\circ} \mathrm{C}$. 'Then it rose slightly and afterwards fell again. Apparently at a sufficiently high temperature $I=\frac{A H}{T}$ as for fecbly paramagnetic bodies. From the experiments he obtained the following results for iron:

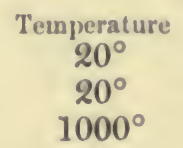

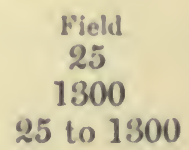

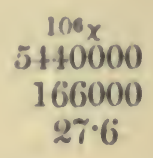

Thus at $1000^{\circ} \mathrm{C}$. the value of $\chi$ is independent of the field and is nearly the same as for air at $20^{\circ}$.*

Similar results were obtained with magnetite and nickel.

Wills' experiment. $\dagger$ In this experiment a rectangular plate of the substance to be tested was hung from one arm of a balance. Its lower edge was horizontal and was between the poles of an electro-magnet as represented in elevation and plan in Fig. 291.

If $\mathbf{A}$ is the area of cross-section of the plate indicated in plan in $(b)$, the force on the plate in direction $x$ upwards is

$$
\mathrm{F}=2 \mathrm{~A} \frac{d \mathrm{H}^{2}}{2} d x=\frac{\mathrm{r} A}{2}\left[\mathrm{H}^{2}\right]
$$

where the limits for $\mathrm{H}$ are the field at the lower edge and that at the upper. But the field at the upper edge is negligible in comparison with that at the lower, and we may put the force measured by the balance

$$
\mathrm{F}=\frac{\kappa \mathrm{AH} \mathrm{H}^{2}}{2}
$$

where $\mathrm{H}$ is the field between the poles. To measure this a "dummy" plate of plaster of Paris with tinfoil strip round its

* For details of the work on iron at high temperatures, Curie's paper (loc. cit.) should be consulted.

† Phil. Mag., xlv. (1S98), p. 432. 


\section{PARAMAGNETIC \& DIAMAGNETIC SUBSTANCES 293}

two sides and its lower edge was suspended in the position of the plate, and a known current $\mathbf{C}$ was sent through the tinfoil strip. If the length of the lower edge was $l$, the vertical force was $\mathrm{C} l \mathrm{H}$, since the action on the sides was negligible, being practically horizontal. Hence $\mathrm{H}$ was determinate by the balance.

The value of $\kappa$ for bismuth was found to be the same within

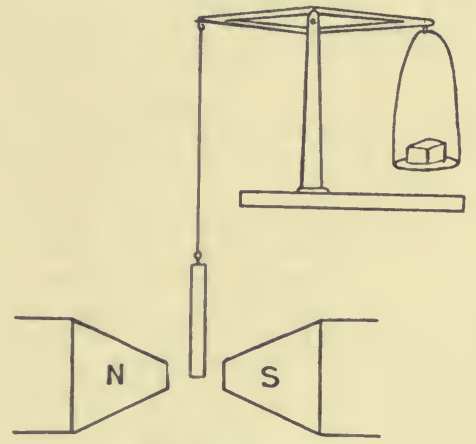

(a) Elevation

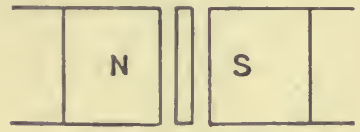

(1) Plan

FiG. 221.

the limits of error with fields varying from 1600 to 10,000 . The following results were obtained:

\begin{tabular}{|c|c|c|}
\hline & & $100^{K}$ \\
\hline Bismuth & • & $-12 \cdot 25$ to $-12 \cdot 55$ \\
\hline Antimony & ${ }^{\circ}$ & -0.714 \\
\hline Sulphur & . & -0.765 \\
\hline Marble & . & -0.7 \\
\hline Aluminium & . & $+1 \cdot 88$ \\
\hline
\end{tabular}

Townsend's experiment.* The method was devised to measure $\kappa$ for liquids, and was in principle like the first method of v. Ettingshausen. A tall jar, which could contain the liquid to be experimented on, was placed within a solenoid which served as a primary coil. Round the middle of this was a secondary coil. There were a compensating primary and a secondary respectively in series with the coils round the jar, and a galvanometer with commutator to rectify the current was in the secondary circuit. The two sets of coils were so arranged and so connected up that when the jar was empty an alternating current of frequency 16 in the primaries produced no current in the secondaries. If there are 
$\mathbf{N}$ turns on the jar-primary and $\mathbf{N}^{\prime}$ turns in the secondary round it, and if the area of cross-section of the primary is $\mathrm{A}$ and its length $2 l$, the mutual induction of primary and secondary is

$$
\frac{4 \pi \mathrm{NN}^{\prime} \mathrm{A}}{2 l} \text {. }
$$

If the jar is now filled with liquid of susceptibility $\kappa$, and if we suppose its cross-section to be $A$ also, the mutual induction is altered to

$$
(1+4 \pi \kappa) \frac{4 \pi N^{\prime} \mathrm{A}}{2 l}
$$

or increased by

$$
4 \pi \kappa \cdot \frac{4 \pi N^{\prime} A}{2 l}
$$

The balance between the pair of secondaries will therefore be destroyed, and a current will flow through the galvanometer.

To measure this increase in mutual induction a third pair of coils was arranged, one in the primary and the other in the secondary circuit, so that the mutual induction, always very small, might be varied from 0 by an amount measurable from the dimensions and positions of the coils. This third mutual induction was so arranged that it exactly neutralised the current due to the insertion of the liquid, and so the value of the incrense $4 \pi \kappa \cdot 4 \pi N^{\prime} A / 2 l$ was known, and $\kappa$ was determinate. Townsend showed that Foncault currents in the jar could not have an appreciable effect.

The value of $10^{6} \mathrm{k}$ for water was found to be $-0 . \tau \%$, agreeing closely with Curie's value $-0 \cdot 79$. 'The susceptibility of a salt solution in water arises partly from the water and partly from the salt, so that, if $a$ is the susceptibility of the solution, $x+0.77 \times 10^{-6}$ is that of the salt alone. For solutions of iron salts this was found to depend only on the weight of iron in the salt and not on the nature of the acid radicle, being proportional to the weight of iron W per c.c. of solution, though differing for equal weights in the ferrous and in the ferric salts. The values obtained for temperatures about $10^{\circ} \mathrm{C}$, were :

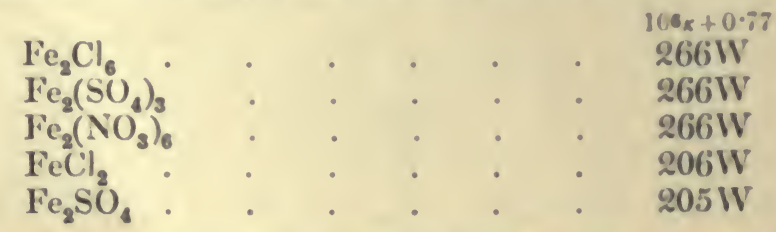

The values obtained for the dry salts were approximately the snme.

All the salts showed a fall in $\kappa$, with rise of temperature of the order of $\frac{1}{2}$ per cent. per degree $\mathrm{C}$. 


\section{PARAMAGNETIC \& DIAMAGNETIC SUBSTANCES 295}

The susceptibilities of solutions of potassium ferrocyanide and potassium ferricyanide were the same as if water alone were present so that when iron is present in the acid radicle it must be at least 100 times less magnetic than where it takes the part of a metal in a salt.

The paper contains an interesting suggestion. Imagine a magnet set spinning about its centre in the earth's field. 'The $\mathbf{N}$ pole will go round the semicircle on the Northern side more rapidly than it will go round that on the Southern side. Thus the end towards the North will be a longer time $\mathbf{S}$ than $\mathbf{N}$, and the average effect at a distance will be that of a reversed magnet. If the molecules of a body consisted of small magnets rotating about their centres under the action of a field, the body would appear diamagnetic.

We may note here that iron carbonyl, the liquid compound $\mathrm{Fe}(\mathrm{CO})_{5}$ discovered by Mond and Quincke in 1891,* is diamagnetic.

Experiments of Fleming and Dewar on oxygen at low temperatures. Dewar had already found that liquid oxygen was strongly paramagnetic. $\dagger$ Fleming and Dewar made investigations on its susceptibility. 'The first method + which they used was an induction method in which the primary and secondary coils were immersed in liquid oxygen and then in gaseous oxygen at nearly the same temperature. But this was not so satisfactory as a second method, $\S$ in which balls of silver, of bismuth, of copper, and of glass contrining mercury, were weighed above the pole of a strong electro-magnet. When the magnet was excited there was a change in weight of $\left(\kappa_{1}-\kappa_{2}\right) \mathrm{VH} \frac{d \mathrm{H}}{d x}$, where $\mathrm{V}$ is the volume of the ball, $\kappa_{1}$ is its susceptibility, and $\kappa_{2}$ is that of the surrounding medium and $x$ is measured upwards. 'The field $\mathrm{H}$ was deternined for different values of $x$ and for different exciting currents by means of an exploring coil connected to a ballistic galvanometer.

'The susceptibility of each ball was first found by weighing it in air with the current off and on, and assuming that $10^{6} \kappa$ for air is 0.024 . To test the method the silver ball and a glass ball were each weighed also in water with the current off and on, and the mean value of $10^{6} \kappa$ for water was 0.74 , agreeing fairly with the values obtained by others. 'The balls were then weighed in liquid oxygen at $-182^{\circ} \mathrm{C}$. with the current off and on. The susceptibilities of the balls were estimated at $-182^{\circ} \mathrm{C}$, and thus the susceptibility of liquid oxygen was found. The mean result was $10^{8} \kappa=+324$, whence $\mu=1.004$.

Curie found that for gaseous oxygen

$$
10^{6} \chi=\frac{10^{6} \kappa}{\rho}=\frac{33700}{\mathrm{~T}},
$$

\footnotetext{
* Trans. Chem Soc., 1891, vol. 59. p. 604.

t P.IR.S., vol. 1. p. $2+7$.

8 lbid., vol. Ixiii. p, 311 (1898),

$\ddagger$ lbid., vol. 1x. p. 283 (1896).
} 
where $\mathrm{T}$ is the absolute temperature. If we assume that this holds when the oxygen is liquefied at $-185^{\circ} \mathrm{C}$. or $+91^{\circ} \mathrm{A}$., and if we take the density of liquid oxygen as $1 \cdot 14$, then

$$
10^{6} \mathrm{~K}=\frac{33700 \times 1 \cdot 14}{91}=42 \mathrm{~g}
$$

The change from gas to liquid, then, makes no great change in the value of $\chi$.

Pascal's experiments. Pascal* studied the magnetic qualities of a large number of solutions, of some liquids, and of some liquefied gases by a method due to Quincke. In principle the liquid to be experimented on was contrined in a $U$-tube with one limb narrow ( $6 \mathrm{~mm}$. diameter), the other wide $(6 \mathrm{~cm}$. diameter). The narrow limb was between the poles of an electro-magnet by which a horizontal field could be established of any intensity up to many thousand gausses. The surface of the liquid in this limb was at the centre of the field and its level was observed with a microscope. The field at the surface of the wide limb was in comparison negligible. Hence when field $\mathrm{H}$ was put on, if the difference of level in the limbs was $h$, if $\kappa$ is the suseeptibility of the liquid and $\kappa_{0}$ of whatever is above it, the force per unit area was as in Wills' experiment :

$$
\left(\kappa-\kappa_{0}\right) \frac{\mathrm{H}^{2}}{2} .
$$

But this balances the column of height $h$. If the density of the liquid is $\rho$,

$$
\left(\kappa^{\circ}-\kappa_{0}\right) H^{2}=2 h g \rho \text {. }
$$

The actual change observed in the microscope is that of the level in the smaller limb, say $\delta$. If $\&$ be the cross-section of the narrow limb and $\mathbf{S}$ that of the wider, it is easy to see that $h=(\mathrm{S}+\delta) \delta / \mathrm{S}$, so that

$$
\left(\kappa-\kappa_{0}\right) H^{2}=2 \frac{S+s}{S} 8 p g .
$$

To avoid measurement of $\mathrm{H}$, the experiment was repented on water with the same value of $\mathrm{H}$. Denoting the susceptibility of water by $\kappa_{1}$, its density by $\rho_{1}$, and the change of level in the narrow limb by $\delta_{1}$,

By division

$$
\left(\kappa_{1}-\kappa_{0}\right) H^{2}=2 \frac{S+s}{S} \delta_{1} \rho_{1} g .
$$

$$
\frac{\kappa-\kappa_{0}}{\kappa_{1}-\kappa_{0}}=\frac{\delta \rho}{\delta_{1} \rho_{1}}=\lambda, \text { say }
$$

* I nn, de Chim. al de Phys., S ser. XVI. (1909), p. 359, and XIX. (1910), p. 5, 
where $\lambda$ is given by the measurements made. Pascal took $\kappa_{1}$ as $-7.5 \times 10^{-7}$ and $\kappa_{0}$ for air as $0.25 \times 10^{-7}$. When coal gas or a vacuum was above the liquid $\kappa_{0}$ was taken as zero.

For a solution under air it follows that

$$
10^{7} \kappa=0 \cdot 25-7 \cdot 75 \lambda
$$

while for one under coal gas or a vacuum

$$
10^{7} \kappa=-7 \cdot 5 \lambda \text {. }
$$

If the density of the solution was $\rho$ and it contained $p$ grammes of salt per c.c. there was $\rho-p$ of water per c.c. Assuming that salt and solvent each produced its own effect, and taking $x$ as the susceptibility of the salt as dissolved,

$$
\kappa \rho=p x+(\rho-p) \% \cdot 5 \times 10^{-7} .
$$

The measurement of $\lambda, p$, and $\rho$ therefore gave $x$. For the modifications of the method for liquefied gases the original papers should be referred to.

From his results with solutions of salts of iron, nickel, and cobalt Pascal inferred that as an ion of a ferromagnetic metal passes into a complex ion or into a colloid its susceptibility diminishes, and it may even become diamagnetic. Hence the magnetic moment of a molecule in a given field is not the sum of the moments of its constituent atoms, but depends on the grouping in the molecule.

With the diamagnetic metalloids he found that the atomic susceptibility $\kappa \alpha$ ( $\alpha$ being the atomic weight) was nearly proportional to $e^{\alpha+\beta a}$ where $\alpha$ and $\beta$ are constants for the same family. With several diamagnetic compound gases, when liquefied he found that $\kappa m$ ( $m$ being the molecular weight) was nearly proportional to $5+n$, where $n$ is the number of atoms in the molecule. In diamagnetic organic compounds his results showed that the different atoms preserved, as a rule, each its own diamagnetic susceptibility, but the presence of oxygen appeared to lead to anomalies.

The electron theory. The following is a brief sketch of Langevin's form of the Electron 'Theory of Magnetism.*

Let us, as a preliminary, imagine an atom which consists of a positive charge on a large central mass, and a negative electron circling round it as the moon circles round the earth. The positive charge will hardly move, and the electric lines of force will sweep through the space round it, all the lines directed outwards from the centre, and their motion will produce a magnetic field equivalent to that of a current along the orbit but opposite in direction to the motion of the electron. The magnetic moment 
will be given by $\mathrm{M}=e \mathrm{~A}$, where $e$ is the electronic charge and $\mathrm{A}$ is the area of the orbit swept out per sccond.

Now suppose that a field $\mathrm{H}$ is put on perpendicular to the plane of the orbit, and in the positive direction, so that if the equivalent current is going round clockwise in the plane of the paper, or the electron is going round counter-clockwise, $\mathbf{H}$ is from above downwards. During the increase in $\mathbf{H}$ there is a negative, i.e. counter-clockwise, E.M.F., and this, acting on the negative electron, gives a clock wise or retarding force. When $\mathrm{H}$ is established the angular velocity in the orbit is less than initially, $\mathrm{A}$ is diminished, and it can be shown that

$$
\Delta \mathrm{M} / \mathrm{M}=-e \mathrm{H}_{\tau} / 4 \pi m
$$

where $\tau$ is the period of revolution and $m$ is the mass of the electron.

Now imagine a more complex atom in which there is a central body with a large number of electrons circling round it in orbits with their aspects indifferently distributed in all directions. On the whole the moment of each atom, then, is zero. When $a$ field $H$ is put on, the effect on each orbit is like that discussed above, but less as the inclination of $\mathrm{H}$ to the perpendicular to the orbit increases. Those going round one way will have their positive moments decreased, those going round the other way will have their negative monents increased, so that on the whole the moment of the atom will become negative. We may suppose that the ordinary diamagnetic atom is of this type, and the formula for the change of moment shows that, in accordance with experience, it is very minute. Further, since there is reason to suppose that temperature describes the agitation of the molecules and atoms as wholes and not the motions within the atoms, we should not expect diamagnetic susceptibility to depend on temperature. Curie showed that, excluding bismuth and antimony, the susceptibility was constant through a very wide range of temperature.

'To explain paramagnetism, imagine a body to consist of atoms in each of which the aspects of the electronic orbits are not indifferently distributed, but that they are grouped more or less about a particular axis. Each atom, then, will have a magnetic moment, like the molecular magnets in Weber's theory. When there is no external field and the body is ummagnetised, the axes of the atoms and the molecules into which they are grouped will be distributed indifferently in all directions. When a field $H$ is put on, it acts in two opposite ways. In the first place it tends to decrease each atomic magnetic moment in the way already explained for diamagnetic atoms. In the second place it tends to pull the magnetic axes into its own line and so to give a positive moment to a mass of molecules. This second effect is rastly 


\section{PARAMAGNETIC \& DIAMAGNETIC SUBSTANCES 299}

greater than the first and quite masks it. If there were no intermolecular and interatomic agitation - that is, if the temperature of the body were absolute zero-the molecules might set under sufficient force all with their axes in the direction of $\mathrm{H}$, like the magnets in Ewing's model. The intensity of magnetisation would then be a maximum and would be independent of the tield when this had reached the value needed to secure parallelism. But molecular and atomic agitation - that is, heat-with the resulting collisions, produces disturbance of the axes.

Langevin examined specially the case of a paramagnetic gas such as oxygen. Let us imagine that we are dealing with a constant mass kept at constant unit volume. Let its moment in field $\mathrm{H}$ be $\mathbf{M}$. Let the moment be increased to $\mathrm{M}+d \mathrm{M}$. The work done from outside on the gas is therefore $\mathrm{H} d \mathbf{M}$. But work done on a gas at constant volume goes to increase its temperature. If we keep the temperature constant we must take away heat $d \mathbf{Q}=$ $\mathrm{H} d \mathbf{M}$. But if $\mathrm{T}$ is the temperature, $\frac{d \mathrm{Q}}{\mathrm{T}}$ is a perfect differential, for it is equal to the entropy removed, $d \phi$. Then $\frac{\mathbf{H}}{\mathrm{T}^{\circ}} d \mathbf{M}$ is also a perfect differential. Now the condition of the gas, including the condition $\mathrm{M}$, is a function of $\mathrm{H}$ and $\mathrm{T}$ only.

It follows easily that $M$ must be a function of $\frac{H}{T}$

$$
\text { or } \mathbf{M}=f\left(\frac{\mathbf{H}}{\overline{\mathbf{T}}}\right) \text {. }
$$

But as far as experiment has yet gone, when ' $\mathrm{T}$ is constant

$$
\mathrm{M} \propto \mathrm{H} \text {. }
$$

Therefore

$$
\mathrm{M} \propto \frac{\mathrm{H}}{\mathrm{T}}
$$

or

$$
\frac{\mathrm{M}}{\mathrm{H}}=\frac{\mathrm{C}}{\mathrm{T}} \text {. }
$$

But $\mathrm{M} / \mathrm{H}$ is proportional to the susceptib:lity, so that this result gives Curie's law. 'This cannot hold, however, down to the absolute zero. 'There we shall have a moment $\mathbf{M}_{\mathbf{0}}$ due to parallel alignment of all the molecules. But of course the gas law assumed ceases to hold before we get to zero temperature.

By a treatment analogous to that by which the mean square of the velocity is found, Langevin found that the magnetic susceptibility should be $\frac{M_{0}^{2}}{3 R^{\prime} I^{2}}$, where $R$ is the gas constant.

The magneton.* P. Weiss, in conjunction with Kammerlingh-

"Weiss, Journal de Physique, 5 ser. I. (1911), pp. 900 and 965. 
Onnes, ${ }^{*}$ made measurements at the tenuperature of liquid hydrogen of the intensity of magnetisation of iron, nickel, and magnetite, and by certain inferences of that of cobalt. At this temperature the bodies might be regarded as saturated, with all the molecular axes parallel. Weiss found that the magnetic moment per grammemolecule was very near in each case to a small integral multiple of 1123.5. 'This value he termed the "magneton-gramme." He puts forth the theory that there is in each atom of these substances at least one elementary magnet of construt moment and the same for all of them, and he terms this the "magneton." Assuming Perrin's value $68.5 \times 10^{22}$ as the number of molecules in the gramme-molecule, and assigning one magneton with moment $m$ to each molecule in the magneton-gramme, we have

$$
68.5 \times 10^{22} m=1123.5
$$

$$
\text { whence } m=16.4 \times 10^{-22} \text {. }
$$

The magnetisations of iron, cobalt, magnetite, and nickel respectively agree with the possession per molecule of $11,9,7,3$ magnetons respectively. Using Langevin's theory with certain extensions, and assuming that it is applicable to solids as well as to gases, certain results obtained by limself and Fö̈ $t$ on bodies above the Curie point, or the point where the permenbility falls almost to 1, appear to fall in line with the magneton hypothesis on the supposition that the number of active magnetons changes. Weiss also uses Pascal's results to show that in paramannetic solutions the magnetisation can be expressed in terms of the magneton.

* Comm. Phys. Lub. Leiden, No. 111. p. 3 (1910).

† Journ. de Thys., ser. I. (1911), Ip. 2 it and 741. 


\title{
CHAP'IER XXIII
}

\section{TERRESTRIAL MAGNETISM}

\begin{abstract}
The direction of the earth's lines of force at a given place-T'o find the declination-To find the dip or inclination-The earth inductorThe intensity of the field-To determine the horizontal intensity HThe vibration experiment-The deflection experiment-Recording instruments-Results-sketch of Gauss's theory of terrestrial magnetism.
\end{abstract}

Is many magnetic and electric measurements it is necessary to know the intensity and direction of the earth's magnetic field at the place of experiment. It is also of the highest scientific interest to determine the field of the earth as a whole, in the hope that we may answer the questions how and where is the earth magnetised and what is the origin of the magnetisation? We shall give here a brief account of the methods usually adopted to obtain the field at a given place, a summary of the results of observations made at the various observatories distributed over the earth's surface, and of the progress so far made in the investigation as to where the magnetisation resides and how the magnetic field is disturbed. As to the origin we have at present no theory of certain value.*

The direction of the earth's lines of force at a given place. It is convenient to describe the direction of the earth's field at a given place by two angles: (1) The angle which the vertical plane through the line of force-the plane of the magnetic meridian -makes with the vertical plane through the geographical North and South - the plane of the geographical meridian. 'This angle is called the Declination. (2) The angle between the line of force and the horizontal plane. 'This angle is called the Dip or Inclination.

To find the declination. Let us suppose that we have a compass needle with axis of figure coinciding with the magnetic axis balanced without friction on the point C, Fig. 222. If the poles are $\pm m$, and if the earth's total intensity is $\mathbf{I}$, we have two equal and opposite forces $m \mathrm{I}$, one acting at each end. These may be resolved into horizontal components $\pm m \mathrm{H}$, and into vertical components $\pm m \mathrm{~V}$. The latter will give a couple $m \mathrm{~V} l$,

* A full account of the Kew instruments will be found in Gordon's Electricity and Magnetism, vol. i. chaps, xiv. and xv.; or in Ency. Brit., 9th ed., vol. xvi. p. 159, Meteorology, 'Terrestrial Magnetism. The results are discussed in that article, and in "Magnetism, Terrestrial," 10th ed., vol. xxx. p. 453. In the 11 th ed. the corresponding articles are under "Inclinometer," "Magnetograph," "Magnetometer," and "Magnetism, Terrestrial." See also Bauer's Land Magnetic Observations, Carnegie Institution, 1912. 
where $l$ is the distance between the poles, and will tend to make the needle dip in the direction of I. But the needle may be kept horizontal at a place in the Northern magnetic hemisphere by putting $c$ towards the $\mathbf{N}$ end of it a distance from $\mathbf{G}$ such that if $w$ is the weight of the needle $w \cdot c \mathrm{G}=m \mathrm{~V} l$. The needle will then

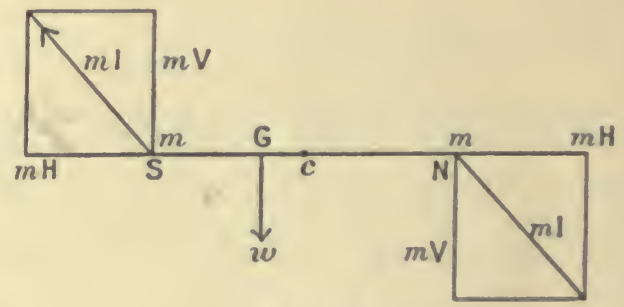

F10. 222.

- remain horizontal even when displaced from the magnetic meridian, for the only other forces acting on it are $\pm m \mathrm{H}$ horizontal. When so displaced these forces will form a couple tending to bring the needle into the magnetic meridian, and it will ultimately settle there. Even if $c$ is not accurately plnced for horizontality, the needle will still settie in the plane of the magnetic meridian.

Let us suppose that it moves over a horizontal graduated circle of which $c$ is the centre, and that we know the geographical North point on this circle; obviously the declination is the angle on the circle between this point and the $\mathbf{N}$ end of the needle.

In practice the magnetic axis caunot be made to coincide exactly with the axis of figure. We may deduce the dircetion of the magnetic

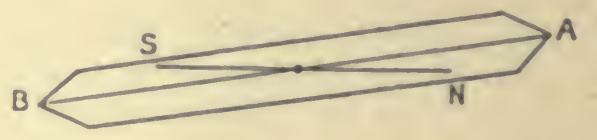

(a)

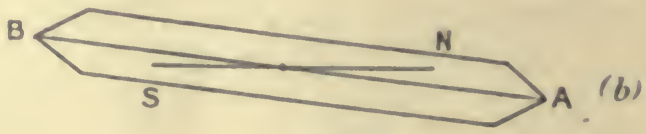

Fic. 223.

axis from the positions of the axis of figure if the needle is first suspended with one face upwards, and is then turned over and suspended with the other face upwards. Let (a), Fig. 223, be a plan of the needle in the first case. We read the direction of $\mathrm{AB}$ 
on the circle. Now turn the needle over about NS as axis and suspend it with the other face up, and let (b), Fig. 223, be the plan in the new position. $\mathrm{NS}$ is still in the same direction, but $\mathrm{AB}$ is thrown as much on one side of NS as it was previously on the other, and the mean of the two readings for the direction of $\mathrm{AB}$ gives the direction of NS.

The form of the apparatus adopted in this country is known as the Kew magnetometer. The needle is a hollow magnetised steel tube about $4 \mathrm{in}$. long and $\frac{1}{2} \mathrm{in}$. diameter, Fig. 224, with a very fine

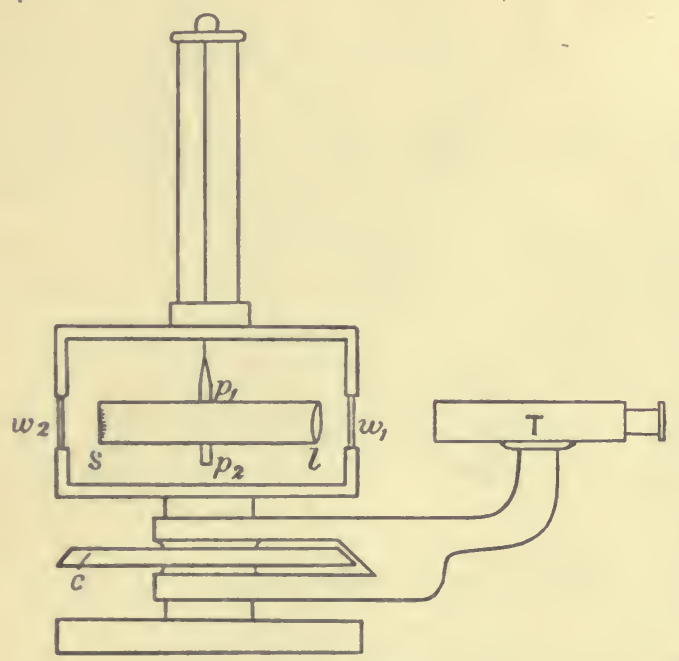

Fig. 224.

horizontal scale on glass at one end $s$, and a lens $l$ with $s$ as its focus at the other. It is provided with two points of suspension, $p_{1}$ and $p_{2}$. The needle is hung up in a torsion box by a silk fibre, as represented diagrammatically in the figure. 'This torsion box is mounted so that it can be turned about a vertical axis, easily adjusted vertically by levelling screws and a level not shown. A telescope focused for a long distance away moves round the same axis, and is provided with verniers on arms moving on a horizontal divided circle $c$. First, it is necessary to eliminate the torsion of the fibre. This is done by placing the torsion box as nearly as possible in the magnetic meridian, and then hanging a non-magnetic plumb bob of the same weight as the magnet and in its place. When the bob has come to rest the magnet is hung up by $p_{1}$, and the telescope is moved round so that the centre of the scale, $s$, viewed through the window $w_{1}$ and lighted through the window $w_{2}$, is seen on the cross wire of the telescope. The axis of figure is the line joining the centre of the scale $s$ to the centre of the lens $l$. The readings of the tclescope verniers are then taken. Say that the mean reading 
is $\mathrm{D}_{1}$. 'The needle is now turned over and hung from $p_{2}$, and the telescope is moved round so that again the centre of $s$ appears on the cross wire, and the telescope vernicrs are again read, giving, say, $\mathrm{D}_{2}$. The magnetic axis bisects the two directions given by the telescope readings, and corresponds to a reading $\frac{1}{2}\left(\mathrm{D}_{1}+\mathrm{D}_{2}\right)$.

For simplicity we shall suppose that by previous observations on the sun some object on the horizon at a distance has been fixed on as the geographical South point. 'The telescope is turned so that this point is on its cross wire and the verniers are read, giving a mean reading $\mathrm{S}$, say. We have then the declination given by $\frac{\mathrm{D}_{1}+\mathrm{D}_{2}}{2}-\mathrm{S}$. The instrument is provided with apparatus by which a sun observation may be taken and the reading of the South point on the circle $c$ may be directly determined, but into this we shall not enter. 'The determination of the declination by different instruments * may differ by several minutes.

To find the dip or inclination. The ideal apparntus for the determination of the dip would consist of a needle with its long axis of figure coinciding with the magnetic nxis, and able to move freely round an axis through its centre of gravity, this axis being supported so as to be horizontal and perpendicular to the plane of the magnetic meridian. A vertical divided eircle would be placed in the magnetic meridian with its centre in the axis of rotation of the needle and its zero reading in the horizontal through that axis, the ends of the needle lying just in front of the circle. 'The reading of either end of the needle would then give the dip.

In practice, of course, these ideal conditions are only more or less nearly approached. The Kew Dip Circle consists of a vertical circle contained in a rectangular box which is glazed front and back, and it is mounted so as to turn round an axis which can be adjusted to be vertical by a level and levelling screws. There is a horizontal divided circle round the axis which gives the azimuth of the vertical circle. 'The vertical circle, shown without the box in Fig. 226, is divided from $0^{\circ}$ at the ends of the horizontal diameter

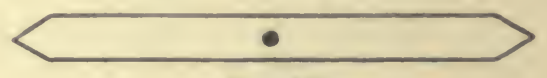

FIG. 225 .

to $90^{\circ}$ at the ends of the vertical diameter. 'The ncedle, Fig. 225, is a flat plate of steel from $3 \mathrm{in}$. to $6 \mathrm{in}$. long, with sharply pointed ends, and an axis is fixed as nearly through the centre of gravity as possible, and perpendicular to the plate. This axis is a thin cylindrical steel rod projecting on both sides of the plate, and is as truly circular in section as it can be made. Almost level with the centre of the vertical circle are two horizontal and parallel agate knife edges, on which the axis of the needle itself can roll, the central line of that

- Thorpe and Rücker, Phil Trans., A, rol. clxxxriii. (1896), p. 16. 
axis being level with the centre of the scale. The needle when in position moves in a plane midway between the agate knife edges.

A cross arm provided with verniers and two microscopes having cross wires in their fields of view can be turned round till, say, the upper end of the needle is on the cross wire of one microscope and the upper vernier is read. The cross wire in the

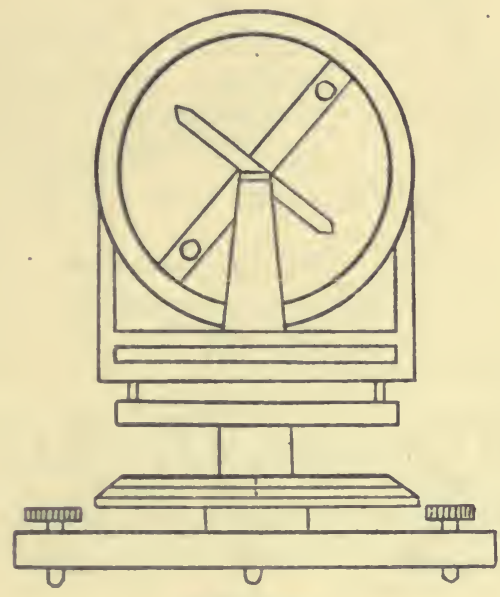

FIG. 226.

Diagrammatic representation of the Dip Circle.

other microscope is then brought to coincide with the lower end and the lower vernier is read. 'The mean reading gives the position of the needle on the scale.

When an observation is to be made, the axis round which the dip circle rotates is made vertical and the needle is put on to $V$-bearings, which are then lowered so as to leave it on the knife edges. We shall suppose that the dip circle is already adjusted in the plane of the meridian, and that the face of the instrument is East. The position of each end of the needle is then read. The mean of these eliminates error due to a small error in the centering of the needle. For if NS, Fig. 227, is the line through the ends of the needle and it does not pass through the axis of the circle, draw N'S parallel to NS, and $\mathrm{HOH}^{\prime}$ the horizontal through ( $)$. 'Then $\mathrm{NON}^{\prime}=\mathrm{SOS}^{\prime}$, and $\mathrm{HON}+\mathrm{H}^{\prime} \mathrm{OS}=\mathrm{HON}^{\prime}-\mathrm{NON}^{\prime}+\mathrm{H}^{\prime} \mathrm{OS}^{\prime}+$ $\mathrm{SOS}^{\prime}=\mathrm{HON}^{\prime}+\mathrm{H}^{\prime} O \mathrm{~S}^{\prime}$, or the mean of the readings for $\mathbf{N}$ and $\mathbf{S}$ gives us the mean of the readings for $\mathbf{N}^{\prime}$ and $\mathbf{S}^{\prime}$.

The vertical circle may not be correctly set-that is, the points $\mathrm{H}$ and $\mathrm{H}^{\prime}$ may not be at $0^{\circ}$ on the scale. The circle with the needle on it is therefore turned through $180^{\circ}$ round the vertical axis so as to face West, and the positions of the ends of the needle are again read. 
Considering this error to exist alone, if Z, Fig. 228, is the zero in the first position, we read NZ instead of NH. In the second position $Z^{\prime} Z^{\prime}$ is brought to the position $Z^{\prime \prime} Z^{\prime \prime}$ and we read NZ". But $Z^{\prime \prime}$ is as

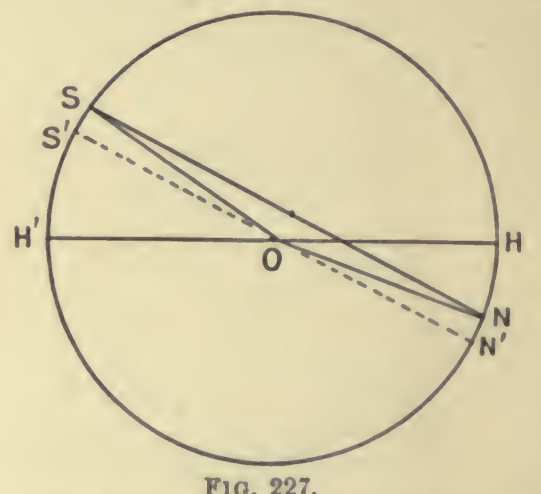

much below $\mathrm{H}$ as $\mathrm{Z}$ is above it, so that $\mathrm{NZ}+\mathrm{NZ}=2 \mathrm{NH}$, and the mean of the readings eliminates the zero error.

The axis of figure of the needle does not in practice coincide exactly with the magnetic axis. 'To eliminate the error thus

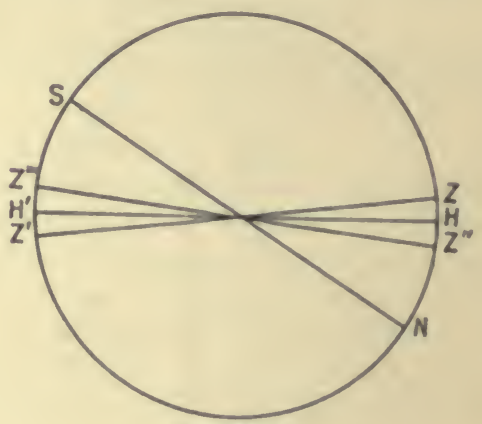

F10. 228.

produced the needle is taken up and its face reversed. The positions of the ends are again read. 'Then, as with the declination needle, it is easy to see that the mean of the readings, with the face of the needle West and with its face East, will give the position of the magnetic axis. At the same time part of the error due to the centre of gravity lying out of the axis of rotation will be very nearly corrected. For if G, Fig. 229, is the centre of gravity and $\mathrm{O}$ the axis of rotation, drawing the rectangle $\mathrm{OAGB}$, the moment of the weight of the needle acting through $\mathrm{G}$ will be equal to the moments of two equal weights at $\mathrm{A}$ and $\mathrm{B}$. By turning the needle over, the moment of the weight at $A$ is reversed, and the 
error due to the component of the displacement of $\mathrm{G}$ perpendicular to the axis is eliminated on taking the mean of the readings before and after turning over. The face of the instrument is then turned round into its original position facing East, and the positions of the ends of the needle are again read. Denoting the aspects of the face of the instrument by $\mathbf{E}$ and $\mathrm{W}$ and those of the face of the needle by $e$ and $r$, we have to read each end in each of the four arrangements $\mathrm{E} e, \mathrm{~W} z, \mathrm{~W} e$,

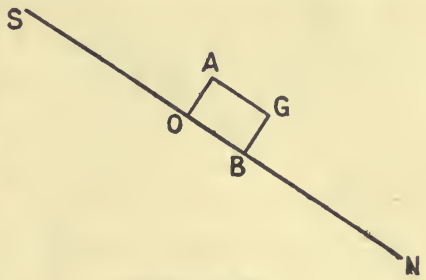

FIG. 229. Ew, and the mean of the eight readings taken eliminates the errors so far considered.

There is still outstanding the error due to $\mathrm{OB}$, Fig. 229, the component of the displacement of the centre of gravity parallel to the axis. To eliminate this the needle is taken out and its magnetisation is reversed by the method of divided touch. It is assumed that the magnetisation is exactly reversed-an assumption quite certainly not fulfilled, but it at least gives us a method of procedure which tends to diminish the error. B is now thrown as much above $\mathrm{O}$ as it was previously below it, and going through the four cases again the mean of the readings with the magnetisation direct and reversed gives as good a value of the dip as can be obtained with a single needle. It is usual to find the value with two different needles and to take the mean in the hope that it is nearer the true value than that given by either separately. In this country, with a value near $70^{\circ}$, skilled observers may obtain determinations with different needles differing by one or two minutes, and different instruments may give results differing by quantities of the same order.

We assumed that the observations began with the circle in the magnetic meridian. To make this adjustment it is usual to set the microscope verniers at $90^{\circ}$, and turn the circle facing $\mathrm{S}$ till the ends of the needle are on the cross wires. The position on the horizontal circle is then read. The needle is then turned round and the dip circle is turned round the vertical axis till the microscopes again sight the needle-ends on the cross wires, and another reading on the horizontal circle is taken. The dip circle is then turned through $180^{\circ}$ to face North, and the operations are repeated. The mean of the readings on the horizontal circle then gives the position of the circle in which the magnetic axis of the needle is vertical and, therefore, that in which the plane of the circle is perpendicular to the magnetic meridian, for in that plane the vertical force alone can move the needle. The dip circle is then turned through $90^{\circ}$ and the needle is assumed to be in the magnetic meridian.

Sometimes the dip is taken by another method which does not require this adjustment. 
Suppose that the dip circle is in a plane making an unknown angle $\theta$ with the magnetic meridian. If $\mathrm{H}$ is the horizontal intensity in the meridian, the effective horizontal intensity in the plane considered is $H \cos \theta$, and if $\delta_{1}$ is the dip observed when the needle is free to move in this plane, and if $\mathrm{D}$ is the true dip,

$$
\cot \delta_{1}=\frac{\mathrm{H} \cos \theta}{\mathrm{V}}=\cot \mathrm{D} \cos \theta .
$$

The circle is now turned through $90^{\circ}$ about the vertical axis and the dip $\delta_{2}$ in the new position is observed. Then

$$
\cot \delta_{2}=\frac{H}{V} \cos (\theta-90)=\cot \mathrm{D} \sin \theta
$$

and $\cot ^{2} \delta_{1}+\cot ^{2} \delta_{2}=\cot ^{2} \mathrm{D}$, whence $\mathrm{D}$ is obtained from observation of $\delta_{1}$ and $\delta_{2}$.

The earth inductor. 'There is a totally different method of obtaining the dip-the earth-inductor method. It is employed at some observatories and appears likely to come into general use. The principle may be given thus: Suppose that a coil in the form of a large ring has total area $A$, counting the areas of all the turns. Let it be laid flat on a horizontal table and be connected to a ballistic galvanometer. The total flux of induction through it is $\mathrm{AV}$, where $\mathrm{V}$ is the vertical intensity. Now let it be tumed over suddenly so that the other face of the coil is on the table. The change in induction is $2 \mathrm{AV}$, and the throw of the galvanometer will be due to a flow of electricity proportional to $2 \mathrm{AV}$. Say that the galvanometer gives this flow as $\mathbf{Q}_{1}$ coulombs. Now let the coil be raised into the vertical plane perpendicular to the magnetic meridian. The total flux through it is now $\mathrm{AH}$, where $\mathrm{H}$ is the horizontal intensity. Now let it be turned suddenly round a vertical axis through $180^{\circ}$. The change in induction is $2 \mathrm{AH}$. Say that the galvanometer indicates a flow of $Q_{2}$ coulombs. 'Then

$$
\frac{Q_{1}}{Q_{2}}=\frac{2 A V}{2 A H}=\frac{V}{H}=\tan \text { Dip. }
$$

The intensity of the field. The total intensity $I$ is never determined directly. If $\mathrm{H}$ is the horizontal component and $\mathrm{D}$ is the dip, $\mathrm{H}=\mathrm{I} \cos \mathrm{D}$. 'The value of $\mathrm{H}$ can be found much mure easily and much more exactly than I, and if $\mathrm{D}$ is known I may be deduced if needed. But in almost all cases we want to know $\mathrm{H}$.

To determine the horizontal intensity $\mathbf{H}$. The horizontal intensity is determined by a combination of two separate experiments. By one, known as the vibration experiment, we find the product of $\mathbf{H}$ and of $\mathbf{M}$ the magnetic moment of the magnet used. By the other, known as the deflection experi- 
ment, we find the ratio of these two quantities. The two results combined give us $\mathrm{H}$, and incidentally $\mathrm{M}$.

The vibration experiment. If a magnet of moment $M$ suspended so as to be horizontal and is displaced through $\theta$ from the magnetic meridian, it is easily seen that the magnetic couple tending to restore it to the meridian is $\mathbf{M H} \sin \theta$, or, if $\theta$ is very small, it is $\mathrm{MH} \theta$, and we shall have a simple harmonic vibration. If $K$ is the moment of inertia of the magnet and if the magnetic couple alone acts, the time of vibration is

$$
\begin{aligned}
& \mathrm{T}=2 \pi \sqrt{\frac{\mathrm{K}}{\mathrm{MH}}} \\
\text { or } & \mathrm{MH}=\frac{4 \pi^{2} \mathrm{~K}}{\mathrm{~T}^{2}} .
\end{aligned}
$$

To find $\mathrm{K}$, a non-magnetic bar of calculated moment of inertia $\mathrm{B}$ is attached to the magnet and the new time of vibration ' $\mathrm{T}^{\prime}$ is observed and

$$
\begin{aligned}
& \mathrm{T}^{\prime}=2 \pi \sqrt{\frac{\mathrm{K}+\mathrm{B}}{\mathrm{MH}}} \\
& \text { or } \quad \mathrm{MH}=\frac{4 \pi^{2}(\mathrm{~K}+\mathrm{B})}{\mathrm{T}^{1 / 2}} \text {. }
\end{aligned}
$$

Equating the two values of $\mathrm{MH}$ we obtain

$$
\begin{array}{ll} 
& \frac{K+B}{K}=\frac{T^{1 / 2}}{T^{2}} \\
\text { and } & K=\frac{B^{\prime} T^{2}}{T^{1 / 2}-T^{2}} .
\end{array}
$$

The value of $K$ need not be redetermined for each vibration experiment.

'The vibration experiment is carried out by suspending a needle like that used in the declination experiment in the same torsion box, Fig. 224. 'The vibration needle, however, is provided with a holder' for the bar B. Corrections, into which we shall not enter, are made for the additional torsion couple dueto the thread, for the arc of swing not being negligibly small, and for the change in the magnetic moment $M$ due to the temperature and for its increase due to the magnetisation induced by the earth's field.

The deflection experiment. In this experiment the vibration needle used in the previous experiment is set to deflect another needle from the meridian. The general principle of the method is as follows:

If the vibration needle $\mathbf{S c N}$, Fig. 230, is set end on, $\mathrm{E}$ or $\mathbf{W}$ of another needle it will deflect it through $\theta$, given by (p. 215)

$$
\tan \theta=\frac{2 \mathrm{M}}{d^{3} \mathrm{H}}
$$


where $d$ is the distance of the centre of the deflecting needle from the deflected needle, when we neglect small quantities. If then we observe $\theta$ and measure $d$, we know the ratio of $\mathrm{H}$ to $\mathbf{M}$, for

$$
\frac{H}{M}=\frac{2}{d^{3} \tan \theta} \text {. }
$$

Multiplying this by the result of the vibration experiment and taking the square root,

$$
\mathrm{H}=\frac{2 \pi}{\mathrm{T}} \sqrt{\frac{2 \mathrm{~K}}{d^{3} \tan \theta}} .
$$

Or another procedure may be adopted, and this is the more usual. The deflecting magnet, always in the end on position, is
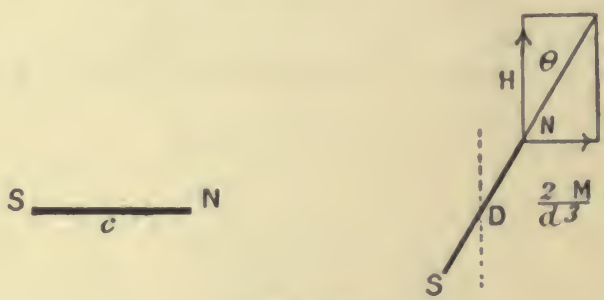

FI. 230 .

turned round the centre of the deflected magnet until the latter is in equilibrium and at right angles to the former. 'Then from
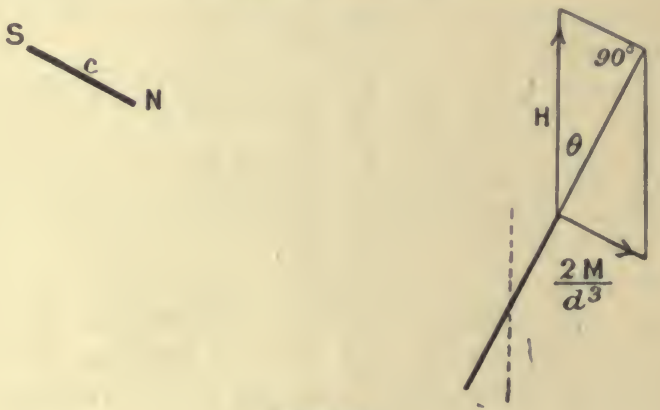

F1G. 231.

Fig. 231 we see that the angle $\theta$ through which the deflecting magnet has been turned round is given by

whence

$$
\begin{gathered}
\sin \theta=\frac{2 M}{d^{3} \mathrm{H}} \\
\mathrm{H}=\frac{2 \pi}{\mathrm{T}} \sqrt{\frac{2 \mathrm{~K}}{d^{3} \sin \theta}} .
\end{gathered}
$$


In using the last method, a graduated horizontal cross bar about a metre long is fixed at right angles to, and just under, the torsion box. A carriage slides along this bar to carry the deflecting magnet, and the distance $d$ is read on the scale of the bar. To measure $\theta$ a short magnet provided with a mirror is suspended in the torsion box of Fig. 224 to serve as the deflected magnet. A telescope with a scale attached replaces the telescope used in the declination experiment to fix the position of this deflected magnet. The deflected magnet is first suspended and its position is observed when the deflecting magnet is not acting. The reading of the horizontal circle at the base of the instrument is also to be taken. The deflecting magnet is then put in position at a distance indicated as $d$ on the bar scale and the instrument, with the telescope, is turned round until the deflected magnet appears in the same position in the field of the telescope. The horizontal scale at the base is again read, and the difference in readings gives the deflection $\theta$. If the observed $d$ were the actual $d$ this would suffice in so far as the simple formula holds. But the centre of the magnet may not coincide with the index mark on the carriage. The deflected magnet is therefore turned end for end. The deflection is now reversed and the mean value eliminates error of centering of the deflecting magnet. The graduation of the bar may not date from a point exactly under the centre of the deflected magnet. The deflecting magnet is therefore moved on its carriage to the other side of the bar to the distance marked as $d$, and the deflections are again taken with the magnet in its last position on the carriage and when it is turned end for end. We have then four values of the deflection, and the mean eliminates the errors considered.

But the formula for the deflecting force $\frac{2 M}{d^{3}}$ is only approximate. A nearer approximation is given by *

$$
\sin \theta=\frac{2 M}{d^{3} \mathrm{H}}\left(1+\frac{\mathrm{P}}{d^{2}}\right)
$$

where $\mathbf{P}$ depends on the distribution of magnetisation along the magnet. We may eliminate $\mathbf{P}$ by taking another value of the deflection $\theta^{\prime}$ at another distance $d^{\prime}$ and it is easily found that

$$
\frac{\mathrm{H}}{\mathbf{M}}=\frac{2\left(d^{2}-d^{\prime 2}\right)}{d^{5} \sin \theta-d^{\prime 5} \sin \theta^{\prime}} \text {. }
$$

The error in the determination of $\frac{H}{M}$ is a minimum if the value of $\frac{d^{\prime}}{d}$ is about $1 \cdot 3$.

* Maxwell, Electricity and Magnetism, vol. ii., 3rd ed., p. 106. 
Values frequently chosen are $d=30 \mathrm{~cm}$. and $d^{\prime}=40 \mathrm{~cm}$.

Corrections for temperature and for magnetism induced by the earth in the deflecting magnet must be made.

Dr. Chree has shown* that the approximation represented by the factor $1+\mathrm{P} / r^{2}$ is not justifiable with the magnets usually employed and that another term, $Q / r^{4}$, should be taken into account. 'The reader is referred to Dr. Chree's papers for a discussion of this point.

The value for $\mathrm{H}$ in this country is in the neighbourhood of $0 \cdot 18$, and different instruments may give values differing by as much as two or three units in the fourth place, say by as much as 1 in 1000 of the whole.

In the regions near the magnetic poles, i.e. the two points where the force is vertical, the horizontal intensity is only a small fraction of the total intensity and too small to be measured accurately. Special instruments which we need not describe $†$ have been devised to measure both dip and total intensity in these regions.

Recording instruments. Besides the instruments which give the absolute values of the magnetic elements, it is usual to equip magnetic observatories with self-recording instruments which register continuously the small changes which are always occurring in the direction and intensity of the force. For this purpose three magnets are used, one to record changes in declination, another those in horizontal intensity, and a third those in vertical intensity. A satisfactory self-recording dip instrument has not been devised. Each magnet is suspended in a dark box, and is furnished with a mirror which reflects a small beam of light from a lamp on to a revolving drum covered with seusitised paper. $\neq$ Each magnet is surrounded with a thick copper ring to damp its vibrations. The declination magnet is suspended so that it is free to set in the direction of the horizontal component. The horizontal force magnet has a bifilar suspension, and the torsion head is turned round so that the magnet is at right angles to the average magnetic meridian. Let Fig. 232 represent a plan. If $\mathbf{H}$ changes in direction only and not in magnitude, the change being through the small angle $\delta$, we shall have $H \sin \delta$ along $S N$ with no turning effect, and $\mathrm{H} \cos \delta=\mathrm{H}\left(1-\frac{\delta^{2}}{2}\right)$ perpendicular to NS : therefore the moment on the magnet is decreased by $\mathrm{MH} \frac{\delta^{2}}{2}$ which is negligible. A very small change in direction is, therefore, without effect. If $\mathrm{H}$ changes in magnitude, say from $\mathrm{H}$ to $\mathrm{H}+\delta \mathrm{H}$, the moment on the

* Proc. Roy. Suc., Ixv. (1899), p. 375 ; Phil. Mag. [6], vol. viii. (Aug. 1904), † See Walker's Terrestrial and Cosmical Magnetism, p. 209, for early forms. Recent forms are described in National Antarctic Expedition Magnetic Obserrations, Royal Society, 1909.

$\ddagger$ For details see references in footnote on p. 301 . 
magnet increases by $\mathbf{M} \delta \mathrm{H}$, and this is indicated by its angular displacement.

The vertical force magnet is provided with a knife edge at its centre, resting on a plane, and is so adjusted that with the average vertical force it is horizontal. It is practically a balance arm. If the vertical component changes, either through change in direction or magnitude of the total intensity, the magnet tilts. Special methods are adopted to calibrate the records on the revolving drum (Ency.

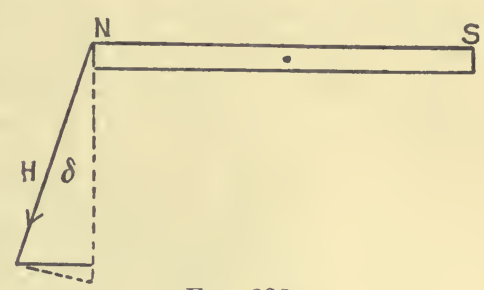

FIG. 232. Brit., l.c.).

Results. Observation shows that the magnetic elements are subject to continual "disturbances." On quiet days, in this country, the disturbances in the declination are not usually more than a few minutes. 'There are days on which the disturbances are greater, and sometimes are so large that they are described as due to magnetic storms. But it is very rare in this country for the range of declination to exceed $5^{\circ}$. When the value of an element for each hour of the day is averaged for a large number of days, it

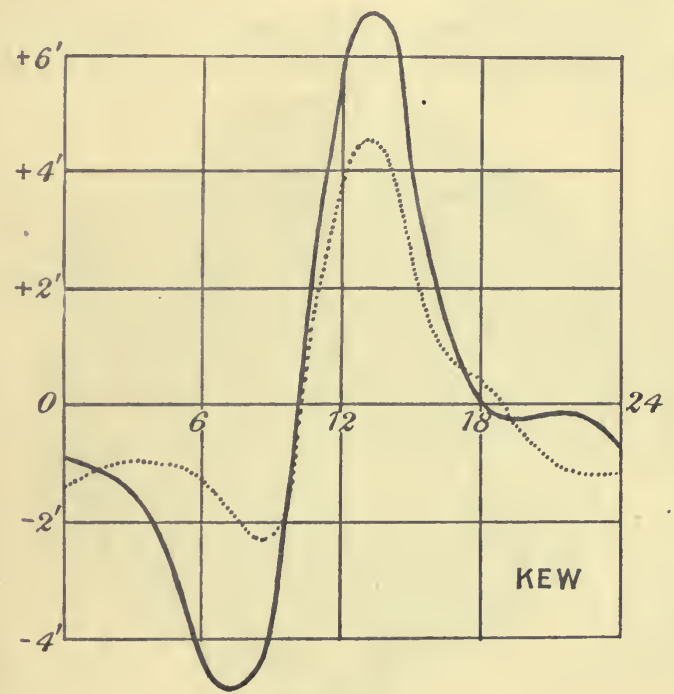

FIG. 233.

Continuous line, snmmer. Dotted line, winter.

is found that there is a diurnal periodicity or inequality differing at different parts of the earth, and differing again in summer and winter. Thus the declination at Kew shows a diurnal inequality 
represented by Fig. 233. There is also a small annual inequality and probably a very small lunar inequality.

If, instead of considering the actual value of an element, the amplitudes of its range are plotted, it is found that there is a connection between the range and sunspots, the range increasing as the area of the sunspots increases.*

In addition to these changes there is a secular change. 'The secular change in dip and declination is well represented by a

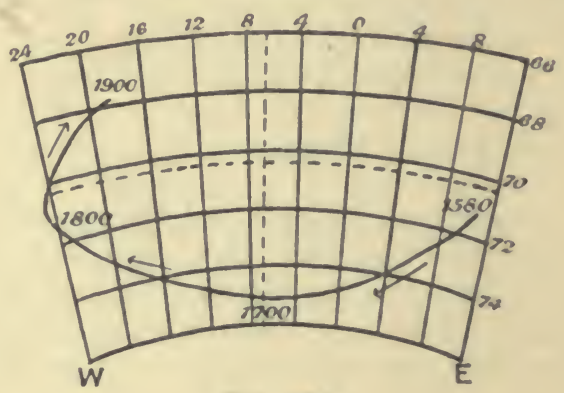

F1a. 234.

method due to Bauer. Fig. 234, gires the changes in the declination at London since $1580 . \dagger$

'These secular variations make it necessary to take a particular epoch when giving the value of the elements at a place.

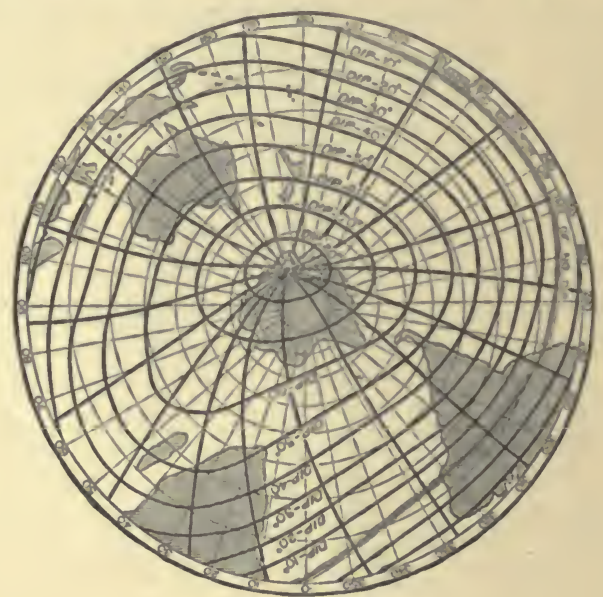

FIG. 235.

Figs. 235 and 236 show the lines of horizontal force (Duperrey's

* For an account of the inequalities see Chree, Studies in Terrestrial Magnetism On the connection with sunspots and auroras, see Ency. Brit., l.c.

$\dagger$ A Table of the mean annual changes in the elements at different positions in these islands is given by Thorpe and Rücker, Phil. Trans., A, clxxxi. (1890), p. 325. 
lines) and the lines of equal dip for $18 \% 6$. It will be seen that there are two poles or points of $90^{\circ}$ dip. That in the Northern Hemisphere in the neighbourhood of lat. $70^{\circ} 5^{\prime} \mathrm{N}$. and long.

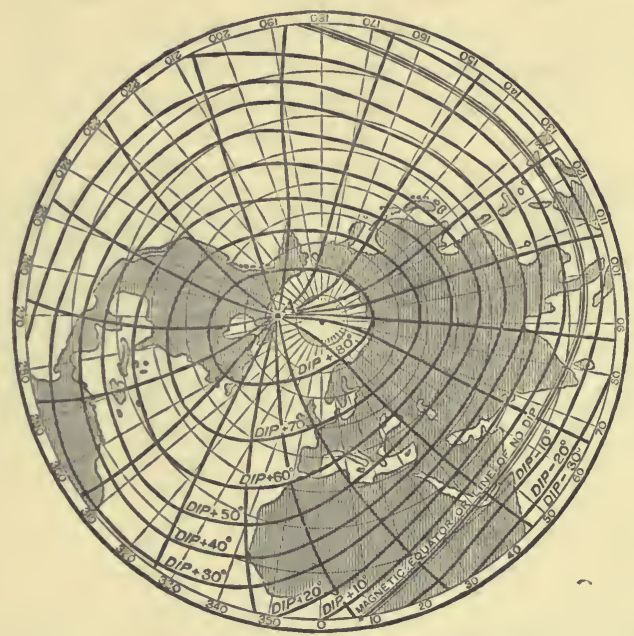

FIG. 236.

$96^{\circ} 46^{\prime} \mathrm{W}$. was reached in 1831 by Captain James Ross. In 1903 the region was again visited by Amundsen, who made a stay of two years, carrying out an extensive magnetic survey there. The

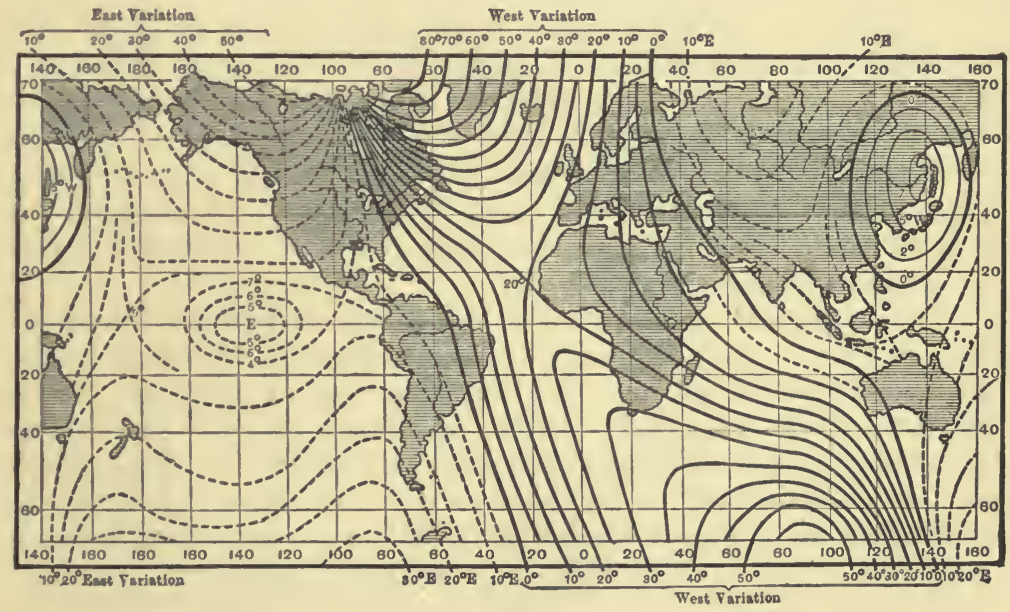

FiG. 237.

South magnetic pole was reached in 1909. It is about $72^{\circ} \mathrm{S}$. lat. and $155^{\circ} \mathrm{E}$. long.

Fig. 237 shows lines of equal declination or isogonic lines, and 
Fig. 238 those for equal dip or isoclinic lines for 1876. 'The figures on the right of Fig. 238 are the tangents of the dip.

Several careful magnetic surveys of limited areas on the earth's surface have been recently made. 'Thus, Thorpe and Rücker made a survey over Great Britain and Ireland in 1891-92,* finding various local deviations due, apparently, to the presence of magnetised rocks. Some of these deviations could be ascribed

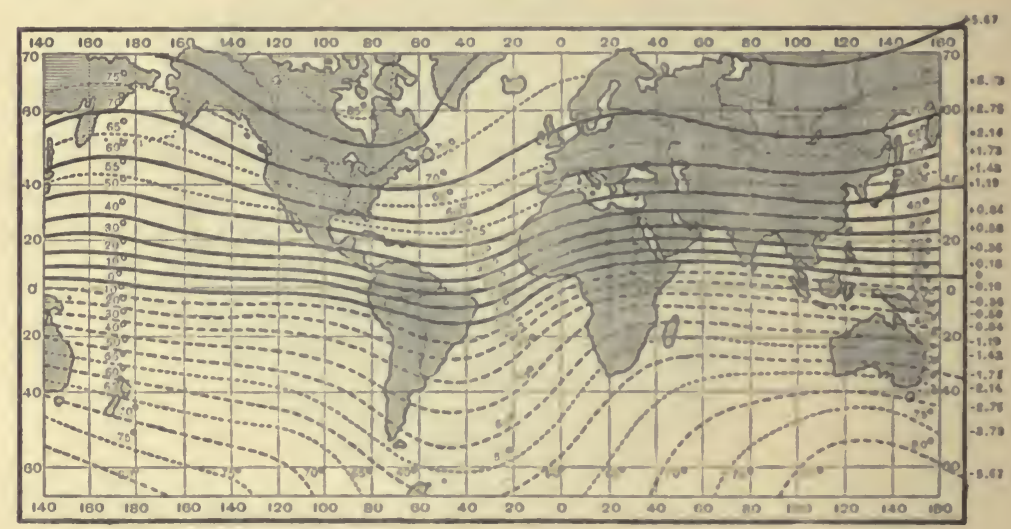

FI0. 23s.

to the known presence of such rocks, and from others the subterranean existence of magnetised matter was inferred.

Sketch of Gauss's theory of terrestrial magnetism. In 1839 Gauss published a paper on the General Theory of Terrestrial Magnetism, $\uparrow$ in which he investigated a formula which should represent the earth's field at every point of its surface. We shall give only a brief account of the principles of the theory. 'The original memoir is well worthy of sturly, not only for the subject-matter, but for the admirable introduction which it gives to the use of spherical harmonics.

Let us assume that the earth is a sphere and that its magnetic field is due in part to magnetisation within the surface, in part to magnetisation without the surface, and in part to electric currents which lie wholly within or wholly without the surface. These electric currents may be replaced by magnetic shells (p. 222), so that the field may be regarded as due to a definite distribution of magnetism. This distribution will have a potential given by $\mathrm{V}=\int \frac{d m}{r}$, where $d m$ is an element of magnetism and $r$ its distance from the point considered, and $\mathrm{V}$ will have a single definite value

* Phil. Trans., A., vol. clxxxviii. (1896).

† Werke, v. p. 119; Taylor's Scientific Memoirs, ii. p. 184. In Lloyd's Magnetism, p. 223, a brief abstract is given. 
at each point on the surface. 'This would not be true if currents existed passing through the surface. For in order that the potential shall be definite the work done in carrying unit pole fiom one point, $\mathrm{A}$, to another point, $\mathrm{B}$, must be the same by all paths, or, what is equivalent, the work done round any closed path ABA must be zero. But if a current $\mathrm{C}$ passes through the area enclosed by $A B A$, the work done round the path is $4 \pi \mathrm{C}$, and this work will be different for different paths enclosing different amounts of current. Hence the work from $\mathbf{A}$ to $\mathbf{B}$ is indefinite and in this case there is not a potential.*

Let us first consider the distribution of magnetism within the surface. Let $\mathrm{R}$ be the radius of the earth, $r$ the distance from the earth's centre of a point $\mathrm{P}$ outside the earth, $l$ the latitude, and $\lambda$ the longitude of the point of intersection of $r$ with the surface. 'Then it can be shown (Gauss, l.c.) that the potential at $\mathbf{P}$ may be put in the form of a converging series :

$$
\mathrm{V}=\mathrm{B}_{0} \frac{\mathrm{R}}{r}+\mathrm{B}_{1} \frac{\mathrm{R}^{2}}{r^{2}}+\mathrm{B}_{2} \frac{\mathrm{R}^{3}}{r^{3}}+\ldots
$$

where $B_{0}, B_{1}, B_{2}$, \&c., are certain functions of $l$ and $\lambda$ known as spherical harmonics. The general form of each of these functions is known. $\dagger$ If the order is designated by the suffix, that of the $n$th order $\mathrm{B}_{n}$ contains $2 n+1$ arbitrary constants.

At a very great distance from the earth the potential tends to a value $\mathrm{V}=\frac{\mathrm{M} \cos \theta}{r^{2}}$, where $\mathrm{M}$ is the earth's magnetic moment and $\theta$ is the angle its magnetic axis makes with $r$. The first term, $\frac{\mathrm{B}_{0} \mathrm{R}}{r}$, must, therefore, vanish, or $\mathrm{B}_{0}=0$; and it is easily shown, too, that in the second term $\mathrm{B}_{1}=a \sin l+b \cos l \sin \lambda+c \cos l \cos \lambda$, since $\mathrm{B}_{1} \mathrm{R}^{2}$ must be equal to $\mathrm{M} \cos \theta ; a, b$, and $c$ are constants depending on the position of the magnetic axis.

Next let us consider the distribution without the surface. It can be proved that the potential within the surface may be put in the form of a converging series :

$$
\mathrm{V}=\mathrm{V}_{0}+\mathrm{A}_{0} \frac{r}{\mathrm{R}}+\mathrm{A}_{1} \frac{r^{2}}{\mathrm{R}^{2}}+\cdots
$$

where again $\mathbf{A}_{0}, \mathbf{A}_{1}$, \&c., are spherical harmonics, but with arbitrary constants other than those in $B_{1}, B_{2}, \& c$. $V_{0}$ is the potential at the earth's centre, since it is the value of $\mathrm{V}$ when $r=0$. Being

* It is now known that there are minute vertical currents in the air, but too minute to be consirlered in a theory which does not pretend to give an exact account.

† Thomson and T'ait's Natural Philosophy, vol. i. p. 187. 
constant, it may be omitted, for in determining the forces we differentiate $\mathbf{V}$.

If we suppose a thin shell round the surface of the earth to be free from magnetism, within that shell the potential will be the sum of the potentials due to external and internal magnetism. 'Then

$$
\begin{aligned}
\mathrm{V} & =\mathrm{A}_{0} \frac{r}{\mathrm{R}}+\mathrm{A}_{1} \frac{r^{2}}{\mathrm{R}_{2}}+\mathrm{A}_{2} \frac{r^{3}}{\mathrm{R}^{3}}+\ldots \\
& +\mathrm{B}_{1} \frac{\mathrm{R}^{2}}{r^{2}}+\mathrm{B}_{2} \frac{\mathrm{R}^{3}}{r^{3}}+\ldots
\end{aligned}
$$

The intensity in any direction $s$ is $-\frac{d V}{d s}$. If $s$ is drawn in the geographical meridian towards the North, ds $=$ Rdll. I.et the component of the horizontal intensity in that direction be $\mathbf{X}$. If $s$ is drawn towards the geographical West, $d s=\mathrm{R} \cos l d \lambda$. Let the component in that direction be $Y$. If $s$ is drawn vertically downwards, $d s=-d r$. Let the vertical intensity $=\mathrm{Z}$.

Then

$$
\begin{aligned}
& \mathrm{X}=-\frac{1}{\mathrm{R}} \frac{d \mathrm{~V}}{d l}, \\
& \mathrm{Y}=-\frac{1}{\mathrm{R} \cos l} \frac{d \mathrm{~V}}{d \lambda} \\
& \mathrm{Z}=\frac{d \mathrm{~V}}{d r} .
\end{aligned}
$$

Gauss began by assuming that the earth's field is entirely due to inside magnetisation, and that the series converges so rapidly that the first four terms are sufficient to express $V$. So that

$$
\mathrm{V}=\mathrm{B}_{1} \frac{\mathrm{R}^{2}}{r^{3}}+\mathrm{B}_{2} \frac{\mathrm{R}^{3}}{r^{3}}+\mathrm{B}_{3} \frac{\mathrm{R}^{6}}{r^{4}}+\mathrm{B}_{4} \frac{\mathrm{R}^{8}}{r^{3}} .
$$

$\mathbf{B}_{\mathbf{1}}$ contains three constants, $\mathbf{B}_{\mathbf{2}}$ five, $\mathbf{B}_{\mathbf{a}}$ seven, $\mathbf{B}_{\mathbf{6}}$ nine, giving twenty-four in all. It must be noted that though these constants depend on the internal distribution, they tell us nothing as to that distribution, for a given distribution of external potential may be produced by an infinite number of different arrangements of magnetism. To determine the constants it would be sufficient to take eight stations at which $\mathbf{X}, \mathbf{Y}$, and $\mathbf{Z}$ were known, if these were knozen exactly. But, of course, there are errors in the values, not only of observation, but also of reduction to the same instant of time. Gauss took twelve stations in seven different latitudes, and computed the values of the twenty-four constants best satisfying the values of $\mathrm{X}, \mathrm{Y}$, and $\mathrm{Z}$ at the stations. He then calculated the values of $\mathbf{X}, \mathbf{Y}, \mathbf{Z}$ for a larger number of known stations all over the earth where $X, Y, Z$ had been observed. The differences between observation and 
calculation were not much greater than the errors of observation. In Taylor's Scientific Memoirs, vol. ii., will be found maps giving the lines of equal dip, equal declination, and equal total intensity according to Gauss's formula. Considering that the observations could not all be correctly reduced to the same epoch, these lines show a remarkable agreement with the observed lines and justify the preliminary assumption that the earth's field is, at any rate, mostly due to internal magnetism. Subsequent recalculation of the constants $*$ has tended to confirm this assumption.

Gauss himself suggested that the disturbances of the magnetic elements might be treated in a similar way, and that it might be shown whether they arose from internal or external changes in magnetism. Schuster $\dagger$ undertook such an investigation on the diurnal variation for the year 18\%0. His work led to the conclusion that the variation was chiefly "due to causes outside the earth's surface, and probably to electric currents in our atmosphere," and that "currents are induced in the earth by the diurnal variation which produce a sensible effect, chiefly in reducing the amplitude of the vertical components and increasing the amplitude of the horizontal components."

- Ency. Brit., 10th ed., xxx. p. 462 ; 11 th ed., xvii. p. 381 ,

† Phil. Trans., A, vol. clxxx. (1889), p. 467. 


\title{
CHAPTER XXIV
}

\section{MAGNETISM AND LIGHT}

\begin{abstract}
The Faraday rotution-Faraday's successors - Verdet's constunt and its variation with wave length-Effect of rise of temperature-Absolnte values of Verdet's constant-Rotation by gases-Rotation by films of iron, nickel, and cobalt-Representation of the rotation by two ejuul circularly polarised rays with opposite rotations travelling with different velocities -An electron theory of the rotation-Confirmations of the theoryMrgnetic double refraction when the light ray is perpendicular to the lines of force-Voigt's theory-Magnetic double refraction in colloidsMagnetic double refruction in pure liquids - The Kerr magnetic effectThe Zeeman effect-Iorents's theory-Zeeman's verification.
\end{abstract}

The Faraday rotation. Faraday opens his paper describing his great discovery of an action of magnetism on light by saying: "I have long hold an opinion, almost amounting to conviction, in common I believe with many other lovers of naturnl knowledge, that the various forms under which the forces of matter are made manifest have one common origin ; or, in other words, are so directly related and mutually dependent that they are convertible, as it were, one into another, and possess equivalents of power in their action." *

He then states that this persuasion had led him formerly to make many unsuccessful attempts to obtain the direct relation of light and electricity. Recently, however, he had succeeded in "magnetising and clectrifying a ray of light, and in illuminating a magnetic line of force," a picturesque description of the fact that if a ray of plane-polarised light travels through certain substances along the lines of force in the field of a permanent magnet or in that of a solenoid through which is passed a current of electricity the plane of polarisation gradually rotates as the ray travels on.

The first substance which exhibited the effect was a very heary glass, a silicated borate of lead which he had made some years earlier. A plate of this glass two inches square and half an inch thick was polished on its narrow sides and placed between the poles of an electro-magnet in such a position that when the current was on, lines of force went, as nearly as might be, straight through the two inches of glass from one side to the other. A ray of light from an Argand burner was made to fall on a glass plate at the polarising

Exp. Rex. III. p. 1 (November 1845). 
angle and then passed plane-polarised in the horizontal direction through the glass close to the poles and in the direction of the lines of force. It was then received through a Nicol prism into the observer's eye. 'The prism could be rotated about the ray as axis, and the rotation could be measured.

Before the current was put on, the Nicol was turned into the position of extinction. On making the current the field of view lighted up. If the Nicol was then rotated, a new position of extinction was found showing that the light was still planepolarised, but that the plane was turned round in to a new position. He found that there was 110 visible effect if the magnet were so turned that the lines of force were perpendicular to the ray. The fields produced by a permanent magnet, and by a helical coil or solenoid in which an electric current flowed, were effective, and as far as he could estimate he found that the rotation with a given length of glass was proportional to the strength of field, and with different lengths of glass proportional to the length. He examined a large number of substances besides the glass, and found that many exhibited the effect, such as flint and crown glass, water and alcohol. 'The solenoid arrangement was useful for the liquids, which could be enclosed in tubes with glass ends, placed along the axis of the solenoid. It was this solenoid method, in which an electric current was effective, which led him to say that he had electrified a ray of light. With optically active bodies such as turpentine or sugar he found that the magnetic rotation was merely added to or subtracted from the natural rotation according to its sign. He was unable to detect any rotation in air.

In every case which Faraday observed, the rotation was in the direction of the current which would produce the lines of force, if we imagine them due to a solenoid surrounding them. 'Thus if the lines of force are supposed to come out from the paper to the reader's eye, the creating current would circulate counter-clock wise, and that would be the direction of rotation of the plane of polarisation. The rotation would be reversed if the lines of force went from the eye to the paper.

Subsequent researches have shown that all substances which light traverses exhibit the Faraday effect, and for the most part with the rotation in the direction which he observed and which is described as positive. But salts of iron and some few others, though not salts of nickel and cobalt, exhibit a negative rotation. 'The distinction in no way corresponds to the distinction of paramagnetic and diamagnetic, for iron nickel and cobalt, though enormously paramagnetic, coincide with the great majority of diamagnetics in giving positive rotation, while the diamagnetic chloride of titanium gives a negative rotation.

'The magnetic rotation differs in one very important respect from the rotation by a naturally active substance such as sugar solution. In the sugar solution the rotation is always "right- 
handed" or clockwise in a ray coming to the observer. It is therefore counter-clockwise to him in a ray going from him. So that if a ray is sent along a solution from the observing end and then reflected back to the observer the rotations in the two journeys are opposite and cancel each other, and the ray emerges polarised parallel to its original plane. But with a "magnetised "ray the rotation is always in the direction of the current which would produce the lines of force, so that for each journey along a line of force the rotation is increased by the same amount. 'Ihus Faraday * found that the rotation for one passage through a piece of glass was $12^{\circ}$, but on reflecting it to and fro so that it made five passages the rotation was increased to $60^{\circ}$.

Faraday's successors. Furaday does not appear to have made further experiments after the publication of his paper, his attention being called off by his investigations into paramagnetism and diamagnetism; but the subject was pursued by many workers. E. Becquerel was the first to observe that different colours were rotated by different amounts, red least, violet most-a phenomenon known as rotary dispersion. By making the magnetic rotation "left-handed" and then passing the rotated light through a sugar tube of suitable length to neutralise the rotation for one colour, it was neutralised for all as nearly as he could judge, or the magnetic rotation was proportional to the natural rotation for all colours. G. Wiedemann used different parts of a solar spectrum as the source of light, a solenoid to produce the field, and a tangent galvanometer to measure the current. He was thus able to show that the rotation was proportional to the current, and therefore to the field intensity within the coil. He found that the magnetic rotary dispersion of carbon bisulphide, for instance, was not very far from being proportional to the natural rotary dispersion in a sugar solution but yet sensibly different, and it was not far from being inversely as the square of the wave length, though again quite sensibly different.

Verdet's constant, and its variation with wave length. Verdet $†$ made a long series of experiments on various substances with an accuracy probably superior to that attained up to his time, and he proved conclusively that the rotation per unit length of a substance traversed is proportional to the component of the intensity of field in the direction of the ray. If, then, $d s$ is an element of the path of the ray in a substance, and the intensity $\mathbf{H}$ makes an angle $\theta$ with $d s$, the rotation $\mathbf{R}$ of the plane of polarisation between $s=a$ and $s=b$, usually expressed in minutes of arc, is

$$
\mathbf{R}=\mathrm{C} \int_{a}^{b} \mathrm{H} \cos \theta d s
$$

* Exp. Res. III. p. 453.

$\dagger$ Quvres, tome i.; or Gordon, Electricity and Magnetism, vol. ii. 
where $\mathrm{C}$ is a constant for the substance for the particular wave length used. It is known as Verdet's constant, though it might more appropriately be named after Faraday. If $\mathrm{V}^{a}, \mathrm{~V}_{b}$, be the magnetic potentials at the beginning and end of the path considered we may put $R$ in minutes $=C\left(V_{b}-V_{a}\right)$, a concise form of expression, though the integral form is the one practically useful.

Verdet worked before the value of absolute units was realised, and he was content to express the constant of a substance in terms of the constant for water. Taking the rotation in water of light at the $\mathbf{E}$ line in the spectrum as 1 , he found the rotations of the light of the various lines as follows. Underneath are the rotation. which would have been observed if they had been inversely as the squares of the wave length.

\section{$W_{\text {ATER }}$}

$\begin{array}{lccccc}\text { Lines in spectrum } & \text { C } & \text { D } & \text { E } & \text { F } & \text { G } \\ \text { Observed rotations } & 0 \cdot 63 & 0 \cdot 79 & 1 \cdot 00 & 1.213 & 1.605 \\ \text { Calculated from } 1 / \lambda^{2} & 0.64 & 0.80 & 1.00 & 1.08 & 1.50\end{array}$

For carbon bisulphide, creosote, and various other liquids the dispersion is about as near to that calculated from $1 / \lambda^{2}$ as in the case of water, departing as notably from it as the wave length diminishes.

If the substance examined absorbs the light of one particular wave length there are peculiarities in the rotation, which were first predicted from theory, and which will be more appropriately described when we discuss the theory now accepted.

Effect of rise of temperature. Bichat was the first to make a careful examination of the effect of change of temperature of the Verdet constant, and he found that in general the constant lessens as the temperature rises, though his numerical values have not been confirmed by subsequent workers.

Absolute values of Verdet's constant. The first determination of the absolute value of a Verdet constant was made by Gordon * for sodium light in carbon bisulphide.

A few years later another determination of the same constant was made by Lord Rayleigh. $\uparrow \mathrm{He}$ used a coil with a known number of turns surrounding a tube of known length containing the bisulphide. 'The current was measured by a potentiometer method, assuming the E.M.F. of a Clark cell. If the tube had extended a great distance beyond the coil at each end the difference of magnetic potential between the ends would have been $4 \pi \times$ number of turns of coil $\times$ current. But as the ends were at points where the field was still appreciable a correction was needed.

'To measure the rotation Lord Rayleigh used a special " half-

* Gordon, El. and Mag., vol. ii. chap. xlviii.

† Phil. Trans. 176, p. $3 \pm 3$ (1885); or Scientific Papers, vol. ii. p. 360. 
shadow" method,* on the general principle now always adopted in polarimetry, in which the field is divided into regions with their planes of polarisation slightly inclinced to each other. 'The sodium light used was polarised by a Nicol. It then passed through a cell containing sugar solution, but a plate of glass was immersed in the solution, and placed so that one half of the beam passed through it, while the other half passed by its edge and so through an extra thickness of sugar solution. 'This half of the beam was therefore a little more rotated than the half passing through the glass, and when the bean emerged from the cell its two halves were polarised in planes making a small angle with each other. Then the beam passed through the tube and into the analysing Nicol in front of the observer's eye. 'The observer either focussed his eye directly on to the edge of the glass plate in the sugar cell, or viewed the edge by a Galitean telescope. To understand the method let us suppose that, to begin with, there is no current in the coil. Let OR OL, Fig. 239, represent the equal amplitudes and
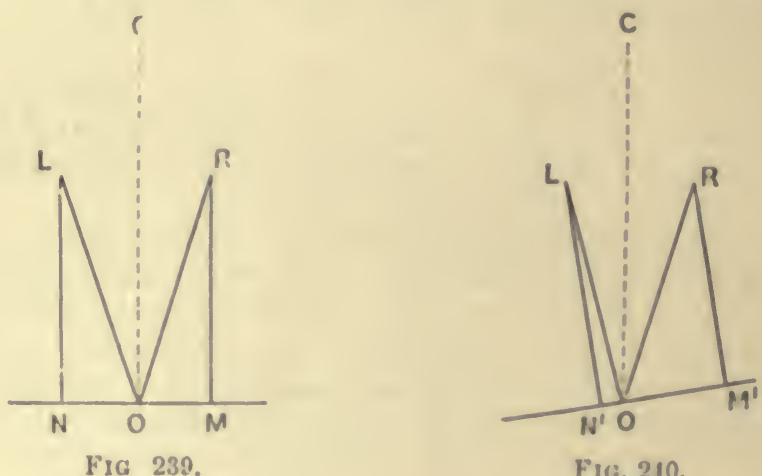

the directions of vibration in the two halves of the field of view. 'The angle ROL, is greatly exaggerated in the figure. It is not more than 2 or 3 degrees usually. Let the analysing Nicol be turned so that it extinguishes vibrations in the direction of $O C$, the bisector of ROL. If MON is perpendicular to OC, and RM LN are perpendicular to it, the analyser, when placed so that it would extinguish vibrations along $\mathrm{OC}$, admits equal vibrations OM ON from the two halves of the field. If it be now turned through a small angle so that the vibrations admitted are $O M^{\prime} O N^{\prime}$, Fig. 240, it is obvious that there will be a great difference in the illumination of the two halves. Hence the position of the analyser for eyuality of illumination can be fixed with considerable aceuracy. When this has been done let the current be put on so that the magnetic field is established. 'The planes of OR

* Poynting, Phil. Mag. [5] ], x. (19s0), p. 18. 
and $\mathrm{OL}$ will be equally rotated, and the analysing Nicol must be turned through the same angle to secure equality of the two halies of the field once more. The angle is read on a divided circle with which the analyser is provided.

The result which Lord Rayleigh obtained for the Verdet constant for sodium light for bisulphide of carbon at $18^{\circ}$ measured in minutes per unit difference of potential is

0.04202.

Rodger and Watson* made determinations of the Verdet constants for sodium light in carbon bisulphide and in water over a range from $0^{\circ} \mathrm{C}$. to $40^{\circ} \mathrm{C}$. for the former, and over a range from $0^{\circ} \mathrm{C}$. to $100^{\circ} \mathrm{C}$. for the latter. 'Their values for the constants were: for carbon bisulphide $0 \cdot 04347-737 t / 10^{7}$, and for water $0 \cdot 01311-4 t / 10^{7}-4 t^{2} / 10^{8}$. 'Their formula for bisulphide of carbon would give the value of the constant at $18^{\circ}$ as 0.4214 , which only differs from Lord Rayleigh's value by 1 in 350 .

Constants for many other materials were determined in terms of that of carbon bisulphide by $\mathrm{H}$. Becquerel, and these may be turned into absolute values by using the constant just given for that liquid. 'The following values are extracted from a table of Becquercl's results given by Gordon, l.c.: carbon bisulphide, 1; water, 0.308; benzene, 0.636 ; rock-salt, 0.843 ; crown glass, 0.481 ; bichloride of titanium, $-0.358 .+$

Rotation by gases. In 1879 both $\mathrm{H}$. Becquerel and Kundt and Röntgen + succeeded in measuring the rotation by gases. Becquerel passed a ray several times to and fro along a copper tube with glass ends surrounded by a coil. Kundt and Röntgen also used a copper tube with glass ends surrounded by a coil. Into this the gas to be dealt with was compressed to 250 atmospheres. 'The glass ends were so seriously strained by the internal pressure that they became doubly refracting. It was therefore necessary to put the polariser and analyser within the tube, each being fixed to the tube at the end at which it was placed. The polariser end was clamped in a fixed position. 'The analyser end could be twisted round, subjecting the tube to torsion, and the angle of twist could be measured. It was found that the rotation was proportional to the density of the gas, so that it could be reduced to normal density at $0^{\circ} \mathrm{C}$. and $760 \mathrm{~mm}$. In all cases examined the rotation was positive. 'The following rotations at N'IP in terms of that of bisulphide of carbon were obtained:
$\mathrm{CS}_{2}$
o
$\mathrm{N}$
0000127
H
$0 \cdot 000132$
$\mathrm{CO}_{2}$
().000232

* Phil. Truns., A., 186, Part II. p. 621 (1895).

+ A number of values by different workers will be found in Winkelmann's Ilundbuch, vol. v.

$\ddagger$ Some account of both researches is given by Gordon, l.c. 
Rotation by films of iron, nickel, and cobalt. In 1884 Kundt* succeeded in depositing on glass films of iron, nickel, and cobalt less than a wave length in thickness which easily transmitted light. When one of these was placed in a magnetic field normal to the lines of force the plane of a polarised ray passing through was rotated in the positive direction-i.e. the direction of the magnetising current-and by an amount proportional to the thickness of the film. 'The rotation increased with increase of the field up to about $17,000^{\circ}$; but when the field exceeded this the rotation did not increase much. 'The maximum rotation of a certain red ray in iron was such that passage through $1 \mathrm{~cm}$. of iron would at the same rate give a rotation of $200,000^{\circ}$. 'The rotation of blue light was less than that of red.

The approach of the rotation to a limit suggested that the rotation is proportional, not to the external magnetising force $\mathrm{H}$, but to the intensity of magnetisation $\mathrm{I}$. With $\mathrm{H}=17,000$ we may suppose the iron to be saturated or that I has reached its limit. In an ordinary non-ferromagnetic substance the intensity of magnetisation is given by $\mathbf{I}=\kappa \mathrm{H}$, where $\kappa$ is a minute constant and $I$ is proportional to $H$. In a ferromagnetic metal $\kappa$ is not constant and I is not proportional to $\mathrm{H}$. I) Bois $†$ plotted I against $\mathrm{H}$ for each of the three metals iron, nickel, and cobalt, using Rowlands's values. He then plotted rotation for a definite thickness against $\mathrm{H}$, using the rotations determined by Kundt and by himself, and found that the form of the two sets of curves was the same, or the rotation was proportional to I.

Representation of the rotation of the plane of polarisation by two equal circularly polarised rays with opposite rotations travelling with different velocities. Let a point $\mathrm{P}$ ' move with uniform angular velocity $\omega$ in a circle radius $a$. Drop a perpendicular $\mathrm{PN}$ on a diameter $\mathrm{AC}^{\prime}$ ' If $\mathrm{P}$

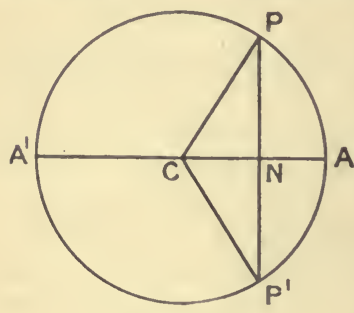

FIG. $2 \sharp 1$. starts at $t=0$ from $\mathrm{A}, \mathrm{ACP}=\omega t$ and $\mathrm{CN}=a \cos \omega t$. 'Then $\mathbf{N}$ has a simple harmonic motion with the same period as $\mathrm{P}$.

When $\mathrm{P}$ starts from $A$ let $\mathrm{P}^{\prime}$ start from the same point with the same angular velocity $\omega$ round the same circle but in the opposite direction, so that $\mathbf{P}^{\prime}$ is always in PN produced. 'Then the sum of the displacements of $\mathrm{P}$ and $\mathrm{P}^{\prime}$ parallel to $\mathrm{AA}^{\prime}=2 x \cos \omega t$, and the sum of the displacements perpendicular to $\mathrm{AA}^{\prime}$ is zero, since the displacements are ecial and opposite. Hence a simple harmonic vibration may be made up of, or conversely may be resolved into, two circular motions, each of half its amplitude and with equal period.

\footnotetext{
* Phil. Mag. [5], xviii. (1884), p. 303.

† Ibid. [5], xxiv. (1887), p. 445 .
} 
A train of waves of circularly polarised light may be regarded as consisting of circular disturbance, represented by a radius of given length travelling round each point in the path and with the same angular velocity for each radius, the ends of the radii being on a revolving spiral. A corkscrew turned round counter-clock wise without advancing exactly represents such a train of waves with counter-clockwise rotation travelling towards its point, and if it be laid lengthwise on a mirror its reflection in the mirror represents the transmission of a train with clockwise rotation. Let $(a)$, Fig. 242,represent the right-handed corkscrew as seen from above, the thick lines representing the upper part. 'Ihen if it is turned round

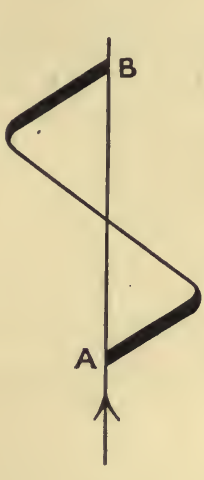

(a)

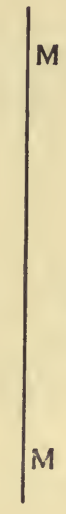

FIG. 242.

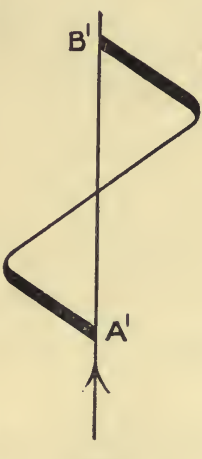

(b)

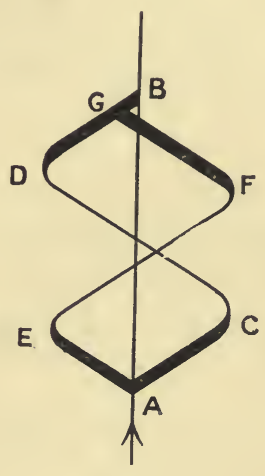

F'IG. 243.

counter-clockwise as seen from $\mathrm{A}$, a given displacement will travel forward from $\mathrm{A}$ to $\mathrm{B}$. If $(b)$ represents its reflection in the mirror MM, a clockwise rotation as seen from $\mathrm{A}^{\prime}$ will send a given displacement forward from $\mathrm{A}^{\prime}$ to $\mathrm{B}^{\prime}$.

If the two displacements in $(a)$ and $(b)$ are combined, they will always give an up-and-down displacement perpendicular to the plane of the paper, or a plane-polarised disturbance.

But now suppose that while the speed of rotation of the two is the same, one travels faster forward than the other, that is, has a longer wave length, or is a steeper spiral. 'Thus in Fig. 243 let $A C D B$ represent the faster-moving and longer wave, $A E F G$ the slower and shorter. At the instant represented they coincide at A, and as the revolution is at the same rate for both spirals they combine now and always to give at $\mathbf{A}$ a disturbance perpendicular to the paper. At this instant they coincide again at $G$, and combine now and always to give at $\mathbf{G}$ a disturbance in a line which is turned round in the direction of the circular motion which has the greatest forward velocity. That is, there is rotation of the plane of polarisation. 
We may obtain the actual rotation by noting that the forward propagation of a circular disturbance with speed $v$ is represcuted by

$$
\theta=\omega\left(t-\frac{x}{v}\right)
$$

where $\theta$ is the angle the radius makes with a fixed direction perpendicular to the line of propagation, the distance $x$ being measured from a starting-point on the line at which $\theta=0$ when $t=0$. For at any fixed point the angle $\theta$ grows at the rate $\omega$, or at each point there is circular motion, and at distance $x, \theta=0$ when $x=v t$, or the displacement which is at $x=0$ when $t=0$ is propagated forward with relocity $v$

Now let an equal circular disturbance, but with opposite rotation, $-\omega$, travel forward with a less velocity $v$ '. 'Then the direction of disturbance at $x$ at time $t$ will be

$$
\theta^{\prime}=-\omega\left(t-\frac{x}{v^{\prime}}\right)
$$

assuming that it is 0 at $x=0$ when $t=0$.

It is easily seen that the sum of two displacements, cach $a$ and in directions $\theta \theta^{\prime}$, is in the direction $\frac{\theta+\theta^{\prime}}{2}$, and is in magnitude

$$
2 a \cos \frac{\theta-\theta^{\prime}}{2} \text {. }
$$

'That is, it is in the dircetion $\frac{\omega n}{2}\left(\frac{1}{v^{\prime}}-\frac{1}{r^{\prime}}\right)$,

and its magnitude is

$$
2 a \cos \omega\left\{t-\frac{x}{2}\left(\frac{1}{v}-\frac{1}{v^{\prime}}\right)\right\} .
$$

It is, then, a simple harmonic vibration in a constant direction at a constant point, but as we travel forward the direction turus round in the positive direction of $\omega$ at a rate per unit distance

$$
\frac{\omega}{2}\left(\frac{1}{v^{\prime}}-\frac{1}{v^{\prime}}\right)=\frac{m}{2} \frac{v-z^{\prime}}{i v^{\prime}}
$$

or if $n$ is the frequency of revolution, so that $\omega=2 \pi n$, we may put the rotation of the plane of polarisation as

$$
\frac{\tau^{\prime}-v^{\prime}}{r^{\prime} \tau^{\prime}} n \pi,
$$

and in the direction of rotation of the faster-moving constituent.

So far this is metely a geometrical representation of the facts 
and at first sight it appears somewhat artificial. But it appears more natural if we assume, as we surely must, that there is some sort of motion round the lines of force in a magnetic field. 'Then a resolution into circular motion would seem to be appropriate.

An electron theory of the rotation. We have now to show that if we suppose that in a circularly polarised ray the electrons are whirled round with the frequency of the waves (i.e. a number of times per second equal to the number of waves passing a point per second), then the velocity will be different for the two directions of whirling if a magnetic field exists parallel to the path of the ray.

According to Sellmeier's mechanical theory, the velocity of a train of light waves in a medium depends on the nearness of the wave frequency to the natural frequencies of vibration of the molecular or atomic systems constituting the medium. By the natural frequencies we mean those of the different types of motion of each system if it is disturbed and then left to vibrate without any external action.

I et $\mathbf{N}_{1}, \mathbf{N}_{2}, \& \mathrm{c}$., be the natural periods of the constituents of the medium. 'The velocity $v$ of a train of waves of frequency $n$ is given by

$$
\frac{1}{v^{2}}=\mathbf{K}+\frac{\beta_{1}}{\mathbf{N}_{1}^{2}-u^{2}}+\frac{\beta_{2}}{\mathbf{N}_{2}^{2}-u^{2}}+\& \mathrm{c} .
$$

where $\mathbf{K}, \beta_{1}, \beta_{2}$, \&c., are constants for the medium.*

For simplicity we shall suppose that there is only one mode of natural vibration $\mathbf{N}$, and that this takes place under an acceleration towards a centre at distance $d$ equal to $\omega^{2} d$ or $4 \pi^{2} \mathrm{~N}^{2} d$. 'The relocity of a train of wares is given in this case by

$$
\frac{1}{v^{2}}=K+\frac{\beta}{N^{2}-n^{2}}
$$

When $u=\mathbf{N}$, the formula makes $v=0$. 'The physical interpretation is that there is at this value "resonance," and the wares spend their energy in setting the constituent systems of the nedium into vibration so that they are absorbed and not transmitted. 'There will be absorption too, in a real system, for some little range on each side of $n=\mathrm{N}$. As we rise from $n=0$, i.e. from waves of infinite length towards $n=\mathrm{N}, 1 / v^{2}$, which is proportional to $\mu^{2}$ where $\mu$ is the refractive index, gradually increases and becomes great as $u$ nears $\mathbf{N}$. When $n$ has just passed $\mathrm{N}, 1 / v^{2}$ is at first negative or $\mu$ is unreal. But when $1 / v^{2}$ becomes positive and $\mu$ real it increases as $n$ increases. 'There is, however, a limit $\mathbf{K}$ towards which it tends as $n$ goes on increasing.

* See Schuster's Theory of Optics for an account of the theory of dispersion and of magneto-optics. Voigt, who has very largely contributed to the theory, is the author of a work on Ma!jneto- und Elelitro-optik, Teubner, 1908. 
We may represent the general nature of the change in $1 / v^{2}$ or $\mu^{2}$ by Fig. 244 .

This theory holds good in the electro-magnetic theory if we suppose that the medium consists of central bodies round which electrons or small charged bodies are revolving, each with a period or periods of its own. We make the usual supposition that the

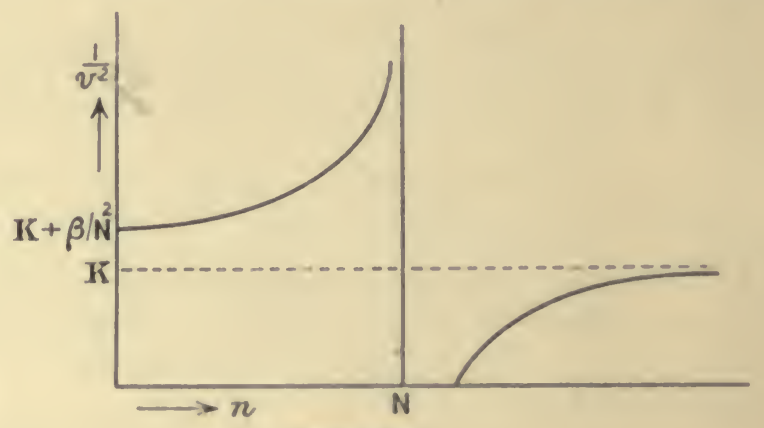

F10. 244.

charge on each electron has the same negative value. We represent . it numerically by $e$ and the mass of the electron by $m$.

Now let a circularly polarised beam of frequency $n$ travel through the medium. We suppose that it mahes the electrous whirl round the centres of their natural orbits in circles with frequency $n$, instead of the natural frequency $\mathbf{N}$, the wave disturbance calling into play a force which, added to $m .4 \pi^{2} N^{2} d$, the natural force of the system, accounts for the new period.

When a ficld $\mathrm{H}$ is put on, let us suppose that its + direction coincides with the direction in which the ray is travelling. If $d$ is the distance of the elcctron $e$ from its centre, its velocity is $2 \pi n d$, and as it is moving transversely to the fieid $\mathrm{H}$ a radial force due to $\mathrm{H}$, equal to $2 \pi n d \mathrm{H}$, will be called into play. The uegative electron moving clockwise is equivalent to a positive current circulating counter-clockwise. Hence the force is inwards on an electron moving clockwise and outwards on one moving counterclockwise. If $m$ is the mass of the electron, the acceleration at distance $d$ is $\pm 2 \pi n d \mathrm{Hc} / \mathrm{m}$. The total accileration due to the internal force and to this magnetic force is

$$
\left(4 \pi^{2} N^{2} \pm 2 \pi n \mathrm{H} c / m\right) d^{2}
$$

and this is the natural acceleration when the field is on.

Putting (1) in the form $\omega^{2} d$, we see that there are now two different natural frequencies, given by $\omega / 2 \pi$, where $\omega$ has the value

$$
\sqrt{4 \pi^{2} \lambda^{2}+2 \pi n H e / m}
$$


for a ray in which the rotation is clockwise as seen from the source, and the value

$$
\sqrt{4 \pi^{2} \mathrm{~N}^{2}-2 \pi n \mathrm{H} e / m}
$$

for a ray with the opposite rotation.

If the two frequencies are $\mathrm{N}_{1}, \mathrm{~N}_{2}$, then

$$
\left.\begin{array}{l}
\mathrm{N}_{1}^{2}=\mathrm{N}^{2}+\frac{n \mathrm{H} e}{2 \pi m}=\mathrm{N}^{2}+\nu \\
\mathrm{N}_{2}^{2}=\mathrm{N}^{2}-\frac{n \mathrm{H} e}{2 \pi m}=\mathrm{N}^{2}-\nu \\
\text { where } \nu=\frac{n \mathrm{H} e}{2 \pi m}
\end{array}\right\}
$$

As the natural frequencies for two circularly polarised rays with opposite rotations thus differ, they have different velocities, $v_{1}, v_{2}$, given by

$$
\left.\begin{array}{l}
\frac{1}{v_{1}^{2}}=\mathrm{K}+\frac{\beta}{\mathrm{N}^{2}+\nu-n^{2}}=\mathrm{K}+\frac{\beta}{\mathrm{N}^{2}-n^{2}}-\frac{\beta \nu}{\left(\mathrm{N}^{2}-u^{2}\right)^{2}} \\
\frac{1}{v_{2}^{2}}=\mathrm{K}+\frac{\beta}{\mathrm{N}^{2}-\nu-n^{2}}=\mathrm{K}+\frac{\beta}{\mathrm{N}^{2}-n^{2}}+\frac{\beta \nu}{\left(\mathrm{N}^{2}-u^{2}\right)^{2}}
\end{array}\right\}
$$

If we subtract the first of these from the second we get

$$
\frac{1}{v_{2}^{2}}-\frac{1}{v_{1}^{2}}=\frac{2 \beta \nu}{\left(\mathrm{N}^{2}-n^{2}\right)^{2}}
$$

- a result which is due to Voigt.

We have taken $v$ as positive for negative electrons, and as $\mu^{2}$, which is proportional to $\frac{1}{v^{2}}$, usually increases as we approach an alosorption band from the long wave side, we may take $\beta$ as usually positive. Hence (4) is usually positive, or $v_{1}>r_{2}^{\prime}$, and a circularly polarised ray in which the rotation as seen from the source is clockwise moves with greater velocity than a ray with counterclockwise rotation. 'The rotation therefore has the same sign whether $n$ is less than or greater than $\mathbf{N}$.

If a ray of plane-polarised light is resolved into two co-existing circular components, and we assume that they have different velocities as given by (3), the clock wise component moves the faster and the plane of polarisation rotates clockwise or in the direction of the current round the ray which would produce the field in which it is travelling.

If $v_{1}$ and $v_{2}$ are nearly cqual, we may put the rotation per unit length (p. 328), by aid of (4), in the form

$$
\frac{v_{1}-v_{2}}{v_{1} v_{2}} \pi n=\frac{\pi n v \beta \nu}{\left(\mathrm{N}^{2}-n^{2}\right)^{2}}
$$


When the rotation is in the opposite direction, as in titanium chloride and in salts of iron, we may perhaps account for it by a negative sign for $\beta$, i.e. a decrease in $\mu$ as $\lambda$ : lecreases, or perhap.s by assigning the opposite sign for $\nu$, which implies that positive charges are revolving.

Confirmations of the theory. Righi* showed that the wave lengths of two oppositely circularly polarised rays were different when they were made to traverse a magnetic field along the lines of force, before the electron theory was elaborated to account for the difference of their velocities. A pencil from a source was divided into two pencils which were circularly polarised with rotations in opposite directions and then ran alongside each other through a tube containing an active liquid and surrounded by a solenoid. 'The two pencils then passed through a Nicol and interfered in the focal plane of a micrometer eyepiece by which the fringes could be seen and their positions fixed. When the current was put on the position of a fringe was read by the micrometer wire. 'Then the current was reversed and the fringes shifted, and by an amount sufficiently near to that which would be expected from the observed rotation of the planes of polarisation in the liquid by the same current.

Brace $\nmid$ made a similar experiment with a similar result. Some time later, by making the two rays travel in an rrangement of

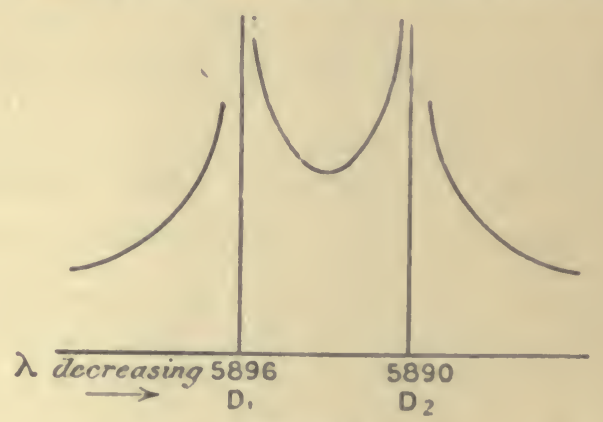

Fic : 245.

prisms to and fro along the lines of force, he succeeded in separating them on energence.

Macaluso and Corbino $\ddagger$ showed that the rotation is in the same direction on opposite sides of a sodium vapour absorption band, and Wood measured the rotation in the neighbourhood of both 1) lines, and obtained results of the kind represented in Fig. 245. But in the case of a solution of praseodidymium chloride,

* Wied. Beiblälter, ii. p. i1: (1878).

t Phil. Mag. i. p. 464 (1901). He gives in the introdnction a brief account of his earlier work, and then describes his method of sepurating the rays.

† Compt. Rend. cxxvii. p. 548; Wood, Optirs, 2ud ed. p. 530.

$\$$ Plit. Mag. [6] xiv. p. $14.5(1907)$. 
a salt with a strong absorption band in the yellow, Wood found that though the rotation had the same sign on the two sides of the

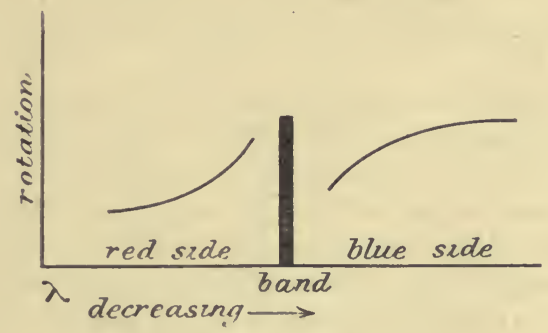

FIG. 246.

band it did not appear to rise towards the band on both sides, but rather to be as indicated in Fig. 246.*

Magnetic double refraction when the light ray is perpendicular to the lines of force. Voigt's theory. We have seen that if we resolve a vibration which is perpendicular to the field into two circular vibrations with opposite rotations in a plane also perpendicular to the field, the natural periods of the two are one a little greater and the other a little less than the natural period before the field existed. If we assume that these two periods exist for a ray travelling perpendicularly to the lines of force in which the motion of the electrons is also perpendicular to the lines of force, the equation for the velocity $v$ before the field exists, which is

$$
\frac{1}{v^{2}}=K+\frac{\beta}{N^{2}-n^{2}}
$$

must be replaced by the less simple form

$$
\begin{gathered}
\frac{1}{v_{n}^{2}}=\mathrm{K}+\frac{\beta_{1}}{\mathrm{~N}^{2}+\nu-n^{2}}+\frac{\beta_{1}}{\mathrm{~N}^{2}-\nu-n^{2}} \\
=\mathrm{K}+\frac{2 \beta_{1}}{\mathrm{~N}^{2}-u^{2}}+\frac{2 \beta_{1} \nu^{2}}{\left(\mathrm{~N}^{2}-u^{2}\right)^{3}}, \text { neglecting higher powers. }
\end{gathered}
$$

'The velocity before the field is established is also the velocity of a ray which has its vibrations along the lines of force, and which is therefore unaffected by the field. Let us put it $r_{p}=v$. If we take off the field, $\nu$ becomes $=0$ and $v_{n}=v_{p}$. 'This requires that $2 \beta_{1}=\beta$. We have then

$$
\frac{1}{v_{n}^{2}}-\frac{1}{v_{p}^{2}}=\frac{\beta \nu^{2}}{\left(\mathrm{~N}^{2}-n^{2}\right)^{3}} .
$$

It will be noted that this is a small quantity of a higher order than the corresponding difference which accounted for the rotation 
of the plane of polarisation. Further, it changes sign with $\mathrm{N}-u$; or when $n<\mathbf{N}, v_{p}>v_{n}$, and when $n$ is $>\mathbf{N}, v_{p}<v_{n}$. 'The theory of which this is a mere indication led Voigt to the result just obtained, and it was verified by Voigt and Weichert in the case of sodium vapour.*

Magnetic double refraction in colloids due to suspended particles. In 1901+ Kerr observed that if a small quantity of $\mathrm{Fe}_{3} \mathrm{O}_{4}$ is chemically precipitated in invisibly fine particles in water, which is thereby rendered slightly hazy, the liquid becomes doubly refracting when it is placed in a magnetic field and is traversed by a polarised ray perpendicularly to the field. 'The vibrations in which the electric component is along the lines of force, travel more rapidly than the vibrations in which it is at right angles. At the same time the former are more absorbed. 'This agrees with the supposition that the particles of the oxide arrange themselves in fine filaments along the lines of force, and act like a Hertz's grating of parallel wires.

Very shortly after this, Majoranat independently discovered the phenomenon in colloidal solutions of iron, and found that it was very notable in long-prepared "Fer Bravais." Cotton and Mouton $\S$ also worked at the subject, examining many colloidal solutions.

Magnetic double refraction in pure liquids. At first Cotton and Mouton supposed that the colloidal condition was necessary for mugnetic double refraction, but they discovered that it is exhibited by nitrobenzene, and it has been observed in other aromatic compounds. The phenomenon is closely parallel to the Kerr electric effect. If $\delta$ is the retardation of one ray behind the other in traversing length $l$ of a substance placed in a field $\mathbf{H}$ which is at right angles to the path, and if $\lambda$ is the wave length

$$
\frac{\delta}{\lambda}=\mathrm{ClH}^{2} \text {. }
$$

$\mathrm{C}$ is the constant of magnetic double refraction for the substance for the wave length used. Cotton and Mouton found that for nitrobenzene $\mathrm{C}$ increases as $\lambda$ diminishes, and that if $\mathrm{B}$ is the Kerr electric constant $\mathrm{C} / \mathrm{B}$ is very nearly the same for different wave lengths. 'This has been confirmed for other aromatic compounds by McComb and Skinner.\|

A theory of magnetic double refraction has been developed by Havelock, $\mathbb{T}^{*}$ who supposes that the molecules are differently spaced along and perpendicular to the field. Cotton and Mouton prefer

* Wied. Ann. 67, p. 345 (1899) ; or Wood's Optics, chap. xvii.

† Brit. Assoc. Repurt (1901), p. 568.

$\ddagger$ Science Abstracts, 1902, No. 1844.

$\$$ Ann. de Chim. ct de Phys. [8], vol. xi.p. 146 and p. 289 for their earlier work; Journal de Pliysique [5], vol. i. (1911), p. 5, for their later discovery.

il Pliys. Rev. xxix. p. 525 and p. 541 (1909).

I Proc. Koy. Sic., A, vol. Ixxvii. p 170, and vol. Ixxx. p. 28. 
to account for the effect by supposing, not that the molecules alter their distances apart, but that they are oriented with axes of some kind along the lines of force. They consider that Voigt's theory will not account for the double refraction which they have discovered, for his theory should apply to all bodies, whereas their double refraction is so far confined to a particular class.

The Kerr magnetic effect. Reflection from magnets. The reflection of light at a surface is not merely a return of the waves at an impenetrable surface, for the nature of the reflection depends on the constitution of the medium within the surface. For instance, the polarising angle differs with different reflecting media, showing that the medium near the surface takes part in the process. Considering this, and further considering that, as the Faraday effect in some salts of iron is already great, it might he expected to be vastly greater in the steel of a magnet, Dr. Kerr* was led to expect some kind of rotation in a plane-polarised ray when reflected from the pole of a magnet.

'To test his expectation he polished a flat as perfect as possible on one pole of an electro-magnet and then brought the other pole near to it, just leaving between them a chink through which a ray of plane-polarised light could be directed on to the surface at an angle of incidence between $60^{\circ}$ and $80^{\circ}$. When the plane of polarisation was either parallel to or perpendicular to the plane of incidence and the current was off, the plane was unaltered by reflection. 'These two planes were selected because, in accordance with the ordinary result of reflection from a metal, the light in any other plane would after reflection be elliptically polarised. After reflection the ray passed through a Nicol to the observer's eye. The Nicol was turned to extinction. On putting the current on, the light reappeared faintly, the plane of polarisation always turning in the direction opposite to that of the magnetising current, and the polarisation was slightly elliptical, as the principle of reversibility would lead us to expect.

In further experiments Kerr worked with normal incidence through a perforation in the opposing pole and found again a rotation, though less in value, and again in the opposite direction to the magnetising current. According to the later work of Kundt, the light entering the metal and finally absorbed by it would have its vibration turned round in the direction of the magnetising current so that we might expect the reflected vibration to be turned in the opposite direction.

Afterwards Kerr obtained similar effects with reflection at surfaces worked on the equatorial parts of a magnet. He summed up his results in the statement that a new small component was introduced in the reflected ray with vibration perpendicular to that of the ray reflected before the iron was magnetised. The vibiation

* Plil. Mag. [5], vol. iii. p. 321 (1877), for reflection from a pole; vol. v. p. 161 (1878), for reflection from the side. 
in the original direction was not changed in phatse by mannetisntion. The new component differed from it in phise by a small angle.

Du Bois* measured the rotation on reflection at normal incidence from mirrors worked on the ends of iron, nickel, and colsalt cores of electro-magnets. The cores were in the form of prolate spheroids. He used this shape because, according to the theory of induction, if without the metal core the field is uniform, the magnetisation $I$, when the metal is put in, is uniform and parallel to the axis. Measuring the rotation $\varepsilon$ and the intensity of magnetisation $I$, du Bois found that $\varepsilon=K I$, and he termed $K$ the Kerr constant. If the magnetisation was not normal to the surfuce, then I was replaced by $I_{n}$, the normal component. $K$ was found to be nearly independent of temperature, and when $\varepsilon$ was expressed in minutes it was found that $K$ was: for iron-0.0138, for cobalt -0.0198 , and for nickel -0.0160 , while for magnetite it was +0.012 . 'There was dispersion, $K$ decreasing for iron from red to violet, while for cobalt there was a minimum in the green, and for nickel a minimum in the yellow of the spectrum.

The Zeeman effect. $\dagger$ The three actions of nugnetism on light which we have already described consist in modifications of the light due to changes in the medium through which it is transmitted, or at which it is reflected when the medium is magnetised. The modifications may be ascribed to alterations of velocity and not to alterations in the frequency of the waves. 'There can be no alteration in ware frequency where there is no relative motion of source and medium and the condition is steady. Now we are to describe an effect of the magnetic condition on the light emitting elements of the source-an alteration in the frequency, not in the velocity, of the wares emitted. An alteration of this kind, sought in vain by Furaday, perhaps observed by fiever, was only certainly found by Zeeman in $1896 . \pm$

Zeenan placed a sodium flame between the poles of an electromagnet which could create a powerful field through the flame. 'The light going out perpendicularly to the lines of force or equatorially was received in a grating spectroscope, the grating having 14,938 lines to the inch and therefore giving considerable dispersion. Before the eurrent circuit was closed the D lines were fine and sharp. When the current was put on they widened perceptibly. 'The red lithium lines from a lithium flame showed a similar effect. 'To show that the effect was directly due to the magnetic field and not to any indirect effect such as a change of temperature through the change of shape of the flame, a tube with glass ends was put across the nugnetic axis in place of the flame. A piece of sodium was inserted in the tube. which was heated so that sodium vapour was formed. A beam of white light from an

* Phil. Alag. xxix. p. 253 (18:0).

† Zeeman, Researches in Magnet-Optics, Macmilla 1, 1913.

\$Phil. Mag. [3], xliii. p. $22 s$ (1897). 
arc was passed through the tube into the spectroscope and the dark D lines appeared. When the current was put on, these too widened. 'The total widening of each of the lines was about $1 / 40$ of the distance between them when the field was about 10,000. As the two lines differ in frequency by about 1 in 1000 , the two sides of each broadened band differed in frequency by about 1 in 40,000 .

Lorentz's theory. Zeeman thought that the electron theory as developed by Lorentz would give the key to his observations, and Lorentz indicated to him a theory of the effect which we may put in the following form.

The principle is that which was afterwards used by Voigt in the explanation, which we have given already, of the change of velocity of circularly polarised light with change of direction of revolution. 'The starting-point is the equivalence of a charge $e$ moving with velocity $v$ to a current element $e v$, so that if there is a magnetic field of which the component perpendicular to the direction of $v$ is $\mathbf{H}$, a force acts on $e$ equal to $\mathrm{H} e v$ and perpendicular to $\mathrm{H}$ and $v$.

If $v$ be resolved into any components and the force on $e$ due to each component be separately considered, the resultant of all these separate forces will be Hev. It is sufficient to illustrate this by the simple case where the field is perpendicular to the velocity $v$, and that velocity is resolved into $v \cos \theta$ and $v \sin \theta$ at right angles and in a plane perpendicular to $H$. The forces on $e$ moving separately with these velocities will be $\mathrm{H} e v \cos \theta, \mathrm{Hev} \sin \theta$, and these will have as resultant Hev. Hence we can resolve $v$ as we like, and the forces acting on $e$ due to the separate components will have a resultant equal to the force due to the actual motion.

Now take the case of a system in which a single electron revolves round a centre under a force towards the centre proportional to the distance. 'The orbit will be in general an ellipse, and it may be in any plane. But the motion can always be resolved into three linear simple harmonic motions along three axes $\mathrm{O} x, \mathrm{O} y$, and $\mathrm{O} z$ through the centre $\mathrm{O}$, and all three will have the same period, though they will not have the same phase, i.e. will not all pass through $O$ at the same instant, unless the actual motion is linear. Each of the linear motions may now be resolved into two equal and opposite circular motions in any plane through the linear motion, each having the same period but with radius equal to half the linear amplitude. If the period is $\mathbf{N}$ and the radius is $d$ the acceleration in each circle is $4 \pi^{2} \mathrm{~N}^{2} d$.

Let a magnetic field $\mathrm{H}$ be created in the direction of $x$. We leave the motion along $\mathrm{O} x$ linear, for it will not be affected by the field, and its frequency will rernain $\mathbf{N}$. The motions along $\mathrm{O} y, \mathrm{O} z$ may be resolved into two pairs of circular motions, and it is most convenient to put these in the plane of $y, z$, each pair consisting of two opposite motions. As we have seen, we may treat each motion separately in considering the effect of the motion in the magnetic field. Consider a circular motion 
of radius $d$ in which the electron $c$ moves round clockwise as seen from the N.S.I'. If its velocity is $v$ it will have an additional force $\mathrm{Her}$ inwards, and so its frequency will be increased from $\mathbf{N}$ to $\mathbf{N}_{1}$. 'The total force to the centre if the mass is $m$ is $4 \pi^{2} \mathrm{~N}^{2} m d+\mathrm{H} e v=4 \pi^{2} \mathrm{~N}^{2} m l+2 \pi \mathrm{N}_{1} \mathrm{Hed}$, putting $2 \pi \mathrm{N}_{2} d$ for $v$. The acceleration is $\left(4 \pi^{2} \mathrm{~N}^{2}+2 \pi \mathrm{N}_{1} \mathrm{Hc} / \mathrm{m}\right) \mathrm{d}$, and the frequency is given by

$$
\mathrm{N}_{1}^{2}=\mathrm{N}^{2}+\frac{\mathrm{N}_{1} \mathrm{H}_{e} / m}{2_{\pi}}
$$

and as the second term on the right is very small compared with the first, in it we may put $\mathbf{N}$ for $\mathrm{N}_{1}$, so that

$$
\mathrm{N}_{1}{ }^{2}=\mathrm{N}^{2}+\frac{\mathrm{NH} / \mathrm{m}}{2 \pi}
$$

A counter-clockwise motion will have an additional force outwards, and so the frequency is reduced. Its value $\mathrm{N}_{2}$ is given by

or approximately

$$
\mathrm{N}_{2}{ }^{2}=\mathrm{N}^{2}-\frac{\mathrm{N}_{2} \mathrm{H}_{c} / \mathrm{m}}{2 \pi}
$$

$$
\mathrm{N}_{2}^{2}=\mathrm{N}^{2}-\frac{\mathrm{NH}_{c} / \mathrm{m}}{2 \pi}
$$

There are therefore three frequencies, the original frequency $\mathbf{N}$ still'remaining in the vibrations along the lines of force, and there are two sets of circular motions in a plane perpendicular to the lines of force and with frequencies, one rather greater and the other rather less than $\mathbf{N}$.

Consider the light going out equatorially. The circular vibrntions will only send linear vibrntions in that direction, for it is in their own plane, and the direction of vibration in the waves will be perpendicular to the lines of force. The vibrations in the source along the lines of force will send out vibmtions parallel to that direction and of the original frequency. Hence, if we receive the light in a spectroscope, we may expect to have a line in the position of the original line polarised in the equatorial plane, or, as it is easier to picture, vilbrating parallel to the lines of force, and this should be flanked by two lines with vibrations transverse to the lines of force. If we cousider the light travelling out axially, then the vibration along the lines of force with the original frequency sends no waves in that direction, but the two opposite sets of circular vibrations send opposite circularly polarised rays, and in a spectroscope we ought to have two lines, one on each side of the original position, and the light in them should be circularly polarised in opposite directions.

Zeeman's verification. Zeeman found that experiment verified these anticipations of theory. But further research with 
stronger fields and better resolving power showed that the effect of establishment of the field was usually considerably more complicated than in the simple case which we have treated, though for some lines there is mere tripling, as described above.* For instance, the sodium line $\mathrm{D}_{1}$ shows a quadrupling in the equatorial direction, the polarisation indicating that the central line is doubled. $\mathrm{D}_{2}$ shows six lines, and the polarisation indicates that the two side lines are also doubled. Lorentz $\dagger$ showed that if there are $n$ degrees of freedom in the vibrating source, all coinciding before the establishment of the field, and so giving one line in the spectrum, we may suppose them to be made all slightly different when the field is established, thus giving $n$ lines. 'Thus in our simple case $n=3$, and each of the degrees has the same period if there is no field. When the field is put on there are three periods and three lines in place of one.

Since Zeeman made his great discovery a very great deal of work has been done by himself and by others in investigating the details of the effect, but these details belong more to optics than to magnetism. +

We shall conclude with a very interesting calculation which Zeeman made, in his first paper, of the value given for $e / m$ by the observed widening of the lines. From the values of $\mathbf{N}_{1}$ and $\mathbf{N}_{2}$ we get

$$
\begin{aligned}
\mathrm{N}_{1}{ }^{2}-\mathrm{N}_{2}{ }^{2} & =\frac{\mathrm{N}_{1}+\mathrm{N}_{2}}{2 \pi} \mathrm{H} e / m \\
\therefore \quad \mathrm{N}_{1}-\mathrm{N}_{2} & =\frac{1}{2 \pi} \mathrm{H} e / m \\
\frac{e}{m} & =\frac{2 \pi}{\mathrm{H}}\left(\mathrm{N}_{1}-\mathrm{N}_{2}\right) \\
& =\frac{2 \pi}{\mathrm{H}} \mathrm{V}\left(\frac{1}{\lambda_{1}}-\frac{1}{\lambda_{2}}\right),
\end{aligned}
$$

and

where $\lambda_{1}, \lambda_{2}$ are the wave lengths of the two side lines, and $V$ is the velocity of light,

$$
=\frac{2 \pi}{\mathrm{H}} \mathrm{V} \frac{\lambda_{2}-\lambda_{1}}{\lambda} \cdot \frac{1}{\lambda}
$$

since in the denominator $\lambda_{1}, \lambda_{2}$ may be put equal to $\lambda$, the original wave length. Now, as $\frac{\lambda_{2}-\lambda_{1}}{\lambda}$ was found to be $\frac{1}{40000}$, for $\lambda=0.000059$, when $H$ was 10,000 , and as $V$ is $3 \times 10^{10}, e / m$ is about

* Schuster's Theory of Opticis, l.c.

$\uparrow$ The Theory of Electrons (1909), 1). 112 ; or Conyrès Inlernational de Physique (1900), vol. iii. p. 1.

A bibliography is given in 'Leeman's work (see p. 336). 
107. Various later measurements of the separntion of the lines in known fields have shown that $c / m$ obtained in this way is a little different for lines in different series, but that it is the saine for lines in the same series. 'The mean value is very near to $1.77 \times 10^{7}$, which again is very near to the mean of the best measurements of $e / m$ for the kathode rays and for $\beta$ rays. 


\section{N D E X}

ALCOHOL, specific inductive capacity, 129

Ampere, hypothesis as to constitution of magnets, 201

Analogous and antilogous pyroelectric poles, 149

Armatures for magnets, 175

Artificial magnets, 165

Atmospheric electricity, 95

Axis of a magnet, 215

\section{BALLEXIDD magnets, 174}

Ballistic method of measurement of permeability, 271

Belli's machine, 17

Bennett's Doubler, 16

Biot, experiments with nickel needle, 203

Bismuth, von Ettingshausen's experiments with, 285

Boltzmann, experiments in specific inductive capacity for gases, 127

experiments in specific inductive capacity for solids, 122

investigations with crystalline sulphur, 124

Capacitr, Mersurement of, 96

of a conductor, 81

Centre of a magnet, 215

Charge, Measurement of, 30 Unit of, 28

Charged surfaces, pall per unit area, 104

Circular disc, Intensity due to, 40

Clouds and lightning conductors, 14

Cobalt and nickel, magnetisation and permeability, 276 magnets, 176

Coil as a magnet, 181

Colloids, Magnetic double refraction of, 334

Condenser, potential and capacity, 87

Conduction, 3 Electrification by, 25

Conductor, Capacity of, 81 Intensity outside surface of, 36 proof that charge on outside only, 27

Conductors, Distribution of electrification on, 26

Consequent poles, in a magnet, $17 t$
Coulomb's direct measurement of forces between electrified bodies, 62 experiments to establish inverse square law for magnetic field, 208

Curie, discovery of piezoelectricity, 153 experiments on suscepibility and permeability, 286

law for paramagnetic bodies, 291

Current, Electric, produces equal positive and negative, 26

Cylinder, Intensity due to charged, 41

Cylinders, Potential and capacity of two co-axial, 83

Potential and capacity of two parallel, 84

\section{DECLINATION, 301}

Lires of equal, 315

Diamagnetic bodies, Induced magnetisation in, 249

Diamagnetics, 203

tend to move to weaker field, 205

Dielectric, 50, 99

Alterations in, when subject to electric strain, 142

constant of ice, 131

Quincke's experiments, 138

Stresses in, 134

Dip, or inclination, 304 or inclination by dip circle, 305 or inclination by earth inductor, 308

Discharge from points, 13 of electrification, 11

Disturbances in magnetic field of earth, 313

Dolazalek electrometer, 89

Drude's experiments on specific inductive capacity with electric waves, 131

EARTH as a magnet, 169, 301 inductor, 308

Earth's lines of force, 301 magnetic field, disturbances in, 313 magnetic poles, 315

Ebonite, Inductive capacity of, 99

Electrification by conduction, 25

by friction, 1, 25

by heating, 149

by induction, 5,26

Coulomb's test of distribution of, 65 
Electrification, Discharge of, 11

Distribution on conductors, 26

Occurrence of the two kizds together, 10

on outer surface only of conductors, 8 positive and negative, 2,25

Quantity of, 24

Units of, 28, 32

Electrified bodies, action between, 2

Electric action in the medium, 48

current produces equal positive and negative, 26

deformation of crystals, 157

displacement (Marwell), 49

double refraction (Kerr effect), 144

expansion in glass, 142

expansion of liquids, 148

field, 32

intensity of field, 32

intensity in dielectric, 108

mechanical action, Law of, 2

strain and intensity, Relations between, 67

strain, 18

strain, direction of, 50

strain, measurement of, 82

strain, molecular hypotbesh of, 58

strain, variation of, with diatance, 68

waves, Drude's experiments on specific indectivo cupacity with. 131

Electrical level, or Potential, $: 2$

Electricity, Atmospheric, 95

Electro-magnetic waves, 19

Electro magnets, 178, 182

Electrometer, Dolaralek, $\$ 9$

Harris's attracted disc, 91

Piezoelectrio, 156

Quadrant, 87

Thomson's trap door, 81

Electron theory of magnetism, 297 theory of rotation of plane of polari. sation, 328

Electropborus, 14

Electroscope, gold-lea\&, \&

gold-leaf, capacity of, $0 \%$

gold-leaf, detection of change br, e

gold-leaf, use of, to indicate equality of charge, 21

Energy, dissipation of, in a hysteresis cycle, 199

distribution of, in a dielectric 104

distribution of, in an electric system, 78

of charge in a dielectric, 103

of a system, 44,76

per c.c. in magnetised borls with constant permeability, 216

supplied during a cycle when perme ability varies, 248

Equipotential surfaces, 14, is

liwing's model (molecular theory of magnetism), 195
Kiwing's measuretneds of magnetlc jer. uneability, :66

FARADAY, divcovery of specile íductive capacity, 02

experiments with cobalt as a magnet, 208

experiments with para. and dia. magnetlc substances, 282

on action of magnetism on light Jy0

Faraday's "butterify-net" experiment, 27

Verro-magnetics, 207

Field, electric, $\mathbf{3 2}$ electric, force on small body iv, 61 electric, intensity ol, 82

Fleming and Dowar's experiments on susceptibility of oxysed, 295

Fiux of lorce In magnetic fiedi, 216

Force on a small body in electric beld, B1

Forces between electrified bodies, measurement of, 62

Forces on magnetloed bodies, $285 \mathrm{et} \mathrm{my}$.

Firnklin's jar, 22

Friction, Rlectribicatlon tr, 1, 95

Yricilonal machisee, 11

Cises as para- or diamacoetlca, 20.

Speotilo ioductive capacity of, 127

Giaugain's rescarche is igroelectricity. 130

Ciause, prool of leverno squaro law (mag. netian), 212

Theorem of, $\mathrm{x}$ :

Theorem aj fillod to tubes of lorce, at

Theory of terrestrial maguetem, 816

Glasa es inswlater, 1 Electrio expunsion in, 142

Gold-leal electroscores 4

capacity of, 27

detection of change by, 6

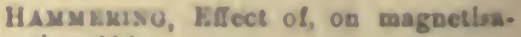
ใIon, 191

Heerwacen, on spectbe inductive cayacity of wates, 130

Hertilan waves, $\$ 9$

Hopkinson on rate of fall of potental in Leyden jer, 117

on specibc indective capacity, 12t

Hydraulic lllestration of magnedic per. meabilisy, 236

Hyatereals cycle, Disulpation ol cnergy in, 199

loop, and energy dissipated in cycle, 276

Nagnetic, ISs

Mecharical model to lllustrate, 279

ICW, Dielectric cobstant of, IS1

Induced magretion, 165

Induction and intensity in air (wag. netic), 230 
Induction by electric charge, 5,26 machines, 14

magnetic, 229

magnetisation br, $16 s, 150$

within a magnetised body, 231

Influence machines, 16

Insulation, 3

Intensity, direction and magnitude of at any point in magnetic field, 220

of field of a bar magnet, 211

of field, representation by lines of force, 37

Inverse square law, general consequences of, 31

square law of electric force, 61

square law of electric force, Cou. lomb's proof, 65

square law of electric strain, 53

square law of magnetic force, Coulomb's proof, $20 S$

square law of magnetic force, Gauss's proof, 212

square law gives force on a small charged body, 67

Investigation of field, note on, 68

Iron filings for mapping field of magnet, 177

Isogonic lines, 315

KELVTS replenisher, 18

Qosdrant electrometer, 87

theory of pyroelectricity, 150

Kerr electric offect, 144

magnetic effect, 335

Kew magnetometer, 303

Kundi'sexperiments with proelectricity, 119

IANGEVIN'8 form of Flectron Theory of Magnetism, 297

Lejden Jar, Capacity of, 96

Jar, charging, 21

Jar, discharge, 21

Jar, rate of fall of potential in, 117

Level surfaces, 75

Iight and Jagnetism, 320

Lightning conductors, 13

Lines and $t$ ubes of force, 67

and tubes of induction in air (magnetism), 231

of force as measure of intensity of ficld, 37

of force, effect of insertion of in. finitely thin metal plate, 80

of force in electric field, 32

of force in magnetic field, 177

of force in magnetic field, mapping

by iron klings, 177

of force of earth, direction of, 301

or tubes of electric strain, 55

Lippmann's theory of electric deformation of crystals, 157

Liguid dielectrics, Quincke's experiments with, 138
Liquids, as para- or diamagnetics, 204 electric expansion of, 143 magnetic double refraction in, 334

magnetic properties of (Pascal), 296

L rentz's theory of Zeeman effect, 337

M.IGNET, axis and centre of, 215

consequent poles in, 174

earth as a, 169

moment of, 215

situation of magnetisation in, 174

saturation of, 175

Magnetic double refraction, 333

field, 176

field, lines of force in, 177

fields, some special, 219

hysteresis, 183

induction, 229

intensity, 211

measurements, founded on inverse square law, 209

recording instruments, 312

permeability, 23.5

poles, 315

shell, field of, 222

shell, potential of, 222

susceptibility, 239

Magnetisation and temperature, 186, 200

and the magnetising force producing it, 181

by divided touch, 171

by double touch, 172

by single touch, 171

by induction, 180

Calculation of induced, 249

Change of Length on, 188

Distribution of, 174

Effect of hammering on, 194

Electro-magnetic, method of, 173

Induced, 168

Methods of, 170

of iron in very strong fields, 274

of iron in very weak fields, 273

situation of, in magnet, 174

Magnetised bodies, forces on, 253 ct scq. sphere, field due to, 226

Magnetism and light, 320

Electron theory of, 297

molecular hypothesis of, 192

terrestrial, 301

Magnetometer, Kew, 303

method of measurement of permeability by, 266

Magneton, 299

Magnets, ball ended, 174

compound, 174

constitution of molecular, 201

electro, 173

fields, of, 178

horseshoe, 174

natural, 165

nickel and cobalt, 176

portative force of, 175

preservation of, 175 . 
Maxwell, eleckro-magnetic theory of light, 120

on electric stresses, 138

on residual charge, 114

Measurement of charge, 30

of permeability, 266

of permeability, Ballistic method, 271

of permeability, magnetometer method, 266

of susceptibility and permeability of para- and diamagnetic substances, 282

Mechanical model to illustrate hysteresis, 279

model to illnstrate residual charge, 118

Medium surrounding magnetic body, stresses in, 261

Molecular hypothesis of electric strain, 58 hypothesis of magnetism, 192

theory and permeability, 241

Moment of a magnet, 215

Natural magnets, 165

Needle, time of vibration of, with small susceptibility, 260

Negative electrification, 2

Nickel and cobalt, magnetisation and permeability, 276

as a magnet, 203

magnets, 176

Paramagnetics, 203

Curie's law for, 291

tend to move to stronger field, 205

Pascal's experiments on magnetic quali. ties of solutions, liquids and gases, 290

Permanent magnets, 186

Permeability, 180, 184, 234

and molecular theory, 241

and temperature, 187

measurement of, 266

Piezoelectric electrometer, 156

Piezoelectricity, 148

discovery by Curie, 153

Pole, strength of magnetic, 210

unit magnetic, 210

Positive electrification, 2

Potential, 42, 72

and capacity in certain systems, 80 at any point in a magnetic field, 217 difference of, 103

Equations expressing continuity of, 236

Instruments used to measure, 87

measurement of, practical methods, 94

of a small magnet at any point in its field, 219

variation of, and intensity, 43

Proof plane, 6

Pyroelectric crystal, change of temperature on change of potential, 161

Pyroelectricity;:148
QUADRANT electrometer, 87

electrometer, calibration of, 89

electrometer, theory of, $\$ 9$

Quantity of electrification, 21

Quincke's experiments on stresses in dielectrics, 138

ReCondisg instruments for magnetic observations, 312

Refraction, laws of, 100

Residual charge, 22

charge and discinarge, 113

charge, Maxwell on, 114

charge, mechanical model, 118

lesinous or negative electrification, 2

Retentivity in magnets, 169

IRiecke's researches in proelectricity, 150

lowland's experiments on susceptibility and permeability, 284

Jubbers for electrification, 1

SolIDS, specific inductive capacity, 122 r $s e \%$.

Solutions, magnetic properties of (Pascal), 296

Sparking, 11

Specific inductive capacity, 100, 120

inductive capacity and refractive index, 120

inductive capacity, Boltrmann's experiments, 122

inductive capacity, Hopkinson's ex. periments, $12 \%$

inductive capacity of alcohol, 129

inductive cajacity of gases, 127

inductive capacity of solids, 122 of ser.

inductive capacity of watcr, 129

Sphere, capacity of, 81 potential due to clarged, 4 :

Spheres, concentric, potential and capacity of, 82

Spherical shell, intensity inside, 40 shell, intensity outside, 39

Storage of electricity, 21

Strain in dielectric, is et seg.

in dielectric, pressure transverse to lines of, 135

in dielectric, tension along lines of, 134

lines or tubes of, 55

Stresses in medium surrounding magnetic body, 261

Surface density, definition of, 52

Susceptibility, magnetic, 184, 239

TEMPERATURE, effect of, on magnetism, 186,200

effect of, on permeability, 137

effect of rise of, on Verdet's constant, 323

Tension along lines of strain, 134

Terrestrial magnetism, 300 magnetism, Gauss's theory, 316 
Thomson's trap door electrometer, 91

Townsend's experiment on susceptibility and permeability, 293

Tubes of force in electric field, 32 of force in magnetic field, 217 of force, properties of, 34-36 of strain, transference from one charge to another, 56

UxIT magnetic pole, 210

Units of electrification, 28, 32

VECTOR resolution of a small magnet, 220

Verdet's constant, 322

constant, effect of temperature on, 323

Vibration, time of, of a needle with small susceptibility, 260
Villari reversal, 190

Voigt's experiments in pyroelectricity, 153

theory of pyro- and piezoelectricity, 156

theory of the Faraday rotation, 329

Vitreous, or positive, electrification, 2

WATER, specific inductive capacity of, 129

Weber's theory of magnetisation, 194

Wills' experiments in susceptibility and permeability, 292

Wimshurst machine, 18

Winter's plate machine, 11 ring, 12

ZEEMAN effect, 336

PRINTED BY 





\section{PLEASE DO NOT REMOVE}

CARDS OR SLIPS FROM THIS POCKET

\section{UNIVERSITY OF TORONTO LIBRARY}

S\&M

A

130 
